\title{
ReF. \\ Z \\ 5856 \\ G53
}




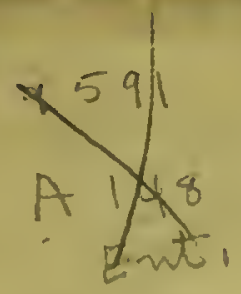

\title{
Cornell aniversity
}

\author{
LIBRARY OF THE
} DEPARTMENT OF ENTOMOLOGY Thew Dork State College of Elgriculture

SLINGERLAND COLLECTION

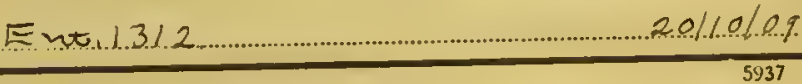

\section{Z $5856 . \mathrm{G} 53$}

Corneil University Library

Lexikon der entomologischen welt, der ca

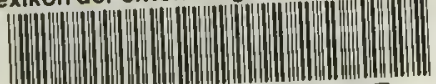

31924018833347 
M.V. Alimgeriano

1896. 
9591

A 148

Ent. 


\section{It $\mathbb{D}$ X I I I I I}

\section{DER}

\section{ENTOMOLOGISCHEN WELT,}

DER

\section{CARCINOLOGISCHEN UND ARICIINOLOGISCIIEN.}

\section{Adressenbuch}

der Iebenden Entomologen und Entomophilen etc.; der Careinologen und Arachnologen sammt ihren Schriften, dann der Naturforscher-Akademien und deren Verhandlungen, der zoologisehen Ephemeriden, Bibliographien, Biographien und Real-Wörterbücher, der offentliehen und Privat-SammJungen der Welt, der Sehriften über Sammlungs- und Aufbewahrungsweise der Gliederthiere, mit doppelten Registern und einer Aufzählung aller entomologisehen, earcinologisehen und arachnologisehen Sehriftsteller von Aristoteles an bis zur Gegenwart.

\section{Bearbcilet}

unter Mitwirkung von achtundfünfzig Gelehnten und Naturforschern

$$
\text { durch }
$$

\section{Johanmes Gistel.}

Doctor der Philosophie und der freien Künstc Magister, der gesammten Medicin Baecalaurens, resign. Professor der Naturwissenschatten und Geographie, mehrer kôniglichen ete. Auszelchaungen luhaber, ordentliclıcs, correspondireades oder fihrenuitglied der náturwissensehaftlichen, medicinischel, phrenologischen, geographlschen, historisehen, landwirthsehaftlichen, literarlseh-philosophlsehen, philomatisehen, Ermunterungs - nnd phinnthroplsehen Akademien, Instltute und Gesellschinten von Amsterdnu, Albany, Angers, Ascoli, Barcellona, Bern, Bordeaux, Boston, Boulogne, Calcultn, Cambray, Cnmbriclge, Deutselıland, Görlltz, Grenoble, Lille, London, Lyon, Montreale, Naney, New-Orleans, New-York, Northumberland, Parls, Perplgnan, Phlladclphla, Regensburg, Rotterdam, Rouen, Sulem, Tonlouse.

\section{STUTTGART.}

E. SCHWHIFRBART'SCHE VERLAGSHLANDLUNG.

1846. 
(a)

$$
\begin{aligned}
& Z 5856 \\
& G 53 \\
& 9591 \\
& \text { A } 148 \\
& \text { Ent. } \\
& \text { Ent.1312 }
\end{aligned}
$$

"Nur eins verdient entschiedenen Hass: die Gleichgültigheit; nur cins volliommene Verachlung: die Sellstsucht; nur eins stete und strenge. Verfoigung: der böse Wille! Alles Andere, cas eines Menschen Brust beucgl, oder in eines Dlenschen Geist lebt, hat gerechte Ansprïche ant Achtung, Belehrung, Sehonung und Duldung."

LUDEN. 


\section{DEN MANEN}

\section{RUDOLPII'S VON JENISON-WILWORTI,}

GRAFEN, OBERSTFORSTMEISTERS, KAMMERHERRN, RITTERS VIELER HOHEN ORDEN ETC. ETC.,

UNSERES

HOHEN, UNVISRGESSLICHEN FREUNDES UND GÖNNERS

IN

INNIGER VEREHIIUNG UND DANKBARKEIT. 
- Lingechad

Ent. 1312

Unsterblicher! den Todten beigesellt, Mit Thränen grüss ich Dich!

Du seigtest mir die rühselhafte Welt Die Liebe, - seigtest - Dich!

Und Alles, ob es längst vergangen,

Noch hält's mein Ilers mit Lust gefangen,

Und wird sur Tröstung mir, sum Weihesegen,

Wenn schon schier alle Fre und' sur Ruh sich legen.

J. Grstei. 


\section{Vorwort.}

Die Adressenbüeher der lebenden Entomologen, 1834 und 1836 zu Münehen, mittlerweile (1835) in französischer Sprache zu Paris und Luneville ersehienen, haben dem Zweeke, der ihrer Tendenz zum Grunde lag, entsprochen; daher aueh vorliegendes Lexikon wieder, wiewohl unter sehr veränderter Gestalt, hinaus in die Welt geht *. -

* Wir schmeicheln uns keineswegs, dass die bereitsersehienenen Adressenbuicher ihres Inhaltes wegeı so allgemeinen Anklang gefunten, das wäre eine Versïndigung an das Publikum, denn beile tragen das Gepräge höehster Unvollkommenheit, zumal fremde Correcturen daran während unserer Entferuung vom Diruckorte pfuschten, wodurch eine Menge personeller und statistiseher Unrichtignkeiten entstanden; die l dee wares, der man Beifall schenkte. Den sehleunigen bedeutendeu Absatz beförderteu cinige Hä ndlerseelen, welche in i!ırem merkantilisehen Walıe es fïr erspriesslich hiclteu, bedeutende Partien beider Auflagen anfzukaufen, "un ihren sogenanuten „Kundsehaften" solehe muzugänglich zu maehen. Die Welt monopolisirt iiberall und der Mikler siud leitler zu viel! - Zufrieden mit denı mangelhaften Bestreben eines uaturforsehenlen Studenten, stenerten vicle Getrene dem guteı Zwecke bei, einchend, dass man zusammenhalteu müsse, Gemeinniitzliebes 7.u gewinueu, und, was die Mülıe des Einzeluen nicht zu erreiehen seheint, unterstütze, nieht aber selbstsïehtig und voruehm krittle. - So hat such (wie man uns sehrieb) Mr. Graf vou Mannerheim, jener ruhmvoll bekannte Entomograph, in eimer Auwandlung von Hegemonie einem dïrrea Namensverzeichniss, welches wir in Jahre 1834 für unseren seligen Reisegefahrten, Hru. Giafen Rud. v. Jeuison-Wal worth, bearbeitet (,Die Insekten-Doubletten ans der Sammlung des Hru. Grafen v. J.-W. zu Regensburg etc. $\mathrm{Nr}$ I, $36 \mathrm{pp}$. 8.), cine so grossartige Autoritit in der Nomenclatur eingerïunt, dass Jixpollenz anf p. 167 et scq. Ules Bulletin des sciences de la sociète des Naturalistes de Moscou, 1837, Nir. VIII (,Eummeration des Buprestides") unserer \|еu eiugeführteu Käfersippenuane" wegen, sich moqu a nt zu ïrgern gerult, iiber tie Ankïntigmng von sieben Werken lakonisirt, endlich (p. 127: Observatians rritiques sur quelques ouvrages entouologiques) über die Entomologen von IIru. Silbermann und Gistel herfïlt. - Wenn wir das allegirte Heft des lullletin selbst gelesen haben werden, soll es an einer Antwort nieht fehleu. Wenu tem, so ist, wie man uns berichtet, so nelımen der Herr Gonvermenr (Alis poor Yorick!) einstweilen die Worte hin aus der Comödie des grossen Dichters:

Consider,

Wheu You above perceive me like a erow,

That it is place, which lessens and sets of.

Cymbeline, Act. 111, Sc. 3.

Man sagte uns, in eincm bayrischen Blatte sey ein Auszug davon enthaiten; auch diesen kenuen wir nicht. (Wir haben in München nnter dem sogen. geleluten Pöbel manehen Feind. - Einer von diesen, Ticfenbachern" besehäftigt sieh umablässig damit, unser. Wirken zu verhöhnen, sogar das, was uns gelungen, a nderu, höhern Geistern zuzuselureiben. Flibustier? wie sind wir fïr solehe Ehre Dir verbunden!) - Car la jalousie, la medisanee, la calomic les preeèdent of les arcompaguent incessamment, et èloignent les prisomes qui ont des droits viritables (Rohinenu-Des- 
Anspruchslos erwartet es anspruchslose Aufualıme. Scinen Werth whd seinen Nutzen wird der Gelrauch bestätigen, seine Mängel werdcn aus dem leztern lıervorgehen. Ueber die frühern Ausgaben ist nicht mehr nöthig' 2 su sprechen. - Die Idee hiezu, welcher das in gleichem Verlage erschienene mineralogische Lexikon von der Feder cines unserer entomologischen Schüler scinen Ursprung verdankt, ist 1822 entstanderi.

Pflichten sind heilige Dinge; ilnen nachzukommen sey uns nun gestattel. - Den IIerren, welche durch Beiträge dieses Lexikon vou Adressen bereichert, die erschienenen berichtigt und hiedureh das unscheinbare Unternehmen werthvoll befördert laben, im Namen der Wissenschaft, der Entoinographen und Entomophilen, noch eimmal öffentliche Danksagung!! Wenn uns als Redacteur dieses Büchleins das Verdienst gebülırt, die Werke eines jeden Scluriftstellers und desseu Aufsätze aus Hunderten von periodischen Sanmelwerken durch eigenhändiges Nachsclılagen allegirt zu haben, das Hauptgerïste des Büchleins verdankt der Leser, der Entomograph wie der Entomophilus, nachbenannten Lebenden und Todten: Albrecht, Herzog von Mecklenburg, Uhlanenmajor zu Berlin ( $\dagger$ ), $A$ u b é (Charles) in Montpellier, BarthéI emy, zu Marseille, Bull $\mathrm{e}$, in Halle, Boudier, in Paris, Cailla ud, zu Nantes, Cantener, zu Colmar, Campo zu Bareellona, Chevrier, Banquier zu Genf, Chevlolat, in Paris, Fléschuez, in München, Freyer, in Augsburg, Frivaldsky von Friwald, in Pesth, Fröhlich in lillwangen $\left(\frac{+}{\dagger}\right)$, Grimmer zu Gräz, Guérin-Méneville in Paris, von II eyden, Senator in Frankfurt, Hecht in Stralsund, II ering \%u Asch in Böhmen, Hope (Rev.) in London, Jenis on-Wa I w orth in Regensburg ( $\dagger$ ), IL $n$ örle in (Jos), Beamter in Linz, $\mathrm{Kr}$ a u s c, Dr. med. zu Philadelphia, Kunze in Leipzig, Landgrebe (K.), Burggraf \%u IIessenkasscl, Le fe bvre (Alex) in Paris, Maximilia n Prinz Wied zu Neuwied ( $t$ ), Macquard zu Lille, Marie tti (Bernardo) zu Mailand, Melly in Manchester, Mniszech (Graten Andreas und Georg) in Wien, Motschulsky (Vietor) in St. Petersburg, Nathusius (Herm.) zu Ilundisburg bei Magdcburg, Nendtvieh (Thom.), Apotheker zu Fünfkirchen in Ungarn, Ocskay d'Oesko, Baron und Kammerherr \%u

voidy: Recherclies sur l'organisation, Puris 1828). - Die Holılköpfigkeit

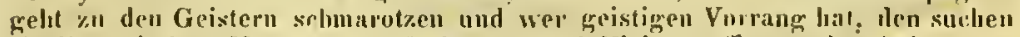
din literarischen Maroleurs inf alle Ant und Wrise, offen und greheim, um Ehre und Reputation zn bringren, wie uns zunïchst schon begeguet ist. Die Afterweisen suehen, so silurt Göthe, vou joder neueu Entdechung ur so gessclıwinle als möglich für sich einigen Vortheil zu \%iehen, inden sie einen cillen Rulum bald in Fortpflanmon, bald in Veruelirung, bald in Verbesserung, gescliwinder Besitznalme", viclleicht gar durclı P'raeocenpation zu erwerben trachten, und dureli soldie Uureifleiten die wilue Wissensehaft unsieber maehen und verwirren, ja ilıre sehönste Blüthe, die praktische Bliithe derselben, offenbar verkïmmern (Nacligelassene Werke. X. Meteore des literariselıeu Hinmels). - In der Naturwissensehaft durehaus ,ist es anf Herrsehen und Beherischen augeselien", - unsere Feinde machen uns berühmt und interessant nebenher. Wir können denselben nur Plilippiken an die Hailse jagen, uII uns, unil sey es der schmutzigste Quark, nichts schenken zu Jassen. (Vgl. unsel Lexikon der lebenden Plyytologen (Botaniker) ete, welches im Manuseripte vollendet ist und im Laufe des Jaltres erscheinen soll.) 
Oedenburg in Ungarn, Passerini (Carlo), Dr. med, etc. zu Florenz, Perty (Maxim.), Dr. med. und Rector zu Bern, Porro (Nobile Carlo di) in Mailand, Richard so n, Dr. med. in Philadelphia, Richter (Judw.) zu Liebenstein bei Eger in Böhmen, $\mathbf{R o g e r}$ in Bordeaux, Rosenhauer, Dr. med. und Conservator zu Erlangen, von Roser, Lcgationsrath zu Stuttgart, Sadler (Jos.), Dr. med. und Custos etc. zu Pesth, Sehrank (Fr. v.P. v.) zu München(t), Seidel (Wenzel Benno) Rechnungsofficial zu Prag, Silbermann (Gustave) zu Strassburg, S olier, in Mazargues bei Marseille, Spence (W. BI.) in Florenz. Spitzy (Jos.) in St. Leonhard bei Marburg in Steyermark. Thion, in Orleans, Ulrich in Wien, von Yest (Leonh.), Dr. jur. und k. k. Gubernialrath zu Grätr, Villa (Antonio und G. B.) in Mailand, de Villiers, Director zu Chartres, Vollmar, Professor in Fulda $(\nrightarrow)$.

Um deın Irrthume zu steuern, möge hier eine Liste der seit mehren Jahren (also während des Erscheinens der frühern Auflagen und etwas oder lange zuvor) verstorbenen Entomologen ihren Platz finden. Ueber Vieler Geburts- und Todesjahr wird der besondere Anhang Aufschluss ertheilen.

A hlens, Aug., Particulier zu Hettstädt (gest. 28. Nov. 1841 im 62. Jahre. - Necrolog: ent. Zeitg. III. 45). A hrens, Professor in Augsburg. Audouin, Victor, Professor der Entomologic am Musée national $2 u$ Paris. Büringer, Cantor in Gunzenhausen. Brongniart, Alex., in Paris. Boie, H., auf Java. Brigtwel, in London. Bosc, in Paris. Büttner, zu Schleck. Ba umhauer, in Aachen. Blumenb a cll, in Göttingen. Besser, W. v., in Kiew (??). Bill ardière, de la, in Paris. Block, C., Freiherr in Dresden. Bonelli, Fr. A., in Turin. Chamisso, Ad. v., in Berlin. Cllrist ofori, de, in Mailand. Fröhlich, in Elwangen. Froriep (Vater), in Weimar. Grim in, in Fbersdorf bei Lobenstein († 1835). Guilding, Landsdown, gest. 1832 auf St. Vincent. Gyllenhal, Leonh. ( berg bei Skara in Schweden). Ila hn, Dr. phil. in Nürnberg. He ine cken, Dr. med. in Madeira. If offmann, F. Kr., Naturalienhändler zu Wallerstein. He ri $n g$, Eduard, in $\Lambda$ sch (Böhmen). II awo rth, $\Lambda d r$. Hardy, in London ( + 1834). Il asselt, van, auf Java. Horner, auf Java. II u $\mathrm{mmel}$, in St. Petersburg. Is er, Carl, in Linköping Je $\mathbf{n}$ is o nWalworth, zu Regensburg ( $t)$. Kollmann, k. b. Medicinalrath (?) auf Java. Kugelan, in Osterode. Kuhl, Dr. med., auf Java. Latreille, in Paris. Leuckart, Professor zu Freiburg. Lämmle, in München. Langs lorf, in Heidelberg. Le ach, William Elford, Esq., in Genua. Michalıelles, Dr. med. in Athen. Macklot, auf Jara. Maximilian, Prinz Wied zu Neuwied. MarkIin, Gabr., zu Upsala. Meyer, k. b. Landgerichtsphysicus zu Vilsbiburg. Me Isheimer, zu Ehrenberg. Millin, in Paris. Nitzsch, in Halle. Oelmann zu Leipzig. Ort, van, auf Java. Posselt, K. Fr. in Jena. Quensel, in Stockholm. Rengger, in Aarau. Ra alten, van, auf Java. Retzi us, in Lund. Roxburgh, W., in London. Schrank, in München. Scheidler, in Wien. Schellenberg, J.R., in Winterthur. Sehmidt, Arzt und Vorstand des entom. Vereins in Stettin. Sellow, in Berlin. Schultze, Lieutenant in Stettin. Sellmann, in Linz. Sömmering, 
in Frankfurt. Say, Thomas, in Philadelphia. Sternberg, C. Graf, zu Prag. Storch, Badarzt von Gastein, zu Glanegg. Swarz, Olaus, in Stocklıolm. Studer, Samuel Lmanuel, Professor der Theologie zu Bern. Tausclier, A. M. Treitschke, in Wien (gest. 4. Juni 1842, $66 \mathrm{Jahr}$ alt). Trost, in Eichstädt. Urville, Dumont d', auf der Bahn yon Paris nach Versaille. Vollmar in Fulda. Vest, Dr. Max von, in Grïz. Van der Linden, in Brüssel. Villers, in Lyon. Vogt, in Wien. Walter, in Berlin. Warre, in London. Westerhauser, zu München. Wiegmann, Dr. med. in Berlin. Z sehorn, Schullehrer zu Halle. Zencker, in Dresden. Zinken-Sommer, in Braunschweig. Zippeli IIs, auf Java.

Nach was wir jahrelang gestrebt, was Fleiss und Lust zusammengebracht - übergeben wir hiemit der naturforschenden Lesewelt. Vieles wird darin und vielleicht Wesentliches fehlen; aber ein Wörterbuch von lebenden Menschen ist auch zll sehr unterworfen sublunarischer Wande lbarkeit! Im Rciche der Schatten wimmeln die Gestade des Flusses, welchen die Dichterphantasie der Hellenen mit dem Namen der I. ethe bezeichnete; unter der Sonne, welche uns bescheint, stehen die Ufer leer und einer geht langsam dem andern nach nach kurzem Wallen. Alle fünfzig Jahre erneut sich die Menschheit und Leben erblüht dem Tod! Während des Druckes sind schon mehre heingegangen und $L$ a ma r ck längst gestorben, Fr. Cuvier, Hardwieke u. s. w., was in der Uebersicht der Autoren mit oder ohne Sternchen bezeichnet würde.

Wie in der physischen also ist auch in der geistigen Welt eine Ebbe, eine Fluth abzusehen. - Die Productivität hat sich zwar nicht vermindert, aber der selbstständigen Werke erscheinen in neuester Zeit auffallend weniger. Den Journalen zugewendet werden die meisten Arbeiten und Entdeckungen; diese sind die reichen Vorrathskammern der Wissenschaft. Rülımlich ist solche Einigung, wie es auch höchst nützlich wäre, eine Sammlung der Unzahl von Flugschriften zu veranstalten, welche erscheinen und so selten oder nie zu erhalten sind. - Die zoologische Literatur im Allgemeinen besteht gegenwärtig grossentheils noch in Zeitungsartikeln, Inauguraldissertationen und monographischen Flugheften; aber ill dem bunten Chaos, aus dem eine grosse Zahl von anatomischen und physiologischen Schriften sich aufthürmt, offenbart sich unläugbar der Keim einer neuen Entwickelung, der von Norddeutschland und Frankreich, lauptsïchlich aber von der britischen Insel ausgeht *.

Man hat, der Curiosität halber, versucht, den musikalischen Adel und den der belletristischen Welt aufzuzïhlen, welcher lezterer 1 König, 3 IIerzoge, 2 Fürsten, 11 Lords, 84 Grafen und Vicomtes, daun noch 38 Gräfinnen zählt. - Aber die naturforsclıende Welt stelit jener keineswegs nach. Sic maclit namhaft unter ihren Schriftstellern: 2 Herzoge (S. K. H. den Herzog Paul Wilhelm von Wïrttemberg zu Mergentheim in Würtemberg; S. D. den Ierzog von Rivoli und Essliugen, Sohn des Marschall Massena in Paris; 1 Fürsten (Friedrich Gïnther,

* Wird nur erst der linmel heiter, Tausend zülilt ihr, und noch weiter.

(Göthe.) 
Erbprinz von Sehwarzburg-Rudolstadt), 1 Prinzen (Carlo Luciano B o n ap a r te, Principe di Canino e di Musignano, in Lueca), 2-4 Lords, 12 Grafen ( $r$. Corberon und Dejean in Paris, Franz $v$. Hohe nwarth zu Laybach, Georg v. Lavea u ii Moskau, Amédée Lepelletic de Sa int-Fargeau zu Saint-Germain en Laye, Carlo Maggi in Mailand, C. G. Mannerlieim zu Wiburg, A. Marmora in Turin, Marsehall in Wien, Ignaz r. Mielzinsky zu Genf, General Romand und Saporta zu Aix in der Provenee), 4. Freiherren (Maxinilian v. Chaudoir, v. Feisthammel, Franz Ockskay d'Oesko, Carl Athan. Walke naer) und 2 Nobili und Marchesen (Carlo Porro und Spinola).

Zu den nichtsehriftstellernden Naturforsehern und Naturforseherinnen gehören: 1 Kaiser (Ferdi u a ud von Oestreich), 2 Könige (speciclle Protectoren: Le opold I., König der Belgier und Friedrie h Wil helm, König von Preussen), 4 Damen (Madame Catharina Caillard und Frau Gräfin V. v. Doguero zu Paris; Demoiselle Augusta Sallé und Uranie Thiébaut de Berneaud); 1 Fürst (Alphons Gabriel $\gamma$. Poreia und Bruguera zu Triest); 1 Lord (Sir Patrik Walker, Graf zu Edinburglı); 11 Grafen (Carmagnola, Ferrari, Gujceiardini, Louis Emanuel v. Jousselin, L. v. K ue $\mathrm{nburg}$, die beiden Brüder Mniszeeh, Morawitzky, Piecolomini, Ranzau und S. Erl. Graf Willelm v. W.); 9 Freiherren (Minkwit\%zu Grunwitz, Hallberg, Caelorn, Gabriel Pronay, Stillfried, Uechtritz, Wellens, Welser, Wimmer).

Herzinnig sey Dank gesagt für die Liberalitït, womit uns $\mathrm{S}$. Hoelfürstliche Durchlaucht, Herr Fürst B u o neampagno ete. ete. auszuzeichnen geruhten. Ohne ganz freier Benützung Hochdessen Bibliothek (wohl die reichste naturhistorische Privatbïchersammluug!) wäre unser Vorsut,, dieses und alle nachfolgenden Lexika, der theriologisehen, ornithologischen, herpetologischen, ichthyologisehen, skolegologisehen und sepiologischen, der malacozoologisehen, der echinodermatologisehen (mit den Akaleplieı und den Supplementen zu diesen entom. Lexikon), und endlich der pliytozoologisehen Literatur herauszugeben, unausführbar geblieben. - Dureh die beigefügten Literatursehätze und deren speciellen Nachweis ist der frühere cphemere Zweek der Lexika zu einem literaturgesehichtlichen erlıöht worden.

Uuser Briefweehsel, weleher rein naturwissenseliaftliehen Zwecken gewidmet ist, wird wohl jührlich 1200 Nummern ausmachen. - Ein höehst nöthiges Bedürfniss für einen Naturforseher ist Correspondenz; keiner gelangt sonst auf den immer grünen Zweig, auf welchem sich die Blüthe Innd die Frucht aller Erfahrung wiegt. Zeichnen muss er können, der wahre Jünger der Natur, er muss ïben Waidwerk jagen und „Federspiel“, darf Sonneubrand und Kälte und Gefahr und lintsagung nicht scheueu. In Winter soll er lesen und arbeiten mit liopf, Aug, Hand, Feder und Griffel. Er muss ausgezogen seyn in die weite Welt und viel, reeht viel geschen liaben, auf den Firnen der Gletscher herumgewandelt, auf Mecren gesegelt und in Almenhütten übernachtet, in llöhlen den Tag erwartet haben. Durch und dureh praktisch kanı er mit Geisteshlarheit Schriften lesen und das Wahre rom Falschen 
darin unterscheiden, da ihu so Vieles unter die Hände und Augen gekommeı. Wir achten, wer er aucl sey und wie vieler Akademieı Mlitglied, wir achten keinen, weleliem nicht alles eigen ist, was einem Forscher geziemt. Aus eiıes Ofenlıokers Feder wird lebenslang nichts als Traum und Schaum tröpfeln, über welchen der Priester der Natur mit Verachtung hinweg bliekt. - Les seules expériences faites sur les clioses dont il est question, doivent servir de bases à nos raisonnements (Reaumur). - Es gibt fïr den Naturforscher nur eine Erfalı $\mathbf{E} 1 \mathrm{~g}$. Opinionum commonta delet dies : naturae judicia confirmat (Cicero de natura deorum lib. II).

Fasst der Leser deu Inlaalt dieses Bïclıleins zusammen, so ergeben sich nachstelıende Zahlenverhältuisse:

Eutomologen, Entomographen, Entomophilen der Gegenwart . . 1312

Diese wolneu an so viel Orten . . . . . . . . . : 461

Entomologische Schriftsteller (Autoren) der Vergangenheit und Gregenwart sind aufgefiilhrt . . . . . . . . . . . . . .

Davon leben noch und stehen im Lexikon sammt iliren Werken : 684

Das Lexikon macht mamhaft von Akudemien und gelehrten Gesell: schaften (mit dereı Sehriftenangaben) . . . . . . . . 429

Solche befinden sich an Orten und Städten, in Zahlen . . . . . 283

Ephemeriden (wovon rein entomologisch 12)........ . . 138

Bibliographisehe Werke . . . . . . . . . . . . . . 164

Biographien . . . . . . . . . . . . . . . . . . 130

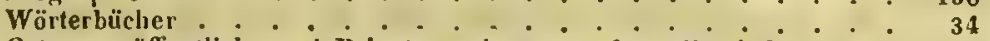

Orte, wo öffentliche und Privatsammlungen aufgestellt sind : . . 634

Syllogistisehe Schriften (über Fung, Aufbewalırung etc.) . . . . 19

Careinologen und Krabbensammler der Gegenwart . . .. . . . 317

Wolnen all so viel Ortell . . . . . . . . . . . . . . . 52

Carcinologische Selıriftsteller enthalt das Lexikon . * . * . * . 644

Davon leben und sind angefuhrt mit ihren Selariften . . . . . . 239

Aufgefïlırt sind Sammlıngen davou . . . . . . . . . . . . 50

Araehnologen cnthält das Adressenbuch . . . . . . . . . . 130

Diese wohnel an so viel Orten . . . . . . . . . . . . . 72

A rachnologische Antoren führt dos Lexikou nit vollständiger Literaturauf 328

Davon sind an Iseben und aufgezählt . . . . . . . . . . 99

In Summa Artikel . . . . . . $\frac{8147}{817}$

Rücksiclitlich der Anonymi und Pseudonymi ergeben sicl aus der. älern Literatur (Roemer, Cobres, Gronovius etc.) und den neuern literar. Handbüehern (Eiselt, Perclieron etc., sowic unsern Scripten) 2:7 Numern.

Möge dieses Lexikon auch zu einem Mittel dienen, so viele sehöne Kräfte zu verbinden, als es Namen vereint auf seinen Spalten trägt, und der Jugend und Studentenschaft, diess- und jenseits der Meere, Belehrung seyn, und zur Nacheiferung des Bestehenden ciuladen. Zum Nutzen reisender Naturforselıer, zum Endzwecke grösserer Gemeinschaft unter denselben, zu Tausch und wissenschaftliehen Verkehr, zur Uebersicht der neuern, newesten und ältesten oder besten literatur ward das Werk gesehrieben. Mö̀re es denn seinen Gang in die Welt nehmen und überall freundliehen Eingang finden und Nitzen stiften, so und so. Quod potui feci, faciaut meliora potentes. 


\section{I.}

\section{ADRESSENBUCH}

$$
\text { DER }
$$

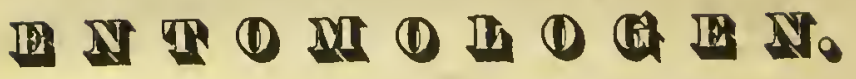




$$
\text { . }
$$


A chermann, Kerfhïndler in Carlsruhe. Sammelte sechs Jahre in Brasilien.

Agnssiz, Louis Dr. M. et Pl.., Professor der Naturgeseliehte ete. z.1 Neuenburg (Scluweiz). Sammelte die Käfer des Waatlandes. - Nomenilator zoologieus, poutineus nomina systematiea generum animalium tam viventiun yonm fussilium anctore L. Agnssiz. Snlodori (Gent et Gassmimu) 1\%43. fase. III. IV. Crustacea, Vermes, Hemiptera et Infusoria. Isis 1843, p. 622. (Die Crustacenn von Bormeister, die Wïrmer von Mieseher, die Hemipteren von Erichson und Germar, die Infusorien von Elirenberg revidirt.)

Arilstudit, Entomolog, zu Abo in Finuland (nicht Esthland, wie der frïhere Verleger beigefïgt hat).

Ainvorth, W., Chimrg und Geo$\log z u$ London. - Rescarclies in Assyria, Babylonia and Clialdaca, forming part of the lebours of the Euphrates Expedition. Loudon by Parker 1838, 8 . 343. t. 5. (Recensirt in der Isis 1841. 1. 804.)

ARvoine, Alberts, Proprietair. $Z u$ La Basséc bei Lille. Rejelıe Sammlung.

Alberti, in Lurea. Sehricb 1841 einen Catalog der Falter un Lueca.

Alerk, H. v., Oberförster zu Duninowo bei Kowal in Polen.

Altwirth, Nonosus, Consistorialrath, Deehant, Sehulbezirksaufseher und Ordensmitglied des Benedietiner-Stiftes Kremsmin̈nster (in Oberöstreich). Würdiger Entomolog.

Au Stein, J. R., Major in Malans, Canton Graubüudten.

Andereg5, W., Kerfhïudler in Gansen, bei Brieg (Canton Wallis). biefert vorzüglielı sehön erhaltene Falter aus Helvetien, I talien und Frankreich.

Auderseh, Dr, med. in Berlin. Faltersnmuler.

Andreossy, Proprictair z.ı Castelnaudary (Aude). Schöne Summlung.
Andrencecti, Dr. med., ju Venedig. Selırieb über die den Oelbänmen schädlielien Kerfe in G. Grimaldi's Rag-. gionameuti apcademici. 1833.

Andrzejowstis, zu Crènenezk (Volhyuien). Entomolog.

Angelint, Bernardino, Entomolog in Verona (nicht Wien). Sulurieb über die den Oliven sehiidlichen Kerfe.

Angelis, P. Moriz, in Stifte Admont in Obersteyermark.

Angerer, Oberlieotenant im 4. Infanterieregimente zu Regensburg. Eifrig im Sammeln der Falter.

Ansiyn, B., zil Harlen. Entomolog und Seliriftsteller. - Cutalogne des inseptes des Pays-Bas (Natorkund. Verhand, vande Mautsel. d. Vetensch, te Harlem. XVI. 1828. p. 1. 125. - Liste supplénentaire des insectes d. P. B. (1. e. XVII. p. 12.)

Antinori, Vincenzio, Director des grosslierzogl. tosean. museo di ficicie storia naturale di Firenze, zu Florenz.

Apetz, Professor in Alteuburg. Mitglird des entomologiselien Vereins zu Stettin.

Arrosto, Franeeseo, Med. Dr. in Messina. Sehriftsteller. Hauptsärlılich Botanikus.

Arvisenet, Negoziant inChâlonsurSaône. Beschăftigt sich grösstentheils mit Käfern und besizt davou eine schöne ond namhafte Sammilung.

Asmus, Herm. Mart., zu Dorpat. Monstrositates coleopterorum. Adj. tabb. X. litlogr. Dorp. Liv, 1835.8.

Aubé, Charles, zo Montpellier. Berülimter Entomolog. - Monographia Pselaphidum. - Ueber dif Metanorphose des Agrilus viridis (Annal. soc. ent. de France 1837, 7. - Isis 1839, 533). - I conngraphic et histoire naturelle des Coliopteri's d'Europe. 8. Paris 1836. Avec 46 plameh. enl. (Ist Contimution des dejean. Werkes.) - Deseription de lcux coliopteres nouveaux des genres 
Plilinmet Hister. (Annal, de la soe. ent. frane. II, 1833, 94-96). - Note sur la famille des Psèlaphiens (ibid. II. 502).

Babiugton, Carl Cardale, Esq. Prof, an Johus-Collegium in Cambridge.

Bach, Lehrer a॥ der höliern Stadtschule zu Boppard. - Einige Bemerkungen über Trypeta signata Meig. (Ent. Ztg. III. 263).

Bacher, David, Cooperator zu Glödnitz in Kärnthen (Gurkthal).

Iirix, C. F. v., Dr. Med. und Professor zu St. Petersburg. - Ueber Galleria cerenua in Permien. (Bulletin acientifique public par l'Aeademie impérialc des Sciences de St. Petersbourg. VII. 1840, p. 178.)

Imin, in Moskau.

Briubridge, William, in London.

Balmn, zu Pricur (in Cliamouny).

Balser, geheimer Medizinalrath, in Giessen.

Brmou, Pharmaceut der Marine zu Toulon. Bereiste Cayeme und besitzl cine anselanliche Sammlung.

Bnase, Gymuasial-Lehrer in Maguleburg. - Ueber die Famna HelgoIands (Kiiferverzeichu). Ent. Zig. II, 77). - (Banse und Matz) Notluus (Osphya) elavipes Meg., bipunctatus Illig. und prueustus Oliv. (cnt. Zeitg. Il, 162). - (Banse, Krasper und Matz), Beiträge zur nähern Keunluiss des Lebens und Fanges ciniger Coleopteren (Entom. Zeitg. III, 29).

Barchard, in Loudon. Ueher die Zellen der Bienen md Wespen. (Amuls of Plilos. IX, (1817) $310 .-$ $X,(1817) 428$ ).

Bnrdet de Iongmesse, in Puris. Schöne Sammlung, Käfer und Falıer.

Bnridou, J. A. F., in Beaucaire (Dep. du Gard). Besitzer einer seliö. nen Faller-Sammlung.

Bnrker-Wepp, P., in Paris. Histoire naturclle des iles Cantries (mit Sabin Berthelot). Paris et Fribourg 1830-10. (Isis 1837, ․ 246; 1839, p. 700-713.)

Bnakley, in London. Ueber Bie. menzellen (Anuals of Plil, X, (1817) 14).

Turliow, Dr, med. und Professor in Breslau.

Barsch, Patrimonialrichter in Hof (Bayeru) an der siichs. Gräızc. Summelt die Käfer seiner Gegend.

Bartels, Dr. med., Hofrath elc., iu Si. Petersburg.
Barthélemny, Conservator des Naturalien-Kabinets zu Marseille. Vorzüglicher Entonolog. - Observations sur le genre Plochionus (Annal. soc. enI. frane. III, 1834, 429).

Bartom, Benjamin Smith, Dr. med. in Philadelphia. - An luquiry into the question, whether the apis mellifiea, or Irue Houcy-Bee, is a nalive of America (Trans. of Plilud. Vol. I1I, N. 24 $1-261)$.

IBnssi, Ritter Carlo di, in Mailand. Im Besitze einer grossen Sammlung italienischer Kerfe, lıa! dieser Entonolog manelı Interessantes selion entdeekt, und in der Versammlung der italienisehen Nafurforselier (zu Florenz i. Sept. 1841) sprach er íber das Studium der fossilen Kerfe und einen Rüsselkäfer, der keinem lebenden ähnliclı ist. - - Sur Je genre Cardiomera (Carabique). (Ammales. soe, ent. frane. Ill, 1834, 429 fig.). - Notice sar une monstruosile du Rhizotrogus castanens. (Annal. soe. entomi. franc. III, 1834,373). - Description de quelques nouvelles espèces de Coléoptères del' Italic (av. 1 pl.), (ibid. 463). - Ueber die Larve von Oryctes und Scolia flavifrons. Vorlrag (1 sis 1843, p. 648.)

Bnstard, Doetor und Naturforscher in Clialon - sur - Sàovue. Schöne Sammlung.

Bnudry de Lozières, Gelelırter und Stifier der Socicty of Seiences and Arts at Capef rançois, in Ploiladelphis. - A niemoir on animal Cotton, or the Insect Fly-Carricr. (Trans, of Phil. V, 1812 , p. $150-160$ ).

Mandry de Balzac, Dr. med. und Professor der Naturgeselichte in Versailles (Rue Montbauron Nr. 18).

Bnuex, Dr. und pensionirter Reetor zu Ingolstadt. Nalurforscleer und gediegener Scliriftsteller.

Briner, Dr. und Kreisphysicus zu Friedwald.

Baumanu, J., Professor der Nafurgeschichte z.u Linzern. Bereiste Surdinien. - Naturgeschiehte für das Volk. Mit 208 Holzsehuitten. Luzern $1837,8$.

IRumhneln, A., in Gotha.

Baumbech, Kriminalrath in Meiningen. Sammirlt Käfer und Falter.

Benudet-Infarge, M. S., Mitglied der Deputirfenkanuer zu Maringe (Dept. Puy-de-Dom).

Bayrhofler, Karl Theod, Dr. 
and Professor in Leipzig. - Betrach- der k. k. landwirthschaftl. Gesellsth. tungen über Erfalırung und Theoric in der Naturwissenschaft .Leipzig 1838. 8.

Beclrer, Joscph, Naturalist in Wiesbaden. Dureh die Ravue entomologique bekannt.

Beckwitl, Juhn, in London. - The hisfory and descriptions of four speeies of Phalaena. (Linnean. Transact. $11, \mathrm{p}$. 1, л. I, Tab. ?.

Beden n, Militärchirurg in Algier. Sammelte viele Käfer in Portugal, Spanien und Afrika.

Belnu, Dr. Medicinalassessor in Stettin.

Beklemicherf, in Kerensk (Gouvernement Penza).

Bell, Th., Esq., erster Redakteur des ,Zoological Jourual" und soust Vice. präsident der "Entomologieal Society" in London.

Beneden, P. J. van, Professor z." Löwen. - Exercices znotomiques. Bruxelles, fasc. I. $1839,4.53$ tab. 4. II. 1830,55 tab. 10 et seq. (Mèm. Acad. Bruxelles XI. et seq.).

Iennett, William zu Capham in Engrland.

Tennett, E. T., Dr. med. in London. - General observations on the anatomy of the thorax in Insects and on ist fontions during flight (Zool. Journ. I, 1824. Oct. 391-397).

Benrad, Daniel, Jugendlehrer in Stullberg bei Aachen.

Benteli, Emauuel, Notar zu Bern.

Berendt, Dr. med. und Arzt in Danzig. - Vorwelilicluc Inseliten in Bernstein eingeschlossen. 1830.

Berge, F., in Stuttgart. - Schmetterlingsbuch oder allgemeine und besondere Nuturgeschichte der Schnetterlinge. Mit $1100 \mathrm{col}$. Abhild. Stuttg. (Hoffimann). 1842. 4. - Käferbuch etc. Mit 1315 colorirten Abbildungen. Stuttgart 1844. 4. Sehr nützlich.

Berulhard, W., Dr. med. in Berlin. - Repetitorium der Naturvissenseliaften oder Aliriss der Physik, Chemic, Botanik, Zoologie und Mineralogie für Studirende der Medicin. Berliu, 1843. gr. 8.

Iernheiu, Dr., Lehrer an der Gewerbeschule in Kaiserslautern nnd Vorstand des stüdtischen NaturalienKahinets daselbst.

meroldiugen, Graf, zu Bresslan (und weil. Prof. Nöss). - Mittheilung über deı Borkenkäter (Verlandl.

Wien. Neue Folge II, 1833 ).

Bertuzzi, Apotheker in Cremona. Bertè, in Puma.

Bertlioli, A mold Adolph, Dr., Professor zu Göttingen. Der Uebersetzer des Werks von Latreille "familles naturelles du règne animal“.

Bertholdy, in Odessa. Entomolog. Bertoloni, G., Professor in Bologna. - Ueber zwei schädliche Kerfe (Galeruca calmariensis nod Cossus aesculi: Nuovi Amnali delle Scienze naturali. Bologna, 1841, p. 212. - Isis 1843, 613). - Ueber den Schaden der Larve von Buprestis fabricii an Birnbäumen (Nnovi Amuali delle Se. nat. Bologna, l. p. 46).

sertolomi, Sohn, in Bologna.

Beshe, B. H., Entumolog und Naturalienhändler in Hamburg. Machte grosse Reisen in Afrika und Amerika. Schr zu empfehlen.

Bescer, V. S., Prof. der Botanik nu Kiew. Sammlung russischer, volhynischer und podolischer Kerfe. (W. $v$. Bessert). - Additamenta el olsservatiun. culae in Tentyrias et Opatra. (Extr. des nouv. Mem. de la soc. inup. des natural. de Moscou 11,4 , avec 1 pl.).

Ietta, Gebruder, in Mailand.

Bevail, Dr. med., in London. The Honey-Jee, its Nntural History, Pliysiology and Manngement, Vol. I. 12. Lond.

Bezel, v., Professor in Stockholm.

Biasoletto, Dr. med. und Apothekerin Triest. Ausgezeichneter Phytolog. Käfersanmulung.

Biedermaun, der redliche Insekteuliändler aus Domo d'Ossola, ist blind und scin Sohn besorgt nuf Rei.sen die spärlich nöhrénden Geschäfte.

Billberg, Gust. Joh., Dr. und Cameralratl zu Stockholm. Der Verfasser der Monographia Mylabrilum (1813). Wahrscheinlich schon lange todt.

Bingley, William, Fntomolor in Loudon. - Fórficula gigantea (Trransactions of the Limuean society, IX, p. 404).

Biot. Naturalist in Paris. - Sur les insectes tenus dans le vile pendant plusicurs jours (Nouv. Bullet. de la soc. plilum. 1817, 44).

isird, C. H., Smith, Rev., zu Buruficld in England.

Bischof, Theod. Ludw. Wilh., Dr. merl. und Professur zu Heidalherg.

Bisclnofr, Igualz Rudolpl, Dr., k. k. 
Ralli, Stabsfeldarzt und Professor der Pliysiologie, Ritter mnd Mitglied vieler gelehrteu Gesellschafien ete. zu Wien (Goldsilhuidgatse $\mathrm{Nr}$. 625).

mischoff, Schreiblehrer in Angsburg. Falter.Sammlung und ejuige Käfer. Angehender Hündler.

mIninville, Heiurich Ducrotay, Professor der Nutnrgeschichte 11. s. w. zu Paris. Gefeierter Zuolog. - Prodvome d'une nouvelle distribution systèmutique du règne animal (Nouv. Bull. de la soc. philom. 1816, 105). - Sur la cousordance des anneaux de Entoniozonires hexapodes adultes (ibid. 1820, Mars 33). - Sur l'organc appelé galètr, galea, dans Jes Orthoptères (ibid. Juin 85).

BInclinard, A., Entomolog in Bordenux. - Bearbeiter des entomologinchen Theils (mit Audouin) zu der meruen Ausgabe von George Cuvier's unsterblicheu Wcrk: Le Regue animal, distribue d'après son organisation, pour servir de base a l'histoile naturelle des aninuax et dintroduction a l'anatomie conpare. Edition arcompagné de planehes gravées, reprèsentant les types de tous les genres, les caractéres distinetifs des divers groupes et Jes Modificutions de strueture sur lesquelles reprose eette classification; par une Rèumiun de Disciples de Cuvier, M. M. Audouin, Deshayes, d'Orbigny, Duges, Duvernoy, Laurillard, Milne Edwards, Roulin et Valencicones. Paris (Forstin, Masson ct Cie.).

BImschke, Dr. nued. in Petersburg. Samnelte in Sithka (Aleuten).

miondeI, I. H., Architekt zu Versailles. Grosse Sammlung. - Mémoire sur une espece nouvelle de Brachelytre du genre Prognate (Amul. des sc. nat. $X, 1827,412$ ).

mlot, F., in Caen. - Propriétés des Insectes des environs de Caen (Mèn. de la soc. Linn. de Calvados 1824, 81).

Blum, W., in Wicsbaden. Lepidopteren-Sammlıug.

IIundell, in London.

Iochuiak, in Elisabethrgrad (Gouvernement Cherson). Entomolog.

Bock, Prediger in Danzig. Sammelt Kerle aus allen Ordmungen.

Bocksch, Markseheider in Wal. deuburg (Breslau).

Tohemann, Carl Henrik, Lientenant (sonst Fälınrich) im k. JönköpingsRegimente zu Stockholn. Berïhnite. Hymenopterolog. - Calodrunus, ge- wus e familia eurculíonidum (1. c. 1837, 218). - Observationer vörande nigra Insecters melamorphos (Vatensk. Handl. 1828, p. 164-176! - En ny art af Jusect-slägtet Pimpla noars larv uppeháller sig $;$ Spindelbon (Stoekl. Vatensk. Ilaudl. 1821, p. 335338, Tab.). - Försok till beskrituing of de i suerige funne Arter, hörande till lusekt slägtet Ceraphron (Vatensk. Arad. Handl. 1831, p. 322-340). Observationes in Derbe genus, una cum specierum quinque novaium descriptiowibus (1. c. 18-38, p. 223-230. - Isis, $1839,756)-D e$ Tinea linncella (ibid. p.. 231-204). - Skandinuviska Pteromaliner beskrifna af C. H. Bobemanu. (Kongl. Vatensk. Handl. 1822, p. 394. 1833, p. 329. 1835, 222.)

Toie, Friedr., Justitiarius, in Kicl. - Uebes das Aufstecken der Insekten (Ent. Zeitg. 111, 22). - Beiträge zur Gesehichte der Kerle cte. (Isis). - Zur Vervandlungsgeschichte inländischer Zweiflügler (Kröyers Zeitselır. 18381840, II, 3-6, p. 234). - Cynegetis globosa und Epilacluna, Generation (ent. Ztg. II, 79). - Verzeichniss dï̈liseher, schleswig-holsteinischer und lauenburgischer Sclimetterlinge (Kröyer naturhist. 'Tidskr. 1, 506. Isis 1841, 115). - Vortrefflichkeit des von heydensclıen Silberdralıtes (Förhandlingar ved de Skandinaviske Naturlorskeres an det Möde etc. Kjöb. 18:1, p. 300. - Isis 1843, 300). - Lupe von Bergkrystall(ibid. Isisl.e.) - Tentluredo costalis fabr. (ibid., Isis 1. e.).-Mierogaster (ibid.) - Apoplyyllus (Cymips) apterns fahr. (ibid. lsis 1. e. - A puphyllus synaspis (ibid. - ibid.). - Entomologische Beitrïge. (Kröyers naturhistorische Zeitschrift, Heft IX. 1841, p. $315-324$. Isis l. e. 893 .)

Boisduval, J. Alphons, Dr. med. ete.iu Paris. Lcpidopterolog. Im Besitze der schönsten Faltersnmmlung. - Entomologic zu Dumont d'Urvilles Reise. - Redactcur der Iconograplie des Coléptires d'Europe. - Notice sur 5 espéces nouvelles de Lepidoptères (Anual. de la soe. linn. Par. VI, 1827). Genera et index methodicus curopacorum Icpidopterorun, Parisiis 1840, 8. (Recens. ent.Ztg. I, 1840, p. 167 durel Keferstein. - Enthijlt 310 Species $\mathbf{P a}$ pilionen, 145 Sphingidem, 241 Bombyciden. 716 Noctuen und 522 Geometriden). 
methodieus, I, Parisiis 1829, 8. (3 Fr.) - Relation entomologigue de la relation dir voyage par Dumout d'Uiville. 5 livrais. in fol. (chaque 5 plancbes). Observations sur les Lepidopteres de Java (Annal. suc. "nt. fiance I, 1832, 416-420). - Anomalie du geme Urania (ibicl. II, 1832, 248). - Monographie des Zygrinides, suivie d'un tablean uretlıodique de classificution des Lépidoptères 8. av. pl. color. (14 fr.).

Uoitard, in Paris, Mamel d'Entomologic. II Vol.

Bomafous, in Turin. - Osservilyioni intorno ad aleme varictà di bachi dà setı. Torino $1825,8$.

momaprerte, Carlo Luciano, Prinripe di Canino, in Lucea, - Osservazioni relative alle larve pregindicevolialla Piaufa de Grano'lurco(Zea Mays) - Osserv. sopra alcume Larvee Tignole dell Ulivo del Dott. Carlo Passerini 1832. - Ceno ni supra le variazioni a eni vanno soggette le Farfalle del gruppo Melitea (antalog. ital. n. 125, 1831, 8.).

Eomin, W. v.: k. preuss. OberPrisident der Provinz Ponmern, Ritter efc.: der vielfach verdiente Protelstor, so win Unterstiitzer des cntomologisclien Vereines zal Stettin.

abonpland, Aime, Botaniker und Reisegrefährte Alex. v. Humboldt's in Amerika, z.ll Paris. Sammelte viel Kerfe (bescliricben von Latreille).

\section{IBomoln, in Mailand.}

Hory de Saint - Vincent, J. B. M., Minjor u. s. w. zu Paris. Beriilomter Reisender, Naturforsclier und Seluriftsteller. - Ueber die Naturalisation der Cochenille in Spanien (Amual. des se. VIII, 105. - Isis 1834, 863). - Beitrige zur Naturgeselichte der maskareniscloen Iuseln (übersetzt v. Bergk).

Butin-Desylles, in Paris. -Ueber die Lebensart einer Spannerraupe (Urapteryx sambnearia). (Aminl. soe. ent. france 1837. Isis 1839,535 .)

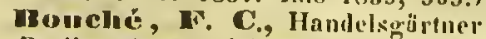
in Berlin. Ausgezeichneter Entomolog, Verfusser gediegener Werke über schäidliche Gartinkerfe u. s. w. - Die Körpertheile der rweifliiglichen Insekten (Diptera) von P. Fr: B o u e hé (Mitg. d. Ges, n. Fr. in Berlin, V, p. 36-45).

Bond, W., in London. Bekannt uus dem Entomological Magazine.

Bondier, Jeiurich Pliilipl, Pluarmazeut etc. zu Montmoreney (an der Dibtef's Lexikon.
Scine). Bedeutende Sammlungen. Bekaum durch verschiedentliclse Arbeiten und Sendungen. - Obesrvations sur divers Parasifes (Anual, de la soe, ent. de France 111, 1834, 327, av. fig. eol.). - Description d'me nonvellesspice de Lema (Mém. de la soc. Linnéene, $1 \mathrm{~V}$, 239, I pl.1.-Deseription du fenre Psam. moechus (Colcopt.), av. fig.

Bongine, l'rosector in Freiburg (Breispau); zeigte in der Versammlnng deutsch. Naturforscher Exemplare vou Mantis religiosa vor, welche um Freiburg an den Reben gefangen wurden. HBowaral , R. P., Dr. med. in Orleans. Besitzt aus allen Ordnungen die Kerfe Fraukreiclss.

Esowerlosula, Jumes, zuLoudon.Berïhnter Physiolog. - Observations on the Circulation of the Blood in insects (Walkers eutom. Maga\%. 11. 3 Avi. $1833,239-44,1$ |ll.).

woyer, Pliarmazent in Aix. - Extrait des observations sur un ver qui se tronve dans l'interieur des pepins de la pomme d'apis; av. fig. (Bullet. soc. philom, 111, 241).

Eoyer de wonscolombe, Proprietäı in Aix (Boncbes-du-Rhône). Vertasser der "Monographia Chalciditum Galloprovineiae" undandrer eutomologischen Schriften. - Ueber zwciScliaben, welche dem Olivenbam seliadeu (Aun. soc. ent. france; Livi: $1-4,1837$, p. 5. - Isis 1839, 532). Gefeiciter Forscher.

IRrsabilln, in Mailand. Ilat die Sammlung des sel. Dr. Caduliui an sich gehracht, beschrieben in der En. mmeratio Caraborum ticinensium 1830 . Meist nur europäische Käfer.

Iz rand, Fr. v. P., Dr. med. und praktiselier Arrt in Neustadt an der Aisch, in Franken. Sammler.

IBrenult, J. Fr., Dr. und Professor cte. in St. Petersburg. - Naturhistorisclie Bemerknugen über Wurzeleochenille in Vergleieh zur Mexikanischen. (Mèm. de l'Acall. de Petersb. 1835, 65, Tab. (Porplyyroplora Hamelii). Pentaminum quormolam monographico. rum insecti myriapoda chilognatha Liltrcillii spectantium prodromus. (Bull. mose. ed. leq., 1833, p. 283-297, Tab.). - Bemerkungen iber die Mund-, Magen- oder Eingeweidenerven (nervus sym pathicus seu nervos reproductorius) der Evertebraten (Mém. de I'Aend. de Petersb. 1835, I, 561-611, Tab. 1-3). - In den Bomer-Akten ist eine seliöne 
Monographie der Sippe Meloë von dicsem Forsclier und Dr. Erirhson mitgetlocilt(Pendant z.u Leach'sNIonographie in den Linnean Tuansactions).

Erants, A., Dr. med. in Rotterdam. - Automisclie Untersuchungem der Skorpionsflicge, Panorpa communis (Tidselurift fur naturl. Gesch. VI, $1839,173$. - Isis 1810, 398). - Tinca lavicella (ibid. p. 321 . Isis l. e.). Lritrag zur Kentniss der cinfaclien Angen der gegliederten Thiere (Tidscluift for natuturlyke Gesclieslenis, uitgegeben door J. van der Hoeven ell W. II. de Vriese. (Amsterd. 1836. IV Bil., p. 135. Isis 1840, p. 379. Mygale ete.). - Beiträge zur Kenntniss der Mundtheile der Hautftügler (Tidssrlurift for natururlike Geschiedenis Vlll. $1 \$ 41$, p. $71 .-1$ sis $18.13,858$ ).

Mraun, Alex., Dr. Med. und Professor in Carlsrmlie. Schuicb n̈ber den Nutzen der Naturgeschichte ete.

Brebisson, in Paris. - Sur un nouveau genre d'insecte de l'ordre des Hyménoptères (Nouv. Bullet. soc. phit. 1818, A out 116).

I3 relim, Pfarrer in Renthendorf. Schilderung eines Ansfuges nacli Lausnitz bei Eisenberg, num die von der Nonne, liparis monacha, angerichteten Verwiistuugen 7.11 selien (Jis 1s41, p. 348).

Ereuni, Jacob, Drechslermeister in Ziirich. Stumulung von Hymenopteren, Dipteren und Wanzen.

Ierignnti, Vincent, Dr. med. in Neapel. - Besebreibung und MIetamorphose der Mïcken, welche die Olive z.crstören (Atti del' real Instit. de. Ineorrag. di Napoli III, 1822,97).

Erocln, J. K., in Mïhlhansen. Correspondenee entomologique (Lucamus) 1 pl. au trait. (Deutsels und Frumz.). Mïhll.. 1823.

IE rotz, Jolıannes, Dr. pluil. zu Heidelberg. - Einleitung in die Gesclichte der Naturwissenschaft. Heidelterg 1842.8.

Browne, IR. G. H. liev., un Dulwich-Colleginn in London.

Erowue, zu Thun (Canton Bern in der Schweiz. Schöne Sammlung.

Bruck, Enil von, in Crefeld. Sammelt cifrig Käfer und lat eine schoure Sammlungr.

Brigmntelli, G., Professor der allgemrineu Naturgeschichte in Pavia.

IBrulle, Anguste, in Paris. Entomolog. - Lapartie des Insectes dans l'ouvrage de la Expedition scientifique de
Morce. Paris 1832. 4 avec $22 \mathrm{pl}$. in folio; col. - Mangel der Tarsen bei ejnigen Kerfen (Institut 1838. 15). - Mémoires sur um nouveau genre de Dip. tíre de la famille des Tipulairs (Xipluma) av. '- pl. col. (Anal. soe. ent. fr. 1. 1832. 205-9). - Sur les transformations du Cladius difformis (ibid. I. 1832. 308. av. fig. col.). - Note sur le G. Xyplutra, formes aux dépens de celui de Ctenopliora (ibid II. 1833. 398).Observat. sur la bouche des Libellulines (ibid. II. 1833. 343-51. av. 1 pl.). Sur un insecte hymcinoptere parasite et voisin du genre Alyson (ihill. II, 403). - Bearbeitung der Crustaceen und Kerfe in P. Barker-Webb, und S. Berthelot's Histoire naturelle des iles (anaries). Paris 1835-41, 4. Atlas in fol. (1sis 1842, p. 698).

Brmuer, Sanuel, pract. Arzt, in Berr. - Reise nach Senegambien und dcu Inseln des grüneu Vorgebirges im J. 1838. Beru 1840. 8.

Brumuer, Karl, Studiosusqu Bern. Der Solun des berülmmten Chemikers. Schöne Sammlung.

Briumer, Kerfhändler in Meyringen (Canton Bern).

mittuer, Franz, Chirmg in Botzen. (Flci-chgasse Nro. 112) Faltersammlung.

Buttucer, Pastor zn) Schleck (Seliooneu?)iuCurland.TïchtigerNaturfor'scher.

Bngrion, Cl. J. J., Banquier ctc. in Lausanne. Lepidopterolog. Note sur le Satyre Styx (Annal. de la sor. ent. de Franec IlI, 1834, 337). (Mit Blandel und $A$. Forel) iber einige dem Weinstock sthaidliche Kerfe im Canton Waat (Neue Denkseliriften der allgeul. Selwweizerischeu Gesellschaft fiir die gesummten Nuturwissensilaften. Neneubarg. V. 1841, [1. 4. I Taf. - Isis 1843$, p. $860-865)$.

muilue, Dr. ph. und Inspector des Naturalienkabinets z.ı Halle a. S. IEntomologischer Sehriftsteller. - Raupenkaleuder. - Vertilgung der Raupe vou Gastroparlua processimea und Fang des Hypudacus amphibius.

Bullenleciner, Conditor in Uffenloeim(Rlocinhayern). Faltersammlung.

Bulow-Hietlı, Oberforstmeister in Stettill. - Urber die Landplage in den pommer'sehen Kieferwälderu. (Gastropacha Pini.)

Bunuet, Lucien, imMarine-MInisterium zu Paris, Rne du Seine-SaintGermain Nıo. 5o. Kerflöndler. - 
Kostbare Sammlung. - Deseription de deux Insectes nouviaux du genre Oodes. (Annal, soc. ent. Fran. III., 1834, 473).

Burger, in Wien. Hymenopterolog.

Burne ister, Hermann, Dr. med. et phil., Professor der Naturgeschichte zu Halle a. S. Wisiensehaftlicher Entomo$\log$ und vie lberiilhmter Srhriftsteller. Hantbuel der Entomologie. I Bd. 1. und 2. Abtlı. 16 Stcindrucktaf. gr. 8, Berlin. 1837. (8 fl. $24 \mathrm{kr}$.) II. 1. Abthl. 2 Kupf. u. Text, 4. 1838, 1839. (25! Bogen; 4 fl. $30 \mathrm{kr}$. 2. Abtlı. (19⿺ Bogen. Neuroptera) Berlin 1838. III. Bd. (Lamellicornia.) 1842. - De insertorum systemate naturali. - Generainsectoruon. Iconibus illustravit et deseripsit $\mathrm{Her}$ manuus $\mathrm{Burme}$ ister Vol. I. Rhynclota. (c. fig. lit. Parliylis gigas) Bcrolini Burmeister et Stange. 1838, 8. (1 Heft VIIl. Pag. et 1 Bgell Text. 4 Tab.) a 1 'Tluls. oder 1 fl. $48 \mathrm{kr}$. rlı. - Zoologischer Hand-Atlas. I.-VII. (letzte) Lieferung. Imp. 4. Berlin 1838-43. - Zur Naturgeschichte der Gattıng Calandra, nebst Besehreibung einer neuen Art: Calandra Sommeri. Berlin, Burm. gr. 4. M. 1 K. 1837. (I Thlr.) - Burmeiwer und Seliaum: Kritische Revision der Lamellicornia melitophila (Germars Zeitschı. Il., Heft 2).-Grundrisa der Naturgesehichte fïr Gymna. sicn ete. 4. Auf. gr. 8. Berlin 1841.

Bus, Bernlard, Ritter du, in Brïsscl (Rue tles Petits Carmes). Coleopterolog

Caehora, Baron de, Seeretaire d'Anbassate, zu Constantinopel.

Chem merer, Hauptstant skassen. koutrolleur in Darmstadt. Faltersammlung.

Caillari, Madame Katlarina, Wittwe Salle, in Paris. "Femne intrepide" sagt Hr. Clievrolit vou ihr. War 1831 mit ihrew Solın in Mexico, wo sie Viel gesammelt von Instetcn.

Cailland, Friedr., Director des Musenus in Nantes. Herrliche SammIung. - Voyagea Méroè et au fleuve blanc.

Campa, Professor der Medicin, in Barcelona.

Carnjo, Benedetto da, KerfsammIer, in Verona.

Compos, Apotheker und Entomolor, in Sevilla.

Centener, Ludw. Prosp., Advoknt Prof. cte in Columar (Judeugasse Nr. 39).' Lepidopterologischer Schiftsteller. Catalogue des Lëpidopteres du Départe- ment du Var. Revue entom. par Sil. bermann I, 1833. 69-94.)

Cantor, Dr. med., in London. Sanmelte in Assan und Bengalen.

Carlier, Brutus, Collservator des Museums in Lütticl uud Protessor daselbst.

Carmaguola, Graf, in Mailand.

Curré, Major zu Metz. Besizt eine selur reiche Sammlung.

Cartier, in Neuenburg. Entomolog.

Carus, C. G., Dr. nied., k. sïchs. Hof- und Medieinalrath in Dresden. Lebensbedingungen kalt- und weissblutiger Thiere. Lejpzig, 1824. - Entdeckung cines einfachen vom Herzen aus besclileunigten Blutkreislaufes in den Larven netzflïgeliger Insecten. M. 3 Kupfert. gr. 4. Leipzig 1828 (3 f.).

Casteluan, Graf v. (vgl. Laporte).

Catuire de Biancourt, zu Strassburg (Grand rue Nro. 136).

Cech, Sammler in Prag.

Cérisy, Lcfebure de, Marine-Officier in Alexandrien.

Clnabrier, J., zu Montpellier. Elltomologiseher Scliriftsteller. - Analyse de la prenière partie du Mémoire sur le vol des Insirtes. Fascieule de 33 pages. - Observations sur quelques partics de la mécanique des nonvements progressifs de l'lonme et les animaux. (Nouv. Bullet. soe. philom. 1820. Avril 49). - Essais sur le vol des lusertes (Mén. du Mus, Vl. 410-476. 4 planches VIl., 297-372. 5 pl. Vill. $47-99$. 3 pl. et 349-403 1 pl. - Rénnis en un seul volume in 410, av. $13 \mathrm{pl}$. $\mathrm{Pa}$. ris 1823).

Clnaut, J., in London (New North Road 13, Critchellplace). Selıriftsteller. (Eutomogical Magazine).

Clumratiuy, Steuereinnehmer in Lyou. Reiche Sammlung vou Faltern, mit vicl Seltenen.

Chn rpentier, Toussaint de, Oberbergratlı zol Dortmund in Westplialen. Berülımter Sclıriftsteller. - Horae entomologicac. Vratislaviae. - Dic Zünsler, Wickler, Schaben und Geistelien des systemat. Verzeiclunisses der Schmortterlinne der Wiener Gcgend, vergliehen mit den in der Schiffermuiller'sehen Sammlıng in Wien befindlichen und von. J. Hïbner in scinem grossenKujferwirke abgebildeten Artendieser Gattung von T' von Clarpentier (R. P., Oberberg ratli) und mit Anmerkungen versehen von J. L. Th, F. Z i ı ken, gen。 
Somm e r. Braunselıweig. 1821, 8. Pag. | de Mègueephale. (Revue ent. I. e. 85). 16 und 178 (Germar's Mag. III. p. 401). - Libellu linae europaene, descriptae et depictue. Cun tabulis XLVIII coloratis. Imp. 4. Lips. 1840. (16 Rtlılr.). Orthoptera descripta et depicta. Fase. 1-VI. 4 maj. Lips. 1840-42. Cum tab. ill. -

Clnuudoir, Baron Maximilian von, (nicht ru Dorpat in Esthland, nicht Lieflaud), in Pertitselieft in Volhyuien. Praclitvolle Sammlung, besonders Carabiden. - Neue Carabicinen (Bulletin des Natur. de Moscou 1842, pag. 832.)

Chancenut, Ileinrich, Baron von Capitain cte., in Arras (Dept. Pas de Caliis). Befasst sieh unit Nikropteren.

Cleavanues, Jak. Aug., Director des Nuturalieneabinets in Lausanne.

Cinavannes, Adrian, Priester in Echallans (Canton. Waat). Ansehuliche Sammlung.

Chevrier, Banquier, in Genf (Rue Corratière, Nro. 27). War in Brasilieu. Nur europiiselse Coleopteren.

Clecvrolat, Anguste, in Paris (Rue de la Ferme iles-Mathurins, Nro. 33). Monographie d'un nouveau genrc dons la famitle des charansonites (Annal soc. ent. Frane. I. 1832,98-108. pl.) - Fontomologie mexique. - Mćmoire sur quelques chasses entomologiques a Fontainebleall (Annal. soc. ent. franc. II. $1833,466)$. - Description de deux nonveaux genres de coléptires de lia fumille des Mélasomes et des Diapères (Leptonycluus et Oopiestus) (Annal. soe. ent. franc. 1833). - Mémoires sur un nonveau genre de Colcopteres de la famille des Milasomes (Revue entom. de Silberm. I. 1833. 25. Fig. Avec une note sur la bouehe de cet insecte, par Guèrin). - Notice sur un nonvenn genre de la famille des Diaperides (fig.) (ibid. I. 30). - Description de l'Odoutopus cyaneus, col. teuebrion. (ibid. deser. 11. 6. av. fiy.). - Description din Nonoclanus tridentatus (fig. eol.) (ibid. n. 9). - Deseription du geure SphinJus (fig. eol.) (ibid. deser. 1.8 ). - Rectificat. du genre l. e. 235. ) - Observations sur les moeurs des Coléopteres du Mexique (Revue ent. J. 1833. 2:.7.) - Description de l'lnea irrorata (Revuc: ent. 1833). - Deseription du genre Dorlonyehus (fig. col. ibid. I. 1833 11. 14-15.) - Deseription du geure Plitynoplère, fig. col. (Revue II. 1834. it. 18.) - Description d'une nouvelle especec| 'liciui regii. 1837. 8. desCaralicyues (Revue ont. $11.1834,114$ ).

Chiaje, Antonio delle, in Ncapel. - Mèmoires sur les animaux sans vertebres du royaume de Naples. Naples 1823-25. 2 vol. 4. (ital.)

Chiaje, Stepliano delle, in Neapel. Memorie sugli animali senza vertebre del regno die Napoli. 4. Bis 1829 sind 4 Binde mit 75 Tafeln ersehienen. (,Ouvrage capitale, ouvrage d'une rare conscienee, enrichi de figures detailles." Cuvier in Fèrussae's Bullet. sc. nat. 1831. p. 265). - Sunto anatomia degli animali senza vertebre. Napoli, 1824, 8. (meist Mollusken). - Istituzioni di Anatomia comparata. Ed. II. Nipoli, 1836, 8. II Bde. in 4.t. 64. (lsis 1843, 681). - Istiturioni di Anatomia e fisiologia comparata. Napoli 1832 . I. 8. 428. 1836 ist dic zweite Auflage ersehienen. In Ganzen 4 Bände.

Chiesn, de, in Pisa. Entomolog. Selir gefülliz.

Children, John George, soust Prïsident der entomological Socicty, Mitredacteur des Zoological-Jourual ete., in London.

CInoisy, Professor in Genf (Rue de belles filles, 39 ).

Clocisy, Gresandtschaftsagent in Genf. (Alice du Lion d'or, 48).

Christy, William, Jun., Esq., in London. - Remarks on a species of Calandra, oceurring in the Stones of Tamarinds. (Trans. of. ent. Soc. of London, Part. I. 1. 1836).

Clark, Braey, Dr. med., Veterinair. nrzt, Professor etc., in Loulon. - Observations on the Genus Oestrus. (Linnean Transaet. ilI. 289. tab. 23. - Philos. Magaz. and Annals of Philos. apr. 1828. 283.) Uebersetzt in Tilesius's Jalubueli). - Dissertation on the Root of the Horse, Part. 2. Loudon, 1812, 4.

Coldstrean, Dr. med., in Loidon. - Article: Inminousness Animals in the Cyelopacdia of Anatomy and Plyysiology.

Collins, Zachïus, Viceprïsident der Sorivtas listoriae naturulis in Philade:lulia.

Colsmanin, Professor in Kopenharren.

Comolli, Antonio, Dr. med. in Mailand. - De Coleopteris novis acrarioribus minusve cugnitis l'rovinciae novocomi 
Coupngno, Ludovieo, Dr. med. Atti della r. Aecademia di Napoli, 1837, und Eigenthïmer des Srhwitzbndes zu Perpignan. Kerfsammlung. Zoolog. Durchforscht seit vieleu Juhren dip Pyrentien mit unsäglichem Fleisse und grossem Erfolge, lund es ist schr zu wünsclien, dass er sich entschliessen möclste, dariiber etwas in Druck crscheinen \%u lassen.

Coute, I. Achille, Professor der Naturgesehiehte an der Akademic z.l Paris, Chef des Bureau's der gelehrten Gesellschnften und der medizinischen Augelegeuleiten im Ministerium des öfentlichıcn Unterrichts. - Règne auimal dispose en tableaux methodiques. Paris (9t Tableatux, comprenint environ eing mille figures: 113 (i. 75 c.)

Coutariui, Nicolo, Graf von, in Venedig. Sehöne Sammlung. Schrieb ïber den Macronyehus. - Memoria sopra la Ceridomya Woeldiikii. Yenezia 1840. fig. (Nuovi Annili delle Scicuce naturali. Bologna pr. Marsighi V. 1841.8. p. 316 ete.).

Corberou, Graf de, in Dresden(?) - Les hylophlthires et leurs enmemis par Riatyeburg. Trudnit de l'allemand par C. de Corberon. Nordli.et Leip\%. 1842.

Corala, A. J., Custos am Nationalmuseum in Prag. Wissenschaftlicher Naturforscher.

Comnelius, Lehrer an der Realschule zu Elberfeld.

Coronini von Cronberg, Michael Graf; k. k. Kämmerer und Her'schaftsBesitzer in Jaybarlı.

Corti, B., in Florenz (?). Citirt von P'nsserini.

Costa, Gabricl, Dr. med. und Kerfbïndle in Neapel (Strada del peregrino). Widmet sich der reinen Zoologie und pflegt diese Wissenschatt mit Ruhm, besonders die Eutomologic, ohne jedoch das Gimze zu vermaellifissigen. Dieser unermüdtiche Naturforscher lat fost alle lrovinzen des Reichs durehwandẹrt; die Terra d'Otwato, die Berge, worauf der Vesuv raucht, der Aspromonte, das südliche Sieilicn und die Umgebungen der Hauptstadt haben ilın geduldig und still suclien selien den Engerling, der unter dem Rasen unbenerkt kriecht, den Selimetterling, der über Blumer flattert, und andere Kerfe, welehe auf den B:ïmen uisteu, oder deu nützlichen Oclbaum bescläidigen. - Degli Insetti movi e. rari della Provineia di Terru d'Otuanto (in IV.) - Fauna vesuviana ossia descrizione degli Insetti ele vivono ne fumajuoli del Cratere del Vesuvio. (Ibil. t836.) - Fanna di Aspromonte e sue adjacence. Ibid. - Fauna siciliana 1839. fase. 1. - Degli Insetti noeivi al Olivo, in Correspondenza zoologiea 1839. Fauna del Pegno di Napoli. Fasc. I. XXX. - Lezioni di Zoologia. Napoli 1838. - Ueber den Zustand der Naturwissenschaften im Königreieh Neapel am Ende des Jalıres 1840. (Isis 1843 , p. 651). - Specie nuove di Lepilopteri del regno di Napoli. 1832. Ueber die Insckten der Oliven (Atti del Instituto dineorragg. di Napoli IV. 1828. 202).

Coulon, Louisde, Director des Muscums in Neuenburg (au Faubourg pres Hospital). Zoolog.

Courtillier, in Saumur (Maineet Loire). Entomolog.

Cramer, Stadtrichter in Stettin. Crepu, Conservator des Museums der Naturgeselichte zu Grenoble.

Curtis, John, \%.ı London. Berühnnter Entomolog. - British Entomology: London 1823-1840. XVI. Vol. 8. With. 770 ill plates. - A guide to an arrangement of britislı Insects. London 1829. 8. - On two Speeies of the genus Elaphrus, lately discovered in Scottland by C. Lyell. (Wilker : the entom Magnz. 11. 1. Sept. 1832. 37.) - Cliaracteres of some undeseribed genera and spe. cies indicated in the guide to an arrangement of british inseets. (Walk. ent. mag. n. 2. Jan. 1833, 186-19!).)

Cuvier, Frederie, Dr. med., Professor rte. in Paris. Hoehberülmtev Zoolom Anatom.

Czilauk, J. voll, Dr. med., Professor und Oberstabsarzt in Jassy (Moldan). - Zustand der Naturwissenscliaften in dem Fürstentluum Moldau (Isis 1839, p. 381; vorgetıugen in der Freiburger Versammlung.). Naturgeseliclite u. s. w.

Czeknmovnky, L., in Kiew. Catalogue des Papillons de Volliynie et de Podolie de la collection du Lyece de Volhynie. (Bullet. Mose. ed. Leq. V. 1833. 1. 187-93.)

Dagonet, Dr. med., in Châlonssur Marne. Sammelt die dortigen Kerfe.

Dslillow, Anders Gustav, Di. med. Piofessor und Amanuens 
bein Naturalienmuseum in Lund (geb. 3. Mai 1806.) - Synopsis Hymenopterologiate Scandiuavirae. Skandinaviska Steklarnes Natur-Historia. Med. figurer, malade efter Naturen och ritade pit Sten af l. All lgren. Andra Krethen: Rof-Sterklar och likar. I. sta Häftet. Lund 1839-1841. gr. 4. cum tabul. - Clavis novi hymenopterorum systematis, anatonia exterua, metnmorpliosi moribusque horum animalium siuml consj. deratis; adjecta synopsi larvarum ejusdem o rdinisScandinavica rum eruciformium. Lundac. 183R. 4.40.1 tab. col.-Monographia Pompiliorum sueciae. Lugduni Goth. 1829. 8. - Monograplia Chrysidum sueciae. Lond. Goth. 1829. 8. - Exercitationes hyinenopteroloricae. Part. 1-6. 1831 - 33. 8. - Bombi Scandinaviac. Lond. Goth. 1832. 8. Prodronus liymenopterologiae Seaudinaviae. Lond. Gotlı. 1836. - Jakttagelser, öfver Scandinaviens Fjärillar. Lond. 1837. 8. - Dc crabronibus Scandinaviris. Lond. G. 1839. 44. - Kort underrättelsc on Scandinaviska Inscetas, allınäınare skada och nytta husherllingen. Lund, 1837. - Beriehtigungen iu der Dispositio meth. lyyme nopt. (Ent. Ztg. III. 214.) - Srandinaviska sterklanes naturhistoria. Lund. 183940. - Chelonus (Isis 1834. p. 779.) (Veten skaps nya Handlingar; Stocklılm) Faunns, Bd. I (neue Folge) 1837). Försïk till beskrifning öfver Hymcnopte 1 slägtet $\mathrm{Ch}$ elonus, med deıtill lıörande Serndinaviska arter (VatenskJlandl. 1832. p. 147). - Dispositio me. thodiea specierum seandinavicarum ad familias Hymenopterorum naturales pertinentium. Part. I. Sphex in sensu Linnaeano. Lundac 1842. 8. e. 8 tabcll. 4. ef uno tab. in 12. - Onyclia och Collaspidia, tvenne för Skandinavikers fauna nya Insect-Slägten, hōrande till Galläple-Steklarnes naturliga grupp. MLonograplisk bearbetıing, at följd af 2: ne planelier eolor. ocli 2: ne synop. tiska tabeller in 4. Lund. 1842. 8. Anınärkningen om Häggmalet (Tillea padella Liiı.); sant en ny art of Lepi. dopters slägtet $\mathbf{Y}_{p}$ solopluus af Samme. (Kongl. Vatensk. Handlinger 1835. q. 30 et 205. Isis 1838 . p. 5 et 20). Clavis novi Hymenopterorum systcmatis adjecta synopsi larvarum cjusilem ordinis Scandinavicarum eruciformium. Lundac, 1835. 4. 40 Pag. cum tr.bula.
Dale, J. C., in Londou, Zoolog. - Observations on the influence of Locality, time of appearance on species and varietirs of Butterflies (Walker ent. Magaz. n. 4. Jul. 1833).

Dallinger, Ignaz, Theol. Dr., in Würzburg.

Damua, Justizcommissarius, in Magdeburg.

Damucil, F., z1t Salzwedeln (Magleburg).

Duanuert, in Abo (Finnland). Entomolog.

Daniel, Advokat, Bürgermeister undStadtricliter, zuRelına inMcklenburg:

Dargelas, Raymond, Professor ler Botanik, der Entomologie etc., il Bordeaux.

Davis, A. H., Es(., in Londou (Nelson-S(juare). - Observations ou Lueanus Cervus (Walker ent. Magaz. n. t. Sept. 1832. 86.)

Debrunt, Niclas Auguste, Ciseleur in Paris. Fleissiger Sanmler.

Debruyn, Otto, Dr. der Medizin, in Müuchen (Miillerstr. No. 23. p. t.) Ausgrzeiplinete Sammlung von Naturalien aller Classen und Reiche.

Defrance, in Paris. - Note sur la Puce irritante (Aunal. des sc. wat. I. 440. Avr. 1824.)

De:Lnun, W., Dr. philos., Conservator des $k$. Miserumis der Niederlande zu Leyden. - Meimoires sur Ics Metamorploses des Coléoptères I. Livr. (av. 1e Planch.) Paris 1836. gr. 4.

Dejenu, Graf von, Pairvon Irankreich, Generallieutenant der k. Armec etc. efc. in Paris (Rue de l'Université, Nro. 17.) - Catalogue de la Collection des Coleopteres. 3 edlit. Paris 1838. Sprries general des Coléoptères de sa collection. 6 vol. Paris 1825, 26, 28, 29, 31,3 3. 8. (58 fres.). llat seine Sammlung - verkauft - familienweise!! (meist an Spinola.) - (Dejeall et Boisduv a l) Iconograplie et histoire naturelle des Coleopteres d'Europe. Paris 182934. Tom. I-IV. 8. avce. 223 platieh. col. (130 livr. environ-12 vol.) - Continue par Dr. Ch. Aubè. Tom. V. Paris 1836. (IIydrocantlares) avec. 41 pl. cnl. - Extrait d'un mémoire lu h la Sociéte philonatioue sur la famille des Carabiques simplicipides (Nouv. Bullet. soc. phil. Décemb. 1825. 187. Mémoir. de la soc. linn. de Normandic. 1826-27. 123.)

DeInuontngue, Alcxandcr, 
Plaamazeut in Paris. Pariscr-KäfcrSammilung.

Delessert, in Paris. Sammeltc auf dem Plateau des Nielgherries-Gebirge etc. in Ostindien.

Delta, in Loulon. Schrifucr. Notc on the habits of insects (Entomologin al Magazinc by Walker. no. 4. Jul. 1833. 385 et 439.) - Thoughts on the geographical distribution of Insects (Walker ent. Magaz. n. 6. $1834.44-54$. $280-86$.)

Demel, Joh., Associe der llandlung Opell und Dcmel in Prag. Schönc Fal. tersammlung.

Demny, H., in Nordwiclı. - Monograplia Pselaphidorum et Scydmacnidarum Brittaniae. Norwich, $1825,8$.

Desberacer, A. F. A., Dr. med. in Gotha. Gab Bechstcins schädlichc Forstinsecten wieder neu heraus.

Descourtilz, Theodor, in Paris. - Description de quelques Insectes nouvcaux décollverts en France en 1825. (Mèm. Soc. Linn. de Paris. V. 1827. 156. av. I pl.) - Mémoirc sur un noulveau genre d'Insectc de l'Ordre des Hemiptercs (ibid. 111. 1824. 293. av. fig.).

Deshuyes, G. P., Dr. med. in Paris(avec Mil nc-Edwards). Histoire naturelle des animaux sans vertebrcs, reprèsentan! les caractères gendraux et particuliers des ces animaux, leur distribution, leurs classcs, leurs familles, leurs genics, et la citation des principales especes qui s'y rapportcot, par J. B. P. dc L amarek. 2. Edition revue et augmentée des notes présentaut les faits nouveaux etc. ctc. 3 vol. grand in 8. (a deux colonnes). Brnxelles, 1837. (50 frs.).

Desinarest, Gä̈tan A., dcr Vater, Professor an der Veterinäschulc zu Alfort ctc. \%u Paris (Rue Saint-Jacques Nro. 161). Bedcutendc Sammlung. Viel Eifer \%.11 Entomologic bat dessen Solu,. - Notes sur les Larves de denx insectes oléoptèrcs (Scolytus limbatus ct Cicin dela campestris.) uv. fig. (Bullet. de la Soc. philom. IIl. 297. II. 86), - Memoirc sul une espece dinsecte des envirous de Paris dont le male ct la femellc ont servi de type à deux genres differents (Drilus) A. 'T. 1. Paris 1824,

Desunonlins, in Paris. Berïlumter Anatom und Schiriftsteller.

Desvoily, Robiucall, Dr. med. in Paris. - Les Dipteres du Canton SuintSaveur (Dcpt. de lYonne). In den Sans, |itrangers. 11. 1830. - Recherches sur l'Organisation vertébrale des crustaces cte.

Diekhofr, Apotheker, Rendant des entom. Vereins in Stettin. Sammelt rifrig Kiffer. - Ueber das Leucliten der Lampyris.Arten (Ent, Zeitg. Ill. 117).

Dierbacle, J. Heinrich, Dr. med. il Berliu (?). - Uehersicht der gebräuchlichsten Arzueimittel des Alterthums, mit besonderer Rücksicht aut die Werke des Dioscorides und Plinius. Ein plıa'makologischer Versuch (lsis 1842. P. 101 et seq.).

Dicesing, M., in Wicn. Bekannt als Scluriftsteller über dic wirbellosin Thiere (Annalen des Wicncr Museums ll.)

mietrich, Kerfsaumler in Prag.

Dill wyy, in Londou. Verfasser des Kertkatalogs von Nepaul. - Catalogue of the Coleopterous lnseets of Swanseit.

Distleri, Steffano, Professorzu Turin. - Obscrvationes variac cntomologicac. (Men. Turin. 1809. 1. 00-95; By'1hus, Dermestes.) - Vespac gallienc histnria.Auctore (Lecta, 15. Nov. 1813). (Mmm. dell' Acad. de 'Torino. 1813. Vol. 22. p. 1-20.) - Fasciculus observationnu entonologicarum. Pars prima. Lepidop: tcrorum observationcs. (Mem. de Turin. 1804-5. p. 147-166. p. alt. Hymen. obs. 1. $166-202$.)

D\&bner, Eduard, Professor der $\mathrm{Na}$ turgeschichte an der $k$. Forstschule in Aschaffcuburg. Käfer- und Faltersammlung. - Ueber das Oclig- und Speckigverden der Insceten (zucrst in der lsis, danı umgearbeitet in Germar's Zcitschrift IV.) - Naturhistorischer Atlas zum Scluugehraucl, nit besonderer $\mathrm{Be}$ ziehung auf Dr. Fiïnrohr's Grundzïge der Naturgeschichte bearbeitit und mit erläut. Texte versehen. Augsb. 1840. Qucifol. I1. 15 Tafeln.

Dbring, Reinhold, Dr. phil. z.n Brieg iu Schlesicn. - Erster und zweiter Bcricht des schlesischen TauschVereincs für Schmetterlinge. Briç $18+0$ und $41 . \mathrm{mr} .4$.

Dognero, Frau Gräfin V. vou, in Paris, - Bcsitzerin einer cleganten Collection von wirbellosen Thicren, wclche sie mejst selbst fïngt und lixedurch ein Andenken an die hörlistsel. Königin Katharina Pawlowna von Wïrttcmberg crneut.

Dohrn, C. A., Renticr und Literat in Stettin. Vorstandsmitglied des cntomologischen Vereins daselbst. 
Domergué de Saint-Florent, Proprietair in Vandoeuvre bei Nancy. Anseluuliche Falter. und Käfersammlung.

Dou, Conservator der Sammlung Linne's und Banks in Londou.

Donzel, Ingo, in Lyon. Prächtige Faltersammlung.

Dorner, Jos. von, Apotheker in Pressburg. - Das Banat in topographiscli-naturhistorisclier Bezichung, mit besonderer Berï cksiehtigun der Herculesbider nächst Meliadia und ilıen Umgebungen ete. Presshurg 1843. gr. 12.

Doubledry, Elwaril, zu Epping in England. War Archivar der cutomological Society in Loudon. - Abstract of M. Strauss. - Durkheim, Considèrations génèrales sur l'auntomie des animauxarticules (Walkerentom.Magaz. 1832, 6-12. 1833, 277-83, 466. Cont. 1. 2. נ1. 7. Jan. 1834, 1-144) - (and Newmann) An entomological excursion. (ibid. n. 1. Sept. 1832,50-60.) - Bearheitete mit Adam White die Insekten zu dem Werke: the Zoologic of the voyage of H. M. S. S. Erebus and Terror: (Vgl. White.)

Donmerk, A. J. L., Dr, med. iu Paris. Bearbeitet die gesammte Entomologie. - Sur le Psalidomya fucicola; nouvelle espèce de Diptères (Aunal. soc. ent. firance. II. 1833, 8993. av. fig.). - Notice sur quelques Monstruosites entomolog. (ilid. III. 1834. 171. fig.).

Downes, H., am Schiff- und Militärmuseum zu Whitehall (Eurland.)

Doycre, Prnfessor der Naturgeschichte an college royale de Heury IV. in Paris. Mitarbeiter an dem nencsten Dictionaire universelle d'listoire naturelle.

Drupicz, Professor der Chemie und Naturgescliclite und Director des Muscums in Brïssel. Ausgezeiclnct als Meusch und Naturforselıer. - Description de 6 especes d'Inscetes nonveaux (Annal. geı, des sciene. phys. IV. 1820. 314. av. fig.). - Descriptiou de espéces d'Insectes nouveaux (de tous orlies. 88 Species.) (Amual. des se. ply ysiq. 1VIII.). - Deseription d'un nouveau gente d'Tusecte de la tribu des Tetrameres (Aumal. des sce pliys. III.p. 181. pl, 39).

Dresé, Naturalist in Hamhurg. Vorkauft selu billig javanisclie und capisclie Coleopleien.

Drefer, Reficrungstefercudarius in Stetiil.
Drewsen, Christ, in Kiel. Sclıricb ïberHymenopicren(Wiermanns Arthiv). - Verzeichuiss der dänisclıeı Arteı der Gattungen Bombus und Psithyros (mit J. Schiödte : Kroyers naturhist. Zeitsclurift 1838, II. 2. Isis 1841, 326.). Bcobachtmong, dass die Larve von $\mathrm{Xy}$ loplagus ater nicht hulzfressend sey, sonteru sich vou Raub mitlue. (Kröyces Tidskrift IV. 1842. III.) - Beolachtung vou Parungeu vou 7 Iclueumonenarten (ibid. und Isis 1843. 64).

Drilupelumanu, in Riga (Liefland).

Drury, Studiosus in Müuchen. Sammiler.

Drzevicki, Dr., k. russ. Hofratls iu Warschau.

Diiluce, Magnus vou, in Lund. De fabrica corporis insectorum Diss.

Dubuisom, Conservator des Natu. valiencabinets in Nantes.

Darour, Lèon, Medicus zu Saint-Séver (Dept. des Landes). IIochberïhuter Naturforselier. - Observatious sur quelques Cicindeles et Carabiques observis en Espagne. (Annal. genèr. des Sc. physiq. Bruxell. T. 6. 1820 , p. 318.) - Description et figure de trois hémiptìres europiens nouveaux, ou mal comus; le G. Cépaloetice, l'rostema, Leptopus (Anbal. Soc. ent. franc. III. 1834,341. pl.). - Recherdies anatomiques et physiologicjues sur les Hemiptères, accompagnees de considerations relati. ves a llhistoire maturelle ef à la classification de res insectes. (Mémoir. des savants étrangers à l'Acad. des Se. Par. 1833, 4. av. 19 planclu.). - Mémoire sur les gemres Xylocoris, Leptotus et Vclia (Ámal. Soc. ellt. fr. II. 1833, 104-118 fig.). - Ueher die Carabicinen und andere Käfer (contin.) (Annal. d. Scienc. VIII. (1826) 5. t. 19 et Isis 1834, 868. Anat. pluys.) - VerdauungsApparat von Auobium striatum (Amal. des Se. nat. XIV (1828.) 219. t. 19. A. - Isis 1834. 1025). - Anatomisclie Untersuchungen ïber die Forficolarien. (Annal, des Scienc. nat. XIII. 337. t. 19-22. - Inis 1834, 1021.) - Risumé des Recherches sur les Hèmiptères. (Anmal. des Se, nat. 2. série I. 232.) - Bcscheribung uml Abbildung einer newen Galfumg Oruitlomyia. (Aunal, des Sricuc. 11al. 253. t. II. f. 1. - I sis 1834, 920.) - Metimorphoses des Mordelles. (Amual, des Seicoe. Matur(II. 1840. T. XIV. 238). (Nennt unjasseud das leceptaculom 
seminis: glande sẻbifique. Abbildnn-| gen sind aus ältern entomol. Schriften copirt. Siebold.) - Beitrag zur Geschichte der Sippe Ocyptera. (Amal. des Se. nat. $X .248$. T. II. f. 2. 3.) Isis 1834, 20. - Ueber Trigulinus: neue Sippe der Schmarot\%er-Insecten. (Amnul. dles Srienc. natur. XIII. (1828) 62. t. 9. - Isis 1830, 204, 1832. VII, T. 14.). - Mémoire anatomique sur une nouvelle espece d'Insecte du genre Brachine (1 pl.) (Annal. du Mussinn d'hist. 11at. Vol. 18, 1811, 70. - Bulletill soe. plitom. n. 58. 5. année. T. 3. 201.) - Recherches anatomiques sirr les Scolies (Nouv. Bullet. de la soc. phil. 1818. 101. - Journ. de pliys.. Sept. 1818.). - Deseriptions des dix especes nonvelles ou peu connues d'Jusectes reeoueillis en Espagne (Aumal. génér. de Se. plyysiq. Brux. T. 6. 1820, 307. 1 pl.). - Anatomie de la Ranatra limeiente ef de la Nepa cendréc (av. 3 pl. lith. (Amnal. gen. de Se. pliysiq. Vol. VII. 194. pl. 105. 106-t07.) Deseription de six especes d'lusectes nouvelles (Coleoptères). (Ammal, des Se. physiq. Vol. 8. 358. pl. 130.) - Description d'une nouvelle espice de Coceus. (Extrait des Amual. des Sc, natur. Tom. 2. 203. Juin 1824.). - Piecherches anatomic|ues sur Ies Carabiques el su1 plusicurs autres Colécoptères, avec fig. (Annal. des Sc, nat. Il. 1824, 462. III. 215,476 . IV. 103. V. 265. VI. 150. 427. VIII. 5.). - Recherehes anatomiques sur les Cigales (Ammal. des Se. nat. V. 155. - Recherches anatomiques sur l'Hipoobosque de ehevanx (Annal. des Se. 1nit. VI. 299). - Description de quelpues Insectes dipteres, av. fir. (I. c. XXX. 1833, 209-221.). - Recherehes anatomiones et consider. ent. sur quelques Jusectes coléptères cle. (I. e. ser. J. p. 52-84).

Duares, Ant., in Paris. - Recherches sur les caractires zoologiques du genre Pulex et sur la multiplicité des Insectes qu'il renferme. (Amual des Se. nat. XXVIJ. 1832. 145-164. av. plancli.

Dejardin, Dr. med. mil Mitglied der Societe philomatique in Paris.

Dunérill, Andıe Marie Constant, Professor der. Pliysiologie, Alialemilier, Administrator an Musenum der Naturgeschirhte etc., zu Paris. Der Antor der "Zoologie analytique" (Paris. 8. 1806) (deuts(h v. Foríp)! - Cisnsidérations gemerales sur la Classe des in-l sectes. Paris (Lévrault), 1823. 8. 272. 60 planel. noir ou color: - Elements des seienees naturelles; oùvrage preserit par arrête des Stotuts de l'universite, pour l'enseignement, dans Iss eollegres. Nouv. edis. avec. 33 planches (représentant plus de 700 sujets diver's) Paris. 1 Vol. grand in 8. (Prix 12 Fr.). - Dissertation sur l'organc de l'odorat, it sur soul existence dans Jes Insectes (Magasin encyclopèdique Il. 435. Bullet. soc. philom. I. 1797. 34. - Plan d'une Néthorle naturelle pour l'étude et la elassification des Inseetes (Bullet. de la soc. philom. AnII. 4, II. an 7, 153.). - Traité èlementaire d'histoire naturelle. Paris 1807, 2 vol. 8. 3 édit. 2 vol. Par. 1825. 8. - Rapport sur un mémoire de M. Bretomneau, initule: sur les propriptes vésieantes de quelques insetes de la famille des Cantharides. (Anual. des Se. Hat. XIII. 1828. 75.) Auszug ditvon in Okens Isis 1834. 10t1. Wurde mit dem sel. Latreille bearbeitet mid ist in vicle Journale ibhergegangen.) Rapport sur un mèmoire de il. Andouin, relatifangenre de Lépidoptère Dosithea (Amual. des Se. nat. 2. serie I. 122.)

Dumuliu, Commis bei der Marine am Senegal. Sammelte vicl in Afrika.

Dur, in Lomdon. Sammlung exotischer Kerfe.

Duncux, James, in Edinburth. - Introduction to entomology, conpreds a general view of the metamorphoses, external structure, anatomy, physiology, and systemit. arrangement of the class. insects, illustr. by 28 colour. plates, with mimoires of Swammerdamn and de Geer. Ediub. 1840, 8. - The natural history of beetles. Edinb. 1835. 8. - The natural history of british butterflies. Edinb. 1835, 8. - 7'he uatural listory of british moths, splinxes, Edinb. 1836. 8. - The natural listory of bees. Edinb. 1840.

JupoucheI, A. J., Vieeprisident der entomologischen Gesellschaft in Frankreich n. s. w., in Paris (Rue ('Assias Nro. 3.) - Hautwerlisel des Charaxes Jasius (Ammal. soc. ent. fr. 1838. 8.) - Isis 1839, 533.) - Monographic des Erotyles (Amual. dı Muséc). - Catalogne de Coléopteres de lile. de Lion. (Mèm. soe limerenne l. bult. 1827,344 , worin noch mche lepidopterologisthe Aufsatte uber Ruupen und 
Falter enthalten sind.) - Histoire naturelle des Lépilloptères ou Papillons de Fiance; par J. B. Godart. Contimce par N. P. A. J. Dupouchel. (Voun Tome VI. an (Suite les Noiturlites) 15 liv. Tome VII. Norturnes 1 et 2, 15 et 19 livr. Tome XIIl. 1. (20 livi.) et 2 ( 12 tivr.) (Prix de la livraison 3 Francs. (Tome 1. 15 livr. II. 14 livr. Ill. 6 livi. IV. 20 livr. V. 16 lv.) Paris (Mequignou). Supplement 30 livr. (a) 3 fis.). (Bell, Zool. Journ. XI. 1827. 472). - Icnnographie des chenilJes (50 a 60 livr.); planches dess. pirr Duménil. - Notice sul me espècc de Tincite dont la Chenille vitet se métamorphose dans la resine du pin sylvestre. (Anual. soc. ent. france. 1832, 300. 20. av. fig. color.) - Description d'une nouvelle espece du genre Amphicoma (Annal. soe: ent. france II. 183\%, 254 (nebst mehen Dissertationen). Revision du genre sutyre en nenf groupes, d'après des caractères tirès a la fois des nervures et desanteones (Amnal. soc. ellt. france. Jl. 1823, 97-103). Genre Adelostoma. (Annal. soc. linnè. de Paris. VI. 1827, 338.). - Raupe und Puppe von Nymphalis sibylla. (Annal. des Scienc. Iut. XI. 331.)

Dupont, Naturalist der Prinzen des königlichen Haunes u. 8 . w. in Paris Q Quai Saint-Michel, Nro. 25 !. S'hriftsteller. Grosuo Sammlung exotisther Käfer. - Monographie d's 't'rachydéres. Paris 1839. avec. $60 \mathrm{pl}$. color.

Durazxo, Don Carlos, in Genua. Entomolog.

Dutrochet, H., Mitglied des Instituts von Frankreich, in Renaud bei Vendône (Loil-et-Cher). - Ueber die Respiration der Kerfe (Annul. de la soc. ent. frane. II. 1833. Bull. 10.) Mémoires pollr servil a l'histoire anatomique et physiologique des Végetanx et des animaux. I gros vol. 8. et un atlas de 30 planehes gravées. Paris 1839. - Mémoire sur les Métamorphoses du canal alimentaire dans les iosectes. (Nouv. Bullet. de la soc, philon. 1818. 42.) - Observations sur les organes de la génération chez les Pucerons. (Annal. des Sc. wat. XXX. 1833, 204.)

Duvau, August, in Paris. - Nollvelles Recherches sur l'histoire maturelle des Pucerons (Mém. du Mus. d'hist. nat. de Par. XIII. 1825, 126140. - Anual. des Sc. nat. V. 1825, 224. Bullet soc. philom. Avr. 1825.)
Dnveruoy, Dr. med. und Professor iu Sirassburg.

Dnvernoy, Dr. med. unil Arzt zu Stuttgint, Carlssirilssc Nin. 3.

Mylira, Graf Collad von, in Breslat.

Ecorfet, Contributionsdirektor in Pontarlicr (Doubs). Sammlung von Ker. fen des Juri.

Edwards, Milne-, Professar, in Paris. - Cahiers d'histnire naturelle (avec Ach. Comte); 6 Cahiers: - (et A ulonin) Untersuchungen über die wirbellosen Thicre der Chansey-Inseln. (Amnal. des Sr. nat. 1828. XV. 5. Isis 1834, 1029).

Wowards, Sir Heinr. Barton, in Salrp (Englani).

Fggenidiner, Beamter, in Triest.

Winreaberg, Hofrath ete., in Berliı. Der hochgefeicrte Reisende in Afrika, Sibirien ete. - Symbolae phyvicne (mit Hemprich). - Prodromus ciner Auseinamlersetzung der Gattungen von afrikanisehen und asiatischen Scorpionen.

Whremlnans, F., zu Gaumwitz.

Elarlezralt, Justizcommissarius in Swinemïnde. Sammlung.

Whrlna rult, Tapezieler, in Leipzig. Schüne Käfer- und Faltersammlung. Handelt auch mit Kerfen.

Eichwold, Ed., Etatsrath etc., Ritter vou, zu Wilna. - Fauma caspio - eaucasiea nomullis observatio. nihus novis ilhstravit. Cum tab. XL. fol. Petrop. 1841. Berol. Heymann 1842. wiselt, Joh. Nip., Dr. medicinac und pract. Alzt in Policza (Böhmen). Der bekannte Entomolog und Verfasser des bis dahin einzig vollstïndigen $\mathrm{Hand}$ buchs der entomologisehen Literatur.

Hisenring, Jos., Geistlicher zu Wallcustadt, (nicht Pfeffers) in der Sihweiz. Lepidopteroloy.

Encely, Melicus, zu Castelnaudary (Dept. de l'Aude).

Endlicher, Dr. Philos., Custos an Ilom k. k. naturhistoriselien Musemm in Wien, Correspondent der k. Akademie in München ; botalischer Küustler und Schriftsteller.

Nngelberg, J. M., zu Giessen (P) Schriftsteller. - Ueber Aphis (A nualen der naturf. Ges. in der Wetterau).

Esins, Faustin, Professor und Custos am Museum in Troppau. 
Erdl, Joseph, Dr. med. und Professor der Physiologie auf der Universität in München. Stummelte, ein Begleiter Schuberts, in Palistina und Acgypten.

Erlacher, Dominik, Kapitular des Benedictinerstiftes in Kremsinünster, derzeit Stiltsagent und Verwalter der Stiftshäuser zu l.inz, luhabel der Selman'schen Sammlumg von Käfern.

Eruesti, Juscpli, Hanptmam im 4. Linien-Regimante zu Regensburg: Hübsche Sanmulung, meist Falter.

Erichson, Will. Ferd., Dr. med., Professor und Custos des Museums efc., in Berlin. Coryphace zonlngiselier Wissenschaft. - Genera et Species Staphliylinorum. Berol. 1840, 8. eum. 5 tab. (3 Rithlr.). - Bericlit über die wissensehaftliehen Jeistungen im Gebirte der Entomologic wälı: d. J. 1838. Berlin 1840, 8.; Idem von $1839,1840,41,42,43$ und 44 . Entomographien, Untersuclımgen in dem Gebiete der Entomologie mit besonderer Benutzung der köı. Sammlung zu Berlin. I. Hlt. M. 2 Kupf. Berlin (Morin) 1840, 8. - Ueber Aphodien (Ent. Ztg. II. 16). - Ueber Volueella bombylans und plumata. (ent. Ztg. III. 113). - Versuel einer systematisclien Eintheilung der Nitidularien (Germar Zeitschr. IV. II. p. 225-362). - Ueber die Elateren, livens. mesomelas ele. (Ent. $\mathrm{Z}$ gr. 11. 6). - Genera Dyticeorum. Berol. 1832, 8. (Walker ent. Magaz. n. 5. Oct. 1833, 501. - Revue eut. I. 1833, 268). - (et B urmeister) Beselireibung und Allhandlung der v. H. Meyen nuf einer Reise um die Erde gesammel. ten Insckten. (Nova Aet. Aead. Leopold. Nat. Cur. Bonn. 1839.)

Excher-Zullikofer, Particulier in Zürich. War lange in Südamerika. Der Besitzer einer der reiehsten und sclı̈nsten Käfersammlung.

Espinasse, J., in London. Bienemzuelit (Philos. Magazine LV. 1820. 329).

Hsstingen, Sr. D. der Herzog von Rivoli und Snhn des Marschall Masseun, in Paris. Selır schönes Kabinet von Naturgegenstîndev.

Estreicher, A., Dr. med, und Professor der Botanik in Krackan.

Etholén, Flottenkapitain in Si. Petersburg. Entomologisehe Sammlung.

Wversmann, E., Dr. med., Professor in Kasan. - Lepidopterorum spc. eies nonnullne movae gubern. Orenburgeuse insolentes (Méu. de Moseou VIII. ou nouv. Mén. II. 349. 2 pl.) - Enumeratio Lepidopterorum flavium Volgaum inter et montes uralenses labitantium (Bullet. de Mosr. etl. Le(l. p. 99-104. (111.) 1831). -- Enumematio Noctuarum etc. (ibidl. IV. 1832: 1. 152-154.). Ueber einige Sehmetterlinge Russlands. Kritische Bemerkuneen und Verbruitumg. (Bulletin de la Sor. inp. des Nafur. de Moseou 1837, Niro. 1, p. 3 (1837). - Verzeichniss der Kerfe zwí. srhen der Wolgn und dem Ural-Gebirge. (150 Wauzen, 55 Scluricken, 32 Bolde.) (Bullet. de Moss. l. e. 33.)

Fyolonx, Fortune, Chirurg erster Classe der k. Marine, in Paris. - Voyage autuur du moude par les mers de I'lude et de Chine, (xircuté sur' la porvette de l'etat la favurite pendant les années 1830,31 et 32 sous le eonimandement de M. Laplaee; publiée par ordre de M. Ie Viee-Adiniral Conte de Rigny. Tome V. Znologie par M. Fortune Lydoux et Paul Gervais. Paris 1839. 195 et 192 P.n. 8. avec Allas. (60 planelies; 45 Kerfe.).

Fack, Rector in Dornburg a. d. Saale. Tausehte Falter im nun aufgelösten entomologischen Tauseliverein zu Aschersleben.

Fageroth, Kupfersehmiedin Augsburg. Sammelt Kerfe und besizt eine nette Käfer- und Couclyyliensammlung. Fngeo, Johı, in Philadelphin. - Urocerus. (Silliman's Americ. Journ. Vol. IX. n. 2. p. 288.)

wahreens, 0. J., in Upsala. Mitarbeiter an Schoenherr's Genera et Speeies Curculionidum. Tom. VII, Pars I. Parisiis 1843.

F'nlderumann, Fr. , Obergäıtuer des k. k. botnnischen Gartens in St. Petersburg. Silmmelte in Russland, Sibirien, Persien u. s. w. - Lettre au Directeur (G. Fiseher) sur la faute d'insectes de Perse. Bullet. mose. ed. Leq. IV. 1832. p. 104-9. - Species novae Coleoptecorum Mongoliae et Sibiriae ineolarum. (Bullet. Mosc. ed. Ley. V. 1833. p. 212-234. Tab. IX.). - Fanira entomulogica trans-eaueasiea. Pars I. II et III. (Nouvenux Mémoires de la sociète imperiale des Naturalistes de Moscou. Tome V. et VI. Arec 16 plancles, formant le Tome Xl, et XII, do la collection. Moscou 1837, 4, - Coleop- 
terorum ab illustr. Bungio in China boreali, Mongolia, et noutibus altaicis collectormm, nee nou a T'urcraninovio et Stsclunkino e provincia Irkutzk missorum illustrationes (cum V. tab. litl. et color.). Petrop. 1835, 4. (Meim. de l'Acad. de St. Petersh. II. 337.) - Ueber einen nèuen sehïdliclıen Rïsselküfer, Ctiorliynchus Marejuardi (Bulletin de l'Aead. de St. Petersb. l. 1835 et 36. p. 179).

Fallew, Carl Frederik, Professor zu Lund. Gefeierter Entomolog. - Hemiptera Sueciae; et Cont. $1-5$ et $6-11$. Lond. Gotl. 1826, 8. - Monographia Tenthredinidum. 1-3. Lond. Guth. 1829. - Monographia Pompilorum Sıeeiae. Lond. Goh. 1829. - Observatioues entomologiente. Lundar 1802-07. 4. - Monographis Cantharidum et MiıIachiormu Succiae. Landae 1807. 4. Scatomyzides Succiae. Imndae 1819. Monographia Cimicum suecise, Hafuiae 180\%. - Oscinides sueciac, Lundie 1820. - Sciomyaides succiate. 1820. Opomyeides sueciae. 18:0. - Ortalides sueciae. 1820. - Jeteromyzides sueciae. 1820. - Rhizomyzidles sueciae. 1820. - Monographia Muscidum sueciae. $1820-25$. - llydromyrides sueeine. Lundae 1823. - Supplementa dipterorum sueciae Loud. Grotlı. 1826.

Fanin, Secretair der answiotigen Angelegenheiten in Minrseille. Zilhlreiche Sammlung. 'Zwei Bridler, zwei Entomologen.

Fraines, P'harmazeut zu Perpignau, - Memoire sur la chenille con. nue vulgairement sous le nom de conque. Perpignaı, 1825, 8. - Notice sır le Cebrio Xinthomerus Hofi. et description de sa femelle (Actes de la soc. linn. de Bordeaux IX. 3 liv. 137). Notice sur quelques l'ricautions à prendre dans la classse des Coléopteris (Act. de lat soe. Linn. de Bord. IV. 255. 1830.) - Ueber die Larve vou Phipidophorus maculatus (Annal. des Sc. VIII, 214. - Isis 1834. 865).

Farkas, Franz von, Advokat zu Grosswarlein in Ungarn. Lepidopterolog.

Fassbeuder, B., Lehrer an der Stadtschule zu Eupen.

Feluler, E. A., in Güttingen. Sanmler.

Felar, Lelıter zu Gunzenhausen in Bayerı. Zieht und sammelt fleissig Falter und handelt damit. - Alplabetisehes
Verzeichniss aller in Boisduvals System der europiii ischen Sclimette linge (Genera et Index Methodicus curop. Iepidopt.) vorkommenden Gattungsmanien mit ibren Synonymen. Erlingen 18:12,8.

welurle, Beanteter bcin k. Oberlandsgerichl zu Hirschberg in Schlesien. Summler.

Peistlinmmel, Baron von, General und Commandant der MunicipalGarde in Paris. Namlafte Sammlungen. Schriftsteller. - Deseription de li Chimaera funebris (Anual. soe. ent. france 11. 1833, 259). - Notice sur le l3ombyx repanda, Hub. (G. Megasoma Boist.) av. I pl. color. (Annal. soc. ent. I. $1832,340-34 \pi$ ).

Feldurer, Naturalist in Prag (?). Simmele in Brasilien.

Felix, l'tarer in Nufenen (Siliweiz),

Nermer, Graf von, in Wien. Hat blos Küfer, aber aus allen Gegenden. Fermerio, G., in Mluiland. Simmolung.

Ferrero, D. M., in Turin. Entomolog.

Fest, Franz, in Magdeburg. Entomolor.

wiclaler, C., Schönfärber in Neuhaldensleben bei Magdeburg. Scluöne und reichlaaltige Saumlung. Zieht Raupen und lat darin grosses Geschiek.

Fieber, Fr. X.: in Prag. Cultivirt die Ilymenopterologie. - Die bölumischen und ostreichischen Cetonien. - Ueber Rhynchoten (Weitenwebers Beitr. f, ges. Natur- und Heilwis:. Hift. 1-3.). - Entomologische Monographieu. Sieben Abhandlungen mit 10 'Tafelı in Steinsticls. Hr. 4. Leipzig, J. A. Bartli, 1844. (2 Thilr.)

Nicimus, Hpinr., Hofrath ete. zu Dresden. - Allgemeine Naturkunde. Zınäclıst für Thieräl\%te und Landwiюthe und als Leitfaden zu Vorlesungen. Dresden und Leipzig, 1839, 8.

Figulus, Dr. nerl., in Breslau.

Fiudel, Arzt, zu Temeswar in Ungarn.

Fimtelumanu, L, Doetor in Berlin (?). - Beitriige zur nähern Bestimmung und Naturgesehichte einiger auf der Kiefer lebender Lophyren (Verhandl. d. Kais, Leopold-Akademie. Bl. XIX, 1839. 1. p. 245. Taf. 25).

Fischer von Jisaldheim, Gotthelf, Vicepriisident der Akademic, Director, Professor etc., in Moskau. 
Horhgefcierter Nestor ller Naturgesebichte, Naturforseher uud SelniftstelJer. - Zoognosia. Mostpume, 2 vol. gr. 8. - Entomographin ruthenica. - Lettre sur le Pliysodactyle. 1824. - De Nycterilio movo genere Hymenopterormm ad fumiliam Tenthrelinctum pertinente. (Act. socict. physicomedic. Mosquens. 1. 1806, 80. - Observation d'un nouvean genrc de Diptères. (Mèm. de Moscon I. 1805, 184; 1811, 198. avee 2 pl. no. XXXV bis). - M(nscum Dèmidoff. Moscou 3 Vol. 4. 1806-7. iv. planch. (Insecta, Tom. III. p. 37-102). - Notice sut quelques inscetes exotiques du MIuseum Demiloff (M èm. de Moscou II. 1809, 43-46). - Sur deux genres nouveanx de Coleoptêres (Mim. de Mose. Il. 1809, 293). - Pogobocerus, novau genus insectormu Caucasi merit. (Mèm. de Mose. Ill. 1812, 281). - Notice sur lc Phlocerus. - Carabus chiysochlorus. (Mcil. de Mose. III. 311). - Observatims sur les Dipteres de Rnssic (Mim. de Moscon IV. 1812). - Gemera Insectorum systematice exposita ef analysi iconograplica instructa. Mosturac, 1821. - Progromme d'invitition a la Sénnce publique de la Sotiete imperiale des Naturalistes qui aura lieu ee 15. Decenbre. Contenant une Notice sur bne mouclie carnivore, nomee Mcleterc, ac compagnce d'une planche; Mosicon 1819. 4. pag. 11. - Adversin zoologica. Mem. de Mose. V et VI, 17 pl. col. - De colcopteris quibusdam novis (ibid. V, 1817, 463-571. Entumographia imperii russici. Vol. I. (cum 26 col. tab.) Mosqu. 1820 . Vol. II. (c. 40 tab.) ibill. 1823. Vol. III. (c. 18 tab.) ibid. 1825. - Notice sur le 'l'ettigopsis, nutvean genre d'Orthopteres, Moscon 1830. 4. e. tab. acu. - Conspectus Orthoptciorum rossicorum (Ballet. des Naturalist. de Moscou 182933. edit. Lequien V. 1833 . p. 323-355. - Aulacoths, genre nouv. de Coliopt. app. aux Seirabacides (ihil. p. 5 Pl. 3. 1829). - Psilotus Hoffmanseggi (ibid. 1. 7. PI. I. 1829). - Denops: nov. gen. de Colcopt. (ibid. p. 9. Pl. I. 1829). - Trichotes de l'lberic (ibid. j). 10. PI. I. 1829). - Cucujos puncticollis et Seydmacnus Flcisthesi (ibid. 14. PI. I. 1829). - Notice sur quelques insectes de la collection de $C$. Stiven (ibid. 64-68). - Description de quelques inscetes colcopt. nouveaux (ibid. 68-70). P. V. - Note sur quelques nonvelles espètes d'insectes (ibid. 9:-98. II. 1830). - Amaleetí al faunam insectorum rossicam (ibid. 4. 1832, p. 154-167. T'als. V.).

wiscluer, Sebastian, Dr. med. und Tucibaryt (fruhcr des Vicekönigs Mehemet-Ali von Aegypten) Sr. k. k. Holeit des Grossfïrsten Maximilian, Herzog vou Leuchtenberg und Fïrst von Eiclıstädt, zu St. Petersburg. Besizt schr siloune afrikanische lusekten, besontlers vollstïudig aus Acgypten, Sennaar und Fussoghli, welche ce bci seincm langen Autenthalte in Atrika gesammelt hat.

Wisclecer, J. E., Edler von Rislerstamm, z. Nixtorf in Bölnmen. Mikrolepidopterolog. Scheoswerth ist dic Einrichtung seiner Raupenzolit. - Abbilluogen zor Berichtignng etc. der Silhucterlingskinde. - Ueber Oelssculeiucria (Hbn., Ploygas; Tro) taarella ond ihre Verwanden (cut. Zeitg. III. 200).

Fiscleer, Overmcer van, in Java. witzinger, in Wicu. Arathnolog. Fleisclier, J. Theoph., Dr. ned. in Mitau (Curland). Coleopterolog und Sihriftsteller. - Trechus sericcus; Colcopt. sp. nov. (Bollet. des Nat. de Moscua ed. I.cq. p. 12-14. PI. I. 1829). Flemuing, J., in London.

Fleschmez, Adolph, Baccilanrcus fer Medizin cte in Minnchen (Löwenstr. Nro. 24). Schöne Sammlıngen aus allen Klassen; wissenschaftlich behandelt.

Fotow, Julies von, Major a. D. in llirschberg.

wocke, W., in Wien. Sehriftsteller. Annaleu des Wiener Muscums d. Naturg. Wirbcllose Thierc. Alth. Il. d. Werkes. 1841.)

wdester, Arnold, Lehrer an der höhcrn Bürgerschole in Aachen. Ein sehr lieber Mann, der besonders Dipteren, Hymenopteren unil Colcoptereu sammelt. - Bciträge zor Monographic der Pteromalinen. I. Hft. Aachen, 1841.

Fontenny, Artillerie-Kapitain, in Lvoll.

Forel, Alexis, in Lausaume. Schritt$s$ eller.

Forster, Albert, in Bromley bei London. Nitarbeiter am entomologieal Magazine.

Forstrtbu, schweliseher Entomo$\log$ auf St. Barthilemy (Autillische Inseliu). 
Foucon, Capitain der Infanterie ain Senegal.

Foudres, in Lyon. Colcopterolog und Hymenopte rolog. Selıöne Samm lung.

Fonlques de Villaret, J. H. J., Capitain der lufauterie in Batignolles (Scine). Schriftner. (Annal. de la soc. ent. de France).

Frauck, H., in Strassbure. - Catalogne des Lepidoptères de la collection de H. Franck. Z. T. 2. Strassbourg. 1826.

Frronk, Hermann, Dr. philos. in Breslan.

Frnuke, Sen., Naturalienlıändler in Leipzig.

Franke, jun., Naturalicubäudler in Amsterdam. Besizt vorzüglich ostindisclie Naturalien.

Frantzius, A. von, Stud. med. in Hcidclberg. Selur wackerer Jïugling, der mit Einsicht Käfer sammelt und studirt.

Fray, Kriegskommissair in Limo. ges (Haut-Vieune). Schrieb cine entomologiselı-physiologisehe Abhamilung.

Fremaye, de la, in Falaise (Dept. de Calvados). Entomolog.

Freyer, Heinricl, friiher Magister Plinrmaciae in Warasdin (Croatien), nun Custos am Landesmuseum in Laybach. Geschickter Präparntor.

Freyer, C. F., Kassier in Augsburg. Lepidopterolog und Schriftsteller. - Verwïstungen der Wiesgriunde in den Ortsfluren Leclılıusen, Stïtzling und Friedberger-Au bei A ugst) u'g durch dic Raupe der Hadena popularis in Mai und Juni 1833. (Inis 1834, 257). Frlänterumg ïher Sphiux Jisulae (ent. Zcitg. Il. 125. Sey ächte Ait (?). Ueber dis iiltere lepidopterologiselse Literatur (Clavis 7.11 Clerck: Icones Insector. 1789). Wiclutig, weil Linne theilweise nacls den Abbildumgen bestimmt lut (ent. Zcitung 1. 75). - Die schädliclısten Schmetterlinge Deutseh. lands, mit 12 ill. Kupfert. î 2 A. $24 \mathrm{kr}$, - Wird mit der Zcit fortgesetzt, da der Verfasser tagtả glich Nenes eutdeckt. Lepidopterologisclie Beobachtungen, Nachrirliten etc. (ent. Zeitg. II. 52 ).Beiträge z.ır Geschichte europäischer Sehmetterlinge ete. Heft 1-24 oder 3 Bändelien mit 144 ill. Kupfert. Augsb. 1828-30. 12. (à 1 f. rl.,) - Die Falter der Merian. Systematisch bestimmt und erläutert und mit Aunerk. vers. (Isis 1842, p. 18). - Ueber die Beur- theilungr von Reaumur's Schmetterlingell, und die Preisselırift von P. C. Zeller in Glogan (Isis 1839. VII. 448. (Isis 1839. p. 728). - Neuere Beiträge z.u1 Schmefterlingskunde. Heft 1-60. 4 Binde mit 360 illum. Kupfert. Augsb. 1833-41. 4. (à 1 f. $24 \mathrm{kr}$, rlı.). Ueber die Stellung und Einreilıung des Pap. Lucina im Systen. (Enton. Zeitg. II. 50).

Freyreiss, Dr. med., dentscher Eutomolog, friilier in Rio Janciro, jetzt in Ekaterinoslawl. Insectensammlung.

Friedrich, in Breslau. - Ueber Hipparchia, Notodonta (Uebersicht der Arbeiten und Veränderungen der schlesischen Gesellschaft fïr vaterländische Kultur in Jahre 1841 p. 100 et seq.) Friedricl, fixiatleer, Erbprinz voul Seliwarzburg-Rudolstadt.

wries, B. F., Professor in Lund. Monograplia Tanyporum succiae Lundae 1823. - Observationes elltomologicac. Purt. I. Lundae 1824. - Beskrifning ofver nya Insekter fran Columbicu. (Vatensk. Akademiens Handl. 1833. p. 320).

Wrivaldsky, von Friwald, Emrich, Dr. med., Arzt und Conservator des Naturalien-Kabiuets 2.11 Pestlu. Herrliche Sammlung. Verkauft und v ertansche tïrki. selue und ungarisclue Kerfe, besonders Falter. Schriftsteller. - Közlesek a' bal. kány' videkén tett termes 'Zettudomanyc utazasrd (Insecta balcaniea in : A Magyar tudos társasíy, Eukönyvei. Második Kütet. Budàn, 1835. 4. p. 235-76. II. Tabul. V, VI et VII. Jul Auszuge nitgetleilt von Gistel, in dessen Fannu l. Bd. neu. Folge, p. 84-93. XVIII.)

Froriep, Dr. med., Solın des sel. Medicinalrathes in Weimar. Ueber die innern Gesclilechtswerkzeuge der vivipareu und oviparen Blattläıse (Dessen Notizen, No. 262. 1839 (40?).

Filrarolna, Dr. phil., Professor der Nafurgesclichte an der Gewerb. schule zu Regellsburg. Früher Pharmazent. Tüclitiger Botaniker. - Mediciniseh-topogr. Besclureibung von Regensburg. (vgl. Hervich, Koch etc.)

Fulilrott, Dr. med. in Elberfeld.

Trentoriel, C. H. Seminarlehrer in Berlin. - Leitfaden zu einem metlodischen Unterriclite in der Tliop- und Menselienkunde (Antliropologie und Zoologie) ete. Berlin, 1840. 8. 
Flixtner, in Leipzig. - Gelocimnisse unl Vurtheile Iïr das Insektenkabinet. M. 1 Kupf. Jeeipzis.

Grimar, Jos. Paul, in Paris. Bekanuf durch dou Antheil an den entomologischun Besehreibungen zur Reise de's Capt. Freycinet mit Quoy (Vergl. diesen).

Anlenzzi, G., Maler in Mailand. Sammlumg lombardischer Käfer.

colle, Flottencommissair bei der Marine in Toulon. Grosse Sammlung vnn Kerfen aus Grierbenland, vun der berberisehen Kïste und vou den balearischen Inseln.

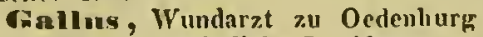
in Ungarn. Ansehuliche LepidopterenSamoulung.

Carduns, in Neulausen bei Münehen. - Die wissenschaftl. Sinmmlungen Münelıens. (Isis 1844. Heft $V$ und VI Unischlag.)

CAruot, in Brest. Bekaunt als Reisender (uIn die Welt mit der Corvette Coquille) und Schriftsteller.

Ganspariui, Ferd., Postdirektor in Montpellier.

Tamini, Officicr in Paris. Samaelt Käter.

Grumbleluau, Naturalist bei der Expedition des Capt. Freycinet, in Paris.

Tramzy,ProprivairzuCastelnandary.

Giebler, Medicinalratls ete. zu Barnaoul in Sibirien. Botaniker und Znolog. Schuiftstelier: - Notne et additamenta ad catalorum Coleoplerorum Sibiriae oceidentalis ef confinis Tatariac, operis: Voyage de C. F. Jeelebour dans les montes altaipues et les Steppes des Kirguises; Seconde Partic, Berlin 1829 et 1830. (Bullet. Mase. ed. leq. V. 1833. p. 235-283. Coleopt.) - Characteristik melıre never sibirischer $\mathrm{Kä-}$ fer, von Schrenk mitgebracht aus der Kirgisen-Steppe (Bulletin scientifique de P'Acad. imp. de St. Petersbourg. VIII. 1840 p. 369. 11. 192.

Gelieu, Jonas von, Pfarrer in Co. lombier (Citut. Neneuburg). Schriftsteller ïher Bienen.

Ceremuinger, Candidatus Medicinae in Müncheu. IKanfte die Sammlung des sel. Westerlanser, reich an cinhei. mischen Giffungen. Sammelt anch Sehmetterlinge.

Cóne, Giuseppe, Professor in Turin (an des sel. Bonelli Stelle). Ueber dic Lebeusart von Osmia, Stig- mus und Myrmica. (Atti della terza Riunione. Firenze p. 398). - De quibusdam insectis Sardinine novis et minus roguitis. t. 1. (Memorie della reale Aecadeaia delle srienee di Torino XXXIX. 1833. p. 161.) - Siggio di una monografia delle forticule indigene. 1832. - Nemoria per servire alla sturia naturale di una specie di Cecidomia ele vive sugli Iperici (Mem. de Torino. 1833. XXXVl. p. $287-95$. - Isis. 1844. 58 .

Reofroysaint-Inilnire,Isidor, Professor der Naturgesclichte z.u Paris. Grosser Naturforseher und wielıti. ger Sehriftsteller. - Zoologie (mit VaPentiennes) zur Voyage autour du Monde sur la Frégate la Veuus, pendaut les nunies 1837, 1838. 1839 et 1840 ; publie par Oidre du Roi, sous les Auspices du ministre de la Marine, par Abel du Petit-Thouars, Cpt. 10 Volumes grand in 8. av. Atlas (Zoologie, emntenant 80 planches gr.).

Geofray Saint-Tilaire, Etienne, Prutessor etc. 21 Paris. - Sur le Systeme intra-vertebral des Inseetes; fascicule de 16 pages (Bullet. soc. philom. 1823, 40). - Memoires sur l'organisation des Inseetes (Journ. eomplementaire du Dietion. des Sc. médieales). - Sur l'organisatiun des in. sectes (Anual des Se. pliysiy. III. 165.) - Cunsidérations philosophipues sur la determination du systeme solide et du système nervenx des animaux articules (Annal. iles Sc. nat. II. 295).

cheofroy de villemeruve, in Paris. Solun des Verfossers der "Histoire abrege dos Inseetes, qui se tronvent aux enviruns de Paris". Hielt sich lange Zeit am Senegal anf, und hraclite vou dalier anschuliche Simmlungen.

Trerlisa rolt, Hermanu, Cmmptoirist zu Bautzen in Schlesien. Sammler.

Geveruan r, E. Fr., Dr. mell., Professor der Mimeralogie etc. zu Halle. Vielberiulımter Natuforselier mudSch riftsteller. - Melolontha rutieurnis, schädliches Inseet (Dess. Ming. de Ent. I. 1. 1. 8-10. - Naturgesclichte des Carabus gibhus, ciucs saatverwïstenden Inseets (Dess. Mag. d. Ent. I, 1. p. 1-8. Tab. I. f. 1-5. - Neue Insecten (Käfer) besehrieben (Mag. l. 1. p. $114-134$.$) - W. K ir by'$ Monographie der Gattung Apion, Herbst. Aus lem Englischen jibersetzt, mit Bemerlungen und eingesclialteten 
Besclupilungen neucr Arten (Dess. Mag. d. Eut. II. S. 114-266. Tab. II., III., IV.). - Tödtuug der Kiffer durelı die Soune. (Dess. Mag. II. S. 339.). - Coleopterorum Species novic aut minus roguitar, descriptionibus illustratae, eum Tub. aeu. II. Halae, impeusis J. C. Hendelii et Filii. 1824. Etiam sub titula: Inscetorum species movae efe. uti supra, volunen primum. Col coptera. (2 Thlr. $16 \mathrm{gr}$ ) XXIV. et 624 pag. ii) 8. - Eine nene Kïfergattung Potamophilus., m. 1 Kpf. (6 Gr.) Monströser Elater variabilis, mit 7 Beinen. (Dess, Mag. II. S. 335. VII. Tab. I. f. 12. - Mückenscliwïlme von Chironomus in Sagan. (Dess. Mag. Il. S. 336.). - Insecten in Bernstein eiugeschlossen, beschriebeu a us dem academ. MincraIieneabiuet zu Halle. (Dess. Mag. d. Ent. II. p. $(1-19)$. - Abhandlung iiber die Gatfung Authidium Fabr. von P. A. Latreille. (Deutsch aus deu Annales du Musemm d'hist. nat. v. Ge r.mat, in dessen Mag. 1. 2. p. 40104.). - Beiträge zu einer Monographic der Rolırkäfor (Donacia Fabr) m. 1 Kupfertaf. 8. (3 Gr.). - Nuclutrïge zu Aug. Ahrens Mouograplice der Rohtkäfer. - Strepsiptera, cine nene Orduung derlnsecten, von Kirby (aus dem Bullet. de Sciene. pliton. de Paris; Avr. 181 5, p. 62 - 64. mit Aumerkungen übersezt; Germar'sMag. II. p. 290-300). - Systematis glossatorum prodromus, sistens $130 \mathrm{~m}-$ bycum species serundum oris partium diversitates in nova genera distributas. Sectio 1. Lipsiac apud Reclam 1810. Sectio Il., 1812, 4. - Synopsis der neu aufzustellenden Sippeu von $\mathrm{Cur}$ culionen (Dess. Mag. II. S. 339). Cantharis fusca. (Germar, Mag. I. 1. p. 134.). - Bemerkungen über Cus: culionid en (cnt. Zcitg. III. p. 98). - Conspectus gener'uu Cicadariun, cum notis (Silherun. Revuc I. $1833,174$. ) Ucher 'outatoma aruminatuu etc. (ent. Zeitg. III. 68.) - Précis d'un nonvel arraugemeut de la fomille des Braclsélytres, par M. Maumerheim. (Silbermann Revuc 1. 1833, 184.) - Uebcr dic Gruppe der kugalförmigen Trugiden (Germar, Zcitsclır. IV. I 109148. Taf. J. f. $5,6,7)$. Mladen im Koclisalze. (ent. Zcitg. It. 127). - Germar (E. Fr.). Bemerkungen uiber Elateriden (Germar, Zeitschr. IV. ctc. in Mii nch('). Reisender in Frank-
rcich, Schwciz, Deutschland, Illyrien,

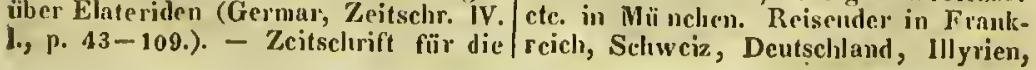

Entomologie. Leipz. 1839-43, 8. I-IV. Bd. - Eiltige Bcmerkungen uiber Curculioniden mit uugebrochenen Fïllicrn (ent. Zeitg. 11l. 2.). - Bemerkmuren iiber Curculioniden (Fortset\%. ent. Zeitg. III. (1842) p. 98-110.). - Coubophoruu Spccies eumueratac al H. Bur. meister. 1 pl. col. (Revue ent. de Sil. hermann 1. 1833. 227-33.) - Observations sur plusieurs espèees du genre Cicalda (avec 8 pl. col.) (ibid. II. 1834. 49). - Deseription du gente Thorictus (ibicl. Il. n. 15). - Description du genre Chirodica (ibid. n. 16). - Deseription du genre Brachyscelis (av. fig.) (ihid. in. 17). - Die versteiuerteu Insekteu Soh. leuhofens, beschrieben. (Verlandlungen der kaiscrlichen leopold. citr. Akad. XIX. 1. 1839. p. 187. t. $21-23$ ill.)

Ceyer, Kat, in Regenshurg. Der Fortfïhrer des prächtigen Faltelwelkes von. J. H ib b $n \in$ r sel. mit Hru. Frühlich sel. zu Ellwangen. Ist von Hervich engagirt, der auch seine Kupferplatten an sich gebracht hat. Bcilrïgc z.11" Sammlung exotisclier Sclimettcrlinge 1818. 4. Erstes Hundert (complett f. 16.30.) Zweites $H$. (compl. f. 16. 3; zum dritten Humdert sind fertig (v. Karl Geyer) $34 \mathrm{BI}$. (ausamu. f. 15. 18.) und zuu vierten 28 Bl. (zus. f. 12. 36 rlieiu.)

curecinto, Pudre Carlo, di G. M. Carmelitano Scalzo, Professor der Botanik zı Messiua. Schriftsteller.

Cimmerthal, B. A., in Bolderan nonvelles cspeces de dipteres. - Besclireibung ciniger weucn in Liefland aufgrfundeueu Inserten, - Métamorplose des Insectes (Diptères. Noctuae occultue. Larva phosphorica; Bullet. de Moscou ed. lequien. $15-19.1829$. - Catalogus systematicus Dipterormm in Livouia observatorum. (Bullct $l_{\text {. }}$. IV. 7832. p. $145-152$.)

Cxioren, in Turin. - Memoire d'Entomologic (Mem. de T'mrin. 1803 -4. p. 215-224; Mordella nova ambigua: ist Rlipidopthorms subdipteJus. Mantis pectini cornis, Linn. Papilio Jasius Liım. 2 Tab.)

chirod-Clemutrsus, Ritter der Ehreulegion und des Ludwigsordens in Baume (Douhs.)

Cristel, Johannes, Dr. phil. und Med. Baccalaur., resignt. Professor bei Riga. - Observatious de quelques 
Dalmatien, Italifu u. s.w., Znolog umıl Sichriftsteller. (Vergal. gelelures Min!. chen int J. 1831. Von A. v. Sibralen; p. 35-39.) Durch ihn mul alie Natara. lienhandlung (Allalbertstrasse Nro, 9), welehe grosse Vorräthe ans der pyreniischen Halbinsel, Frinkreich, slem gamen Norden, Russland, aus dem Süden (besonder's Sicilien und Griechen. land), damn aus Asien, Mfika (Acegypten, Senegal und Cip) unl Amerika (Nord- und Sind-) beständig unl frisech bericht, kanı Jeder grosse und kleine Partien un alie billigsten Preise und im 'Tausphe prialten. So die Gliederals Wirbelthirre. - Antimachus, novim Colpopterorum genos, e familia Tenebrionidum (Isis 1829. Ileft 10, p. 1055-58, Taf. III, Fig. A). - Reise des Grafen Rumlolph von Jenisun-Wal. worth dureh Siid-Dentschaland uud NordItilien. Besehrieben von J. Gistrl. I. Theil m. 6 Abbihl. München, 1835. Cotti. 8. Xil. et 384 Pager. Bil. Il, 111. - Vollstaindiger Abriss iler Natırgeschichte. Nach den besten Quellen und pigenen Beobichtungen bearbeitet (Ilandbuch fiir alle Stimle vom Oberlieutenant $A$. v. Schadel. Miinrli., 1834. 2 Bde. Bal. l. p. 1-112). - Systeux Insectorun: secundum elisses, ordines, genera, species, emn caracteribus, synunynis, an notationibus, locis ef iconibus. I. Coleoptera ; Fisc. I. (Mantivlıora-Dromien.) Cum tab. Monachii, 1837. XVI et 64 Pagg.gr. 8. Fasc. II. Bernac, 1838. Dromira-Zophium. p. $65-113)$. Miseellen (zoologisehe) (Faunus !.1832. Acis $\mathrm{Nr} .1-2-3(1835) ; \mathrm{Nr} .4, \mathrm{Ni} .5$. Nr. 1. 1837.) - Die jezt lebenden Entomologen, Kerffeude und Kerfsammler Europa's wnd der übrigen Contimente. Muinclien 1834. 16. IV. u 80 Pagr. - Dasselbe Werk, das Hr. Silbermann ins Französische ribertrug und ansehnlieh bereicherte; $z$ weite, n:tch der framzös. Ucbersetzung ma llen nevesten englischen und deutselien Original-Beiträgen, sebr vermehrte uml verbesserte Auflage. München, 1836. (Weber, Ph. J. Batyer) 5 Bogeu olle. 103 P'nge. in 16. - Naturlistor. Artikel zuu Tasehen-Conversations-Iexikon, luerausgegeben vou eiller Gesellschaft Galebiter XIl., XIIl., XIV. BH. Augsburg (Scblosser), 1832, 16. - Generiв Megralupi speries (Fautus, mene Folge I. (1837) 11. 111-121.). - Fanta von Parteukirehen, in dem Werke von Dr.

alstel's Lexilion.
G. L. Dieterieh: „ıler Kanixer-Brunnen bei Partenkirchen. Münch., 1834." - Enlomologisclie Notizen (Thon: Archiv f. Enfomologie). - Einige natnrhistoriselie Bemerkungen voul dillre 1826 (lsis 1827. Bl. XX, p. 625-27. Sucke, Kerfe). - Fauna der A1, in Auselm Mart'u's Trpographie und Stat stik des Landgerielits Au bei Mïnchrol. Münclıen 1837,p. 119-121. - Enumeratio Colfopterortur agrio muncensis. Diss. inang: MInatuii 1829, 8. Ang'ustac Vindel. 1830, 8. (vgl. Isis 1829, p. 1058). - Systematische Uebersiclit der Wanzen und Cicaden der Umgebung von Mïnchen. Gesammelt und bestimnit v. J. G. Miñchen, Finsterlin, 1837, 8 . is Pagg. (Faous, neue Folge Bul. I. (1837), p. 98-111; olme dic vielen Varietäten: 71 uene Rhyuchoten-Species, 38 nenc Cicaden, Aphiden: in Ganzen 109 Species; dabei 4 neue Genera. Die lnsecten-Doubletten aus der Sammling des Hin. Grafen Rmilolph von Jenison- Walworth, I. I., Käfer. Mïnelsell, 1831, g\%. 8. - Hemip'eren- und Orthopterenfune aler Siluweiz. lat mul litlungraphit worden. Epselien $1838 \mathrm{im}$ Herbst. - Insekten in menseblichen Körper. Lin Wörtclien zu seiver Zcit. (Bayr. Nationalzeitung 1836, 11. 199, p. 825). - IKifer der Gegend um Zilsmarshausen. Zusitze. Y Isis 1829, II ff I I, p. 1130! - Schefflarn. Das Heilbad und dic Ungebung. Gealenke-Mcin für Kurgiste. Miünchen, 1837, s. (Geognosie, Flora und Fanna). - Handburh der Nitturgeschichte für alle St:inde. Beri, 1838, 8. Heft 1, 2, 3. (Sucke, Vögel, Lurehe, Fische). Ist unbeendigt geblieben und kan nirbt in den Handel. - Mesoclastus paraluxus, cine neve Fanilie, Sippe und Gittung uns der Ordumug der Kiffer. Minmehen, 1836, m. I Abb. 8. (Faunus l. (n. IF.) p. 54-59. Tals. 1). - Entomologische Beobachtungen (Isis 1829, Heft 10, p. 1067-70). - Kritisehe Revisionen und Ergiuzungen zu Selurank's Euumeratio insecturum, fauma boien etc. Aus dem Nachlasse des Seligen. (Faumus, nelle Folge I., 1837, p. 5-20.) - Beitrag zor geographisehen Verbreitung der Kuffer (1sis 1829, Heft 11, P. 1129). - Die schãdlichen Insecten tles Ilanses null der Fhor (Herthi, 1837, 11.. 30). - Hertha. Zeitschrift liir Naturgesetrichte, Physiulogie, Naturlehue, Astró. nomic, Läider- ind Völkerkunde u. s. 
w. 1837, 4. I., 11., III. - Ueber die höhere Bedeutung der Naturwissenschaften, ihre Zweeke und ihrun Nutmen (vorgetragpn 1837 in der Oberelasse des alten Gymnasiums zu Münehen: abgedruckt in der Herlla No. I., P. 3 -15). - Auszuige ans minem zoologiselien Tagebuche vom Jahre 1829. (Isis 1831, Heft 5, p. 496-99). - Etwas grgeu die Cliolera.Insecten und fïr die Clıolera (Bayr. Laudbötin. 1836, No. 140). (Gegen Dr. Viale, der Dip. teren als ansteckend be\%cichucte). Die bayrischen Alpen. Reminiscenzen einer Gebirgsteise (Lesef rüchte belehr. und unterl. Inl. $183 \mathrm{I}$, l. Bd. 7. p. 97 -109). (Flora, Fauna und Sittengemälde). - Entomologisehe Fragmente (Isis, 1831, Hft. 3, p.301-311. - Zur geogruphischen Verbrcitung der Käfer (Isis, 1831, Hft. 3, p. 248). - Eutoinologiselue Notizen (Faunus I. (1832), p. 128-151). - Kerfe im Copal ciugeschlossen (Isis, 1831, Hft. 3, p. 247. - 2 Käfer und 2 Diptera). - Verzcichuiss von Kätern, welche in Spanien ete. gesammelt wurden (Isis, 1832, Heft 2, 11. 148-153), - Einige Falter uus der Gegend von Cadix ete. (ibid. p. 153). - Die vorzü̈rlich lästigen Kerfe Brasilieus. Besclir. v. V. Kollar. Im Auszugc. (Faunus, Bd. II, 22-37). - Correspondenz-Auszüge (zoologisclic) Faunus 1, Acis. No. 1, 1834 , n. 2, n. 3 (1835), 11. 4, 11. 5, 11. I (1837), 11. 2. - Literarisclie Notizen (Faunus, Bd. I., 46-49. - Acis 1834. I. und II., III. (1835) IV., V., I. (1837) II. - Nekrologisches (Faunus l., p. 49-56. - Acis Nr. 1. - Nr. 2, Nr. 3. (1835) Nr. 4, Nr. 1 (1837) $\mathrm{Nr} .2$ ). - Correspondenz-Auszüge (entomologisehe; Faumus l., p. 41 - 46). - Brmerkungen und Zusätze zu Latucille's "Gevera crustaccorum et Insectorum (Geschrieben 1828.) (Faunus $1 ., 1832.122-128$ ). - Culenpterorum species nova (Jsis, 1829, p. 1131). - Faunus. Zeitschrift für Zoologie und vergleichende Anatomic. I. Bd., Miïchen, 1832, 8. 11. $1 \mathrm{Kpf}$. Mit Intellifenzblutt Acis. 11. Bd. ib. 1835. Mit Intell.-Bl.: Galleric denkw. Naturforscher und $1 \mathrm{Kupf}$. III. (nene Folge I.) Münclı., 1837, in. 3 Kupf. und Beiblätern. - Die Todtemular. Naturgeselichtliclıe Notiz. (Vaterliindisebes Ma. gazin fiir. Belelırung ete. Mit Beitriigen yon Advokat Ellbalier, Dr. Gistel, Kanzicidirektor Hardt, Regieruugsti- rektor v. Obernberg, Ministerinlsekretair Schmidt etr. 1. Jahrg. Erlangen (Palm). 1837, 4, p. 496).

Cincli, G., Professor der Naturge. schichte und Botanik zu Sicua.

Corser, L., Realschullehrur zu Darustadi. - Von der Uebercinstimmung zwischen deu Characteren der Pflanzen und der an ilmen loheuden Inseeten, im Besonderen der Schmetterlinge. (Isis 1842, p, 6). - Parallele zwist:hen der Klasse der lusecten unul dem gesimmten 'llhierreich (ibid. p. 13).

CInsewald, Archivar im Ministerium zu Cussel.

Ciloger, Carl, zu Breslau. Ento nolog.

Todet, Lonis, in Berlin. Bereiste und durcliforsclite Russlaud, deu Caucasus u. s. w. Sammlungen vou dilher.

Fodet, Carl,in Neuenburg(Stliweiz). Entomolog und Botaniker.

Godmau, John D., Med. Dr. und Professor der Naturgeselichle in Franh. lin-Institut und Professor der Anatonie und Physiologie an Rutger's medieiniselıem Collegium r.и Philadelphia.

Colbdew, Antsratlı in Rügenwalde (Pummein). Sammlung.

Goldruss, Georg August, Professor der Zoologie und Miueralogie zu Bom. Zoolog und Sciniftsteller. Entumeratio Insectorum Eleuther athorum capitis bonae spci totiusque Africae, descriptionibus iconibosque monnullarum specierum novarum illustrata. 1804. Erlangae, typis Kunstmanuianis. 38 Pagg. in 8. $1 \mathrm{~K} \mathrm{pft.}$

Coodal, Joseph, Rev., im ento. mologischen Collegium zu London.

Cory, Ritter Hypolite Louis, Capitain der Cavallerie in Paris (Rue Tronchet No. 7). Entomolog und Seluiftsteller. Ausgezeiclunete Käfersamunlung (18-20,000 Species'. - (Mit Percheron) Mlonographic des Cetonims. Description des Meloloulba lactea et spinipennis. av. fig. col. (Revue ent. de Silherm. I., 1833, Nr. 11-12). Description de l'A reoda maculata; av. fig. (Revuc ent. 1., 1833, Nr. 13). Monographie du geme Sisyphe. Paris, 1833 , 8., avec 1 tab. ( $1 \mathrm{fr} .50 \mathrm{co}$.).

Gotsch, Pfarrvikur in der Nöhı ing bei Gmind in Oberkäruthen.

Gombert, Lev, Beanteter hei des Stener-Adninistration in Strassburg. Schöne Sammlung.

Goudot, Kevfländler in Pariş. 
Bereiste Mudagasear und dic Geyend um 'Tanger und Spauien, so wie din Jura. Brachte ausserordentliche Sammlungen uach Hause.

Conld, Jolu, Esq., in London.

Gonrean, in Paris. Entumolog und Schriftsteller - Ucber die Suídulation der Kesfe. (Annal. de la Soc entom. de France. Livr. 1-4. 1837. 3 . l'lnstitut 1839.) - Ueber die Stridulation der Mürcken wilhrend des Fluges (Amual. sor. ent. 1837, 11. - Isis 1839, 53.1). - Stimnorgane der Kerfe, besonders über das Summen. (Rrvue entomologique.)

Greels, in Paris. - Ueber dic Er. selieinung der Cebrionen in gewissen Jahreszeiten (Amal. soe. ent. fr. 1837, 4).

inracerer, Heinr., Director der Steinkohlengruben zu Escliweiler.

Gerrar, Di: med. und Apotlieker zu Laybach in Krain. Entomolog und Botaniker.

Tremer, Buchbiuder in Berlin. Mittel gegen Böcherläuse. (Entom. Zcitg. I., 62.)

Grape, Prappositus von Karasuando und Enontekis in Lappland. Description of eight new Species of Indian Butterflies, from the collection of general Hardivieke (Zool. Miscell. by Gray, 1831, 8.).

Gravenhorst, J. IJ. Clir., Di. med., gelieimer Regiernugsuath und Professor der Zoologie nud Director des Museums zu Breslau. VielherïlımIer Zoolug und Seliriftsteller. Iehmenmonolugia europaen. Pars I. Vratisliviac, sumtihus Autoris (Lipsiac apod L. Voss) 1829, XXXI el 827 Pagg. II. Tab. in 8 maj. Tomus 11. 989 Pagre. Tomus III, 1097 Pagg. - Monograplia Ichmeumonum perdestrium, pracmisso proemio de lransitu et mulabilitite specierum et variatorum. 8., Pagr. VIII. et 110. Lipsiae, opud Gösilien (Germar, Mag. III., p. 401). Monographia Iclusemonom pedemontanae regionis. (Men. Tor. Vol. 24 , p. $275-389$ ). - Monngraphia Coleopterorum micropternom. Gottingae typis Heurici Dictrich 1800, 8. I'ager. XVI., 236. 12 Pay. Ind. und Syn. 1 Tab. - Coleoptera microptera brunsvicensia nee non exoticostum quotquot exstant in collectionibus Entomolugorum Brumsvicensium, in Gener n familits et species distribuit Dr. I. I\% G. Graveulorst, Brunsvigiale, 1802,
8. r.XVF. und 206 Pagg. in gr. 8. Hellwigia, nov. ius. genus. (cum tab.) (Nov. art. soc, nat. cur. XI. P. II., 315 - 322). - Ueher die Gattung Stapliylinus. (Germar, Zeitschrift f. Entomolngic I. 1810, p. 210). - Verglcirhende Zoologic. Breslau, 1843, 8. 687 (Isis, $1843,627)$.

Tray, Georg Robert, Esq., Secretair der entomologieal Society in Loudon. Zoolog mud Srhriftsteller. - Insecta (Kings Sorvey to Austrulasia). The Eutomology of Australasia in at series of Monograplss. Part 1. Containing the Monograph of the Genus Pliasma. London, 1833, 4. (8 Taf. u. I 6 Arten. - Extatosoma, Trigonoderus, Podicanthus, Diura, Ctenomorpha, Bacteria (Latr.) et Bacillus). - Descriptions of several species of Autralian Phasmata (Trans. of ent. Soc. of Lond. 1836. p. 45, 1. - Faumus, Bd. I. neue Folege p. (2.1).

Ciñ rebe, Ielirer der Naturgesehichte in der Academic zu Eldena bei Gruifswatd.

Grecene, Copley, Dr. med. in Boston (Vercinigte Staaten).

Griftitlı, Edward, Esq. in Isondon. - Cuvier: the Animal Kingdom.

Gxibuofrsluy, in Georgiefsh (Goul. vernement (aneasus).

Gíries, die Bröder Jacobns und Johammes, L'rofessoren und Cloorlierren ules Bemedictinerordens zu St. Peter in Salzburg. Botaniker and allgeliende Zoologen.

CAmimer, Carl Heinr. Benj, pensionirter Wappen-Arhivar in Graez. Fleissiger Samuler und gründlieher Beobarhter. Sehricb sehon 1836 cil Werkchen über den Fang der Steycrischen Kerfe, allein es fander sich so wenig Theilnehmer, dass der Drock desselben bis jezt unterblicben ist, wo es denn unter dem T'itel: Steyermarks Colcopteren, mit Einhundert seelss meu besthriebenen Species, zu Gritz 1841 in 8. ersdijenen ist (bej Damialu I fi.). Gírolumanu, Franz, Naturalist in Palermo.

Grons, Rechungskammerrevisor in Wienbaden. Faltersanmlumg.

Groscumsu, Staltpifarrer in Wiudsheim (Franken). Sammelt die Käfer und Falter seimer Gegend mit Verstand und wissenschaftlicheou Streben. Entdeckte maneliesSelteue und Neue.

Grenloer, Mich., Concipist und Bau- 
Ingenieur in Wien. - Darstellung der forstsch ïllichen Insekten. Naeh ilıren Kennzeichen, ilurer Lebensökonomie und ihrer Selıädlichkeit; damn pract. Anleitung zu iltrer Vertilgung. I. Abtheil. (entlı. die dem Nadelloolz sehällieheu Arten), Wien, $1842,8$.

Crillis, Mariano, Professor der Medizin in Barecllona. Seluriftsteller und Entonolog.

Grundlach, Dr., in Cuba.

crumer, in Wien. LepidopterenSummler.

Puence, Achilles, Advokat in Paris (und in Chartres). Entomolog und Schriftsteller.

Guérin-Meneville, L. E., Professor etc. in I’aris (Place da Pantlicon No. 3). Gründer der Socicté cuverienue, des Magasin de Zoologie (reiclı all Meisterstijcken der Kunst und gediegenell Abliandlungeı), und der Ieonographie du Regue animal de Cuvier etc., geschmijckt mit Leistungen seiner Kiinstlerland. Viele Werke zoologischer Art zieren die letzterm. - Notice sur les Metamorplioses des Ceratopogons. Mugasin de Zoologie. Paris, 8. Annee I. 1831. (40 pl, enl.) Annee Il. 1832. (avec 59 pl.). Annce III. 1833 (av. 42 pl.). Annee IV. 1834. (av. $38 \mathrm{pl}$.). Annee V. 1839. (avee, 49 pl.). - Ueber cine Müeke, Bolitoplila einerea. (Annal. des Sejene. nat. X. 399. t. 18. f. t. 2. lsis 1834, 926). - Note topographi(gue sur quelques inseetes coléopteres, et Deseription de deux espices dis genres Badister et Bembidion. (Bullet. de la Soe. plitom. 1823, p. 121). - Genera des insectes, ou exposition ditaillèc de tous les eharactères propres a chaeun des genres et cette elasse l'animaux; par Mssr. E. Guciriu et A. Pereheron.6. Livr. A Paris clie\% Mèguignon ete. 8. (h $10 \frac{1}{4}$ Bogen 'Text und je 10 Kupfer von Delarue.) (a Livi. 6 Franes).

fiturtler, in Wipll. Sammlung.

Gulciardini, Pietro, Graf, in Florenz. Lepidopteren-Sammlıng.

Gnillnumé, in Paris, und abwedsselnd auch z.1 Cluteandun (Eure et Loire; Rue de Blois). Scliriftstelter.

Gumd Iach, J. Christ., Studiosus seientiarum naturalium zu Marbnrer (Kurhessen). Eutomolog und emsiger Sammler. Collection von Kerfen aller Ordnungen.

Cumdelach, Ferd. Willı, in Kas- sel.- Die Naturgeschiclite der Honigbienen, durelı langjührige Erfalırungen ermittelt. Kassel 1842, 8.

Gurlt, Dr. und Professor in Berlin. Guttuanu, Dr. ned. in Rutibor. Fussenbance, Cooperator in Treffling bei Mliilulstadt coder in Flattach) in Oberkïrnthen. Kiffersamnlung.

Hagen, Herm. Aug., in Königsberg. - Synonymia Libellularum europacorum. Regimonti $1840,4$.

Haiberg, Hermann Siegburg, Freiluer voll, in Clammeregre bci Chan (Nicderbayern). Kerfsammlung.

Iralidny, A.H. von Dublin, in London. Scluriftsteller. - Hymenoptera britannica, Lonclon 1839, 8., I, 16. (Isis 1840, p. 407).

Haltumeyer, Georg, Dr., I'rofessor und Cnstos am Johannemm zu Grätz.

Hamel, J., k. k. Staatsrath ete. in Petersburg. - Ueber Cochenille an Aramat und niber Wurzelrochenille im Allgemeinen (Minoires de l'Acad. de St. Petersb. Tom. 111, 2 P., Vle serie, 1834 , p. 9-65). - Naturhistorische Beunerkungen siber Wurzelcochenille im Vergleiclt zur Mexikaniscluell etc. von J. F. Brandt. (Lbond. p. 65-69, 'Tab. II.) - Ueber Oestius der Antilope Saiga (ebeudas.).

Hamuncerschnidt, C. L., Dr. Jur., k. k. IIofkanmer-ProkuraturBeinnteter etc. in Wien. Ploysiolog, Zoolog mel Scloriftstelles. Agronom. Ueber die Entwickelung der I'flanzenanswirclise durch lnseeten (250 Answioltse Isis 1834, 721). - Lebensart verschiedener Insectenlarven (1sis; 746). - Scolytus Founi (domest.) und seine Larve (mit rigentl). Blase am Nacken) (1sis 1834, 744). Monstrositäten von Carabicinen (Isis 183.1, 736). - Zwei nene Coleopteren (Latridius und P(ilium) (Isis 1834, 737). - Cecirlomyia Tritici et Bromi (1sis $1834,719)$.

Inampe, Dr, med, in Wien (Bancrnmarkt im lichtenliergiselien Hause).

Irandschuch, Cluristian, in Erlangen. Simmelte mit $\mathrm{K}$ iister in Dalmatien.

IInulea, in Itohenelbe am Fonse des Riesengebirges. Sammler und Händler. Durclustreift julurliclt das Riesengebirge fleissig, un Kerfe z.ı saunmeln. Olme niblere Kentuiss und blass auf eineu kleinen Eiwerb bei Licb- 
habern bedacht, findet man bei ilum immer eiuen Vorrath vou kerfeu aller Ordnumgen und Klassen wild durelcinandes gesteckt, aber mitunter manelses seltene Stiir.k : nnd Reiscunde, die il jene Grgent hommen, mögen ihn besuehen ( $\mathrm{D}$ eme l).

Enuslow, in Lonton.

Hanson, Samuel, Esं. in L,ondon.

Herdviche, Thomas, General. Major ele. in London. Zoolog nnd Reisender in Indirn. Seloriftsteller (1,iunein Tiansaetions). - Deseription of a new genus of hemipterous lusect Ptilocerus. - Observations on the loves ant the ants, and the Aphides; w. fig. (Zool. Journ. IV, 1828, 113',

Hexris, Tl. W., Bibliotlukar an der Harvard-Universität in Newlaven. - Besthreibung des Verzeichnisses der nordamerikanischen Schwälmur. (T'he american. Journal of Stience and arts by Brnjamin Silliman. New-Haven 8. 13d. XXXVI, "1. 74, Juli 1839 , p. 282.)

Tharis, T. W., Naturalist in Boston. Siluritisteller. - Upon the ceonomy of some American Speeies of Hispa. (Boslou Journal of natural History. P. 1. - No. 11, 1835, p. 141, ॥. K.).

Hertensclucider, Udalrieus, Vorstelier der Stiftsbibliothek uml k. o. ö. Professor an der philosophiselhen Leluranstalt z.u Kloster Kremsmünst('y.

Eartig, Th., Dr., Forstrath und Profissor in Braunscluweig. - Zweiter Naehtrag zur Naturgesthiehte der Gall. wesple (zu Germar's, Zeitstlır. Bd. Il, IIft. I, p. 176-209, Bu. III, Hft. 11, p. 322-358). (Germar's Zeitsehr. IV, II, 1. 395-423\%. - Ueber verschiodene Kerfe, vorketragen in der neunten Versammlung des naturwissenselaftlielien Vereines des IIarzes zu Blankeuburg (lsis 1839 , p. 666). - Hymenopterulo. gisclue Mittheilungen (Ent. Zeitg. 1, 19: Blattwespon). - Ucher die Familie der Gallwespen (Grmar, Zeitsclurift tür Eutomologie II, 1840, p. 176).

Inatliel, W., zu Militseh in Schle. sicu.

IInrtmann, in St. Gallen. Schönc Collection schweizeriseher Kerfr.

Harzer, August, in Dresden. Guter Kerfzeidher (zu dem mikrolepidopterulogisilien Wo ake des II. Fischer von Risslerstanm), cifriger Cullector und Siluriftsteller. - Der kleine Sirlumetteslingsjäger, 1828. 12.
Mnazer, C. A. F., NaturalienMaler und Kupferstecher zu Dresden.

Houf, W., Dr. M., Redakteur des Morgenblattes in Stuttgart. Berïlomt Uurelı selor gediegene Arbeiten.

Duulon rt, in Basel - Der Ameisenkrieg (Zeitsehrift der Basler-Hochsthule $1825,62)$.

Haupt, in Ekuterinoslawl. Entomolog.

Ilawpt, Dr. phil. und Inspektor der Nituralic'nsanmlung (Linderischen Stif. tung) in Bamberg. Coleopterolog.

Faupt, in Lodenan (Srhlesien). - Wer legi die Eier zo den Drolnen? (Abhamll. der naturforsthenden Gesell. schaft zu Gürlitz, Bıl. 1II, Hft. J, 1840, p. 17. - Isis 1842, p. 697). - Auf welche Weise wird das Schwärmen der Bienen am sichersten befordert? (Ebendas., wo im But. II, Hft. 1, 1836 aurh Fiedrich Schlüter's Abhandlung ïber Ovisugen der Insektenwelt vol:kommt.)

Hauschikn, Apotheker zu Essex in Ungarn. Coleopteren - und Faltersammluing.

Fansmann, J. F. L., Professor in Göttingen. Srhriftsteller.

Heath, T. M., Naturforscher in: Nadras.

Heclit, I. A., Dr. med, in Stralsund. Hübsehe Sammlung norddeut. scher Kiffer.

Heeger, E. Maristratsrath in Mödling bei Wien. Begann die Käfersippen in Figuren herauszugeben, vou denen ziemlich gelungene vorliegen. licitrïge \%ur Selmetterlingskunde oder Abbildungen und Besehreibungen neuer sizilianisclier Sclimetterlinge. Wien 1838.

Itecr, Oswald, Prof. der Naturgeschichle auf der Universität und Conservator uler Esilher - Zollikofer'sehen Kerfánunlung in Zirrich. Kerfploysiolog und Finuist. - Fauna Coleopterorum lielvetien. Pars 1. 1. Turiei 1838, 8. (bis jetzt 2 Bänle fertig bis zum Ende (lsis 1839,71$)$. - Observationes entomologicae, continentes metamorphoses coleopterorum nommullorum adlue ineognitas. Turici $1836,8.36,6$ Tab. (Isis 1839, p. 71). - Dic Kïfer der Scliwciz, mit besonterer Berïtksichtiyumg ilırer geographiselı'" Verbreitung. i. Thl. 1. U. 2. Lief. Neuchatel 183739, 4. (Lul. Zeit. 1, 104). - Anfiage über die Maikäferflugjalre (Ent. Zeit. 11, 142). - Zur grogr. Verbreitung 
der Sehweizer Käfer (Ent. Zeitg. 1, 109). - Ueber geogr. Verbr. der Aphodien der Alpen (Ent. Zeite. I, 110).-Ueber Vertreibung and Vertiluming dev Laubkifer und Inger, Zürieh, 1843, 8. (3 Bogell.)

Ilefuer, Joseph vou, Dr. pliilos. und Gymnasialprofessor in Minchen. Der bekannte Seliriftsteller. Sammelt.

Legetscliweiler, Joh. Jikob, med. Dr. und Arzt in Riffersweil (Cantou Ziiriels). - Dic Verwiistınıen der Obstbäume durch Insekten betreffend. (Denkschr. der Seliweiz. Ges. p. 65137, Ziirich 1829, I, 1 \%

Hegewitscli, E. D. H., in Hannover.

Meidenreiclu, Superintendent in Weissenfels a. d. S. (Herz. Saclosen) Lepidopteren.

Mell, Alex., D. Pliilos. und Custos am k. Naturalienkabinet in Münclıeu. Selur fleissiger und grïndlicher Beobachter.

Meld, D. med. in Mïnchen. Melır Concholug. Solm des genanuten Zoologen.

Melfer, J. G., med. Dr. in Smyrna (friiher zu l'rag). Entomolog. - Nova species curopaca Trichidum (Anmal. soc. ell. frnnce $11,1833,495$, fig. col.).

melmoance, Lithograph, in Mïnchen (Lerchenstrasse). Simmler. Miteht hübscle Abbildıngen von Kül'eru.

Aemanerich, Nituralist in Berliu (?). Sammelte in Denerary.

Menming, Joh., k. k. Hof hirurgus und Kollegienrath in Yetersburg. Entomolog.

Mentz, N. M., in Philadelplia. Remarks on the use of the Maxillae ii Coleopterous Insects, with an Account of two species of the family 'Telephoridae, and of thice of the family Mordellidac, whiel ought to be the type of two distinct generi (Trans. of PlitJid. III, 1830, j. 458-d(i1). - Deseription of eleven new speeies of NortloAmerican Insects ('Trans. of Plibad. JiI, II. ser. 1830, 1) 253-59. Plat. 2). - Description of new spreies of lnsectes (Coleopteris) of North-America. (Journ. of Aead. Philad. V, 373, Febr. $18 \pm 7$, fig.).

Texing, Carl Eduard, Apotheker, Clemiker und Entomolno 21 Kleinroto-

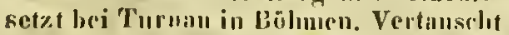
und verkanfi unginliselle und sinddentsclo Kerfe, Lizicht Falter aus Raupen. etering, Professor in Stettin. Beitriege zur lusekten-Fauna Pommerns. Die poumerschen Falter (Ent. Zeitg. I, 146 et seq.). - Die Falter Prenssens (Ent. Zeitg. I, 52-54): Mittleiluıgeu aus den Gebiete der Lepidopterologic (Eut. Zeit. I, 13). - Xylina somniculosa (Ent. Zeitur. II, 165).

Meranes, Candidat (1836), z11 Burby an der Saale.

Merold, Manritius, Dr. med. und Prot. der Naturueschirlite und Pliysik an der Universität zu Marburg. Schriftsteller. - Eutwicklungspescliclite der Selonetterlinge; inatomiseh und physiologiseh bearbeitet. Mlit 33 illum. und schw. Kupf. Cassel und Marbure 1815. - Untersuchungen iber die Bildungseseschichte der wirbellosen Thiere im Eie. 1. Th, Von Erzeng. der Spinne im Eie. M. 4 Kjft. (Dent. und Lat.) Majburg 1824. fol. 2. Lieferumg. Frankf. 1840, mit Tab. VI-X und XiV fol. - id. latine. Francof. 1835, fol. - I'lyssiologisclae Untersuclunngen über daskiïckengefüss der Insekten (Scluriften der Ges. zll Marburg 1823. 1. Bd. 41). - Disquisitiones de animalium vertebris carentium in ov o formatious. Franeof. II, 1838, Fol. t. 6 (Isis 1839, p. 231-240; Recension).

Iterrich-Gchä trex, G. A. W., Dr. med. und Stadtgericlitsarzt zu Regensburg. Braelute durelı Kanf die Summlung des sel. Jenison-Walworth an sirh, die sonst ein Theil der Gysseleniselien gewesen. Verkauft europäische IKerfe. Entomolog und Selıriftsteller. - Panzeri fanna insectormm germaniae (Fortsetzung seit Heft 86; bis jezt sind 186 Liefermugen erschienen). - Nomenclator entomolugicus. Verzcichniss der europiziselien lusekten; zur Eileichterung des Tauschverkelurs mit Preisen verselsen. l. Heft 1834 (Isepisoptera et Rhyogota) II. Heft 1840 (Coleoptern, Orthoptera, Dermatoptera et Hymenoptera). Mit 8 litlı. Taf(c)n. 8.

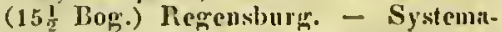
tische Bearbeitung der Srlmetterlinge von Europa, als 'Text, Revision und Supplement zu J. Hiibners Summlung europäischer Schmetterlinge. 1. und 2. IIeft mit 20 illum. IKupfertaf. vou C. Geyer. gr. 4. Regensburg 1843. Fortsetzung der Halbu'schen IVauzenwerkes. - F ï rorolı. Topmernhie der Rogensburger Gonend. (Verzeichuiss det animalia articulata.) 
Merrick, Edward, C., in NewHaven (Nordamerika).

Werturig. Dr. med. in Berlin. Ueber die Bremsenlarven in Magen der Pferde, von Dr. Nımanu, aus deo Holländischen ïbersetzt. Berlin 1837, 8., 140, T. 2, ill. (lsis 1840, p. 301 ).

Heyden, C. H. G. v., senator der freieu Stitd Frankfurt (a. M.) und Direktor der Senkenberg'sehen naturfurselienden Gesellschaft daselbst. Coryphitee der Entomologie. - Extrait del' onvrage de P. F. Bonche sur les Insectes, utiles et nuisibles (Revue ent. II, $1834,47)$; nnd viel Anderes des interessantesten Inhalts.

Meyer, F. C., Stadtsehreiber in Lin ehurg. tentomolog. Sammlung curopiiselier Kerfe.

IEeyshan, F. C., in London.

Hildebrandt, Professor in Diisscldoif.

Minciss, Joh., in Prag. Kerfsammler.

Miss, Pastor in Gsteig hei Sarnen (Stliweiz). - Selirieb über SehweizerLepidopteren in der Revue cntomologique.

Hobliouse, John Lam., in London. - Some accuint of a Juurney into Albania, Romelia, and otlier provinces of Turkey in 1809 and 1810 . London 1812. 4.

Elochut, ans Cassel, Inspeltor bei dem hotan. Garten zn Kiew. Ornithologische und chtomologische Sammlungen.

I Fibtzer, Förster zu Kleinschmalkalden.

Hipfner, grosslı. hess. Oberappelations-Gerielitsrath in Darmstadt. Schlienkte seine kostbare Sammlung dem naturhistorisiben Kabinet daselbst.

Moeven, J. van der, Prof: in Rotterdam. - Neuer Unterseheidungscharakter fïr die Libellulen und Aschnen. (Amnal. d. sc. nat. XV, 1828, p. 423 , t. 11. Bd. - Isis 1834, 1037). Sur les erochets des ailes rhez les Splinx et les Plaulèues, av. fig. (Bijdragen tot de natuurkund. Vitensila. Jl, 1. 2, 273). - Remarques sur l'organisation interne du Taupe-grillon (ibid. V, eal. I, 99). - Systematische Beschryving van eenige Inseeten van Noord-Nederland, 27, 8.40. - Oratio de aueta et cmendata Zoologia post Jinnaei temporil. Lugduoi 184'2, 8. beschrribung von Bupestris Duleoi,
Papilio Payeni und Coliast Verhuelli. (Tydschrift for natuurlike Geseliedenis, nitgegeven door J. van der Hoeven en W. H. de Vriese. Amsterd. 1838, Bd. V, p. 334.) - Einige geschichtliche Bemerkungen ïber Filter mit Raupenkijpfen (Tydssehrift for natuurlike Geschiedenis en Plyssiologie, dor J. van der Hueven en W. H. ile Vriese. Leyden VII, 1840, Ileft 1-4, Taf, 7, VIII, 18.11, p 271). - Beschreibung einiger nener oder wenig bekannter Falter (lbid. p. 276, 'Taf. $5-7$ ill.).

Mofruamu, Nafuralist aus Ham. bure, io Paris (Quai des grands Augustius No. 19). Verkauft billig.

Hofmanu, Dr. med. zu Meissenheim a. Rhein. Entomolog.

Moffmannsegg, J. C., Graf vou, auf Rammenan bei Dresden. Reisender und Entomolog, dessen selbat gesammelten Schälze grösstentheils aus Portugal, Spanien und den Pyrenäen, im Berliner-Musem sich befunden. (Vgl. Illigers Magazin). Der Graf ver. kauft Jinamisehe Käfer. - Veber das Leucliten der Fulgoren. (Magaz. d. nit: Fr. zu Berlin 1807, I, 1. 152-156.)

Iofrmeister, Pfarrer zu Kleinschomalkalden. Stifter des 1837 ins Leben getretenen entomulag. Tanselsvereius zu Ascluersleben (ähulieh dem botanischen zu Erfurt).

ISolnewvarti, Franz, Graf von, k. k. Kämumerer und pensionirter Gubernialiath zu Laybael.

Hollandre, Bibliotheknr zu Metz. Sammlun von 2800 Speries ellropiiseher Käfer und 2000 Species Falter.

Moleme, in London.

Holzer, Joseph, k. k. Finanz. Wach-Oberkommissïr in Marbura (Steyermark).

Ifombron, Chirurg in Paris, Der Bearbeiter Jer Zuolngie (mit Jaequinot) in dem Werke vou Dumont d'Urville: Voyage an pole Sud et dans l'Orcinie, sur les Corvettes l'Astrolabe et la Zilee, (wellole Reise er mitmachte).

Dominornt, Postdirektor zu Digne (Dept. des Basses-Alpes).

Iooler, J. Esq., Dr. med. zu Glaserow. Entomolog.

rope, F. W., Esq. et Rev., iuLondon (Upper Seymour Street, Purtmann S(quarre), Vicepriisident der entomolorisclien Gesellschaft daselbst. Ungebure Sammlumg vou Coleopteren und Hemipteren. Sihriftstellerund Reisinder. 
- Deseriptions of some listhertloo uncharicte rized exotic Colenpteri, iljefly frou New-IIolland. (Trans of ent. Soc. of Joudon 1, p. 7.) - Synopsis of Nepanl Coleoptera. - Desideratil in aler judischen Entomologie. (Calcutta-Joumul 1, 1840, p. 61.) - Monograph of the Elateridus genus Camposteruns (Ammals and Magazine of natural history. Vol. 8, n. 53 : Febr. 1842 , p. $953-54$. Zusiitye hiez.川 gab Germar: Zeitschr. IV, $99-189)$.

Hoppe, David Heinrich, Dr. phil., Prof. "tc., in Regenshurg. Botaniker und Schriftsteller. - Enumerstio insectorum elytrutormm cirea Erlankam indigenorum see. syst. Fubr. Erlang. $1795, \mathrm{gr} .8$.

Moruschnch, F., Dr. med., Prof. und Dilektor des botanisclien Gartens z.n Grejfswald. Botaniker mod Schuftsteller. - Insecta alpina (Actal leopoldina. Mit Hoppe besclurielurm).

Hornung, E. G., Apotheker in Aschersleben. Eiuer des Mitstifter des entomolog. Tauseluvereins. - Auregung zur aufmerksamen Beobachtung kler in und wuter der Rinde der Bäume lebenden Insckten. (Ent. Zeitg. I, 16:) Rliynchites honguricus Fubr. (Ent. Ztg. I, 62). - Ueber einige in don Betclniissen vorkommende Käfer. (Ent. Ztg. 111, 114.)

Horsfheld, Thomas, med. Dr. in Juondon (ostind. IIans). Eutumolog und Schriftsteller. - A descriptive Catalogue of the Lepidopterous Inspets, contained in the Aluseum of the Honourable East India Company, illustrated by eolonred figures of new species and of the Metamorpliosis of Indian Lepidoptera: with introductory observations on i general Arrangement of this order of Inserts 4. pp. \&0, Jlates. IV.

Honndud, in Montpellier. SamnJuın.

Finwit, Enfomolog, in Juondan.

IInber, Fr., in Lansatnue. Be rïlımter entomologiselier Seluriftsteller. Peelerehes sur les moeurs des fourmis. 18t0. - New observitions on the natural history of bees 3. edition 12. Ediubourg, Lognan.

IInbere, Peter, in Genf. Brrïlumter Forselier. - Ilistoise du Trachusa dori: (Apis aurulputil P'ulycri). (Mcin. de Gincs. II, 1, 1\{23, 1. 145-161. aver lig.)

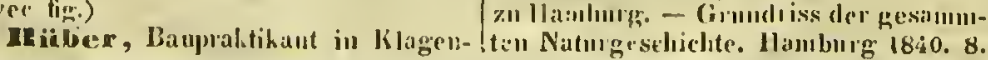

EnauboIdt, A lexander Freiherr voll, W. . Rath, Excellenz, in Berliu. Diluchlie, E., Dr. ph. Ind Prof. zo Jena. - Réde iibre den Lifluss de. Nafnrwissenselaften bei Uebernalımo dles Protectoruts an der Univ. z॥. Jüla (6. Ang.) $18+2$, Leipzig.

IIgen, C., Pfarrer 2.1 Naurod in Nassall.

Henlioff, Lndw., med. Dr. in Basel. Hymenopterolog nud Selurifisteller. Singulorum generum Curculionidum unam alteramve speciem alditis iconjbus a David Lahram ad naturam delineatis illustravit (Lat. et geımaniee) Bascl, 1838. 8. - Samunlung scliwcizeriselier lusckten. In bjeferungen. Entomologica (de lymenopteris: - lsis 1832 , p. 1198,1834 , 1. 370). - P»ppenluïlle von Bombylius mijor, aufgefinden und besehr. (Isis 1834, B36, Tif. XII.)

Ingpen, A., in London. - Instruetiuns for eollecting, rearing and prekerving british Inseets. i.ondon, 1827, 10. fig. col.

Isensser:, Emil, Dr., Hofrath, Prof. ete. z.u Berlin. Die Geschisclute der Medirin und ibrer Iliilfswissensilaftem. 11. Thlı. t. Abtheil. Die Nuturwissenselaften. Berlin 1842, 8.

Jnckson, James-Gray, in Loudon. - All Areonint of the cmpire of Maroceo and the district of Suez etc. Londou $1809,4$.

Jucoloson, Jac., Pastor in Kopenlalgell. - De platacna noctua gamma. Regium. 1829.

Srequin, Dr. med. in Wien. Solın des sel. hocligefrierten Pliytognosten. Botaniker und Keıfkenuer.

Jnequinot, Marine-Clirurg zu Paris (vel. Ilombrou).

Jickel, J., Stud. theol., in Nürnberg. Kennt vielc Kïfer gut und sammelt fleissig. Zejehnet Kerfe wunderschön.

Jiiger, G. F., Hofrath ind Professor in Stuttgart. - Upber den iclativen Wortl der Naturvissensclafien fiil dje formelle Bildung der Jugend. Festrede. Stuttgat 18t1. 8.

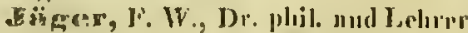


Jizer, Benediti, in Petersburg. Sammier.

Jiigeri, in Kranchthal, bei Bern. Bunseln, C., Kanzlist \% Breslau. Jnu, G., Professor in Misiland. Entumolog. Tanselt mud verkanft Na inralien. - Catalogo dej iusctti coleotteri. -

Janer, Dr, Professor der Medicin in Barcelona.

Inquier, Militionelirurg in Caycuine. Sammiclt.

Jardine, Sir William, in London. - Naturgeschichitlidies Kabinet des Thierreiches, VIII. Entomologie. I. Thl. - Anch unter dem 'litel: Naturgeschichte der curopäisclien Schmetterlinge. 1. Tagfolter. Bearbeitet vom Ritter mind Naturtorscher Fr. Trritscluke. Pesth 1840, 8. mit 34 Kupfert.

Javet, Curl, Zeidher in Paris. Tiirlutiger, fleissiger Sammler, der die Sache mit liebe betreibt.

Jenisou, Graf von, Major des $\mathrm{Cu}_{1}$ vallevie, im Dienste des Herrogs von Ilessen, zu Pbiladelphia. Samunler.

Jensen, Henning in Rign (?). Nova dipterorum genera (Bullet. - 124 de MosenII ed. Jeq. IV, 1832, p. 145).

Jenyus, Lconlanrd, Rer, in London.

Jeru, in Iprwich. - The Butterfly collectors vade mecum, or syuop)tieal table of english Butterflies. Ipswich 1824, 12. edit, 2. 1827.

Johnun, Seine kaiserliche Hoheit der durchlaurhligste Erzherzog von Oesterrei h ete., der Grüuler des pachtvalleu Johiuneum zu Grätz, il Gant\% (Steyermalk).

Jenes, William, in Dublin (?). A new urangement of Papilious. (Linn. Trans. II, p. I.)

Yordano, Don Ignazio, in der Abtey von Publet in Barcelona. Sanmelt mit Eifer.

Jordt, llans, zu Katharinenhof bei Finushurg:

Domsseliu, Louis Emanuel Graf vou, Cnvalleric-Capitiu zu Versailles. Besitzt cine selır strhöne Sammlung.

Inlie:u, Stauislans, l'rofessor der chinesisclicon Spraclie am College de Fonee z.ll l'aris. Der Uchertrager des Chinesischen Werkes: Ueber Miulherrhammzuclst und Erzichung der Seidenraupen deutselı vou live Ludw. LiudHer auf Belchl Sr. Maj. des lionigs rou Wintemberg. Stutgart und T'ibing. 1837, M. 1 Kıрा 8.).
Junler, F. C. , Oberfinanzhanzlist in Kassol (sonst in Hanum). Epilarhun chrysoneliua, Jeren Nahromg und Furtphanzung. (E). Zeitg. I, 2.) - Ordicstes querens ls. und dessen Volkommen (Ent. Zeitg. 111, 63).

Jnrine, Scbastian, Suln des beriilumten Nannes gleich. $N$ in Genf Besitzt eine sehöne Simmlung.

Knas, N., Staatsminister in Kopenhainell.

Waden, Director in Dresden. Besit\%t ciure ausgezciclunct reicle SammIma Lepidopteren mind Coleopteren und Vïgel.

Enitendouch, J. H., Lelier zu Aacheu. - Mnuographic der Familien der Pflanzenlïuse (Pliytnulhthires). M. Abbild. Aaclien 1836, s.

If burg.

Innold, in Altenburg. - Den Obstbaiumen schödlieder Insekten (Mittheilangen aus dem Osterlande, 1837).

IXnpp, IIofruth, in Heidelberg. Sammelt mit Lust Falter.

Anreliue, in Orenburg. Entomolog, Reisender und Sichriftsteller. Catalogne des Carabiques du Proviue Mazenderan d'Astrubat redigée par Baron W. de Cla a doir: Bulletin des Natural. de Moscoul 1842, N. IV). Catalogne des roleopteres pris dans les Steppes des Kirgises, mitre le Vnlga et I'Ounal (Bullet. Mose. ed. leq. p. 45-47 (nduexis pretiis) 1829).

Inudfuss, Dr. med. in Halle. Voryingliclı Botaniker.

I aup, Dr. plijos. mod Custns am zonlogis(ben Musenu in Darmstadt.

- Dis Thierrech in scinen Hanptfoll men. Darmst. Bd. III, 2.

Deferstein, Gericlitsuth in Erfurt. - Selur hedentemde Sammlungen von Faltern. Fiante vor vinigen Jahren die Zschorn'sedie Samnlung. Naturgeschichte der sebindlichen Insekten, mach eigenco und fremden Er. falorumgen. I. Thlıl. 8. Eifurt 1837. U'cber den ummittelbaren Nutzen der Inscliten. Erfirt 1827, 4. - Note 2.11 Stephens Catalog (Revie ent. 1, 1833, 135) und m.1 Kollar's (ihid. 190). Nites sur les momis de eluclques leepidoptires du Briesil (Revue ent. I, $1833,243)$. - Ohservations sur le Cureulio grunarius (Revue cut. 11, 1834, 115). - Fugen an dus cut. Pubikum 
(eut. Ztg. JII, 90, Jepidopt. Honie.). - Miselia jaspidea Douz. (ent. Zeitg. 111, 215). - Flïchtige Bemerkungen iber Buisdural's Genera und ludex meth. Eiı. Lepillopiterorm, 1840 (Ent. Zig. J, 166).

Ke:Iele, Oberlelurer om Gymuasium in Ratibor: - Paorung zwisclien Meloloutha vulgaris und hippocastani (Isis 1834, 737).

Meller, Adolph, Kaufuaun in Reutlingen. - Ein eifiger Sammler; maclite diesen Sommer desslaalb dic Reise nach Oestreich, Steyermark und Italien. Jat moist Schmetterlinge.

Hellner, A., herogl. sächs. Goth. Revierföster zu Finsterbergen, im Thüringer-Walde. - Ueber Vorkommen seltenur dentscher Käfer im 'llıüinger Walde (Ent. Zeitselır. II, 128 ).

Herl, Joseph, Kaufmann im Markt Wolfrathshansen (Landger. gl. N.) in Oberboyern. Sammlung von tyroler und bayrisch-alpineu Kiffrrn.

Hiloustine, in Moskall. Entomoleg.

If idal, John, in Loudon (?). - Alatomy of Giyllotalpa (Philos. Transact. 1825, part. 2, 283, pl. 15).

Iriesenwetter, H. v., Cand. Jur. in Lejprig (kleiue Fleischergosse 242). Selur griudlicher Culeopteralog, voll dem wir nus, woferu ilun seine Verhältuisse nicht oblualteu, vicl versprechell diisfen. Er sammelt die kleiuen Käfer, vorziglich die Brachelytren mit grossem Fleiss und vieler Uinsicht und hesucht die Salzsece'u bei Halle ofter in Jahr. - Beitriige zur Monographie der Gattung Heterocerus (Gemar's Zeitschr. IV, II, 194-224, Taf. 1II). - Ueber den Colymbetes cousputus, stum ('nt. Zeitg. III, 88).

Hindermnnn, Adalhert, Vater und Solın, Keıflü̈ndler in Ofen (Clıj. stiauen-Vorstadt, Siliöpfungsgasse No. 105). Bereisten Russlaud, Syrumien u. 8. w. Solur zu empfelileu.

Mirby, Willion, Rector von Barlanm, in England. Eerilimter Entomolog und Seluiftsteller. - (mit Spence) Elements of Entomology. - Sitten Jer Thiere (Bridgevater Stiftung). - Moun. grophio A pum Auglise 1802, P. I, XXII. Pagn. Vorr, 112 Pagm. Einl.: II. (deutsch von Illiger. - Ipswielı Magaz. V, 1806 , 28). - Deseription de quelquesinsectes qui servent a devellopper la doctrill de
M. Williau Mac-Lray sur Jes offinités et anologies des insertes et des champiguons (Bullet. d. sc. nat. Mals 1823). - A description of some colcopterous Inscets in the collection of the Rev. Hope (1 pl.) (zuol. Juul'u. JII, 1828, 520). - The raracters of Clinidimm a new geuts of Inseres in the order enleoptera (fig. cal.) (Vigors Zoul. Jouru. V, $1828-29,6)$. - Strepsiptera a new order of Inseets ete. (Linu. Transact. $X I, I, 85$, III, tab. VI, VII ; 8 (Bilttungen). - Century of Insects, includiun several new genera efe. (ihis. XII, $375, n$. XXVIJ). - The claracters of Otiocerus and Anotia, two new genera of hemipterous insects (Ciradidae); (ibid. XIII. 12-24, IJ, Tab. 2.) (Eine genane Anatomic der äusseru Organe stellt die schöngestochene'lafel vor.) - A history of three Species of Cassida (ibid. III, 7, 11. 3). - Ammophila, a new geums of Iusects Hymenoptero ete. (ilid. IV, 195, t. 19). - History of Tipula Tri. tici and Ieluneumon Tipulae etc. (ibid. 230 ef $107,1.4-7$ ). - Some obser: vatious upon Insects that prey upou Timher, with a short history of the Cerambyx violareus (ibid. V, n. XXVI, p. 246). - The Genus Apion etr. (ibid. IX, 1, n. I, Tab. I). - Descriptious of seven new species Apion (ilid. $X$, n. XV, 347). - Loftre explicotive de de la mote sur la structure des tarses des coléopteres tetrameres et trimeres - Philosophical Magaz. Apr. 1825, 267). - Sur la strueture des tarses des coléciptères tetromeres et trimeres des entomologistes f'rançoises (Philosopl. Mayraz. Mars 1825, 193). - Some remarks on the nomenelature of the Grylliua of Macleay (Vigors, Zoolog. Journ. 1, 429). - Some further remarks on the nomenclature of Ortloptera, with a deseription of the genus Scaphura (w. 1 il. eol.): (Vigrors zool. Jouln. II, 1821-26,9). - A brief Jescription of o puir of remarkable hormed mandibules of an Jusects. e. fig. (ibid. n. 5 . Apr. 1825, 20). - A description of I wo new species of coleopterous Insects belonging to the Genero Cremastorlieilus and Priocera (ibid. II, 1825-26, 516). - A deseription of some new Genera and Species of Petalocerous Colcoptern. (PI. eol.) (ibid. III, 1827, 145) - Ueber Thiere, welelie sich vou mineraliselier Substauz e rnililireu. (Pliilos. Magoziue Vol. 6t, 1823, 3; 
Dermestes vulpiuus im Asbesth, anch in Mlumien; durchwüblet den Amianth.)

Mercher, Apotheler in Butyen. Besizt die Kerfe der Fauma um Botzen.

In ittel, Baldnin, Dr; mell, Professor der Chemie in Aschaffeuburg. Botamiker und Selıriftsteller. - Mémoire sur les Purerons (Amul, de la soc. limm. de Paris. Mni 1826. t. V. 133).

I irkup, in London. - Account of Tenebrio manritanicus (Trans. soc. 'utom. Lond. 1812, 329).

Clingel afer, Oberlicutenant, in Darunstadt. Einer der fleissigsten unt gründlichsten Sammler und cin schu fiederer Mann. Er besizt in den rillzeluen Fangmethuden selir viel Praxis und eigenen Erfindungsgeist: $\mathrm{cr}$ hat unter Anderem in seiner Gegend Stromatium strepens Nachts mit der La. terue in meluen Exemplaren gesammelt. Nur Käfer ist der Iulaalt sciucr Silmomlungell.

IIopselh, J. E., Gymmasial-I.ehrer in Breslun. Isepidopterolog unt Seleriftsteller. - Dir Entomulagic z.ur Zcit des A ristoteles (Isis 1834, 744).

Klug, Friedr., Dr. med., Medizi. nalentlı, Director des k. Museums tler Naturgesthichte ete. in Berlin. Hnchverdienter Entomulog und Schriftsteller. - Bemerkungen bei ter Zerglietlerung cimes Zwitters der Melitaen dillyma nelst der Breselureibung der Zwittrim Berliner Museum. (Verhandl. der Ges. naturforseliender Frde. Berliu, l. Hit. VI. 1829, 363. 'Taf. 15). - Procopia, novim Inscrtorum Orthopterorum gemis. Berul. fol, 2 tab. tol. - Versuch ciner Berieltigung der Fabrieins'sclien Gattungen Scolia und Tiplia (Weber und Molır's Buitr, z. Nat. I. p. 8-41. lI. p. 167-217. - Pterocheilus, cine mene lnsekten.Gattung ans ter Klasse der Pimaten (Weher und Mloh's Beitr. 7. Nat. I. p. 143-157. Tal. 3. f. 1-3). - Absonterung einiger Raupentödter unt Vereinigung derselhen zu cines newen Gattung (New. Sibr. der Berl. Ges. Naturf' Fille. III., p. 555-67). Finige neue Piezatengattungen (Mag. d. G. N. Fr: in Berlin, IV. (1810) p. 31-16. Tol. I). - Versul'h ciuer systematisclien Feststellung ter Familie Panorpatae (Abhandl. d. Akudenic d. Wissenseh. zu Berlin, a. d. J. 1835. Berl. 1838, 4. p. 81. Taf. I.). - Speriıs A pranialum fomiliale novas, descripsit, gencrumyjue characteres adje- gensb. 1833-43.16. (a Huft 24 lith. Kpf.). rit Dr. Fr. Klng (Mag, do Ges. Nat. Fr. zu Burlin I. P. 26:3-65. Tab. VII. f. $2-5$ ). - Oxaca, cinc neue Gittung ansdir Ordnumg dir Piczaten. Von Dr. Fr. K l I g. (Mag. der maturt. Ges. zu Birl, l. p. $261-63$. Tab. VIl. f. 1 a. -r.). - Ueber die Gesilulectitsversehientenheit der Pirzaten. Von Dr. Fr. Klug. Erste Hälfte der Fabrie. Gattungen. (Magaz. ler Ges. nat. Fr. z.1 Berl. I. 1807, 1. 68-81). Andere Hälfte Il.(1807) (ib. S. 18-63.) - Dic europïischen Arten der Insecten-Gattung Lenicospis. Näher liestimmt vou Dr. KI ॥g. (Mag. d. G. wat. Frde. in Berlin vI. p $65-71$. Monograplia Siricum Germnniae atque generum illis anmumeratarum, auctore Dr. Fr. K Ing. Cum tahulis aencis coloratis VIll. Beroliui impensis Fr. Schuippcl, 1803. XIl. et 64 Pagg., 4. - Die Blattwespen mach iloren Gattungen mud A rten zusammengestellt. Vou Dr. Fr. K Ing. (Mıg. d. Ges. mat. Fr. zil Berlin II., j. 261-287, Tab. VIl.-Bd. VI. p. $45-62$ et p. $236-311$, Vll., p. 120 -132 , vill., p. $\$ 2-84$ et $110-144$, 179-219, 273-308.) - Versileli ciner Diıstellung ler Familien umil Arten der Blattwespengattung Cimbex, Fabr. Vou Dr. Fr. KIng. (Verhamil. der Berl. Ges. I., 18:9, 1.. $71-98)$. Nacbricht von einem neuen Silmaroxerinsekt auf ciner Andrene. Von K Ing. (Mag. d. Ges. nat. Fr. zu Berlii IV., p. $266-70)$. - Ein neller nerkwïrdiger Henops, besclirichen vou Dr. Fr. K ling. (Mag. nat. Ges. zu Berl. I. p. 265-267, Tal. VIl, f. 6. Henops Waxelii: Diptermun.).

Indrrleiu, Jos., BaudircktionsPruktiknnt in Linz. (Hauptplatz Nu. 218). Bedentende Sammlungen von Käfern und Faltern. Eifriger Sammler; durehfurschte die Donau-Gegenden, Salaburg cli:

Funflumel, Cand. phil. in Botzen. Sammelt Käfer.

Kocle, Karl, Dr. phil. in Ienn. Fauma vou 'I lin̈ringen umel den angräilzenden Provinzen. l. Sueke (mit Origiunlzcielmumpru von Dr. Ermst Silhenk.) Heft I. II. itc. m. \& col. Alib. Jena, 1841,8 . (Die wirbellosen Thiere fol. geu nath.)

Koclı, K. L., pension. Forstrath in Regensburg. Tiichtiger Zoolug. und Schrifisteller. - Deutichlands Crustacecn. Myriapoden und Amatmiden. Re- 
- Die Aracluniden. Gelreu naelı der $\mathrm{Na-}$ tur abgelbildet. Fortsetzung des Hahu'schen WVokes. Nïrubg. (Hahu gab 2 bile. $1831-34 .-18$ il. licraus).

Moch, Fr., Hofökonom in Stuttgart.

Kächdia, in Miilllatusen. Hat pine shöne Sammlnug. - Correspondence futomologiene (sur Lucanns Cervus, Limı. (Mïlilhuıser, Rissler, 18:3.)

Id bliler, Schullehrer zu Sclumiedeberg in Schlesien. Éutomolog.

Kalleker, Albert, Dr: med. in Ziin ich. - Briträge zur Kcuntuiss der (ieselulechtsverhältuisse und der Siamenllissigkeit wirbelloser Thiere, nebst einem Versuch äber das Wesen und die Bedeutung der sogenamuten Saamenthiere. M. 3 Kulf. Berlin, t84t, 9. - Observationes de puima insectorum genesi adjecta articulatormu evolutionis cum vertebrintorum eompratione. 'Turici (Meyer), 1812, 4. 31. tab. 3. (Isis $1843,74)$.

Kdonig, Rector mul Proliessor der Oelonomie mil Naturgesichichte in L,inz.

Ifurte, l'. l', in Berlin. - Die Stricll-, Zng. oder Wander Heusclitecke, ihle Beschreilunng, Verluecoung in jelzigen und lirulesu Zcilen u. s. w. Berfin, Riicker, 1828. S.

Kohl, Silhwertfeger in Stuttgart. Jiin cifriger Sthmetterlingssaumler und hat seine Sammlnug seliön grcordnet.

Hokeil, frichieh, k. k. TluxamitsOllizial in Laybuch (nicht meler in Klagenfutt). Simmlung krainischer und kiiluthiscluer Käfer und Fulter, so viel wie romplet. Besiyt die kïmthiscleen und krain'srhen Conchylien in seincr schönen Sammlung. - Beobachtungen siber die in Krain umd Kärnthen vorkommenden Saturnieu (lsis, 1843, IJeft 3, 1. 134-t39). - Eutomolngisclue Notizen (ihisl. J. 139).

Xolb, tehrer in Stmttgart. Sammelt in- und auslämdiselıe Käler, und besitzt ansserst schöne seltent: Exemplate.

Mollar, Vincent, Inspector an $k$. k. Musenu der Naturgeschichle in Wien. Berüluniter Entomolug und Schuritistel. ler. - Nonograpluia Chlamydum. Cum tab. aer. col. 2. Viennae 1826 , in fol. - Ueber die Waldstrohenle (Plal. N. aquilinas als lieind des Wcinstockes (Noue Folge d. Verhumd. d. k. k. Jandw. Ges. Wien. III). - (Kollar mill Ha m. merselimidt) ïber Tinea grauclla. (Verluandl. d. k. k. Landwirthsehaft. Gegellsehnt in Wien. Neue Folze 2.
Hefl.) - Systematiselıer Kalalog der Selumetierlinge des Erylic'\%oytlıums Oestreicll. Statistik von Oestr. u. d. Eus. Wien t832. 8. II. Rd.) - Grylleu (neup) (Buitr. zur Landeskunde vou Oestreicl. III.). - Ballsiliens vorzijglich lästige Insckten. Wien t 832 . Fase. iis 4. tab. col. (zucrest in Polls Reise, danu so und in Fuunus l. (n. F.) 1837. mit Zusatz voum sel. Vollmar.) - Lepidopterorum Brasiliap Species novae. (Anualeudes Viency Museums der $\mathrm{Na}$ turgeschichte. Wien, II. (1×39) I. 2 t3. l'al.) - Lopliyrus piniperda iu allen Starlien.(1sisl834.7 t4.Waldverheerend.) Eoller, Ge. Gottlieb, in Göttiugen, - De Cicindela cumplestri. Gotiingate, $1836,4$.

Hórnatzla, in Breslat. - (Mit Neustä (dt) Abbildung und Besclireibumg der Selimetterlinge Dentschlands. Heft I-IX, Breslau i840. 4.

Hortum, Dr., A ryt zu Stolberg. Moss, in Lilybacls (i').

Ponzmichteherf, in Kamtschatka. Eutomolog.

Aruckevitzer, Dr. med. in Wien. - Linumerntio systematica currulionidum archiducatus Aust riae. Diss. Vindob. $1842,8$.

WÁtí mer, Jos., Di, med. und Badearzt za Kreutlo (im bayer. Gebirge, iu Olserbayeru). Sammler und Schriltsteller. - Das Bad Kreutl. Miinclien 1823. 8.

Krassew, C. R. A., Freiherr von, in Berlin. - (Mit Dr. Ed. Leyde) Lehrbuch der Naturgesehichte fiir Gymnasien. 1. (Zoologie). Berlin 1842, 8.

Ifrasper, Lehrer am Domgymma. siuu। *u Mngdeburg (Cf. "ent. Ztg. IIt, 29).

Ardnse, Pastor z.n Tanzalel (Thiiliugen :').

Hrause, Joh. Wilh., Pfarrer in Jena. - Fauna von Thü̈ringen und deu augrenzenden Provinzen. IV. Sclimetirilinge. Heft 8. M. col, Kupf. Jena 1810,8 .

Irams, Ferdinand, Dr. philos. und Custos ann k. Naturalienkabincte zu Stuttgint. Sammelte dic Thiese aller Ordnungen auf seinen beseluwerlichen Reisen im Kaffernlaude, am $K u p$ II. s. w., welclie alle in dem von ihm so trefflich cingerichteten Mĩuseum der würtlembergisehen Hauptstadt aufgestcllt sind. 
Mrelos, in Sïdafrika, anf dem Cup. Simmler fiir dis Berliner Museum.

Irenzer, S, Jugendlehrer z. Montjoic.

Kress, Landarzt zu Aschlonch bri Burnindhein, in Frankel, Sammelt mit grosser Licbe und Umsirht Fulter und besonders Kiffer. Wackerer und anspruclisloser Minul.

Mretsclemar, Dr. und Professor in Franlitint. Burnilumter Zoolog.

Hriechbanuter, Dr. med. in Minmeher. Selar griundliehes' Forscher, hauptsiichlich der Käaler. Schrich) eine Dissertation über die Cerambycinen um Minchen, 1845, 8 .

KrUsuanu, D. W., Lelorer an der Girnisonssilule zol Hamover, - Einer der ansgezeichnetsten and verstïndigsten Faltersammler. Er besitzt auch in Ausspanuen der Falter eine grosse Geschicklichkrit und sammelt alle iibrigen Kerfe. Sehr anschulich ist seine Käfersammlung.

Kritiger, M. S. Dr. philos.in Berlin. - Naturinsturische Ditrstellungen aus den vorziiglich. naturhisturisclien Sehriften. Nebst biograpliscli-literar. Notizen über die Seluriftsteller I. s. w. Mit 7 Portrïts ete. in 4. Berlin 1842, 12.

Mrutselı, Dr. der Medicin iu Leiprin.

Inrizscl1, K. L. , Professor zu Tharand. Schriftsteller iber Bostriclius ( $t 825$ ).

Mrymicliy, J., Professor in Kliarkoff. Eutomologisilher Schriftsteller. - Litterae, Direntori G. Fischer datae (Coleopt. nova Siloirias et ex reg. Charkov. (Bullet. de Mose. ed, leq. p. 54-64). - Enumeratio Coleopternrun Rossiae meridionalis etc. (Bullet. mose. ed. Ieq. (V) 1833. p. 16i-187).

richler, Fricdrich, Stadtumsikus in Ausbacli. Ein thitiger Leppidopternlog. Besitzt pine schöne, ziculich vollstîndige Faltersammlung.

Muenbure, F.Gruf vou, in Wien. Entomolog.

Iilster, J. C., Dr. phil., Professor an der Griverbescluble in Erlangen Bereiste von Oktuber 1841 his Septem. ber 1842 Dnlmatien mol brarlite vicle Kerfe, Vögel und Conehylien heim. Verkant die von Waguer in Algier, Bona ete. gesammelten Coleopteren, Systemutisclies Verzeiehniss der in der Umgegend Erlongens heohachteten Thiere. I. Heft. Wirbelthiere, Mollus-
Lem und Käfer entbaltend. Eulangen 18.0. fr. 8. - Natulhisturisthe Reis(berichfe aus Dalmatien Iml Nuntrnegro. (Isis 1842, 84i, 1843, 654-665.)

Ihranemer, Ferdinand, Dr. med. in Mijnclust. Besitzt pine kleine Coleopterensammlnng; ist ibbrigens ein sehr gediencener Plivtulag.

Kunles:, Pastor in Wissgoldingen bri Schwäbischgmind in Wüittemberg. Entomolng.

Bdumze, Gustav, Dr. med. und Protessor ete, il lepipatr. - Berïlımter Eintanolog und Schriftsteller: fefeiert als Botaniker. - Entomologiselue Frarmonte coler nene Sibriften ter naturforsclienden Gessllschalt жullalle, 11, 4. Heft). Hille bei llendel lstK. 8., p. 76. (Germar Magwy. IIT, p. 408.) Inpido, k. k. Buchhiltumgsieclinonessatl, in Brïnu. Entomolog. Niclifplanenanure, 1825,8.

Iyber, Joh. Friedr., Diaknnus a.1 Eiscnbery in Sachsen. Entomolog $11 m \mathrm{l}$ Siluiftsteller. - Einige Lerfahromgen und Bemerkungen iber Blattliuse (Germar's Mar. 1, 2, p. 1-40). - Beiträgo zour Verwandlungesgeschichte einiger Kiferarten, 1. Besehreibung der crsien Stande des lancekifors, Iuthrilius forratus Herlost (Germar's Mingaziol der Entomologie: 11, J. 1-16, Till. I). 11. Verwandlung:seneschichte des Siliwaizdiistelkifer's, Hlelops ater Fabr. (Germar. Mag. II, p. 1-21. Iab. I, fig. $1-12)$.

Inyer, zu Kronstadt.

Mabrenu, David, in Basel. Maler undentomologiseher!Siniftiteller. (Vgl. lmholf). - Fimma incluetiea.

Lanelaat, Dr. med. in Paris. - (ot Aulonin) Anatomie d'une la rve apoderte. (Mim de la snce. d'hist. mut. de P'ais l.)

Eur clunane, F. H. A., Dr. mell. in Bramsrliwrig. (Vergl. Lind ucr.) Seloriftsteller.

Tancondare, J. Theodor, zu Dijon. Berillumer Kerfphysiolog. Reisender in Amerika mol Schriftsteller. Introduction a l'eutomolumie. I. 'lome. Paris, 1834. 8. av. 12 planrhes. Notice sur l'Entomologic de la Guyane francaise (Ammal. sor. ent. France 1. 1832. 348-66). - Notice sur les hatbitudes des Lepidoptives diurnes de la Ginyane (ibill. 11. 1832. 379), - (avec Boisduval) Fauncentomologiquedes environs de Paris, on Species qui se 
trouveut dans un rayon de 15 a 20 lieues anx aleutonrs de Puris. Paris 1835. 1 vol. in 18. avec figir. br. 8. Fr.) - Memoire sur les habitudes dis Coléptères de l'Amerique merid. (Ammal. des sc. nat. XXI. 1830, 140).

Uacoste, ju Paris. - Buffon classidue, ou Résume d'uistoire naturelle cte., ouvrage neuf, orne de 150 plauches. 2 vol. 8. (Prix 9-18 Fies.)

In Trasea, M., in Madrid. Anenidades naturales de las Espanas. Madrid 1821. 8 .

Tregemanu, Joluan, in Lund. Apromizides Sufeiae. Lundae, 1823.8.

Walmude, in Bordenux. Seliriftstelles iber Lepidopteren.

Indu rek, J. B. F. de, Mitglied des Iustituts, Professor ete. iu Paris. Hochbeributer Forselies. - Histuire uaturelle des animaux sans verfibres. (Denx. edit. T. 3 par Desl a yes cte. Rodiaires, vers, iusectes. Paris 1840. 8) (Vergl. Desbayes.) - Plilosopbie moologique. ou exposition des rousiderations relatives a l"histoire naturelle des abimaux: á la diversité de leur urganisation et des facultes qu'ils en obtieuneut; aux causes pliysiques qui maintienueut en eux In vie et dounent lieu aux mouvemens qu'jls executent ; etc. 1 Vol. gr. 8. Bruxclles 1837 (12 Frs.). - Sur deux nouveaux gemes d'Inseetes de la Nouvelle Hollonde (Chirosinlis et Panops. Anual. dit Muséum d'list, mat. 'T. IV. 383. PI. 69).

Trambertliod, Jolıann Carl, Offizier bei der Kanffalurtei-Marine in Burdeaux. Hat eine Kafersummlung; verkauft alielı davon.

Inanderectue, E., Burggraf und Castellan der Orangeric in Hessenkassel. Entomolog. Instructive Sanmilumgeu.

Ianger, StaltverorducterzuHlirseloberg, in Sclulexien. Sammler.

Iungatore, Vater, Negoziant zu Lalur (Breisgau); Bruder des sol. Cousul. Besizt eine der sclröusten Lepiclop. terensammlungen, zu deren Vervoll. kommnung er weder Geld norls Zeit selient. Kenut die Falter selsr gut und ist tein gauz vortremliuer Mann.

Langatengel, Leluer iu J,jpzig. Fleissiger Sommlev und eis guter Ruupenziolser. Maclıt jöhrliclı cutomologiselue Reisen und linudelt mit Korfen.

Lanier, in Paris. - Nene Kiffer rou der Insel Cuba. (Revue zoologique 1838: Sept., Nov.)
Inporte, M. F. I. de, Graf von Casteluau, in Paris (Rue de l'Université, No. 75). Liuer der grundliclasteu entomulogischen Seluriftseller. - Bearbeitete mit H, Gory das Werk: Histoire maturelle ct Iconographie des In. sectes, publices par Monographies sépariens. Puris 1837-1841. Ell 45 livraicons (aver 168 planclu. eul.) (A 6 Fres.). - Norice sur un nouvean genre de l'ordre des Homopties (Heteromo(us) (Aumul. soc. ent. france. I. 1832. 95-97 pl. col.). - Memoire sur quelques nouv. genres de l'ordre des Homopteres (ibid. I, e. $221-31$ pl.). Memoire sur cioquante espedecs nonvel. les on pen commus d'Juscetes (des tons ordres) (ibid. 386-415). - Essais d'une revision du genre lampyre (ibid. II. 1833. (22-153). - Etudes entomo. loginues, on deseription d'Insectes nunveiux (Camassiers); 8, av. 4 pl. color. (6 Fres.). - (et B rulli) Notice sur un nouveau geure de la famille des Cluarançous (Mein. de la soe. d'hist. It. Paris IV. Sept. 1828. 197). - Notice sur le Macrotuma, nov. grin. d. Diptères (Muscides) (Aumal. I. C. XXV. 1832. 457. $1 \mathrm{fig}$.).

Lascerre, Hrinrich, Auditor in Genf. Entomolog. Herrliclue Simmlung.

Inneret, in Paris. - Memoire sur les larves des Coleopteres aquatiques:- avee Miger. (Bullet, de la soc. plitom. III, 229).

Eavean, George, Graf ron, Seeretär der k. Gesellsclioft der Naturforseher in Moskan. Entumolog und Scluriftsteller. - Considerations sur les principanx Oroomes des Insectes (Bullet. de Moscour. 1829-33'.

Hatrolue, B. Heinich, in Philadelphia. - On two species of Spluex, imbabing Visginia and Penusylwania, and probably exteudiug through the uniled States. (Trans. of Pliil. VI. 1804. j. 73-79. (Splsex caerulea Lin. et penus ylvanien, Latrobe.)

Isnnbevis, Dr. med. und Plıysikus in Wiirzburg. Sammolt Käfer.!

Leanticer, Advokat (?), zu Marscille. Schönc Sammulıng.

Lelons, Paul, zu Rourn. Eutomo. log und Reisender in Columbian, Car'tlagena u. S. w. Grosse Sammluug.

rechner, in Niimberg. Insckteu. Inindler und Antiquar.

Heconte*, Joln, Ingenieur-Capilin der vereinigten Staoten zu New-York. 
Entomolog, Rrisender (im Norden) ond Srluiftsteller (Amunls of the Lyermu of unt. hist. of New-Youk). - 1conoamplie des Lepidoption (avee Boisduval). - Deseription des quelques nouvelles espéces d'hosetes (Culiopt.). (Aminals of the Lyeemm of notor. hist. of New-York. Dee. 1824. 169).

Hedchonr, C. F. von, k. k. Staitsrath etr. iı Mü̈lchen. - Reise durch das Altili-Gehirge und dic Soongorisehe Kircisen-Steppe. Berlin 1829 30. 2 Tilile. 8. (Vergl. Gebler.)

Hedebur, Prenierlieuteuant in Königsberg.

Lederer, Julius, in Wien.

Ledonx, in Paris (?). - Deserip). tion d'une nouvelle espece du genre Enoplimu (Anual. soc. ent. fr. II. 1833. 474. fig. rol.'.

Hees, J. C. Entomolog in London (?).

Lefebvre, Alexander, Sckretiis der entomologischen Societĭi von Frankreich, in Pal is Roe de Provence No. 19). Beriihuter Reisender, Entomolow Ind Schriftsteller (besonders iiber Lepidoptereu). - Deseription de divers insectos incilits recueilles en Sicile. Paris 1827. pl. (Anual. sor. linn. Par. VI.). - Cbaractères distinctifs entre quel(jues Sutyres enropéens. (Amial. soc. Cllt. France I. 1832. 80-91. tab. color.) - Description des espises de Lipidoptieres woefurues des lades ori. entales (Zool. Joorn. n. 10 Apr.-Sept. 1827. 205). - Description de trois Papillons nouvellement observés (Anmul. soe. limn. Par. V. 18:26).

Letblein, Dr. med. und Professn der Zoologie etr. auf der Universitiot zu Wiirzburg (Frankell; Bayeru).

Telnumin, J. G. C'sr., Dr. neel. und Professor in Hambork. Berilhonter Botaniker und Schriltstelles.

Leiner, Stadtratlo, in Constauz. Zonlog und Sifhriftsteller. Sammlung von Foltern ete. - Die Fisclie des Boden. sees. - Verzeichuiss der Sibmetterlinge um Coustanz (Isis 1829).

Leisler, Commerzicuroth in Hanau.

Lekenx, in London. - Vertilgung der Haltiken ("Transactions of the entomolog. Soricty.).

Le:opold I., Se. Mnjestät, König der Belgier, in Brïssel.

Leopolat II., S. k. Hobeit, Grossleezong vou Tostana, z.l Florenz.

Leprige, Ch. Theod. J. G., Deputirtes: in Darney. (Vogesen).
Depelletier de Saint-Furgan, Graf Amedee I. Mich. v., z.ll Siniut Germain eo Lsiye bei Paris. Berilinoter entomolowischer Srhuiftsteller. Munugaplia Teuturediunn synonymia extricatio. Paris 1823. 8. - Memoire silr le geme Goryles de Latrille (Alivil. soc. ent. Fratue 1. 1832. 5279. 1 (ab.). - Observatious sur l'ouvrage de M. Dalilbom sur le Bombus de la Soide, uvec les earacteires des geures Bomlos et Bitlyyrus. (Aunal. sor. est. France I. 1832. 366-382). Deseriptions de trois nouvelles esplèces de Cimbex (ibid. 11. 454\% - Finne fronçaise. Ėutomologie. - Mèmoire sur qurlques esperces d'Jnsectes de la Section des Hymenoptères. av. fir. (Anbal. du Mus. d'list. nat. VIl. 1806. 115-129). - Observations sur l'neeauplement d'luscetes d'espères difièrents I Amalyse des travaux de l'Aead. roy. less Sc. p. 1827. Plyssiy. 56). Encyclupedie méthorlique (Artirle: Hymenopteres). - R (mancjues sur Irs caractères donues par Mt. Klug an greme Syzigonia (Hymeunt.) (Anual sor, ent. France II. 1833. 456. r. fig.).

Lepriene, Pharmazout in Paris. Reiscuder in Alvika (Senegal), wo er simumeltr. Botaniker.

Lequien, Bu(d)änller in Paris ( Quai des Aurustins No. 47). Sehrieh iibrer Antlia ite. und gibt die seltenen, thenem entomol. Werke heraus.

Lerlue, C. v., zu Kopenhagen.

Leronx, iu Versailles. - L'art entomologique.Porme. Versailles 1814.8.

Teschenand, in Paris. Reisender Naturforscher und Correspondent des Museum d'listoire maturelle.

Lesson, Marince-Plarmazent in Paris (Rue St. Jaques No. 166). Berülınter Reiseuder (tum die Weli), Naturforseler und Srhiftstelles. - Illustrntions de Zoolngie (thorils Weichthicre, theils Kerte ( 8 . Hft.): Tetropluthalma cliblocusis PI. X XIV. (Fanil. Litenuid.); ob syuonym mit Chiosognathus Grantii Stepli. in Transartious of the Cans. bridge philosoploiral Society?; Sagra Buquetii von Corhinchina.) - Centurie de Znologic. Pal is 1830 . 8. av planch.

Lennis, Professor in Hildesheim. Samuclt vou den Kerfen besonders Kijfer und davon meist nur deutsehe und Nordeurapäer. - Ueher dic Larven van Brueliytarsus sinbrosus Fahr. (ent. Zeitr. III. 190). - Verzeichniss 
der in Fiirst. Hildesheim mol dem angrenz. Harze aufyefundenen Blattwes. pen (ent. Zeitg. III. 42).

Lewin, John William in London. Olsservations respecting some rale british Insects (Liultean Trans. III. p. 1. tab.). - The insects of Great Brititin systematically arranged, arecurately engraved, and peinted from nature ete. (franz. Ind englisch). London 1795. 4. 46. pl. col.

Lewis, R. H., Esq. Entomologischer Scliriftsteller in London. - Desicriptions of some new Gruera of Britisly Homoptera. ('Transactions of entomolog. Soc. I. p. 48. Faunus I (1837) p. 125- (28).

Letziner, in Breslau. - Ueber Pterosticluns cordatus, Clurysomela cerealis, fingida( Fontwirkelumgsgeschicbte) (Uebersicht de' Aibciten ete, der' seblesisclien Gesellschaft 1841. 1. 100\%.

Leyde, Ed., Dr. plitos. Ind Lehrer an Berlin. Schuriftsteller. (Vgl. Krassow.)

Lleeraninier, Ferd. Dr. med. zu Point a Pefre nuf Gualelonpe.

Lhotaky, Naluralist, in Brasilien. Sammolte in Neuhollnud.

L'Innilier, Advokat in Genf(Grand Mezel 251).

Liclntenstein, in Mitnu (Curland). Linduer, $F$. W., in Braunschweis. - (Mit Di: F. H. A. Lachunnn) MnIerische Naturgeschidlute der diei Rei. ehe fiir Schule und Hans. In Heften. 4. Brallnsthweig 18:10-44.

Liuk, H. E.. Dr. med., Professor der Botanik, Hofratl, etr., in Berlin. Hocligefeiert als Ploytognost. Reisender (iil Griechenland, Itilien etc.) and Seliriftsteller.

Isincke, Lehrer an der höhern Bürgerschuls: zu Stettin.

Isinz, J. M., Steuereinnehmer in Speyer (hayr. Unterpfilla). Elirwiriliger und kemutuissreicluer Minn. Selır schüne Sammlung (iiber 6000 Species Käfer alleiil).

Lodrliges, J., Esq., z.u I.ondon (Hammorsmith.) Inluaber der grossen Nursery.

Wusw, H., Dr. med. und Professor in Posen. Hat eine schöne $\mathrm{K}$ äfersilmmJung, die er in Preussen mud Ungarn zusainnenbrachte. Beschäftigt sichı vorzäglich und gnnz wissensehaftlich mit Dipteren. 1843 war er in Kleinasicn. - Entwiekclung'sgeschichte der Gele- ehia lappella Lin. (Entom. Zeito. III. 257). - Horae nnatumicie. Beifritge \%ur gebanern anatomischen Kemtniss ter niede'lu 'Ihierklissetu. Alth. I. Hft. I (it). 2 'l'afelu). Posen (trine). $15 \mathrm{Sgr}$. 1. Abthlg, and s. t.: Eintomotomicn. (Rerension v. Siebold in lirl.: cut. Zeitg. II. 168) Anat. d. Genital. der Dipteren. - Ucber die in Grosslierzogthum Posen aufgefindenen Zwejflügler; cin Beitrag zur gewauern kritischen Bestimmung der europioischen Arlell. (Isis 1840. p. 512-584). Ueber die Gaftung chrysotoxom (ent. Zuits. II. 136). - U(ber cine cigenthümliche Nervenverbindung an den weitlichen Genitalien der Insekten (Dipt.) (lint. Zeitg. II. 74. tab. I. f: 17). - Ueber die Gaftunr Saltella iberhaupt nut uber S. sculdllaris besonders (Ent. Zeitg. II. 182). - Zur Entwickelungsgeschichte und Anatomic von Buprestis Marjana (Ent. Zeiter. II. 34. 'Tab. I f. 1-9). - Syiphus dispar. Neu (Eut. Zeitg. II. 6). - Bemerku»gen viber die annfomischen Verbilitnisse der Neu ropteren. (Gerumar: Zeitsclur. IV. II. 423-434). - 'Irypeta stignia und cometa, 2 nene enrop. Dipteren (Eit. Zeitg. 1. 156). - Benerkungen über die in der Posener Gegend einhcintschen Arten meluer Zwcifiigler-Arten. Posen; 1840. 8.

Libue, Chr. Lud. W., Ilofschinu. spieler in Wiru. Hocbberïhnt als dramatischer Kiinstler. Studierte in Ilalle. - Diss. inaug. (pracs. Curt. Spreugel.) de partibus, quibus insecta spiritus ducunt. Hallac 1814. 4.

Lomler, Kammerrath in Meiningeu. Sammelf nur Käfor.

Lonegi, in Mailand. - Della Abitazione dei Coleotferi, e dei mez\%i acconzi per farne caccia.

Lapez, Don Salvador, Almosenpileger in St. Antonio de la Florida bei Madrill. Sclırieb über die dem Weinstock sehïllichen Kerfe ete.

Losann, Matteo, in Turin. Ueber die Amcisen in Piemont (Memoive della renle Accademia dolle Scipmze di Torino XXXVI. 1833.p. 307. Isis 1844, 67).

Lovetzliy, A., in Moskau.

Lucas, Ht, de Verdun, in Paris. - Ilistoire inaturelle des Iépirlopteres d'Enrope. Ouvrage orue de près de 400 figures peintes d'apres nature par A. Nuël. Paris 1834. - Lssai str 
une Monographie du genre Thelyphone (Guerin Magas. 1835). - Notice sur le Bombyx de l'llierarium (Ammal. des Se. nat. Août 1830. 473). - Observations sur It's males de quelques espèces du genre l'erle, qui sout privis d'ailes on le's ont très-courtes (ibill. XXVII. 1832, 453).

Ianciani, F., in Castelnuovo (bei Cerboli). Schricb 1838 fïr die Atti dei Georgngli (XIII) über die dem Welscli. korn ( $Z$ ('a mays) selhädlielien Larven. (Vol. Passerini und Isis 1842. 369.) - Notice sur le Bombyx de l'Hicraeiunn. (Annal. des Se. nat. Août 1836. 473.)

Huczot, Fr. M. Jul., Ober-Ingenieur des Brüeken- und Strassenbaues zu Paris. Schriftsteller über Kerfe. Sammlung (darin viel von Terra nova).

Tund wis, Baron von, Banquier in Capstadi, am Cap der guten Hoffnumg. Scine reichen Sammlungeu sind zu Stuttgart, Tübingen, Frankfurt und Darmstadt anfuestrolli. Lir verdient hief'ïr das grösste Lob.

Liben, Rector z" Asehersleben.

Hibbert, A. G., in Breslau. Sammler.

Hymen, Richard, Messingfabrikant in Stolberg.

Macnire, Banquier in Constanz. Schöne Lepilopterensammlung.

Nuccary, Ange, Dr. med, in Nizza. Entomolog und Seliriftsteller. - Mémoire sur le Scorpion de Cette. Memaire sur le Bombyx Pavonia.

Mrelery, Wilhelim Sharp, derVater (sonst zai Hobartstown in Vandicmensland, als sein frei erwilltes DoInicil), jezt Commissiir und Richter in Havama. - Notice of Icratitis citriperda, an Insect very destructive to orange (tab. col.) (Zool. Journ. IV. 1829. 475 -82). - Horac entomologicae or essais on the anmuluse animals. London 18192t. 1 Vol. in 2 prarts. 8. e, fig. (cdit. mouv. Lequien. Paris, 1833). - Ueher die Mucken-Larven (the philos. Magaz. II. (1827) 178. - Isis 1834. 791). Remisks on the devastation occasioned by the Hylobius Abictis in lir plantation (Zoolog. Jouln. I. 1824-25. II. IV. 144). - Besclureibung der suidafirikaniselien Amulosen, enthalten in A. S mith s Illustrations of the Zoology of SontliAfrica (Hit. 3. 1839.) - Remarques sur lidentite des ecrtains lois genéraGist心's Lexikun. les observès dans une distribution naturelle des insectes et des ehampignons. (Trans. of Limm. Society of London, XIV. 1823. part. 1. p. 46 . Bullet. des Scicuc. nat. et Geol. Febr. 1525). Annulosa; in fase. III. 1838. (Isis 1843. p. 929); Heft XIV - XVIII. - On the Inseets ealled Oistros by the ancient Greeks, and Asilus, hy the Romans. (Linn. Transact. XIV. II. 353.). - Annulosa javaniea. Loudon 1825. 4. c. fig. (edit, nov. Paris (Lequien) 1833. 8). - Notes on the Larves of the diptères (Pliilosophical-Milgazine, Sept. 1827. 178.) - On the Oestrus of M. B. Clarek (Zoological Journal, V 1828 -29. 18). - Explanation of the conparative anatomy of the thorax in win. ged inscets, with a review of the pre: sent state of the nomenclature of its part (Zool. Journ. V. 1832. 45. - Trad. fiane. Annal. des Seienc. naturelles XXV. 1832. 95-161. Avec Notes par V. A udou in 2 plates).

Nracleny, Alexander, Sohn, in Ha. vanna, auf (ler Insel Cuba.

Mncqunrt, Jean, in Paris. Berïlumter Dipterolog und Selniftsteller. - Dipteres exotiques nonveaux ou peu connus. Paris 1. 1. 2. 1838. Il. 1. 2. 1840. (Isis 1843. 941). - Insectes dip. tères du Nord de la Firance (Mèm. de de Ia soc. roy. des Se., d'Agric. et d. Aits. de Lille. 1825-28. 8. avec 2 planch. (Ailes). - Dipteres (Suite de Buffon). - Monographie des insectes Diptères de la famille des Empides. Lille, 1823. - Histoire naturelle des Insectes (Diptères). Paris 1834-35. 2 Vol, av. pl. color. (Isis 1943, p. $876-79)$.

Maess, Cand. jur. zu München. Colcopterensammlung.

Mrenss, Superintendent und Pastor 7.11 Neden bei Cleve.

MifrkeI, Friedrich, Cantor in Stadt Wehlen bei Pirna (süchs. Selıweiz). Gediegener Entomolog und ScluriftstelIer. Schrr wertlivolle Simmlung. - Anfrage und litte (wegen Zusendung;) vou Myrmecophilen. Characteristik 5 never Myrmedonicu, Alcoehara etc. (Ent. Zeitg. III. 142. - Das Uebrigo erscheint weilläufiger in Germar's Zeitschrift.) - (MärkeI und Sehmidt) Triarthron Märkelii (Anisotom.) (Ent. Zeito. I. 141.)

Ingegi, Graf Carlo, in Mailand. - Transinnto d'una memorie sopra un 
nouyo metodo di far nascere con mig- / une nouvclle espèce de Cecidomie. lior csito i vermi da seta (Opuseoli Scelti, XIII. 77-81).

Hehieg, E., Arzt und Conservator des Cabincts zu Châlons sul Marne. Sammlung französiseher Käfer.

Mnilie, Arsenue, zu Rouen. Selıöne Sammlıug.

Mrckenzie, G. S., Dr. med. zu Ediuburglo. - Notice sur les Inscetes qui apparaisent tout a coup $\mathrm{cn}$ grand nombre sur les arbres (Edinb. Journ. of Seienc. Janv. 1826. n. VIl. p. 37. Bull. des se. nat. et geol. Avril 1827).

Malinowaḱy, Kapitäı von, zu Pechau bei Magdeburg. Entomolog und Seluriftstellcr. - IBcitrag zur Naturgesehichte der Vesja Crabro. (Mag. d. Ges. naturf. Frnde. in Berliı II. p. 151-157.) - Elementarbuch der IIIsektenkunde, yorzinglicl, der Käfer. Nebst einer Anweisumg, dic Insekten zu erkeunen, zu bestimmen, zu finden, aufzuspjessen, zu sammeln, zu stellen, aufzubewabren und zu versendell. Quedlinburg, bei Basse, 1816. 8. pagg. VI. und 228. (Germar's Mag. 11. p. 327.)

Melo, Clarles, in Paris. - Les insectes. Almauach pour l'annce 1822. Paris. 24 (avec quelq. planch. colèopt.'. - Les Papillons. Alinauach pour 1817. Paris. 24.

Mranger, Amtseluirurg zu Warubrumn in Schlesicn. Entomolog.

M нmu, Josepll, Maler, friber zu Reichsstadt in Bölumen, jetzt zu Wien (Vorstadt Hundsthurm, Johannaerasse Nr. 153). Emsiger Falter- (Microlepidopteren-) Sammler: Leobrehtet die Metamorphoscu, erzielit Raupen und Falter und colorist die Tafeln zu Hinn. v. Fischer's Werk. Handelt mit Miero. lepidopteren und ist ïusserst billig und elirlirh. (Vergl. Isis t840. J. Umsching). - I Mnererinein, Carl Gustav, Graf von, Gouverneur der Festung Wihurg in Finnland. Berülumter Schriftsteller. - Obscrvations relatives aux sexus des coléptères liydrocanthares en général et speciellement de l'Hydaticus verrueifer. - Description de quaraute nouvelles especes de Searubacides du Brèsil. Avee 2 pl. col. 4. (Mèn. de Moscon, Vli. on nouv. Mem. I. 28.) Eucnemis, inscctornm genus monograplice tratatum iconibus illustratun. Petrapoli 1823. Lipsiae 1825. 8. c. tabul. - Memoire entomologique sur

(Nèm. de Mosrou II, 180-84. I pl.) - Description de six nouvelles espéers de Carabes de l'Armenic tur'qur. (Bullet. Mosc. cl. Icq. 1830, II, P. 70 -77.) - Enumration des Buprestides etc. (Bulletin des Sciences des Natur. de Moscon. 1837, Nr. VIII, p. 167.) Observatious critiques sur quelques ouvrages entomologiques (ibje. p. 127 . - Notices sur la Collection de M. Dejean ribid. n. IV.). - Käferfauna der alcutiselien Inscln (ibid.). - Alc̈moire sur la recolte d'inscetes Colénptères. Fuite ell 1842. (ihid. 1843, Nr. 1.) Prieis d'un nouvel arrangenent de la Famille: des Brachelytres de l'ordredes ins. col. (Mćmoir. pr. n l'Academ. des Sc. de Petersh. I, 1831, p. 415-502. (lu Juin 1830 !).

Marclinual, sen., in Clartres. Besitzt ciuc der grössten und sehöusten Sammlungeu in Europa.

Mrariett, Bernardo, Geistlicher in Miriland iCoutralli della Biblioteca Ni. 3147). Tanscht luswetcu und ist zuverlïsxig. Schöne Sammlung.

\section{Nerin, i" Noskau.}

Matrlevid, William, in Loudon (?). Eirtomolog und Selurittstcller. - Ub. servation on the Curculio Tifolii, or Clower Wecvil, a smal lusect which infests the heads of the cultivated clower, and destroys the sccd. (Limenn Transact. VI, p. 142, tab. 5 et p. 147.)

Marlog, C. P. J. B., Medieus in Auriol bei Marseille. Sclur verdient um die Wissenschaft.

Maranor, Dr. med. in Constanz. Schöne Käfersammlumg.

Mnranora, Ritter, Graf A. de, I॥ Turin. Entomolog; öfrer cilit im philosophical Magrzine. Schriftsteller. Voyage en Sardingne ou Description statistique, ploysique et politique de cette ile, ivec des recherelics sur ses productions naturelles et ses antiquites.s. Secoude idition. Turin (Boeca); Pai is (Bertrand) I. 1839, 8. 528. Atlas tal. 10, fol. 11, 18.10, p. 595. Atlas. t. 41. fol. (Recens. in Okens Isis 1842, p. 626).

Minrselnall, Ginf von, in Wien. Wird die Orflupteren der Sclubert'schen Rrise bearbeiten.

Marohall, Thomas, Esq. in Birmingham. Schrieb üher Kerfe für das entomological Magazine.

Mraxingu, Thomas, in London. 
Beriihmter Entomolıg und Schriftsteller. - Observations on the Plualaena B. Lubricipeda elc. (Limneau T'allsact 1, 67, tab. 1, 1-4. - Reminks of the Musca Pumilionis (ibid. II, 76. tab. 15.) - Observitions on the oeconomy of the Iehneumon Manifestator. (ibicl III, 23, t. 4.) - Observations on the luseets thet infested the Corn (ibid. Ill, 242.) - History of 'Tipula Tritici ete. (ibid. IV. 230 und 268.) - Some (Observations on a linsect that destroys the Wheat. (ibid. 1X. 156.) - Description of Notoelea, a new gen. of col. Ins. from New Holland. (ibid. IX. 283. t. 24-25.) (20 Species.) - Buprestis splendens (Linnenu Transact. IX. 399. t. 32).

Mnetinl, von, in Petersburg (?). Citirt in den Essais entomologiques von $I I$ u m mel.

Martyn, Vater und Soln, in Paris. - 'Traite sur les ruches h l'ail libre. Paris 18:6. 8.

IIaslemnihofr, in Wiazma (Russland).

Mntz, Lehrer, am TaubstummenInstitut \%u Mlagdeburg. ( $V_{g}-$. B anse.)

Mintzeck, Dr. Leluer der Naturwissenschatten an der Realsehule zu Breslau. - Ueber Pseudopliana europ. und Pentatoma rulipes (ent. Z1g. IIt, 69). - Niceruphororum momographiae partienla lma. Diss. Viatislaviue 1839.

mayburger, Lehrer in Salzburg.

Mayer, Gustav, Revierförster zu In-Zeli bei Traunstein (Oberbayern). Tiichtiger Zoolog.

Nayer, Johanu Edler vou, Dr. med. und Leibarzt Sr. Durclulaucht des Hospodars des Firstenthums Moldau, Michael Sturdzá in Jassy.

Mayer, Carl, Revierförster zu Eurasburg hel Augsburg. Sammlung.

Mryer, Franz von, Ritter destranzösisclien Verdienstordens der Trcue in Mïnclien, Vorstand des roulogisclien Tausclivercius (Adalbertstrasse No. 9, parterre). Vor Kurzem eischien ill eillem besondern franzosiseh abgefussten Cirrular Folgendes iber den Zweek und die Einrichitung des vortıeflichen lnstituts: P. 1?. Haben wir die Ehre, anzuzeigen, dass duch cincu Zusam. mentritt rechtlicher Zoologen und eben so sacliverständiger Sammler und Reisender ein Verein sieh gebildet habe, welchersich zu $\Lambda$ ufgabe maehte, grusse Sammlungen vou Gegen- ständeu aus allen Gebieten der Natur, der Zoolngie, Botanik und Mineralogie, so wie von einschligigen Büclıeru und Kupferwerken anzulegen. - Diese Summlungen sind dazu bestimnit, unter die Naturforseher nller Nutionen und die Licbhaber der Natur vertheilt zu werden. Die Ant mol Weise, wie dieses geschieht, ist folgende : Jedermann, welcher DoubletI'n vun Thieren, Pfanzen, Minerulien und naturhistorischen Bïehern, einzeln uder in Mehrzahl, besizt, sendet die. selben franco anden Clief des unter. zeichneten Cunitè's ein; unfrankirto Zusendungen gehen une röffnet zurüek. - Der Eiusender legt ein Verzeiehuiss sciner Desideraten für seine eingrsendeten Saelien bei. - Dieses Venzcicluiss wird vun dem Comité nit dene'l der vorhandenen (cingesendeten) Grgenstünde verglichen und der Finsender enlält entweder binnen Jah. resfrist seine Sendung, insoweit dieselbe niclit komnte äquivalirt werden, zariick, oder er erhält diejenigen Gigenständle, welehe er gewïnscht liat, wein selbe vurhanden sind, olne weitere Auslagen, nur doss er die Kosten des Pustuorto's zu tragen hat. - Das Comite kann versicluern, dass es siel bereits in Besitze eines bedeutenden Vorrathes aus allen Ländern der Erde befinde und schon jezt im Stande sey, den Wïnschen selır vieler Desideranten prompt entgegenzukom. men, wie untcrfertinte Belege ausweificu. - Des Comitè's Hauptzweck ist: a) Verbreitungder Naturgegen. ständeunteralle Naturfurscher nller Länder; b) Aufhebung des bislaer grösstentheils und, mit wenigen Exeeptiunen, duréh Betrug, Wucher und seluüde Industrie geleiteten Naturalienhaudels, desseu Ergebnisse bisher der Wissenschaft melir machtheilig nls föderlich waren. - Jeder Naturforscher, sclbst der unbenittelste, erlangt anf diese Art in kuzer Zeit anselur. liche Sammlungen ohue Mühe und we. scutliche Kosten, zngleich weit zu ver. lässiger als auf irgend einem Privatwege. - Das Comite nimmt, im lialle Jemind gar nichts besizt, was ex z.um Tausche bieten künnte, auch Auf. träge an gegen Credit; bei gänzlich u ubekanteu Persunen nur dann, wem vorher linlängliehe Cautiou 4 . 
gestellt oder der Betrag des Bestellten in Loco angewiesell worden ist. - Das Comite wird fïr sorgfältige Verpackung der von ilum nusgehenden Gegenstïnde Sorge tragen. Es wird voransgese\%t, dass schlecht conservirte Naturalien, so wie unpraktiseh gepackte $\mathrm{Z}$ us e Idungeu in keinem Falle berïcksiclotigt werdeu können und der hiedorch herbeigeführte Defekt dem Zusender auhcimfällt. - An Büehern zälılt das Comite 1600 Werke und 900 Doublettell, an Säugthieren 300 Species (200 D.), Vögelı, 1600 Species, mit first gleich so viclen Doubletten, Ampluibien (in Weingeist), 700, mit 400 Donbletten ; Fistien: 500 Speries, simmtlich in Spiritus vini, in 1000 Exemplaren; an wirbellosen Thieren eine noch (IIIberechnete Menge, besonders cine ungeheure Anzalil vou Insecten, (gegen 40.000 Exemplatien) und Concliylien, Radiarien u. 8. w. - Ehrlichen Bïcher-Antiquaren und Händlern von Nafuralien, denen es un Baarzah. lungen z.u thun ist, will das Comite gegen unfrankite Einseudungen von Buichern, Naturalien und Briefen gerne ilsr Verkäuflielıes in Commission nedmen; kann aber sich erst $n$ is el $A$ b. fluss eines Jalues verbinden, das Nielitverkaufte remittiren oder Rechnung und Zalslungs-Anweisungen stellen zu lassen.

Die ausserordentliche Theilnahme, welelie dem Untermelmen bisliet zu Theil geworden, sichert cin rasches Fortschreiteu scines als aucli des allge meinen grossen ' $\mathrm{L}$ wekes, und die Mitgliedersehaft sowohl als auch die Privatspenden, welche dem jougen Unternehmen zugeflossen, stellen ein änserst gïnstiges Prognostikon fïr die Wissenschaft und deren Förderer. - Wir ladeu alle redlieh. gesinnten Nafurforseluer und Naturfreunde zur Theilnbane ein und fordern sie auf, lem edlen Streben nur förderlieh, niemalshinderliebzuseyn. Miirehen am 20. Janutr 1843. D as Comité des naturhistoriselen 'Jusehvereitues. (I.S.S). Der Agent des Verciues und des privilegirten Naturalienlagers: Francois le Mayer (de Zürich), Citoyen français, Chevslier del' orde de la fidelité (dı Inonis VIII) a Monic (Adalbertstrasse Nr. 9 parterre). Folgen viele Unterschriften und Recomandations-Aensserungen. Din in der Isis 1844, Ileft III (Umsehlug) duretı llur. Gar du us skizzirte Sammlung von Naturilien, einzio in iluer Art und zoll Begriïudung cines Privat-, vorzinglich aber öfientlichen Schul. Musenms geeignet, ist im Lager zum Verkaufe ansgestellt, und wird $11 \mathrm{~m}$ cinen üusserst billigen Preis abgetreten. - Sic enthält meistens Insecten (in 90 Kïsten). Dic Thiere besteben ans 1403 Generibus und 6754 Species, in 9624 Exemplarien, welche XV Ord. nungen angelüiren; Pflanzen sind (meist nus den Alpen) 1925 Species in 2690 Exenplarien (537 Generia) vorhanden, und 21 Geseblechter Mineralien, in 99 Spreies (203 Excmplarien); alles auf das Beste conservirt. Die ganze SammIung enthiilt 1960 Gencra, 8778 Species und 12,527 lixemplarien. Wir künuen sie mit Vortranen bestens empfelilen. - Specielle Cataloge liegen vor.

Nayerlnofer, in Müuchen. Fing 1834 dell Bothrideres contraptus um Miinclien.

Mazy , Pfarrer zu Irsingen bei Tiït'klinu. Sammelt aus allen Ordnungen Kerfe, besonder's Lepidopteren.

Mediei, in Mailand. - Singgio di storia maturale de moutr. Jegnone.

Megrerle von Mihlufeldt, Joh. Karl, eister Kustos des k. k. Museums xu Wien. Beriilnnter Entomolor, Concholog und Schriftsteller. - Catalogus Insectoruın qune Viemnae Anstriae die 14 et sq. Dec. 180 t anctionis lege distribuntur. - Bemerkungen, Berichtigungen und Zusatitze zut Illigers Zusätzen, Bericlatigungen und Bemerkungen zu Fabricii Systema Eleutheraforum. Linz 1812 , 8. 44 S. 8. (16 Gr.) (Siche Germar im Mag. d. Ent. I. Bd. 2. Hlft. p. 135, IV).

Meigen, Johanı Wilhelu, Lelurer z.u Stollberg bei Aichen. Beriilinter Dipterolog, Isepidopterolog und Seluriftsteller. - Systematische Besclureibumg der hekmuten enropiiischen zweilliggeligen Jusekten; 7 Theile 8., Parg. 412. Kilpfer XIll, Hamm. 1826. (Der VII. Bd. ist der letzte. Cf. Isis 1839, p. 159). - Europäisclue Selumetterlinge. Aaclıen, Mayr. 4. 1827. Mit Litlogr. (Bel. Zool. Journ. XI, 1827, p. 473).

Me:iss, Fr. v., Insektentändler; demalen it Algier.

Meismner, Professor zu Basel, Falter. 
Melly, A., Esq. in Manchester (in Liverpool, nach Demel). Iintomolog und Schriftsicller (Guèrin Mragasin). Sammelt ansscbliesslieh nur Käfer. Genauei Kenner davon. Seine Samunhune soll melir Species als die des Grafen Dejein enthilten (besonders Exoten).

Mellet, Pfarrer 7.u Concise (Sr:lıweiz). Sammelte die Kiffer des Jura.

Téuétries, J. Francois, Diretitor des k. k. Naturalien-MIuseums жu Petersburg. Bereiste mit Langsolorf Brasilien, war in Taurien etr. Beribunter eutomologiseher Schuiftsteller (vgl. Mle. moires de l'Ac:ul. de Petersburg, de Moscou ete.) - Russische licrfe (Bull. de l'Acidl. des se. de St. Petersburg, I, 1835 et $36,1 \% 180$ ). - Neue Kerfe aus der Türkci (Bullet. de St. Petersburf 1,1835 et 36, p. 149). - Zypaenit Wirdemamii (Mem. de l'Acal. de Petersburg VI ser. III, 1840 , p. 50, tnb. 11, f. 10). - Catalogne d'inscetes reereillis entre Constantiuople et le Balkan (Mems. de l'Aead. des sc. de St. Petershurg VI Ser., Tome 1II, 1840, p. $1-52$, tab. 2 , col.). - Neue Mlotte ans Turcomanien, Bullet. sriemtilicque de St. Petersb. 1X. 1841, p. 40). - Lssai d'une monogiaplipe du gemre Anacolus, de la famille des Longicornes. (Ins. Col.) (Mein. de l'Acad, de Petersb. VI. Ser. 111, 1840, p. 277-295, Tab.). Observations sup (juelpues Le pridopteres du Bresil (Mem. de Moscon, VII, ou nouv. Mém. I, 181-196, 3 pl.). Catalogue de quelques Lepidoptères des Autilles; avee lit description de plusieurs e'spèces nouvelles (ibid, ed. leq.. V, 1833, j. $193-212$ ).

Jineinger, L., zu Freiburg in Breisgau.

IVerlaci, Joseph, Officiant bei der k. Staatsschuldentilgungrstiasse in München. Ansgezeichucte Faltersammlung. Kault Fulter und tauscht auf Verlangen.

Netnxn, Telemaco, in Rom. (Mit Rolli) Osscrvazioni naturali intormo alle cavallette nocive de la eam). pagnit Romana (Schricken): Romac 1825, 4. lab. eul. - Histaire de deux Larves d'oestres de l'orcille l'un prysan (memoire di Zoologia medicale. Roma 1833,8.. 61-71. cun tivola).

Vetzuer, in Frankfut an der Oder. Lepidopterensiammlung:

Mevers, Dr. med. und praet. Arst in Berlin.
Meves, in Kiel.

Meycullorf, von, in Petersburg. - Voyage d'Orembourg a Bockhara. (Zoologie) Paris 1825, 8., fig. col.

Meyer, Hernann, Dr. ined. und Privatdocent in T'übingen. - Urber den Bau der Hornsehale der Käfer. (Muillers Arehiv.)

pleyer, Fr. A. P., Dr. med., in Krecuzburg.

Meyer, in St. Gallen. Sammlung: schweizeriseluer Käfer.

Heyer, L. R., Hauptmann in Burgdorf (Canton Bern). - Ueber Identitit und Separation einiger Rhynclooten (Ent. Zeitgr. II, 82). - Velzeichniss der in der Selnweiz einheiniseben Rhynchoten. Bevorwortet von S rll inz. I. Hft. (Capsini). MI. 7 ill. Taf. Solothum 1843,8.

Micer, J. J., Professor in Basel. Eutomologischer Sehriftsteller. Seluöne Sammlung. Beabsichtigt eine Monographie der Buprestiden erscheincu zu lassen.

Micluivisly, Ignaz, Graf von, in Geuf. - Mèmoires sur ume Larve qui devore les Helix nemoralis et sur l'insecte anquel elle dome maissance (Drilus flaveseens). (Mèm. de Genev. II, $1,1823, p .1-13$, avec fig. - Annal. des se. natur. 1, 67, av. fig. Bibliotheq. univers. 1823, 137).

Misger, Felix, in Paris. - (Avec La ucret) Mémoires sur les larves d'in. sectes coleopteres aquatiques. Jre Mèm. Sur le grand Hydrophile, av. I pl. (Amnal. du nuséum d'hist. nat. XIV, 1809,441 . - Nouv. Bullet. soc. philom. 11. 32, I1, 74).

Milsau, J. Chr., Dr. med. und Professor in Prag. Beriilumter Reisender (in Brisilien), Pliytolog, Zoolog und Seluriftsteller.-Monographia Bombylio. rum Bohemiae. - Entomologische Beols. nclitungen, Berichtigungen und Entdeckungen (N. Ablinndl. d. bölun. Ges. III, Plifs. 108-136).

IIIIbert, Maler und Correspondent des Musenms der Naturgesehichte z.u Paris; sonst in Nordamerika.

Millard, S. W., in Bristol. Outlines of hritish Entomology, in prose and verse. Bristol 1821, 12.

Mriler, Techniker in Wien. Artigo Sammlung. Sammelte sphr fleissig in Kaintlun ind Oesterreich.

Ninlswitz, S. A., Frciherr von, zu Gruuwitz in Schlesicu. 
MnIszech, die Grafen Andreas und Georg, von Kreminiee in Volliynien, derzeit in Wien.

Moller, Dr., Compagnie-Wusdarzt in Cissel. Hat eine Sammlnng.

Momici, l'rofessor der Zoologie in Parma.

Monlet de Lnroche, Postufficiant in Vendône.

Moravitzky, Graf vou, Oberliententut in Regiment Kronprinz zu München. Lepidopterolog. Grosse Samm. lung vau enropiiischen Faltern.

Moris, Professor in Turin.

Moritz, Lebrer zll Hage bei Frierack. War in Westindien. Sammelte auf St. Tlomas, in Columbien rte. -

Morrea, Professor in Liittielı. Ueber die Phosphoreseenz der Lanpy risnoctiluea und splendidula (Atti della lerza Riunione degli Scienziati italinni, tenata in Firenze del 18.11. Firenze 1841,4 . p. 306).

Motechulgky, Victor von, $k$. k. russiselser Gardeoffizier in Petersburg. Bereiste 1839 und 1840 Siherien. Schrieb über Pselaphus. Sammelte im Cancasus. - Deseriptim de quelques eaJeptères reeueillis dins une voyage au Cauease et dans les Provinees transcaucasiennes russes.

Moux-Deloclie, Fr., zו Paris. - D'une Résine employée par l'Abeille - la construction de ses gateanx (Mem. de Turin 1804-1805, p. 123-157, av, Plan).

Mitilemprordt, Dr. philos, und Lebrer der Zoologie mnd Bolanik an der höhern Gewerbsrhule zu Ilannover. Jepidopterensammlung.

Muine, in Odessa. Entomoloz.

muller, Johamure, Hofrath und Professur in Berlin. Gefuierter Anatom. - Die Augen und das Selien der In. sekten, Arachniden und Crustacecn (Auszug aus dem bekannten Werk). (Aunal. deg sr. nat. XVII, 1829, 225.)

Inthller, Oberförster zu Rötgen bei Montjoic.

Miller, Rod., Pastor zu Grindel. wald. im Canton Bejon.

Mulle: , Pl. W. J., reformirter Pfarrer zn Óleubacl bei Mainz. Entomnlog und berulumter Scluriftsteller. Benerkungen über cinign Iusckten (Germar Mag. d. Ent. II, p. 200-290). (Käfer: Limnius, l)asyierus, Begattung der (xoden). - Lepfinus, Dünnkäf-r, eine neue Käfergattung (Germar Mag.)
II, p. 266-273, 1. 1). - Naelitrag zur Besehreibung der num Odenbarli gefund(nen Selolammkïfer (Limuins) in lllig. Magnz. V, p. 205 und 206 (Germar Magnz. II. 273-275, 11.2). - Ueber dio netr Käfergallung Dasyeerus (Germar, Mar. d. Entom. II, p. 279).

Nutller, A., Kreisheamteter und Cunservator zu Brünn in Mälıren. Zoo. log und Seluiftsteller. Virl ebrlieh.

Metuch, Joh. Casp., Dr. med. in Basel. Simmelt fleissig.

TPY if nter, Gust. Wilb., Dr. philos. und Aufselier des k. anatomiscluen Musemus zin Halle. - Allgemeine Zoolo. gic. Halle $J 840,8$.

IDulsant, in Paris. - Les Iamel. licornes de France. - Lettre a Julie sur l'Entomologie, survis d'une des. eription methodique de la plus grando partic des insectes de Franee; 2 Vol. iu 8. Ornés de 45 pl. par Duménil (18$24 \mathrm{tr}$.$) .$

Ninck or Fosenschold, Eb., in Lund. - Prodromus Faunae Coleop. terorum Lundensis. Praesid. Sueno Nilssou. Pait. I. Lundae 1835.

Meranceld, Apotheker zu Rieine. - Vuber Nebria lateralis (Ent. Zeitg. I, 140).

Niurray, W. C., In Swansen. Selurieb ïber Coleopteren.

IIInsclal, Prediger in Kntolow (?) bei Fredland in Merklenburg-Strelitz. Sehreifrig und thätig, lieferio er Manelıes zu Freyers Werken.

Munsehi, S., Ptarrer in Merklen. burg-Strelitz. - Ueber das Winterleben der Honigbiene und einige durrls die Einathmung derselben bedingto Erseluciumben etc. (Abhandlumgen der uaturforseheuden Gesellsclıaft zu Görlitz, Bd. II, 1, 1836).

Nagezaun, Pater Albert, geistlieher Ratlı: Ábt des BenediktinerStifter und KIoster zu St. Peter in Salzburg. Zoolog und Oryetoguost.

Napoll, L., Apotheker in Triest. Nardo, J. D., Dr. med., in Ventedig. - De cantharidibns (Isis 1834, 671). - De naturia agendique mndo sub. stantiarum epispasticarum comparato eonsideratarum. Po ogramma (Isis 1834, 753 ; Vesienutia).

Natlusius, Her'mann, zu Hundis. burg liei Nagdabug. Zoolog.

Nutly, Postolficiant in Warasdin Fallersaminlung. 
Netterer, Johann, in Wien. Beriilunter Reisender (mit Dr. E. Pohl in Brasilien), Entontogg und Schriltsteller.

Serale, G. P., in London. - Observations on the study of lintomologr, with a short Account of the early stages of Bombyx versicolor (Trans. ent. soc. Lond. 1812, 323).

vees ron Fsenbeck, C. Ch. G., Dr. med. und phil., Professor der Botanik auf der Universität zo Bopslau Ind Prises der k. Akademie der $\mathrm{Na}$ turforseluce z,11 Bomm. Viellerilhuter Phytolog. Eutomolog und Sehriftste-llet. - Die Fresswerkyeuge der Insekten, geseliricben in Juni, 1814 (Isis 1818 , 1. $1385-1406$ ). - Ilorac pliysirac berolimenses. Bomne 18:0, fol. e. 27 tinbul. - Isapton femoralis, rine nene Icheumoniden-Gattung "bebs eingen Bemerkungen iber verss:hicdene unter Ophion stehende Ichnenmonidenarten. (Magazind. Gesellschaft uat. Fr. in Berlin. VIII, p. 45-53, Taf. 1, F. I, II, a-c). - lelunenumoues adseiti in genera et familins divisi a Dr. Nees ab Esenbeck (Mag. d. Ge's. nat. lor. zoll Berlin V, (1811) 1) 3-38, Tal. I, II, VI, l. 183-222. - VII, p. 2:3-277, Tuf. $7,8)$.

Negri, P., Dr. med. in Bologna. - Memoria sopra il bineo, che devasta $i$ scminati di frumento. Bologna pi. Nobili, 1833 (in Atti de Georgofili. Firenze. XI. Vgl. Passerini in der Isis $18.2,368)$.

Nendtvich, Pharmazeut z. Fïnfkirclie» (Süd-Ungarn), Bedeutende Faltersimmler. Fruher leidenschaftliher Butiniker.

Semuing, Professor und Dr. phil. in Constanz. - Upber ein den Wein. traubeu höehst schïdliehes vorzügliclı anf der lnsel Reirhenau bei Constanz pinlieimisches Insect (Tinea Uvae) 2. Aufl. (Erste 1811). Mit 2 ill. Kuplertaf. fr. 8.

Nemuing, Kajefan, Wundarzt zu Holsenfurt bei Budweis in Bölunen. Schon hejahrt. Sammelt ïusserst fleissig Kerfe.

Nenling, Lehrer in Aschersleben. venschel, Dr. med. in Ofen.

Nenstadt, in Breslin.

Vewusun, Edwaid, Zoolog zu Deptford in England. Der Verfasser do" Monographia Aegeriarum Augliac (Walker eut. Maguz. u. 1, Sept. 1832).
- Sphinx vespiformis, an Essay Lond. 8. (Exeerpt. Walker ent. magaz. n, 1, Sept. 1832, 44). - Entonological notes (Valker I. c. II. 3, Apr. 1833, 283$28 \times$ n. 4, 413, 11. 5, 505, n. 7, 1834, $200,11.8,313)$. - Osteology, or external anatomy or Insects (ibid. n. 4, Jul. $1933,394-433$, ․ $6,1834,60-$ 92, 2 plat.) - Atteinpled division of britisl insects into natural orders (ibid. 11. 9 , oet. $1834,379-431$ ). - A familiar introduction to the listory of inscets. London. With numerous illustrat. 8. Lond. $1840,125$.

Vicolai, Erm. Aug., Dr. med. in Halle. Entomolog und Sehriftsteller. Colcopterorum species agri Haleusis. Halie 1822. 8.

Niclere, Franz Antou, Dr. med., in Prag. - Bcohachitungen der ersten Stïnde von Sesia culieif. und mutillacf. (ent. Ztg. I1I, 68). - Bölmens Tag. falter. Prag 1837.

Nicolet, H., in Neuenburg. - Reeherches noul servir à servir à l'histoire des Podurelles. Neuchatel 1841, 4,88 , tab. 9, col. (Mem, soc. helvetique VI. - Isis 1842, 871).

sicoli, in Mailand.

Nicolsou, zu Upsala (?).

Nicpold, Hauptmann, in Cassel.

Niunrod, Pustor, in Quenstedt (Harz). Entomoloz. Spraelt in der z.hnten Versammlung dis naturwissensehaftichen Vereins des Harzes zut Blankeuburg über das hïufige Ersehei"le" vou lchneumoneu ete.

Nisete:o, zu Citta-Vechia auf der lnsel Lesina in Dalmatien.

Norlier, Chitrles, Bibliothekar am Arsenal und berïhmter belletristischer Sehriftsteller in Paris. Sammlung:

Nibrdinger, Hermann, Candidat der Forstwissenschaft zu Stuttgart (Oberfunariatl gl. Namens in der linde).

Nordlinger, Stadtrath in Tühingell, Ein iusserst cilitger Schneterlimgssammler:

Fordmann, Alexander von, Professur der Naturgeschichte am Lyceum Richelien ill Odessa. Beribmter Zoolog und Sehriftsteller. - Symbolac ad monographiam staphylinorum. Petro. poli, 1837, 4. Cun 2 tab. (Mén. de l'Aead. de Petersburg 1837, IV, livr. 1, p. 1). - Mikrograplische Beiträgo z.11r Naturgeschichte der wirbellosen Thicre. 1. Heft. Berlin 1832. - Stimm. 
Apparat bei Splinx atıopos (Bulletin del' Acad. des sc. de St. Petrrshourg 1, 1835 et 1836 , p. 164 . - Isis 1839 , p. 641).

Nonris, Thomas, Esq. in Iancashire (nach Silbermann in Rotrales bei Manchester).

Norwich, A. H., in Brenen. Entomolog. Sammlung.

Nowleky, von, in Thorn. Lepidopterolog.

Numannu, Dr. med. und Direetor der Thierar\%neischule zu Utrecht. Schrieb jiber die Bremsenlarven. (Vol. Hertwig.)

Nuttse, D., in London.

Dekskay d'Deksko, Baron von, k. k. Kanmerherr etr. zu Oedenburg in Ungarn. Berïhmer Entomolog und Schrifisteller. Bedeutende Sa mmlungreuropäischer (besonders ungarischer, tiirkisclier etc.) Käfer. - Orthoptera novil, illustravit. (Acta Aead. Iropolil. Bl. XVI, 1833, p. 959, in. Anm. von Silummel).

Dherleitner, Igu., Brauer in Miinchen. Grosse Sammlumgen vou Küfern und Sclumetterlingen.

Cberts, Johaunes, Entomolog in St. Petersburg.

Drermanu, Paul und Cliristian, Tuchtabrikanten zu Stollbere.

Dken, Lorenz, säelis. Hofratl, Dr. med. et phil., Professor dev Naturgeschichte ete. in Zürich. Ilochgrfcierter Niturphilosoph, Zoolog, Oryktognost. Anatom und Scliriftsteller. Stifter dor Versammlung Ieutscher Naturforscher und Arrzte. - Einleitung in dic Eutomologif : oder Elemente der Naturgeselichte der Insekten. Vou WVilliam Kirby und W. Spence. Mit Abbil. dungen, Bd. I-IV. M. Kpf. 8. Stuttgart, in der Cotta', chen Buchlandiung 1823. - Nafurgeschiclite. Jena. Allgemeine Naturgescliclut: f. a. St inde. Stuttgart (Holfuann) 1833-38. Mit Atlas. Die Zoulogic bestrlit ans 8 Bänden (IV, V, 1, 2, 3; VI, VII, 1, 2, 3).

Mirexs, J. F. M., von, Gesandter in Birlin( $P$ ). Entomolog.

\section{Moskau.}

Dininnusen, Gustav von, in

Dpiz, P. M., med. Dr. zu Prng. Der Grïuder ciner Nituralien-Tanse.jAnstalt (hanptesichlich lïr Pflallzent). Grumdgesclic:ktø. Botani, lls und Entomolog; selur thäfig und fleissig. $-\mathrm{N}_{i 1}$ turalicutuusch, Prag 1820, -12 II eftc. 8 . horst.

Dppermana, Dr., in Delmen-

Arkniguy, Alride Drssalimes d', in Paris. Der lierübmte Reisconde durch Siidamrrika voll 1826-1833. - Voyage dans l'Ameivue meridionale. Paris I, 1834, 672, II, 1836, 4. (l.sis 1839 , 406; Müncluner Gelelirt. Anzoig. 183\%).

Arlich, vou, k. presss. Premierlicutenant in 2. Garderegiment in Kreuznisch an der Nahr." Sammelt vorzüglich Kiffer.

Dtto, Nafuralist in Brrlin (?). Sammolte auf Cuba.

Dugspurger, eliemuliger Offzier der Scluweizrrynde in Paris, mu Bern. Schöne Simmlung vou Käfru; eifiger Sammler.

Pagenstechex, Rudolph, 'Kaufmann in Bremen. Lepidopteren.

Palliardi, Dr. med., zu Franzensbad hei Axch in Bühuren. Brschricb 2 Dekaden ueuer Carabicinen und be sizi rine sehoine Sammlnng europäischer Käfer.

pander, Gerard, in Petersburg. Beriihmter Reiscuder, Naturforschire und Schifisteller.

Parreyss, Ludwig. Naturalienluäıd. ler in Wieu (Leoproldstadt No. 600). Bereistr im Jahr 1823 die joniscllen Inscln (Corfn, Paxu, St. Nanra, Ceplinlonien mind Zante); im Jul11 1829 Odessa und pan\% Taurien mit der K rimm, und im Jal). 1831 Lingland, Croatieu, die Bukowinn, Dalmaticu (Ragnsa umd Cattaro). Hr. Drmel sclurieb (April 1835), Parreyss sey Director einer Fa. brik in Nïhren geworden.

wascant, in Mosran. Entomolog. Pascerimi, di Patrizia. Carlo, Profrusor mul Conservator an Museum dej Naturgeschichte in lilorenz. Beriilım. ter Zoolag und Schriftsteller. - Trug in der Versammlung der Naturforsclier zu Pisa (1839) iuteressaute Bemerkungen vor über die Larven und Puppen von Scolia flavifrons. - (Osservazioni sulle larve ete. di Seolia flavifrons. Pisa 1840 , 8., 16., tav. 1. - Continuazione delle Osservayioni etc. Firenze 1811, 4, 7 tav. Alogedruckt in Okeri's Bericht darülıer: Isis 1341, p. 639). Moltiphieatione in Firenze della Paroraria cucullatil. Firenze isil, fol. s. 'lav. 1 rol. - Osservazioni sapra la Sphiux atropos. Pisa 1828, 8. (Antologia 
nov. Dee. 1828; Anual. d, seienc, nat. XIII, (1828) 332. - Isis $1830,206$. - Giornale seientifico di Pisu 1828). - Osservazioni sul Baco damegiatore delle ulive, e sulla Mosea in (ui si transforma (Oscincs oleac) (Giormalc agraric toseano, 11. 10, Firenze 1829, 8. 1 tav.). - Osservazioni sopra alellue larve e Tignole dell' ulivo. Fase. vou tt Seiten, 11 Tab. (Ginrn. agr. Tosrano n. 23). - Ueberden Schaden, welchen ein Kerf den Olivin vermisacht hot in dem Dorfe Castiglione della Pescaja-1838. 8, 16 (Atti dej Grorgofili XVI. Auszongeweise in der Isis 1842, 371 ). (Osservazioni sopra dhe li1setti nocivi, Lytta verticalis et Apate sexdeutata. Firenze $1810,8.28$, tav.Rapporto sopra il brucu che in quest'anno devastil $i$ seminati di frmmento etc. Firenze 1833, 8. (Vol. Negri, in Isis 1842 , p. 368.) - Aleme no.ivfie sopra unat specie d'insetto dell gen. Thrijs. ete. Firenze 1834, 8. (Atti dei georgofiti X11. - lsis 1842, 368). - Rapporto sulla Memoria manoscritta de Sig. F. Luciani di Casielnuovo sulle larve danueggintrici del grano siciliano ete. Firenze 1835, 8. (Isis 18.12, 369). Memmia sopria gl'insetti etc. Firenze 1837, 8., 12. (Direse Ablandlung: "iber einige dem Welschkorn, Weizen, Anis und Minngold selıadliche Kerfe" (steht ancls in Alti dei Genregofili $\mathrm{XV}$ und anszlnswejse in der Isis $($ K42, 370).

Pand Wille elna, S. H. der Prinz, Hersog von Winttemberg, z.n Mergenlleim. Hoclbberilinter Ripisender (in Nurdameilia $(822-22$ - ), Niturfurscher und genialer Reisebesplueiber. Grosse und ausereztichuete Sammlungen.

P'nulet, in Paris. - Flore et Fanne de Virgile. Paris 1834, 8., fig. color.

Pearson, J. T., in Calcutta. Ucbel pineu ostindischen Ribenkifey (Haltiea nigrofusca) (T'ransaetions of the agriculfural Society of India IV. - Isis 18.13, 809).

Peck, Willian-Dandijlge in Boston. - Mémoire sur une espèce de Rhynchene gui ronge les pins (Collection of the Massacluusets Hist, soc. Vol. IV. - Zool. Journ. I1, 1825, 487). Natural Ilistory of the Slug-Worm ('Tenthredo) (ibid. V, 280. Boston 1799, 8.)

Pecluoll, Vietor, Dr. med. in Florenz. - Bohrer-Falle (Trivella) um kleine Erdthiere zu fangen (Ginrnale toscano 1, Nn. 3. 1841, 216. - Vergl. dariilor Boie, Isis 1843 , 11, p. 970 und Isis 1843, 1. 668).

peil, Will., Kaufmann z.ı Külı.

Peiroleri, G. L., Kriegskommissiil und Oberauditor bei der kün. Rechmungskanmer in Turin.

Eellatier, in Nijni-Nowgorod. Entomolog.

Pelzer, Jol. Ant., Messingfabrikant zul Stolberg bei Aiteliell.

mercheron, Acluille Remy, in Paris (Ruc des griands Augustius). Berilunter Entomolog. - Monographic des Passales. Paris 1835, 8. (avec 7 pl.). - Bibliographie entomologique, comprenant lindication par ordre alphabethique de noms l'auteurs 1. des ouvrages elc., 2. des manographices ete. 2 Vol. Paris et lumdon 1837,8. (I Vol. XII it 326 I'ag. II Vol. 376 (14 Fres.). - Monograpluie des Cétoines et genres voisins, format, dans les familles naturelles de Latreille, la division rles Sraribees merlituphiles; par 11. II. Gory et M. A. Percherou. 1. Ljivraison. A Paris, J. B. Baillière, libraire, a Londres, même maixon, 219 , regent-street, 1833,73 Pagg. 7 Planclies. 8. Con trititera de gré à grié del' exemplaice qui contiendra les velins originalx (envirun 150 Fr. par livirison) 410 Pargo. 72 fig.

Perleb, Carl Julius, Dı. phil. und med., Professor der Naturgesehichte und lotanik z.u Freiburg in Badeu. Lehrbuch der Naturgeschichte. 11 Bde. Freiburn 1826-31-35. 8 .

Perolli, Carlo, in Turin. - Essuis sur l'organisation externe et interue des insertes. Turin, 1808, 12.

perriejres, August, in Bordeaux. Siluiftstelter.

Perromd, in Mailand.

Perty, Maximilian, Dr. med. et phil., Professor der Naturgeschiehte etc. an der Hochschule zin Bern. Berïhmer Zoolog und Seloriftsteller. Alfgemeine Nafurgeschichte als philosuphische Ilımanitätswissensehaft. 3 Bde. Beru 1837-39, 8. - Ohservationes nommullae in coleoptera hudiae oricutalis. Monachii 1831 , 4. e. fig:Delectus animalimm anticulatorum, quue in itinere per Brnsiliam amis 181720 jussu et auspiciis Maximiliani .Josephi I. etc. peracto collegerunt Spix et Mirtius. Digessit, Descripsit et 
pingenda enravit Dr. Max. Perty. Monachii. Imp. Editoris 1830-34, 4.

Petit, Louis, Exp. in London.

Fetterson, Carl WVilh. in Lund. Phifomyxide's et Ochtidiae Sueriar. Lilıdac 1823.

pelliucer, Jcher in Hameln.

Plabllugl, Nofuralist in Berlin. Sammelte in Peru.

Plallipai , Rudolyli Amand, Dr. med. in Berlin. - Diss. inaug. de otthopteris berolin. IS30, 4.

Piazzi, P. D. Ginseppe, Präisidenı der Akademie in Neapirl.

Pickering, C., Esif., Curator der entumulngical Sociefy in Lomblon.

Picofaunina, Graf, in St. Petersbu'g (?). Sammelte in Califormien.

Fictet - HBaraban, F. R. alules, Soln, Administrator und Professor der Zoologie an Musemm z.⿲丿丨日 Genf. - Ilistoire naturille generivale el partienlière des insertes nenroptères. I. Monographic (Perlides). Geneve et Paris 1841, ช. I1. Nonographie: (Epluemerines). Recherches pour semir allhistuire ot a lanatomin des Phryganides, onvarge qui a remporte le prix Duvy fonde is (iconeve pour encourager l'etudo des seiences plysiques et naturelles. Genève, gr. in 4., aver: 20 tables (24 0 . l..). - Notices sul les animanx wonveanx uu pen counus du Musce de Genève. Geneve 1843, 4. - Vortrag bei der Versammlang Ifer Naturforscher in 'luriu 18.10: Dallukanal ein Untersehied der Neuropteren (Isis 18.12, p. 251). - Description des guelepues nonvelles expeces d'insectes du bassin du Jėuın. (\$lèm. le Geneve VII, 1835, 1. 173-191, arec 1 planche) (Orthopthera. Nemoura ete.) - Descriptions de quelanes nouvelles especes de Neilropteres du Musce de Genève (Mem. de la son. de phys. et d'hist. 11. de Genève) 4. I pl. col. - Note sur Ies organes respiratoires de Capricornes. (ibicl. Imprimcicic A. I. Vignier, rue du Rhone, maison de la poste).

Pierret, A., in Paris. - Sur le Polyommate Ceronus (Annal, soc: cut. France 11, 1833, 119-121).

Plrugruluer, in Linz. Entomolog.

Plienlinger, Dr. med. und Sckietäs des landwirthschaftlichen Vereins fïr Württemberg, iu Stuttgart. - Der Maikifier als Lâve und Kïfer. Stuttgart 1844, 8.
Porlewin, in Wien.

Pacy, Pl., Advokat in Paris. Bereiste Cuba und beschriel rine Cellturie Falfer dieser Insel. (M. Kupfern. Pavis 1s32, 8.) - Observations sur le rin des Iexpidopteres de la tribu des crepusculaires w des noeturues (Anual. sor. elt. Fiance, 1, 1832, 91-91).

IDolal, Kunfinaun und Stadtaldermicill in Glat\%.

Poncenta, Peter S. du, Priisident der amerikanischen philusophiselıen Akndromie ete. in Philadelplia.

Hounz, Isarent, in Turin. - CoIroptera salutiensia sive Ennmeratio methudica eoleoptrorom, quac ill ag ro siluticusi reperiuntur ete. (Mém. de Turin 1804-5, p. 29-95, 2 Tab.; auch nene (iattungen.)

Pomzomi, Milrchese Ali, in Mai. land. Simmlung.

Forein und Brugnerin, Fïrst Alphous Gahr. v., k. k. Geheimerrath, Kämmerer nud Gonvernenr des küstenlänclischen Guberuialgebietes zu T'riest. Porro, Nobile Carlo, in Mailaul (Confrada St. Giovanni, No. 1808). Bevilunter Entomolog und Srlyriftstellep. - Bibliographia malueologica (arbeitet divalis.

Pouret, in Paris (?). - Mémoile sur quelques inseetes de Barbaric. f.lournal de physiq. 1. 30, p. 241-45, T. 31, p. $111-116$.

Freasl, Jol. Swatoplue, Dr. und Professor ill Prag. Der bekannte Botanicus. Seliune Simminluner vou Reiferu. IPreysle:r, Bergmeister zal Zbirow In Böhnen: einer der ersten und besten Colenpterologen, A watomen und zoologischen Schriftsteller Bölumens. Ein herliclues Werk vou ihu mit genauen, vergriossentru Abbildungen der Fresswerkzene, Fuilıler, filüzel n. s. w. sall icls bei ilm im Manuseript. Aus Mangel an cigrnen peruniairen Mitteln und bei nicht linläinglich gesicherter Subscription wird dieses Werk wohI kaum erseleeinen, was selar zu bedauern wïre (Demel in Prag).

Prévost-dBuval, P. L. A., Negoziant in Gonf. Sclüue Sammlung.

Promay, Freilier Gabriel vou Thot-Prones und Blatıitza, ungariseher Magnat in Aska.

suzos, Militär-Unterintendant in Paris. Sammelte anf Saint-Jean l'icdde-Poril.

Pyday, Dr. med., iu Ofen. 
Guoy, Jean-Renc-Constant, in Par is. Berihmtey Reisender. - Voyage da I'Uranie et de la Phyaicieme. Zoulogie conjointement avee $G$ a ima 1 . Paris 1824, 4. (2 planches; insectes).

Rnimonil de In Sagra, Professor der Natorgesshichte in Havanua.

Inmbir, J. Peter, Dr. med. in Pais. Kundiger Kerfsamuler und Mirarbeiter an den Ironcs des Lepidopteres d'Europe von Bo isduval.Notire sor plasirurs espèces de Lepidopteres nouveaux du midi de la France: av. 2 plancl. (Aunal. des scienc, d'obEervation II, 255, Mai 1829). - Funna d'Andalonsie, moj mohere Kataloge.

Themalohr, K. A., Di. med. z.1 Schloss Beichlingen in Thüı ingro. Bckimnter Kerfanatom. - Ablildungen zor Anatomie der Kerfe, 18u8. - Kleine Abhandlungen ang der Anatomis und Physiologie der Insckten. (Mag. des Ges. nat. Fi: zn Berlin IV, p. 287292 V, 386-90.) - Anatomic des Darmkanals und der Geschlerhtstheile vom Carabus monilis (virlnelu cancellatos Illig.) (Tab. IV, V) (Mag. naturf. Fr. zo Berliu 1, p. 207-222).

Runtzau, Graf von, in Lcipzig. Entomolog.

II anzort, in Kerensk (Gouvernement vin Penza'. Eutomolog.

Rasclo, Heiurich, Dr. und Conservator am k. Naturnlienkabinet zu Chri. stiania.

Mntlalke, Heinrich, Professor cte. zu Königsberg. Der berühute Anatom. Srlnicb unter anderm anch jiber die Sexualorgane der Libclluliden (1832). Konigsherg 4. Mit 3 Kupf. VI, 38.

Ratzeburg, J. T'. C., Dr. med, mod Professur der Naturgesi hielite zo Neristade-Eberswalde. Sihriftsteller und bekanter Forsclier. - Gastropacha pinivora Tr., häufig mit pityocampa verwedhselt. (Ent. Zeit. I, 40.) - Ueber die Lebensweise der ISâfer, welche der Kiefer (Pimss sylvestris L.) sobädlich werden. (Verhandl. d. k. Leopold. Car. Akad. d. Naturf. 17. Bd., 1. Abtl. 1835, 1) $421, \mathrm{~m} .2 \mathrm{Kpf}$ ) - Die Forst-lısıkten oder Abbildung und Beschloribung der in den Wäldern Prenssens und frr Narlibarstaten als schädlich oder nitalich hekannt gewordenen lnsckten. I. Die Käfer. Berlin, 1837, 4. Mit 22 theils ill. Kupfertaf. - Dic Waldverderber und ilure Feinde, oder Besehrei- hung und Abbildung der schädliclisten Furstinsekten nud der vibrigen schädlirhen Waldthicre, nebst Anweisong zol ihrer Vertilfung und zur Schoumng ihrer Forinde. Mit 6 stahlstichen; eni. und 2 Lithogr. Beslin 1841, 8. - Nachl. trag lierzn, oder Verionlerungen der 2. Ansgabe. Nibst I entumologisclicn Vademerum (i) gi'.4.) und Ilolzsrhnitt. I.cx. - 8. Berlin 1842. (Ist bloss cin eigener Abdruck aus dem grassen Werk.) - Forstnaturwissenschiftliche Reisen durch verschiedene Gegenden Dentsch. lands. Mit Anhang von Prof. Dr. Ferdl. Sulu uze zu Elcena. Mit 4 illum. Taf. und uldhren Holzschnitt(n), Berlin 1842, F10. 12. - Bitle an Forstmïuner, Gärtner ete (wugen Forstins.; Eut. Ztg. II, 6o.) - Untersurbung des Geschlechts. Zustundes bri den sogenannten Neutris der Bienes und úber dip Verwande. srhaft derselben mit den Königinnen. (liseopold. Akten; Bonn 1833, p. 6II, Taf. +7). - Mittheilungen ïber die Ratzebuig'sche Inscetensimmlumg zu Nenstadt-Eherswalde (Lint. Zivitg. III, 1361. - Eine dntwort anf die Frage des Hrn. Prof. O. Heer betr. dell Lebens-Cyclus der Maikiifer) (ent. Ztg. III, 39). - Diplerolugische Mittheilangen. (Ent. Zig. III, 93; 2 Gallniicken (Ceciclonyia) entdeckt. - Apussere Geschleclitsunterschiede bei dor Maulwurfserille, mobst Mittheilungen cines noch nieht bekaunten interessanten $\mathrm{Zn}$ ges aus ilurem Lclicn (Ent. Zcitg. 111, 2(iz). - Medizinische Zoologie. Berlin 183\%. 4. (nit Brandi).

Rau, k. k. Beamteter zu Brünn, frilur in Finme. Entreekte 1825 die Suturuia caecigena.

Mnve, B., Dr. med., in Köln. Büffon's simmtliche Werke nebst den Sapplementen derselben, mach der Clas. sification des G. Covier, mit zoo Stahlstichen, mindestens 900 Thiere dajstcllend, iblersetzi und mit de ut möthgen Erläuterumgen versehen. Düsseldorf $1837-39$.

Read, W. H. R., Esq. in London. Teeliemmacher, Pfarrer in Wegscheid (Unterbaycin); fribler in Zwiesel.

Tedtenbre che-r, L., Dr, med. und Adjunkt am k. k. Museum in Wien. Illietrationes et descriptiones Colcopterorum novorum Syrioe (p. 1-20 in Russegger's Reise, auch cinzeln bei Selweizelbart in Stut(gart 1843).

Tees, Dr. med. (Eugland). 
Teich, G. C., Dr. nnd Professor der Medicin z.n Berlin. Fintomolog vou grossc" Gelehrlhrit. Hat rine grosse Kiffersimumlun $(20,000$ Speries $)$. Bemelimugen iber die Lebensverlatiltnisse der Coccinellon iberhanpt nud der Coprinella lieroglyplica Fubr. (flexunsa) iushesoudere. (iMag. d. Ges. nat. Fr. in Brolin. 11I, p. 288-296), - Beitrag zur Lorlure von der geomraphischen Verhrritung der Insakteu, inshevondere der Kïfer. Merlio 1833, 4., 36 (Acta Acad. Nat. Cur. XVI, p. 805). - MIiıtissac ln-ectoluul iconibus illustratac. Norimb. 1797, \&. Collu 1 tinh. color.

Reiclne, iu Paris (geh. zu Lille). Samumelte in Bengalen.

Feichenbncik, H. G. L., Dr. med., Hotrall, Profissor efe. in Drisden. Virlberülunter Botaniker, ZooJor und Sillriftstelles. - Vnnographia Pselaplumutu. Lipsiac ap. Voss. 1816, 8., 79, 2 Tilb. (lsis 1S2x, p 1566).

Reinaluard, H., Professor der Zoologie in Kopculaggen (nicht Stockholin).

Reinusarelt, C. G. C., Dr, med., Pro:e-sor der Naturgestlichte etr. zu Leyden. Berilumter Amphibioloy mad Schriftstiller:

Teissig, Oberforstsekvet ïr in Darulsladt.

IRemascleanidt, Obcrlelurer in Breslan.

Tennie, Jannes, Professor derZno. logrie an kön. Collegrum in London. - Alpluabet of insects for the use of Beginners. Lond. 1832. (Walker ent. Magra\% 11. 1. Siept. 1831.60\%.

Ftemsslanex, Jer. van, Dr, med. ju Philatelpilia.

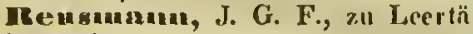
bei Lüıehurg.

Bequieu, in Avignon.

Iicinter, Erust Jleiur., Leluer zu Frankfurt a. d. 0. - Lecitfiden der Zoulogie fiii höhere Bürgersclunlen. Franki'. a. \&l. 0. 1843, 8.

Rielrter, H. L. F., Oberforster in Kliitz bri Stettin.

Michter, l. F. W., Iustitutslelırer in Breslan. Entomolog nul Schriftsteller. Tausclit Kiiler und Finler. Kritisches Verzeirhniss der bislıer bekannt „ewordenen sehlesischeu Sthmetterlinge. Als Britreg zu ciner Fanna dieses Landes (Germar's Mlag. d. Eut. 1, p. $72-114$ ).

Richter, zu Königsberg. Schöne
Faltersammlang. - Harpyia Milhauseri und Diplutera ludifica (ent. Zeitg. II, 59). - Zu Diplitera ludifica (ent. Ztg. III, $165 \%$.

Fiedetex, Ludwign, Candidat der Theolonge zu Sehloss Licheustein bej ligerin Bölımen. Lepidopterolog. Sclı̈̈ne Silmumlus: gen.

TRied1, in l'rag. Simmmler.

Rielı, Hanptsfaatskasseneontro. Ieur in Kassel. Ausgezeicluneter Kenner der Kaifer; lat cine selır schöne Simmulung sowohl Europice als Exoten und arbeitet mit grossem fileissund vortrefflicheo Genauigkeit. Hat mehre Anisotoma anformulen, die unser, leider zol früh verstorbener, Frenud Dr. Sirlumilt in Ger mar's Zeitselurift besiluieben lat.

Ficese, Mr., zu Frankfurt. Tauselıt nud verkanft Lepidupteren.

Ieiley, in londun.

Mivoli, Hermog von, Friirst von Massena, in Paris.

Rohincenu - Desvoidy, J. B., Dr. nucd., in Saint-Saveur (Youne). Belaunter Scluiftsteller. - Essais sur la tribu des Culievdes. (Mém. de la soc. d'hist. Uat. de Paris IlI, 390, Oct. 1827). - Mémoire sur les Miodaires (formant 1 Vol. des Men. des Savants etrangers del' A ('ad. des sciene. Par: 4.) - Re(herelies sur l'organisation vertébrale des erustaces, des aruchuides et des insectes (av. I pl.). Paris 1828, 8. Sur l'harmonic des espèces de coléoptores tetramdres avee le regne vigeital (Bullet. sore plitom. 1826, Der. 192). - Classificition des inseetes d'après Ies larves, less ailes et les bonclies.

Eonert, Corl, in Clience bei Liittich, in Bel rien. Entomologischer Schriftsteller. luteressaute Samunlung. Description de trois nonvelles esperess du genre Diastata of unc nouvelle espire du genre Opomyza (Amual. soc. ent. de Frante, III, 1834, 459).

zockstroh, Ileiurich, Dr. und Recturzı Königsherg (?). Schrieb : „Anweisung, wie Schuretterlinge gefungen etr. werden müssen". Leipzig 1825.16.

Roe, Lieulenant in Loudon. Sammelte viel Schöncs und Seltenes von Kerfen auf seinen Reisen in Austral. asien. Hope lat Mchreres davon in den Transactions of the entomological Soeiety bekinnut gemaeht.

Roetzer, in Saunen (Canton Bern). 
Toemer, Landricliter in Aub| (Bayeru). Der bekamule Botaniker.

Bogerer, Theodor, Negoxiant in Bordeals. Selir glosse und frachloulle Sanmulumge. - Justructiuns à l'usage les peusounes qui voudaients s'ueruper à reenoillio des insertes punr les rabiurts d'lisfaire nalurelle. Bordeanx, 8. - Descriptions de six especes nouvelles de Pajillums. (Bullet, l'hist. nat. soc. I,inn. de Bordcallx. I. Livr, 35.)

Thogersom, in Lourlon. - Unber Glow-worm (Lampy ris) (l'hilas. Mizyaz. I.VII, 1821, 53).

Iiroget, Prier Mareus, Dr. med., in London.

Relett, Loren\%: I,plurer in dem Priger'schen Erziehungs - Institut zu Tricest (Salzmolo Nr. SS 8 ). Entomolog. Sicilianische mod exotiselıe, wic illyrische Kerfe. Hamblelt damit.

Relli, Plarmazent in Rom (veryl. Met i $\times a)$.

Loumare, Balthasar li. de, General cte. iu Paris, Schöne entomolo. giselie Samulumg und ansgezcichurte Bibliothek. - Note sur la larve du Leptis veruileo (Dijtire) av. fig. (Anu. soc. Pnt. France II, 1833,498$)$. hing.

Fomalds, in London. - Fly.fis-

Hondani, in Parma. - Insetto ditfero, momoria proma. Parma 1840. tul. (Novi muali delle seicnze naturali. Bologna 1841 , 1. 398.) - Vorschlitg \%u rimer Klassifitiation du curopiischen Zwoifligher nach Familien. (Nuovi ammali 1. e. p. 257. - Isis 1. e. 6 /4.)

Rerdurl, Rudolph, PfarreviuSeen bei Winterthu: $\tilde{A}$ usserst thätiger Sammler von Falteru, dro gute Excmplare billig verkuuft.

Finsembarer, W. G., Dr. Inred. und Conservator an naturlistoriselien Museum der Universität zu Elingen. Beriilumter Schriftsteller. Bereiste 1838 Ungarn; 1840 und 42 liess er manz. Tyrol durelisuchen und 1843 die Gegend $n$ In Fimme. - Scine Sammlung puthiilt gegen 10,000 Gattumgren rutopäischeı" Käfer: Exaten salu" wenige. Ueber die a 1 und in alten Kânen lehenden Käfer (eut. Zeitg. III, 162 -165). - Entomologische Mittheilnn gen (ent. Zeitgr. 1842, 111, 33). - Dic Lauf - und Schwimmbifer Erlangens. Eil. 1844, 4.

Hosensebdr, Dr. med., in Lund, schichte in Leobschütz.
- Prodromis Fanna colcopterorum L, Imindensis.

Iesentinal, Fr: Chr., Dr: med. mad Professor zu Greifswald (nicht Halle). Bercilumtor ki cofpliysialom, Anatom und Seluiftsteller, - Ucher das Gertuchooryander Stuben-und Selumeissticere (Muscan elamosticy el carmaria). (Reils und Auteurietls Archiv f. Pliys. 1811, X, p. 427. - Germal Milg. I, 1, 1. 138.)

Eoser, C. L. F. v., greleimer Legitionsmath und Kanzleidicchtor in Ministerium der auswiitigru Angrle. genheiten in Stuttgint. Riblumliclo bekumuter entomologiseliep Folscher und Seluittsteller. Ansugzuichuct schöne Sammlumer. - Dipteren Wültemberges (183 1, Cotrespondenzblatt). - Bemerkingen ïher dic Naturgeseluiclite dos Geschlechts Xyloplangus (Nufurwissenschaftl. Abliarllung('u 11. 2. Jlefi, 1828, 185). - Verzoichuiss dev in Würtemlierg vorkomumendeu Kïffer (Correspoudeumblatt des laudwirtaschaftlicleu Ver. pins, 1838$)$.

di osmuatissler, C. A., Professor iu Tharand. Schriftsteller. - Die Forstiusetern. Lcipzig 1830-35. 8 .

ncostang, Caximir, Dr. med., il Symphoropol (Tinien). Eitomolug.

Rotembach, in Schinfeu. Lepidopterensilmulumer.

Rotermanel, H. IV., Conservitot des Naturalien-Kabinets in Breslau.

ERotla, Dr. med., in Müuc-len. Sammolte in Angypten und Palästiua: als er Schubert begieitpte cte. Ist als Militärnrat engagint in Bombay und Cal. cilta.

Eetli, Franz, Coaperator z.u Sogritz hei Dällach in Kärutheu, Coleopteren.

Rovedic, Proprintill a.l St. Denis auf dev lusel Bumluan (Markarmias).

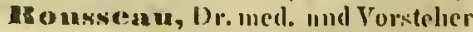
der Anatomie im Pfanzengarten in Paris. - Geschlerhisveisiluiedrnheit hei Dermestes (Porus des of au Bauchseite des 3. und 4. Abdominalscgunents, ungrehon vou cimen Brisuhal Hanre. (Guciriu, Revur zoul 78. - Lrichson Wiegul. Aiclive f. Nale. V. Jahı'g. VI. Hft. 332) Dr. Schuidt und Dr. v, Sicbolal, iil der (unton. Zeit: I, 137).

IBonx, P., Maler und Conservator des Museuus der Niturgescliclute zu Mirseille.

IRticher, Jehrer der Naturge. 
Ithdiger, Dr. philos, in Bresslan. Tuppel, Eduard, Dr, etr.iu Frunkfurt a. M. Der berühnte Reisende in Afrika und zoolagische Schriftsteller: Seine liberalitit überliess die gesinnmelteu Niturschitze der Stadt Frankfort als Gesclienk.

Pusdd, G. T., in Losidon. - Ob. servations on Hesperoplilus arenarins and on Zabrus gibbus (Walker entom. Magnz. 11. 7. A pr. 1834. 180).

IEmy, Georg Carl, Dr., ord. Praf. der Reclite (finilier Professor der $\mathrm{Na}$ torgesehichte ete. zu Kerzthely) in Grail.

IEnnde, Wilhelu Hermann, Dr. med. in Halle. - Brachyelytrorum speejes agri ha lensis. Dissert. inang. Hallac (Lips. Voss.) 1835, VIll et 32 page. 8. (8 Gr.)

Russegger, Joseph, k. k. östreichischer Bergrath und Vicedirektor der k. k. Berg- und Salinen-Dirertion zu Hall in Tyrol. Der berillumte Verfasser der lurirlichen Reisen in Enropa, Asien und Atrika, mit besonderer Riicksicht auf die naturwissengelaftlichen Verhältnisse der betreffenden Läıder, unternonumen in deu Jalıren 1835-1841, IV Bände. Stuttrart, bei Scluweizerbart. Nit Atlas in Folio.

Restlkuk, zu Gadulming, in England. Schriftsteller. - Some observations on Bliglit (Walker ent. Magaz. 1832 und 33). - The other End of a trip to the isle of Wight, (ibid. n. 7.)

Ruthe, Oberlelirer (sonst in Frankfurt an der Oder) in Berlin (vergl. Troscle l).

Danere, Di. med. und GymnasialOberlehrep in Braunsberg. - Abnormitït von Prionus coriarius ó. (preuss. Provinz.-131. XX. Aur. Ent. Zeity. I. 48). - Zoologisehe Tufeln. gr. Fol. Braunsb. 1810.

Sachse, Dr. med. in Leipzig. Entomolog.

Manller, Jos., Dr. med. und Professor der Botanik, Custos an NaturulienKabinet des ungariseloen Nationalmuseums in Pesth. Botaniker ond Zoolog.

Salibers, Carl Reginuld, Dr. med. und Professor der Naturgeschichte auf der Universität zu Abo (Finnland). (2. Auflage p. 81, hat: in Seliweden!). Beriilimter Entomolog und Scluriftsteller. - Periculi entomographici, Spe- cies insectorum nondum deseriplas propollells, Aboae 1823. P. 1, 2, 3, 4, 5. (Thon, Areh, 1l, 205, p. 1\%.) - Diss. entomol., inserta femica encmerans. Helsingf. 1817-1844. Park J. 8 maj.

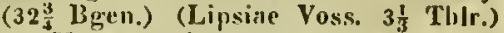
- Novae coleopterorum femicorum Speeies. Helsingfors. 1834. - Dissertatio ueademial, observationes quasdam Historiam Notonectidnm illustrantes pro. popositura: Aboae 1819. 4 pagg. 15.

Salaberg, Ferdinand, Solu, Dr. med iu St. Petersburg.

thinson, in Paris. - Voyage an. tour du monde par les mers de l'Inde et de Cline, exécuté sur la Corvette Favorite, 1830, 1\$31, 1832.

Amint-goilaire, Auguste de, in Paris. - Relation d'un empoisonnement ransc paı le miel de la Guèpu Iecheguaua av. fig. (Mémoire sur Musé(um d'list. nat. de Par. XII. 293-348. Extr. Bullet. de la Soe. philomat. 1824. Mai, p. 74.)

Sallé, Augusta, in Paris. Tochter des lierühmten Entomologen; war selion als Kind mit iluer Mutter in Mexico. Sallé, in Paris, Vater und Solnn. Sammelte in Mexico eine grosse Menge Käfer (nicht 30,000, wie die 2. Edition p. 81 irrthiimlieh bencerkt).

Salzaman, deutsclier Botanist z.I Montpelliev. Kerf- und Pflinzenländler.

Samonelle, George, in London. - Entomological Cabinet. London 1832. Nr. 1-4. 12. - Entonologist's Usefu! Compendium. London, 1819. - General Directions for colleeting and preserving Exotie lnserts ete. London, 1826. \&. with plates. - Nomenclature of b ritish entomology, alphabetically arranged. Lond. 1819, 8.

Annder, Reetor zn Hildesheim. Sunmelt mit wissenschaftlicliem Eifer. Sans, Mariano, Pıoprictär in Barcellona. Schriftsteller.

Suportn, Grafvon, zu Aix in der Provence. Lepidopteren. - Ménoire sur trois Lipidopteres des genres Orgya, Phlogophora et Satyris. (Annal, soe. ent. France IIl. 1834. 183.)

Sattler, Universitätsprofessor in Pesth. Acussorsl lieber, gefiilliger Manu. Sammelt alle Kerfe, vorzïglich aber Himipteren.

Saulcy, lngenieur in Rouen. Entomolog.

Sauley, Bruder d. G., Offizier 
bei der francösiselen Mariue in Tonlun. Sammelec in Perin.

Samulers, W. W., Esq. in Landon. - On the IIabits of some Indian Insects (T'Talls, of ('llt. Suc. of Lond. I. c. p. 60, l.).

Sinnter, Joh. N(p)., Dr, med. in Winterthur'. - Besclucibung des Getreicleschionders (Tipula corealis), eines dew Getreidehau böclist schädlichen Insekts, saumt Vorscloligge zu sciucr Veutilgung ete. Winterthur', bei Steines 1817, 8. Pнв. 47 แ. I Kupf. (Germar, Mag. III. 366.)

Savi, Prolo, in Pisa. Berühunter Zoolog.

Saviguy, Jules César, Mitglica des Instituts (Sect. d. Zoulogies, in Versailles. Beriibmter Nifurfurscher und Reisender in Argypten. - Mèmoires sul' les animaux salls vertébres. Aver, planclo. P'aris 1816. 'T. II, 8. av. pl. (Extr. Bullet. soe, philoni. 181 t. Dec. 168.) - Ouvrage de la Commis. sion d'Egypte, partie des insectes (plauches eur. $50 \%$

Snxevern, IV., Mirler zu Clansthal im K. Hannover. Hymrnopterolog. Ueber Lyda. (Eut. Zeity. I, 16.)

Selnacelel, Revierfïster zu Burgwinllecim in Franken. Grosse Samulung.

Achnun, Herm. Rud., in Halle. - Analerta entomologica. (Disserta!io inauguralis). Cum tabula nencal ef roIor. 8. Inaj. Halis Sax. 1841 (velgl. Bur me is ter). - Beitrag zor Konutuisis der norddentsehen Silzkiter (Germar's Zeitschl'. IV, I. 174--191). - SymboJae ad monogiaphian scydmacuoutum insectorum gruts. Halis 1841.

Feluayer, in Briissel (?). Sammelte. in Van Diemcnsland.

Schemlcenberes, F. C. A., Dactor der l'litosophie und ller ficirn Künste Magrister, Berur-Ingrnirnr ete. Bekannter Scluriftsteller (Mineralogic mol Statistik) und Reisender dur't gan\% Europa, in Münelien (Carlsthor Roudell Nr. \%), Grosse entomologisebe und sehöne oryctognostische Sitmm lungen. Das Publikum hat von diesem) (alleu. tiefelenkenden und unermudet forschenden Gelchiten noch viel des Ungewöhnliehen zu hoffen.

Scluenk, Fr. v., gehcimer Secretär zu Waltershausen bei Giessen.

scineuereck, W., Kerfhändler in Leipzig.
Schilliug, Kerflın̈uller in Bres. lan (Albibserstrasse $\mathrm{N}$. 7 ).

schilling, Conservator des Museums in Greifswalds. Eutumolog nud Schriftsteller ïlır Henipteren, Neuropteren etr.

Cenilling, Gustav, Professor am Magdalenen-Gymuasium zu Breslau. Belinunier Eutomolog. - Holucnemis, Ćarabicorum geous novum et species nova H. Gravenhorstii. (Bcitrïge zal Entomologie vout Schlesictr. 93. 1 IK.) - Die Galtungen von Apion, Otiorhyuchus; dann Apiatien (Uebersicht der Arbeiten ete. der stblesischen Ge. acllscluati fo vate lïudische Cultur i. J. 1841. p. 100). - Cimex domestivis (alati) (lsis 1834. 738.). - Einflhcilung der Ftiigelfeedern bei den Lepidopteren (Isis is.31. 7.35).

Schalling, Saml. I.cbrel in Bresbu. - Anstubrliche Naturgeschichte des Thier-, Pffuzcu- und Mineralieirhes. 4 Bde. mi! 276 Albild. 2. Ausg. Bieslan, 1843. gr. 8.

Sclinuner, Di., Director am na. turlive o:ischen HIuscum z.u Strasshurer. Schidsale, J. ri, Dr. med. etr. in Kopculaterly. - Gencra ne Speries of Danmarks Eleutberata, at tjene sum fauna for deune Orden og som Indledniog lil dins Aluatmuic og llistorie. 1. Bind 2. Decl. Med 9 Kobbertavler. !jöhreuhavi, 1842. Lex. 8. (Kostet 6 fl.) - Brobacheungen ilier das ungepaarte Srlilumdnervensystem bei Acilius sulcatus (aurl in Kröyer's Weok inber Diincmats lusecten mit frein Kiefern I. p. 382-3) - Bcobarlitungen ilber die giffahsondernden Drissen der Piezatc" (ibid. VI. ubd Isis 1843. p. 6567). - U: ingefässsystem von Silpba ete. (ilid. und lsis 1. c. 66). - Zusammenstellung dev dăuisclıen Pompilidac (Naturbistorisk 'Tidskrift. Udgivet af Heni ik Kröyar. Bol. I, IIft. 4. 1837. \%. 313-344. - Isis 1841) - Ueber (ine noue Gattumy der Bracouartigen Irhoenumenel. (ibid. Bd. I, II ft. 6. 1837. p. 596-605. - Isis 1841. 185.) - Verzcichniss der diunisclien Arten der Galtungen Bombus und Psithyrus (ilsid.1838, Il, p 105-126. Vgl. D rewsen.) (Isis 18\$1. p. 325). - Ichnenmonidarum ad Daniae faunam pertinentiun genera et speeies novae. (Magazin de Zoologie, d'Anat. comp. et de Palènt. par Gué ri n 1839. Tidskr. af Kröyer III, 1840, p. 96-100. - 
Versuch riner monographiselen Darlerung der in Dämeniatk bislıer enteleckteu Ajten dec Insereten. Gattumg Amara. (Isis 1840. - Tilskrift van Kı̈̈yer I, 1836.)

SeInleInels, Jugendlelier zu Escl)weiler, luei Stollbeig.

SeIneicher, Jolı. A. und Matl. Lud.: Messingfabrikanten zu Stollberg.

Selnioifer, J., in Oldenburer. -

Sellliter, Fr., Sailermeister iu Halle. Sammlung vou Kiferu und Fal. tern. Beseluaftict sich auch (loaupri:säclılich) mit dem Studium dev Cunchylien. - Ueber die Begnttung von Lophyrus und Ovisugen der Insectenwelt. (Ablıanl. d. nat. Ges. Gürlitz II.)

seliznerreld, Guaf vou, kulless. geleim. Staatsminister in Fulda.

Aclemide, Regicrungarath in Stettin. Eutomulog und Scluiftsteller. Sam. melt Käfer und keunt besomiles die Rhynehophoren gut. Stehi mit SrhoenJeru" in Verbindung. Will näelsstens eine Voubritung dev dentschen Carabess (Genus Carabus) in der entomologisclien Zeitung publiciren. - Ueber das Aufsterken cler Inscren fii sammlunger. (Eut. Zcitg. von Stettin I. 178.) - Ueber Larve md Puppe der Cicindela eampestris Lin. (ent. Ztr. Ill, 270, Tinf. Fig. IX-X.) - Bemerkungen, den Otiorliyuclus niger betreffend. (ont. Zeitg. Ill, 110.) - Verzeichniss bölnnischè Käfer (Ent. Ztg. 1, 28).

Sclouidt, Hermann Maximilian, Jr. med. und Arzt in Prag. Berühmter Entomolog und Schriftsteller. Diss. inauc. zool. cle Pselaplis faunae pranensis eum anatomia Clavigeri. Pragac, 1836. 8. e. 2 tabul. (Eime ausgezeciclunete Arheit.)

Sclounidt, Robert, Dr. med. in Breslau. - Silpharum menographiae particula 1. Vratislaviae 1841.

Sclnmidt, Ferdiund Joseph, Kaufmann in Laybach (Vorstadt Schiska). Bekannter Entomolor, Coucholog und Schriftatelley (Faunus, Acta Acid. 11. Cur. Bommae). Grosse vaterläudische Samnilungeu.

Selomidt, praetiseher Arzt in Breuen.

Sclumidt, Rudolph, Graveur in Weissenfels. Besitzt cine der schönsten Sammlungen von seltemer Rejuleit, dabei ist sic zicmlich vollstïndig.

Selnunidt, Ilermann, Kaufmann in
Gera. Bereiste deu Siiden. Falter. S.mmuluug.

Aclnumit, I1. R., Dr. philos. und Oberleluer in Dinzig. Sammelt Falter und Käfer srlur fleissig. - Grundriss der Natusgeschichte fiir die ohern Klassen lı̈̆erer Büróselıuleu. 2 Abth. Dawrig, 1843.

Scliuuidt, A., Privatlelirer in Er. furt. - Haudiuclı der Naturgeschiehte. Mit gegen 400 es läut. buntgrediuckten Abliflangan. Erfurt, rr. 8. 1843 und foùlıel:

Schnuidberger, Joh., Chorherr 2» St. Flouian iu Steyermark. Pono$\log$ und Srouftsteller von grosser Autorität. - Driträge zur Olssthaumzucht und zur Nilurogeschichte der den Obsthämmen schädlichen Jusekten. 4 Hefte. fr. 8. Liuz 1836.

Sclumitt, evang. 'Pfarmer und Dr. Thcol. iı Mininz, Sclıöıe Käfrosanmulumg. - Beiträge zur K emutuiss der in Wespennesteru lebenden Insceten. (Eut. Zeitg. III. 18.)

Sclnuita, Jol. Will,, 'Tuelufabrikant in $S$ ollbere bei Aaclieu.

GeJnatbel, Dr. nied. in Ostende. Acliocin, Pastor zu Dürben il. Kuılaurl.

Selnoen, Dr. med. mnd practisclier Arzt iu Hof (Bayeın; sãchs. Gräuze). Sammelt die Käfer seiner Gegend.

Schoentuerger, Basilius, Kipitulaj des Bencolictiner-Stiftes Kremsmúuster und k. k. Professor am Gyumasium daselist.

Selnoenherr, Cp. Jos., k. Consmelzicmrallı zu Skasa mul Sparresater bei Stockluolm. Geleierter Eitomolog und Schuiftsteller. (Dic neueste Monograplic des" Cureulionides zählt 3000 Gatturgen!). - Symonymia insectorum. Genels et Species Curculionidum cun symonymia lujus familiac. Parisiis. Bis jetzt VI 13ile. 1842, - Curculionidum disposita methodica eum generum characteribus, deseriptionibus atque observatiouibus variis, sen prodromus ad synonyuiac insectorum. Part. IV. Lipsian apud Fleisclıer. 1826. 8. 338. (Jsis, 1827. 1. 874.) - Synonymia insectorum. 3 Bde. 1806-9, 8. (Im Ganzen 10 Bde. mit obiger. 1806-43.)

Sichott, J.J., in Frankfurt. Raupenkalender, oder systematisches Verzeichniss aller Raupen, welche in Deutselıland bekannt siod. Mit 6 illum. Steintafelu. Frankfurt a. M. 1830. 
Selhott, in Wien. Sammelte mit Natterer in den Trapenlïndern.

ficloottstedt, k. Geyeraleommissions-Srecetariats-Gehiilfe iu Stendal.

Selnoww, J. F., Professor der Botanik an der Universität zu Kopenhagen. - Naturschilderungen. Eine Reilie allgemein fasslipher Vurlesungen. M. Tafeln. Aus dem Dän. Kiel. 1843. gr. 8.

selureibers, Carl Ritter v. ete., Direetor des k. k. Naturilien-Muscams zu Wien. Viclberilunter Sehriftsteller umd piner der gefälligston Männer seiner Zeit. - Deseriptions of some singular Coleopternus lusects (Limenu Transactions. Vol. VI. p. 185, Nr. XVI. tat). 19, 20 I1)( 21).

Sehreiner, Instifutions(luef zu Ribeauville (Dept. de Haut-Rhiu).

Sehrenlk, in St. Petersburg. Sam. melte $184 \mathrm{I}$ in den Steplen und Ge. birgen der Songarey, die H. M énétries heselurieb.

Schricliel, Pharmazeut in Carlsrube.

Schroeter van her Inolk, Professor zu Utreeht. Bekannt dumbli scine Abhandlung riber dic Organisation und Lcbensweise der Larve vou Oestrus Equi, in einer Sitzung der deutselien natarforsehenden Gesellschaft zu Heidelberg vorgetragen.

Selmbert, G. H. voll, Hof- und Bergirath, Ritter und Professor, auclı Conservator cles k. Kabinets der Naturgeselhiclute in München. Reisender und bekannter Scluriftsteller.

Schuch, Dr: med. in Regensburg. Sammelte in Grieehenland vicle Kerfe, Spiunen, Vögel ete.

Selnile, Willelm, Gärtmer des königl. württemb. land- und forstwirtl. schaftlichen Instituts zu Hohenheim. bifriger Sammler von lnsekten aller Ordnungen, wetelse er vertauseht und verkauft.

Schihı, Joh., Papierfabrikant zu Dïrell.

Gehifppel, Friedrich, ehemals Buchlıändlè und Kaufmann in Berliu. Berilumter Entomolog. Grosse Sammlumg (von Mikrokoleopteren meist).

Sclnuarmann, Theodor, in Briissel (Rue de la Clancellerie).

schulthess-Discher, Caspar, Negoxiant in Ziilicl.

Sehultz, Carl lleinrich, Dr, med., and Professor in Berlin.

Gintul's Texiliun.
Sehultz, Friedricli, Dr. phil. und Apotheher in Bitehe (lisass). Botaniker. Beginut selur fleissig die Coleopteren seiues Wohnorts zu sammelu.

Sehultz, Ćarl Heimich, Dr. meel. IInd Hospital-Arzt zo Vejelesheim in Rlieinbayerı. Sammelt Kïfer.

Selnitze, llofiatl zu Gicifswalde. Bekaunt dureh scine interessante lintdeckung eines in der Daclurimnen-Erde lebenden Thierehens (Macrobiotas llufelaudii).

Selnulz, J. H., Oberlehrer in Berlin. - Groudriss der Zoologie mod Bo. tanik. Zam Gebrauch in Gymmasicn. 2. Anf. Berlin 1843. 8.

Sclumiz:, Conservator in Lipipain. liandelt mit allen Arten von Naturalien. Besitzt eine ausgezeichnet sehöne ornithologisthe Sammloug (in Hamburg fiir I 1800 tl. erkauft).

Gehnsmuel, Th. Emil, Professor in Breslan, - Hiommerschmidtia vitti. tic (Syrplridae Dipt.) Isis 1834. 739. Beiträge zar Entomologie. Heransgegeben vou deu Mitgl. d. entom. Sert. der schles. Ges, für vaterl. Caltul. Leipzig 1829. (1. Schilling, Hemiptera. 2. Gravenborst, lehnemmonidae. 3. Schilling, Holocuemis, nov. Carab. genus. 4. Schummel, Dijteren.) - Versuel einer genaen beselureibung der in Schlesien einlueimiscluen Gattang Raphidia. M. 1 K. Bresl. 1832. 8. (II dels Beitr. 1. Heft.) - Ueber die Sippscluaft der Syrphiden (Uebersicht der Arbeiten und Ver. ïndermugen der schlesischen Grsellschaft fär vaterlïudische Cultar i. J. 1841). - Dryomyza Zawadzkii (Isis 1834. 741).

Schuttleworth, aus England, in Jolimont bei Bel'n. Botaniker seines Haupt-Faches.

Schwah, Commd Lodwig, Dr. med., Hofrath und Professor an del" k. Viterinairselunle »n München. Die Oestracideu der l'ferde, Rinder und Srltafe. Minch. 1840, 4.

Selw waet riehen, Fro, Dr. med. Ind Professor der Botanik in Leipgig.

Sehwurzenberg, Oherst-Forstrath in Hessenkassel. Schöne KäferSammlumg.

Celinveigerer, A. F., Professol in Giesnen (?). - Handbuch Jer skeletlosen mgegliederten Thiere. Leipzig. 8. (Herabgesetzt zu cium THIr.) 
Seeger, M., Jugendlehter in Stull. bern bei Aachen.

seidel, Wenrel Bemo, k. k. Sta atsbuch haltume:s - Rechummgs-Oflicial iil Pray (Jleuwadgilsse Nr. 32). Bo'ilniker. Selurieb entomologisclle Aul'sit\%e etc. in Dr. We it en w clier's Iseitritipen z.m. Natur- und Arzoni-Kunde, and bearbeitete sonst die Orthopteren.

ceelig, A., Sclullelurer z.n kitrin(ueu (bayr. Franken). - Naturgeseliclitliebe Tabellen. gr. Fol. Würzl, 1842.

Seiler, in Sehaffhausen.

sellier, in Toulon. Samulumg tropischer Kerfe, gross umel reich au Gattıugen.

Gelys de Lougchanps, Elm. de, in Laittiels (Belgien). - Catulogne de Lepidoptieres de la Belgice - Nonographie des Libellulidees d'Europe. Paris et Brux. 1840, 8.

Seng, Frouz, Dr. med., in Wien. Entomolog.

serrres, Marcel de, in Moutprollier. Berülimter Kerfjlyysiulog, Anatom ond Schriftutcller. - Memoires sur les yeux lisses et les yenx eomposins dis Insectes ete. Moutpellier, 1813,8. (Ueber die Augen der Inseten ans dem Fran\%. von Dieffeubach. Berlin 1820,8). - Obscrvations sur les maseses du vaisseau dorsal daus les aninilux artieulés. (Mín. dı Mıs. IV, 149-192 et 313-380, 2 Pl., V, 59-147; 2 pl.) - Observations sur les diverses parties du tube intestinal des inseetes. (Annal. do Mus. XX, 1813, 48-89 et 213-339.)-Comparaison desorganes de la mastieation des Othopter.s avec cenx des autres aninaux. (In Mimoires du Missium, 1809.) - Uelier div fossilen Arichniden und Inseeten der Sïsswasserschichten von Aix in der Provence. (Aumal. des sc. nat. 1828, XV, 98 , Isis 1834, 1032).

Sexinge, J. C., Botaniker in Lyon. - Notice sur quel(jnes monstronosités d'insertes. Av. 1 pl. Paris, 8.

Serville, Aurlinet de, in Patis. Beriilumter Eintomolog und Scluriftsteller: - Les derniers livrnisons des insectes de l'ouvrage de P a l $\mathrm{i}$ s o t de B callvois (avee Palisot). - Nonvelle classification de la famille des Lougicones. 3 Partics (Anuales soc. enton. de France. 1I, 1833, 118-201 et $528,111,1834$.) - Irttre adelressie au Direeteur G. F iselier (Bullet. de Moscon ed. leq. 'Tom. V, 1833, 1). 355-57. Sur Orthopteres.)
Neyerfert, Otto, In Stutgart (Lindenstrakse No. 21). Palters.mumlumg.

Geyfried, Ritter voll, fürstl. von

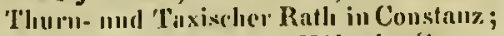
sonst zu Regensburg. Hibsclie SammluII-

Sirnaniu, Artillerie-Capitnin etc. iu Loirent. Veöllentliclite silur Interessantes ibeo die Sitten der Faltor ete.

sincppara, in London. Zoulog und siluitistellere.

Slinckard, W. E.. Esq. in Lon(lou. Zoolog und Schufisteller. - A new observation mon the llalits of the indigenous aculeate Hymenopter:t, gugrested by M. de St. Firneran's Paper. "1pon the gemus Gorytes in the lirst Number of the Amales de la Societi rut. de France. (Trans. ol ent. Soe. of London I, 1. 52). - El('ments of british Entomology. Cont. a general introduction to the science, a systemational deseription of all the meneril, aud a list of all the species of britisli losects, with a history of theip trallsformation, hahits, economy and distribution, with ontliue finures of the families and their lave and puphe, and explanation of the teehuical terms, and full directiuns for collecting. Part. I. Illusirated witl fifty wood-ents. Londou 1843 , gr. 8 . (Leiprig bei Weigel.) - Coleopterorum Delineationes. Lon(lov.

sieber, F. W., Dı: med., in Wien. Der berillute Reisende in Aegypten, Palästina. Schriftsteller, Sammler und Naturforscher. - Beschreibendes V' 1 zeiclusiss der in den Jaluren 1817-18t8 auf eincr Rrise durch Creta, Aegypten und Palistina gesammellon Alterfliimer und auderer Kaust- Ind Natur-P'odukte, nebst einer abhandlung iber igg ptixche. Munieı. Wien 1820, ช. (Entomologie). Sieble, Naturforscher zu Cliristiania in Norwegen.

Sieloola, C. Theodor vou. Professor der Zonlogie und verinleidlenden Anatomic Direetor der zoologisclien Abtheilung des Universititsmusemms z.u Erlangen. Beriilumter Ploysiolog und Seluiftsteller. Sommelte in Damyig dic Insecten der Provinz Prenssens mit grossem Fleisse und gibt jezt einen Catalog daríber beraus. - Falter Preussens (Prenssisclu. Provinzialblitter.Juli 1835 mal Nov. 1839). - Ueber dic Fadenwïrmer der Insecten (Ent. Ztg. 111, 146). - Bemerkumgen iiber einc 
de Bacillus Rossii bewolunende Selımarotzer-Larve. (Germar's Zeitsclir. IV, II, 3.99-39.t, Taf. 1, f. $\left.t-\downarrow_{\text {. }}\right)-$ U.ber das Receptaculum Seminis der Hymemoptrecu-W Weibchen (Germar, Zeitschı. IV, II. p. 36:-389, Taf. I1). - Larven dess Meloë Proscarahacus (eut. Zlg. I, 177). - Beitrïge zur Niturgeschichte der wirbellosen [thice. (Auch unter dem Titel: Neueste Scluriften der uaturf. Ges. \%.1 Danzigr. 3. Bd, 2. HIt:ft, 4. 1839.) Dissert. de Oxybelo uniglumi et Miltogramma conira. Eilang. 1840. - Ueber die Litlven der Neloiden (Ent. Ztg. II, 130.). - Ueber Xenos sphecidiarum (ii) Ammophila sibulosa und Miseus eampestris). (Neueste Selurften der uatuifurseleuden Gesellschaft iu Diızig III, 2.). - Bearbeitet gegeuväitig dic Kerfintestinen, woru wir ibm cinige Beitrïge gegrben haben. -

sibermann, Gustav, Bucl.drueker und Aduminstrator am zoolo. gisclsen Museuı э.и Strassburer (Place Saint-Thomas, Nr. 3). Gediegenes entomulogischer Selurittsteller und Redaeteur der beliebten Revue entomologicue (scit 1833), welele: leider nielıt melus fortgesetzt wird. - Mimoire sur la chasse aux eoliopteres ete. (Revurent. I, $1-17,53-68$.$) - Hermaplirodisme$ de Papilloms (ib. 50). - Dese.iption du geme Oclontopus (ibid. Nr. 3-6). Deseription del' Otior'lyonchus substriatus (ib. Nr. 7). - Quelque ide pour fuciliter les travaux de synonymic (ibid. 13t), und cine Menge anderer sclätzbarer Abhandlungen.

sillimsus, in Loudon. Redacteur des ameriean Journal of seienees.

Skrimshire, Th., in London. Reuarks ou some rare Inseets found during a fleceh at Wisbeeh (Transaet. ent. soc. Lond. 1812, 315).

Slovitz, J., in Petersburg. Sammelte in Persiru. - Sur les preitendues punaises de Miana (Argas persi(:us) (Journ. de St. Petersburg 11-23. Sept. t828, - Frorieps Notizen XXII, Ni. (6).

sinee, Capt. Walther, E. H., in London (Baker-Street, Portmann-Squarre). Seliöne Sammlung.

sodorrshy, Carl H.Gcorg, Dr. med., zu Riga in Liefland (2. Auflage p. 88 saget fïlselslich in Culaud). Bokannter Entomolog (Microlepidopterolog') und Selurftsteller. - Lepidopterorum microp)teroruu Species t3 novae (Bullet. de
Mosc. ecl.leq. P. 19-29). - Lepidoptera Livuniae olsservata (ibid. 47-54, 1829).

Solier, Ingenieur-Capitäı zu Mazargues bei Marseille. Der Verfasser virler in den Denksehriften französiseluer Societiiten zerstreuten Ablandlungen. Flcissiger Samuler, Namlaafte Samulungen atus allen Classen. - Essiais sur les Buprestides (avec planches au tratit) (Anual, suc, cuit. France II, 1833, 261-316). - Obselvations sur les 2 genres Brachinus of Aptions (ibid. 459). - Deseription d'uue nouvelle espece de Gyrinus (ibitl. 16.1). - Notice sur des apparitions l'Orthopteres dans les environs de Malseille (Alulal. soe. ent. France II, 1833. 486). - Observations sur la tibu des Hydrophiliens, et principal'ment sur le genre Hydrophile de Fabricius (ibit. III, 1834. 299). - Extrait d'une lettre sur les tarses des Lougicomes (il)id. 400). - Essais d'une division des Colioptires licteromeres et d'unc monoirraplie de lit famille des Collapterides, iv. 4 plancl). an trait (1, c. 479).

Sommer, M. C. Kaufmann in Alloua. Eutomolog. Herrliche, brillantc Sammlung und grosse entomologische Bibliothek.

somofr, in Kamiehloff (Gouvernement Perm). Entomolog.

sorf, F. L. A. W., Dr. med., zu Rudolsticlt. - Disquisitiones phy siologicac circa respirationem insectorum et vermium. Rudolstadt 1805. 12.

Sowerby, James Carle, in London. Mitredaetcur des Zoological Jour. nal (vou B (-11).

sowerlyy, G. B., in London. Mitredacteur des Zoolorrieal Journal.

Spangenterg, G., in Hannover. spatzenceler, Dr. med., in Silzburg.

Spence, William, Esq., in London. Hochgefeierter Eutomolog und Schriftsteller. - An Introduction to Entomology : or elements of the natural history of insects: with Plates. By William Kirby M. A. F. R. and L. S. Rector of Barham, and William Spen ee, Esq. L. S. Third edition. Vol. IV, London : printed forLo ngman, Hurst, Rees, Orme and Brown, PaternosterRow, 1818. 8. (Second. edit. 1816. I; XXIV und 5 t9 l'agg. II, 1817. par. $329,8$.$) - Observations on the Dis-$ ease in Turnips terment in lluklerness Fingens and Toos. Ilull, 18t2. 8. - 
Ohservations on a made puactised in Italy of exelurling the Common Ilousefly from Apartements. ('l'rassict. of the ent. societ. of Lond. Yol. I, l'. I, 1. 1.) - The ohjections nguinst the Corn Bill refuted. Lomdon 1815. 8. Tortrix woeberata seluadet hiswrilen den Aepfelb)iumen (ist ancli besclu icben im Plitus. Mayn\%iue Vul. 59, 1822. p. 439). - Manograple of the moritish species of the genus Choleva (Lium. Trausactions XI, I, 123). - (18 GuttuIIgev.)

Apence, William Blondel, Est., acswärtiger Seeretiir der entomolugischen Gresellsehaft in Loudon, Kuinstler etc. in Floren\%. Sclutieb cinen vortreffliclien Anfsatz. in den Seluriften genamoter Gesellscluft: - Remarks on the Passige in Heroclotus referied to in Mlr. Spences's Paper, real at the A pril Meeting. (Transact. of ent. Soc. of Lond. I, p. 7.)

Spence, Robert Heinrich, zu Florenz. Entomolog. Schüne Küfersammlimg. Bearbeitet gunz. besonders die Carabiden. - Description d'une notvelle espèce de Carabe, av. fig. (Amal. soc. cut. Frabe. Il, 1833, 500).

Speyer, Otto, in Pyomont. - Lepidopterologische Beiträge (mit Dr.s dol ph Speyer) (Isis 1843, 161-207.)

Spezer, Adolph, Dr. med., in Pylmont. - Lepidopterologische Beitıä̀n (Isis 1838, p. 277 und 1839, p. 89-110 und 1843 , p. 161 und $243-265$ ). - Berichtigungen und Zusät\%e zu den Srlomettrilingen von Europa (ibid.; bis 126).

Spinola, Maximilian, Marynis von, in Genua (Contrada della Sto. Catherina, Nr. 13). Berilumter Entomoloy und Schriftstelley. Kaufte das Meiste vou Dejeau's Sammlung. - Insecta Liguriac (1809). - Memoire sur la Ceratiue albilabre. (Aunal. dı Mus. d'hist. wat. IX. 1807. 236-248. N. Bull. soc. pliil. 11. 4, $1^{\text {re }}$ ant. I. 64.) - Essais d'une nanv. classification des Diplolepaires (ibicl. 17, 184, 138). - Sur un Coléoptìre monstrueux, 1835. 8.9, t. 1. - Sur au groupe de Buprestides, 1836. 8., 22. - Compte rendue des Ilyminopteres en Egypte, 1837. 8. 437-546. - Sur les espiees des geu res Heruspis et Aemarodera. 1838. $\&$. 303-395. (Anuales de la soe. entomoIng. de France.) - Custuni degl'Imenotteri e posto dei Sireciti. Genova 1843.
8. 31. - Osservazioni dei Prioniti. Torino, 18:3. 4. 33.

Epitzy, Joseplo Nicolans, Eotomo$\log$, in st. Leculated bei Ninlourg in Steyermarl. Schöne Simmlungen; lroIIm) istuer Verkeler mit ihm.

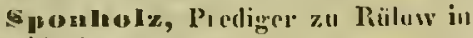
Meckleulumig.

Spensel, Rittmeister in Bamlerg. Samuelt Palter.

sproble, ill Golılis (Srlulesien?). Beobnclitung an einer Locusta viudissima (Abbandl. der vaturforsclenden Gesellseluaft \%u Gürlit\% III, Ileft 1, 1840. P. 698).

Stadtuiller, Mart. Fr., Goldselunicd in Augsburg. Seltüne Faltersammlım,

Stäger, C., Justizroth in Kopenlagen. - Systematistlaes Verzeichniss de $1^{\circ}$ in Dibemalk bisher grefundenen Dipteria (Kröyer's Tidakjift III. I. 1840. p. 1-58). - Kı tiveloe Bemerkungen zur Anlliatengatfum Oeydlamia Meig. Zun äehst nit Rücksjelit anf vinge Behauptungen das prof. Löw in dessen Ablandlung: ,uber die im Grosslerzogthum Posen aufgefundenen Zwriflïg. ler", Isis 15.10. S. 512 ff. (in K rö yer's Tidskrift. IV. 1\$42, IIft. 1, p. 98). Notiz über das Haustellum bei den Gattungen Enpis und Rlınuphomyia, linsielttlich der riehtigen Bentheilung desselben zur Bestimmumg der Arten (ibid. als Inbalt der Verhandlungeo der skaudiuavischen entonologischen Gesellschaft). - Däıisehe Dolichopoden. (K rö y e r, Tilskrift, Bıl. IV, Heft 1 , 1842, p. $1-44$. - 1sis 1843 , p. 56 -58$.

Stmunins, Fr. H., Dir mel., in Berlin. Entomolar und Schriftstellev (Dipteren Schlesiens ete.). - Olsservationes de speeciebus nonmulis generis Mycethophila vel novis vel minus cognitis. (C. 1 tab. col. IV ratisl. 4. (Silberun. Revue 1, 1133, 273, Exr.).

Starli, Geometer in Mituclaen. Sammelt Kiffer.

Stark, J., in Eliuburgh. - Elements of nitural history, adopterl to the present state of the science. Ediub. 1828. 2. Vol. 8.

Steetz, Di. med., in Mambure.

Stefraleny, Gustav, in Berliu. Entumolog und Schriftsteller: - Tentamen Mosograpliae generis Byrrhi (Geruar's Zeitschrift, IV. 1. p. 1 $-42)$. 
Stertens, Heinricli, Dr. medl, in Breslan. - Monita puaredam de speetebus migris Jchurmmonum. IVratisl 1829. 4.

Ate-Ifens, Iorsfmeister yu Dïren.

Stesiu, Aprotleker iu Cliarlottenhura. Sammelt alle Orelnungen der Kerfe (scollist dic Isoproden).

Siteiu, J. W. v., Jugendlehrer zu Gemarke.

steinkircherer, IBeneficint in Miinchen (Ni. 9, Maximilians-Plat\%).

Gtenz, Carl und Anton, Vater und Sulı, Kerfläudle in Vien (ersterer) und Veusiedel am See.Zu recommandireu.

Stepliens, J. F., in Loulan. Ber rïhuter Entomolon und Schriftsteller. Simmulum aller Kerfe Euglands. Nomenclature of hritish insects etc. Isond. (1829). 12. Edit. 2. 1833. - 11Justrations of Britislı Eutomology; or " synopsis of indigenons lnsects: $\mathrm{Nr}$. 1-VIII. Witl Pates 8. Idondon 1827. (B (०1), Znol. Journ. X1. 1827, p. 470). - Drscription of Cliasognathos Grantii. Cambridge 1831. 4. - Anoplotes pte. (Luolom. Jour. 11. 12. 1828.502.) - A systrmatical Catalome of britislı lusects. London 1829.

witerter, Cli. Gottl., Dircctor iu Beri).

Steven, Christian, Ritter von, k. russischer Etatsiatl cte, Director d's butanischen lustituts zu Symphoropol in der Krimm. Beriihuter Entomolog mud Schriftsteller: - Deseription de dix insectes de la Russic meridionale (Mim. de Moscon I, 118-130, I ll. I. 11). - Tentyriac et Opatru collectionis steveniana nune in museo nniv. mosy. (ilid. VII, ou nouv. Mém. 1, $81-100)$. - Deseription del' Elater Parreyssii ete. (ibid. VIll ou nouv. Mém. II, 79-94, 2 pl. Bullet. II. 153).

Stenvens, Jolı C., in Lundou. A Catalogue of the extensive nud voluable Collection of Inseets of the late Ad. II. II a worth. Jundon 1834. 8.

Stiller, Erduanu, Selmllehrer in Solnurnudorf bei Görlitz in Sehlresien.

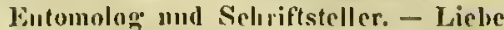
dej Amoisin zu jhren.Jugro (AbhandJumgrn der unturfurshendeu Gesell. sclunl z.ı Görlity, 1. 2, ן. 16).

Stiltreied, C. Freilew vou, z.ı llisschberer in Sichlssicu.

Stjernovall, Emil vou, Offieicr der enurasiselien Aruec, meler. Orden Rittri, in Petersburg. Saumelte im Cincasis. ?
Atolluerser, Audicis Gial 7.11, k. Forsturister zo lumenenboich bei Mout. joic.

Stroltenluor, Gebrüder, Turlıfabrikanten in Stolberg.

Sitoltz, Obergeommer. in Mïmrhen. Lepidopteren aus Raupen zichend.

Strack, Di. phil. nud Professor \%" Ilalle. - Einime Be\%iehumgen, in wolrhen die Insecteu zu den ïbriren Thierklassen und den Pflanzenriche stelıen. (Neue Auual. d. Wetterau Ges. 1. 1. 1. $65-8.1$.

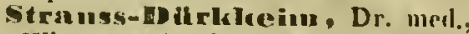
in Winter zu Pavis (Rue Copeau, $N_{1}$. 4), und im Sommer anf dem Landginte Frötluillerbeistrasshurg. Berïlum. ter Anatou mad Plısiolog. Seine zalılreiclıen, selurfsimigen Werke simd zum Theil besonters, zum Theil in lin Mémoires dı Musenm d’list. mat. zı Paris ersclienen. Die Anatomie Ies Maikäfers gelıört unter die musterbli. clın Iscistungen in diesem Fnehe. Consideration gineralus sur l'anatomie compared des animanx articules. Paris 1928. 1) 346. (Mpist iber Ceustacen.) - Jutio sur Ie Mémoire de M. MI ii I. ler sur la vision du Haumeton (Aunal. des se. nat. XVIII. IS:9. 463). - T'njté proticpue et theorique d'anatomie comparative, (comprenant l'art de dissidue les animanx de toutes classes of los moyens de conserver les pieces [ulitoniques. l'aris $1842,1,8.434$, II, 435, |P. 4. (Isis 1813. p. 623.)

Gireimz, Promedicus und Regic. rungsiatl in Linz.

Stremlie:I, Airgust Vollintl, Dr. med. etr.y in Berlin. - Uelice dis.Stellutir der Brachyelytren oder Stapliylinen im natïrlichen System. (Isis 1839. p. $126-138$.

Strth hims, Seminariul-Lelorer in lifurt.

Strace, G. A., zu Pulkova, o. Mitml. der Akademie zu St. Peterkhirr.

Struev, H. v., Elrenmiturlicel d. li. Ahademie zut l'eterslourg, T,egationsiatl in IIamburg. - Ueloce dio limust Sichunetterlinge nach dem Loben abyudricken. (Ammal. der Wetterin. Ges. III. 1. 1812, 1). $188-1$ (\$3.)

Stacluegrati, Plofessor zu St. Petershinim. Entumolog.

Stencu, Jakob, Kupfersteclier in Ninuberg. Vivelverdienter Litomolon und Botaniker, dessen selıriftstelleris.luru und vorzinglielı küustlerisclıen Leistuı- 
gen unilhertremirl, sind, Grosse, be-

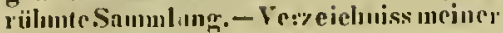
Insckteusammlung. Nür'uberk 1796. 12. M. 4 ill. lipfot. - Verzeichuiss meiner Insektensammulnugr oder entomologisches Haudbuch fiij. Liebbaber und Sammler. Niirub. 1800. 8. M. I ill. Kpfr. - Cacalog meiner Inscktensammlung. Nörmb. 1826. 8. M. 4 ill. Kpf. - Catalog der. Käfersammlung von J a cob Stu rm. Mit 6 ausgem. Kupfert. Nïruberg, 1843 bcin Verf. (Panierstrasse 5, Nr. 709). XII mind 386 Pagg. gr. 8. Deutselslands Fanua ju Abbildungen mit Beschreibungen. V. Abth. die lnseckton Nïrnb. 14 Bile. 1805-39. 8. Mit 287 ill. Kptitaf.

Sturalza, Michael, Hospodar des Fiiistentlums Moldan (seit 1834), Dureh. laucht, z.l Jussy.

Giekow, Gustav, Professor ete. z.n Halle. - Systematische Encyklopädie und Methodologie der theoretischen Naturwisseuschaften. Halle 1843. 8.

suckows, F. W. L., Dr, med., zu Manulıein (niclit Heidelbers). Entomolog, Kerfanatom und Schriftsteller. - Ueber die: Ueberwinterung der Insekten. (Heusinger's Zejtsehrift fii) organische Pliysik 1827. nov. p. 597.) Ueloer die Semblis bieandata mod lutaria (ibid. II. n. 3, 1828. 265). - Ueber die Respiration der Insekteu, besonders ïbev dic Intestinalrespiration der Aeseha grandis (ibid. II. n. 1. 1828. 24.) Naturgesclichte des Maikifer's (Verliandlungen des badischen landwirth. schuftlichen Vereius z.u Ettlingen. 1823. 12. Hft. M. Kpf (.). - Digestions-A pparat der Insekten (Heusiuger's Zeit. schifift, lli. p. 3. 1828). - Ansitomisillphysiologiselse Untersuchuugen der Inseiten und Krostentbierc. M. $10 \mathrm{Kpf}$. Heidelb. 1818 , I, 4. - Ueber die Organe der Fortpflanzung bei den Iusek. ten. Mit 6 Kupf. (Heusing. Zeitsclur. f. org. Pliys. II, 1828, 23.)

sulfrinu, Dr. plifins. und Direetur der Pealseluule in Siegen (Westphaleui). Ausgezeiphueter Entomolog. Wird die Donacien bearheiten und gegenwiitig trägt ev Alles yusammen, Wals Dr. Selomidt bereits über dic Oedemeriden geschrieben hat. - Fongencute "u] genanern Kenutuiss deutselier Käfer. 3. Gyıinus. 'Eut. Ztg.I, 82. III . 219.) - Die Caraben des Regieroungsberioks Amsherg etc. (Germas's Zeitselur. IV, 1, 149-172, Tab. IV). - Vavictäteu vou
Ciciudela coupestris. (Ent. Zeitg, I,

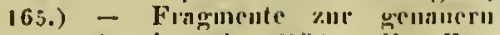
Kenutuiss deutsider Jialer. (Eut. Zeg. 1I, 19.) - Bemrtouneu iber cinge deutsclue Rüsselkäfer mit Bexirlutum auf Schönherr. (Enton). Zeitg. I, 6 el 44 ete.) - Ueber Micropeplus. (Lint. Ztg. I, 139).

Sundewall, J. C., Dr. und P'rofessor der Orkonumie in Lutud. - Ueber eime Fliegenlarve in der menschliehen Haut (Fürlandlingal ved de Skandinaviske Naturforskeres 1841. 295. - 1sis 1843. 298). - Oeconomisk Afluabdling om Insekteruas äverkan pa vara Skogar. Lund 1826. 1. - Ueber die Zalil und reogruphiselie Verbreitumv der Coleopteren. (Förhandlingar ved de Skandiuaviske Naturforsker'es. Kjöhenh. 1841, p. 283. - Isis 1843, ґ. 291.)

Swninson, William, in St. Albans (Grafschaft Hertfort) in Englaud. Berülumter Zoolog und Selurifiateller. Zoological Miscellany. - Essai d'une classifiention naturelle des Lépidoptères diumes. - On the history aud natural arrangement of insects. Tond. 1840. 8. - Zoological Illustrations, Nr. 1-4. Loudon 1820-21. 8. - Hints relative to the present polins et future prospectus of clltomologieal soriety. (Wallicr the ent. Magaz. 11. 7, Apr. 1834. 190 -200.) - Verwandtseliaften der leppidoptera diurna. (The philos. Magaz. I, 1827, 180. - Isis).

Groboda, Kerfsammler in Prag. Sylkes, Will. Heinr., LicutenintColonel, Viceprisident der entomologisehen Gesellschaft in Loudon.

Trams, zu Abo in Finnland. Entomolog und Reisender in Russland.

Tascher Ile In I'ngerie, Graf von, iı München. Samulungeu vou Kerfen, Münzen und dergl.

Telnertkofr, in Toula (Russland). Entumolog.

Teichgriber, Schlossverwaltep z.u Arnsdorf bei Solnumedeberg in Sehlesicu. Entomolog.

Telehi, Juseph, Graf vou, Gonverneur vall Sicbeubürgen und Prij.

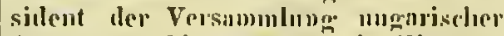
Acrzte Ind Niturtorseluce in Klansenbu1n (Ungatu).

Thareaniu, in Trknzk. Eutomologr.

Thicencuanan, L.. Dr. med. ite. iu Kopeulangen. - De araro qunodau in Alga vivali (Chiouyphe densa 'H.) 
vivente. (Fürlanudlingar ved ıle Skaıldinaviske Naturforskeres andet Müde, der looldtes i Kjöbeuluven fra den 3 ilie til den 9de Juli 1840. Kjöbenlaven 18.11. 8. (421 s.) 1. 282. - lsis 1813, p. 291.)

Thie:rse:lh, Erust, k. Oberffirster in Eibenstoek (Sactsen). - Die Forstkäler oder vollstiiudige Naturezeschichte der vor\%ün-lichsten den Gebiressforsten selıïlliche॥ Insekten ete. Stutg. 1834 mis 2 Kupf. 8. (1 fl, $36 \mathrm{kl}$. oder 1 'Tlıl.).

Thiébunt ale Bernanul, Ars., in Paris. - Description d'une nonville espèce de Dorthesia existente aux ell virons de Paris. (Mem. soc. linne. Par. III, Sept. 1824. 285 av. fig.)

Thiébsud de Berueand, Mademoiselle Uranie, Tuditer (des ubigen) des beriilumter Sekrutairs der Societe linnemue in Paris. Lepidopterensammulıng.

TInomai, C., Dr. plit. nud Direktor des Museums zı Wieshaden. Gesclichte des Vereins tïr Naturkunde iin Her\%ogthum Nassaul mud des natur histurischen Musenus z.u Wiesbaden. Mit Vign. Wiesbaden und Siegen 1842. gr. 8 .

Thon, Theudar, Dr. und Prolessor z. Jeni. Bekannter Kintumologr und Scluriftsteller, so wie Kerfaciehner, Entomologisehes Arehiv. I. Bal. I-III. H(ft. Mit Kupfertifelı. J'וna 18261828. 4. (I. 45-92 bez. S. I Kupf.) Tiselienbuch der deutsehen Schunetterlingskuude. 2 Bde. in 8. m. K. Jena bei Aug. Selomidt 1826. - Abbildumaren ansioindiselier Insekten. I. AlutheiInngr: Käfer. Jena bei Krüker 1S26, 4 S. 4. I 'Taf. (Isis 1827, p. 1077.)

There:y, G., Pharuacent in Humhurg. Bereiste Brasilien. Seine Summlung ist brillant, grösstentheils brasiliseh.

Tiede, Justiz-Commissarius in Jacobshagen.

Tiedenamn, v., Gutsbesitzcr auf Ruseacrin bei Dankig.

Tilesslus, W. G., llofrath, Dr. med. mol Arat in Leipzig. Berihinter Reisender, Naturforsclier und Scluriftsteller. Besehrieb in K insenstern's Reise Wasserkerle. - Jahobuch der Naturgeselichte zur Anzige und Prifung neuer Eutderkungen umul Beabachitıngell. I. Jahromg in 10 tKupfertafelu. (Mchr ist niclı erschienen.) Leiprig. 1802. 8.
Tileslus, G., Dr. etc. in Raveuna. Silniltsteller.

Tisclicer, Kul val, Geleitsman, zo Sehoudau (Sithlosiselie Silhweiz). Entomolur. Kertiniler numl Selniftstrller. - Taseheubucli fïr Schucterlings-I_iebhaber. -

Todal, Rubert B., in Londun. The Cyrlopaedia of Anatomy and P'by. siologry. Vul. II; A. - Ins. Lond. I83s -39 (bis 1841) 1015 Pagg. 8. Dazıl liefern Bell (Th.) die zoologischeu Artikel, H all (Marshall) dic iiber Itiberuation etc., Jones (Rymer) viber Gisteropoda, Newport (G.) iiber Insekten, Owen (R.) iiber Entozooen, Sharpey über Echinouleruatic etc.; ïber die Generation Thousou (Allen).

Trontmann, in Prag. Sammler. 'Treviranns, L. Christ. (Soliu), Professor etc. z.u Bremen. Herausge. ber der Beobachfongen ans der Zontomie und Plonsiologic ( $v o n$ G. R. T'e evi. i i II II s). Bremril 1S43. 4.

TrentepolnI, J. J., Di. med. in Kiel. - Revisio rritien lchuemonis: specierum quae Kiliate in Cl. Fubricii unsen adlut: superstites sunt. Sert. I. Diss. inang. Killate 1S25, A. (lsis 1826. 55. 216, 293.)

Trieplese, superintendent in Gar\% (Pouncrus), Senior des entomol. Virpcins in Stertin. - Einige Bemerkin!gen über Éphemera Flos ac|lise Illiw. (Eistom. Zcilg. I, 51.)

Trinlcer, Joseph, Juwelier in Stuttgart (Bazar, Nr. 38), Eifriger SammTer von lusckten aller ()idnungen, besonders der Lepidopteren.

Tripaldi, Andrea, in Nerpel. Sur quelques lusectes qui font dessecher fes branclies d'olives et devorent la pulpe de lenrs fruits ef sur la maniere de les detruire. (Atti del real Inst. di incorrag. di Napoli, Toul. III, 1832, p. 139, Bull, des sc. nat, et geol. Janvier 1826.) (Phloiothribus?)

Trosehel, Frank Herm., Dr: med. in Berliu. - (Mit J. Fr. Ru(hr) Wirgmin's und Ruthe's Handluclo dep Zoologie. Naelı den Fortschritten der Wisseuschalit ibloratbeitet und vermelirt. Berlin 1843. \& $r$. 8 .

Trosehlie, L. von, Rittmeister in Halherstadt.

Truelnet, Michel, in Paris. Traite complet du Cliermes av. 2 pl. Paris 1811.8. 
'T'achernikh, iu Si. Petersburgy. Samumelte in Californien.

'Eitrk, vou, Schulrath in Potsdam.

Wuclutritz, M. F. L., Freileri vou, 7.11 Birslau.

Whersperger, Dr. med. und Aizt iı Mimplıcn. Selı̈ıe Sammlunğ exotiscluer Käler.

WIrelic, Rechnumgsofficial in Wien. Berilimter Entomolog und Srhriftstelleı. Herliche Sammlumg, besunders Mi('roroleopteren (uureuropaiiselue). Reiste vicl in Oesterreich und im Littorale. (Verul. Germar's Fauna Europae).

Euderwood, Medicus in London. Saunelt besouders Araeluiden.

vallot, J. N., Dr. med. in Dijour. Entomolog und Solnriftsteller. - Natice des insectes qui se voient sur la vigue rt vivent a ses depens. (Mèu. de la snc. linn. Par. I, 1822, 248.) - Mimoives sur quelques diptères du genre Coridomye. (Amalyse des travaux de l'Acind. des sc. 1827 , p. pliys. 59.) Nouvelles esperes de Cochenilles. (Aead. des sc. et bell. lettres de Dijon, Aût 1829, 30.) - Observations entomologiques. Paris 18t2. 8.) - Ueber das Betraren vou Authibus marmoratus. (Amual. des sciene. nat. XIII, 1828, 68. - Isis 1830, 205.)

Varand orfr, v., Regieruugsseere. tiil in Arusberg.

Visselet, M., in Paris. War in Mexiko.

vanaloner, in Paris. - Obscrvatious su la léthargie periodique des hroilles de papillons Euphrosine ut Dia. (Anual. soe. liun. Paris Sept. 1827,374.)

Eeclui, in Mailand.

Verdint, F. J., Dr. med., in Delenout (Delsberg) (C'antou Betn, Jura). Siluriel über Kerf-Metamorulıoseru. Mémoire poul servir a l'histoire des Simulies, gemes d'inseetes de l'ordre des Dipteres, famille des 'Tipulaires. Avec 1 planel. 1821. (Nalum. Auzcip. der Seliweiz. Ges. 1822, 11. 9, p. 65.)

Verleln, Dr. med., in Elisabetligrad (Cliersou). Entomolog.

Verranx, Naturaliculaindlev iu Paris. Viel exotisclue Käfre u. a. K.

Viala, Plarmazeut zu Castoluaitdiry (I)ept. de l'Aurle). Simmuler.

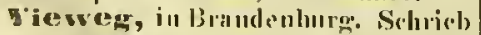
lin tabellarisches Verzeieluiss dev iu (ié Mark Brandeuburg cimleimischeı Sclunesterliuge. vigors, Nirolaus Aylward, Esq. \% Cliclsca hei I,ondan. Zooloer und Schriftsteller: - Descriprions of some rare, interestiug, or hithertho uncharacterised subjects of Zoology. With 4 pl. col. (Zoolog. Jouru. I, 1825, II, III.)

Ville, Fratelli Autonio et Giovanui Battista, in Mailand (Coufrada della Sillo n. 5558). Entomalogen und Kerfhäıdler (schr zu empfehleu), sowvic Scluriftsteller.

villamove, Professor der Zuolo. gie zu Madrid. Srlıöne Sammloug.

villaret, Foulque de, in Paris. Menoires de 1 nouvelles espèces de Tentlıredines (av. fig. col.) (Aumal. soe. ent. France I, 1832, 303-308). - Deseription d'une nonvelle espece du grenue Lampyris (Amual. sor. ent. France II, 1833. 352).

villiers, Franz vou, Capition der Infanterie, Director des Museums \%u Charties. Entomologiseluer Seluriftstel. ler. Sclurieb iber Zyriinen mad unter andern (nit Guence) die Tablenux synoptiques des Lepidoptères d'Europe. Paris 1835. 4. (â 10 Fres.)

Villiers, Adrian Prudent von, Bruder des obigen, zu Montpellier. Sclı̈ne Sammlung. - Notice sur trois Lepirloptères incidits on pen eounus de la Frane merid. (Ammal. soe limejen. de Paris Nov. 1826. I pl.). - Observation sur le Bombyx pityocampa de Godart (Anmal. soc. ent. France 1, 1832. 201-203). - Observations su I'éeaille pudique de Godart (I. r. 203-20:).

Virmomal, Naturalist in Berlin (?). - Sammelte in Brasilicn.

VIadsinirsliy, in Nertsehinsk (Gouvelu. Irkuzk). Entonolog.

vourel, Clırist. Fricdr., BüclierAntiquar iu Leipzig. Sammler und Siliriftsteller. - (Nielıt unter sein(m) Nimen) Schmetterlings-Cabiuet f. d. Kinder. 10 Hefte nit vielen Kupf(u'u (1823-28). - Raupenkalender. Berlin 1836:

Vogt, Heinr., Talıakfahıik-Besitzer in Mannlueim. Srlı̈ıc Sammlın.

Voigt, li..J, I Iufratl und Professor ete. zu Jewa. - (Mit G. W. Bisr.hoff,

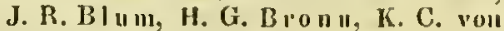
Leouluard, F.S. Leu c lia r) Naturereschichte der diei Reinlue (aurl untor speeielleren 'löitolu). Stuttant bri Srluwei-

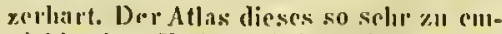
pfolileuden WVrkes wird pratis gegelun. - Das Thierveich georluret nacb sejue 
Organisation. Als Grondlage dor Natureseredichte der Thiere nual Einleitung in die vergleinluende Anatumic vou Barou von Curier. Nich der 2. reruehrt. Ansuabe ibbersezt 11. s. w. Sechs Biiude. Leiprig 1831-43. 8. (18 Thir.)

VoIz, Prieeptor in Stottgart. Conchylieu- Imul Inscctensaumlung.

Vozelvichinsliy, in Riazan (Russland).

Wrachter, Stadtdecan in Mcmmingen. Faltersimnulnng.

waickel, Franz, Lehrer am Forstiustitute zu Melsungen (Clutrhessin). Sammelt, mit der Naturwissenschaft vertrant, eifrig Käfer und Schmetterlimgr.

DEaga, in Warschau. Entomolog. vagner, Carl, in Bingen.

Werager, Rudolph, Dr. med. und Professor z. Götting gen. Berïlumter Ana. tom und Pliysiolog. - Icones plyssio. logiene. Lripzig, 1843. Imp.-.4.

Bvanguer, Mori\%, Dr. med. ans Angsburo. Macht gegenwärtig cine naturhistorisclic Rrise ans scluvalre Meer. - Reisen in der Regentseliaft Algies in den Jithren 1836-38. Leipz. 1841. 8. 3, Bole. Nit I Kupferatlas.

voraner, Andreas, l'rofessor zu Miinchen. - Berieht über die von Selı \|bert, Rotlı und Erdl vou Acoypten und Palästiua mitgehrachten Thicre. (Gelelute Auzeigen der kön. bayr. Akad. zı München 1839, 11. 37.)

valaloerg, P. F., in Stockholm. - Britrige zar Kenutniss der scliwedisctuen .Zweiflïgler (Kongl. Suensk Vetenskaps-Academiens Ilandlingar för ar 1838. Storkholm 1839, p. 1-23. - Isis 1841, p. 440.)

Iwailes, George, in London. Catilagne of new Inseets, found in Caste Eclen Dean etc. (Walker ent. Mag. 1832. 199.) - Notice of the halits of Charaxes graminis. (ibid. n. 2, 1833. - Observations on the Enicoceri (ibid. Apr. 1833. 3556, ) - Observations on ignis futuus (Lampyris). (Ihill. 11. 4, 1833. 350.)

Ernitz, ITofrath in Altenlunra (Osterland). - Bastarderzerumg vou Falteril (Isis 18.34. 720).

v8ralcelledel, in London. - latro. dnction to the natural history of Insects. Inoulon 1816.

valclnmer, F. II., Dr, med. mil priakischer Arzt zu Carlsrulie. - Der practiselic Naturforscliev. 5. Abtheilung. Carlsinlie 18.13. 8.

Eralcker, Francis, Esq. in London. - The entomological Magazine. London 1832. - Monographia Chalcidum. 'Ejusd. Mag. ent. n. 1, Sept. 1832- Oet. 1835.) - Observations of the british species of Sepsidae. (ibid. 1833-34.) - Obscrvo on the brit. spec. of Pipuneulidae (ibid. n. 8. Juli 1843).

walrord, Thomas, Est] in London. Eutomolor und Schiffsteller. (Linnean Transactions, 1808.)

valker, Sir Patrik, Graf zu Edinburgh.

Ivaliner, Polizeibeanter und Kerflı̈ndler iu Geuf (Temple 165). Meist schweizerische Lepidopteren.

Wnikenner, Baron Carl Athan. von, Prïsident des Instit uts zu Paris (Ruc de Fanbourg-Poissomiere, Nr. 87). Der beriihmte Arachnolog. - Fanne parisicune, inseetes, on histoire abrigie des inseetes des environs de Paris, classées d'après le systeme de Fabrieius, precerlec d'un discours sur les insectes en genéral, pour servir d'introduction a l'étude d'entomologie; accampaguée de sept planches graveses; Paris. Ann. XI, (1802). 2 Vol. CLX et 301-438. 8. - Mémoires pour servir a l'listoire natorelle des abcilles solitaires, goj eomposent le genere Italicte. Paris, che\% Didot 1817. 8. pag. 90, av. 1 pl. (Germar Mag. III, l) 409).

VraltI, Joseph. meil. Dr. und Professor zu Passan (Unterhayern). Bekanuter Entomolog, Reisender (in Spanien, Siid-Frankreich u. s. w.) nud Sclniftsteller. Ilandelt mit Naturalien aller Art, mit Waffen, Büeliern u. dogl. - Kïfer $11 \mathrm{~m}$ Passau (Isis 1838, p. 273, 1839 , p. $221-227$ ). - (W alt) und Schnidt: Cistela sulplurea, cigene Art verseluieden vou C. bieolor (ent. Zeite. III, 70). - Entomologiselıe Notizen. (Ent. Zeitg. 1II, 31.) - Neue Zweifliigler aus Minelien (in Gistel's Zeitschrift "Faumus", wo Melues vou ilm norlı s(elit).

Wralton, Joln, in Jondon. Entomolnerical raptures (Walk. ent. Magn\%. Ni. 8. Jul. 183.4. 277.). - Cąpfure of noctural Lepidoptery (ibis.).

Eraplex, Emil, in Mülnlıusen (am Rheiu).

Wastel, Gregor, Oberlientenant 
der k. k. Militïr-Montonr-OekonomieCommission in Prag. - Niclitskostende, verlïssliche Motten-Vertilgung, oder: das Nöthigste aus der Naturgescliclute iler Mottell. P'rag 1841. 8.

venterlnonse, G. R., Esq., Archivnr der entomologischen Gesellschaft z.n London. Bekannt als tïehtiger Entomolog dureh mehre Anfsiitze in dell Scluriften der entomologischen Gesellschalit zu London. - Descriptions of the Larvae and Pupae of various speries of Colcopterous Insects, (Trans. of eut. soc. of Lond. 1, p. 27). - Monographia Notiophilon Angliae (Walker ent. Mag. Nr. 2. 1833. 202-211). - Deseription of the Lnrva and Pupa of Raphlidia ophiopsis. (Trans. of ent. Soe. of Lond. 1.) - Monographin Hyd'aenarum Angliae (Walker ent. Magaz. Nr. 3. Ap)r. 1833. 292-96.). - Deseription of some Coleopterous Larvac (Walker ent. Maraz. 1834, 373).

Uravre, in Nellenburg. Entomolog.

Weideubneli, v., Dr. med., praetiscluer Arzt in Hexenacker bei Regens. lıurg. Grïndlieh.r Forseber. Selır schöne Käfer-Sammlumg.

Veideulotrer, Sammler in Prag. Eceidunum, Sammler in Wien. veigel, l'astor, in Görlitz (?). Zoolug und Schriftsteller (Faona silesia('i).

Weigel, v, Professor und Direktor der Naturaliensammlung der Universität zol Greifswalde.

Eveisenfeld, J., Cand. Theol. in Bamberg. Sammelt mit Einsieht und Liebe die Käfer Frankeus.

Weissenlnoru, von, Oberlandes. gerichtsratls in Eofort. Hat eine grosse Faltersammlung. Sammelt, olıne Geld y. selienen, alle Varietüten Zwitter, Missbildımgen u. dghl.

Welleus, Baron de, in Brïssel.

Welser, J. C. Freiherr voll, 7.1 Gunzenhausen in Bayeru.

TEelwitsele, Friellich, Medicines in Wien. Bereiste Kärnthen und Steyermark. Käffersammlıng.

Wenizer, H. Iر., Jugendllelurer z.l Milhlheim nm Rhein.

Wertlemannn, in Basel. Sammlung schweizerischer Kerfe.

TEesuas. $C_{\text {., }}$ in Briissel. Entopolog. - Ennmeratio methodicn Orthoptrorum Belgine (Ballet. de l'Acad. de Brux. 1838. V. 587-597. 'Tul.) Monographie des Odynères de la Bel. gie. Bruxell. 1833. 8. ar. \& pl, - Sur un cas de renversement de la jambe, complicjoe de bricuete rlez un rolé. optere (Bulletiu de l'Acad. de Brux. 1838. V. $662-661$. Tab.)

Testermanu, B. A.: Negroziant zil Kopenlangen. Futomolog und Reisender (in Ostindien).

westwood, I. 0. , in r,oudan. Berühmer Éntomolog und Schiftsteller. - Monography of l'aussidac (Transactions of the Linnean Society). Deseription of a minate Colcopterous Insect, forming the type of a new sulb. gellus allied to Tomieus, witl some observations upon the nffinities of the X ylopliaga. ('Trans. of ent. Sor. of Loutl. 1.) - Observations on the saltatorial powers of Inseets, and upon the britislt Coleopterous geuns, Chomgus. (Walker ent. Mngaz. 1833, 288.) On the structura of the antenua in the order Aphaniptera (ibil. $359-$ 363). - Ueber l'aussidac (The philos. Magaz. VII. 1830. 92. - lsis 18.34. 80.1. 23 Gattungetr.) - Descrip. tion of the Nost of a gregarions speeies of Butterlly from Mixico. (Tratus. of ent. Soc. of Lond. I. I. c. p. 38.) - On Caprification, as practised upon the ligs in the Sontl of Europe and the Isevant (Transact. of the entomol. Society II. I'art. IV.). - Mell('ospis. (Vgl. Germar's Zeitschr. II. Heft. 1839. 237.) - An introduction to the modern elassification of Insects. I Vol. 1839. - Oliservations "pon Siagomum quadricorne of Kirby, and the other portions of the Brachelytia. (\%ool. Jouru. III. 1827. 56-66. 1 jll. Vol. Ill. Palt. II.) - Deseriptions of several niw species of lusects belonging to the Family of the sacred Bectles (Transact. of Zool. Soc. Lond. 1837. II, 2, 1838, $155-163$, t. 29 . - 1sis 1839,140 ). Memoir upon a parasitic Species of the Cynipidae. - Remarques sur les thorax des insectes (Zool. Jonirl.). Menoir on the Connexion between the Land and Water Cimicida, and description and fignre of Encephalus com. plicans. - Additional observations to, and roprections of a paper upon the geuts Stiphylimus of Limuens (")ool. Journ. III, 1828. 504). - On the Clialrididare (1 plnt. - Zool. Jourur. IV, 1828, 3-30), - Obs(rivition ont the genus Scaplura, witl descriptions of two new Speeies ("/ool. Journ. IV, 1828, 
225). - Olservatian upon the genus of Culeopteruns insects Cienostoma (\%,ool. Ionri, V, 1829-30,53). - Obsersition upon the Notoxidar, a family of Coleopterous Insects, with characters of two new british generin ete. with fig. (Zool. Journ. V, 1829-30, 62). - Chametêres des Amydetex (Zool. Jourı.). - On the affinities of genus Clinidimu of Kirby (with. fig.) (Zool. Jourll. V, 1830, 213.). - Let1cothyreus Kirbyanus, Bolax Zoubkovii, Loxopyga bicolor (Guerin, MIngas. de Zool. IX, 70, 71, 72 Fir.). - Trochalonota badia (Guerin, Magas, 95. Fig.). - Ueber fossile Flügeldecken \%ol Stunisficld. (Annal. and Mag. of Nittual-Hisfory. London, 1841.)

vetter, Jolı. Bnpt., Dr. med. in Gicssen. - Abhandlungen und UnterAnchungen ans dem Gebiete der $\mathrm{Na}$ turvissensehaft, inshesondere der Biologic. Ein Versuch, Giessen. 1843, Hir. 8 .

Drite, Adam, in London, - BeNeluricls die vall Capitiiı Grey im König-Georgs-Sund (Australien) gesammelten Inserten in clessen Jounal of two lixpeditions, daun anch (mit Edward Doubleday) die des Werkes: T'he Zoology of the Voyage of H. M. s. S. lierchus and 'lerror under the rommind of Capiain Sir I a mes Cl ark Ross ete. during the Years 1839, 40, 41, 42 and 43. Edited by John Richardsou and John Edward Gray. (Eirterer bearbeitete die Fische, letzterer dic Sucke, Reprilien, Conrhylien und Corallen; G. R. Gray die Vö. gel mal Bell und Goodsir die Crustaceen.)

vider, F., Pastor in Berfelden am Odemwalde. Schıriftsteller. Sammelte für das Senkenbergiselse Museum in Frankfurt.

vilebel, K., Dr. med., Professor und Vieepräsident der naturforsehenden Gesellschaft \%u Hauburg.

revedenann, C. R. W., Professol Uer Mediziu, Elatsrath u. s, w. in Kiel. Der hochrefrierte Nestor der lintomologie, Anatom mul Zoolog, Siduifisteller und Reisender (Constantimopel, Lurupa u. s, w.). - Amaler:ta entomolorica. Kiliate 1824, 4. 1 Tish - Nova Dipterorum genera offert iconibusque illustrat. Kiliac Halsatorum $1 \times 20$, in 4. Vlil and $23 \mathrm{~S}$. -- Diptera exutica. Pars I, tabulis aeu. duabus.
Kiliae 1821, 8. - Neue Inserten vom Vorgebirge der guten Hoffumg. (Dessen Zool. Magin. I, 1, 1817, 40.) - Diptera. - Ueber einige neue Fliegengatlungen (ibid, I, 1, 57). - Zwcihumdert neue Käfer von Java, Bengaleu und dem Vorgebirge der guten Hoffunng (ibid. 1, 3. 4, 1819, 15 \%, 11, 1, 1823, 1). - Brasilienische Zweiflïgler (ibid. 1. 3, 1819, 40), - Besehreibung nener Zw ciflügler aus Ostindien und Afrika (ibid 1.). - Aus Pallas dipterologischem Nachlasse (ibill. 1818). - Zoologisehes Magazin. 4 Bde. 1817-1826, Kiel. 8. - Archiv fiir Zoologie und Zootomic. IV Bde., jedes 2 Stücke", m. K ff. Brannselwweig $1800-1805$, 8. - Nachrichten voll den zoologisehen Arbeiten französiseler Naturforseher, ans dem Tagebuche der philomatisehen Gesellschaft zu Paris. (Dessen Archiv. IV, I, (1S04) p. 126-150 and IV, II, p. 180.) - Zoologische Bemerkumgen aus G. A. Olivier's Voyage dans l'Empire ottoman, l'Egypte et la Perso Tom. 11. Paris all 9. (Ebendas, p. 150 -156.) und vicle andere Abhandlı!!gen. Melir als die Hiilfte von Meigen's Muckenwerk ist von ihm.

vicrzloick, Peter, Magister Pliarm., Chirurg. et Obstetr., k. Bergwerlis- und Kamernlwundarzt 7.u Oravitza im Banat. 'Tiielitiger Botanilier und Eutomolog. Sanmelt alle Kerfe, vorzüglich Käfer und Falter; nur Schade, dass er so cinsam in der grossen Wïste steht.

Wrilnelm vontwiintentuerg, Se. Erl. Graf, 7.u Stutgart.

vviluelus, in Sarepta. Eutomolog. Villuens, Dr. med. und praet. Arzt in Bremen.

Uvilkin, S., in Norwich. Entomo$\log$ und Schiriftsteller. Scliöne Sammlung. - Catalogue of some rate Inserts in the Collection of S. Wilkiu. Norwich 1816,8.

trill, Dr. med. in Leipzig (?). Britrïge zur Anatomic der zusammengesetzten Augren mit façettirter IIorllhat. Leipzig 1840.

Trilson, J., in London. - Illustrations of Zoology. Loud. 1831, Fol. 2 Tal.

TVinmer, F., in Breslan.

Wioumer, loseph Baron von, in Prag. Ausurecichurte Sammlung curopiiischer Lepidopteren.

Winkter, Ed. Dr. ete. in Leipzig (i'). - Vollstindiges Reul-Texilou 
der medizinisch-plarmazeutischen $\mathrm{Na}$ turgesclij(lite mind Roliwarienkunde. Juprig $18: 10-42,8$.

ETintlurn, W. v., Kaufuann in Habubrg. Eutomolog.

Dintheru, $A$. v., in Hamburg. Entomolog (Diptera). Ausgezeiclunete Kerfsammlung aller Klassell, beson. ders von Käfern, Dipteren und Faltem.

Wood, William, Esq., in Lon. don. - Illustration of the Liuncan genera of Insects. London 1821, 2 vol. 12. with 86 plates color: - Yudex entomologieus or a complete illostrated Catalogue of the Lepiclopterous linsects of Great Britain. London 1833, 8.

trredow, Hauptmann in Neapel (zeitweise in Clumr). Eifriger Sammler.

Tundran, Dr. med. in Wien. - Beobachfungen ïber die Erdflïhe (Verlandl. der k. k. Iandw.Ges. zoWien. Neue Folge. III.).

Wylk, H. J. Baron van der, Geneval, Mitglied der Ritterseluaft der niederlindlisclen P'roviuz Ober-Yssel, z.แ Manuleim. - Er und scine Frïllein Tochter sind die cifrigsten Beförderer der Naturkunde.

Tintart, Pharmazcut etc. zu Pratz de Molo (Dept. des Pyrenees orient.). Sammlıng.

Fanez, Professor der Naturgesilhiclite u. s. w. in Barcellona, - Schrift, stcller.

rvan, Melclior, zu Digne (Dept. des Basses-Alpes). Entomolog.

Trarel, William, Esq. in London. Zoolog.

Wacher, Polizeidirector in Lembere.

Zauders, I. W, in Köln. - उсjtrigge zur Geschichte der Thiermeta. morpliose. Köln 1807.

vensolski, Alexander, Dr. und Professor z.l Przenysl (ostr. Galizien), Neue Kaifer alls rlen Karpathen (Memmosyue, 1833 (?) - Carab.Zacheri, Zawadskii, P'achygaster Kratteri und P'eryphus Cikowskii). - Zwei Gencrationen von Noctua Moneta (Isis 1834, 737).

Zuelue, F. S., Oberforster zı Borutin bri Ratibor, - Rhagium indagator-Zwillinge und Seardia mediella. (lint. Zritg: I, 61.)

Jeiz, in Lcipzig.

Zeller, Dr. in Frankfurt a. M.

Theller, P. C, Oberlehrer in Glo- gan. - Ueber die Schmetterlingsarten in Ratzclourg's Forst-luserten 2 'Thl. 1840. 1ent. Zeity. I, 185.). - Brsclureihnng ler Gelechiella lapella Linn. (cul. Zeilor. III. 259.). - 'Tabaksaft z.um Insertriltölten ete. (ent. Zeiter. I, 110). - licitı ig zur Kenotniss der Volucella plumata InI bombylans. (ent. Zcitg. III, 65.) Versucl eines naturgemässeu Eintlıejlung der Solaben (Isis 1839, p. 167221). - Dytiscus oder Dyticus, Cillosoma ete. gen. neutr.? (ent. Zeitu. III. 67 p.) - Nachviclit ïher die Sec. felder bei Reinerz in entomol. Beyie. lung (ent. Zeitg. II, 171). - Kritisclle Bestimmung der in Degecr's Abland. lung zur Gesolichte der loserten enthaltenen Lepidopteren (1sis 1839, p. 243-349). - Beurtheilung der Fieyri'sehen Preisatbeit viber Reaomir's Sclumetterlingr, lsis 1838. 737. (Isis 1839 , p. 448-470.) - Beitrag z.115 Kenutniss der Dipteren ans den Fumilien: Bombylier, Anthracier nnd Asiliden. (Isis 1840, P, 10-78.) - Lepidopterologiselie Beituige. (Isis 1840. p) IIt -143 und p. 208-248.) - Vorlitufes einer vollst aindigen Naturgescluiclute der Pterophoriclen, ciner Nachtfilterfit. milie. (Isis 1841, p. 755-79.5 ind 827-893.) - Dipterologiscle Beitrige. 11. Abtheil. (Isis 1842. p. $807-8.18$.) - Die Symonymie der Hufuigelselaeu Filterarten (Isis 1844, p. 16-49). Monograplice des Gemus Hyponomeuta (Isis 1844, p. 199-238. Tif. 1. 2).

Zaendriui, M., Professor der spe. eicllen Naturgesclichte zu Pavia.

menneck, Professor der Jindlwirthschaft in Stuttgart (Gerberstrasse Nr. 25) IIübsele Sammlong von Kï. fern, Schmetterlingen, Flipgen, Biencuund Wespenarten. Beabsichtigt cine Besclureibung seiner Sammlung lieraus. zugelsen. - Eintheilung der Raupen zul' leiclitern Bestimumung Jer gefundenen inlündischen Atten (ent. Zeitg. I, 115).

Zetterstedt, Joh. Will., l'rofessor der Zoologie in Jallud. Bekanuter Scluriftsteller. - Fanma iusectorum Lapponica Hammonac, 1828. - Lip. siae 1840,4 . Mit Fase. VI (1840), 4. $902-1139$ ist das Werk gestlulossen (Isis 1839: 760). - Diptera Srandinaviae disposita et desceripta. Ton. I, Joludae 18.12, II, 1843: 8. I Isis 1843, 873.) - Mothopterat Succiac. Disposita

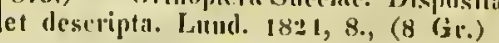


Zueyocent, Victor, in Strassburg. Sammler.

miegler, Franz, in Wien. Itymenopterolog. Ausgezeichnete Sammlung. Steht z.muI Verkanf.

Ziecter, Anton, Oberlicutenant in Linien-lufauterie-Reriment zu Rcgensburg. Faltersammler.

Zillich, Hauptmann in Prag. Sammolt Falter.

Vinumermann, Cluristian, in Plibadelphia. Tüclitiger entomologischer Selniftstelier. Sammelte in NordAmcrika. - Nomograplice der Carabiden. I. Stüek. Halle (Anton) 1831.8. - Dio Amireı, Ein Beitrag zur Monograplie dieser Sippe. (G istel: Fammus, Zeitschrift für Zoologic und verglcichende Anatomic. I. Bd. I. Heft. Müinclien 1831, 1-69.). - Ueber Masoreus (Gis tel's Faumus. I, 2, p. 119.)
Mippe, Custos an Muscum in P'rag. Vipser, Professor zu Neusohl in Ungaru.

Zoblikofer, Dr. med. in St. Galleu I Schweiz). Sanmlung.

Tscelnorn, Carl, Seluillehrer in Halle. Sammelt Kerfe jeder Art.

Zublsor, Basilius von, Justizrath in Moskau. Prä́chtige Sammlung (meist russiscluc Arten). - Notice sur un nouveau genre et quelques nouvelles espèees de Coléoptères. (Bullet. de MIoscou ed. Leq. p. 29-45. PI. III, 1829.) Nouveaux Coléoptères recueilles en Turcménic (ibicl. Tom. V, 1833, p. 297-323.)

Zwwick, von Glarus, frülier zu Sarepta, gegenwärtig in Schafflausen. Entomolog und Reisender.

\section{NACHTRÄGE.}

Anyot, iu Paris. - Moluris Picrreti 122 papes, avee 16 planches, conte(Gueviu Magis, de 'tool. IX, 129). Histoirc de la Cigale (Silberumann Revue ell. IV, P. 60-71).

Aymari, A., zu Le Puy. - Reste zahlieicher Kerfe in den Mergel der Gypsformation zu Puy. (Amal. de la sociètè géologique de la Franee, 1835 , 1V.)

Erssi, in Mailand. - Malacogenster Passerinii (Guerin Magas. de Zool. IX. 99. Fig.).

Beck, Heinrich, Dircktor des Na. turabicukabincts Si, Maj. des Königs C'li istia u von Dimemark, in Kopeuliagen.

BIanchnrel, L., in Paris. - Notice sur les metamorphoses des Coleopteres du genre: Télephore: (Guevin Ma. pas. de Zool. IX, pl. 108, 1-6.) Mantis chlorophaca (Guirion Mag. de Zool. IX, (35).

Enoisdnval, in Paris. - Coroegidiou horreus gGnetin Magas. IX, 127. Fig.). - Fame cutomolengipue de Madagistar, Bourbon et Mirurice. Pantie des Lépidopicies, avee des noies : les mocurs, pilr M. Sganzin. Panis nant 100 figures. Prix 32 fies.) Description de deux Leppidoptères nonveaux d'Espagne (Rev, ent. 11, p. 120 $-124)$.

Erignoli, Giovanni di, P’ofessor der Botanik auf der Universitit zu Reggio. - Schriftsteller. - Edirte 1836 eine Brochure über fossile Crustaceen und Radiarien und hat eine wohigeorduete kerfsammlung.

mrotlerip, Willian John, Escy. in Loudou. - Zoolog und Sehriftstefler. - Beschreibung ciniger fossil 'u Crustaceen und Radiarien von Ijime Regis in Dorsetshive (Trunsictions of gecolog. sor. 1835).

Brulle, Anguste, in Paris. - Obscrvations critipues sul lat synonymie des Carabiques (Revue ent. 11, 10. 89 -11.1). - Ilistoire naturelle des Insectes, traitaut de leur organisation et de leurs moeurs en general, pits M. V. Andou in, professenu-administratente aII Museum ol'histoice maturelle de P’iris,

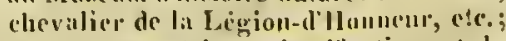
ef conprenant leur classilication it lia description des esperees, par M. A. Brullé, aide-nituraliste au Mluseum 183.1. (Un volume très-grand in 8. de cte. Pais (chez l'illot), 1835, 8. 
(Le tout aecompagné de planelies gravées sur?aeier.). (Voyez: Revue ent. III, p. $515-220$ ).

Bnrmelster, in Halle. - Mèmoire sur la division naturelle des Punaises terrestres (Geocores), consi. déres surtout relativement it la strucfure des auteunes (Revue eut. II, 527. Planehe 16). - Monographic des Genus Darnis (vgl. Silberuanu).

Clnardiny, I. C., in Lyon. Description d'une nouvelle Zygène (Revie entom. IV, p. 194-195. Plinelie).

Charpentice, Oberbergrath zu Dortumul. - Esper's Naturgeschichte der Schnetterlinge; fortgesezt. - Jede Liefermug mit 2.8 illam. Kupfertaf. gr. 4. Erlangen. (Erscheint noeh.)

Cleverolnt, in Paris. - Scarabacus Anubis (Guerin Magas. IX, 139 et 140. Fig.). - Dryophilus anobioides (Guerin Magas. IX, 3. Fiv. Jet. Wurde vou uns $n$ m Miunclien, von Grimuer um Gräiz entdeckt). - Une nouvelle Mígecephala (Revne ent. II, p. 83). - Quelques observations sur la famille des Carabiques (Rev. ent. II, p. $11.1-115)$.

Cinristol, Jules de, Professor der Naturwisseuscluften an der Akademie 211 Dijolt. - Sammelt eifrig.

Corla, in Prag. - Selırieb, uns unbekaunt in welehem Journal, iiber einen fossilen Seorpion im Kolılengebirge bei Clımle in Bölımen.

Crivelli, Balsamo, Professor der Naturgeschichte in Mailand.

Debey, Dr., praktiseher Arzt zu Aaclen. - Beabsiclıtigt Einiges über die Sittrn der Kerfe eto. Inerauszugeben, vvorüber das Manuskript bereits vollendet ist.

Desmarest, in Paris. - Hypocephahus armafus (MCloclastus paradoxus, Gistel). (Guerin Magas. 24. Fig.)

Desportes, Naceisse, Conservator des Museuus der Naturgeschichite zu Mans (Dept. Sarthe).

Dencyey, Cliester, Professor an Williau-Collegium zu Roehester (Massachuxetts).

Diceld, Franz, Professor der Oekonomie und der Naturgeschichte in Brïnn. - Lelubuclu der Naturgeschichte. Brï̈u. 1835. 8.

IDepont, in Paris, - Encirrus

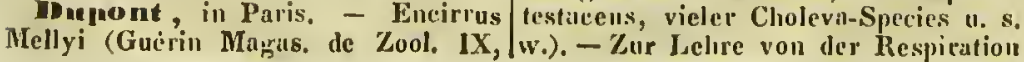
testiceus, vieler Cholevil-Species u. s.
w.). - Zur Lelire von do' Respitation

47. Fig.). - Nariclus opalus et olivacens (Guirin Magas. IX, 128). Heterosternus buprestoiles (ibill. IX, 10. Fig.). - Polybotris eroesus (ibil. 77). - Malloderes microecphalus (ibitl. 125. Fig.). - Callipogon senex (ibid. 33. Herrliclıes Gemiilde).

Eins, F., Professor und Custos am Natualienkabinet zu T'roppau.

Faldermsnn, in St. Petersburg. - Bercicherung zur Käfeckunde des russischen Reiches. Peterslurg, 1836. 8. - Additanuenta entonologica ad Fanum rossicam (Nouv. Mem. de la Societe impèviale des maturalistes de Moscoll IV, 1836, 4.).

Nerrara, F. Abbe, Professor der Nafurgeschiehte an Jer Universitit zu Catunia.

wischer von Traluhein, iu Moskan. - Bibliographia palacontologica animalium systematiea. Edit. 2da. Mosjuac, 1834. (Ein selur diekleibiges Werk ïber die Literatur uller fossilen Thiere.)

Géné, in Turin. - Eiı Aus\%ug Jer Scluritt iber dic Kerfe Sardinirns findet sich in Silberminns Revuc (IV, p. 269-273), alls der Revne zoologique.

Germar, in Halle. - Insertorum protogarae specimen, sistens Insectu Carbonum fossilium. (Fanna Ins. Europ. Fasc. XIX) Hillae 1837. - Ueber die versteinerten Insekten des Jurascliefers von Solulenlofen (Isis 1837). - Descriptiou de deux navelles Cigales (Rev. cnt. IV, p. $71-73$ ). - Observations sur plusienrs espèces da genre Cicade (Rev. ent. II, 1834, p. 49-83. ['anches). - Synonymie de Coleopteres (Rev. ent. IV, 1) 112-113).

Tervais, Amannensis am Mrusimm d"historic naturelle in Pflanzengarten zII Paris.

Cistel, Johanues, in Müuchen. -

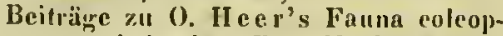
terorum helvetica. Dem Verfusser der. selben brieflich mitgetheilt, als z. B. die Eutdrekung von zelu nenen Ptilien (In den Feften: die Kiffer der Schweiz. Neuehatel 1837 , steht unrei u e e j и \% i g e Gattung.), das Vorkommen des Necrophlilus subterraneus in Selueckengehäusen um Bern, des loptiuns 
der Ammlosen. Als anatomlsch-physiologiseluev Prodrom einer umfassenden Munographic etc. Mit mehren IIolzschuitten. (Wird demnächst erscheincu.) - Neuc Entrleckungen in Reiche der Natur. (Zellenbauen der Anthoplioren in den lecren Gehtusen der Weinbergsseluncele (bereits vorgetragen in einer Sitzun der naturforshemelen Gesellscliaft zu Beru, 1838), Pupplengebären der Acherontia Atropos in Gespionsten, Fadenspinnen des Avion Empiricorum: Vurkommen eince neuen Grylle un Beru (Herbst 1838), Gr. sylvarum, einer neucn Nafter un Bern und eines neucu Frosches, entcleckt am Sclueideck im Juli 1838 u. s. w.). Auseluliclse Beiträge zur Monographic der Müncluner Ceranbycinen, ciner Dissert. inaug. pro gradu Doct. in med. von Dr. med. K y i ech b a u u cr. Muinelien, 1S44. 8.

(x)ey, in Paris. - Hyboma rubri. pennis (Gucion Magas. 37. Fig.). Cuniatus uimropumetatus (ibid. IX, 120). - Hamaticherus suturalis (ibid. P. 1). - Buprestis Buquet (ibid. 61. Fig.). - Attelalius sumptuosus (I. c. 119). Anacolus quatdinaculatus (ibicl. IX, 31 ). (Mit Percheron, zu Paris.) Descriptions de quelques nouvelles espèces de Cétoines de Madagusear, par les auteurs de la Monographie des Cétoines (Revue ent. III, p. 122-130).

foncenu, in learis. - Sur les sons produits par ecrtains Inseetes (Rev. elit. III. P. 101-109).

(indiy, Alonzo M. A., Professor der Naturgeschichte und der Cliemie am Tenehers-Seminary zu Andover (Massachusets).

(Anray, in London. - Trictenotoma Cluildrenii (Guérin Mag. IX, 35. Eine uniblsertrellidiche Abbildung.).

Guérin, in Paris. - Mierorhipis Dumerilii (Gucrin Mitg. de Zon)I. I. Figr.). - Cladupliorus Iatelialis (ibiol. 11). Aterpus pipa (1. c. 98). - Cimpe l'byllocerus (Revue zoologigac. I. Silberm. Revue IV, p. 259). - Gemre Lissomus (I. C. p. 260-62). - Sur le gemre Panssus (I. e. p. 262-64), - Sur le genve Trochoide (I. c. Revue ent. IV, p. $264-65$ ).

It: Iol, Alexander, in Mlinelies. Vou ilnu wirl in Stutfutt, heillallherger: clseheincu: Demonstrative Natellye'scluichte. Vollstindiger Unterricht im Sammeln,
Prïpnriren,Klassificiren, Verwahu eumd Demonstriren der Naturkörper aller drei Reiche. Nebst Beselereibungr der Lebeusweise der Thiere, sowie îrer Köruerhaltung bei allen Bewegungen. Gan\% aus eigenen Erfiudungen und Beobachtumen genommen. Nit einer Vurrede von S eliubert. 7 Tafeln Abbildungen.

Meyden, C. N. G. von, Senator in Frankfurt. - Entomologisclie Beitrige. Frankturt, 1838. 4. (Ueber H cmiptera inphidina.) - Recension von de Laporte's „Essai d'une classifcation systimatique de Yordre des Hémiptires" (Revue ent. Il, p. 4\$-47).

Mope, in Lonton. - Description d'une Cerambycin nouveau (Revue ent. III, p. 71-72. Fig.).

Intton, in Newcastle (upon Tyne), Srkretair der naturlistorischen Gesellsclaaft daselbst.

Jolnnstou, John, Professor der Naturgeschichte an der Wesleyan-Universität zu Middletown (Conuceticut).

ICferstein. - Beobachtungen ïber den Curculio granarius (Uebersezt von G. Silbermann in dessen Revue ent. II, P. 115-120.).

Irircliner, Di. med. und prakt. Arzt zu Bauberg, Vorstand der naturforschenden Geselisehaft daselbst.

Kolenati, Friedrich von, Dr. med. in Wien, - Zu Hoheuheim, der berülumten Landwiollsehafts - Akademie

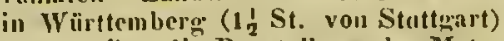
ist von ihu die Darstellung der Metamorphosis des Bombyx Mori ete. in watürliclsen Excmplaren zu selien merksiïrlign.

Iomink, de, Professor an ler Universität in Lütticlu. BekannterSthriftstellar. SammeIt lierfe. - Mémuiro sur les Crustarè fossiles de Belpiyue. (Extr. des Mim. de l'Aead. ray. d. sciene. (le Bıux. XIV.)

Kredcowitzer, Ferdinaud, Dr. med., lebt in Pesth mach neuern Nachrichten.

Iancoranire, Tlı., ist un Professor der Zoolegie an der Universifiit zu Luittich. - Der 2. Batml der Indroluction is l'Eutomologie, conprenant les principes gencrinx de lianatomie et de la ploysiologic des insectes, des détiils sur leur moeturs, et uu 
résume des prineipnux systemes de classifieatiou proposés jusqu'à ce jour pour er's animallx, ist ersclitenen 1837 (in 8. volı 681 Pages und mit Kupfern zu Paris bei Rouet). - Sur la priorite des noms en Eutomologie (Rev. ent. IV, 1837, p. 223-230).

Laporte, in Paris - Enicotarsus viridipennis (Guririn Mag. 35. Fig.). - Oplopes atriplicis (ibiol. 20). - CaIieuemis Latreillei (ibid. 7). - Recension des entomological Magazine von W a lker (Silberm. Revue ent. II, p. 40-44. - Wir sind geuöthigt, nachtriglieh zu bemerken, dass wir von Hin. Silb e rma un's sehr vermisster Revue entomologique nur folgende Hefte besitzen : Tome II, 7, 8, 9, 10 (Pulis 1834); 1II, 13, 14, 15, 16 (Paris 1831); 1II, 13, 14, 15, 16 (l'aris 1835); IV $19,20,21,22,23,24$ (Paris, 1836 -37). Gistel.). - Une nonvelle Meracephala (Revue ent. II, p. 83). Etudes entomologiques, ou deseriptions d'inseetes nouveaux et observations sur la symonymie (Rev. ent. IV, p. 5-60; 11I, p. 157-181). - Observations su1 Ia tribı des Cicindélites (Revu ent. 1I, p. $27-40$ ).

Lefebvre, in Paris. - Polyommatus oftomanus (Gueicin Mag. IX, 19). Ephippiger maerogaster (ibicl. 5).

Tdequien, in Paris. - Amallopo. des scabrosus (Guérin Mag. IX, 74).

Teunis, in Hildeslicim. - Synopsis des Thierreiches. Hannover (Hahu), 1844. 8.

Locklinrt, Direktor des naturhistoriselen Museums und Ritter der Ehreulegion, zu Orlcaus.

Iancas, in I'aris. - Pediculus PJocae (Guérin Mag. IX, 121).

M. (aus Rouen). Ueber das Betragell der Larveu von Lampyris (Ammales des Scienc. VII (1826), 353. - Isis 1831. 850).

Macedo, Jose da Coste de, ferpetuellor S'ckretair del königl. Akadenuir del Wisseuschaften zu Lissalbou.

Mnpes, J. B., Professor der Naturgeschichte an der National-Academic zu Designe (New-York).

Velly, in Liverponl. - Passalus Goryi (Guérin Mag. 56. IX, Fig.).

Mis isliug, Theodor, l'ofesvol der Seidenzucht in Iohenheim (bei Stutt-

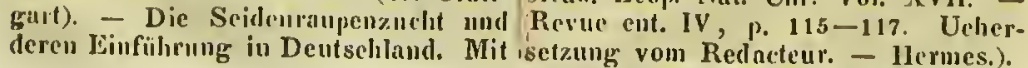

meliren (11 illuminirten und seliwaryen) Zeichunngen. Stuttgart (llallbrrucer). 18.1.1. XX et 2.17 Pagg. 8. - Ist selit zil emplehlen.

Mosch, Direktor des NaturalienKabinets der Ritter - Akademic zu Liegnitz.

Nees von Esembeck, in Breslau. - Hynenopterorum Ichueumonibus affinium monographiae, grenriti curopaca et species illustlantes. 2 Vol. Stuttr. et Tubing. (Cutta) 1834-30

Nevport, G. D., in London. (Vgl. Tod d, p. 71 dieses Lexikun's).

Desterlen, Dr. med, und Leibarzt des Fuirsten von Hohenlohe in Oehvingen.

(1) rers, vou, ist in Berlin.

Arbigny, Ale. Dessalines d', soll in La Rochelle wohnen, nicht in Paris.

Partscli, Paul, Dr. nied, und Direktor des k. k. Naturalienkabinets in Wien.

Intuchurg, in Nenstidt-Ehersselkifer (Nov. Acta Acad. Nut, Curios. 1835. - Revue ent. IV, R. 113-115. - Hermes).

Remssluer, Jeremiah Etienue van, Dr. med. und Professor der Geolorie am Athenenm zu New-York und Pi:isidenl des Albany. Instituts, in NowYork. - Fossile Crustaecen von NewJersey (Amuals of the Lycemm of Niv. York, 1825). - Seine Sammlung von Kerfon ist bedentend.

Doser, in Stutgart. - Ueber eine im Fleisclie der sthwarzen Kirsclien vorkommende Insekfenlarve. Stuttg. 1836. 8. (Revue ent. IV, p. 132. Ist $U_{1}$ tulis Cerasi).

Suncerotte, C., in Luneville. 183.). 8.

Sclibeiermácluer, Dr. med. und Diuchtor des Naturalieu- Museums in Dirrustadt.

Gelnumidt, in Laibirlı. - Art, Nichtschumetterliuge zul fangen (Nov. Act. Acas. setzung vom Redncteur. - llermes.). (Revue ent. III, 1835, P. 95-96). valde. - Ueber die seluädlielıen RiisEliments d'histoire maturelle. 1. Paris. 
Gotanxin, Artillerie-Capitain und Commandaut des Forts vou SainteMarie, in Loirent. Lepidopterologiseher Seluriftsteller. (Vgl. '13 o i s d u v a 1, i॥ de॥ Nachträge॥ dieses Lexikons.)

silfoermann, in Strassburg. De l'Instinet des Insectes. Traduit de l'Introduction a l'Entomologie, de M. M. Kirby et Spence (Revie ent. III, 1835, p. 7-71). - Lettre de M. Beske sur les Lépidoptères du Biesil, suivic d'observations de M. Kefersteiu. Traduite de l'allemand (Revue ent. IV, p. 73-103). - De la nouvelle éditiou du Catalogue de M. Dejcan (Revue cnton. IV, p. 124-131). - Degâts oecasionnes par la Geometra pinaria (Revue ent. II , 124-127). - Uebersetzung der Keferstein'sehen Schrift: Observations détuchees sur l'apparition des Lépidoptères (Rev. ent. II, p. 137 - 189). - Les Latridius des euvirons de Munich, par J. West erhauser; traduit de l'allemand du Journal "Faunas" par M. Gist el (Rev, ent. III, 109-122). - Monographic du genre Darnis, Fabre, par M. le Dr. H. B urmeister. Traduite du manuserit allemaud (Revue ent. 11J, 1837, 1. 164192. Planche). - Uehersetzung der Abhandlung ïber Byturus tomentosus und fumatus, aus Gistel's Faumus (Rev. eut. V, p. 192-194). - Die Uebersetzung des ganzen entomologischen Theiles der Reise voll Dr. W al I I maeh Spanien ete. (Rev. ent. IV, 1837, p. 137-164), - Reeensiou und Auszug von Klug's Bericht über eine auf Silbermann).
Madagasear veranstaltete Simmlung von linsekten aus der Ordnung Coleoptera (aus den Denkschr. d. Eeil. Akademic. Starker Quartband von 136 Seiten unit 5 color. Kupfern, 1833). (Revue ent. III, p. 132-147. Wichtig.) - Recension von Ma equal's : Histoire naturelle des Inseetes - Dip. teres. I (ibid. p. 147-153). - Ausz.ıg aus K I $n g$ 's Jahrbüichern: Aperçu des Histiroides. de la eollection du Muséum de Berlin par M. Erielısou (Rev. ent. III, p. 181-215). - Sur la priorite des noms en Entomologie (als Anhang zu Isacordaire's (vgl.) Schuit' in der Revue (ent. IV, p. 239-242). Uebersetzung des Aufsatzes iber Amara vou Zimmermann in Gistel's Fau. mus I (Revue ent. II, 189-231).

Spinola, in Genua. - Deseription d'uI Macraspis nouvera (Revue ent. 111, p. 130-132. Planehe).

villa, Ant., in Mailand. - Coleopteru Europae dupleta in Collectione A. V. Mediolani, 1833, 8. Adj. : Coleop. terorum species novac et supplementum. Mediol, 1835. 8. - Tauseht uแr, verkauft aber nieltt. Das gilt auch von dessen Herrn Bruder.

Waltl, in Passau. - Ueber dus Sammeln exotisclier lusekten. (Fanmus voll Gistel. I. 3. Heft, p. 166-170.) - Reise dureh Tyrol naeh Oberitalien, Piemont und dem südlichen Spanicu. Passau (Pustet). 2 Bde. 1835. 8. (V 


\section{Akademian und gelehrte Gesellschaften und deren Schriften.}

Anrau. NasuriorbchendeCantonalgese!lscis ri:

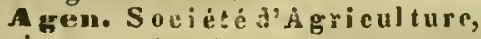
Seiences ché arts.

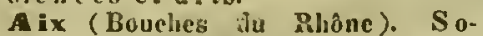
ciété des Sciences. - Procès. verbal de sa séance publique du $1 \mathrm{t}$. Juin 1825-jusq. 1834, \&. - Acade. mie des Seiences.

Albany (New.York). Whe Lyceum of natulai hist org. - Trausactions of the Albanj-Institut.

Altenbers. Iraturforsehende Gescllschat: des Osterlandes. - Mittlueilungen aus Jem Osterlande. Von cier natuirf. Ges. zu AJtenburg. Il] Ede. 17, 1. Heft.

Anerike. Versammlung der amerikuniselıcu Naturforsclıcr. - Die erste Versammlumg wnrd, wie man uns versichert lat, in Micligan in Jalire 1841 abgelaalten.

Aunens. Academie des Scien. ces. - Compte rendu des travanx.

Amsteralam. K. niedelli indi. solies Institut der Wissensehaften und Kiinste. - Verhandelingen der cerste Klasse van uel hollandsch Institut van Wefeuschappen, Letterkunde en seboone Kenaten te Am. sterdam. Jirl. 1812-25, 7 Deel, 4. Nieuwe Verhandelingen der ecrste Klasse, I. - VII. Drel (14 Vol.) Amsteriam $1827-38,4$.

Angers (Maine et Luire). Sociéte d'ugriculture, scieuces et nrts. - Aendemie des Sciences.

Angonteme. Sociéte d'Agri. cul ture. -- Amnales. 1821-44,8.

Antwerpen. Akademic der Wissenseluaften.

Arezzo. Academia di Scicnac, letlerec arti.
Arras. Société royale académique.

Athen. Naturforsebende Gesellschaft. (Sirit 1835).

Aviguon. Socièté des lettres, arts el seiences.

IInuberg. Naturforselende Gesellscilıaft. (Gestiftet 1833.)

masel. Naturforsclieude Gesellselıaft. - Bericlite über die Verhandhugeu derselben $1834-36$ (Frilier: Nova Arta ete.). 4.

IBtavia. Soejiti des Seiences et des arts. - Verhandelingen (seit 1820).

mengalen. The BengnI Society for inquiring into the history and antiquitics, arts, seiences andliterature of Asia. - Journal of the Ariatic-Socicty of Bengal. 8.

Eerlin. K. Akademie der Wis. senschatten. - Abhatullungen der kön. Akudenie do." Wissensehaften zu Berlin. Erseheinen srit 1802. - Gesellseliaft naturforseliender Freunde. - Der Gesellsrhaft naturforschender Freunde z.11 Bcrlin Magazin für die neuesten linteckmmgen in der gesammten Naturkunde. lirster Jahrgang, 1807, Berlin. Realb. q. 317 Pagg, 8 Kupf. Zweiter Jalurgang 1808, 326 Pagk., 8 Kupf. Dritter Jahrg. 1809, 320 Pagrg., 9 Kupf. Vierter Jahrg. 1810, 322 P'ingr., 9 K. Fïnfter Jahig., 1811, 424 Pagng. $10 \mathrm{~K}$. Serlister Jalug., 1814, 318 Pagg. 5 K. Siebenter Jalirg., 324 Pagg., 7 K. Acluter Jahrg. 1818, 312 P'uggr., $10 \mathrm{~K}$. Ist Fortsetzung der Seluriften muter dem Titel: Der Gesellselaft naturforscliender Freunde zu Berlin neue 
Schriften. Erster Band. Mit 4 Kupf.|chnselts History Soeiety. Vol. Berlin 1795, XII. IV., 380 S., 4. I (1822) - XXX. - Collection of Zweiter Bd., m. \& K., 1799. XXVi the Society of natoral history. II. 458 S. Dritter Bd., In. 7 K. - Jourmolof natur. history. 1801 , XII II. 612 S. Vierter Bd. nI. 7 K., 1803, XII II. 428 S. - Beobarlitungen und Enfdeckungen nos der Nafurkunde vou der Gresellsehaft Daturforscheuder Freonde z.u Berliu. Berlin (2. Band, 1788) 8. - Mittlucilungen ans den Verhantlungen der Gesellsthaft nafurforschender Freunle 211 Brrlin (1. Jahr; 1836). Ereheinen in geringer Bogenzalıl jiihrlieh in 8. Geographische Gesellschaft. Garten b a u-Gesellseh uft.

Bergamo. Aleuco. - lustitu1n. - Sociela agraria.

Meru. Naturforseliende Cantonalgesellschaft. - Oekonomiscle Gencllschaft. - Gibt Schifteo heraus.

Bexnncon. Academic des Scienes.

Boulogme-sur-Mer. Sucićté l'agrienlinre. - Procès-verbal de sa séanee publique du 12 Juillet 1824; gelut bis 18.14. ill 4.

Bourg. Sociéti d'histoire nat in relle.

Bourges. A eademie des Seienees.

Brenen. Naturforseliende Gesellselu aft.

Brescia: Societa de la sto. ria llaturale.

Brealau. Sehlesische Gesell. selıaft l'ǜ vaterländiselı Cultur. - Unbersicht der Arbeiten und Veränderungen der sehlesischen Gesellschaft fiir vaterlïndische Cultur in Jalure 1841. Breslall, 4. (Isis 1843, 640.) - Jahresberichte der entomologischen Section der sehlesischen Gesellschuft fïr vaterlïndische Cultur zu Breslan, 1839, 18.10 und 1841. - Na. 1 urforseliende Gesellsehaft. -

Bristol. Institution of Seien.

cemburg. Der wisseuschafliche Verein des Harzes. (Hielt 1844 seine 15 . Ver8ลmmlung). - Protokolle (1sis).

Bologna. Instituto. - Me. morie dell' Instituto. 1806, 4. - Nuavi Anuali drlle Scienze naturali. Bologna: I. $1838,8.479,1.13$.

Eombuy. Sueiely. - Transactions of'Bombay. 4. Lond. 1819-23, 3 Vol.

moun. Kaiserlieh-leopoldiuisch-karolinisehe Akademie der Naturforseler. - Nova Acta physicn-medica Academiae Cuesareac Lcepoldinae Natorac curiosorum. Bonnae. Die Nova Acta erscheinen seit $\mathbf{8 2 3 .}$ - Niederrleinisehe Gesell. seluafi fïr Natur-und Heilkunde.

Borderux. Société de Midiciuc. - Notices de ses travaux, depuis le mois d'aôt 1824, josqu'an 1844 (par .11. Dnpuch-Lapointe). 8. - Programme des prix decernes. 4. - Société liuncenue. - Bulletin d'hist. nat. de la sor. lin. de B. - Société de Sallé el l'histoire naturelle. - Journal. III vol. - Academie royale des Scienes, belles lettreset arts (Gestiftet im Jahre 1816).

Bosteu. Aeallemy of the Seienees. - Memoirs of the Americun Acudemy ol Arts and Sciences. Vol. 1, Bostun, 1785, Vol. 2, Part. ecs.

IBrock In maseu (Hanuover), Gesellseltaft nat urfous che uder Freunate.

IBrilgee. Gesellschaft der Nafurwissenschuft. - Amuales de In Socièté des Sciences naturelle do Bruges (Belge).

Brihun. Mälı riselı-schlesisehe Gescllsehaft für Ackerbau u॥d Naturkuude.

Brussel. K. Akademie der Wissensehaften. - Nouveaux llimoires. - Bulletius des Séances. Aunuaire de l'Academie royale des Sciences et belles-Leltres de Bruxelles. Cinq. Annece. Bruxelles, 1839. (Enthält meist Necrolog(e) - Aumales geuerales des Sciences Pliysiques. I.

Buclinrest. Gelehrte Gesellsehaft. - Naturhist.Gescllseluft.

Cren. Acadimie des Seien. ces. - Mémoires pour les anmes 1823 et $24 ;-44,8$.

Cahors. Acadćmie des Scien. ces.

Colcutta. Medieal and pliysieal Suciety. - Transuctions of the medical and physical Society of $\mathbf{C}$. 1. Charlestown, 1804, 4. - Massu.| Vol. 1-8. Calc. 1825-36, 8. - Asijtio: 
Rescarches, Vol. I. Caleutta, 1804, 4. - A siatie Society. Journal of asiatic Sucicty (begoumeu 1829). - Calcutti-Glaings of Science. 8. - A g ricultural Society. Transactions of the Agricultural Society of India. VII.

Colvalos. Société linnéenue. - Mimoires.

Cambray (Flandern). Socièté d'èulation. - Memoires pendaut lanuic 1824-1844. 8. - Société d'histoire naturelle.

Cambrian (Wales). - Iust it ution of Scieuce.

Canbridge. Society of phi1050 ply.

Capstualt (an Cap der guten Hoffnumy). Naturhistorische Grsell. schnlt. Wahrscheinlich durch den verdienten Baron v. Ludwig, ejnen Wiìrttemberger, grgründet.

Cassel. Cluturfürstlich laudwirthseliaftlicher Verciu.

Cotnuin. Acadcmia gioenia di Scieuze naturali. - Atti dell' Acad. Catan. III, 1829. - Alessi ( $G$ i u s.) : Relazione academica per l'anno $X$. dell Acad. etc. dal Segretario gene. rale. Catania 1834. 8.

CntsldII (New-York). The Ly. cenu of natural listory.

Cette. Societed'histoirenaturelle.

CVAlons-sur-marue. Societ è d'Agriculture. - Comptes rendus de ses tinvalux de 1807 à 1844 . 8. Societe d'histoire naturelle.

Clinmbery (Savoyen). A cadémic des Sciences de Chambery c ns Savoyc.

Clathon. The philosophical und litcrary lustifution.

C'liserlentewio (Sïl-Carolina). Literaryaud philosoplical Acad. $\mathrm{cm} y$.

Cherburg. Societed d'histo ite naturelle.

Cluavarl. Socictáceonomica.

Cluristiauian.

Niturwissenschaftlicher. Verein. - Pliysin2uaphiseher Vereil. - Nyi Ma. faxin for Nafurvilenskalserne, udgives if deu physiografiske Forening $\mathrm{i}$ Christianiu. llid. I-III, 1838-42.8. (Isis 1843. 8.37).

Clubr. Naturforschende und ökonomisehe Cantonal. Gesell. scluaft.
Clueluntl (Ohio). Westeru-Muse un Socicty.

Clauntloai (IIamover). Forstseli ule.

Clermont-Forrand. Academie des Sciences. - Compterendu des travaux.

Coisulura. A kademie der Wis. senschaften.

Compiégne. Société d'agri. culture.

Corlc. Instifutiou of Scienre. Cortona. Sonst war eine Gesellschaft daselbst, von welelier S a ggi (di Diss.) Berichte seit 1742 iu 4. herausgab. Ob nocl eine da existirt, ist uns uubekannt.

Janzig. Naturforselieude Gesellsehaft. - Sulhriften (seit 1767). - Nelle Sammlungen (seit 1778). Neueste Schriften der maturforschenden Gesellschaft in Danzig. Halle bei Rengerer, B. I, seit 1820, Hft. 3, 1824, 210 , und 5 Stf. Hft. $4,1825,146$, 3 Kuffl. II. s. w.

Dhinemark. Versammlung der skandiuavischen Naturforscher. - Förhandlingar vid det af Skandinaviska Naturforskare och Liikare hallıa möte $\mathrm{i}$ Göthehorg Ar 1839. Götheborg, Norberg, 1840, 8. 188. (Aunzug aus den Verhundlungen: Isis 1843, p. 207.) (Erste Versammluug Amu 1837). - Forhundlingar ved de skandinaviske Naturforskeres andet Nöde, det holdtes i Kïbeulavn fin den 3 die til deu 9. Juli 1840, stor 8 , Kjöbenlavn (Reit\%el).

Darinstait. Der Grossherzoglich hessisehe Laudwirtlisehaftliche Vereiu. - Gartenbauverein.

Dentscilnnit. Vers a m m l u $\mathrm{ng}$ der deutsebeu Naturforscluer u ud Aerzic. Gestiftet durch Okeu 1822. - (Die antlichen Bericlite über dic Versaumlungen dentseher Naturforscher und Aerzte (jedesmal localites wechselud) erselienen und crscheinen in der Isis). - Versammlungen wurden bisher gehalten: 1822 Leipzig. 1823 Halle. 1824 Wüı)ırg. 1825 Frankfurt a. M. 1826 Dresderi. 1827 Müuchen. 1828 Berlin, 1829 IIridelberg. 1830 llamburg. 1831 krine gelialtell. 1832 Wien. 1833 Bresliul. 183.1 Stuttgart. 1835 Bonn. 1836 Jena, 1837 Prag. 1838 Freiburg. 
1839 Pyrnont. 18.10 Erlangen. 1841 Bınuselıweig. 1842 Mainz, 1843 Grä̌. 184.1 Bremen.

Dijou. A cademie der Wissen. scliatteu. - Mémoires de l'Academie de Dijon. 12 Tom. Dijon (seit 1769 und 1774, 8.).

Dốle. Société d'agriculture. - Extrait raisume des procés-verbaux de ses 4 preniers seances. Dôle, 18.2, 8. - Nonvelle Extrait 1844.

Dartreclit. Naturforscliende Gescllselati von Sidhulland.

Douni. Societè royale et centrale d'agriculture scieuces et arts du Departemeut du Nord.Academie des Sciences.

Drceissigncker. Socint $\mathrm{tät}$ der Fứ: "ud Jagitiunde.

Dresiles. Gesullschaft fïr $\mathrm{N}$ ut ur-\|nd Heilkumbe. - K $\ddot{\text { u }}$ iglich öknuomische Gesellschaft.

Drontlecier. Gesellscliaft der freieu Künste ete. (fither sehou bestand, scit 1765, cine gelchrte Gescllschinti daselbst). - Königliche unurorschende Gesellseluat. - Die $110 \mathrm{rwegiselie} \mathrm{Gescll-}$ seluatt der Wissenschaften.

Dablier. Royal irisl Academic. - Irausactions of the royal iuislı Acadeuy. Vol. I - XIX. Dubliu 1757 - 18.11, 4. - Proceedings of the roval irisli Acarlemy (schou 180.1) 1836-39. Part. I-lli. Dubliı. - Royal Dubliu Society.

Derisum. Society of uatural history.

Ealimbergli. Royal Society. - I'rasuctious of royal Society of Edimburgh. Tome $1-X I V$, Edinb. 1788-1840. - Procecdings of the roy al Society of Edimburgh. Nr. 1-18 (1832-41.) Edimhtrgh. 8. - Werneriau uatural history Society.Memoirs of the Weru. nat. list. Soc. of Vidiub. $1811-24, \mathrm{~V}$ Vol. 8.

Femuleu. Naturforseliende Gesel Ischatt.

Wengelnut. Britische Association \%ur Befirdernug der Wisse $\|$ scliatien (for the avaneement of sciences). (Sitzungen in verschiedenen Stïlten.)

Erfurt. Noturforscheude Ge sellseliaft. - (Seit 1799.) - Nova Artal. - Akademie uïtzlicher IV isseuse hulteu.
Frangen. Ply sicalisch.me. dicinische Gescllseloaft. - Kameralistisch-ökouomische Gesells r laf t.

Ettlingrin. Badiscluer I andwithsehafticher Velein. - Ver. liandlumgen. 1823 (t. Jahrg. 12 Hefte).

Wure. Sociéte libre d'Agriculture, Scicnees, arts et belIes Iettres du Departement de l?.

Dvrenx. Socicte d'agricul. ture. - Journal d'agricultue, me. dicine et Sciences access. de Oct. 1824 $-41,8$.

Ferrora. A cademia nedicocli ilurica.

Forenz. Academia dei Georgofili di Firenze. - Societit agraria di Toscana. - Giornale agrario. Atti. 8. - Lyceo della hístoria naturale. - Academia fiorameutan a.

Srankfart a. M. Senkeubergiscilie naturforsclieude Gesellschatt. - Selırifteu und Abhandfungen: - Museum SunkenbergiaIIm. Abhandlungeu aus dem Gebiete der beschueibenden Niturgesflichte. Von Mitmliedern der Senkrnbern. natuforscl. Greellscbaft in Finukfut a. M. 1-III nud Suppl. III. Heft 2. Mit vielen Tafeln. Finnkf. a. M. $18.36-44,4$.

Frnalufert a. d, 0 . Landwi ill. schaftlicher Ceutralverein.

Freibure in B. (tosellscloaft

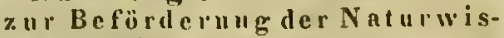
senseliaften.

Freylower i. d. Seluw. Cantoun gesellseh aft der allgemej. uen scluweizerischen Gesell. sc li aft.

Tencive. Socicte Cantouale de Pliysique et d'Histoire naturelle di. - Mimoires de lia Societe de pliysiques et d'bistoire maturelle. de Genêve. 'Tume l, prem. part. Genêre, Paschoud, Paris, maisou de Commerce, 1821. et seq.

Fent. K. Collegimm. - Na. turforseheude Gesellschaft vou F lande in. Gestiftet linde 1833 . Socicte d'agriculture et de Bot a n i q ue de. - Messager des Sciences ct. $1-10$ livr. $1825-44,8$.

Cocuns. Academia delle Scieuze, Lettere e Arti di Ge. llova. 
GIarus. Naturforschende Cantoulgesellseliaft.

Adbrlitz. Nafurforseliende Gesellschaft. - Abhandiungen der nafurforschende" Gesellschnft zu Görlit\%. 1. Bitnd, 1., 2. Ifeft, Görlit\%, 1827, 8. Mit Steindruck. - Oberlausitz'sehe Gesellsclaft der Wissensehafteu.

Tindtingen. K. A kad emi e. (Gest. 1750.) (Seit 1752 wissenschiftl. Gesellschaft.) - Acta soc. scient. 1752-54, 4 vol., 4. - Nov. commeutaria 1771-77, 8 Vol. et nov. enmment. 1779-1808, 17 Vol. - Recent. comment. 1808-27, 5 Vol., 4. Götting. gelelortc Anzcigen. 8 .

Coritz. Johann e um (Museum) der Naturgeschichite und des öffenfl. Unterrichits.) G('stiftet durch Erzherzog J olı a n, K. H., an 16. Juli 1811. Kaiserlieh königlielo Gescll. selıaft. - Steyermärkisehe Zeitschrift. Grät z.

Grenoble. Sociètè d'histoirc nat urelle.

fridiningen. Naturhistorischphysisch-chemisehe Gesell. seliaft.

Mang (Südbolland). Naturforseluende Gesellsehaft. - Verluandelingen (seit 1780). - Gesellschaft der Diligentia für Naturgeschichte im IIเล․

Hanarem. K. Jolländiscloc Gesellseliaft Jer Wisseuselat. ten. - Nederlandsche huishondelijke Mantschappij. Anfang 1825, 8. - Naturkundige Verhandelingen der hollandsche Maaischaappy der Wetenschappen te. - Haarlem 1841, 4. Seit 1759. Verbaudelingen, nitgegeven door Teylers tweede Gennotschap. Twree en twiutigrste Stuk. Haarlem, by Bohn, 1836, 4. 40 Bogen, Tal. 21 in Folio.

IInile a. S. Naturforschende Gesellsehaft. - Neure Schrifteu der naturforselienden Gesellselıaft zou Halle. 1. Heft hotanischen Iulialts. Halle, J. C. Hendels Verlag 1806, 50 bez. Seiten, in 8. (scit 1783). 2. H. (Zool.)

Mambings. Naturforselicude Gesellschaft. Gegriandet im Jahre 1837.

Manas. Wetterauisehe Gesellschaft fiir die Nalurkunde (scit 1809). Aunalen. Tausch- und Haudels bureau.
Hannover. Der Gartenbau. verein.

Havanna. Agronomische Gesellschaft. - (Ramon de I i $S$ af $r$ a:) Memorias de la institueion ugronoma de la Hahana.

Heinelbers. Natulhistorisehe und medizinisehe Gesell. seliaft.

Melsingarors. Soeiti des Spienecs.

Hudson (New-York). The Ly. ceum of natural listory.

Innsbruck. Ferdinande - Zcitselurifti fiir Tyrol und Vorarlberg 8 Bde. Inusburuck 1825-34, 8 . - Nene Zeitselurift des Ferdinindemus fïr Tyrol und Vorarlberg : herausergeben von dessen Curutoren. VIII. Bile. M. K. I. Ch., 8. Imusbruck 1835-44. - Kaiserlich königliehe La ndwirthsehaftiche Gesellschaft fïr Tyrol und Vorarl berg.

Itallen. Versammlung der it alienischen Naturforselier und A erzte. Die erste Versimmlung war 1839 zu Pisa, die zweite 1840 in Turin und 1841 in Florenz.

Sinssy. Naturforschendo Gesellsehaft der Moldiu.

Jaucr. Vgl. Scluwcidnitz.

IX dingsbers. Pliysicalisel. bkonomische Gesellschaft.

Kopenlnagen. K. dii niselue wisseusehaftlielue Gesellscluaft (seit 1790). - Oversigt over det kougelige danske videnskabernes Sel. skab. Forhandlinger efe. in Aaret 1840, af H. C. Oersted. 38 Pagre, 4. Skrivter af Nafurhistorie Selskabet. 1. Bind, 1. Heft. Kjöbenhann, tryekt Hos Hnfloggtryktlerne. r. N. Möller 1790. 6 Kupf. (Z110 Entomoingrip.) Det Kongelige danske Videnskaberues Selskalss nafurvideuskabelige of milthematiske Aflandlinger. VIII. Thelle Kjöbenhavn 1841, 4., mit 33 Tafelı. (1sis 1843, p. 732.) - Skandiuavisclue entomnlogische Gesell. seluaft. - Verhaudlungen der skandinavischen entomologisehen Gesellschaft, mitgetheilt von J. E. Sc hiödte, Seeretiir der Geselischaft. (In Kröyer's Tidskrift.) - 'Tidskrift for Nuturvidenskulb 10 Cah. Kioihoul. $1824-K \ddot{0}$ niglich dünisehe Akademie dev Wissensehaften. Gestiftet im Jilur 
1742. - Gesellseliaft zur Ausbreitung der Naturlehre in Dïne ma rk.

Laibacls (Kraill). Acudemia operosorum.

Uansanue. Socicted des S cicnecs pliysiques et naturelles du Cantou de Vaud (seit 1784). - Rapport de ses travaux depuis le premier Août 1824 jusq. 1844, 8. Mémoires.

Leipzig. Naturforsehende Gesellseluaft - Schriften. - Miscellanea lipsiensia. $1670-1819,59$ vol., 4. - Fiirstlich Jablonowsky'sche Gesellschaft ler Wissen. seliaften.

Leyden. Akademie - Anmales Academiac Luyduno-Batavac. 4. (1834 liat C. G. C. Reiuwardt, d. Z. Rector herausgegeben.); seit 1817 bis jetzt. - Verbandelingen over de matuulijke Geselhiedenis der Nederlandsche overzeselie Bezittingen. Door de Leden der uatuurkundige Commissie in Oust-Indie en andere Schrijviers. Zoölogie. Nr. 1. Leyden 1839, 9 Bern. m. 10 Tnf.; Nr. 2, 1840, 13 B\#11. m. 10 Taf, Fol. (Enthalteu Arbciten von M iiller (Sucke des indisehen Arehipels) und Sehlegel (auch mit M. gempinscliaftlielı: über Simia satyrus, Pittil (Avis) und Crokodile des ind. Arehipels u. 8. W.).

Lengo. Dentsclier Apothekerverein. - Archiv des dentschen Apothekervercins im nöıdl. Deutsehıl.; 40 Bde., bis jetet. Lemgo 1828. - (Ma ma cryeugende Insecten; Bd. 2.1.)

Wile. Societe n'agriellure - Recueil de ses travaux pendant les aumees 1819-22. 1 vol., 8. Lille 1823 - 44. - Programmes. 4. - Mimoiles. 1825-28, 8. - Sociétè royale des Scieuces, de l'agricul. ill ic et des arts.

Himoges. Soeide d'agricul. t 1 re. - Journal dagrieulture. $1823-44,8 . \quad-$ Academie des Seicnces.

Tisstobn. K. Ak ademic der Wissellsphaften. - Memorias. XII Tomes. Lisbon, 1797-1837, in 2.

Hivorno. Academia medicoclirurgica.

Tubuen. Akademic der Wissenseliafien. - Ammales Aeade. mine Invani. $1823-25,4$ vol., 4 . Lomdon. Royal asiatic So- vaux pendant le 1. Semesire de 1824,
Triatic Researches; on in Bengal for inquiring into the history ete. of Asia. Vol. I, printed verbatin from the Calentta edition. Isondon prin. ted for J. Sewde etc. 1795. - Society of Literatur. - Transactions of the Society of Literatur. 1827-34, 4. - Philosophical Socicty. - Philosophical Transactions of the royal Society of London. Seit 1665 bis jetzt. - Zoological Socicty. - Iransactions of London Zoologicill Society. Vol. 1, II, 1836-38. Wird fortyesetzt. - Royal Society of Literature of the Uniled Kingdom. - Transactions ete. Vol. I-IX. Lond. 1831-44. - Proceedings ete. Vol. I, 1835. - Ammual Report ete. 1837, II. - Proceedings etc. Part. I-III. Lond. $1833-35,8$. - Transactions ete. Vol. I. Iond. 1833-35. - Entomological Sociot y. - The 'Transactions of the rutomological Society of Loudon. Vol. I. London 1836, 8. will. 24 col. plat. The new moologicallnstitution. - Wernerian uatural history Society. - Memoires of the Wer. meriau Natural history Society, Vol. $\mathrm{I}$, and past of Vol. 2. 8. - The Zoolegiral Jourual. Von eirea 1822 an. Conducted by 'Il. BeIl, Jolun George Children, Jones de Carle Sowerby and G. B. Sowerby. Lundun, - Wird noch furtgesetzt. - T,in. neall Society. - liansnetions of the Linnean Society of London (seit ctwa 1790). - Royal iustitutiou of Great britain. - Society of llatural history.

Lucea. Acadrmia discienze, Jettere et Arti.

Liltioln. Socicte libre d'em lation de Jiege. - l'rocès-verbal de seanee publique du' 29. Janvier $1825-44,8$.

Enua. Plysiograplicebe Societiit. - Sundewall (C.): Physiografiska Sällskapets Tidskrift. 1. Bandet. Lind, 1837; $(-38$, 4 Hefte in gr. 8, 384 Pagre., m. Abbild. (Isis 18.12, p. 936 et seqq. Zeitschrift der physiographischenGesellschaft z.n I.und.)

Huzeri, Cantonalgesell. schaft.

Hyon. Acadimic des Seiences. - Compte reudu de ses tra- 
par M. Rogny et pendant le 2. Semestre pal M. Achard-d almes ete. 8. - Programme des prix. 8. - So. ciete d'A grieulture et d'lijstoire naturclle (Athentemus. Compte renda de travaux depuis le 1 Mars 1823, jusq. 1844, 8.

Jiracom (Saône et Loire). Sociét d'A oriculture, Scinuces et belles lettres. - Conpte rendu de travaux peudaut l'anné $1824-44,8$.

Madrid. Academie der Wissenschaften. - Memorias de la real Academia de la listoria. Vol. 1-7. Madrid 1796, et sequ. 4 .

Mafra (Lstremadura). A eademie le. Wissensehaften.

Mainnul. $A k$ ademie der Wis-P seissilaften. - Memorie (spit 1819, 4). - Opuseoli Scelti. - Antologia itoliana.

IIrinz. Rloiniselue Gesell. schaft der Pliysik und Niturgeschichte.

Manchester. Society of $n$ ataral history. - Literary and philosoulieal Society. - Memoires of the Literary and Philosoplical Socicty of Mancliester. I. et II. Series, Vol. 1, Manchester 1804-5, 8.

Ununlueim. Naturforsclicude Gesellschaft. - Jahresherichte des Manulieiner Vereines.

Mans. Sociètéd'A griculture, Seiences et A rts. - Seance pabli. que du 30. Juin $1825 ;-44$. - Compte des trivaux de la Soc. dep. Juin 1824 jusq. 1844, 8 .

Mantua.

Sricu\% c.

Academia delle

Marburg. Gesellschaft zur Belörderilg der gesammlen Nat ur w isseuseluafteu. Gestiftet 1816. - Seluriften, scit 1823.

Mnrylund. The MirylindAeadeiny of Science und Literatule. - Proceeding of the Mary. land-Aeademy of Science and Literature. Baltinore.

Metz. Academie rayale des Scicuces, arts et belles lettres. - Compte-reudu des truvaux de l'Academie des Seiences.

Nisexieo. Gelelite Gesellselaft. - Registro trimestro (seit $1832 ?$ ).

Midulelburg. Naturforschen. de GeselIseliaft.
Minden (Prenssen). Vereiu fiir angewandte Naturwissell. seli a f ten.

Nitam. Sociétécoulla la ise des lettres ct arts.

Mrodenn. Societi italiuna lelle Scienze, residenle in M. Memorie delle Soc, 180.\}, Vol. I-XI, 4.

Mdgalin. K. Akademie der I,andwirthsobaft. - Mömlin'vilie Aunalen der Landwiotlsschaft. Vou der k. mensisehen Akadenie der Laudu irth schaft zo Möglin. 1830.

Moutauban. Socicté d'li stoire nat urelle. -

Montpelier. Societe d'hi. stoire uat arelle. -

Montresi. The natural lı i. story Society.

Mous. Academie der Wissenschaften.

Moriz. Societe d'histoire natirelle de l'ile Manlier (gegriiudet 1830). - Memoires de lis so. cieté d'listoire naturelle de l'isle de brance.

Moslontr. Societe imperiale des $N$ at aralistes. - Bulletius rotr. Annec 1837. I-VIII. Moss. 18.37, 8. 1838, I-Ill. etc. - Socicti imprriale d'economic rurale.

Mihnchen. Akademie der Wissenseliaften. (Gestittet 1759.) - Denkseluiften der k. bayr. Akad mie de. Wissensehaften (seit 180y); in 4. - Grielurte Auzcigen. - La ul dwirthschaftlieles Verein ï r das Kön. Bay eru. - Woclienblatt desselbell. Seit etwa 1810.8.

Nancy. Societe royale dos Seielles, LettresArtset d'angieulfure. - Memoires de la So. eiete royale des Seiences, Lettres at Arts. - Précis des travaax de la Societé royale des Scienees, Lettres et Arts de Nancy. De 1813-31, Vol. VI, Nancy 1816-35,8. - Soeicte d'Agricalture. - Le bon cultiva. teur. Nr. 1-25. Call. 1825,8.

Nantes. Societe academique. - Jouruil. 1. Vol., 1825, $(1-4) 8$. Procès-verbal de siance publique du 19. Decenbre 1824. - Compte rendu de travaux, durint le cours de cette annie. Von $1825-1844,8$.

Nenpel. A eademia delle Scienze di Napoli. (Gestiftet 1750. ) - Atti della reale Academia neapo- 
lilaun delle Scienze. - Reale Iustituto d'incorraggiamento alle Scienze naturali di Napoli. Atti. Nupoli 1820-28, 4 Vol. - A cendemia di aspiranti Naturalisti. (Gegründet durch O. G. Costa.) Acadrmia Poutoniana. - Atti dell Acc. P. Napoli. 1832 -34. 4. Vol. I, 1-5. Heft. II, 1-2 (seit 1810).

Nemenlures. Sociéte des Seibnces naturelies de Noufcli âtel. - Agassiz. Bericlit iiber die pliysienl. Arbeiten der Gesellschaft (Mém. de Neulchâtel 1. 1836).

Nev-Bury (New York). The Lyceum of natural history. Transactions of the Albany-Instifut.

Newenstla: Natural history Society. - Transactions of natural history Suciety of Neweasile, 18:9. Nortern Institution.

New-Blaven (Connetient). Co n:necticut-Academy of arts and Sciences. -

New-Brienas (Louisiana) Lyce un of natural history.

Nev-Tork. Lycenm of naturul history. - Aunals of the Lycenm of natural History of New-York. - Literary und philosophicalSociety. - Clinton's, (De Witt.) Disconsse delivered before the Literary and Philosophical Society of New-York on the 4. of Mai 1814. New. York 1815, 4.

Nimes. A cademie des Scien. ces.

NortlumberInud.

Society of llatural history. - Trausactions of the Society of natural history of Northusuberland. (Erscheinen auch vou der in New-Custle und Durham unter diesem 'litel.)

Nurnbers. Naturforscheude Gexellscli aft.

Orlenns. Socicté royale des sciences, lettres et arts. - An. nales. Tome I. (1819) -1844.

Dxford. As hmole Socicty.

Fradua (Lombaid. - vellet. Kön.) Imperiale réale Acalemiadi Scienze, Lettere e Arti. Nuovi Saggi lell' Apademia di Paflovi. V Vol. in lol. mils.

Palermo. Sucicta dri Naturaliste. - Acadeniadelle Seienze, belle Lettere ete. - Riale Instituto d'lucorragiamento delle Scienze naturalinel regno delle due Sicilie.

Paris. L'Institut de France. - Memoires de lilustitat national des Scienees et des arts. Seienees matliematiques et physignes. Toue I-XiV, 1298 -1815. - Arademic des Seipnces. - Mènoires de l'Arademie (srit 1816i. - Histoire de l'Amademic royale dros scienc., depuis 1666-1790, av. If.s Mémoites de Mathematique ef de Ploysique. Pa is 1701-93. 164 Vol. 4. Av. jil. - Memoires pres. p. divers Savans; Scienc., mathem. et ploysiques. - Mé. moires du Muspiun d'histoire naturel'r. 1815-19. - Amuales des Seiences natorelles. 'Tome I, 1824. - Arehives dn Musemum d'histoile naturelle, publicess par Ies Professeurs de eet étublissenuent. Vol. 1, 2. Paris 1839-41. - Anuale. do Moscum national d'histoire naturelle par les Professeurs de eet Etablisscment. Oavrage orne do Gravures. A Paris, cliez Levranlt, Sclinell et Compagnie, Strassbonre, clic\% levrault et Conil. Tom. I-XXI, 18ก8-15.4. Noov. Anniles du Mus. d'hist, wat. seit 1832-36. IV vol. 4. Av. pl. - Socièté Linnècune (fond mu 1787). Némoires de la Socièté Linureune de Paris. - Compte renda des travanx de la Soc. Lill. par M. Arsenuc Thjé. bant de Berneaud. Paris 1826, 8. - Socirié entomologique do France. - Anuales de la Sociptè putomologique de France. 8. Paris 1832 -44. XX Vol. nv. pl. (Prix de l'abonnencut 30 Fres. - Alle 3 Monate erselseint rine Lieferung : 4 maphen cinen Band voll $400-500$ Seitell mit Kupfern (vgl Anszug: Isis 1842. 123 -35. - Das Institut theilt gleiehfalls viel daraus mitı. (Wir missen mit Bediumeru bemenken, dass in gauz Bayern und weder in Würlemherg ete. anch mur pin eiuziges Expuplar der Annales de la soeicité entomologinjuo de France zu funden ist. Dil wir noch als Professor der Naturgescliclite und der veroleichenden Gengraphic in dir Srliweiz, Icbten, war es uns gepliiekt, in Gruf pin Exemplar, das ungefïlir bis zom 6. Hefte des Jalurginugs 1837 riug, zu benitze॥ umd riu vollstïndires Reperturiom daraus z.n zielıen, wie wir es fruilıer mit den Philosephieal Tran. snetions und denen der Liunfan Society mit Notzen unternommen haben. Es ist dalscr Alles das uoch nachzusehlagen, 
was in diesem Buche nielut fehlen gollte. - Diese Milse wird dem Ee. sitzer des kastbaren Werks Icicht, dem Nieltbesitzer vou gar keinem audern Nutxou scyn, efwa den auseremmuen, etliclie Namen von Scluifieu und die Seluriften selbst nicht z.t wissen. Gist(l.) - Socicted dhistuire naturmlle. - Mémoire de la soc. d'luist. nat. de Paris. (llat scit dem Julur 1831 den Namen : "Societé des seiencess uaturelles de France" angenommen. - So cie eti pluilounatique. - Bulletin des scienc. Monitl. voul 1791 nn bis jert. Societe a natomiqu cieté Cuverienne. - Revue zooIngique pap In Socièt cuvericune Dircet. Guerin-Mleneville. Nr. 1. Januar 1838, 8 . (bis jetzt fortgesetzt). - Société géologique. Bulletio de la sae. géal. de France. Scit 1830. - Societé de geograplíe. - Recucil des Voyages et ales Mémoires publiècs. Sae. VI. Paris. 4.

Pran. A a d cmiedes S ciences. Perpiguan. Societé plitumat i que.

Pesaro. Academia a graria.

Pestlk. Societas eruditia hulgarica. - Plannu et Statuta Socictatis. Pestini t8.31, 4. - A magyar IIIdos túrsasáy aliaprajza a es reudszahasái. Budau 1831, 4. - Magyar tudos tríkastiyi nevküniv. 1833, Budau, 8. A Mngyar tudas túrsasay cukünivei. E!sö Kütet. Budan, $1833,4 .-$ L i $и$ dwirthseluftlicher Vereill.

Pluiladelphin. A meric a n p hilosophical Sacicty, - Trallsactions of the American Plilosaphical Sacicty, held nt Philadelphia for promnting useful Knowledge. Vol. I, the second edition corrected. Philadelphia. print. by R. Aitken. etc. 1789 , XXIV ct 407 Pagg., 4. VIl Plat Vol. II, (prin. edit.) $1786, \mathrm{XXX11}$ et 397 Pagr., V Plat.; Vol. III, 1793, XI, 370 Pagf., V Plat. : Vol. IV, (by Thom. Dolisoun) 1799, XXXIX, 5.31 Pagg. ct 42 P. Ind., XII Plat.; Vol. V, 180?, XXIII, 328 Pngg. Xili Plat.; Val. VI, Part. I, 1804 (Jane Aitken), XV et 190 Pnoge., IV Plat.: Vol. VI, Purt. II, 1809, XXIV, 19t - 428 Pagg. - Transactinus of the American Philosaplical Society; held at Pliladelphia, for promoting useful Knowledge. Vul. I, lew series. Philadelpleia by $A$. Small, 1818 , XXIV et $\$ 7$ (Eulogium), $\$ 54$ Pagr., 13
Plates; Val. II, new series, Philadel. phia 1825, XV, 503 Pagg., 10 Plat. 4. Fol. III, Philad. pr. by James Kay, 1830 Xil ct 51t l'agge, 19 Plat: Vol. IV , 1834, 524 l'agg. , 30 Plates. (Velu' haben wir davon uiclit g(selicu.) - A caI e m y of $N$ at ur al Seicues. Jaurmal of the Academy of Natural Seiences of Phitadelphin, 8. Vol. IV. Part. II, Nos. 11 ind III. - M a el II ri a n Ly cu Maclurian Lyearome to the nrts and Scienees. Pliladelphia 1827-29. Plilade Iphin MI ss cum - Socicty. - Frankliu- lustitut. pietsfiela (Mnssaelmsetts). T lie Lycen of naturnl history.

pisa. Sosieta dei Litteriti. - Giormale ligustieo dei Istlerati di Pisa.

Histoja Reale Acudeaindi Scicure.

Plymonth. British associa. tion. 18.41 .

Ersg. K. lähinische Gesell. schaft der Wissenscla ften. Abhandlungen. - Uebersirbt sämmtlielıer in den bislerigen Bände॥ von Abhandluugen verkummenden Aufsütze, Prag 1823, 8. - Ahhitullungen weuer Falge. 5 Bde. Prag t824-37, 8. Gesellsoliaft des vaterlïndisehen Musenms. - Monatschrift d. Ges d. vaterl Mnsenms (scit 1827). Jetat fulat die Zeitselurift den lifel: Jahrhüeluev des böhum. Mıs. f. Nntırund Vïlkerkınde: Geschichte, Kunst IInd Literatur (seit 1830), 8. - Akademic ler Wissenschnften. K. k. patriotisels-äkonomische Gescllselaft fïr Böhuen.

Pressburer. Akademie rur Befürderung derk ïnste "u d Wiskeuselinften.

Poitiers. A cadcmie des Seicn. ces.

Poys. Sociéte d'agricultırc, seicuces, artset commeree.

Frovidence (Rhode-Island). Franklin-Socicty.

Amebec. The litcrary and historical society.

Mennes. A cademic des Scien. ces.

In heims. Collège.

Inga. Association littéraire et pratique. 
Rochelle. Aeademic royale. Minoires de l'Acalemic royale de la Rochelle, 2 Tull. Paris (seit 1774).

Hone. A cademi a d clleScience ¿ Ro แII.

Rostock (Meckleuburg). Der Mecklenbuledische patriotiseltakanomischlo Vereill. - Meckleuburesiselic uaturfarselende Gesells $\mathrm{c}$ hif $\mathrm{at}$.

Rottcrelans. Saciéte des Sciences pliysiques et natu. relles en Necrlilude. - Bulletin des Sciences physiqums et natulelles eII Nerrlaude, redigre par $\mathrm{Migucl}$, G. Mulder et Weuckelael. Rutterdau, 1839, Idiviaison I-VI. Utredit 1810, livi. 1, 8. (lsis 1843, 789). 'E1scheint alle 2 Monate in 5 Bugen.) - B atavischc Gesellselaft fii $\mathrm{N}$ at urk II II d c.

Romen. Academie des Scien. ces. - Précis allalytiques de ses travanx prudaut les aumers 1804-i4, $\&$. - So cicitéd'écu oulation.-Comptrs rcudus allutels depuis le 9 . Juin 1809 jusques et compris 181:, 8. (av, pl.).

Rovigo. Academia di scicutifialcteuia de i Concordi.

Dalnt-Ietienne, Societé d'agriculturc. - Bulictind'industic agricole et inaunfacturierc. 182244. 8 .

St. Crallen. Cantoualsgesell. scli aft.

St. Fetersburg. K. k. Akademic der Wisseuschaften. - Mémoires. VI Seric. Sciuces mathematiques, folysiques et uaturelles. T. I-IV, 1831-37. 4. - Meimoires ete. prescutis par divers Savans. 1, 1832, 4. - Rceucils dos artes des Seances publiqucs, des aunees $1831-4.4$ 4. - Dir k. Akademie der Wissenschlaften wald iin Julure 1724 grestiftet. - Socièt imperialc libre écouomique. Societe pla aruacentigue.

Salem (Massurfusetts).

Iudia ulaile socicty. - Tle Essex connty uatural history soeiety. - Jourual of the EssexCounty Natur. sor. (seit 1836). 8.

Sruminiato. Socictid dei Euteletie.

Sclnaflenecn. Cantonalgesellsuhatit.

Sclnveidnitz mul Jnmer. Schlcsisch-patriotisch. ökono- demie royalc des Sciences, In- miselie Societiit der Fiirstent thii ue S. und J.

Seliveiz. Allgemeineschwcizproiscle Gesellseliaft der Naturforscher. - Werhselt alljïlurlich ilue Versaumlungsortc. Die erste fund Statt 7.1I Geuf (18t5). - Im Jalir 1829 war sis iul Hospliz an St. Bernlard (vgl. Ziiricli).

Sevilla. Akademie der Wisseusclu alf $\mathrm{c}$ u.

Siens. Acadcuia. - Atti dell' Arisdruia. $6 \mathrm{~V}$ ol.

Sknudina vièn. Vgl.Däne ut ark. Singrua. Ak ademie der Wise ncuseh aften.

Solothurn. Cantonalgesell. $x \mathrm{c}$ h i ft.

Stettin. EntounogischerVerciu. - Entomologischic Zeitung, heransocgeben vou dem entomolngischen Vercin in Stettin. Stettin. 8. Jahrguile 1840 - 44. 1. Jalirgang 18.10. Strttin (Becker) 8. 192 Pang. 2. Julirgillng 1841. Mit I Kupfer. Loiplaig (Flcischer). Redact. Dr. Sch mi dt (t). 194 Pagg. 3. Jalugang 1842. (Mit L Kuif. I ifid. 280 Pagr. 8.

tochlıolu. Kön. A k adem ie de l Wissenselu aften, - Kongl. Vetenskaps tradeuicus Handlingar. (seit 1741. 23 vol.: dallu: Nya Hindl. 1780). - Dic k. Akademic wurde 1739 gestiftet. - A rsbcrättelser om vetenskapernas fraustegr 1831-44, 8. - Arsbcrättelsel on myare Zoologisku Arbeten ocls Upptiekter etr. af R. Fr. Fries. 1837 etc. 8. - Liuncische Gesellscliaft.

Sitrnshourg. Sociète d'hi. stoire naturelle. - Mémoires de la Sucieti d'histoire naturelle. III, 18301840. Av. planch. - Société d'Agriculture. - Journal. 1824-33, \&. Societedes SciencesduBus-R hiu.

stenterert. Lan dovirthsehaftliclier Verein. Gestiftet 1817. Verhaudlungeu. (Unter der Rediction des beriihustcu Dr. Plieninger, Sccretär des Vercines.) - Naturlorschende Gesellschaft. Gegriindet im Sep. tember 1844. Fribler bestaud schou cille Grsellschaft, welche Abliandlungen herausgab; sic hat seit 1828 anfrehört.)

Thanand (iu Lizgebirge). Forsta ka d cui i c.

Tomlonse (Hante-Garome). Aca- 
scriptions ot belles lettres - Le proces-verbal de sa Seance publique du 25. Aoît 1825 ete. jisq. 1844. 8. - Socièle d'Apriculture de Toulousc. - Jourual des proprietuires ruaux du Midi de la Friner: 1 à 12. (1825) 8. 1825-44. - Procis-verbil le sa Siunce publique teune le 24. Juiu $18 \% 5$ dans la salle des ilhustres du Capitole. 18.14, 8.

Trevixo. A tenen.

Trier. Gesellseliaft niitalirher Wisseuschaften und Unter. suchungen.

Triest. Academia romaua 50 ciaca a Trieste.

Troy (New-Yolk). The Lyeenm of natural listory.

Troyes (Dept. de l'Aube). Societe d'ugrienlture des Seicnces, artsel belles lettres. - Mi. moires pour $1824-44,8$.

Turin. K. Akademie der Wissensebaften. - Memoricete, XXXIV Tom. 4. rseit 1788; friher, scit 1759 - 65, Miscellanca). - Die Akademic ward $\mathbf{7 8 3}$ gestiftet. - S Iciptn agiraria. - Mrmoric della Socicta auraria di Toriuo. - Calendario georgico. 8.

Waline. Academia uraria.

Wpsala. K. Societät der Wissenseliaften. - Nova acta regiae Sueietntis Upsaliensis. Vol. X (1834) 4. - Die Akadenie ward zu Gothen. bure 1725 grstiftet.

Utica New-York). The Lycenm of natural listory.

Utrecint. Naturforscliende Ges.lIschuft. - Nova Aeta. 1821.

Valemcicunes (Norl). Socicte d'Agriculture, des Scienecs et des A rts.

Veroma. Soeieti italiana delle Scienze. - Memorie (seit 1782). - Academia di Agricol. Iura, Artie commercio.
Vesomi. Société d'Agricul. tilic.

Wurselnau. Physikulisehe Gesellsehaft nnd k. k. Gesell. schuft der Freunde der Wissenschuften. - Mcdicinische Gesollse haft (gest. 1821).

Wastington. Columbian In. stitution.

Weilburg (und Pyrmont). Verciuliir Naturkunde im Herzog. lhum Nassan.

West-moint (New.York). The Lycecum of natural history.

Wien. K. k. Akademie der Wisscuseluaften. - Aumalen des Wiener Musenus der Naturgesehichte. 1. Bd., I. Im) 2. Abtlı., II, 1, 2. 4. Wien $1835-40$. Gesellechaft zin $r$ Laudeskunde Oestreichs unter de $r$ Enns. - Beiträure. 1834. Kais. köll. Josephinische Aka. demie (Juseplinuno).

Wilnn. K. k. mediciuische Gesellschaft.

Wurzburg. Nut urforschende Gesellselift. - K. philosoplo.medizinische Gesellschaft.

York. Institution of Science.

Zuthrich. Schweizerische Gesellseliafl fïr die gesamuten Naturuisseusehaften. Hat ihren Hnuptsitz in dieser Stadt. Sie ist gegriiudet duch Goss e iu Jahre 1815. Denkschrifien. 4. - Die " ие и Denk. schriften erscheinen seit t837. - Verhandlungen der 16. Ver-ammlung. St. Gullen 1s31, 8. - Actes de la 17. Sess. ¿ Gevive 1832. - Atti delln Sessione 18, in Lugano. 1834 ete. Vel. die Protokalle der Sitzungen und dic Ausziige aus deu Verhandhungen der Cantonalgesellschaften in Arrhiv d. Gres. zu Ber'n und deren Verzcichuisse im Yerz. d. Bïcher dies. Ges. p. 33 .

\section{Sclilussbe merkung.}

Die Nachtrïge zu dieser Uubersicht der Academien splıe nach in therio. loginchen Lexikon. 


\section{Ephemeriden, oder periodisch erscheinende Sammelwerke von Gelehrten *.}

\section{A. Zeitschriften für Eutomologie.}

1) Annales de la soc. ent. de France. (Vgl. Akademien.)

2) Entomologiselne

Zueltung vou Sicliiı. (Vgl. Akad, u. gel. Geselliscli.).

3) Fermar. Ziritschrift fïr die Entomoiogic und Magazill. (Sirhe unten.)

4) Silbermann. Revue entomolugique. (Vgl. Silbermann untell.) (Hat aufgehört.)

5) Walcher. Entomologiral Magazine. (Ist eingegangen.)

5) Thon. Entumolugisehes Archiv. (Hut nufgehört und auch der Redacteur.)

Dies sind alle entomologisehen Journale, welche in einen Zeitraume von 66 Jahren erschienen sind.

\section{B. Zeitschriften für allgemeine Naturgesehichte}

$$
\text { (7oologie). }
$$

Alunnucln der Fortorhritte in Auericnn Jourmal (vgl. SilWissenschaften, Kü̈sten, Manufaktn- liman).

ren und Handw. v. 1797- (1807nur? Smerlean Iepertory (vgl. Wir kenuen auch norh einen 13. Mapes).

Bd.). (Vou Bus $\mathrm{el}$.)

A.lpina. Von Carl Ulyss. v. Salis iu Marschlius und Jolı. Rud. S teinmiller. Wintertior 1806-9 ete. 8. M. Cl. u. Kupf. 4 Bde.
7) Dienen-Zueitung. ( Vgl.

8) Klug. Jahrbüicher der Insek(Seil inst mir ein Band.)

9) Illiger. Magaziu für Insekteı-

10) Fussly. Magaziu fïr die Liebhaber der Enlomologie. (vgl. unten

11) Schmeiler. Neurstes Maguzin fïr die Licbhaber der Entomo. (ef. Sclunvider). (Eingegangent.)

12) Scribn. Inurnul fïr die Lieblaber der Inscktenkunde.
Amic: (G.). ( Vgl. Giornale toscano.)

Annlectem. ( $V_{\mathrm{gl}}$. Mayer.)

Ammalen der Naturkunde. (Vgl. Rudolphi.)

* Vergl. die Sehrifien der geielirieo Vereine und Akademien. - Wir linben auch etwas fltere Weike aufgenommen. - Uebrigens fudet muibsames Forselien und Zufall oft zerstreute Fragmenls IIIs dem Gebiete der Zoologie in gauz fieteroganen Jouruaien. - Wer snlite Z.

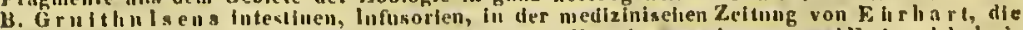
galiziachen Kurfe in durt.emberger $M$ eul os y ne (die wir meggelassen) undFriva idzky's

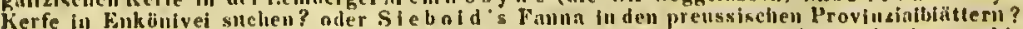

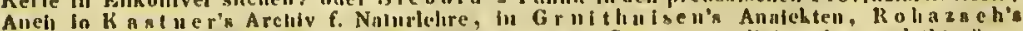
uligem. Zeitung, in B $\|$ e h $n$ er's Repertorim, in den Garten. medlzininchen und therärztIfehen und piarmaceltischen Zeitungen ergilt sleh Munchen, - Wir habeu jeduch weggelussen, was eigentlich ifich zun Faehe gelibri. 
Annsies des seienees d'observation (vgl. Raspail).

Anuales des sciences maturelles, gons la Diruction de M. M. A ud o u in, Brougniartet D nmas. Av pl. Paris $1824-33,30$ vol. 8. Nonv. Seric. (Seronde); redig. par $A$ ud onin et Milue Edwards pour la Zoologic. Par. Seit 1834.

Anunleg frmmenises et étrankers d'unatouie et de playsiologie etc. par La $\mathrm{r} e$ ut et $\mathrm{B}$ azin. Strasb. 8. Seit 1837.

Annali delle Srienze del Regno lombardo-veneto. Parlova. 4. Tab. 8. Anuali di storia naturale. Bologna. In Heften.

Annail universali(vgl.Omodei).

Amuals and magrazine of ratural history, including Zoolugy, Botany and Geology. Conducted by IV. Jur. dill c.

Amuals of plitiosopley (vgl. T'housun).

Antologia. Milano 1828.

Antologia delle Seienze naturali. Napoli.

Arehiv, Eutomologisches (vgl. Thon).

Arehiv fiir Naturgeschichte (vgl. Erichsou).

A relhiv fïr die systemutisehe Natulgeschichte vou Dr. Friedr. We be $r$ und Dr. A. M. H. Molir. Mit (3 schiv., 2 zuasgem.) Kupf. Erster Bnnd, erstes Stürk. Leipzig bei Selıüffer. 1804. X und $153 \mathrm{~S}$. gr. 8.

Archiv fiir Zoologic und Zootomic von C. R. W. Wiedemann. 5 Bdr. Berlin und Braunscluweig $1800-6.8$. - Zoulugisches Migaziu. Bd. I-IV. Kiel t817-20. 8. MI. Kupt:

Archiv fär die Physiologie von J. Clor. Reil. 12 Bde. Halle 1796-tsis. 8. M. Kpf. - Dentselies Archiv fïr. Physiologie. Ilalle 1815-18. 8. M. K. (In Reils Fusstapfen trat Meckel Ind, als dieses dis Zaitliclie seguete, unser gefeierter Johanues Mii Iler.)

Arelniv fiir Anatomic und Playsiologie von J. F. Meckel. Seit 182634. Leipzig (Voss). (a Jahrg. 4 Hefte.) Vgl. Mille l.

Asintic Jonrmal ('The) and montloly register for british and forecign India, China and Australasin. Niew serics. Vol. 31, 32. London 18.98.

Antautic Journal. 1831 .
Autonin (vgl. Annales).

Authenrieth ( $v$ gl. Tübinger Blätter).

IBnrtin (vgl. Bienenzeitung).

Enzin (vgl. Annıl. franç.).

Beitrige zur gesamuten Natur. und Ileilwissensehaft vulu Dr. W. R. We it ell we ber. Prag. 8. (Seit etwa 1836 ; his jezt $6-7$ bände mit Tafeln erschienen.)

- IEcitrigge zur Landeskunde Oest. reichs unter der Enns. Heransuregeben unter Veranlassung der niederöstrei-hischen Stände vou einem Vereine. Wien. 1. 1832. 8. 312. 2 Th, $4 \mathrm{Cl}$. und Holaschu.

Beitrige zur Naturkunde. Heransgegeben von Dr. und Prot. Friedr. Weber and Dr. D. M. H. Molir. Erster Band. Mit 7 Kupfert. Kicl, akad. Buclihdlg. 1805, XII und $356 \mathrm{~S} .8$. Zweiter Bl. Mit 4 K. Kiel, Aug. Silmidt. 1810. VI und $400 \mathrm{~S}$.

Bibliotineque universelle de Geuève.

Bieneuzeitung. Eichstädt 1845. 8. (Borenweise erschrinend.) Redacteure: Dr. Karl B a rt l, k, Landgerichtsarzt und Ant. Schmidt k. Lehrer am k. Srhullehrep-Seminar zn Eiclıstidd.

BIachwood, Edinburgh Magazine. 1837. Vol. 41, 42. Lond. 8. Vol. 47. Edinb. 1840. s.

mialnville (B. H. M. Ducrotay de'. Journal de Pliysique, de Chemie, d'histoire vaturelle et des Arts, avec des plinches en taille-donce. An 1818, Tome 86. Paris (Conrcier).

Bohmenterger (vgl. Tübinger Blätter).

Borhlıaugen (vgl. Magazin).

Bory de St. Vincent, Ammales prinerales des sciences pliysigues (av. Drapinz et v. Mous). I. - VII. Brux. 1819-21. 4.

Boubée (N.). Bulletin d'histoire naturelle le France. Paris $\mathbf{1 8 3 5 .}$

ERrali un (vgl. Magazin).

Brevster, the Edinburgh Jour. nal of Science. Ediuburgh 1824--30, 12 Vol. 8.

Britisin Meview (vgl, Review).

Eromguisert (vgl. Anuales).

Dndletin d'hist. II. de Fr. (vgl. Bonberi).

Bulietin univerael de Baron de Fè oussar.

Infulint (vgl. Giornale tosc.) 
Buscle, Mugazin der ncuesteu Entdeckungen. 18 Bde. (Vgl. Alma a a $\mathrm{cl}$ ).

Bytragen (vgl. Hall).

Calcutta-Jauruhl of maturn] History by .luhu M'Cle II n nd. I. 1 \$40. 8. 699. Tul). 12 (1 sis 1843, J. 804-837).

Caras (vel. Zeitsrluilit.

Cattaneo (vgl. ['iliternieo).

Correspountenza zoologica. Napoli $1839-44$.

Dismeminntar of Useful Knowledur. I. 1828 bis jezt. l'lilndelphiir.

Dublin-Review (vgl. Review).

Dumas (volgl. Auuales).

Wulmhourgli Mognxine: (vg-t. Blackwood).

Edinbourgh Journal (vol. Brewster).

Wdiuhurgh Journal (vgl..Jaucsou).

cintomological (Tlie) Magazime (vel. Walrker).

Ericluson, Archiv fïr Niturgeachiclite. (Gegrïndet vou A F. A.W iegma ॥ n.) InVerhindung mit mehen GeIelırten. V Jalırgänge. Berlin 183640. 8. M. Kpf. Forfsetzung vou Prof. Erichson. Seit Märr In40 (vom 6. Heft 1810 an). - Der Jahrg. Ieseleht nus 52 Bogen in 6 Heften (6) Fill I . (Nicolai erunissigte den Preis der V crsien Bände von 30 auf 16 lithl.) Bd. I oder Jahrg. I ersehien 1835. - Bestelit norlh.

E'Expérience. Paı is. Erschrint in Numeru von je Monat z.॥ Monat.

Fnums (vgl. Gistel),

Fentherstounuglu. The Mouthly anterien , lunrual of Geology and natu ral sciener. Philadelphia. Srit 1831.

Férussane (vgl. Bulletiu).

Nichus (vgl. Zritsiluiften).

Frobel (vyl. Mittheilungen).

Nroriney) (vgl. Notizen).

Foreign yunterly Reviev (vgl. Review).

Nuessiy (vgl. Magazin),

Germar, Zeitschuift für dic Entomologic. Begonnen 1838 (bis jezt V Bände). - Magazin der Futomalogic. Bd. I-IV. Hille 1813-21. 8.

C्रlorgini (vgl. Giornale tosc.).

Aiornale dí Palermo (vgl. Mortillara).
Giomale di Pavia. In Heften.

ciloruale (Ninovo) de, Litterali.

Pinn.

Giormale sripntifico di Pixil.

Gixinruale toscann di sicuze mediche, fisiche a naturali, direftu dai Professorig amici. Bufaliui, Ginr. Ciui, Puccinutti, G. Savi e P. Savi. Pisa pr. Nistri. Fascieule I, 1840. II, 111, 1841. (Isis 1843. 665 -674.)

Sioruale puciclopedico di Napoll. Unc int unr der Julurgang 1819 bekanut.

Giste: 1, Fnunus. Zritselırift für Zrologric und vergl. Auatumic. 1832-38, III Bite, 8. 1. Heft. (Isis 1834. 146). II. Heft. 1834 II is 1834.882 ). III. Heft (1sis 1837. 824). - (Dirke Zeitsclırift entlıäl manclıen vielıtigen Britray zur Zoologie und ist demjenigen mentbolurlich, der in dieser WVisscusiluaft fortsclureiten will, $O \mathrm{keu}$.)

Juleanings (vol. Josse).

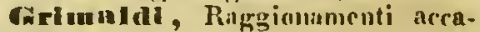
demici.

GẢ érim-Veneville. Magnsin de Zoulogir. l'auis. 8. fig. ill. (Anzeige: 1xis. 1*.12. $50 \mathrm{rt} 20.3 \mathrm{et}$ scyq.)

Bil. I, 1\$31, cutliält: Schurecken nud Kerfe ; beillerscits 40 ill. Tafelı.

Bd. II, 1832 : Thiere als allen Classen. Sucke, Vügel, Lurrehe, Clustaecen, Krife.

Bd. 1II, 1833: Sucke, Vügel, Crusta('een, Pselapliden, Kerfe.

Bd. IV, 1834: Sucke. Vögel, Concliyliru, spinneu, Kiffer, Lans und Melasomeni).

Bd. V. I 835 : Sucke, Lurche, Nilfisclae, Mollnsken, Telyphomus, Geivais' Myriapoden, Käfer, Canopus nuוl W ${ }_{\text {a11zen. }}$

Bd. VI, 1836: Sucke, Vüyrl.Schurekru, Cinstacern, Arncluniden. Iseuchten des Veers, Käfer (Tracliyderes, Telephous, Panborus, Bryaxis).

Brl. VII. 1837: Sucke, Versteinertingen, Vögel, Lurche, Schalthiere, Kerfe, Crinoiden.

Hall (H. C.), W. Vrolik, en G. T. Mulder: Bytragen tot de Nnturkundige Wetensehappen Amsterd. 1826.

Itnamerschuiht (vgl. Zritschrift ).

IAmrtig (vgl. Jahresberichte).

Hecer (vgl. Mittheilungen).

Heuminger (vgl, Zcitschrift für organisclie Pliysik). 
IUpfuer (vgl. Magaziu).

moeven (J. van der) en Vriesa (W. N. de). Tijdsehrift voor natumlike Geseliedenis on Plyysiologic. I-IV Deel. Lcilln, 1833-38.

Illiger (vgl. Masnziओ).

Indicatore? suluse Sicua. 183644. (Dieses Jourual, hesomlers fïi Plyysiologie etr., erselueint in Heften.)

Invitint (Is'. Journal general des aociètes et travaux scientifiques de lai France el de l'étrauge. 1. Sicction. Seimor. mith. phys. et naturelles. Paris 1835-42. 4. (Ersiheint blattweise in Folio alle 5 Tage und ist von grosser Wielitigknit.)

Isis (vgl. Oken).

Jacquenin (vgl. Minerve).

Jahrbnch der Naturgesclichte (vel. Tilesius).

Jnhresberichte (vgl. Nilsour).

Jahrbihcher der Insektenkunoin (vgl. Klug.

Jali resberichte über dic Forstwissensclaft uncl forstliclue Naturkunde iu Jalue 1836 and 1837, I1(b)st Originalabhandluugrou aus deu Gobicte dieser Wissenschaften Eine Zeitsilurift für Forstleute, Waldibesitzer und Camerulisten. Heransgegebeu von Furstrathe, Professor ete Dr. Theodur H in 1 tig. I. Jalırging, 4 Hefte. Berlin 1838-43. $r \mathrm{r} .8$.

Jamenon (R.), The Eliuburgh new philosophica I Jourual. Vol. I. (1819)-XIV(1826. Dis uene begimt mit Bd. I, I826, - VI (1828) VI-VIII (1829). Vol. XII - 24 Heft (1832) XIII-XV (1833) (vergl. Isis IN32).

Jardine (vgl. Amnals and Magilzilue).

Jesse (Edward), Gleanillgs in Natural history. 2 Vol. 4. witl fig.

Jonrual asiatique. Paris.

Journal fiir Natuı- und Heilkunde von der nıedico-eliirurgiscleen Akademic zu St. Petersburg. Petersb. (Grifif). H. 1-4. 1839-4t.

Jugel's miversal Magazine and Munthly miseellany. Edited by F. A Catty. No. I-IV. Lex.8. Frankf. a. M. 1843 (Juge I).

IMIug (Friedr.). Jahubiielier der Insektenkunde, mit besouderp Rüek. sicht auf die Sammlung in könig lichen Museum in Berlin. I. Band mit 2 ill.
Kupferu. fr. 8. Berlin 1834 (4 f. 3 kr.). (Mrlar ist nirlut erschicuen.)

If rbyer (Ileurik), Naturlistorisk Tislskbrit. Kaprolongen, 8. Srit 1837 -44. M. Kpf. (Ausserordentlielı reich und in jeder Berichung zu cupfolulen. - Dureh cin Versehen (und wie leiclst gelit von etlichen 'Tausenden Flug. b!:tteru ciurs vroloren!) ist der Name eliess: hedentenden Gelelirten aus den Lexikon entferut geblipben.)

Ionurent (vırl. Aunales françaises). Heach (vel. Miscellany).

Cielntenberg (L.Clı.). Magazin fïr das Nroestr aus der Physik und Naturgeschi life. 1'2 Bele. Gotlat 1781-99. 8. - Miggizin fïr deu ueuesteu Zustand der Notmkande von J. H. Vaigt. Jena and Wroinar 1797-1806. 8. N. viel. K.

Lichtenstein (vgl. Seudungen).

Henden (.J. C.). The Magarine of natural history and Jourmal of Zonlugy, Botany, Mineralagy, Geology and Metcorology. Vol. 8, 9. Londou 1836. 8.

Luncifero (Jo). Ginmale spientifieo, artistico, lefteratio. Napoli.

M'Chellunel (vgl.Calcuttis Journali. Margasin de Zoologir (vgl. Guciriu). Mrgazin fïr Insisktenkunde vou K. Illigre. Bd. I-VI. 1801-1808. Branuscluweig. 8.

Magnaian ('The philosophieal) and Anuals of philosophy (vel. Thiomson). Magrazu (neurstes) fǜ Entuınolegir ("gl. Siluncid(or).

Mngazin, zoologiselses (vgl. WieIIIIIIII).

Thengazin fïr die Natarkunde Helveticus vou Hojpfuer:

Nagazin. rlscinistles, zur Erwriterang der Natarkunde vou Bolk-

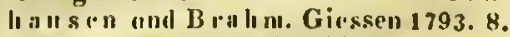
Magnain fïr das Neweste (vgl. Liclutenberg).

IIagnzin für die Liebluaber der Entomologie voll J. C. Fiissly. J., II. Bd, Ziirich and Winterthor 1778-79. 8. M. 5 ill. Kpf - Neues Magazin. 1., 11. Brl. Ziuriel, 1782 und 85. 8. i1. $\mathrm{Kpf}$.

Mugazin of nat, hist. (vgl. Londou). Mnagazine (The ent.)(vfl.Walcker). Magnaine (The) of nat ural history. Conducted by Edward C'liarlesw o rtl. New Series. Vol. I, II. 1838. London 8. (Gelit nuel fort.) 
Mapes (J. B.). American Repr Ttory of Arts, Sciences and Manufac. tures. New-York.

Mnyer (Di. A. F. J. C.). Allillecten fïr vergleichende Anatomir. M. 7 Tit. gr. 4. Bout. Bd. I. 183\%. Bd. 1I. 1835 .

\section{Mreckel (vgl. Archiv).}

Metropolitan Magaxine. Loulou.

Memier (V.). La Revie synthétique; Sciences, Litératıre, beatux Arts, Industrie. Par, 1840. V-VIl. 8.

Minerve, ou choix des Memoires Jes plus importants, qui paraissent dans Jes pays étrangers sur les siences naturelles, publie par E. Jae que mi n. Paris 183t.

Miscellnuy, The Zoological, bring descriptions of new, or interesting animals, by William Elford Leach, M. D. F. I. S. A. W. S. ale illustrated with eolunred figures, Irawa from Nature, by R. P. Nodtler. vol. I, Londou: printed by B. M. Millon, bouvstreet: for E. Nodder et solt. 34, Tavis tock-street, Convent.Garden; and Gold by All. Booksellers $1814.114 \mathrm{bez}$. Seit. u. 60 illum. Knpf. 8.

vol. 2. 1816. 154 S. 60 Kupf. ;

vol. 3. 1817.151 S. $30 \mathrm{Kpf}$. (Zin) ken in Germar's Mag. II, I. 316-325.)

Miscellany, The naturalists, by the late Dr. G. Shaw, complete in Twenty-four Volnmes, containiug 1064 coloured Plates; priee 33 C. 175. 1799 (vel. Shaw).

Miscellany, British (vgl. Sowerby).

Mitheilnmgen ats dem Grbicte der theoretischen Lidkunde. Hevausg. v. .l. Fröbel und O. II e r. 1. Het. gr. 8. Ziirich 1833.

Niszellen (vgl. Fnunus von Gistel, Beibl. z. I3. I, 11, 111).

Mohr (vgl. Archiv u. Beiträge).

Monthly american Journal (vgl. Featherstomingh).

Monthly Review (The) (vgl.

\section{Review).}

IDortillaro (Vineent). Gioruale di scienze e Anti per la Sicilin. Pulermo.

Millter (Dr. Johanne's). A reliv für Anatouie, Pliysiologie u. wisseusch. Medicin. In Verb. mit m. Gel. luerausgegeben. Seit 1834. (AIs Fortsctzung des Archivs von Aleckel, so wie chedem Meckel's Ar. Continuation des vou Reil war.) 8. Mit Kpf.

Giatel'n Lexikon.
Mnlder und Mall (vgl. Bulletin).

Napoli (vgl. Progresso).

Natnrforscher (Der), herausgegeben von J. C. I. Waleh, spiiler vou J.C. D. Selireber. 30 Thle. Halle 1774-1805. 8. M. Kpf.

Neme mordisclne Beitringe von Pet. Sim. Pallas. Peterslung und Leipzig. 1781. 6 Bde. in 8. (Forts. d. alt.)

News. London.

New-Idomdon meehauies Register (1, 11, 111, IV sind uns bekanut).

Nilsson. Jihresberichte über die 7.0ologischen Arbeiten und Entdreckungeu iu der ganzen Welt. Seit Dalmans Tod 1828 in den Denkschr. von Stoekholm (erster 1829).

Notizen, Literarische, von J. Gistel (Dess. Faunus, Beibl. I., Il., 1I. Bd.)

Notizen, Neue, aus dem Gebiete der Natur- und IIcilkunde, gesaumelt u. mitgetheilt von Ludwig Friedrieh v. Froriep. Erfurt bei Cos siun 1838, in Commission bri dem G. H. S. pr. Landes-Industric-Comptoir z.t Weimar 4. (Medizinalrath v. Froriep sel. brgitun damit im J. 1821 und brachte diese rrichhaltige Zeitung bis auf cirea 50 Bde. Sein Sohn setzt sie nIII im Groiste seintes Vaters fort.) (Der Jahrg. 3 ก. $36 \mathrm{kr}$.)

Derstedt. Oversigt. Kopeni. 1841. Uken. Isis. Encyclopådisehe Zeitschuift. Leipzig bei Brockhatus. Vom Jalue 1817 bis jezt. 4.

Bmoeter. Aumali miversali.

Opuseoli seclti. 18 Vol. Milano.

Dpmscoli scientifichi, $37 \mathrm{Vol}$. Milano. Fuse. $[-X V 111,1817$ rte.

PnIIns (vgl. N. nordisclu Beịtı̈ïgr). Politip pes (vgl. Tliomsum).

Politecnico, II, ripertorio mensile di studj applicati alla prosperita c coltura socinle. Redigin von Dr: Cilt aneo. Milano 18.39. 8. (Sind etwa 20 Faseikeln bis dato ersehienen.) EDomencer (Protessor auf der Dini: versitït z.1 Zürich: $\dagger$. Sehwcizcriselse Zritsehrift fiir Natur- und Hrilkunde. Ziiriclı. I Bl. 4 Hefte. 18.34. 8. II, 1 \$35. Prenssisclue Provinzialblintter (vgl. Richter).

Frogrtsso, Je, delle seienze, delle lettere et delle arti. Opera periodicis 
compilata per la cura di G. R. Napoli. Vol. 16-18. Napoli 1837. 8 .

\section{Runaterly (The) Jourual of}

Sripuce. London.

Quesmeville (Dr.). Revuc scimtifique $\mathrm{Mt}$ industriplle. Paris 1840.8. (E) scheint nonatlicls, Juhrg.: 20 Fre.)

IRngglonamentl (vgl. Grialdi),

Raspail et Saigay. Annales des scipnces d'observation. Paris 1828-29. 2 Vol. 8.

Hecorals of general science by R. D. Th o m 80 n and Thom, D. Thomson. London 1835-44.

Itecuell des Min. d. Med. d. Clinurg. pt Plıarm. milit. XI.

Recucil des Savas étrangers.

IReil (vgl. Archiv).

IR. puertario (E1) ameripano. Tom.

1-3. Londies 1826-27.

Mepertorinu fiir Anatoaic ete. (vel. Valentin).

Teports of the Meetings of the britisch Association for the avancement of scienes. Londun.

Reviev (T'he Mouthly). 1840. Vol. 1, 2. Joudou 8.

Itevle: $w$, The british and foreign quaterly. Vol, 21, 22. London 1838. 8.

Review, The Dublin. Vol. 8.

Revoe britanni(ue. Paris.

Revice de deux mondes. I-IV.

Bruxilles 1838. 8.

IRevire entomologique (vgl. Silberma $n$ ).

Hevene scientifique (vgl. Quesneville).

Revae syntheitique ( $v g l$. Meunier).

Iticloter, 0 . W. L., vaterländisches Archiv tiir Wissenschaftell und Agrikultur oder preussisclie Proviuzialbi:iter. Königsbere. 8.

IXIcoglitore italiano a stranicro ossia rivista mensuale curopira di scienze, Iettere, belle arti, bibliografia e varieta. Vol. 1, 2. Milano 1837. 8 .

Itivistn eurapen. Nuova seric del Ricoglitore italiano e straniero, ossia rivista mensuale curopea di scienze, lettere, brlle arti, billliogrifia e varictà. Vol. 1. Milano $1830-44$.

Thodolulil. Schwediselse Annalen der Naturkuude.

Snigny (vyl. Raspail).

Bally (vgl. Alpina).
Snvl (P. et G.) (vgl. Giorn. tose.). Aclumialt (vgl. Bienenzerturg).

seloueider. Neuestes Magazin fiir die Lieblaber der Eutomologic. Heft I-V, 1791-95. Stralsund. 8.

Selireluer (vgt. Naturforsiber).

seluweizerlsclice Zeitsclurift (vgl. Pommer).

Scribn. Jourmal für die I,icbhaber der Insektenkunde. Heft 1-1II. 1790 $-91.8$.

Senduugea der kurländisclieı Gesellscluaft lïr Literatur und Kunst. Besorgt vou lichtenstein, 'I'rautvefter und Paucker. M. K. (Reylicr). 1840. 4. Nr. I-XX. 160. (Lepidoptera Kur- und Livlands p. 116. Vgl. Lievig).

Slunw (vgl. Misecllany).

Silberuaun (Gustave). Revue ("ntomolonique. Seit 1833-37. Paris 1. Sirasb.; I-IV. Hat leider aufgehört.

villimnn, The ancriean fournal of Science. New.York and N.-Haven 1816-29. 15. Vol. 8.

Sowerky's (J.) British Miscellany. No. 2-12. Londun 1804-6. 8.

Steimmiller (vgl. Alpima).

swalusou, W. (vgl. Znol. Jouru. und Zoological Illustrations).

Taylor (vgl. Magaziuc).

TInusen (R. D.) (vgl. Records). Thouson (The) (vgl. Records).

Thonsou, Th. (Profissor in Glasgow). - Aunals of Plilosophy or Magazine of Chemistry ete. Vol. I-XVI, 1813-1820. Lomdin (Baldwiin) 8. New Srries by R. Phillips and J. G. Children. Vol, XVII (Vol. I) $1821-\mathrm{XXVIII}$ (XII) 1826. (Viel iiber Thiere.)

Tinn (Dr. Th.) Entomolngisches Arehiv. 2 Bdc. in 8 Heften. M. Kpf. 4. Jena 18:27-28.

Tidskrift (vgl. Krüyer).

Tiedemnmu (vgl. Zritsehrift).

Tijdselorift (vel. Hoeven).

Tilesius (W. G.). Jahrbueh der Naturgeschichte zur Anzeige und Prüfung neure lintdeckungen und Beobaclitungem. T. Jahrge. (Melır ist nicht crselicu.) N. $10 \mathrm{Kpft}$. Leijuzig 1802.8.

Trantvetter (vgl. Sendungen).

Trevirhuns (G. R.) (vgl. Zeitschrift.

Treviranus (L. Clur) (vgl. Zcitschrift). 
Tibluger deikterer fiir Nafur. unil Arzucikumle van Antentietl mul Bohue uberger. 6 Stïcke iı 2 Bdı. 'Tübingen 1816. 8.

United service Jam rum I. Ioud.

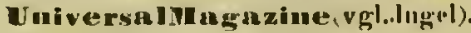

Valewtin (G.): Reprertarium fiir Anatomie und Plivsiolugie. Kritisulue Dirstellung fremder und Ergebnixse eigener Forscliung. 9 Bände. Jalı̈. 1834 in 2 Abth. gr. 8. Bern (Huber). Existint seit 1836 (1. Bd.?.

Verlinanderingen over de nutuurlijke geschiedenis der Nederlandselıe overzeeselıe luesittingen, door de leden der Natumrkunde commissie in Oostludie en andere Silurijurs. Iste cu 2de Attevering. Zoclogie, No. 1. Botanie, No. 1. gr. Fol. (17 B.. n. 20 lith. z. Th. illun. $\frac{1}{2} \mathrm{gr}$. Folio-Taf(In). Leiden 1840.

Vizors (vgl, Zool. Journ.).

Voigt (Julıan Heimrich) (vgl. Lielıtemberg).

Vrlese, W. H. de (vgl. Moeveii).

Vrollek (vgl. Hall).

Wricle (vgl. Naturforselıer).

Walcker, The entomologieal Magazine. Isondon. I-III. 1832. 8.

Veloer (vgl. Arehiv und Beitrige). Veitem veber (vgl. Beiträge).

vricedemann (vel. Archiv).

JVicginamin (vgl. Evichson).

woclnemblatt der $k$. k. Landwirthschaftsgesellschaft von Tyrol und Vorarlbergr. Jahror. 1843, 1844 (jeder 52 Nrn.). gr. 4. Jüsprick (Wagner).

TorreI1 (vgl. Zool. Journ.).

Zeltachrirt (W isseusiluaftliehe) der Busler Hoslischule. Herausgegeben von Lulirern der B. Hocliselı. 1, 2, 3, 4, 5. Basel 1824.

Zestsclurift, allgemeine istreichische fïr den Landwirth, Forstınann und fïrtuer. In Verbindung mit mellren Gelelirlen und Ermumlen der Lani. wiothschaft, herausgegeben von Carl li. H a m m e rsclumidt. Wien gl. 4. $X-X l l$. Bd, bis 1840 ).

Zueltselnrift fiir Anatomic und Pliysiologie, heransg. vou T'i ede ma 11 , G. R. nnd C. L. Treviraus. 4. Seit 1824-29. Darmstadt. 8. 5 Bıle. Zeitaclirirt fïr die organistie Pliysik von C. F. He usinger. Von 18:7-29. 111 Bde. 8. Eisenach. (Selır gelıalt vull).

Zeitsclnrirt fiir Natur - und Ileilknude. Heransregeben von den l'rofessoren der ehiourgiseli-medizinischen Akademie in Diesten D. D. Ca rus, Fieinus, Frake, Kreysig, $O$ l l , Ra a lıig, Reielıenbach, Seiler. 1. - V. Bd. Dresden 1823-27. 8. M. Reg. - Neue Zeitsehrift f. N. 1. . vou A mu ou, Choulaut, Ficiums, Il uase, Kreyssig, I, öwe, Peeli, Prilz, Reie he $n$ bach und Seiler. Dresd. 1829. M. K 1 if. 8 .

Zeitsclerift fiir die Entomologie (vol. Germar).

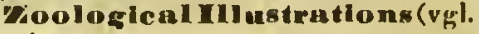
Swailusoll).

yoological (The) Journal. By Vigrors, Yalrel, $S$ wainsou ete. Londaii 1824-44. 8. W. fin.

Zoolorienl Tilnstriotions, or original Figures and Descriptions of new rare, or uthers wise inferesting Animals, selevted prineipally from the Classes of Ornitholugy, Entomnlogy und Conelyyliology. By William S w n ins on. London, printed for Baldwin, Cradfock and Joy, Paternoster-kow. 1820. 16 umbez. S. 6 grem. Kupft. 8.

Nr. 2. 1820.12 unb. S. 6 Kpf. 8.

Nr. 3. $1820.12 \mathrm{mmb}$. S.

Nr. 4. Jail. 1, 1821. Lond. 12 lnb. S., 6 anvgent. 'Taf.

New llluslr. Ibid. $1832-33$.

\section{Schlussbemerkung.}

Die Einsehaltungen und Ergänzungen z.ı vorliegender Uebersicht der Ephemeriden sind in Lexikan der Theriologen maphulesen. 


\section{Bibliographien und Literaturgeschichten *.}

Achard. Cours de bibliographie. 3 vol. Markeille 1807.

Ammal, Britisl, and Epitome of the progress of seimee for $1838-44$. Ellited by Rol,. D. Thomson, M. D. 12. London (Leip\%ig, Weigel).

Anzelgen, Göttingiselıe gelelute. (Redigirt vom Hofr. Heeren.) Göttingen (Vunleuhoeck), 8. Der Jahrgang, oder 208 Stiek, kostet $13 \mathrm{fl} .12 \mathrm{kl}$. Erseheint seit 1786. - Hartmann bearbeitete $2.11786-182.2$ pin Register.

Anzeiger, Literurisclier, nebst Intelligeurblatt fïr die Natur - und Heikunde des In- Ind Auslaudes vou W. Januar. 18.12. 8. 16. (Monallieh 2-3 Bogen. Preis 3 fl.)

Anzeiger für Lileratur der Bihliothekswissenschaft. Jahre. 184.k mit Antoren - und Bibliotheken-Registern. (Vou D. J. Petzliold). \&. Diesclen.

Antiqunas: und deren Cafaloge. - Unter die vorzinglichsten gehöien in Dentscliland, Italien, Frankreiel, England, Holland ete. fulerende: Alt. heer in Utrecht, Asluer in Berlin (sehr zu emplelılen; gibt jälurlich cinen selırstarken Catalog hernus); Br ö nu er zu Frankfurt an Main (hat rin grosses Lager und betreibt das Geschifift grossfrtig und mit aller Einsieht, 1-2 Catuloge in Jahr); $B_{\|}(s c h$ in Angslurre (grosses Lager; curpf(hluugswïrdig zu jedeul Auftrag. - Verzcichniss von gebundenen Biiehern. Alle Jahre erseheint einer von cirea $8-10$ Bogen); Lackingtun in London (a list of Works by I. 8. (et 4.);
L ämmlin in Sehafflausen (sehr gewisseulaft and prompt, wie auch Benteli, Antiquar z.u Beru. - Dic Preise scheinen gegen Dortselıland etwas elevirt. Vereichniss von älteren und neneren geb. Büclsern, Landcliarten cte. vou IIcinr. Laimmlin. 8. (alljähr. lich); Lougman in London (a Catalogue of books. Isond. In jedem Jahr 8. mind 4. Ein bherans reiches Inud volisıändiges Lager ist bei diesem verstïndigen Manne \%u finden); IA nch tmans in Leyden (Biicherratulog des auticju. Verlags v. L. in L. - Selir billige Preise hat man von diesem rechtichen und muterichteten Bucllhändler zu rrvarlen. - Catalogne des Livres. 8. Oefter im Jahr. - Ungeheures Lnger); Mensel in Koburg (verkendet ancli C'ataloge und soll billige Preisestellen); Molin in Florenz (Cifalogo della Letteratura ete. di Aniiquarin M. in Firenze. - In Italien ist dey Buchlinudel, unansgebildet wie er ist, ein antiquarischer nud modernel zugleicls); Nestler in Hamburg (Catalog der Buch. und Autiquariutslandlung. 8. Prompte Bediemung und sehr billige Preise); Renouard in Paris (vgl. Renonard); de Romanis in Rom; Sanrla in Madrid: Steyrer (Clem.) jun. in Miinchen (Anr.eige vou Büchern. Residenzstrasse No. 10, gegenüber der Post. Wïrhentlich in 4. In Müueheu der eingige eigentI iche Antigunar, ausurestattet nit reielier Bücherkemutuiss und grossem Spraclischatz. JIerrliches ausgesuebtes

- Die literatur, nls ein Jlebel der Ge chichte, findet sich xo zlemlie h vo 11 a tandlg

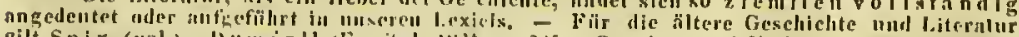

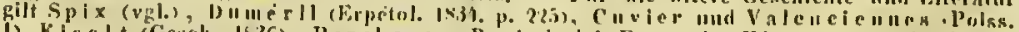

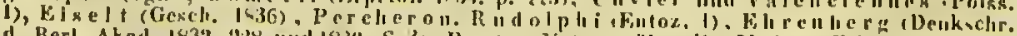

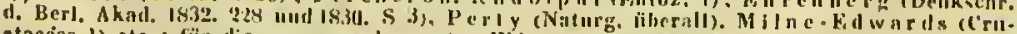

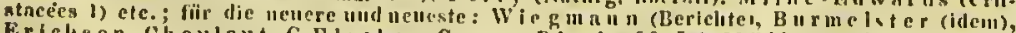

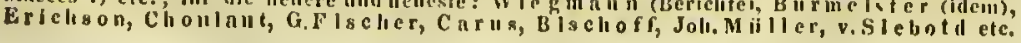


Lager und die billigsten Preise. Ausxerdem sind noel drei weniger bodpeteusle Antiquare iu Müuchell)? W p i gel iII Leipzig (vgl. W.); Wild in Uircrlit.

Aslrer, A., Biblographieal essay on the collection of voyiges and travels; edited aud published by Levinus HuI sius. Loudon 1839. 4.

Avema rius, L. Allgenncine Bibliogrephic tï̈r Deutsclılaud. Leipziz. 8. Srit 1830. Erscheint bogellwcise, wör.lıestliclı.

Farbicr. Dictionnaire des onvrages anonymes, 4 Vol. Paris 1800-9. 8. (Enthiilt ausscliliesslich nur frnnzösische und lateinisclse Bücher).

Beckmann. De histo:ia naturali veterun lihollus pr. Goetting. 1798. 8.

Benthowshi. Polnische Literatur. 1814.

Beselce, J. M. G. Versuch einer Geschichte der Naturgeschichte. 1. 'l'hl., aligem. Geschichte der Naturgeschichte in elem Zeitraum von Eiscllatfung der Welt b s auf das Jalu nach Clur. Geb. 1798. Mitau, Vf. 1802. 8.

Bibliograplice de la Franee.

mibliotheca physico - medica. Verzeichuiss wichtigre iilterer ete. als seit 1821 in D. getle. Buicher ete. Lexikon-8. Leiprig (Voss) 1833 (1 fl. $12 \mathrm{kr} . \mathrm{rh}$.$) . 1835.8. 189$ (16 gr.).

Bibliothecar medico - chírurgica et pharmacentico-chemica (belgica) ab 1790 id anu. 1810. Cur. L. S. A. Holtrop. Hagae. 18.2. 8.

ibliotices oeconomica, oder Verz. bis 18.10 , von linslin; fortgesezt vou Eugclman॥. 2. Anfl. 8. Leipz. (Engelur.) 2 fl. $24 \mathrm{kr}$.

Bibllotlueca Veterinaria, oder Verzcichuiss uller brauchbaren efe, bis zur Mitte 1824 in Deutsehland erschicjicuen Bücher, von 'Th. C. Fr. Ensliu. M. Reg, 8. Berl. 1827.

Biblintheence britannique. Genève $1796-1815$. 8 .

Bibliotlucque universelle seicuces, belles lettres et arts. Seit 1816-41. Genève. 8.

RIbtter fïr literaisehe Unterhalfung. Jahrg. 18--18.13. Iferausgeber Heinr. Brochhsus. 365 Numm, M. Beil. gr. 4. Leipr. Brocklians.

Elatter, literariselo und kıitisclie, der Bärseulsalle. Redigrit von F. Nicbour und Di: Sudwig. Iernusge. uchen von Gerliard von Hostrup und Solil. 21. Jalırg. 1844. 156 Nin. gir: 3. Hamburg (Peitlies).

BInfT. Dic Leistungen der Medicin in Deutsilul. 1, 1832.

Blnume. Itpr italirum.

Billuer (G. R.). Bibliotheea st.liptorum listoriae naturalis. VIt Tom. Lips. 1785-99. - Systematisel literarisches Haulbuch der Naturgesclichte, Orkononie und der verwandten Wissenschaften. 2 Bde. Leipzig $1785-90.8$. 8 .

Brunet. Manucl du Libraire. III Vol. (1)ie nenere Auflage ist noek nicht vollendet.)

Brydges. Britisl, bibliograpler. Loudon 1818. - Censurn lituritria. Lond. 1805. (Nicht zu empfehlen. Auch Clarkc's Werk hat wescntliche Mïngel.)

Incluhind ler-Zueitung, Süddeutsche. 1. Jahrg. 1838. $52 \mathrm{Nrn}$. gr. 4. Stuttgallt (Hoffmann). Soll noch erscheinell.

Euclion, J. A. B. Courses eu Suisse et dans le Pays de Baden, avee des notices sur pllusicurs manuserits des Bibliolhé(ues publiques on partieulière's ete. Paris (Gide). 1843. 8. (8 fr.).

Bulletin du Bibliophile. 1837-38. Paris 1838. 8. etc.

Tiscelt. A linanacl der Fortseliritte in Künsten, Wissenselaften cte. I-X. Bil. sammt Register, $179 j-1810.8$. Erfurt.

Calcutta Glainings of science. Catalognc genèral de la litirnture française. Anne $1837,1838$. Paris 8. Wird fortgesezt.

Catalogue of the library of the London Instifution. London 1813. 8.

Cataloges librorum impressormm bibliothecte bodleionac in Acallemia oxoniensi. IV Vol. Fol. Oxonii 1813.

Clierbullex (Joël). Revale critique des Livres nouveaux. L̀rscheint in Monntlueften. Paris et Genève. (Cherbuliez.) 8.

Chondant (Lud.). Bibliotheea me. dico-historica, sive Catalogus librormm listoricorum de re medica et scicutia maturali systcmatieus. 7 maj. (17 $\frac{1}{2}$ B.). I.ips. 1842 (vgl. Rosenlia um). Geschichte der Anatomic (Picrer's medi\%. Würlerb. III). 
Clarke (Adau). Bibliographical Dictionary. Loud, $\mathbf{t} 820$.

Cohres (J. P.). Deliciac coluresianae. Büchersinumlum zur Nafurgeselichte. 2 Buld. Allgsb. 1782. 8. (Sehr miitzlich und fleissig ausgrearhei(et).

Corhyn, Fred. The inlian review of Works of science and Journal of foreign sience and the alts, embracing mineralogy, geology, uifural history, Physic. efe. Silem (?).

Dieterlch. Verzeichuiss der Disserfationen alter und neuer Werke von Dieferich in Götliugen. 8. (Sehr genau abrefasst und von bibliogra. phischem Werth).

Dryauder (L.). Catalogus Bibliothecae historico-naturalis Jos.Banks. Y Vol. Loudou 1797-1800. 8.

Wbert. Allgenneines bibliographiselies Lexikon. 2 Bde. Leipz. 1821 30. 4.

Welao de la Litténature française. Prumière Aunée 1841. Leipzig; Brorkhaus ef Aveuarius. (Wochentl. I Liefg.

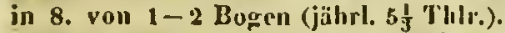

Elchlnorn. Literaturgesehichte. 1824. 8.

Elselt (Dr. Joh. Nep.). Geschichte, Systematik und Literatur der Insektenkunde ete. Lejpzig 1836. 8. (Das beste Werk bis jetz.t.)

Erselı (T. S.). Literatur der Mathematik, Natır- und Gewerbskunde u. ${ }^{2}$ w. Seit der Mitte des 18. Jahr. hunderts. Neue Ausgales. Leipzig 1828. gr. 8. - Allgemeines Repertoriun der Literatur. 1793-1807. Handbuch der deutselsen Literatır.

Pleblg (J.) mul Nune (B.'S.). Bibliothek der gesamuten Naturge8chichte. 2 Bde. Frankf. 1789-91, 8.

Flsclier (F.). Bibliographia paIaeontologica animalium systematica. ed. alt. Mosquae 1834. 8.

Flecher (Gotth.). Zoognosia. Mosquac 1813. III-tum Vol. (Unsere Ausgahe ist in 8. mod enthiilt tlie s̈ltere Literatur vollstindig. Bekmntlich ist dic Auflage l. W. verbrannt.)

Fax, G. Turvishenal, Syuopsis of the Newiastle Museum, Inte the Allan formely the Tunstall or Wyelifte $M_{n}$ scmm. Newciastle 1827. 8. XXII et 312. Plates.
Frorlep's Notizen aus dem Gohiet der Natur- und Heilkunde (vol. Ephemeridin, p. 95).

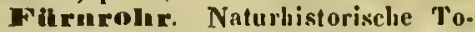
pogrituhe vou Regensburg (vgl. das Lexikou). Wir verweisen hier auf alle Topographien von Grgenden mul Monographicul von Stälten, ivorin der abgcsthiedeuen und kuiftig wirkenden Menselsen Gedärlitniss wird.

Germar und DHem. Uebersicht der entomulogischen Literatur von 1790-1818 (Isis 1818).

Fersdorf's Reperturium der Literistul.

Gistel. Literarische Notizen im Fumus mul in den meisten bayrisclien lournalen. - Wir bereiten seit dem 17. Jilhe (1826), wo wir die Uhiversitat bezogen, ein Handbuch der Geschichte ler resammten zomlogischen Literafur vor, mnd ersucheo alle Naturforscher, welche es mit der Wissenschaft halten, um Zuseudumg ilıer kleinern Werke und Dissertationen gegen Tausel von Naturalien und andern Bïelsern; wir ersuehen die Literaten un die Titel und genauere Beschreibungen von alten seltenen naturhistorischen Druckwerke॥, Manuseripten, Gemälulen; wir leren ihnen alles ans Herz, was diesen Unteruehuen, anch wenn wir es nicht zu Stande bringen, nützlieh werden kann und vergelien rejchlieh, was uns geboten wirul. - Reise des Grafen Rudulph von Jenison. Walworth durch Dentschland und Italien. Beschrieben ulurch dessen Begleiter und Aral Joh. Gistel. Miiıelı. 1835. 8. I, II. M. Kирf.

Godin. Table alphabetique des matieres contenus dans l'histoire et les mémoires le l'Academie roy. des sıiences. Paris 1734-84. Tone IIX. 4.

doxttlnger gelelirte Anzeigen. Vgl. Anzeigen.

Tùmoving. Bibliotheea regni aninalis nfque lapidei. 1760. 4.

fillnther (J. D. med.). Allgemeines Literatur-Repertorium oder ein mountlicher Nachweiser der in sümmntlichen wissensehaftlichen Jourualen undZeituneren Deutschlauds enthaltemen Ablandlungen, nach den rinzeluen Muterien geurdnet, in Verhindung mit meluereu Gelehrten. Jena (Mauke); 
seit 1843. 8. (Monatlich cine Liefg. Jalug. 36-40 Bgen. 2 Thir. Aucl jede Abtheilung besonders. Jilirlich 12 Gros(hen.)

Itibnel (Dr. G.), Citalogi libro. rum manuseriptorum, qui in hibliotle(is Galliae efe. asservantur. 4. Lips. (Thener).

Hain (L.). Repertorimm hibliographicum, in quo libıi ommes ab inte typographica inventa usfure ad anum MD 1y. expr. recensentul. Stullg. 8. 1-111 et seq. 1826 et seq.

Inaler (Albr. von). Biblintheca anatomira. II Vol. Tipuri 1774. 4.

Hallische allgemeine Literaturzeitıng.

Elarris's (W.). Catalogue of the Library of the Royal lustitution. Loudon 1809. 4.

Heinsins (Wilh.). Allgencines Büclicrlexikon. Herausg. vou $O . \wedge$. Schullze. 4. Leip\%. An cin Dut\%end Bïnde.

I Tensinger. Ginndriss der Encyrlopidip und Mettodologie der Naturund Heilkunde. Eisenach 1839. 8. (Ausgerichuet.)

CHurielos (J. C.). Verzeichniss der Biicher, Landcharten etc. Begonnen 1805. Ersclicinen noch alljilurlieh in 2 Licfg. (Jamuar - Juni, Juli - Dezember). Leipz. 8. (it $54 \mathrm{kr}$.).

11 orme (Tlı. Hartwell). Introduction to the study of bibliography. 2 Vol. Lond. 1814. 8.

Index to the Library of the Lyceum of natural history of NewYork. 1830. Printed for the sociely by J. Seymour. II et 72 pagg. 8 niaj.

Jïck (H. J.). Ziveites Panthicon dev Lituraten und Kïnstler Bambergs. Voni 11. Ialirl. bis 1843, IV und 128 S. Bamberg. 8.

Jaln rbilicher, Mediziniselie, des k. k. östr. Stuats, von Dr. J. N. Edl. v. Raimann, S. C. Fiseler, A. Edler v. Ross und J. Wisgrill. M. Taf. gr. 8. Gegen 50 Bäıde bereits. Wien. (Grerald).

Jnhrbikcher fïr wissensehaftliche Kritik. Herausg. vou der Soc. f. w. Kritik in Berlin. Jeder Jahrg. 12 Hefte oder 2 Bde. 4. Berlin (Dunker)
(Kostet blos 21 A. 36 kr.? Gar nicht (liner!):

Jalirbilelier, Heidelherger, der liferatur, unter Mituv. d. 4 Fic. von F. C. Sehlosser, Munke und Clir. B a cr (seit 1807). Neue Folge. à Jalirg. 12 Hefte. 8. Heidell.

Juhrhileler, Wieuer, der Literafur. Herausg. von J. L. De iula ardstriu. Srlhon über 100 Båndc. 8. W'im (bei Gerold).

Jahrhilicher, Hilliselie, fiir die Wissenschaft und Kunst. Red. Dr. A. $R$ "ge und Dr. Tlı. Eelıtermeyer in Halle. Seit 1838. 4. Lepipig.

Jussen. Briefe ïher Italien, vorz. den Zustand der Arzneikunde und Naturgeschichte hetr. Diisselderf 1792. 2 Balc. 8.

Julims (G.). Bibliotheca romana. Opus uno volumine L. Lips. 1843. (Blockliaus).

Mnyser (Ch. G.). Vollstïndiges Bülierlexikon. VI Vol. I Supplensent zu Kayser's vollstindigem Lexikon, enthalend die Erselieinumeren der Jahre 1833-1840 incl., so wic dic Berichtigungen der 6 Bïude. I. Abtlı. Bogeu 1-69. A-K. Lcipzig 1841. 4. Das Sachregister, 2. Hälfte, ersclnen 1838 z.u Leipzig.

Kirloy and Spence. Introdnction to Entomology. Loudon. IV Vol. 8.

I frakam. Rozmaitonci Naukowe. Wissenschaftliche Mamnirhlatigkeitru. Krakowie No. 1. 1828. II. 1829. III. 1831. 4. - Miscellanea Cracovicusia. Fase. 1, 11. Crucov. 1814-15. Miscellanea novi. Fase. 1. Cracov. 1829. 4.

Ineng. Allgencines Handwörterbuch der philosophiselien Wissenschaften. Bd. Ill und IV. Art. Naturwissenschaft.

Gichtentlıal. Manuale biblio. graphico.

linck ( $\mathrm{T}$. W.). Versuch einer Greschichte und Physiolngie der Thiere. 2 Thli. Nenc Ansg. Chemuitz 1815, 8. Litern ry fingzette. London.

Literntur-Zeitang, Berliner.

Literatur-2ueitung, Allgemcinc, herausg. vau Burmeister, Duncker, L. H. Fricdlïnder, J. G. Gruber, Laspe yres, M. H. E. Meier, Niemeyer, Pott, Rödiger. \& Jahrg. 
12 Hefte mit Erg.- und Intelligenzbl. 4. Halle. (Ausgezeichnet.)

Literntur-Zueitnng, Jenaische allgemeine. 4l Jahrgg. 4. à 12 Hefte. Jena.

Litceratur-Zeitung, Iscipzincr. Red. von Bl ïm ner, Krum. He inrotlı, Rosenuinll cr, Pülitz und Lrándes. 4. Heipz. (Breitkopf).

Literntme-mintt von W. Men. zel. Beilage zum Morgenblatt. Stustgart (Cotta).

London and vestuminster neview.

Lomion (The) Catalogue of Buoks with their sizes, prices: since 181 a - 1839 . 8. London by Rubert Beut.

Lowndes, british librorian. Nr. 5-6. London 1839. (Mehr lıaben wir nicht.)

Ludivig (Chr. Fr.). Historine anotomiae ct pliysiologiile eompinantis brevis exposilio. Lipsiac 1787. 4.

Manuedisshrift for Literntur. Kopenl. $1833-44.8$.

Magazin, Holländiselıes, der Noturkunde (vgl. S ch midt).

Mnnget (avec Le Clere). Bibliotheer anatomica.

Marie (J. de). Algemeine Bibliogrophie für Deutschlond. Lcipz. 18.13. 8. Erscheint monatlich.

Marx (Dr. K. F. H). Götlingen in medicinisther, pluysiseher nud histovischer Hinsicht. gr. 8. Göttingen 1827.

Mickiewiez (A.). Vorlesungeu über slavisrhe Litcratur and Znstände. Gehalten in Collegedo France. Deutsch. 2 Thle. Gr. 12. Leipzig (Bruckhaus). 1843.

Mittheilnugen aus $\mathrm{dcm}$ Reise. tagehuch cines deutschen Naturforsehers. Englond. Basel. 1842. 8. 476. (Isis. 1843. 545.)

Moreni. Bibliografia ragionota dello Toseana. Firenze. 1805.

Moretti (G.). Bibliotheca Agraria. T. XII. Pavia 1829. (Mebr hiabeu wir dovou nicht geschen.)

Mensentalog von Leiprig.

Nau. Bihliothek der gesommten Naturgesehichte (vgl. Fiebig).

Sopitsch (Dr. C. F.). Chronologia in literatura medicinac, sive Re. pertorinm de medicinat, chirnrg. ete. hist. et literaturia. II Vol. $8 \mathrm{moj}$. Norimbergate (Stciu).

Dhen (vgl. Germar.

D'Ex eilly', B., Catalugne of the sub. jerts of uatural history iu the Museum of the Dublin society. Dublin 1s03. 8.

Peignots (Gabr.). Dietionnaire maisonné de bibliologrie. 3 Vol. Paris $1802-4$.

Percineron (A. R.). Bibliographie entomologique, rumprenant l'indicntion par ordie alphohéthique de noms d'outeurs 1) des ouvrages ete., 2) des monographies et memoiles eontenus dans le Rerurils, Jourunux etc. 2 Vol. Palis (Jaillicre) et London. 1837. 8. (14 Fr(s.).

Drnck und Ansstattmig fast verselswende: rinch. - I. Vol. XII l'uges Vorrede, 326 pig. Text Yon Aalborg-\{lioy). Vol. li. 376 p:Ig. - Die ersten 140 enthilten noch die Suite von Ruoy, resp. Rabeu - Zxchusius: png. $14-216$ euthalien die Anourumes von A - 2. - Auf Png. 217-219 Eletien die Die. tinnuars; nuf prig. 219-239 dic onvrages perindiques; anf pag. $22 y-243$ die publicalions de socicles savautes; vou $243-373$ rcicht die Table des Articles par ordic de Maliere el de Chrnnologie (d. l. Skelet der Eimtheilung dor Büclier und der Auloren in Momographi, Mlcrographi, Fuunisten, in specielle, in Ama. tomen elc.); also das Drittel des Bandes davon cingcuommen. - Auf pag. 37.3-77 cou fiu) stelien die kratu, deren Anzalıl ma (gewiss!) seclisma! lulter steigt.

Mun vergleiche muen unsere Angalnen; am besten die jelurift sclust.

Es ist fuviss ein selir verdienstlelieg Unteruehucn, alier so lange die alten Saeluen in den Werken und \%eitscluritten niclut geden. let sind, iu Detnil noel, sn rieuliel, unaus. fíticlı, und, wenu man anders die ewig wicder. kelırenden Frazeseiclıen lielen kanu, die jeder Wissenschaft Sichaudfecken siud, immer nocli wielıt gen ïg e

Die Ablamallume p. 5 (obtou) ist vou $A$ h reus (f lu Augsumg. - B e ck (p. 20) ge.

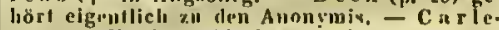
I o I (p, Gs) lst fehlerliatt ind doppeli vor handen; auf pag. 64 aber correci gesclirlebu. - Covily ms (73) sclueint uns mit K ort 11 1140) iclenlixch, - Dnuske (79) is keiu Personaluane, helsst nur d $h$ u 1 sc li. Deleanur. - De br aw (\$0). Der Name De r ba w (s-2) Ist fehlerlıaft, identisch mit ersterm แ॥d แm॥ gelösclit uerden. - Escliseholtz (10L). Hier felitt es (wle bel vielen anderi) an der gelıörigen Werkanzahl. - Niclı Nro. 7 ist zu sebzen: lieschreilumg des inmern Skelets elui. ger lusekteu i(jyllotulpu). Darjut 1820. -

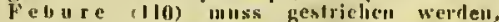
l'ag. 241 stolit der Name richtig gesrlorlebu. - Gistel (136). Ili.r siud un a Arlikel angegeben, es fululen die Augabeu vou $2 x-3 \|$ Werken und klpineru Ablandlumger. Vgl, das lexikun. - Jag. 148 fir Nosograplite lics Mo. nographle. - I63 lies IInr - stut) IR. (RIIbriulues - 169 ist II eer piuzuschntien, der unter 0 s wald p. 304 stelit. - 183 ll u ber 
(Fr.) conf. II + 68. - 195 cinzusclialten: J a. rocki. - 20I schelnt Fremies deul IIrn. $K$ eferstein zunelacilt. Wir laaisen IIclit (belegenhelt zu verglelcken. - 21 (z\| K I ug) selze: 14. Kinferdonbletlen des Berl. Mns. 216 zu A setze naclt de: cherille. -238 ist le enin te de l, evean falsch. Fir lieinst: I, aveall (16. Conte del und bils Citict heisse: Bullet. de Moscon ed. leq. 169). Wn Ist di:n Cltat von Nr. 3, p. 31.1? - 272 muss M Mr. I g I i lceissea. - 275 M e gerle ntat Meyerle. kin alfer lock voll abijelus, - $277 \mathrm{Me}$ "nII ville komat 2 Mal vor. ef, II, 192 Viller T li f éry slelit der Name recht, - $28 \%$ Mos c h a 1 ouss gestrichen werden. Kommt muter Muscliel trochuial richıig vor. -291 Muxal statt Muratih. - 295 Naezen Ist riclitig. - 300 overbech umins gestrieheu werdell. Cf. 30t, wo er richitlg steht. Der dicranf fulgende Nucue lat verhunz und muss

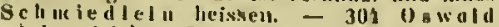
whd eestricliell. S, oben (1Ieer) - $30 \mathrm{~d}$ l'all. digelius: ller alphiahetivcluen Consequent genilss ulelit hieher, souteru zut p. 307, vor Pancer, - 316 l'ictet stott l'iclet. - 317 unss PInteau gallz gestrichen werdel, deun er hommt po 307 rlchilger vor.

11. - p. 5. Das Citat cines nnderii (?) Rayger kontmi hier zom eweiten Male vor. - De locustin volantibus ef. p.9, - II strelche RIfferschyl. Lis ist dle Postponttio al Hegetschweller - 12 schalie lin etn، $-24 \mathrm{Sad}$ off streiche. Kommt riclitig unter Sodoffkky wicder (7I) vor. - 26 SaIIs muss bleibell, aber M a s chlius lu I. Bd. gestrichen werdel - 39 statt Se hiffer setxe Sclilflerm îlled - nd statt Schmiet I ein setze Seholedlelu. - 37 statt $S c$ hwa $t z$ setec Schwir $12 .-64$ bei Serville; Nr, 8,11 mind 12 sind el 11 arbeit. Fo ist elic grosser Feliler des Verfasmers dep Biluliographic die Alhoudlungen so unuiizlich in vicle Numueru zu splittern, in wirl dle biteratur IIII so melir ell d los. - 84, Nr. a Ist uiclit von St uriu. - 59 stalt $S_{x}$, itz setze S Iovit $x_{0}$ - 108 anpassend hielier gesetat Valel. Jat berelis anl D, 119 gehórig piagerellt. - I?I statt WaIt: W IIII. Hebei zolan: 2, viber Sinmeln exot, Kitler (Faunus); 3. Dipteren us Müıchen (Frimunk) 4. Reise durch Spanicu etc. (vgl. die Nacliträge f. d. Lexikou IInd den A rtikel W alil t.38 bei Zim me runn 1 wach Nr. 3 zu setzen daus le Fanmus de Gistel 1, 1837. 4) viber Masoreus (Fnumn 1837, 1), - 143, Nr. 12 ist achon da, nello leh nielıt irre - 143, Nr. 28 lot unter Keysuler zn stellen - is?, Nr. 26 in bereils allf $\mathrm{p}$. 20 . Nr. 27. - 153; ebellso Nr. 33 (cf. Germ. ) 195). - 169. Nr. 14 da. Geliort lil den nlulin. betiscleen Nameatext als Vertisser II off.

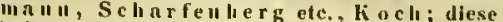
laalien die cutomolag. Ilefic (Frankf, 1803, lier. allsgegeben und uieht $S t$ u ru, - Nr. I5 alu. falls school vorlanden bei Mo ell e r mud mit Recht. - $169, \mathrm{Nr} 3$ schon da Cf. L.J II II g li. - 187, Nr, 14 gehort muter dic Rubrlk Dictlonnaires. - i9s. Nr. 20 schon da. Vergl. Geral. und ll. 153, Nr. 33. - 201, Nr. 19 vol1 Pallis? - 211, Nis. 85 scholl dil, welln wir reclit uns besimen.

Unter dell nuvrngea peiriodiques knmut Vicles vor, was schon bei dell Autores steht.

Vol. II. p. 22! statt Huzlagir lese If eusiu ge r. - 23it. Dic A blındlungen und Denkschriften der k. bayrischen Akademic sind disselbe unter 2 Titeli. Futomologisclies wiissten wir (ausser Splx: Scutelltgera) nichts darin zn suclient und so aticl in manchen anderu l'ut.' eationen.

Ad. 243. Die 8 Bogen der Talile des arIlcles siml, als lumiorze, weittiutige und zelt. ranbeade Zugaben - vergendet, dis Buch dadurch vertheciert ind doch \%ulu Waliren nicht vollkonituen weholfell. Wäre alles nach der voranschenden Division de la Tuble glelch systimatisch eiuregistrirt wordin, das Gase wïrde branchliarer gewarden seyn. soost istes nlelit uelir unil wenlger als cill Bueheriatalog, in Eile lobll Ilast corrigirt, ohne Feite, Correctur, ohile Kritik, mul dirum voller orthngraphl scher Zeit- iud snchfehler, kostspielig, voll stindig stheinend und ulehts werimer als voll. stindir. Deutschland Ist lleu Verfasser eine terra incognitı, ule die Tlulere, tiber welche die altcu Aunten geschrichell. Sull Lich werden In der Bibliographie miinsed die Vorhănge van \& o o In gen aufgezogen werdeu, die Deutung iler aliculb el te vor s l cligegatinen seyn.

Za dein siebeutumdert und vierzelunseitigen Ungchiner haben sich Artikel gescliligen, die dem Reiche der l'tantasie (l'ocsle), micht ober der ernstell Wissenschaft allgehören: Arrikel, die In die Quacksallerbulen der Walleuvergifter nud Stubenkuechte gelüren, und auserdem noch Artihel, dle so weng in dle kintomologie passen, als ein Spanferkel zum Getlïgel. - I, at reille gub den Fingerzeig zur bentung der alten Sclirltisteller iil sciuer Alhund hug vou der Baprestis der Alten -; wir wollen ancli das Unsre tluun und dano erct neit unsier Bibliographie hervorgehen. Sie soll anders werden - oder nie erschelnen. Die Un . zllverlïniakeit des W Wrokn vouP, int zal gross.

Dle Clironologie hut so, wie sie Percheron gab, keinen Werth ttul es wird bei ziealicher A neinanderrcilunog der mal orltistorisclien Scluriften in systematisclier Folge so ziemlich hervortretend die Anclennitit, das Jus perpe. tuum der Filtomologie, dis wie nllex Rech nur nuf deut lapiere stelit. lus Detail einzu. geteen ist itleht anders moglicli, ale das ganze Buch "umzarbeitent

Im Autorell-Iudex (p. 29.3) steht bei Ba* vire Srhraith nicht - und dus ist stark! bir Fauna loolea wird hutentilich desslantb noch lange lelien, p. 3il heisst es: Macrodactylus stutt Macrouyehrn - p. 330 bel Hymenopteren de Sucde felilı Brandstelis (I, 45); (b)endernelbe 3.38 bei den bienen.

Die Feliler des Textes labin sich fortge. ptlanut in der Table $n$; den 1000 . Tlieil getsen die Errata davoll all. - Es ist schade un das sclibine P'apier und das Geld, und un die Millie der Alischrint - ual die 'Zelt - : um Allex ist sclimule. Was felilt? Niır on !bu Selurlftateller und Scluriftell; die Zuverlissigkeit, die Cor. rectheit - wit eilucu Wort Alles. whs elu (1)uts sclumächt mul verewigt. Des Guten mud Nictalichen iat bellr wenig: dle ldoe allein iat liiblich. Die Alısfïlırmus ist nuf elnen lrr. giutg geratlien, - Der Ilr. lierallsgelıer haltte

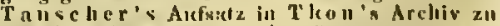
Gcsicht nchmen sollè, Fs wïre eine l'nppe. rei fewesell, ob so odwr son! und eits auch ob friiher nder apäter erachienen - ; so alıer Iatifen die Scliriftsteller IIu die Welte, Ias Eirste 7It gi'wluneu, well der Preis anch deat Stirkaten gilt - uicht aber deon Lönfer, deul es au Kraft gebrlelıt zu laulen und ansanlialten.

Was die biographisclieu Notisen anbutrilit, so erkencen wir mit Dank an die Biographic Latrellle's und die xylngraphlsclee zugabe liezu (des unsterhliclien Meisters Bildniss). eracliten en aber dea Kwerke uicht besunders 


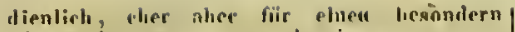
Alnituse wo mian Werke dis Namen aulrialueu kimule nnelı C:Homalogie mit Bemotheilumen in Fragurn-

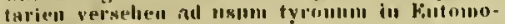
logia.

Sin vivl er ibler die lienen lant, doumelı reiche die literialis mielıt als. - Velser dir

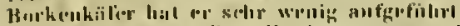

Lemerhungeu viluer ilie Aunuymes (ne 141)

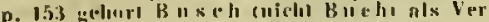
fissev. Also z.11 dru Aulorem. Jizu Busehs

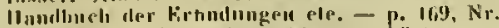

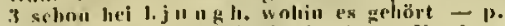
174. $\mathrm{Nr} 2$ and 3 simal eith Bueh. - Nr. 5 zu

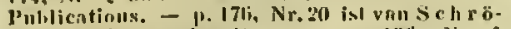
ter mul knmine lueroits vur. - 1!i, Nr. 5 chense, - 2114. $\mathrm{Nr} .19$ mbelesn. - 201, Nr. 211 elecnso. - 20j, Nr. 4j kommt alsppelt vor. vide Nr, 28, - 215, Nr. 64 vou is clirbter und kummi bereils varu beill Nimeu var. 2(10. $\mathrm{Nr} .72$; vide 91. - 210, $\mathrm{Nr}$. $\mathrm{KH}:$ alles ist bei ilime Podiara. Was larve vall (:a II tharls

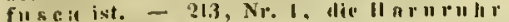

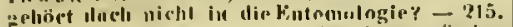

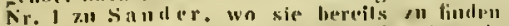
int. - 5fi. Selowall Nr. I mud to ist cloppelt

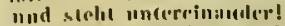

Veloer die bienen ist die I.iteralur bis ine Griule reschipll; sogar Ablinudlungeu

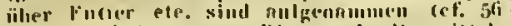
II. Nr. 4:4 Annuyna). Bie Luacksalberuiltel. lie kein Meusel| inrhr narlischlagen kann. sind

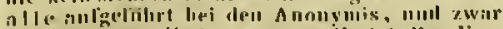

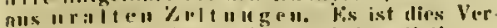

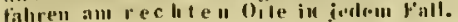

A 11 t. A i \& $\| \theta^{\circ}$ selibirt nicht hicber.

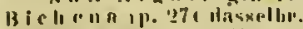

Perehereil zersuliltert viele zasnmmon-

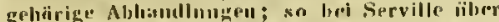

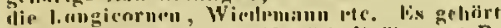

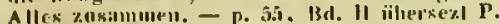
den 7itel einer $A$ bhandlum: von .1. 1). Siclinll\% im nenen Hauli. Masazlu; Nacholeht vou deu

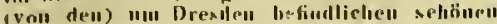
Grumbe und van len darlunen verlandenen Selceulicicen der Nalar, nlan: Sinr mu In'au vert (camble?) qui se Irouve anx enviroms de Dresde - ! !

Perty (Maximilian). Allnemeine Naturgeschichte, als plilosnphisclle itud Ilmunuitätswissenseluaft liii Naturforselier, Pliilosoplien und diss liölier ger. liildefe Publikum. III Bile. Beru (Fiselier) 1837-41. gr. 8. (Dieses in jeder Hit:sicht ansgureichmete, mejsterlafte Werk entluilt auclk fiir die Literatur kosthare S'lhit\%e.)

n"etseñ. Rapporto ace. Napoli 1828.

Pertzluble (Dr. Julius). Dir Biblioflicken Denterliands. 2 Anfl. Diesden (Adlor) 1845. 8. 201 pilger.

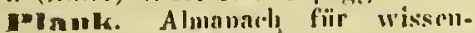
srlhaftlich gebildete Thieriirste. (Die ganze Haussiingethierliteratu" quoad Veter.)

Auérard, J. M. Ia France lileraire, on dietionnaire bibliograpluique des savauts, historicus et gens de let-l tres de la frause ainsi, que des litlé litents btratuers qui out ierit en forncais plos pantionlierement les XVIII et XIX siecles. Vol. XII. Paris 1813.8. - Jal literature irangaise coutempo. (aille 1827-38. Vol. I. Livr. 3, 4. P'illis 18i0. 8.

Iteciarentoerg, Chevaliur etr. ate. baron do. Anumaire de la Biblioblichue linyale de Belgiqur. 1. aunees. gr. in 12. Brilxelles 1840.

IR a-monurel. Catalogue des Livies de Midlevere, Chimerge. Anatomie, Physologie, Histoirs naturelle: Jlyysicjue, Clomie, Planmarie, qui se trou-

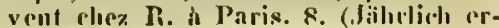
s.heint rin faar Mal rin solelfor Cotalug dieses ansegredidueten antiquariscrlen Lagers.) - Calalogne d'un ama(1) Par. Pars.

Etepertorimu, Allgemeines, der neursten in - "unl autsläudiselen l,iteratur. Horanstrgeben vou siner (jesollschaft Groluhter, besolgt von C. D. Herk. Elstheint scit 1819. 8 .

Tevertorimm, Leipiger, der dentsrlien nud ansliuldiselon Literatur. Unter Milwikune der Universitiit Iseipzig lecrausqegeben von E. G. G e rsedorf. Jolure 1814 in 52 Heften. gr. 8. Idripzing (Brocklıans).

ERensw. Repertorinm Commentatirmum a soe. lir. ete. Göltingare 1 sol. .1. (Zoologia partim).

Boserilbanum (I)r. Jul.). Additamenta ad. Lucl. Choulnuti bibliotlueram medien-histori-am. 8 maj. (6 Bogren) Halis Saxon (Lippert).

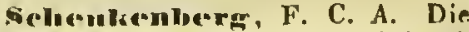
lebenden Mlinerilogen. Stuttg. (Silhwri. z.ellart). 1 843. 8. (Dieses Buclu verdankt nnserer Anciferung sein Entstelien, sn wie die liec zuerat vou $1 m$ s ansnegingen war.)

ichenchzer. Dibliotlieca serip. forrom.

Selmuidt (J. A.). Hollïndisehes Migazin der Naturkiunde, gesammelt ans physischen, clomischen mul maturhistoriselien Srluiften, die iu der hatavisclien Picpublik erserleciuen. II Balc. M. Kpf. Frankf. a. M. $1802-3.8$.

scelnmialt, J. $\wedge . \mathrm{Fr}^{\circ}$. Hnodbueli der Bilulinflackwissensclualt, der Literator' und Bücherkmule. Wrimily 18.80. 8.

Schucialer. Beitrige zur Naturgesshichte der Nlle'u.

Sclirank (F. v. P. v.). Angefïhte 
und hemizte Schriftsteller (inn 111. Bole. Ist (iiber Mineralogrie) ersehienen in

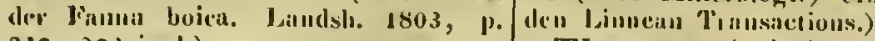

$3.17-37.2$ illel.).

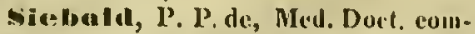
pluriam soe. membr. - De historiac nitturalis in Japonia stitu, nee non de anguento comolumentisque in decomste ferscrutationum exspentandis dissertit. tio, cui acuedunt spicilegria Filumas: Jisponicace Bataviac 1824 (Isis 1827, 135).

bilhermana, Gustave. Eummeilo tiou des Entomologistes vivants; suivie de notes sur les collurtions entomotogivues des musces d'Lurupe ete. avec la tible des jesidenees des eutomologistes; par G. S. Paris (Rorel) et Lumeville 1835. 8. (6 fire.).

simane ( $G$. di). Cullexione delle opere in disletto mijuoletano. Ninpoli 1826. 3 'ouI. 8.

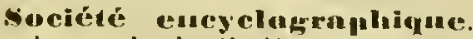
Cialalogne de la Socide encyclonraplique des Scirnces midicales. Bruxelles (Rue de Flaudre Nr. 155) 1835 et sery.

Ajix, Joh. Geschichte und B(urrtheilungr aller Systene in des Zoolo. gie uach ihrer Entwiekelungstulpe vau Aristoteles bis unf die ge'genwiintige

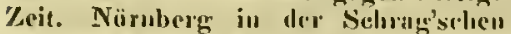
Burlıaudlumgr. 1811.710 \$. 8.

spremerel (Kun) Versul ciuel

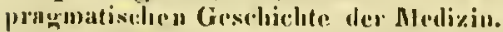
5 Bele. Halle 1792-1803. 8. (1,itriaturselbiet\% zatr Zuolugie und Auntonnic:.)

sericker (W, Dr. pliilos. in Berlin). Alphabetisclue Debersiclat aller fïr Natur - und Hoilkumb introssanten Orte, mit Rïcksicht unl Literatur uml

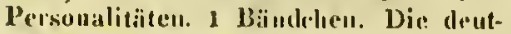
nelien Bundesstanten, Avel, unter Iru Titel: Allgemenues Reisetasclenburl, liiir Aeryte und Naturlorsches. Mlit

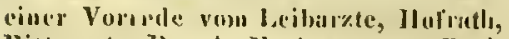
Ritter etc. D): A. F. A m m 0 в. Berl. (Liobmanu) 1841. 251. 12. (5! bogr), II. is lillilr.

Hinclaser. Systrunatische Encyelobäidie und Methodologie der theoretist:hen Naturwissenschaften. Mialle 1538. 8.

Tamseliere (A. M.). S(yjitoruu cutomologinormu liscyelupedia systematica. (Findet sich jin 'l'luon's Arelsiv 1, 2, 1. 39 und ixt selur brav; leider aber un Couspectus. Llwas Aeloulieles
Thomson (vgl. Ammal).

Verlaser von natulhistorischen V Y erken voryugsocise laben: in

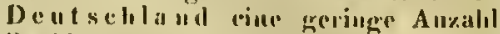
Burlıäudler, doren Namen lier antzufiilaren elemedr iilentlïssig ist, weil sie ja Jedermatur kesunt. - 13 rei den Antipuaren ist das etwas anderes; diese sind weniger bekanm mul haben sich erst in menerer Zait soliwanghafter gemacht. - Iu Eusla II Miissen

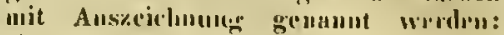
Voorst (Bouksiller to iles Kocilogival Society of Lomblon), B las: $\mathrm{k}$ and Arustroug (Fureign Bookstllers tu

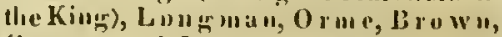

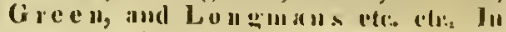
finutue ich \%eichuten sich ans: Deiterville, Croclatre, Laillicre, Gide, Fortin, Masson, Miquigk-

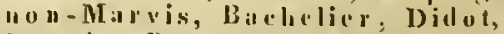
I. equ i en, Royetefe; in II Ivetien, Clierbulie\% жu Gent (und P'aris) etu.

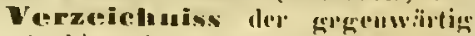

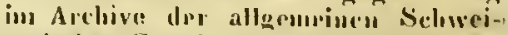

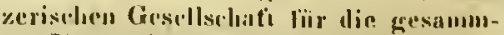

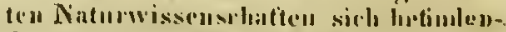
den Briclier (Mamussipite) nut Bilduisse, Aulat 1536 (Berk). 30 l'itg. 8.

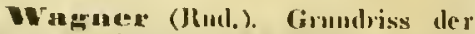

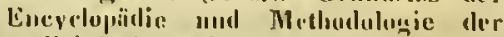
medizinischen WVissenselualten. Erlangen $183 x$.

Wrate (Rob.). Bibliotheen britauni. cai. I,oud. $18 \mathrm{t9}$.

Ereigel (T. O.). Cintulug, n(neter, ciner ansyewislaltu Situmlum vou Bijelaera, «u lablen liei $T$. (). W. grr. S. (Vlll mal 483 S.). Leipxig. is.1.3 (2 fl. $2.1 \mathrm{~kJ}$.).

WVillems (.l. li.). Belupish Musenus vaur tarelen lettertiuste car ale

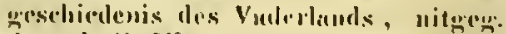

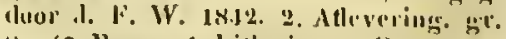
8. (7 18. II. 1 litls. in 4.) (ient.

Drintler. Lifleratura scientiac rerum maturalium ju Dania. Is29. 8.

Taecheray (Gimf v.). Katalou allaty ungüriscluen Workr. J'estl, 17!19 - t807.

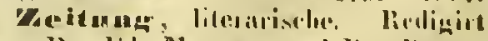

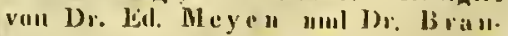
des. 11. Jalarg. 18.11. 52 Niru. «ู. 1. Berliu (Duuher.) 


\section{Biographien, Gedächtnissreden etc. *}

Arzelins. Biographie davon lu jet modene ete, Redigé par unc société Kongt, Vetenseaps Arad, Haudl. Stoekh. de gens de leftes et de Savints, 52 1836, p. 342-345, - Handl. f. A. 1837. p. 263-266, - Hamb, IImpait. Corresp. No, 2 t.

Agassiz. Vgl, Conversations-Lexikon der Gegrowart und cine andere. (jedoch ilın nicht betreffende) Noti\% bei Periottet.

Alsrens' Nokrolng. - Entomologische Zcitung. Stettiil III, p. 45.

Amonymus. Vita Joamis Pleilippi Вгеушi $\mathrm{i}, \mathrm{S}, \mathrm{A} .8$.

Anonyuns, Zum Andeuken nu Joh, Christ. Dan, Edl, vo, Srhrelued (Noirnberg. Minaz. I. Ilft. 1820).

Anouymo. Some Aecount of the late PeterCollins o 11 , London 1770, \&.

A rago (vgi, Pediul).

Audouin (V.). Note sir George Cuvier (geb, 1796 am 23. Ang.; gest. 1832). (Annil. кос. ent, fr. J, 317).

Andubou, Bingraphiselse Skizze.

Isis 1837,922 .

Inck (A.). Gedïehtuissrede auf $\boldsymbol{H}$. Arch, mol Ritter ("nll von Linmi, A. d. Silhwel. Stocklı. I1. Upss. 1779.

BaIdinger, E. G., Bingruplieu je»t lebemder Aerzle und Naturforscluep. Jena $1768-70, x$.

Banlus (Josepli). Selue Biogrophie in 'Jiliochsphit. Mag. Vol. 56. 1820, f1. 40.

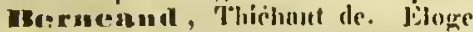
listorifue de $\Lambda, M . F, T$, Pulikot de Benllvois, Paris 182t, 8, (1) Arehiv

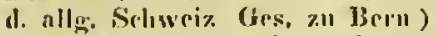

Eiograplio universelle, ancienue

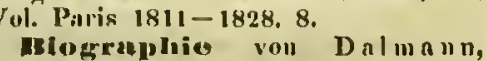
Thunberg, Lj"॥ gh etc. in Stock. lolm. Vetensk. HandI, 1828, p. 224, 242, 279; feruer Biugraphi ofver Ca bi Fredrik Fillen i Viltensk. Handling 1830, 1 . 272). Sundewill (1831), Retzius (18'2, 464), Ekström (1831) damn ibill. 1826, p. 350-359). (Payku IIs Bingraplic.) Vul. Allegirtes.

ABlograplife, Medizinisehe, oder vollstiindige Nathrichten von dem Leben und den Seluritten der Aerzte, Wund-

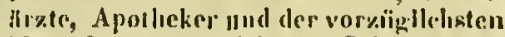
Naturfor'schinr, welche ais Seluiftstelier bekanut worden simul, t, Bd, 1. Heft. A. II. d. T.: Blogutiphie der Aerzte. A. d. Frnnzös., mit einigen Znsitzen

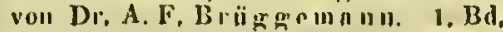
gre, 8. Hahbrestadt, J3rïggemanu. 1829. (4 Hefte hilden 1 Band, das ganzo Werk Imfilsst 6 Bde.).

Alogeraplice ofver l'rof. Dr, An. ders $S_{p}$ is rimain, (Stockh, Vat, Handl, 1820, p. 393.)

ABIuncubacl, (J. Fr.). Zum

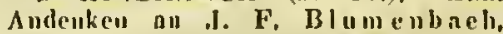
Eine Gedifelitnlsarede, gelıalten in der Sitınng der k, Akademie z.u Göltiugen, Göltingen 18.10. 4.

MBo.janins. 'Torltenopfer (Isis 1828, p. 601. (Vorl. Jielıwald.)

IBumelli (val, Genci).

IBomet's Bingraplice (in Perche. rou's Bibliouraphic),

Borson (vg.l. Gené).

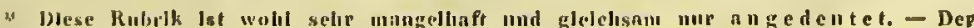

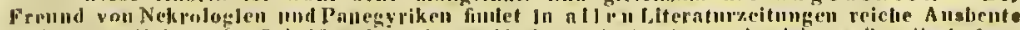

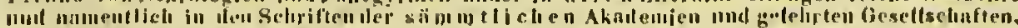

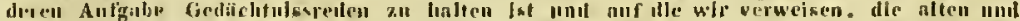

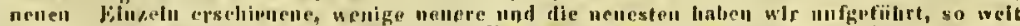

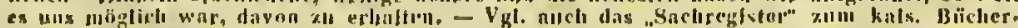
l.exik. I.pz. 18.69, 4, 220-225 It, p. 4.3f. Firuef: KI. AI, B a a der's I.exlkfil verstorb. Jinyr.

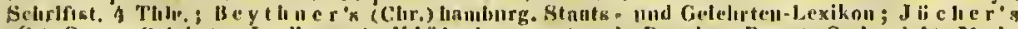

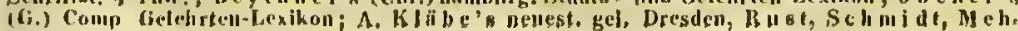
c 4 แ 
Eray (Graf de). Biograplic vou Martius (Flora odev bot. Zeitumg).

Brelum. Mich a lielles (Skiz.e). (1sis 1837, p. 689).

Eroustais. Rede an G all's Grab (Isis 1829, p. 1315).

Buguoy als Plbilosoplı und Diclıter (Isis 1828, p. 602-10).

Callisseu, modizinisches Sehrift. tseller-Lexikou (Naturforscher). 25. Bd. Leipzig. Hermman 1840 (?).

Camper (N. A. G.). Nutice de la vie et des écrits de Pierre Camper. Paris 1803. 8.

Candolle (A. P. de). Notice sur la vic ef les cerits de F. H uber. Genève 183". 8. - Leben und Schrif. tell von Franz. Huber (Ediub. new. ph. Jourm. XIV, p. 283-97). - Nolice listorique sur la vie et les travaux de M. Desfontines. Gendve 1834.

Carey (verl. Voigt).

Chrunchael (vgl. Leben).

Clinje. De prestantissini equitis Poli viti. Neap. 1827. Fog. con, ritr. e vign. - (vel. Redi.)

Cuvier's Biograplife (lsis 1832, p. $1303-2 t)$. - Vgl. Audonin. - Bivgritphische Skizzce (Kröyers Tidskr. I. 1836). - Vyl. D il v y. - Snbseription zu dessen Denkmal (Isis 1833, s68.)

Coleridge (Hartley). Biographin horealis; or Lives of distinguished Northeris. Loud. 1836. 8. Illustrated with Purtrits.

Collinson (vgl. Anonyuns).

Conversations-Tuexilcon. Leipz.ig, Brockliaus.

Daluasum's Biographic, - Kongl. Velenskaps Acad. Handl. 1828. p. 224.

Davy (Inuplary). Cuvier (Ediub. 11, phil. Jonnn. XV, 1-34).

Desfontuines (verl. Cindolle, de). Duunéril (verl. Reden).

Duveruny. Notice historique sur les oinves et la vie de Mr. le Baron de Cuvier, Paris 1833.

Ebel (vgl. Locher),

Eicluwsil, Eduard (Dr. und Professor). - Memaria ('larissimi etc. Land. Ilen!. Bojani. Recitavit. Vilnae 1835. 4. 57. 1 T'ah. (Isis 1836, 555).

Wloge listorique de M. Rielı ard. Par G. Cuvicr. Mem. d. Mus, d'hist. nat. 'T'. 17, p. 349.

Elintribu (Kongl, Vetensk. Handl. f. A. 1831).
Wmanert (J. H.). The british biography: containing bricf and aceurate aecounts of the lives, aets and writings of the most remarkable persons of the britisls mation from the Years 36 before Clrisi to the Years 1810 after Clirist cte. Gütting. 1820. 8.

Eutomologische Zeitung des entomologischen Vereins zu Stettin (vgl. Gel. Ges. Gyllenhal's Necrolog $(1,111)$. - (Alirens' Necrolog (ibid. III, 45).

Fallén (Carl Fredrik). Vetensk. IJandl. 1830, p. 272.

Feller (Fr. X. de). Biographie universclle, nonvelle edition de Veren. nes. 12 Vol. 8.

Filrnrolix, Dr. A. E. Gedïchtnissrede bei der 100jülur. Jubelfeier der Doctor-Promotion K. V. Linne's. 8. Regensiburg. Dessen Topogr. Reg.

Gail (vgrl. Broussais).

Gallerie der vorziogliclssten Acrzte und Nalurforscher Deutschlauds; naelı neuen Originalzeichunugen gestochen und licrauserebeb vou F. R o sum is ler. 1. Heft. Gutlia (Pertloes in Com.) 1831 (Wir laben mur dieses.)

Fene, Ginseppe. Necrologin di Stephano Borson (1833?). - Elogio storien di F. Andr. Bonelli. Torino. 4. - Necrologia di Guid. Gartieri. Torino.

Geofroy. Ueber die Seluriften Gütlue's, welche seinen Namen als Niturforscher reclitfertigen (Anual, d. sc. nat. XX11, 188). - Vurl. Redell.

Germar, Magazin derEutomologie. (1, 2. p. $192-193,1,1$, p. 1:10.)

Tistel. Acis. Intelligenzblatt zur Zcitsclurift Fonnus. I. 1, 2, 11. 3, 4, 5, Ill. Nr. 1, 2 Bd. - (Vgl. Wagler, Spix, Müller.)

Giortou (Johiu). A general biographical Dietionary. A new Edition. Lond, 1836. In thice volumes, large 8. - An Appendix may be lad to the first Ellition. 8.

Goexe (J. A. E). Dr. Friedrich Ilciurich Wilhehn Mintini's Leben. Berlin 1779. M. de'ss. Bildn. 4.

Gyllenlual's Nekrolog. - Vgl. Entomologische Zeitung. Stettin. I, 111.

Inibertsma (H.) de Lecuwenhöckii Meritis ete. Lugd-Batav. 1843. 8. 70. Tab. 2. 
Marless, Dr. C. F. Die Verdienste / dit H. C. v. Il all. 8 maj. (Groningae). der Frauen un Naturwissenschalt, Lipsiac (Barth). - Caroli Linnai Epi-

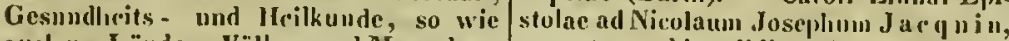
auch un Lüuder-, Völker-und Menschen- ex autographis ediclit. $N$. I. Eques a kunde, vou der iltesten Zeit his auf Seli reibers, praclatus est notinsque die neneste. Ein Beitrag zur Geschichte adjecit St. Endlicher. Vindubouac geistiger Cultur, und iler Nittur- um (Gerold) 1841, 8. 167. (Isis 1542, 465.) Hi cilkunde insbesondere. Mit Abbild. - Vyl. Stöver, Suith und B ii ek. g1: 8. Güttiugen. Vaudeulıöct u. R. 1830.

II aral wiclce + (l'roecedings 1835! Ihrsse (vgl. Zeitgenosseu).

Huvemaun. Ueber $\mathrm{B}$ oja u us (Isis 1829, J. 905).

Mitzig. Golebrtes Berlin.

Ioceven (van der). Nachrichten viber G. R. Trevilau us ('Tijdselur. f. Ilat. Gesthied. IV, 211).

Howue. Biograplice (Brewster, Taylur ete. The Londou it. Edinb. phil. Maga\%. 111. 1833, p. 136).

Ituber (Fr.) (vgl. Candolle).

Jomy (vgl. Redeu).

Mmach (Aug. Wilh.). Desseu Le. bensstizze (Germas's Magaz. III).

IuhI (11.) (vgl. Nees ab Eseubeck).

Latreille. Eröffunumsrede der entomolog ischen Gescllschatt vou Frankreich zu Paris. 1832 (Aunal, de la soc. I, p. 22-34).

Jatrell le's Biographic vou A.P P p. clieron (vgl. dessen bibliographic entomologique).

Lebeu des Hauptmanus Carmi. chacl (Ellinb, new phil. Journ. IX. Oct. p. 113).

Iseben und Teln raneinmengen berïlumfer Plıysiker an Eurle Jes XVI. und am Aufange des XVJ. Jilubunderts, als Briträge zur Geschichte der Physiologie in engerer und weiteres. Bedrutung; lacrusegegrben vou $\mathrm{Tl}$. A. Rixuer und 'Tl, sieber. Ileft VI. 'll. Campauclla. - vll: J. B. v. llelmont. Mit 2 Portr. uncl einer Stcint. gro. 8. Sul\%h. I. - VIII. Iloti 1819-29.

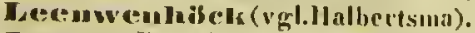

Juems: (De). Notice historipue sur Audve Michaux (Amual. du Mus. III, p. 1911 .

Limué Carolus a. Limuaci, C., Epistulac iucditue, addita parte commereji liternoij inediti, inprimis cirea rem botaucau, J. Burmami, N. 1. Jummani, Dillenii, Ilalleri, Ślmuidelii, J. Gesueri, Oederi, Pallasii, Vanclcllii et 'T'uunbergii; aunis 1736-93. Ex litteris aulographis edi-

- Li gen über sich selbst, mit Ammerkingen und Zusitizen vou Afzeli lls. Aus dem Schwed. vibers. v. Carl Lilppe. Mit einer Vorrede von C. A. R ululphi. Nebst Linne's Billuiss murl Handselurift. Berliu 1825; bei Reimer. 8. XXIV und 260 S. (Inis 1827, 970). Das seluwed. Original lieisst: Lgenlitudige Auterekningar af Cinl Limanens om siz siclf med aumärkningra orl tillïgre af Adlan A $\mathrm{f} z \mathrm{e}$ li Ils. Stochlıolm 1823. XXIV und 248 S. 4. m. Kupft. - A general virw of the writings of Limnarus by Richard Pulteney, M. D.; the 2. Edition by Will. Grorge M a lon, M. D. itr. Lourlon 1805. 4.

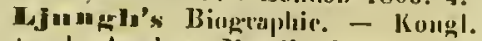
Vetenst. Academ. Hiandl. f. A. 18\%, 1. 279.

Leclner-IBulber. Nekrolog des 11. J. G. libel. Zürich 1831, 8.

Lom raless. Notices sur la vic litteraire de Spallanzmi. 1\%. Paris 1800 .

Manlen, P'l. van der. - Dictionunire des hommes do: letires. dess sicvans el des artistes de la Belgique. Bru. xelles 1838, 1 Vol. 8.

Mregiliveny. Lifes of eminents Zaologistes et.. Lidiub. 1834.

Mair, Dr. W., vergleichende Wüdigung der Verdienste J. Tl. D (es agulier's, W. Gravesaudes mad P. vau M ns semboek's Im die lixperimentalplyysik. or. S. Müucluen.

II rsigli (Comte de) (vel. (luincy).

Martimet. Silunilt's hulliind. Magill. I, Srite 212-226.

Mantimi (vel. Göze).

Nartion (vgl. Sclurank's l,(ben).

Marx. Wirrlipung des Theoplinustus von Holıenliciu (Gött. gel. Anz. 1840,97 ('t sef.).

Medici. Vita di Carlo Mondiui. Bologna 1830. 8.

Mensel. Lexilion der vom J. 1750 -1800 verstorb. Silhiftsteller. Lyiz. 1815,8 .

Mielonhelles (vgl. Brelıu). Mondini (vgl. Medici). 
Mous (J. B. vau) (vel. Quetelet). Maille r's (J. Fr. Aug. Clurist.) Lehen vou Dr. lulius (.I. Gistel). Miinclı. 1838. \&. Mit Silhouret

Mussentbroe:k (vgl. Mair).

Ninturerorsclneer, Dieschwedisehen, Karl l'eter 'l'humberg nucl Joh. Will. D il manu. Ans den Abliandiumgen der hänigl. Akademie der Wissenschafien 7.1 Storkloolı1. 8. Stralsumd.

Neluobogiselue Notizen von J. Gistel (vel. dexsen Zeitsehrift Fannos I, 11, 111. Arisi.

Vees ab Wrendonek. Viti Henrici Kulllii (Nov. Att. Acad. Nat. Cur. $X 1,1 . X V 1-I, X I V)$.

Selseolos, Nener, dev Dentschen. Srit 22 Jaliren, also 22 Jahrgiiuge, jeder in 2 Theilen. M. P'ortr. 8. Weimar (Voigt). (Unsere Biographien Dr. Fr. A. C. Miiller's, Wagler's ete. sollen hierin abgedourkt seyn.)

Nownek. K. G. Schlesiselues Schrifisteller-Lexikon. 8. Breslan 1837.

palivet le Denuvois (vgl. Bermeand).

Panzer (G, W. F.). Quacdam de J. G. Volcamer. Norimbergate 18n2. 4. - Flora oder botaluisele Zei. tı1ı. 1829. Nr. 25. 7 .luli (t 28 . Jnıi 1828, 75 Jalı alt. Vyrl. den l3rief an uns vou $S$ tur in im Filuls I, p. 51).

Marncelsus (vgl. Minrx).

Pariset (E.). liloge du Baron C. Cuvicr. Paris 1833 ; 8. aver 111 bean portiat de Cuvier. (Virl. Duvernoy und Wagner. Intelligenzblatt der allgem. Literat. - Zeitumg No. 45. Juli 1832.)

Presunier. Lobrede auf Cuvier (Eviul. new phil. Journ. XIV, 339 $-367)$.

Paykinu. Kongl. Vetensk. IIandl. 1826, 1'. $350-359$.

Pereluerou (vel. Latreille, Bonnet und licillmuri).

Perentert. Nutiese historiques sur Per roftet et $A$ gassiz. Lausanue 1831. 8 .

Peeurerlonela (vgl. S(luhert).

Ipietet (MI. A.) (vgl. Vaucher).

Poli (vol. Chinje).

Prevast (P.). Notice de la vie et des ouvrages de Bened. Preivost. Gendve 1820. 8.

anctelet. Notice historique sur J. 13. van Mons. Bruxclles 1843.
Auiney (L. D. C. IT. D.). Memoires sur la vie de Mr. le Comte de Marsigli. Zurie. 1741. 4 Vol. 8.

Ramsey (in London). Biogrnphische Noti\% niber J. E. Su ith, Prïsident fler Limne'schen Gesellselaft (Brewster Ed. Jouru, 1, 1829, 1-26.) IRnchlue (vgl. Tiseher).

Renumur's Biographic in Pereherous Bibliographie entom.).

Heden an Cuvior's Gialı: vou A rago, Geoffroy, Dumeril, Villein in, Jo ny. (Annal. d. sc. n. XXVI, p. $394-415$, wo p. 415 Plane zor Errichtung eiues Mouments für Cuvier.)

IRedi. Vgl. dessen Neerolog vou Delle Chiaje. (Necrolog de' soc. ord. del R. Instit. d'Incor. Nip. 1822, 4.)

IRetrins (A. J.). Kongl. Vetensk. Handl. f. A. 1822, p. 464. - Tallaallit da liomel. Carrol. Aead. Natural Kammare, 11. Junii 1811, da fromlidue von limue's Brostbild darstades upsattes.

TRielanarl ( $($ gnl. Cuvirr).

Rixner (vel. I I cben).

Eosminier (vgl. Gallerie).

Aay (Thoaas). Biography. "(Wir wissell niclit wo und wann dieselloe. ersehienen. Der sel. Prinz Max. von Nenwied selurieh uns davon und versprach, sie uns zol selicken.)

Selnelnt. (Holländisches Magazin

f. Nitulkunde; vou Sclımidit I, p. 205.)

Gelıaden, A.v. Gelehrtes Münehen.

Selunirer, Fürurolır's uaturlistorisclic 'l'opographie vou Regensburg.

Schrank's Leben. - Martius: Denkrede allf Fr. P. v. Seliank. Müneloen 1836. 4. - Aeis. Beiblatt z.ur Zeitschrift Faunus von Gistel. 1. 13d. meue Folge, Nr. 1 \%. 2. Miiuel. 1837. Mit Sebrank's Bilduiss von John in Kupfersticl.

Selubhert, D. G. H., Peuerbach und Regiomoutan, die Wiederbegriinder cincr selbstständigen und unmittelbaren Erforscluung der Natur i॥ Enropa. Eiur Aurele an studiereude. Jünglinge. 8. Erlanger.

Sclurbder. Lebensgeseliclite des Wohlscligen Ifru. Hofraths Jol Erust Inuan. W a leh. Jena 1780 . M. Bildn. 8.

Sieber (vgl. Lebeu).

Silbermana (G.). Les lintomologistes vivants. (Gelört wohl anch mit Fug in diesen Abschuitt, insoferue es Charakter, Scliriflen ete. beriihrt.) 
Emlths, Sir J. P. Selection of the Correspondence of Limnaeus and otlier Naturalistes, 2 Vols. London 1821. 8.

Suitla (J. E.) (vgl. Ramsey).

spallamzanil (vgl. Lourdes).

Sprarrunnu. (Kongl. Velensk. Acarl. Handl. 1820 , p. 393 . - Vgl. Biogroplice.)

Spix's Lebensskizze. - Gistel: Gallerie denkwiirdiger Naturforschev (Bd. Il des Faumus), unit Spix's Bild. niss.

Sterler (Jos.). Biographisclie Notizen (Regens. bot. Zeitung von Ho lipe und Fii rnrohr. Jalurg. 1832. Nr: " p. 32 und Faunus 1).

Stdiver, Lcben Cirl vou Linne's.

2 Bände. Hamburg 1790. 8.

Sumdeval. Kongl. Velensk.Handl.

f. A. 1831 .

Thienemanu. Auf Gütle's Tod (Isis 1833, p. 178).

Thumbergs Biugraphie. - Kongl. Vetensk. Acarl. Ilandl. f. A. 1828, p. 242.

Tischer (K, vo). Tudesfall voll Joh. Goltfr, Rnsehke (Germar Mag. II, S. 343).

Treviranus, G, R. (vgl. Iloeveli).

Vancher, J. A. Nécrologic sur M. A. Pietel. Genève. 8.

villemin (vgl, Reden).
Volgt, J. Lehensbesclireibung des Dr. Carey (Kuöyer's 'lidskrift).

Wagler. Ueber Cuvier's Lei. stungeul (Isis 1833, p. 1-25).

Vagler's Leben. - Gistel: Gallerie denkwiirdlger Naturforseher. (Beibl. z. Falıus. Nr. I II. II, 1835. Wagler's Bildn. fundet sich als Titelkupfer zulu I. Bd. des Fauluus. 1832. - Bayer. Lillidbüt. 1832.

Walclı (vel. Schüter).

Witstenfelal (Ferd., Dr. philos., Lehrer der orientalischen Spracheu, Sreretiir an rler k. Universitaits-Bibliothek tıl Güttingen). - Geselichte der urabischeu der\%te und Naturforscher. Naeh deu Quellen. gr. 8. 1 Taf. Göt. tintren 1840.

Zachinelli (G. M., Dr. Med, Prof. und Sanitiitsrath zu Pudua, $t$ ). Dessen Neerolon in Giornale Toseano. III, 1841 , p. 237.

Zeitganossen, ein biographisclies Magazin für die Geschichte unserer Zeit. Redigirt unter Verantwortlichkeit der Verlagshundlung von Prof. F. C. A. Hasse. gr. 8. Leipzigr, Brorkliaus, 1. 11. 2. Bol. it 8 Ifefte. 1828-31. Gilt als Furts. d, 1816-27 ebend. erschiencuen "Zeitgenossru", erste Reihe 1. -6. Bd. 24 Hefte und nene Reilio 1.-24. Heft oder der ganzen Folgo 25.-48. Heft. 


\section{Realwörterbiicher *}

Deblen (Stephau). Real - und Verbal-Lexikon der Forst - und Jagdkuude mit ilıren Hülfswissenschaften. VI Bde. Lex.-8. Frankf. a. M. Sauerläıder. $1840-42$.

Conversntions-Lexilon, Rhrinisches. 3. Aufl. 12 Bde. gr. 8. Cölı $1834-36$.

Conversntions-Iuexikan. Leipzig (Bruchlous).

Cyclopredis (Tle) of Anatomy and Physiology. By Roliert B. T'od i. Vol. I-II (A-Ins.). Lond. 1838-39. (Erscheint noch.) 8. (Vgl. unser Lexikon p. 71.)

Dictionnnire, Nonveau, d'histoire naturelle, appliquec aux arts, principalement a l'agriculture et à l'éconamie rurale of domesticjuc. Par une Societe de naturalistes et d'agriculteurs. Avec des ligures tireses des trois règues de la nature. I. Edio. Puris. 24 vol. in 8 : 1803-1804. (Crustaces par Bose ct La treill c.) 2. Edit. av. 150 planch.; 36 Vol. Paris 1816-19. (Insectes et Crustaces par Lat reille).

Dictionma ire: classique dhistoire natucelle par differents naturalistes. Av. pl. Paris. $17 \mathrm{Vol}$. 8. (lusectes par Latrcille, $A$ ulouin et Guerin.)

Dictionnare dassique des sciences naturelles, contenant un choix des meilleurs articles puises dans tous les dictionnaires qui ont tratè de ees seicnces, aummente des travaux et docouvertes effectues depuis leur publication; mis en ordre et rédige par Dra. piez; ouvrage orné de 200 planches en taille-douce et coloriées, 10 Vol, grand in 8. imprimes à deux calonues, eont cnant le nuatiere de 72 Vol. in 8., qui paraitront en 48 livrissous (anx prix de 4 $\mathbf{F r}$. la livraison. Prix de plan1clies 2 fr. la livr.). Erscheint gegenwì rtig.

Dictionmaire des scirnces uaturelles, dans lequel on traite methodi. quement des dillerens êtres de la nature, considères, soit en cux - mêmes diapres l'etat actuel de nos comaissances, soit relativement a l'utiliti gu'cu peuvent retier la mediciue, l'angriculfure, le rommerce ef les ilrts: suivi d'mue Biographic des plus celibres naturalistes etc. Par plusirurs professivurs du Jardin du Roi el des principales Ecoles de Paris. Vol. I-V. 1804. Vol. VI-XXX ite. ef suppl. 1816-25. (Histoirr naturelle des Crusticres el des lusectes par M. L a troille, Leacli, Desmarest et Dumeril.) Planches. (Sous la direetion de Mr. lurpin.)

Dietionmaice: dles scicuces natlirelles, daus leguel on traile mèthodiguemeut des differeus êtres de la mature, considéres soit en eux-mèmes, d'apris l'etat actuel de nos comnaiseances etc. par pluse professeurs du Jiurdiu du Rui. LX Tomes ef XI Taur. Planches. Strishour (Levranli) et Paris. 1816 $-1830.8$.

Dictionnnire chronologigur et vaisomb des Decouvertus, inventions, perfection nemens, observations nouvelles et importations en France, dans les scieuces, la litterature, les arts, l'agri-

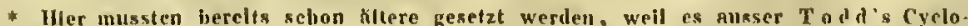

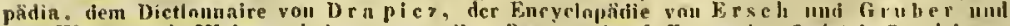
dem Wörterbuch (Weimar) keine ncuen gibt. Der angehende Futomolog fiudet in $B$ a $h$ in a sen's und liliger's Versuci einer zoologischen Terminologie, M b́ I ie r's Lexicnn entamologicun. KIrlıy's und Spence's Kntomoingie, sowie in den Compendien vou Burneister,

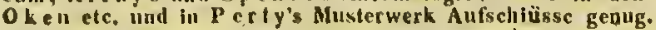


culture etr. de $1789, a$ lit fin de 1820 etc. Par. 1822- - 4.17 Vol. 4.

Dictiomunires de Merliciue et de Chilurgie pratiques; par MM. A 11 a la I Begin, Bla nd i i, Bou illand, B oll. vier, Cruveillior, Cullerier, Devereic, Jugis, Dufuytren, Fovilla, Guiboul, Jolly, L, alle.

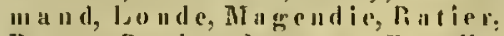

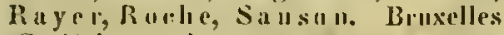
(Socicite incveloure). 8. 1838.

Diefiennsairet Iaisonme of mivernelle cies nuimaus, on lo Regme anjmal, consistaut en Quadrupedes, Cetaris, (O) istux, Poissous, lusertes, Vers, Zooplyytes, ou Plantes animales; lems propuictes en mediciue: la classe, la fimille on l'oudre, le genre, l'espesere uvec Ins variptes, mi chaque amimal ist range suivant les diflïroutes metboles ou munveanx systimes de MM. Li川 D. L. C. 1). 13. (De Lil Cliesnaye-Des Buis: 4 Vol. ia t. Pall. 1759.

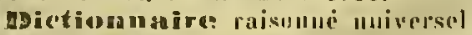
d'Jistoime Jibluselle, coutenant l'histoire des aniuand, des venditux et des mimiralux, ot rolle des (on)s colestes, des mitimes of antles principonx pluenomèues de la nature, avee l'histoire et fie descripition des drogenes simples tirces des trois rigures, of te ditail do lents usigess daus la medicinc, dans

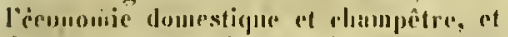
daus les atts et metiens: plus me 'Tablo cuncordante des mons latius. et le jeenvoi aux oljuls mentiomues dans ret

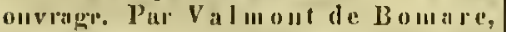

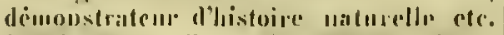
J. Edit. iu s. Par. 1764. - II. Edit. 8. Par. 1768. - III, Eal. 9 Vol. 8. Par. 7775. - IV." El. 15 Vol. 8. 1791. Y. Ell. \& Vul. d. 1793.

Bictionanaires des seiener's mudicolps. LX Vol. Jar. 18t2-22. 8.

Drapiez (vgl. Dicliomane (·l).

Daninburarl, Eucyciopucedian (iel. Fincyolopaledia britturnicas.

Encyctepenedia brittunira or a dietimany of ants: scieneses and mixcellineous litteriture. lid. 4. Vol. 22. Edimburglı. 1810. Vol. 1, Jal. I. Ab

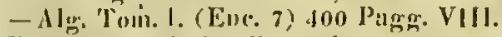
Plat. 4. Vol. 1, Jill. 1, 40t -799 , Pis. XX1X Pit. Vol. 11, P. I. 100 Pig. LVII 1'at. (j). 26 : Räderthiere). Vol. II, P.II, t0 $-: 99$ jag. XCl Plat. Vol. III, P. I, 409 pag. C Pl. Vol. III, Guclin, Horucr, Munke, Pf aff
I2. II, 401-806 pag. CXI PI. (Mehr Bïude kiımen ıus nichı zu (Gesiolıt.)

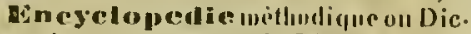

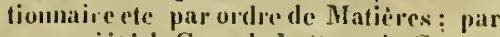
moresociededo Gens do Lettres, de Savaus et d'Artistes; précidé d'un vocabulaire muiversel, servent ile Table poü tout l'onviage, oruee des Purtraits de M. M. Dirlerul et d'A I em be pt, premiers liditeurs de l'Encyelopealic. Euevilopedic mithodique. Histoire maturelle. lintonologie. A Paris chez Panlinuke "te. Lieje che Plombenx. 1787-1225. 4. 10 Vol. (Die ersten von Olivier, die letztern von Latleille, Godal, Lepellet ie r-Saiut-Far. gra (ill e in.) 397 Pauclus.

Eresclopidis, Allgemuine, des Wissemischaflen und Kinnste, in alploabetisclier Ordmuma: vou gemanmen Scluitstellem lonabeitet und beraus:-

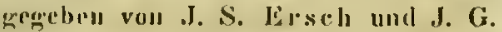
Giuber. I. Section: A-G. Ilernusg. von (iubes. 36. 'Ml. Epimarlas- Esrypue. 2. Sin.: H-N. Hzeg. von A. G. Ilolliminu. 20. Thl. Invagiuatio

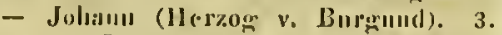
Siect.: $\mathrm{O}-\mathrm{Z}$. Herer. vou M. II. H. Mejer I. L. F. Kinut\%, 16. THI. Pencela - Perigymun. Mit Clanrlen mol Tafela. 1.riprier 1818-4.3. 4. (Cinstu Exemplar gelıl bis Par. - Der leviilunte B armeister beabeitet die rutomo. lugischun, iberliaupt \%oologiselien Arrilial. olie ilnemaceliend simal.)

Eucy andienic, Nuturhistorisclıe, der Clionesull (rel. Isis 18.32, t099).

Gincyclogacedia, or a new mujversal dictionary of arts and sciences. V. $t-16.4$ 1, 1,iul.

Fincyclapseries d'liducation, on expositim abriegre ete. des Sicicuces, des Ants at des Metiens. Redier. s. lat

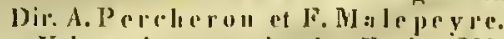
c Vol. s. Av. 400 plancla. Paris i Me(quigumu- Misvis). (sis,

Hirell mud frimber (vigl. Lineycy clopindice.

Disclnesatribur. Nya äkonomisk Diclionarien. Furtereser vou 01.

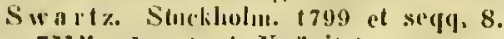

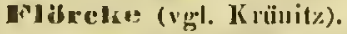

Gxelrlex (J. S. T.). Pliysikulisches Vörterbucl. Neo beaili. v. Brandes Gumeling 
6 Bde. Mit Kupfertaf, gr. 8. Leipz. Schwickert.

connelin, Ph. F., v. a. Onomatologia historiae naturalis compl. oder Wörterb. d. Natg. Ulu (Stettin) 1758 -77.7 Bile, gr. 8.

Mandwdrterbuch, Medizinischchirurgiseh-pharmacentisel- - und naturhistorisclies, zur Verteutsehumg der Frendwörter und Kunstausdrüeke. gr. 16. Wien 1843.

Jomrulan, A. I. L. Dietionnaire raisoni, étymologique, synonymique et polyglotte des termes usitis daus les scicmees maturelles. Paris (Bailliere). 1834, 2 Vol. 8. (Prix 18 fres.)

Kabinet der naturlyke historien, wetenschappen, konsten en landwerken. Deel 1-8. Austerl. 1719-23.

Ir rans (L. A.). Kritisch etymol. med. Lexicou. 2. Aufl. Göttiug. 1826. 2 Bile. 8.

Ihriimitx's (Dr. Joh. Georg) ökonomisch-technologisehe Eneyclopädie, oder allgemeines System der Stats-, Stadt-, Ilaus- I. Landwirthsehaft, mol der Kunstgrsehiehte, in alphahet. Ordmung. Früher fortges. v. Friedr. Jak. u. Heinr. Gust. Flörke, unt jetzt v.
Dr. J. W. Dav. Korth. Mit I Steintafel (in ru. ${ }_{2}^{1}$ Fol.) u. 190 Thle. MI. Portrait. gr. 8. Berlin (Krause).

Manuel du Naturaliste. Ouvrage utile aux voyageurs ete. Paris 1770 . 8. ( Dietionnarium ehenienm edidit. Gistel.)

Nemmich (Plı. Andr.). Allgemeines Pulyrlotten-Lexikon der Naturgeschichte. 2 Bde. Hamburg 1793-97. 4.

Vicolson. Anteriean Eneyelopaedia.

Rees. New Cyelopaedia. Philadelphiti.

Ripetti, E. Dizionario geografieo, fisiro, storico della Toseana ete. Firenze $1833-34$.

Scluwartz (vgl. Fischerström).

Todd (vgl. Cyelopaedia).

Woirterloncli der Naturgesehiehte, den gegenwärtigen Stande der Botanik, Mineralogic Ind Zoologie ange. messen, 12 Bdle. gr. 8. mit Atlas in 4. Weimar 1824-44. Ist noch nielıt volleudet. 


\section{Uebersicht der öffentlichen Museen und Privatsammlungen Europa's, Asiens, Amerika's etc. *.}

Amelien. In Stadtarehive befindot sich eine Sammlung von Natoralien. - Privatsammbunen sind die des Dr. med, und Arzt Debey, Lebrer Förster, - Dr. ned. Lesoine, - Leluej Kalta ubali.

Arran. Die Sammlung der Sitadt und des seel. Re $\|$ grel, welehe walırselieinlich nun verkinft ist.

Abo (Fimland). Das UniversitätsMlusinum. - Die Sammlungen des Hru-von Alistedt, - Dammert, - Dr. und Professor Cirl Reginald Sali I horg, - T am s.

Adumbot (Obersteyermark, an der Ens). - Sammlumg des berilomten Benediktinerstiftes.

Ageru (Garonne). Summlung des Monseiguenr Saint-Amand. - Das Museum der Ackerbauresellschaft.

Arraun (C'roatien). Sammlungdes Semimarimos.

Äx (Dept. der Rhonemiindungen). Das Museum der Naturgescluichte de Stadt. - Das Moseum der Akademie. - Dic Samulungrn den Gesellschatt der Wissensehaften. - Ausserdem sind nuzufüluren dic Sammlungren des Proprietair Boyer de Fouseolambe, - Pharmazeuten Bo y e r, - und Grafen von $S$ a port $\mathrm{t}$. Stadt.

A.juccio (Corsiea). Sammlung der

* In dieser Rubrik sind die Sammlungen derjenigen resp. HIt. Naturforselier ete. aufgeführt, welelue von Seite $77-81$ als Nachträge mit eingeseholtet worden sind - Bemerkingen führt, welelie von Seite 7 biher dic Samulungen von audern Autoren sind bei ihreustelen angegeban wel, Briefe ïber

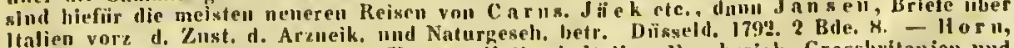
Dr. W., Reise durch Denseliland, Ungarn, |Bolland, lialien, Frankreich, Grossbritanien mo

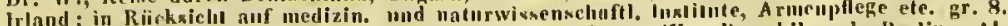
Berlin, T C. F. FnsIIn, 4 Bde. 1831-33, - Sir icker, W., Dr, philos. In Berlin, AlBerlin, T C. Fo Hoiltude interessaiten Orte, mlt Rïcksicht anf phabelische Uebersieht aller fiur Nafur- Ind Heilkinde interessuiten Orte, mit Ricksicht anf Jiteratur und Permonalitäten. I. Bindelien. Die dentsclian Bumdesslinaten, - Alleh unter dem Titel : allgemelnes Relactageboch fíi Aerzte und Naturforacher. Mil elner Vorrede vom J.elbarzte. llotrath, Ritter ete, Dr. A. F. v. A n mo n. Berlin (Llebmamn) 18\$1, gr. 1'?, (51/2 Bog. n. $s / 6$ Rthlr.) 


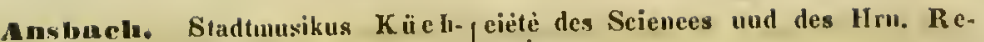
Ler hat viue Sammlung.

Antwerpen. Die Sammlung der Akalemie.

Arrsts (Dept. Pas de Calais). Capitain IIciurich Barou von Clıanveant ist hipr seiner schönen Collection wege॥ \%.u hesuchen.

Arnsberg. Regierungssecretiirv. Varendorff brachte ein niedliches Cabillet zusammen.

Armother (bei Schmiedebere in Schiesient. Die Sammlung des Schlossverwalter Te i cligräbcr.

Aschafreuburg. Eine SammInug der schädlichen Forstinsckten besitzt die k. Forstschule. - Dic Sammlung des $k$. Lyeeums. - Lehrer Däbner und $\mathrm{Hr}$. Dr. und Prnfessor Kittel vermelıren noch täglich ihre Summlungen.

Aschbnch (bci Burgwinthein in Frankeu). Landirzt Kress hat ein Kabinct entomologischen luhalts.

Aschersteben. Apothekm Hor. nung. - Die Collectinncn des Rector Liiben, - Lchrer Neuling.

Aska. Der ungar. Magninat, $\mathbf{H r}$. Freilicr Gabrici vou Pronay Thot-l'ranes mad Blatnitza Icgete sich ein Kabinet an.

Athen. Sammlnng der naturforschendell Gesellschaft. Diesc vou Bayern sestiftete Gesellschaft vereinigt die Fauna turciea, hellenica und argvetiaca. Der verdiente Oflizier H1: Fr. v. ZLentuer, Ritter efc. hat viel gesainmelt.

And (Bayeru). Landrichter Roemer ist im Besitze cincr ausgezeichneten Sammlung.

Augsburg. Kleine instruktive Sammlung vou der Forstkultur und dem Landban schädlirhen Insekten iu der k. Kreisland wirthsphafts- nnd Gewerbesehule. - Sammlumy tler Stalt- und der polyteclinischen Schule. (Die Samulung des scl. Prof, Ala ons ist Eigrenthum der Stadt.) - Dic Sammlungen des Apothekers Alten, - IBisehoft, - Kupterselumicd Fageroth. - Kassier Freyer, - Goldselumied Sialt miiller, - Dr. M. Wannes.

Anriol (bei Marseille). Medicus Marlog hat einc, vorzïglich entomologische, Colfection.

Antun. Die Sammlung des einen Scuninarimus.

Avienon. Dic Sammlung. der Soq แ ic n.

Avilu (im Königreich Alt-Castilien). Die mbedentroule Sammlung der unbedcutsamen Universität.

Baden (an der Scliwöelat bei Wien, im Vicrtel nuter dem Wienerwalle). Sammlungen mehree dortigen Naturalienhïndler, welche sich aus verschiedenen Gegenden wähnend der Barlsaison durt anflialten.

Eneza (im Königreieh Iacu). Sammlıng der Universität. Klpin.

Inatiunce. Collection of Academy of Maryland.

Gisunberg. Die schöne Sammlung des vor ct wa 12 lahren verstorbuen Director Li ll der: Legenwärtig Eigentluum (dureh Vermärchtniss des Begrounders) des Staates unter dem Titcl: "Linderistle St iftull". Conservator der Linderiselen Stilung ist, seit $183 \mathrm{~S}$, D1. Ha $\mathrm{H} \mathrm{pt}$, ein selur lüihtiger Forscher: - Die bedeutende Samminng des natnrtorsehenden Vercines laselbst, wovon S. k. Hoheit ler Kru tor ist. - Der maturforseliende Verein entstand in J. 1833. - Weiter haben Samnlungen folmende Privaten: Dr. und Inspuetor II aupt, - Di: med. Kireliner, - Rittmeister Spunsel, - Caul. Theol. Weisenfold. -

Banz IScluloss in Frankell, Sr. k. Hoh. Hem Herzom Maximilian zugehörig). Sammlune im Sollbsse, einer vorualigen berühmen BencelietinerAbtei. - Dir Sammlung der grisstentheils versteinerten Thiere, nach Cuvier's System geordnet und durelı den Camzleirath Thlı edori sehr vermelite lat der lientamisactuar Ho. IIerd z.110 Aufsicht Prlualten.

Burby (a. d. S.). Herme s'sche Sammlung.

Barcellom (im Fïrstenthum Catalonicn). Sammlung der Municipalitit. - Prolessor Campa, - Professor M. Griills, - Professor II inner, - Dou Ignazio Jorduno, - Proprietä Mariano Sans und Professor Y anez besitzen anschuliche Sammlnugen.

Enrlenun (England). Rector W. Kirby's reiche entomologische ColIection.

Barnaonl (Gouvern. Touns; Sibirieu). Stuats- und Medizinalrath v. 
G cbler liat meist Rossiea in seinem Kahinet.

Basel. Stadtmuseum. Die Samnz. lung der natuforschenden Gesellschaft, - dami des Professur Hauli ari, Dr. med. Ludw. I m hoff, - Maler David Labram, - Professor Meisner, - Professor J. J. Mieg, - Dr. med. J. C. Müuch, - Werthenmann.

Batavia (Java). Das reiche Musemn der dasizen gelehrten Gesellschaft.

Bntignoles (Scinc). Capitin Foulques de Villaret hat schon lange ein entomologisclies Cabinet.

Banue (Doubs). Ritter GirodChantran s' Sammlung.

Bantzeu (Selulesien). Comptoirist Geihárdt hat cine Sammlong von etlichen taısend Käfern.

Bayreuth. Sammlung der k. Landwirthisehafts- und Gewerbeschnle. Schr inlaltsicich. - Das grosse Museum des ( $\dagger$ ) Grafen von Mïnster (meist Petrefaetii).

Beanealre (Dept. du Gard). B a. ridon's Sammlung.

Beer Telden (Odenwalde). Pastor Wider's Kabinet.

Belem (westlieh vou Lissabon, an der Mündnug des Tujo). Hier befindet sich, mach der Versichernng eines Spaniers, in der Abtei der Hieronymiten, einc ziemlich grosse Sammlung von Nituralien.

Beichlingen (Scluloss in Thüringen). Dr. med. Ramdohr's Samus. lung vou Ciustacern und Insekten.

Bergnno. Athenäแm.

IBerge (Kloster-) loei Magdeburg. Dic Sammlung der Scluule in ehemaligen Benediktincrklostergebiude.

Berlin. Dic Sammlumg der Akademie oder das öfentliehe Museum; der grössten eines in der Welt. Sammlung von versteinerten Thieren des Director Klö de n, Mirjor v. Turte und Sr. Exe. des Baron Alex. v. Humboldt, dis aus allen Ordnmoen Thierschätac enthält. - Es finden sieh im Lokale des pharmazeutischeo Vereins und sogar des Cadetten-Corps naturlistorische Sammlunger. - Zu den Privatsammlungen gehören die des Dr. Auderseli, - Dr. med. Bernhard, - IIaudelsgïrtner F. C. B o uché, - Dr. Dierbach, - Hofrath Ehrenberg, - Professor and Custos Ericlison, - Dr. Fintel mant.
- Semiuarlelirer Gabriel, - Iouis Godet, - Buchbinder Graff, Professor Gurlt, - Natmralist Hemmerich, - Di. Hertwig, - Hofrath ind Professor Isensee, - Medizinalratl, Director etc. Fr. v. KI ug, - Körte, - Freiherr v. Kassow, - Dr. Kriiger, - Dr. und Lehrer Le y de, - Hofrath Di. Li uk, - Dr. und Arzt Mevers, - Hofrath und Director Johimnes Müller, - Gesandten $v$ Olfers, - (?) Naturalist Otto, - Nafuralist Philippi und Dr. med. R. A. Philippi, - Dr. und Professar G. C. R c je l, - Oherlehres Ruthe; des eliem. Buehhändlers $S$ e hüppel, - Professor Sc li ultz, - Oberichrer Selu uIz, - Stef tala"y, - Dr. med. Sticubel, - Dr. Troscliel, - Naturalist Virinoud.Die Sammlung der Gartenbau-Gesellschaft enthiilt sehr interressante Entomologica.

Bern. Das bürgerliche Stadtmuseum (an der Bibliothek). - Universitütssammluıg. - Notar E. Benteli, - Dr. Sam. Brinner. - Studiosus Brunner, - Offizier Ougspurger, - Rector und Professor, Di. med. et phil. Max. v. Perty, - Director Stettler und nocle melire Bürgec besitzen selıäne Sammlungen.

Resançon. Die Sammluug des Akademie der Wisseusehoftell.

Bessastuler (am Holmerlafen anf (ler Insel Island). Sammlung der gelehrten Schule. Meist islindische Thiere, Pflanzen und Mineralien.

Piei. Das Naturalieneabinct Jes Hrn. Vaeat.

ubingen (am Rhein), Carl W a g. ner's Sammlung.

MEruinglam. Naturaliensamm. lungr des mechanischen lustituts und des Thom. Marsh in II Eisy.

Eisiu (bei Tüplitz). Sammlung im Selılosse des Fürsten Lobkawitz.

witche (Elsiss). Dr. und Apotheker Fi: Schu It z lat ein Kabinet.

Boldernu (bei ltign). B. Ginmorthal's Sammlung.

molognn. Universitiats-Sammlung und die des lustituts; des lProfessor Bertoloui und Solun, - Dr. Negri.

Bombery. Sammlang der gelehrten Gesell sehaft.

B̈omin. Die Sammlungen der Universität, des uaturhistorischen Semjnariums und der niederrbeiuisclien Ge- 
sellseluaft fiir Natur- und Ileilkunde. - Dir Collection des Professor Goldfiss.

Boplorel (am Rhein). Dic Sammlung der liälern Stadtscliole und des Lehrer. Ba cl.

moraleanx. Museum academicum. - Mnseun der Mnniripalitit, der Linmésclien Gesillsilaft und dev Sociète do Sinte ef d'listoire naturelle. - In. habel von Sammlungen sind: Entomo. $\log B 1$ in nehard, - Prufessor Ray. moud Dargelas, - Lalaulle.Oftizier Lamberilzod, - Aug. lerrieres, - Negoziant Theodor Roger.

Boratiu (bei Ritibor). Oberfür. ster $\mathrm{Z}$ clue besizt einr artige Sammlung. Doston (Vercingte Staaten). Aeademiselies Mnscum. - Die Simmlung der Gesellseluaft für die Naturgeselrichto etre, die des Dr: Copley Grene, Nituralist Harris, - W. D. Peck. - Die Sammlungen des Dr. med. und Naturforscher Webster.

Beotzen (Tyrol). Chirnig B iitt. uer, Apolheker Kireher und Studiasus K noflauch haber entomologisclie Samulıugen.

BBodrg. Die Sammlung der Sociefi d"histoire naturelle.

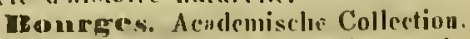

Esrandeaburg. - Vieweg's Siunulum.

Derasilien. Naturalist Lhotzky hat. unbekannt wo, bedenteude Sammlouren zusammengebracht. Walusebeinlich bereits anf europiiselaem Boden.

Ermunsbereg. Dr, und Gymuasialoberlelurer so it ing e lat eine Käfersammlın!

Is ranuscloweig (Wolfenhütel). Das Museum der Naturgeschichte im Collegium Carolinum. - Mnseum des Herzags. - Die Sammlungen dros Forstrath und Professor's Hartige, Dr. Lat chm it n und F. W. L, induer.

Bramea. Städtisches Minsenm. Die Simmlong der niturforselsenden Gesellschaft. - Norwieh, Kaufmani R. Pagenstecher, Arzt Schmidi, Protessor L. Chr. 'l'ueviravus (Solni) und Dr. Wilkens liaben interessantp Samulungen angelegt.

Erescis. Die Simmlung der Societi de la staria naturale.

IEresian Die Sammlung der 1702 gestilfeten kitholischen Universitä. Die: Sammlung der schlesischen Gesill. sehaft fïr vaterliandische Cultur. - Dic
Sammlnog der Realscliule und der na. furforsclienden Gesellseliaft. - Die Na. meld der Privaten. die Kahinete (entomologisclie meist) hesityen, sind: Professor Di. B a rkow, Graf ron Be roldlinge "Graf Cumbad r. Dy li r n, Dr. Figulus. Friedrieh, Dr.H. Frauk,

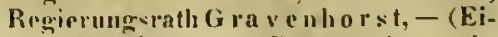
"le्I Berrilf vou Gravenhorst's Sammlung griebt dessen Schrift: Vergleichende Uebersicht des Limui"sclurn und riniger neurn zonlogiscleen Srsteme. Nebst dem Verzeichniss der zoulogiswlen Sammlung des Verfassers uncl deu Besilureibungen neller liliere. (Böttingen, 1sez. 8.) C. Gloger, liallzellist J a e useh. Gymnnsial.Lcluer Klopsili, Korllat\% ki, Jactiner, L ïblielt, Dr. umbl Leher Mat\%ch, Neustadl, Dr. Profressor und Prïses Ners v. Esenbuek, OlectMedivinilratl Dr. () tto: Oberlelierer Reudscltmidt, Institutsiluree E. F. IV. Richter, Conservater Roter-

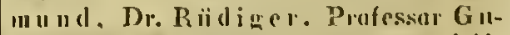
stive Sul illiun, Lecher Silm. SchilIi ng, Kerflüudler Srhilling. Dr.

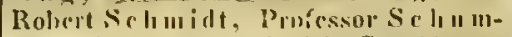
mel. Dr. mude tleiurich Steftens, Frrilure ve Ucelot rit\%. lis. Wimm er. Erest. Gallu al's Sammlung.

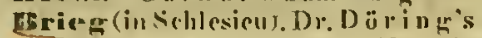
Collictiont ind des sphlesiselien Tauschverinills.

Baristol. Museum des Instituts. Dic Simmlumg Milla rd's.

EEroraley (bei London). Alb, Forster's Sammlum.

IB riigse: (FIandern). Dic ausclulirhe Sammlmurg dev naturtorsheuden Gesellschaft.

Brilou. Stadtmuseum. Das MIıscum dev milıriseli-sorlulesiselien Gesellselialt. Hr. Professar D i cbl, Rechnungsratl K upido. Kreisheauter Anton Miller und Beamter Rau besitzen atuscerdem nach Sammlungen.

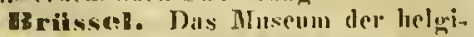
schen Akanlemie der Wissenschaft. Sr. Mlaj. Ra e polal I. Kämig der Belgirr, Director und l'rofessor D rapie\%, Beruhard Ritter du bus, Sirhayer, Sclinermanu, Barou vou Woliens aul C. W estma a l besit\%en die anseluliehsten kiahineice.

IE rismague. Seluensnertle. Samnlunge der Baronin von Wälmout io Selilusse bei Brumagur.

Bucharest. Silmunlung vou allen 
Niturgegenständen, angelegt durch den derzeitigen Hospodar der Wallachei.

Burgdorf (Cantou Bern). Hauptmain L. R. Meyer's Samnilung vou linsckter.

Burgneld (England). C. H. Smitl, Bird, Rev. hat eine schöne Insektensamumlung.

IBurgwintheim (Franken). Revierforster Seli id el's Sammlung von wirbellosen Thieren.

Caen. Das Museum der Akadenie. Blot's Sammlung.

Cagliari (Sardinien). Sammlung der Universilït daselbst.

Caloork. Dic Sammlung der Akademie daselbst.

Caicutta. Ueber Sammlungen und Museen daselbst, vel. Caleutta-Jourual I. p. 137, voll II'CleIland. - Mureum of asiatic Society. - Musemm of agricultural Society und der medieal and physical Society. - J. T. Pearson's Sanım?ung.

Calinar (Seliwedeu). Museum des Gymuisiums daselbst.

Cambrian, Museum der wissenschaftlielicu Institution.

Cambridue. Universitïtssammlung. - Sammlungeles John.s. Colleginm. - Professor C. C. Babingtan's Sammlung.

Caploman (England). William Bennett's Sammlung.

Capistndt (Afriku). Landesmuseum, gegrundet durch den Wiirttemberger Freiherrn von $J_{d}$ ulwig. - Sammlungr der naturlistorischen Gesellsehaft des Cap der guten Hoffmug. - K rebs'sche Sammlung und des Banquier Barouv. Lildwig.

Carisloure (in Sicbenhürgen). In Jalıre 1798 errichtete der Bisehof Griff Igna\% Bathy any discellost in der Festung eine hibsche Sammlung.

Carlsrulue. Sammlung im Gymnasium illustre. - Sammlung in der grossherzoglichen Residen\%. - Sammlung des polyterhuisehen Instituts. Unbecleutend. - Dic Niedellage des Händlers Apkermann, die Sammlung des Professor Alex. Brann. Scluiekel und Dr. med. mud Arzt Wa/chuer.

Crondsstadt (Würmeland; Sehwe(en). Naturalieneabinet des Gymnasiums.

Casan. Universitäts-Museun.

Cassel (Niederhesseu). Sammlung von Naturalien in Musenm auf dem Friedriblsplatze, - Die Sammlumg der uatufurschenden Gesellsulaft. - Zuhemerken sind: Arrhirar Glasewald IIId Hauptmann $\mathrm{Niepold,} \mathrm{welehe}$ Samm/nngen besitzen.

Castelumurary (Aude). Proprietair Andreossy's Sammlung und die des Medieus Encely, des Proprietuir $\mathrm{G}$ a u r. y und Pharmazeut Viala.

Castelnuovo (bei Cerboli). F. Luciani's Sammlung.

Catauin (Val di Demona, in Sieilien). Museum der Universität und der gioeniselien Akademie. - Die Samm. lumg des Professor F errara.

Cayeme (France équinoxiale). Militiirehinrg Jaquier's entomologiselse Collection.

Cntwkil (New-York). Das Iyceal. Mu-cum.

Cervera (Catalonien). Sammlung der Universität.

Cette. Die Sanmlung der naturforschenden Gesellsrhaft.

Chalans sur Mnrue. Naturaralipurabinet (ConservatorD. M a hieu). - Die Sammlung des Dr. Dagonet mud des Arzts nud Conservator's Mah i e u (besondlers).

Chàloms sur Saône. Negoziant Arvisenet's und Dr. Bastard's Sammlungen.

Chambery (Savoyen). Sammlung der Airademie des Sriences.

Chomboral (Depit. Loir et Cher, auf (1.m Insel des Caussou). Sammlung iin präiclitigen Sclılosse.

Chambray. Sammlung der naturforsclumblen Gesellschaft.

Cinmeregg (Niederhayern). Freiherr von Hallberg-Broieli's sorgfillig bestimmte Sammlung.

Charkaw (Slobodisclie Ukraine). Sammlung der 1803 gestifteten Universitit. - Professor Kry ni cky's Sammlung.

Charlestovn (Sïl-Carolina). Museum der Literary and philasophieal Society. - Die Privatsammlungen des Professor Sliepard und des Doetor Wu rilemann.

Cin rlottenbura. Apotheker Stciu's Kabinet.

Chartres. Advokat Guenéc's (hier und in Paris) und Marchand's (sen) Sammlung und die des Capitain Franz de Villie es.

Chateaudun (Eurc et Loire). 
Gullla umé (hier und abwechselnd zu Paris) hat eine Sammlung.

Cheisen (bei Londoni). N. A. Vi. gors, Esq. besizt eine uuspezeichnete Menge von Naturalien.

Clecmunitz (Sachsen). Die Sammlung der Gewerbs- und BaugewerksSihule.

Chenes (hei Luttich in Belgieu). Cayl Robert's Sammlung.

CInristimin (Norwegen). Museum der Universitit und des physiographi. sclien Vereins. Die ganze nurdisclee (bisouders norwerische) Faund entlaltend. - Die Kabinete des Dr. und Conservator Heiur. Rasch und des $\mathrm{Na}$ turforkcher Sie bke.

Chur. Contonalmuseum und dic des Hauptimann W red ow.

Cincinsti(O)ho: Nordamerika). Die Samulung derWestern-Museum. Society. Die Sammlungen der Herren Lea und $A \cap t h \cap n v$.

Citta-Vecluin (Insel Lesina in Dalmatien). Dr. Niseteo's Sammlungr.

Clanstlas (Hannover). Sanmlung der Forstshule und des Malers W. Sn$x \in \operatorname{con} n$.

Clernuont (Auvergne). Museun der Akidemie dascllost.

Coburg (Salisen-Cobnrg). SammImor des akademisclien Gymnasions, genanut Casimirianum.

Coimbra (in der portugiesischen Iandsrbaft Beim. an Mondego). Sammlung de. Universitiit; gross und scliön.

Colmar. Alvokat Cauteruer's Sommlung (meist Lepidoptera).

Colombier (Canton Neuenburg)

Pfarer Gelien's Sammlumg.

Concise (t. (liweiz). Pfarrer Mel. let hat eine huibsehe Käfersammlung.

Constuntimopell. Gicsandtschuftssecretiir Baron von Cachoru hat sich cine Insekten- und Lurch-Sammlung :ugrelent.

Coustanz. Stadtrath Leiner, Banquier Macaire, Dr. med. Marmor, Dr. und Professor Ne nI in in und Ritter von Seyfried besitzen artige Kerfsammlnngen.

Copenhagen (vgl. Kopenlagen).

Corilova. Sammlung des verstorhenen Dr. Itaz erini ans Italien. Soll verkinft seyn.

Corfu (Jonische Inselu). Universitäts-Snnוmlıng.

Cosfela (bei Mïuster in Westplıalen). Sammlung des Fürsten Salm.
Crefeld. Die Kăfersammlung des Hru. Emil vouB ruck. - Die Hön ing. ha o s'sche Sammilung.

Cremona. A potheker B e rtazzi's Coleopterensammlung.

Crénemezlk (Volhynien). Andrzejuwsky hat eine huibsehe Collection.

Cinbr. Dr. Grundla eh sammelt noch anf dieser Insel und das bereits Zusammengebrachte soll betrāchtlich seyn.

Darmstadt. Museun der Naturgesehichte. Mit amerikanisehen Exoten bereichert durch die grosse Dutation des Hrn. v. Höpfner. - Die Sammlung des Hauptstatskassenkontrolleurs Cä un ure r, des Realschullehrer $\mathbf{G} I$ aser und Oberappellations. Geviclitsruth v. Hijpfuer, sowie des Dr. und Custos Kaup, des Oberlientenant Klingelhöf er und Oherforstsecretür Re issig, Dircetor Schleifer.

Denzig (Westprenssen). Das grosse Naturilien-Kabinet der Stadt und der naturforschendeu Gesellsehaft. - Sammlung des Dr. med. Berendt, Prediger B o ckund Dr. und Oberlehrer S. h m i d t.

Daruey (Vogesen). Deputirter L e$p$ a ife liat viel Seltenes aus der ento. mologischen Welt.

Delemont (Bern). Verilat's S:mmolung.

IDeluenunget. Dr. Oppermanu's Naturalien-Kabiuct.

Deptrord (Enuland). Edward New in a nu's Coleopteren- und Hymenopterensimmlung.

Designe (New-York). Dic Sammlung der Nitional-Akademie. - Die Sammlung des Professor Majpes.

Digue (Dept. des Basses-Alpes). Postlirektor Ho nn orat's nuselmliche Srhïr.e aus der Natur. Melcli. Yv a n's Sammlung.

Dijon. Sammlung der Akademie der Wisaenschaftell. - Professor C'hri. stol, Lacoste, de Lamnrek, Lanier, Di. Vnllot habeu Sanımlungen.

Douno d'ossoln. Biedermanu (Sohn) lnt dort stine, meist piemontesiselien, Vorritthe.

Douruescleingen. Die schöne Sammlung des Fïrsten von Fiirste nber.g.

Dornbnre (a.d. S.). RectorE a ck's Samm!nng.

Dorpat. Universitätsmuscum. - 
Dr. N. As mus hat eine Auswalıl von Kerfen.

Iortuund (in Westphalen). Toussaint de Charpentier's entomologisclier Reirhthum.

Dortreclut. Sammlung der natur. forselienden Gresellschiaft vouSiidholland.

Dreinsigneker.

Auschuliehe Forstinsekteusammlung des Instituts.

Dresulen. Naturalienkabinet in dem Prachitgebaiide, der Zwinger, welclier aus 6 Pavillous besteltt. Sehenswerth. Viel Exoten. - Director davon ist der verdienstvalle Hofrath Dr. vou Reielsenbach, der es auch in einer besondיru Selarift beselurirben hat. Medicinalrath Dr. Ritter von Carus, Graf v. Corberau (waluselicinlich); Hofrath vou Ficiuus, Maler Aug. Harzer, C. F. A. Ha rzer (wird wobl nur eine und dieselbe Persou seyn). Director Kade u, Hofrath und Professor Di:v.Rriehen ba $\mathrm{e}$ h besitzen luibsche und wohlguordnete Sammlungell.

Droutuciun (Norwegen). Sammlung der Akademie dro Wissenseluaften.

Dublin (1rlaud). Grosse Sommlungen der Universität. - Königliches MInsenm. - Eine Collection lat noels W. Jones.

Dif rbe en(Curland).Pastor Se boen's Sammlıng.

Diiren. Des Papierfabrikanten Sehiill Sammlnng und jene des Forstmeister's Steffens.

DukJa (an der Jasiclka im Jasloer Kreis: Galizicn). Scllöne Sammlung im Bernhardinerkloster.

Diisseldorf. Professor Hildebrilndt'selie. Sammlung.

Duninowe (bei Kowal in Polen). Obertörster H. v. Alers lat Insrkten.

Dux (in Lritmeritzer Kreise ; Bölımen). Naturaliensammlung in dem sehenswerthen Schlosse.

Ecluallang (Waat). Drs Priester Ad. Chavannes Collcetion helveti. scher Kerfe.

Ediuburgh (Königreich Schottland). Herrliclie Sammlungen vou Niaturalien im Lokale der Universitït. Collectinneu habeu ferner: James D unkan, Dr. Mackenzic, J. Stark, SirPatrick W a l k e r, Graf zut Edinburgh.

Fibenstock (Saclssen). Oberfür. ster's Thierseh Sammlung.

Eichstioldt (an dre Altuïhle, in Bayern). Die bedeutenden Sammlun- das richtigste bestimmt); damn die des gen uus Brasilien, aufyestellt in vormaligen biselüflichen Rersilen\%s:hlosse. Sie sind ein grossartiess Andenken an die Bemühlumgen und den Eifer für die Naturvissiduseloaft eines Naturforsthers, cles hüchstselimen Königg A ng us t v ou Portugal: grhorcnen Herror von Lruclitenberg. (Vgl. G istel's Bingraphic Wauler's in dem ersten Bande des "Fannus".)

Finsiedeln (Cauton Schwyz). Sammlung des Benediktiuerklosters.

Eiscunterg (Sachsell). Diakon K y lar r's Sammlurig. recs und Han in laben Samolungen.

Elbcrfeld. Inlurer C orne li inssoche und Dr. Finhlroth'selie Colleetionen.

EIdeua (bri Greifswalde). Die Sammlıng der Akademie und des Lehrer Gichr.

El isabethgrad (Clicrou). Bochniak und Dr. Verlein sammeln schon lange und haben viel des Soltenen zusammengebraldat.

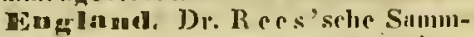
lnng. Ist ans London, eimrm Selureiben zonfolge, nacli cince andern Stadt translocint worken.

Ganoutedis (Lappland). Grappersche Sammlung, riue Qurlle mancliet entomologischen Atbeit (ist mulsckaumt, ob er zu Kartisuando oder Euontekis seine Wuhuung anfgeseluligen).

Evpiug (iu Eugland). Edward Double dity lut dis riuce, "fine Collection of insects" wie uns Smith scluriobt.

Defurt (Prenssen). Die Sammlung der Universitit. gestiftet 1392 - Bedentend ist die S:mmolang Iles Geriobtsratles Kof'orstein. - liines besnehes werth sind die jiugst angelegten SammIungen des Privatlelueer A. Selimiclt, Scminarial-Lelures Striibing mud des Laudesgerichtsirath v. Wrissen hor n.

Erlanges. Universitits-Musem. Recich an ruroptiischen Inschten. Eine hedentende Anquisition wird sie durch Hrn. Rosenhauer's Sammlung (cirea 10,000 enropaiselue Kerfe) rrhalten. Director Prot: Siebold: Custos: Dr. Rosenlaner. - Die Sammlun des Professur Nartius, des Holl. Clur. H a ud selu ue la, Dr. und l'rof. K üster, Dr, med. und Conservator W. G. Rosenla auer (schr pechlaltig und auf
Euatérinosiawl. Dr. Frey- 
beriihmten Director und Prof. Dl, v. Siebuld.

Wrinsturumn (Schloss und Marktflecken im Viertel unter dem Manhardsberge). Sammlung des Fürsten Prosjier v. Z $i$ un $\%$ ullorf.

Wscluveiler (hei Stollberg). Director Griser's Sammlung und des Jugendleluer Schle hek.

Essex (Ungarn).

Apotheker

H a usclak a hat sich sehou vor viclen Jahren cine Colcopterensammluug angelegt.

Fsslingen (im Neckarthal), Samm. lung des Schullelurer-Seminars, - Sanmlinn dec Ober-Realschule.

Disterlaze (Oedenbuiner Gespanschaft in Ungaru, an Neusiedlersee). Die kostbaren wissenseluaftichen mod Kunstsammlungen im fürstlich Esterloazy'sche\| Sclulusse.

Típen. Lebrer Fassbeuder's Sammlumin.

Purresbers (hoi Augsl)urg). Re. vierfirstec Carl Ma yer hat sehon vor melureu Jahren eine Sammlung, besonder's von schädlichen Forstkerfen, augelegt.

Falaise (Dept. de Calvados). I a Frenaye's Samumlung.

Felalsbers (in deu Viertel unter dem Mamulardsberge, in dem Laude un. ter der Enns. in Oestreich). Collection im Schlusse des Fürsten von $L i c h t e n$. stein.

Ferrara. Sammlung der Universitiit.

Finsterlnergen (im Thüriugerwalcle). Revierfötster Kell Ie r's Sammlunn.

Wattracle (Kiiluthen). Conperutor Gussen bauer hat eine Sammlung vou östreichischen Kerfeu, (Lis ist bis datn noeh nieht ermittelt, ob dieser oder Treffliun sein Wolunsitz ist.)

Florenz. - Sammlung der Universitiit und die Sr. k. IInheit H copol d II., Grosslierroy von Toscana. Die entomologisclic Sammlumg des Grossheryogs ist unter Passerini's Leitung im Entstelien. Bis dato meist Exoten, durcl Farbenschmuck ausge. zeicluet, wie durelı Grösse; dann jene des Direetor V. Antinori, B. Corti, Graf Pietro Guiciardini, des Professor mul Conservator Carlo Passerillidi Patrizia, Dr. Victor Pechioli, Willian Blondel Spenee und Rubert Heinleh Spenee, des Dr. Targi oni.

Frnuldfurt a. Mr. - Die Sanmlung- ier Senkenbergischen Stiftung. Eime der grössten und wisseuschaft. lich georduetsten in Deutsehlaud: dureh Rïppel mit ungeheuren Schätzen hereiehert. Director ist K retz m a r. - Privatsammlungeu sind die des Senator $\mathrm{H} \mathrm{rn}$. vou He y deu, Hrn. Dr. uul Professor Kretsclimar, des Hrn. Riese, des hochberülumten Forschers Hrm. Dr. Erluard R ii ppel, die J.J. Sclı ot t'sche und die des Dr. Zelle r.

wraulufurt a. d. (D. Metzner und Tëlitê E. H. Ríçliter sind hier die eiuzigeu, die Sammlungeu besitzen.

Frauzensbad (bei Asrlı in Böhmen). Di: und Brumenarzl Pallia rdi liat schon vor 30 Jahrem cine Collection geliabt.

wreiburg (Breisgau ; Baden). Samulung der Uuiversitât. Nirht selir reichbaltig au Kerfen. - Sammlung der Gesellschaft für Befürderung der Natırwisseust:laften. - Ueber das akademische Muscum lat Hr. Prof. l'e rle b eine eigen: Beschreibung erscheinen lassen. Prosector b ougine und Profissor Dr. C. J. Perle b haben wissenschaftich geordnete Cabinete.

Friedwald. Kreisplyysikus Dr. B a 11 er's sammlung.

Evitluwiller. Strauss-Dïrkh e im (vgl. Paris) hat hier cineu Theil seiner Sammlungen und Präparate.

Fitnfkirchen (Siid Ungam). Pharmazent Nendtvieh's Saumlung.

Fulua. Del churhessische geh. Statsmiuister Graf vou Sclumerfeld legte sich eine schiine Collection an und ist ein eifriger Befördererder Naturwissenschaften.

Fadalming (England), Rustiens (ist wahrscheinlich ein Nomen adoptivuul). Soll rine nicht geringe Anzahl von Culeopteren Englauds besitzen.

Gramsen (bei Brieg; Wallis). Kerfhändler W. A nderegg's Vorràthe.

Gantz (Steyermark). Se. k. Hol. der dur(hl. Evzherzong von Oestreich, Jo h a n n, besitzen Ansgezeichnetes aus allen drei Naturreichen.

Fanrz (Pommern). Des Superintendenten Tripke Samulnug.

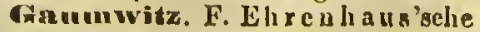
Sammlung. 
Gefle (Stapelstadt in Gïstrikland in Sthweden, an bothnisehen Mecrbusen). Gymnasial-Sanmlung.

Gemarke. J. W. Stein besizt eine uette Sanmmlung.

Gienr. Stadtmusenn. Sebr reiche und brillante Simulung unter Pictet's Conservatorium. (1836 vermelirt dureh Aukauf von Prof. Louis Sordet's Simulung.) - Alles durch freir Beiträige seiner Bürger zusamnengetragen. Herrliches Genf! - Als sehr sehüne Sammlungen sind anfzufiiluren die des Hrn. Banquier Ch e v rier (Rue Corratière. Nur Europäer; aber ziemlich vollständig), des Professor Chu is y und Gesandtschaftsagenten Cbo is y; die wichtigen Sebast. Ju rin e's, Anditor Lasserre's, Advokat L'buillier's, des Grafeu Ignaz vou Mielzinsky, des Negozianten l'révostDuval, des Professor F. R. Jnles l'i i:tet (Solın) und endlich des Polizeilieamten Walluer.

Geut (Flandern). Sammlung der Municipalitit und der naturforsclienden Gesellschaft in Flandern. - UniversitätsMuseum (besonders orientalische Conchylicu). Athenäumssamulumg.

Geman. Don Carlos Dnrazzo's Sammlung ist selienswerth; die des Marquis Max. de Spinola ist wobl eine der reichsten Privatsammlungen in der Welt.

Tieoreriefsk (Gouv. Caucasus). G j jibot'fsky's Sammlung.

Gxera. Kaufmann llerm. Se h midt'sches Cabinet von Insecten.

Cxicusen. Petrefakten-Sammlung des Pfarrer M $n g$ nus, darunter fossile Kerfe und Krabben.

Giessen. Sammlung der Univer. sität - (gestiltet 1607). - Fernere Cabinete sind angelegt von dem Medizinalratl Balser, Hrn. Eng elberg, Prof. Seliweigger und Dr. Wet. ter.

Flasgow (Sïdschottland). Sammlungen von ausserordentlichem Werthe, iufgestellt in Gebiumle der Universität. - Sammlung des meehaniral Institut. - Dr. Ho oker's Esq. ausgezcichnete Käfersammlung, vorzagglich aus den Tropell.

Antz. Kaufmann und Stadtaldermanı Pohil hat ein Naturalieneabinet.

Filauchan (vor den Thoren von -Halle). Die Sanmlung von Naturalien der gelehrten und Realschule und des grossen von August Hermann Frauke gestifteten Waisenlauses.

Glocinitz (Kiiluthen). Couperator B a c h e r hat cine uied licheSammlung.

Glogran. Oberlelirer Zall r r'selic

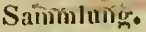

Cabrlitz (Schlesien). Sammlung der naturforstienden Gesellsehaft und des Hin. Pastor We igel.

Gx \&rz (Friaul). Oeffentliche Sammlung des Gymnasiums.

Gr̈bttinuen. Mnsenn der Universitit. - - BI II on en bae h's Sammlung. - Die Collection von Professor Berthold, F ehler, Professortl a usmann, (i. G. Koller, Professor Rudolph Wagner.

Gulhlis (Sehlesien?). Spröde'sche Sinmlung.

(rotlen. Die Braun'sehe und 11 of sche Sammlnng, vereinigt mit dem Naturalien- und Kunstkabinet im Residenzschlosse Frieden st e in. dieht an der Stadt auf einer Anhöle. - Dic Sammlungen des Baron von Sehloth e i m und Dr. Seetre e. - A. B a umh a ch's Sammlung, Dr. Desberger's Sammlung.

Gothenbnrag (Seluweden). Oeffentliches Musenm. - Gymusialsammlung. Gottweila (Oestreich). Sammlung des Benedictinerstiftes.

Gran. Dr: und Professor Georg Carl $R$ nmy besizt ein interessantes Cabiuset.

Grannda. Universitïtssammlnug. Reichlualtigund meist Exutica enthaltend. Grỉitz (Steyermark). Die vaterländisehe Sammlung des J o han u e um's. Fast alles, was Tyrol und dic Steyermark bictet. - Wappen-Arehivar C. H. B. Grimmer'sche Sammlung. (Europäische Colcopteren.) - Die Sammlung des l’rofessur und Custos Hal $\mathrm{t}$ m ey er.

Creirswalde. Dic Universitit hat cine instructive Sammlung. - Die Sammlıngen des Dr. und Professor, sowie Director Hornsehueh und DI. und Professor Fr. Clir. Rosenthal; daun des Couservator Sehilling, Hofrath Se hultze und Hro. Weigel.

Grenoble. Stältiselies Museum. - Conservator's Crepu Sammlung.

Grindelwald (Berner-Oberland). Pastor Rud. Miuller lat sich cine Sammlung, vor mehren Jahreu schon, anyrelegt.

Gribingen. Sammlung der Universitiat (gestiftet 1615). 
Treosenvaraleln (Uugarn). Advokat v. Fa rkas lat rine Sanımlung uIIgariseher Coleopteren.

Eranwitz_dSchlesien). S. A., Freilewr virkwit zesizt eil Insektenkahinet.

Ansteig (bei Saarucu in der Seluweiz). Pastor Hliss'selic SehmetterlingsSammlıu世.

finuzenhauseu (Bayeru). Lehrer Fehr'sche Lepidopterensammlung mud die Käfersammlong des Baron vou We lser.

Irange: (bei Friesack ; Ubi? Wahrseheiulich soll es heissen : in Friesland). Ischer Mo ritz lat rine Sammlnurg.

Harurlean (Siidloolland). 'T a y lersches Musenm. - Naturaliencibinet der batavischen Societat der Wissenselhaften (gestiftet 1762). - 13. Anslyn's Sammlung.

Talberstadt. Sammlong des Dr. Nicnlai und des Rittmeister E. von Truselike.

Mall 'Tyrol. Bergrath ond Dirertor Jos. Russegger'sele Sammlung.

Haile. (a. S.). Dir viammlung der 1694 gestifteten Universitit. Reicli und instruktiv. (vgl. Glaucha). - Dum die Naturaliensammlung desthatigen Inspector Dr. Buhle, des refeierten Professor Dr. Bulmeister, die hekanute grossartige Sammlung des berïhmten Director and Piofessor. Germar, des Dr. K a u I fus s, Dr. uorl Aufselier If uinter, Dr. med. Nieola i, Dr. med. W. H. Runde, Praf. G. Suckow, Dr. Herm. Rad. Seh a um, Sailermeister Fr. Schlüt ar, des Prof. Strack umel Schullehrer Zs selio or II.

In mburg. Stadtmoseum. Sammlnug des naturwissensehaftliehen Vereines, - Sammlung des JohaunesGymnasiums. - Röding'sches Museim. - Struve's Sammlungen. Die ungeheuren Vorroithe des ehilieben, weitgereisten und gehildeten Naturalieuhüudler's Beske, des Naturalisten Dregi, Dr. und Lehrer J ï ger, Dr. und Professor Is elim ann, Dir, Sl ee tz, Legationsrath v. Struve, Pharmaze of Thorey, Kaufmann W. v. Winthem, A. v. Winthen und Prof. Wiebel.

Hanelu. Lelırer Pflïmer.

Trusa. Versehiedene Seltenheiten aus der Natur sind aufbewahrt in den weitlïufigen Sehlosse. - Das Museum der naturforschenden Gesellschaft der Wetterau. - Commerzienrath I cisI e r's Sammlıng.

Manuover. Hege wit seh, Lehrer $K$ r ösman $n$, Dr. und Lehrer $M$ ii hlenpfordtund Spangenberg haben Cabinete. - Sammlung des Gartenbauvereins.

Ilavanna. William Sharp Macleay (Vater), Alex. Macleay (Solin) und Professor Raymond de la Sagra haben grosse Sammluugen aus alleu Zonen der Lïde.

IInve de frrace (Dep. d. BasSeine). Sammlung der Monicipalität.

Heidelbers. Die naturwissenschaftliehen Sammlongen der 1386 gestifteten Universität. - Die Samnlıug der Gesellsehaft fïr Nator- und Hcilkmude. - Dic wissensclaftlich geordnete Sammlung des Dr. und Professor Bisch nff, die Petrefakten-Sammlung des berühmten B ronn, die Sammlungen des Dr. Jol. Brotz, Studiosus Frant zills und Hofiath $K$ app.

Meiligenkreux (im Wienerwalde, Oestreich). Die Samnılung des Stif. tes der Cisterciensernonuen.

Gerununstadt (Sicbenbürgen). Die Petrofakteusammlung Brncken. tla l's (mit fossilen Crustarcen ete.)

IDeruaisami (Scliweden). Im Gymnasinu befudet sich cinc Sammlung.

Hexenacher (bei Remensbure). Dr. von Weidenbato'sche Käfersammlung.

Mildeshein. Stadtmusenm. Des lieruilniten Professor Lennis Sammlung, so wie des Rector $\$$ a uder. Dirschberar (Schlesien). Beamteter Felirle, Major von Flotow und Stadtverordueter Langer liaben Collection'u.

IInluemelbe (anu Riesengebirge). Sammler H a in k a's Verkaufsvorrithe.

Mohenfurt (bei Budweis in Bähmen). Wundarzt Kaj. Nenniog loat auselunlieh viel böhnisehe Naturalien gesimmelt.

Molnenlnein (bei Stuftgart). Sammlung von in der Forstkunde und Aprikoltur wichtigren Kerfen des land. wirtlisehaftiehen Institots daselbst im Schlosse (parterre), des Professor Theod. Mơgling und des Gärtner Scli ïle.

Hof (Bayern, siichs. Gränze). Der Dr, med. unil praktisclie Arzt Selooen liat viel Sehönes von Käfern, wie auch der Patrimonialriehter Bars ch. 
Momberg vor aler Milite (Fïr. stenthum Il essin). Sammlumig des ForstLehrinstituts daselbst.

Mudson (New-York). Das Museum des Lyceum of intmral listory.

Hnil (Eugland). Collection of mechanical Institute.

Mundisburg (bei Magdeborg). Herm. Nat la sius besizt cine Sammlung vou Thieren aller Clussen.

Ingenbroich (bei Mlontjoie). A. Graf zu Stollber g'sches Museom.

Ingulstaut. Rector Dr. Bauer's Sammlung instructiver Naturgegen. stïnde.

Inuspruck (Unter-luntlaal, Tyrol). Ferdinandrum. Inländisches blos, aber ziemliclı viel. - Summlung les Ly eroms.

Inverness(Nordscloottland). Sammlumg der vortrefflich eingeriehteten Akaaenie.

In-Zzell (bei Truonstein in Ober'bayeru). Revierförster G. MI a yer's Pflanzen-, Mincralien- nnd Thiersamn. lung.

Ipswiell. Jerm's Sammlung.

Trkozk. Thermin's Käfersammlung.

Trsiagen (bei Tärkheim). Pfarrer M a y r summelte manches Seltene.

Jncolos Ingene. Justiz-Commissarius 'Tiede hat ein Museum vou uiedern Thieren.

Jussy. Sammlung der naturforsehendeu Gesellschaft zu Jassy (Moldaii). Slürke aos 90 Arten bestchend, worunter Elephas asiaticus, Myrmeco. phaga jubata und Ornithor'hymehus paradoxus. Aus der Sammlong von Vöguln, die writ reicher ist, fiillt ein lecrlicher Lophophoriss anf: Lulleche fist alle mol Janischen und ein herrlicher C'roeodilıs niloticus; Fische der Moldau, besonders ein grosser Aceipenser; Kerfe wenirge. Dem berïlomten Professor

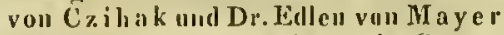
verdnukt die Gesellschaft die Sammlung sowohl ihr Entstehen, als auch diese werth vollen Sidratze, welehe dureds unauspesezten Eifer tantioglici sieh vermelutt. - l'rofessor Di. J. v. Czihak riclitet fïr sicls und das Museum des Hospodar's Sammlungen ein. - Dr. med. und Leribit\%t Joh. Edler von II a yer ist gleiclifalls bemülat, immer melu beizutreiben. - Ifospodar M. Sturdza's Samulung.
Java. Overmeer van Fiseher's erbeutete Schïtze.

Jena. Sammlungen der (1548 gestifteten) Universitat : ferner die Sammlung im Sehlosse. - Dr. und Professor II is clike's, Dr. Kocli's, Pfarrer' Krin us e's, Professor Theod. Th ou's, Hof rath und Professor Voigt's Sammlnugen, moist von wirbellosen Gesehïpfen.

Jolimont (bei Bern). Seluottlewortli's Pflawzen- und neuangelegte Kerf-Sammlung.

Ir misers Innterı. Sammlung des rheinischen Apothekervereins. - Städtiseles Naturaliencabinet (Vorstand Dr. 33 ('n he im). - Die Simmlung des Dr. und Lehrer B e in h e i u.

Eamichloff (Gouv. Perm). S o. mo t'f's Küfer'sammlung.

Kantsclnatlan. Kouz miclitcheff'sche lusektensammlung Sibiviens.

Throsunudo (Lappland). Priipositus Grape's Sammlung (vgl. Enontekis).

IRsan. Professor Eversmann's Cabinet.

Irassel (Hessen). Gundelachsche Collection. - Oherfinanzkanzellist J unke r, Burgeraf nnd Castellin Lit ndgrebe, Compagnir - Wunderyt Dr. M̂̈öler, IItuptstatitskassenkontrolenr Rieh I und Oberforstrath v. S chw arze n berg besitzen hübsehe Kerfsammlungen.

Ifatliarincinlof (bei Flensburg). Iraus Jordt' sche Sammlung.

In atshIII (New-York). Das Museum des Lycemm of natural bistory.

Ifendal. Natoralien-Museum.

I Keremsli (Gouv. Penzi). B ek lemicheff's Sammlung von Kïfern; die des IIn. von Ranzoff.

Helel (Holstein). Sammlung der Universitait (gest. 1665). - Sammlung des seligen Fabricius. - Städtisches Naturnliencabinet. - Bedeutende Sammlongen finden sich in den Wolnungen der II. H. Justitiarius Friedrich Bo i l", Dr. Drewsen, Meves, Dr. Trentepo hl and des liebenswürdigen, hoch. verdicuten Etatsrath Dr, vou Wiedema III.

Kiev (Russland). Sammlung der k. k. St. Wladimirs-Universitit (gestittet 20. Nov. 1833). - Museum für Nalturkunde der Sfadt. - Die Sammlungen 
des Professor B esser, L.Czekanowsky . luspector H oc lo ut.

chitzingen (Bayr. Franken). Srluul. lehrer Seclig hat cine Kerfeullection. Ixind (bei Bergen in Norwegen). Sammlaum des: I'isstor $S$ a rs.

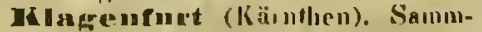

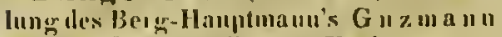
und des Banpraktikantı Il ü bu er.

IIansenburg (Siebeuhïigen). Samulung des errussen akademischen Lyceums und die des Grafen Joseph v. Teleli.

Hapiarotosetzt (bei Thurnan in Böhune'u). Apothcker C. L. Heriug's Sammluug.

Edeincinuabalden. Fürster Hölt \%er"sile mul P'forter Hofmei. ster'sulde Sammlung.

I I cisterutenaburg (an der Donau, iu dem Vientcl untro elem Wiencrwalde). Reiche und solu iustruetive Samulunz des dasigen herrlichen Augustinerstiftes, velches anch die erzherzogliche Krom verwalirt.

Ialintz (hei Stelliu). Oberföster Ricliter sumundte prenssisclie Kerle.

Kälar. Stadtahiouet. - Kuแfuaun W. Poil's, Dr. med. Rave's und J. W. Z II ude o' 's Simunlungen.

Gobuigsberes. Das naturhisturi sele Mroserum der 15.14 gestifteten Uni-

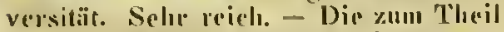
selır grossen und wichtigra Saramlungeu vou 11 . H u gr e u, Premierlieut(mant Led ohur, Professur llejur. Rathke, Richter, Di. und Rectur Il. Rocks t $\mathrm{r}$ al (wahl'sehoinliclu).

ratloen. Div Simmlung llıo Durchluudat der regientuden Frau Iferzon:in.

Iropenlangen (Insel Secland). Samuluugr der Universitit (grestiftet 1475). - Dis Museum. - Sammlume deg diunischer onfumologisthen Gesellsclapft. - Das Nufuralien-iNuscum des Köuigs von Dänemark, als Kroupriuz augreleget uul vou Directur Beck rou. servict: in linsenherer. - Dibeclor B e ck sull eine eigene Sammlung besitzen, wic cine solche hubeu Prof'cessol. Co ls su a u 1 , Pastor J. Jacubsou, C. v. Leerke, Stantsmiuister v. Ka as, Professor II. Reiula a dt, Dr. uul Prof. Se hiödte, Prof. $S$ rlucuw, Justizratl $S t$ iiger, Dr. med. Thi e Westerm ann (nusgereichnet durch Schïuheit in Conservation efe.).
Mechlenburg-Strelitz). - Prediger M us ch l'sche Sammlung.

I raucluthal (bei Beru). Jäg gi's kleine Colropterincullection.

Iraclian (Freie Stadt). Sammlung der Universität und jeue des Professor Estreicher.

Krensmilnster ('Traun-Viertel im Lande ob der Eus, Oestreich). Die Sinnulung der prär-htigen und selur reichen Benediktinecabtei aul dem Hö̀nel üher dem Marktflecken. - Die SammInng(0) des Comsistorialraths N. Altwirtlo und des Capitalaren Dom. LirI a clier; dinn des $\mathrm{l}^{3}$ rof. Udah. Ha rtenschueider mal Professor und Capicular Basilius Se lo oen bejoger.

Bu rentle (Oberbayemi). Badearat

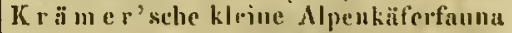
von Tegernsee und Kreuth und der Environs.

In reuzburar. Dir. Hir. Meyer's Simmolung.

Irenguach (un der Nahe). Des k. preuss. Premierlieutenants v. O r li c h Sinแmแ1แ11\%.

Ifronstudt. K yber'selue Käferrollection.

Idn nassée (bei Lille). Proprietär Alb. Alavoinc's Sammlung.

Msder (Breisgau). Negoriant La ll gsd o r f"s (Vater) brasilische CuleopterenSammlung.

Esabibsich (Herrogthum Krain). Stadtunuseum. (Ständisches Museum.) Wus es hat, verdankt es der Grossmuth seines Sehmidi. - Sammlung des Baron $v$. Z 0 is, des Grafen Mich. Corouiui, Custos H. Frey er, dpotheker Dr. Gruf, Franz, Graf vou Holienwarth, Tixantsoffizial Fr. Kukeil, Koss und des mermuideten Kanfunatu Feril. Jos. Selomill.

Danduach (Oestleich). Sammlung des Benedietinerstiftes.

Hancaslire. Die Sammlung des reichen 'Tl. Norris Lisq. (vgl. Rotíales).

Iunuilslunt (Unterbayern), Sammlung der Landwirthschafts- und Gewirbsschule.

Lanusane. Stadtmuseum. Die grnuze Simmulung wolılbrstellt, klein. Dic Sammlungeru des Banquier bugnion. Dircetur. J. A. Chavanues, Alex. Forel, Fr. II ub e ru. P. II uber.

Heeds (England). Sammlung des

Kotolow (?) (bei Friedland, in mechanischen Lnstituts. 
Heertae (bei Linehurg). R e usma un'sche Küfer-Sammlung.

Leipzig. Universitäts-Sammlungr. Die Universität wmrde 1409 gestiftet. Dr. mnd Professor B ay $r$ hoffer, Tinpezierer Erlıardt, Naturalienlaandler Frauke sen., Gärtuer, Cand. jur. H. v. Kiesenwetter, Dı: und Professor Kulze, Dr. med. Krutseh, Lehrer Lantrstengel, Graf v. Rantzau, Dr. Saehse, Kerfhindler W. Sehenereck, Conservator Schulze, Professor Schwaigrielıen, Hofrath und Ritter v. Tilesius, Antiquar Vo. grel, Dr. Will, Dr. Ed. Winkler und endlich $\mathrm{H}_{1}, \mathrm{Z}$ ejz besitzen melir oder minder grosse Sammlungen vou T'hie. ren efte.

Leith (England). Collection of mechanieal Institute.

Lenherg (Gallizien), Lyccalmuseum. - Die Sammlung des Polizeidirectors Z a he her.

Meobschiitz. Lehrer Riìke r' sehe Sammlung.

Le: Pryy. Sammlung des Hrn. Aymard.

Leyden (Sïdholland). Herrliehes Musemu der Municipalitiot. Ansserordentlielı. Fast alles, was die fremden Welten hieten, in Mehrzalıl. Emropäer am wenigsten. - Das Musenon der 1575 gestifteteu Universität. - Die anselınfichsten Sammlungen sind die der HH. Conservator Dr. D e H a a n nnd Dr. und Professor Reinwardt.

Libourne (Durdogne). Muscum.

Dielnewstein (Schloss hei Eger in Bölımens). Candidat Ludw. Riehíer's Faltersammlıng.

Licennitz (Schlesien). Die Sammlung ver Ritrer-Akademic und des Director $\mathrm{Mos}$ osli.

Limoges (Hant-Vienne). Kriegskommissî̀ I' ir ay's Sammlung.

Iinkdping (Ostgothland). Sammlung des Gymusiums.

Einkoin (Eucrand). Dis mechanische Institut dasclbst liat eine Sammlnng.

Limz. Bandircktionspraktikmut Jos. Knörrlein's, Reetor mud Professor König's, Pirngruber's nnd Promedieus und Regiernngsrath St reinz's Sammlung.

Lissahon. Dic Sammlnog des States und der Universitiat. Tropisclie Kerfe. - Macedo, der Secretiir, hat eine Collection, die eines Besuches wirklich wertlı ist.
Liverpool. Sammlung von A. Melly. (Es ist noch niedt hestimmt, ol, Hr. M. z. Manelsester oder Liverpool wohulaft sey.)

Lodemnn (Schlesien). H a upt's Sammlung.

Liben (Belgien). Naturhistorisches Museum der katholiselıen Universität. Soust uieht sehr wesentlieh: nun durclu 13 a u ml a n e r's Sammlmng reich. (Conservator: van Bencden.) - Des Prnfessur P.J. van B eneden's Sammlung.

Loirent. Sgranzin's Sammlung.

London. Diss britische Museum. Sehr reiche, in einem schönen Loeale autgestellte Sammlungen von Naturalicu, Kunstsachen und bedentende Bibliotheken. - Samulung des Collegimms der Chirurgen, der zoologischen und entomolorischen Societiiten ete. Prachtvoll. Limue's Sammlung bej Sir Banks. - University. Aus allen Zouen. Die Sammlung de's entomologieal Collegitums (Conservator: Rev. Jos. Gooda). - Die Sammlung des DulwiehCollegium. - Die Sammlıng drs KingrsCollegre. - Sammlung des mechaniselien lustifuts. - Bullok-Musenm. - Sammlong des Herzogs von B u e k i ugham. - Sammlang des Lord Egerton. Folgende haben Sammlungen: W. Ainworth, William Bainbridge, Barchas, Barkley, John Beckwith, Th. Beli Esq., Dr. med. Bennet t (melur Wirbelthiere), Dr. B evan, Will. Biugley, Blundell, W. Boud, J. Bowcrbank, Broderip, R. G. II. Rev. Browne, Dr. Cantur (meistostindisclue), J. Chant, John Georee Children (grossentheils Exotiea), Will. Christy Esq., Dr. Braey Clark, Du. Coldstreat m, Johu Curtis (dic Fannie Englands), Dale. Davis, Davis Esq., Delta, Dillwy u, Couscrvatur Dan, Dun (walırstheinlich pins mit vorigen), J. Espiunsse, J. Fle mming (vicl Vögel und Kerfe), Rev. Jos. Good a l (zum griissten Theil Käfer), Gould Esq., G. R. Gray Esq., Edward Griffith, Ilaliday (libuds Fauna insectormm), Hanslow, S. Hau. s o n Esq., Generalmajor Th, $\mathrm{H}$ a ld wi. cke, Holme, Hape Esq. at Rev. (Be. sitzt mehr, als Deje a u besass und wir glauben, melı noch als Spinali hnt), II eysham, John Hob house, Dr. Thomas Horsfield, Howitt, A. Ingpen, J. R. Jaekson, Sir William Ja r. dine, Jenyns Rev., Kidd, Kir- 
k upp, Lees, Lekeux, J. W. Lewin, R. H. Lew is Esq., Loddiges Esq., William Markwik, Thomas Marslum, G. P. Neale, G. New. port, Dr. Nuttal (Exoten), Louis Petit Esq., Curator C. P'iokrring Esq., W. H. R. Re ud Es(l., Professol" James Renuie, Riley, Licutenant Roc, Rogerson, Dr. P. M. Rouret, Roualds (imeist Neuropteren etc. yum Behufe seiner Angelfiseherei auf Salmen), G. 'T. Rudd, George Samoue Ile, W. W. Saunders Esq., Sheppard, Shuekard Esq., Sillimans, Tlu. Ski imshire, Capt. Walli. Smee, James Sowerby und G. B. Sow e rby, Willian spence, J.F. Stephens (Alles, was die buitische lusel belerbergt und ausserordentlich viel Auslïndisches), John C. Stevens, Präsident Sykes, Robert B.'To d d, Medicus Under w ood, Wailes, Wakeficld, Francis Walker Esq., Walford, Jolu Waltou, G. R. Waterlu ouse Esq., J. 0. Westwood (selur reiche und kostbare Collec. tion), Adam White, Wilson, Will, Wuod Esq., Will. Yarrell Esq. Man unss staunen, disss Englaud Alles sammelt und jeder Gegenstaud da so viele Liehhaber findet.

Luncen. Sanmlung des Carl Lucian Bonaparte, Prinz von Canino: des H. Alberti.

Wibeck. Dic Simmlungen des Pharmacus K ind und v. Linden ber $g$ : Bürgrormeister.

Lilinebure. Dic Sammlung des Museuns der Ritter-Akadentie, - Stadtsclircibers Heyer.

Lilleville. Dic Satmmlung $S$ au. cerolte's.

Lnud (Sehoouen, Sehweden). Museum vou Naturulien der k. Universität (gestiftet 1666) auf der Bibliotlick. Sammlungen des Freiherrn v. Giille r. $\mathrm{krug}$ und des Professor $\mathrm{Nilson}$, des Professor Dalilhom, Magnus v, D ii. ben, Professor Fallè Prof. Pries, Johamn Lagemunn, Munk of Roseuschold, C. W. Petterson, des Dr. neel. Rusenschold (walirscheilelich identisch mit Munk of Rosensehold pag. 54), des Dr. und Professor Sundewall und Professor Zetterstedt.

Hittich. Universitätssammlung. Vieles, doch selır cinseitig. - Dic Collection des Lector de $\mathrm{K}$ o $n \mathrm{i}$ in, des Conservator Brutus Carlier, Professor
Th. Lacordaire, Selys de Lougchamps, Professor Morreu.

Luxenburg. Künigliclies Athenลีum. - Profensor Ba una n n's Sammluug.

Wyon. Dic Sammlung des Stadthauses. Schön. Vieles rübrt vom seligen de Villers her. Aus Frank. reich (I'rovence) und ltalien, sowie der Srhweiz. - Diss Museum des Athenä. uns oder der Gesellschaft fül" Ackerhatu und Naturgeschichte. - Dic hübscheu, z.tum Theil prächtigen Sammlungen des Stenereinuehmer Chaldiny, Hrin. Hugo Donxel, Capitän Fontenay, Foudras und Seringe.

Macerata. OeffentlicheSammlung. Madras. Nuturforscher's Heatli crbeutete Schätze.

MIndrid. Stadtmuseum. Vicles aus allen Ordnungeu, dorh alles fast unlestimmt. - Sammlung im königl. Schlosse Bucu Retio, am entyegrugesezten Ènde der Stalt. - Das Künigliche Museum der Wissensihafteu (dariu das vollstïndige Skelet des Mrgatherium). - La Gasca's und des Professur Villa ll o va Sammluug.

Marra (in Portugal). Summlung in rer Bibliuthek des hüuigl. Schlosses (dem andern Eskorial).

II agleburg. Sammlung der IJauptscliule daselbst, des Gymutisiallehrer's B : use, Justizkommissarius D a mm, Fr: Fest, Lehrerk $r$ a sper und Leluer M a tz.

Mniland. Museum der Naturgesehichte. Insekten der Lombardei (meist von Villa, Marietti, Posto, Jan, Cristofori). - Musenu dell lustitute del reguo Lombardo-Veneto. - Die Simm. lungen des Ritter Car lo di $\mathrm{B}$ a s si, B l'u mbilla, Gebruider Betta, Bonola, Graf Carmagnola (selur schöin), Dr. Antonio Comolli, Professor 13. Crivelli, Graf v. Ferrurio, Muler G. Galeazzi, Professor Jan (soll nach Parmn gekommen sein, wic uแs Gruf Bentiniversicliert hitt). Longi, (iraf Carlo Maggi, Pitle Beruardo Mia. rietti (selir reich au Lombardeu), M $\mathrm{e}$ diei, Nicoli, Perraud, Marchese Ali Panzoui, Nobile Carlo Porrn (schön), Vechi, der Bruder Antouio u. Giovauni batt. Villa (vollstäudig die italienische Coleopteren-Fuma, wohl conservirt und bestimmt).

Mrainz. Sammluig des Lyeeums 
und des naturhistorischen Vereines, des Di. und Pfarrer S chmitt.

Valaus (Bündten). Majors Am Stein Summlung.

IIfanchester. A. Melly Esq. (Vol. Liverpoal) hat pine praclitvolle Sammlung.

FI tumbeins. Seit 1833 besteht ein Verein tür die Naturkunde. Sekretiir Dr. Kili a u. - Die Sammulıur der Stadt und des maturfursclienden Vercins. Proteetorill ist die Grossherzogin Stephania. - Die Sammlnngen, welelic sieh noclo in dieser atumuthigen Stadt befinden, gehören dem Dr. med. Suckow, Fahrikherrn Vogt, Gencral vau der Wyoli und Fräulein To eliter.

Nans (Dept. Sarthe). Museum der Naturyeschichte. - Die Simmlung des Conservators Desportes.

Mantan. Sammlung der k. Akade.mie der Wissenschafien.

va arbur. Sammlıng der 1527 gestiftef"u Univessitit. - Sammlung der Gesellsclaft zolr Beförderumg der Naturtiunde. - Die Sammlungen des Studiosus Gundlaeh und Di. und I'rofessor M. Herold.

Marburar (Steyermark). Oberkommiscïrs J. H u I a r S S

Mrarsclalins (hei Chur in Bündten). Die v. Salis'sche Sammlung.

viaria-Taferl (in dem Viertel ob dem Nanulartsberge unter der IEnns, in Oestreic(l). Bei unserer Anwesenlıit an diesen lrohen; wcit berïlımten Wallfahrsorte brsahen wir die Natu. ralienhude cines lermmachenden Iländlers, dessen Namen wir verlaren haben. Er hatte hïlische Sachen und war sehr billig dianit.

Minringe (Dept. Puy de Dom). Beaude -Lafarge's schöne Samm. lung.

II aros-Vasnaliely (Neunarkt; im Land der Szekler, Siebenhuirgen). Oeffentliches Minerulien- und Thierta. binet.

Marseille. Naturhistorisclies Museuil. Alles aus der Dauphine. Simmlung des Lycrums, Sammlumin des Mons. Roux. - Athenec des Sciences et arts. - Conservators Bartlélemy, Ministerialsecretiirs F am in, Advokats Lantir, Maler und Conservators P. Roux Sammlungen vou Belang.

Vazargues (bei Marseillp). Jngenicur-Capitan Solier's Sanumlung. Ausgezeiclmet.
Mechlenbura-Strelltz. Pfarrer S. Mussehl's Simmolung. (Iden. tiscli mit den in Kotolow :?.)

Meiningen (Sachseu). Oeffentli-hes Nituralienkibinet und Samulung des akademischen Lyecums. - Die Sammluıg des Kriniualraths B a unbael und Kammeraths Lomler.

Meissenlesm (Rhein). Dr. Hoffma ll "'s Collection.

MI-Isungeu (Chuthessen). SanmInng des Forstinstituts, des Lelirers W ï ekel.

Melın. Die Sopiètè d'agriculture lat eine putsprerlicude Sammlung.

JEemuingern (Bayern). Stadtlekaus W a d h te " Sammlung.

Fersentleim (Iaxtkreis in Württemberen). Dic Sammlungen des um die Wissenschaft selır verdienten Naturforschers und Reisenden. Sr. Hol. des Herzog Paul von Wüittemberg, im Srhlosise. Sie siud ciner eigenen Re ise weitlt.

Vessina. Dr. Franeeseo Arrosto und Professor Padre Carlo Giacinto besit\%en sizilianische Sammlungen und Mehres ans Alrika, das wir gesehen.

Metyringen (Bern). Hä̀dler B r ü̈nne r's Vorrillice.

IEetz. Die Sammlung der k. Akademic. - Najor Calre hat ciuc luubsche Samulung Insikten. - Bibliotlekars Hollaudre Sammlung und die Sammlung des Prïsidenten v. Simon.

Inexilou. Muscun der Gesellschaft fiir Wisenenselaften und Künste.

Nodalletown (Counceticut). Die Simmulutuer der Werlayan. Universität. - Das Cahinet des Prof. Joh nstoll.

Militsch (Schlesien). Hartlieb's Käfersit mm

Mitnu (Kurland). Sammlung des rikademischen Gymunsums. - Dr. Fleincher's und Lichtensteiu's Sammlung.

Miblling (bei Wien). Magistratsratlis Il eeger ausgezcicluete Santmling.

Monk (Marktferken, an der Miinduug der Mölk in die Donan). Die wissenschaftlichen Sammlungen der reichen und prächtigen Benediktinerabtei anf desn Berere euthalten me kw ïrdige Schïtye aus den drei Reicleen der Natur und, verdienen deu Besueh des Reisenden und Gelehrter, weleher hier mit grosser Hospitalitit aufrenommen wird, wie solelies in fast allen lilösteru and 
Abtelen der östreichiselıen Erbblande und anderwärts der Fall ist.

Mllonte Varclni (bei Pisa). Museum der Arademia della Val d'Arno daselhst.

Ilontjoie. Jugendlehrers Kreuzer kleine Sammlang.

Montmorency. Pharmazent N. Pl. B andirr's prislitvolle Collection.

IIontpellier. Museon der natorwissruschaftlichen Gesellschaft. Sammlnugen des hekannten Moquin T'and ou, C. A ube's, J. Cla b r i er"s, Houn a ud's, Postdiretitors Ferd. Ga sparini, Salzmann's, Mareel de Serres', driau Prodent de Vil. liers' Sanulıugen vou grösstem Interesse. Man weiss uiclit, was interessanter sey, die Sammlungen oder die Inhathe' derselben.

Moshan. Universititssamulung. Reich. - Moseum Demidoft. - Die Sammlung Balı's. - Die PrivatSammlung des Staatsrathes Fischer vou Waidlı e im. - Die ansehulichen Collectionen Khloustine's, des Grafen G. von Laveuu, A. Lovetzky's, Marin's, Gustav v. Ol nhauseu's (ling 18:7 2u sammeln an in onserer Gesellsehaft, unter unserer Leitung), Pascant's, des Jostizrath Basilius v. zub b of $f$.

Muhliateen. J. K. B roeh's Sammlung, jene Köehlin's und des Emil W apler.

Minhluein (a. Rhein). Jugendlehrers Weniger Sammlumr.

Minclien. Die Simmlıngen der k. Akademie der Wissensthoften (Eigenthum dos States). Mit diesen sind vereinigt die Sammlıngen der Universität (eliemals zo Landshot). Unbedeutend; Käfer meist brasilisch und wenig: übrige Ordnungen unbedentend, indessen ist cine prachtvolle Faltersimmlung (Geschenk des seligeı von Mulzer) vorhanden and einiges Hübsche ans den Tropen. Simmlıng der folyt cehnischen Selunle. Unbedentend. - Die vom verstorhenen Präpalrator Franz. Fessel crkatuc kleine Sammlung im polytechnisehen lnstitute. - Die ansehnlichen entomologisehen, theriologischen ete. Sammlungen des zoologischen Tauschvereins in der Adalbertstusse Nr. 9 bei der nenen Universitit. - Die Saummlung des Dr. med. Otto De Bruy" (Müllerstrasse Nr. 23, im Hanse des Branutweinbrenners). Sie stelit, da der Herr Dortor auf grosse Reisen siclt begebe" wollen, zum Verkaof zwarans, wird jedoch nicht jedem Kïufer, ler Zersplitterung wegrn, abgrlassen: dagegen dem wissenselzaftlich Gebildeten, dem Naturforscher oder wolıl gar eimer öfentlichen Samm lung anf das Billigste. Sic enthält, wir der Prospectus, den Hr. Gil rdulls im 3. Hefte fler Is is von 18.44, Umschlag, zeigt, ciue grosse Menge von Thieren, deren Anzahl bisher teträehtlich vermelut worden ist: 18 Genera mud 24 Species Sulke (Theria; Mammalia); 12 Genera und 12 Species Vögel; 14 Grnerit und 16 Species L йе he (Amplitia): 5 Genera, 5 Species Fische: 7 Gencril, 9 Species Krebse (Entomostracil if Malacostra(a): 5 Genera, 29 Species dxselı (Isopoda, Tretracera) : 5 (icnera, 29 Species Tausendfiisso (Myliapoda, ITe(racera); 24 Genera, 64 Species $S$ pinuen (Acra, Arachuida) oud 2 Genera, 5 Species Podnren (Apterudiera); 7 Genera, 12 Species Wirrure (Vermes intestinales etco); 1 Gellus aus den E ( l inodprom at en, 3 Gencra sus den Cephalopoden (mit 3 Speries) und 41 Genera (mit 97 Species) W cie ht hi è e - Mallosca et Couchylial), dinnu 5 Gruera Pflanzenthiere (Zoophyta) mit 17 Gattumgen. Mit den lusekten: XV Ordnungen, 1,403 Genera, 6.754 Species in 9,624 Exemplarien!! Pfla nzen therrliel, conservirt und meist alpine) sind 531 Genera und 1,906 Species in 2,671 Exemplarien vorlanden. St ei ne (Mincralia) 20 Genera, 99 Speries ond 203 Irxemplaria. Sumemirt man Alles ond nimmt einen kleinen Supplemont von P'llanzen noch hinzo, so ermibt sich cine Zahl von 1,960 Gencra, 8778 Species mit 12,517 Exemplaricu. Die " $11-$ tomologige he Sammlung ist, wie man begreift, die umfissendste und hat in neuester Zeit dnrell Aryusitinn einer neven einen asserordeutlichen Zuwachs erhalter. Nacli der eatalogisirten Total-Recapitulation enthalt sie $2,437 \mathrm{Spe}$ cies Pentimeren, 414 Hoterumerom, 1,676 Tetrimeren, 113 Timeren und 16 Dimesen. Ausgezciclunt sind unter den Kiffen rine Menge ganz uence Genera sowie Species, die Minutichora maxillosir, 3 Mrgacephalen und 1 ()xyeluila ; 53 Species Cicindelen (coucinna Drj., Dumolinii, Dej.; fimbriata, Dej. ; vicina Dej., anlicon Dej., remalis); $t$ Colliuris, $13 \mathrm{Sp}$. Braehimus (Benuroisi 
Dej.), 3 Graplipterus, 2 Singonen (hru. nipes), 8 starites (turricins Gistel und guineensis Dup.), 11 Clivineu (mandibulacis Dej.), 1 Cartprus, 3 Procrustes und 91 Species Carabus (nnit 156 Exenplaren), 1 Pelophila, 17 Clulaenius, 2 Epomis, 29 Pterostichus, 3 Perens, 1 Acinopus, 3 Selcnophorus, 1 Hypolithus, 4 Cynatopterus, 1 A gabus, 3 Cyelous, 1 Cybister, 53 Staphylinus, 2 Cafius, 1 Platyprosopus, 1 Dianous, 1 Evaestlee. tus, 1 Osorius, 2 Hypocyphtus, 3 Lomeclusat, 3 Stemocern, 5 Jalodis, 4 Acmaeodera, 3 Clırysoeliroa, 4 Steraspis, 1 Éuelıroma, 1 Conognatlia, 2 Lampetis, 3 Capmodis, 5 Dicerea, 5 Aneyloeheira, 2 Eurythyrea, 2 Pristiptera, 4 Lampri, 3 Clurysobothris, 13 Anthaxia, 9 sphenoptera, 17 Agrrilus, 2 Melasis, 1 Phyl. locerus, 1 Lissomus, 2 Sphaerocephalus, 1 Tetralobus, 2 Pericallus. 1 Conoderus, 1 Dima, 6 Agrypuns, 5 Pyrophorus, 2 Chalcolepidius, 1 Steatode rus, 1 Carliorhinus, 1 Eetiuus, 1 Rhipidicera, 1 Nycteus, 4 I,vgristopterus, 1 Charactus, 1 Nyetophanes, 3 Pygolampis, 3 Drilus, 2 Callianthis. 42 Cantharis, $10^{\circ}$ Malthimus, 30 Mislachious, 1 Epiplovta, 42 Dasytes, 1 Melvris, 1 Tillus, 11 Trichodes (ernbroniformis, snyyrnensis ete.), 1 Enoplium, 5 Ptilinus, 8 Xyleti. nus, 5 Dorratoma, 1 Oehind, 13 Anobium, 1 Dryophilus, 1 Hedobia, 19 Ptinus, 1 Mastigus, 11 Scydmaenus, 14 Nerrophorus, 3 Neerodes, 30 Silpha, 1 Necroplilus, 1 Agyrtes, 15 Cho. leva, 4 Peltis, 1 Colobieus, 9 I ps, 30 Nitidula, 13 Cerrus, 2 Micropeplus, 1 Encaustus, 2 Episcapha, 30 Crvptoplia. gus. 11 Ptiliun, 1 Mernatonı, 1 Trinodes, 1 Elachistes, 52 Hister, 2 Dendrophilus, 3 Abracus, 1 Hetaerius, 1 Hololepta, 33 Byrrhus, 1 Limuichus, 2 Georyssus, 6 Elmis, Parnus, 7 Heterocerus, $3 \mathrm{Hy}$ drachus, 4 Ochtlıebius, 3 Hydracua, 1 Spercheus, 8 Ateurhus, 2 Sisyphus, 5 Coprohius, 26 Copris, 9 l'hanaeus, 51 Onthophagus, 10 Onitis, 2 Onitierllıs, 75 Aplodius, 2 Psamuodius, 1 Aegialia, 7 Trox, 21 Geotrupes, 1 Hybosorus, 1 Orphnus, 10 Oryctes, 2 Hoplites, 6 Searubaeus (Hercules, Actapou), 2 Heteronyelius, 1 Chalepus, "2 Rutela, 1 Euchlora, 1 Trigonostoma, 7 Meloloutlıa (loololenda). I Aneylonycha, 1 Schis.nuycha, 2 Philorhloenia, 1 Ceruspis. 1 Hymenontis, 1 Chasmatopterus, I Di. rlielus, 7 Amphicoma, 1 Authipna, 1 Goliathus (mieans), 4 Gymuctis, 3 Gna- thocera, 1 Amplistoros, 2 Diplognatha, 37 Citonia, 1 Orthognathus (prionoides), 1 Psallieerus, 1 Aesalus, 10 Passalus, 2 Erodius, 3 Zophosis, 13 Pimelia, 3 Pncliyscelis, 1 Tracliydroma, 1 Adesaia, 1 Platyope, 2 Moluris, 1 Tracliynotus, 2 Seperlium, 2 Eurychora, 1 Moriea, Akis, 1 Elenophorus, 2 Seotobius, 4 Seaurus, 1 Cephalostomus. 2 Tagenia, 1 Laena, 5 Teutyrin, 2 Thalpophila, 1 Anatolica, 1 Gnathosia, 3 Acisba, 1 Scotinus, 4 Asidn, 1 Gnaptor, 11 Blaps, 1 Aenntliopus, 1 Platyseelis, 1 Isorerus, 4 Pedinus, 2 Heliopatrs, 6 Pandarus, 4 Philax, 1 Pacluypterus, 13 Opatrun, 2 Microzaum, 1 Leiehenum, 3 Crypticus, 1 Trachyseelis, 2 Phaleria, 1 Hypsoderes, 2 Boletophagus, 6 Anisotoma, 1 Tetratoma, 1 Pentaphyllus, 3 Ncomida, 1 Diaperis, 2 Epilampus, 2 Cossyphus, 1 Ieterophirga, 2 Uleiota, 3 Hypophloeus, 1 Sarrotrium, 1 Corticus, 1 Xyloborus, 1 Coxelus, 1 Eustroplius, 3 Orehesia, 2 Dirrien, 1 Myretoma, 1 Meria (Gistel) (M. phalerioides a. Corfu), 1 Hypulus, 2 Serropalpus, 4 Melandrya, 1 Pytho, 1 Epitragus, 1 Geohorus, 2 Pczodóntus, 4 Iphthiuus, 2 Upis, 1 Plateia (Holdii Gistel Isis; - Pl, orientalis Deliaau), 9 Teupbria, 1 Nycteropus, 2 Toxicum, 1 Heterotarsus. I Camaria, 1 Splieniscus, 1 Poecilesthus, 1 Saeran. gudes, 3 Adelpluis, 14 Helops, 11 Stenorhia, 1 Sphacrotus, 1 Amarygnus, 1 Prostenus, 2 Allecula, 3 Myretocheres, 6 Onuphilus, 16 Cistela, 5 Ceratias (Gist(1. Monocerus, Meg.), 1 Orlitlienomus, 4 Phipidophorus, 10 Mordella, 10 A uas. pis, 1 Cissites, 18 Meloc, 4 Cerocoana, 3 Dires (eonfluens, Klug), 2 Decatoma, 27 Mylabris (trifaseiata, bifasciata, pallipes, liolosericen, eleryi, galamensis, pubescens, salonica, cantigua, couspicua, Dejenuii ete.), 1 Lydus, 2 Oenas, $6 \mathrm{Ly}$ tt a (collaris, rlialybea, lutea), 8 Epienuta (Dusaultii), I Tetraonyx, 3 Zonitis, 1 Nemogurtlıa, 1 spalus, 1 Sitaris, 1 Calopus, 1 Ditylus, 4 Asclera, 11 Anogrodes, 5 Salpingrus, 4 Rhinosimos, 10 Bruehus, 1 Caryobórus, 3 Spermaphagus, 1 Clıoragus, I Rlinomaeer, 49 A piou, 1 Rhamphus, 7 A rrhenodes, Raphirluyehus, I Belophorus, 3 Bientus, 2 Ceorephuilus, 1 Cylas, 1 Oxyrcynclus, 7 Braeliycerus (lateralis, Besseri, Algirus, sordidıs, Lutosus, SeaI ratus, Foveocollis), 2 Entimus, 1 Psalidiun, 4 Thylacites (fritillum Pz. Turcia), 6 Naupactus, 1 Lagostomus, 3 
Cyphus, I Platyomus, I Phaedropos (rnudidus), 2 Eustales, Diaprepes (fa. melicus, marmoreus Gistel), 8 Chlorophanos, 2 Aunemerus, 2 Tanymecus, 19 Sitona. 1 Siderodactylus, 1 Eognathus (viridanus. Java), 18 Polydrosus, 5 Míetallites, 1 Entyus, 22 Cleonis (ocularis, guttiferus, plicatus, albicans, marmorntus\}, 3 Hypsonotus, 2 Lordops, 1 Eurylobus, 6 Aloplus, 5 Baryaotus, 1 Minyops, 1 Epicaerus (nebulosus. Mexieo), 1 Tanyspliyrus, 6 Hylobius (areticus, fatous), 7 Molytes, 6 Plinthus, 21 Phytonomus, 3 Coniatus (Villac Gistel. - (C. repandus Villa.) Lombardia), 32 Phyllobius, 6 Trachyphloeus, 6 Omias, 6 Peritelus, 78 Otiorliyuchos, 1 Tylode. res (chrysops aus Steycrmark), $1 \mathrm{Hy}$ phantus, 1 Agraphus, 20 Lixus (Dumolinii, binodulus etc.), 17 Larinus (Jenisonii Gistel, irroratus), 1 lleomus, 2 Rlinocyllus, 2 Heilipus (Dr.jeanii Buq. - Gistelîi Jenison), i Pissodes, 8 Thamnophilus (Cer'asi, barbicornis), 7 Eririhinus, 19 Dorytoanss (Pertyi et Grimmeri, Gistel), 1 Grypidius, 1 Hydronomus, 4 Ellescents (rufipes, Duf. Tauria), t Bracliyonyx, 8 Authonomus, 11 Balaniuus, 10 Ty shius (euprifer, sericeus ctc.), 1 Sibinia, 2 Acalypens, 5 Pliytobius, I Anoplus, 19 Orchestes, 1 Auchonnx (morbilinsus, Gistcl. Columbia). I Myorthinus, 3 Cholus, a Ameris, 2 Alcides (dentipes), 13 Baris, 3 Centriuus, 6 Cryptorliyurhos, 1 Camptorlinus (lineatocollis, Dej.), 2 Coelosternus (ophionotus, Dup. v. Madagascar), 5 Acalles, 1 Desmidophorus (Hebes. Ind. or.), 1 Mrecocoryıus (varipes, Wied. India or.), 4t Ceutorlyyuchus (trimaculatus, Litura, scutellatus, horridus, Noctis, Serratulac), 3 Campylirhyuclus (bruehoides), 1 Monunychus, Zygos (Asio), 1 Piazorus, 1 Orobitis, 8 Cionus (Blattariue etc..), 5 Gymnactron, 1 MIcciaus, 2 Nanodes, 1 Sipalus (mrandis, Gistel. Java), 14 Culandra (Weidenbachii, Gistel. Braz. - Phoeniris, $\delta$ 9 , rubiginea, sericea, luctuosa), Dryophthorus (Lymexyloa), 6 Cossomus, 10 Rhyncholus, 1 Nov. Gen., 13 Hylurgus (elongatus, defessus), 2 IIylesinus, 1 Camptocerus (acueipenuis), 3 Scolytus, 25 Bostrichus, 1 Platypus, 2 Pnussus (trigonicornis et deuticornis), 9 Apate (brazilieasis Gistel, Chevrieri, clongata, seregunlensis, Carmelita, Gistcl), 3 Psoa, 8 Cis, 1 Sphindus, 21 Latridius, (distinguend us, depressus, exilis, lim.! batus), Myeethophagus (Popull, Tetratoma): 3 Triplyyllus, 3 Syuchita, 2 Cerylon, 6 Moaotoma (striatum, fulvipes, Walworthi, Gistel ans Steyermark), 8 Rhyzophagus, 1 Bitoma, 1 Buthrideres (contractus), 4 Lyctus, 2 Colydium, 6 Silvanus (scaber, nov. Sp.), 1 Monopis, I Calyptobium (Villae Porro. Lombardia), I Megagnatlus, 6 Trogosita (grandis, Gigns, acnea, virescens, Lebasii), 1 Cucujos, 4 Laemophlocus (mouilis, nter, ferugineos, testiceus), 2 Brontes, 1 Deadrophagus (crenatos. Sueria), 2 Sphondyla, 1 Accanthopho. ros (serraticoruis. Ind. or.), I Enoplocerus (armillatus), 3 Ergates, 1 Mallo. doa, 1 Macrotomn (palmata), 1 Acgosoma, 1 Tragosoma, 1 Prionus, 1 Pyrodes, 4 Lissonotus, 9 Trachyleres, 1 Dorracerus, 6 Hammaticherus (dentirornis a. Guinea, Heros, Weleasii, Miles etc.), 3 Purpuriciaus, 2 Auoplistes, 2 Rosalia (pompejana, Gistel. Styr.), 4 Aromia, 2 Callieltrouna, 1 Promeres, 1 Otflıostoma, 1 Compsocerus, 1 Disanlax, 2 Cosmisoma, 3 Clırysoprasis, 1 Eryphus, 1 Hespcrophanes (holoscriceus), 1 Criocephalum, 4 Isarthrou, 1 A scoum, 2 Hylotiupes, 14 Callidium (alni, rufipes, humerale, thoracicom, solitudinis Gistel; nus Bayern), 22 Clytus (brazililiensis, scalaris, Zebra, smyrnensis, senripunctatus, cylindricus, trifasciatus, pollinosus, gibbosus), $1 \mathrm{Gra-}$ cilia, 1 Onchomerus, 3 Obrium, 1 Nov. Gen., I Certallum, 1 Stengyra (tricolor), 1 Deilus, 1 Rhiaotragus, 1 Odontocera, 3 Sphenopterus (femoratus, Stev.), 3 Molorchus, 1 Acrocinus, 1 Dryoetencs, 1 Tragomorphus, 1 Stcirastoma, 1 Acaathoderes, t Lagocheirus (araneiformis), 5 Astynomos, 1 Alcidioa, 3 Leiopus, 2 Exocentrus, 4 Pogonocerus, 2 Tacaiotes (Jherminieri, Dup. aus Cartluagena und Scalaris Fab.), 3 Monohammus (litteratus 2. Afrika?), 1 Omacnutha (Gigas; voun Seacgal), $3 \mathrm{Mo}$ rimus, 1 Diastocera, I Sternodonta (regalis), 1 Ceratites (jaspidea), 1 Onrilleres, 1 Hypioma, 2 Crossotus, 1 Coptops, 13 Nlesosa, 1 Gnoma (atomaria, Juva.), 24 Dorradiou (pimrum, Morio, angustatum, atrum, quadrilineatum, Liacolà, eruciatum, holosericeuu, exornatum, laqueatum, sericatum, decipieas, cruciferual and graceum), 3 P'armena, 1 Callia, 1 Hathlia, 1 Aanesthetis, 2 Colobothea, 6 Saperda, 4 Oberea, 15 Phytoccia (humeralis Gistel, aus der 
Tîrkei, rufimana, Ephipplun, caerulesccus, ferrea), 3 Amplionyelıa, 10 Agafanthia (Cynarae, Cardui, marginella, leueaspis), 1 Cometes. 1 Vesperus, 6 Rhagium (nebulosum, Gistel), 2 Rhannusium, 5 Toxotus, 13 Paehyta (interrogationis, chathrata, strigilata), 4 Strangalia, 11 Stenura (auruleuta, jubeseens, villi(a), 15 leeptura (hastata, tomeutosa, cincta, unipunctata), 4 Grammoptera (anmulicornis, Gistel aus Mähren), 29 Donacia, 1 Haemonia, 2 Sagra (senegaleusis), 1 Megascelis (flavipes, Dej.), 1 Orsodachua, 2 Anrhenia, 16 Lema (brunnea, viridis, nitidosa, Leprieuri), 4 Aluruss, 4 Hispa, 2 Polyclialea, 1 Diseomorpha, 1 Engenyssa (grossi), 8 Cyrtonota, 1 Ischyrosonyx, Dorynota, 3 Échoma, 3 Deloyala (miliaris, Fab. Java; diapliara Salılb. Sierra-Leona), 2 Coptocycla (Zona Fab., Wagleri Gistel. Bra\%), 21 Cassida, 1 Aplosonyx (allicoruis. Java.), 3 Coelomera, 12 Adimonia (Villae, brevipenuis etc.), $?$ Galleruca, 1 Rhaphidopalpa, 1 Nov.Gen., 1 Malacosona, 4 Diabrotica, 1 Agela. stica, I Phyllobrotica, 10 Luperus, B Oedyonycluss (Klugrii), 10 mophoita (lineata. Afrika), 1 Asjucella (viridicollis, Buq. Seneg.), 6 Graptodera (jantlininennis Dej, Senegal.), 3 Cacoseclis, 12 Crepidodera, 12 Pliyllotreta, 6 Aphthona, 9 Teinodaetyla, 7 Dibolia (eamprorum et hrevissina Gistel, a. Ungaru), 4 Psylliodes, 3 Plectroscelis, 3 Balanomorpha (tarda, Märk.), 3 A pteropeda (Hederae, orbicularis, leodiensis), 1 Podragica, 2 Argopus (haemisphaericus et Cardui, aus Spanien), s Doryphora, 1 Elytrospluaera (testudinatria Dej. aus Columbia), 4 Deuteroeampta, 1 Zygograuma. 1 Calligrapha, 1 Euparocha (amoena, Dej. Colunbien), 7 Timarclıa (italica, rugulosa ete.), 54 Chrysomela (caerulea, crassimargo, Russin, rircumeincta, Bauksii, Salviae, liricae, fulyida, mixta, orwata, grossa, aurulaus, sicula, diluta (te.), 11 Oreina, (plualerata, gloriosissima, Cacaliae, speciosa, senecionis, venusta, tristis, cyanea, nivalis Gistel, Pyrenaeen, curnleo-lineata Duf.), 8 Lini, 2 Entomoscelis, 6 Conioctena, 4 Spartophila, 1 Nov. Gen. (Chrys, xautlioptern Perty.): 4 Plagiodera (multipunctata Dej.; Tyrus, (Gistel.), 2 Gastropliysa, \& Phaedon, 3 Helodes, 2 Colaspis, 6 Calcoplıara (Klugii, viridipeunis, eribrosa, granulifera), 2 Pleurutaca (purpurea, Gisitel, Braz.?), 2 Thyshe, 3 Endocephalus, 3 Eunnolpus,
1 Euryope, 1 Eubrachis (eureulionoides Dej. Senegal.), 2 Lamprosoma. 4 Clytlura (sparsimaeulatit Gistel, Afriea) , 13 Latbidustomis (taxicurnis, bipunctata, bino. tata, Olnhausenii Gistel), 3 Luelnaria, 4 Maerolenes usencgalensis), 1 Mrlitonoma (seita Dej. Senegal.), 4 Coploeeplaala (inuptialis Gistel. Anstria.), Sumaragdina (limbata Stev, Rıss. mer.), 6 Cyaniris, 2 Pachybrachis, 2 Protophysus, 42 Ciyptocephalus, 1 Disopus, 1 Erotylus, 1 Sareumorphus, 1 Oligacorymus (cireundatus Buq. Colombia), 1 lillipticus: 3 Aegithus, 2 Iseliyrus (mexicanus, lubrus), 2 Myeotretus, 3 Triplax, 15 Phalaerus, 11 Agathidium, 3 Clypeaster, 3 Hippodamii (clatlıata, Dej. Chili.), 2 Anisosticta, 21 Coceinella, 2 Hyperaspis (senegalensis), 1 Mieraspis, 3 Chiloeorns, 1 Synonyelia (versiro. lor Fabr: Java.), 6 Epilachna (ehrysomelina, Elaterii, flav of seiata, humeralis (Latr. ans Peru), circumcincta), 3 Cynegrtis (meridionalis, I'arr. Corfii), 1 Exoplectra, 18 Seymus (quadrilunatus, arcuatus), I Nundiua, 2 Coceidula, 2 Eumorplous, 3 Corynomalus (érneiger, Latı., Carthagena.), 2 Nov. Gen., 10 Lycoperalina (Inumralis Gistel, Austria; fasciata Fabr., quadripustulata, cervina, 4 meue Speeies.), 1 Dajisal (bipunctata), 1 Dasycerns, 1 Tyrus (insignis), 1 ('temistes, 1 Pselaphus, 4 Bryaxis, 3 Bythinus, I Tychus, 2 Euplectus, 3 ('laviger. Rhyssodes europaeus und alles Neue noch unverzeichnet. - Hr. Debu un ist auch geneigt, jede Sammlung besonders abaugelien, als z. B. dic Vögel, Fische, Käler, Schricken ete. für sich. - Unter den Orthopteren (11 Genera, 44 Species und 85 Ex.) findet sirh manch Neues. Interessant sind die Nenroptera und Trichoptera (21 Gencra, 53 Speeies in 68 Exemplarion: letytere 4 Genera, 18 Speeies, 24 Ex(mpl.). Viel Srhönes, Seltenes und Merkwürdiges entlaält die Sammilung von Hynienopteren (86 Genera, 474 Species, in 718 Ex(-mplarien); dic Falter sind minder \%allirciclo und belaufen sich unr aut" 81 Genera, 349 Species in 429 Exempln I ien; mit Supplement 511 Sp., 591 Exempl. Ziemlich gleichlaltig ist die Colleetion der Dipteren; 85 Genera, 348 sprecies in 593 Exemplarien; aber unbedeutend die der Homaloptruen (4 Gen., 4 Sprc., 6 Lxempl.), Aplaniptera (1 Geu., $1 \mathrm{~S}_{\mathrm{p} .}$, 2 Ex.) und Apteren, mit 5 Gencribus vou Thierliuusen, 5 Species in 6 Exem- 
plarien. - Möge diese ansgezeichuet sehüne, wohlehialtene und uut das Serupulöseste rirhtig bestimmte Sammlüg cine ihrem hohen Werthe entsprerhende bleibronde. Stätte finden. Känfer wenden sirli an uns. Wir laben Vollmarht, dic Sammlung zu veräısscrm. Gistcl. - Student Diury, Professor Eril. Bacealanreus Fleschuez, Studiosus Ge mum inger, Dr. Gist el, Prafessor v. Hefuer, Cisstus Dr. Hela (Vatter), Dr. med. Held (Suhn), Lithograph II elm situ e r, Dr. Krie chbauin er, Dr. medl. Ferd. K แ $m$ m $\mathrm{er}$. Stantslath v. Laclebour, linaz Ritter v. I a y cr (sehr anselunlich). Ma y erhofor, Officiant Jos. Merkel, Cand. jur. MI ics. Oberlicuteuant Graf v. Morawitaky, Brituer Oberleituer (sehr schö̈n und kos(bar), Dr. Roth, Dr. Carl Selenkenberer (heiu), Bergrath v. Selublert (selir mubedentend), Rath and Professor S( ) low a b (hat uur Bremsell. Oestrus), Geometer St ark (S'llmetterlinge), Bencficiat Ste inki relnerer, Obergeumeters toltz (Faltersammlung), Graf Taseher de In Pagerie, Dr med. und Arzt Ullersperger, And.

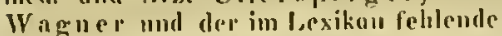
Hr. Oberlientenant Walther laben Sammlumgen, wie anch unsel Freund, Ritter Dr. Keil zu Schwabing, weleher lange Zeit in Afrika (Guinea) und Ostindien snmmelte.

Ilituster(Westphalen). EineSanmlung im Seninarium dasclbst.

Nancy. Muscum der königliehen Gesellschaft der Wissenschaften.

Niantes. Stirltisches Musemum. Die Sammlung des Director Cailla ud und Conservator Dubuisou.

Nianumurar. Buclıbinder Kämpfer's Sammlung.

Naurod (in Nissau). Pfirters IIgen Jusckicusimumlung.

Veapel. Sammlung der Universität und k. Akatlemie der Wissenscluften, des. Dr. Vineent Brisanti, Antonio delle Chiajc, Stephann delle Chinje, Dr. G. Costa, des Prisidenten P. D. G. Piazzi. - Sammlung des Professors Ruggieri, Andreil Tripaldi, Hauptmann Wredow (vol. Chur).

Nedeu (bei Cleve). Collertion des Superintendenten und Pastors Ma erss. dsimirsky's Sammlung von Annulosen.

Nenunurg (an der Donau). Samm. lung der landwirthsschaftlichen Schule. Deufist riz (Taborkreis in Böhunen). Des Barons von IIneluberg Sammlung.

Vienenburg (inder Selıweiz). Professor Agussiz, Cartier, Direetor Louis de Coulon, Carl Godet, H. Nieolet und Monsieur Wavre besitzen Sammlungen.

Nemialdemsleben (bei Magdeburgn). Sehöntärbers Fiekler Kätersammlung.

Veuliansen (bei München), Garduus'sche Sammlung.

Veusiedel an See. Anton Stenz's Naturalienvorrätle.

Veusolul (Ungarn). Professors Zipser Sammlung.

Nenstadt (an der Fischa, im Viertel unter dem Wienerwalde). Die Sammlung der berülumten Militior-Akademio 2.111 Untervicht der Züglingt: (iu der chemaligen kaisislichen Burg).

Seuntadt-Fincrswalde (Brin(lenburg). Die Sammlung der Forstanstalt daselbst, unter den verdienten Ratzeburg. - Des Dr. und Professurs Ratzcb urg Sammlung wordo vor einigen Jahren an die Forstanstalt verkauft.

Nieustadt (a. d. Aisch in bayrisch Franken). Dr. B r a in d's Sammlung vou P'flanzen und Käfern.

Nenvied. Die Sammlung des sel. Primzen Maximilian von Wied, im Silhlasse.

Nevi-Aberdeen (Mittelschottlaud). Sammlung der Universität, resp. des Colleginns.

Nevi-IBury (New-York). Das Museum des Lyecum of natural history und die Sammulung des Dr. Med. G o ol m a 11 m.

New-Castle (Eugland). Samm. Inng der Nordern-Institution. - Die Sammlung des Secretair 11 u tt on.

Nev-1haven (Nordimerika). Die Sammlung der ILarvard-Universität. Sammlumg des Yalc-College. - Die Kiffrrammlung des Bibliothekar Harris mnd Edward Herrik.

New-Drleans (Lonisiania). Das Musemm des Lycenm of natural history. - Die Callertionen des Professor Ridde I und des IIrn. Jitmes S a ul.

Nev-rork. Ingenieur-Capitains John Leconte Sammlung. 
Nijni-Mowgorod. Pelatier's Collection.

NJesky (bei Muskau in der Lausitz). Sanmlong der Herruhuter Brïder-Geunpimle.

Nixdorf (Bölmmen). Fis cher's Edlér vun Röslersta in bedeutende Faltersamulung (nuch in Wien).

Nizzr. Dr. Ange Maceary's Sammlung. - Die Sammlung des Apothekers Risso.

VBblnring (hei Gmünd in Obcrkärn. then). l'farcvikar Gotselu's Samm. lung: meistentheils Kïfer.

Norwich (Norfolkshire). Museum. - Die Sammlungen der IIII. De uny und Wilkin.

Nownsolsk. Die Sammlung der Unterrichts-Anstalt des Grafen Strogan off.

Niirnberg. Sammlung der Stadt, der polytechnisehen und landwirthschaftIichen Schule. - Die Collectionen des Studiosus Jaeckel, Antiquar Lecloner und die weltberihmte Käfersamm. lung des Naturalienhändlers mul hoelıberïlımten Kupferstechers Jiliob S t u rnı und seines Bruders.

Nufrenen (Schweiz). Pfarrer Felix'sche Sammluugluelvetiselser Colcopteren.

Deleubach (bei Mainz). Pfarrer Pl. W. J. Mäller's Käfrersammlung.

Dulessa (Gonv. Chersou). SammIung im Lyceum Riphelieu. - Dic ColIectionen von Berlholdy, M ïh r und des Akademikers Professor Dr. Alexander Nordmanu.

Dedeuburg (Ungarn). Wundarzt Gallus Faltr rsammlong. - Die eutomologiselie Sammlong (Eoropäer), des genialen Baron v. Orkska y d'Ocksko, eines unserer tüchtigsten und heharrlichsten Forscluer.

Dels (Sclulesien). Die Kunst-und Naturaliensammlung in weitläufigen Sehlosse.

Deliringen (Hohenlohe). Dic Sammlunar des Leibarztes und Dr. Med. Oesterlen.

Gren. Die Kerfhändier IK iuder. ma n " (Vater und Solin) hahen grosse und ausgezeieluet gut couservirti Vor. rätlıe zu äusserst billigen Preisen. Dr. Neusuliel's und des Dr. ined. P y l a sammlung.

DIf-Aberdecen(Mittelschottland). Sammlung des Universitäts-Collegium.
DIdenburg. J. Schlo ifer'sche Käfersammlung.

DI inditz (Mäliren). Sammlung des Lyceums und der Ritlerakademis.

(Dravitza (Banat). MagisterWicrzb ic:ki's brdeotende Kïfersamımlıug.

Drenburg. Kariline's Kerfsamml ung.

Drleane. Dr. med. R. P. B ouland, Director Lockhart haben Summlunger.

Osmabritcle. Gymnasial - Sammlour.

Dssel (bei Töplitz in Böhmen). Sammlung des Klosters.

Dstende. Dr. Schnabel's Kerfsammlung.

Dviedo (im spaniselien Fürstenthum Asturim). Universititssammlung.

Dxford. Muscom der Universität und des berühnten Buckland.

Palna. Sammlung des bisehöflieluen Seminariums. - Sammlung des Grafen da Rio. - Sammlung der Universität.

Palermo. Sammlung der Universität ond der Akademien. - Naturalist G rolı ma n n's Naturalien - Sammlong (zum Verkaofo).

Puluna (auf der balearischen Insel Mallorea). Samulung der Univer. sität.

Pampeloun (im Königreich $\mathrm{Na}$ varri). Unbedeutend ist die Collection der Universitit; meist pyreuäische Vorkomm nisse.

Pastolorf (bei Sehlepenstadt). Sammlung des P'rediger-B all custedt. P'uris. Musèum ilhistoire naturelle (in Jardin de plantes). Vor viclen Jahren ist davou eine Besehreibung prschienen, welche anch in's Deutsche ülrersezt worlen ist. - Das Museom der Societe plibomatique. - Die Sammlong der Sociète d'histoire naturelle. - A m yot, A. A y mard, Bardet de Louguesse, Pl. Barker-Wepp, Ars. Thiéba ut de Beru a ud und Mademoiselle Unanie Thiebaut de Beruat, Naturalist Biot, Professur H. Ducratay do Blainville, Dr. Alplous Boisduval, Boitard, Aimé Bonpland (meist Pflanzen), Major Bory de Sa intVincent, Botin-Dessylles, Aupuste Brulle, Brobisson, Lorien Bu y uet (ansgezejelnetes Cahinet zom Ifandel), Madane Catharine Caillard, Auguste ChevroIat, Prot. J. Actille 
Comie, Dr. und Professor Frederie Cuvier (aus allen Ordnungen), Graf von Dejean, Alexander Del a montague, Delessert, Theodor Des. courtils. Ciselenr Debrant, Defrance, Dr. Dissliayes, Professor G. A. Desmarest, Desmonlins: Robineau-Desvoidy, Fran Grîfui Doguero, Dr. Don merk, Dr. Duja ${ }^{\circ}$ in, Professor And. M. Coust. D ume ril haben mehr oder wenigerorosse Snmmlungen; fermer Prïsident D Iponchel, Nafuralist Dupout, A. Duvau, Marine-Cirurg Foruné Ey. doux, Professor Milne-Edwards, General Baron von Feisthamnel, J. P. Gaimar, Offieier Gaubil, Naturalist G a udieha ud, Isidor Geoffroy Saint-Hilaire, Professor Etienue Geoffroy Saint-Hilaire, Geoffioy de villeneuve, Gervais, Kerfhändler Goudot, Capitain Ritter H. L. Gory, Goureau, Graels, Advokat Gue ué (vel. Chartres), Professor G॥ é rin Me us ville (prächtig), Guilla mè (vgl. Clıâteaudıu), Naturalist ll o ffmann, Chiruru Honbron, MarineChirurg Juequinot, Zeichner Javet, Dr. Lachat, M. F. L. de Laporte, Comte de: Castelnau, Laucret, Leloux, Alex. I ef ebvre, Pharmazent Leprieur, Buchuäudler Lequien, Le sehen and, Mariue - Pharmarent Lesson, H. Luens, Oberingeniem. Fr. M. Jul. Luezot. - Ermidend ist die Aufziilılung und sie ist noch nieht ru Ende. Es laben noch Samnilumgru: Jean Mit equart, Cliarles Ma Io, MI a rt vu (Vnter und Sulun), Felix Miger, Maler Milbert, Fr. Moux-Del oelie, Mulsant, Bibliothekar Ch. Nodier, Alcide Dessalines d'O rbigny, P anlet. Achille Remy Pereheron (sowolıl ausgezeichmete Bibliothek als Sammlung), A.P ier re t, Advokut P o ey, $P$ ouret (walirselieinlich noeh $z u$ P'aris), Militiir-Unterintendant $P \| \mathrm{O}_{0}$, JeanRend-Coustant Qu oy, Dr. med. J. Prter R itubur, Reiche, Sr. D. der Herzog vou Rivali und Esslingen, General Balth, E. de R umand, Di. ned, nnd Vorsteher imPfanzengarten Rousseau, Sainson, Auguste de Saint-Hilaire, Auguste Salle und Vater und Solın gl. Namens, Audinet de Serville, Prof. Strauss-Durklein (verel. Fröthwiller bei Strassburg), Michel Trucliet, M. Vasselet, Vau. louer, Verreau, Foulque del
Villaret und Barnn von Walkenaer: Dr. und Prof. Duvernoy.

Parma. Ocffentliches Naturalienkabinet. - Petrefakten.Sammlung der Professoren Guidotti und Jan. Die Samulıngen der Grafen Sa nvitale und Linati, die voin Berté, Profescor MI ou i $\mathrm{i}$ und Roud a $\mathrm{n}$ i.

TPassin. Dr, und Professur's W sehöıc Küter'sammılung.

Pavin. Die Sammlung der Unlversitiit und der Professoren $\mathrm{Br}$ ug $\mathrm{n}$ ate lli und Zendrini.

Peclanu (bei Magrleburgr). Capitain vou $M$ a lin ow s k v's Sanınılung.

Wergarse (ill Biscaya). Die ükonomische Gesellarbaft hat hier, an ihrem Versamılungsort, eine Samnlung angelegt.

Périfueux (Dep. der Dordogne). Städtisches Museum.

Ferpiguan. Dr. Ludov. Com. pagno und Pharmazeut Farines hahen wunderseliöne Sammlungen.

Pertitscheff (Volhynien). Barous Maximilian von $C$ handoir reiche Samulungen von Kerfen aller Art.

Perugin. Muscun der Akademie und Universität. - Sammlung des Professur Can ale.

Pestl. Das Museum der Univel. sität. - Das Nationalmuscum. Die Collectionen des Dr. und Conservator Emrich Frivaldsky vou Friwald selar gross und s(luŭn), des Dr. und Professor Jos. Sa dl er.

Plniladelphin. Die Sammlung des rudger'sthun medieinisehen Collegiuns. - Die Sammlung des Frankliu. Instituts. - Grosse Colleetioneu luaben inne: Dr. Benj. Snith Barton, Prüsident Zachaens Coll in s, John F a g go, Professol Di: Godma $\|$, N. N. He $\mathrm{t}$, Major Graf von Jenis on, Latrobe, Baudry de I, ozières, Peter S. du Ponceau, Dr. Jer. von Rensslaer (vide New-York), Christ. Z i m me r. ma $a n$.

Pietsfield (Massaclussets). Das Museum des Lyeeum of natural histıry.

Pilsen (Bühmen). Das Museum im'Sclilosse des Graf Zellenky.

Pisa. Sammlung der Universität. Die Collertionen des de Chiesa, Professor Panlo S uvi.

Policza (Bühmen), Dr. med. Ei. se it's Samiling.

Foint a Iétre (auf Guadeloupe). 
Dr. Ferd. Lherminier's gesammelte Natmalienvorräthe.

Pontarlier (Doubs). Contribntinns-Director E co ffet hat pine kleine Sammlnug.

Ponte de Lima (in der Iandscluaft Entre Duero e Minko in Portu(aal). Sammlung der ökonomisclien Gesellsehaft dnselbst.

Fotsilam. Sammlung des Baurath v o u K rïger; des Scluulrath v o u Tïrk.

Posen. Dr. und Professor Lö w's Sammlumg.

Prag. Das böhmixche Nationalmuseum, das Professor Zippe geurdnet und besehrieben liat. Die Monatwehrift der Gesellseliaft des vuterliindisrlien Museums und die Jalırbiicher des bolımischen Museoms gehon viele Ant. schlïsse viber die darin entlualtenen $\mathrm{Na}$ furschätze. - Die Summlmug der Fiau Gräfin Marzin. - Die Sammlune des Fürsten Coloredo mul des Grafen Ca n a l. - Sammlung der Universität (geetiftet 1348). - Samulıng der Akadeunie und des Benedictinerstittes Stralı of, mit ansehnlicher Bibliothek. - Prag hat eine Menge vun Privatsammlunge" anfzuweisen, als da find die von $\mathrm{C} e \mathrm{ch}$, des beriilunten Custos A. J. Corda, Joh. Demel, Dietrich, Naturulist Felduer, Naturforseber Fieh ar, Joh. Hineiss, Dr. und Professor Mikun: Dr. Fr. A. Nieker1, Dr. medl. Opitz, Dr. und Professor J. Sw. L'essl, Sammler Riedl, Official Seidel, Dr: med. und Arzt H. M. Selumidt, Kerf. sammler $S$ woboda, Trautmann. Oberlieutellant Wastel, Weidenh offer, Joseph Baron von Wimmer, Hauptmann vou Zilliel vud Custos Zipe.

Pratz de Molo (Pyr. orient.). Pharmazeut $X$ at art's werthvolle $K a ̈$. fersammlung.

Pressiburg (Niederungarn). Oef. fentliehes Stadtmuseum. - Apotheker Jos. yon Doruer's Sammlung.

IPrenre (Chamouny). B al m a's Faltervorrätlie.

I"rovidence (Rhode-Island), Das Musenu der Franklin-Society.

Przenyal (im üstreiclivelien Galizieu). Die Sammlung des Professor der Plıysik und Mathematik Dr. Alex. Za $w$ a dzki. lung.
Fyrmont. Die Sammlıng des Me. diciualıutss Dr. Mruke, Dr. Otto speyer, Dr. Adolf Speyer.

Quenstedt (Harz). Pastor Nimrod'sclie Sammlung von Anuulosen.

Mamanenan (bei Dresdeu). Graf von H oft minti segg sche newerliclist arquirirte Sammlung.

Tathbor. Gymnasialsammlung, wetclie Tus Hru. A. Keleb heseluieben worden ist. - Die Collectioneu entumologiselser Art vou Dr. Guttman I und Oberlelirer Kele l.

Tavenua. Tilesius hat eine Sammlıng you allen möglichen Natır. gereustündin.

Regensburg. Die Sammlungen des k. Isyceums, angelegt dur(eh deu voutreffichen Boianiker Hru. Prof. Dr. F iै r u rolır: dic der k. Kreislandwirtl. seluafts - Ind Gewerbssilunle. - Oberlieut. A ngerer's Srlumettrilingssammlung und die des Hauptmann Ernesti; Di: und Professor Fiirn rolır, Maler Carl Ge yer, Do med. u. Sialtgerielsts. al at Herrieli-Scluaffer (Grosse Colleetion aus allen Classen: kanfte die von uns geordnete und bestimute Jeuisousclue Sammlung), Dr. und Professor Hoppe, Forstrath Koeh, Dr. med. Schue h, Obmilient. Aut. Ziegler luben Colleetionen, wie unch der Bürger. meister und Apotlieker Eser (in Stantnmhof), Kanonikus Emm (-riel, (†?) und Seminarinspektor W e in.

Teegrio. Die hiibselıe Sammlnng des Prolessor $\mathrm{B}$ riguoli.

Relnne (in Merklenburg). Bürgermeister Da niel's Sammlung.

IRenaud (bei Vendôme : Luir et Clier). Akademiker 11, Dutroeliet's Sammlume.

Tentliendorf. Pfurrerl3 rehm's Kiter - und Vögelsammlumg.

Reutlingen. Kaufmann Ke II e. I's Samunluux.

Teval (Estliland). Sammlung der dortigen Ritterakademie.

M locine. Apotheker Murdfield's hübsehe Collection.

Theineck (am Budensee, Seliweizerseite). Die Sammlung des verstorbenen Pfarrers Steinuniller wird dort anfbewahrt.

IBinzan (Russland). Vuzelvichinsky's Samnlung. 
Tubenuvllle. Des Institutionsclefs Solireiner Sammlung.

reiferaveil (Cant. Ziirich). Dr. Hegetschweiler'silie Snmmlung.

Eiga (Lieflaud). Das Naturalien. kabinet der Stadthibliothek und des Lyccums. Driiupe!man u, Hemuing Je asen, Dr. Carl So do ffeky labeu Sammlıngen.

Foclre:I le: (La). Naturalit:mnuseum daselbst. - Dio Sammlu., des Moits. Dessulines d'Or biguy.

Toclsester (iu Berkshire im Staate Massiclumsetts. Die Sammlong des Williancollegiums (Conservator Cliester Dewey).

Edeteren (bei Moutjoiv). Oberförsters Miiller Sammlung.

IRom. Museum der Universität. Samuluug rles Professor Carpi, Telema t: Metaxi, Pharmazut Rolli.

Rouca (bei Muntebello in Veronesiseheu). Die samunluug (uneist vou Petrefakteu) des Gincondo C uv a gio».

Fostock (Grosiluerzogthum Merkleubur. Schwerin). Sammlung der Univer'sitiit, gestiftet 1419.

Rotrules (bei Manchester). Th. Nopris' Samulung (unbestiumt ob da oder zul Lancasltire).

Rotterdum. Dr. med. Brants und Prof. vou der Hoeven sind in Besitze silıüner Sammlungeu.

Howen. Anunyuls (M.), Paul Lebas, Arsenne Maille mud Ingenieur Sauli:y laben Kiifershmmlungen.

Mendolatadt (Soliwarbury). Naturalienkabiuet im Sclulosse L II d w igsb 11 r p. - Kahiuet des fiirstlicloen Grvmnasiums. - Des Hofintles Werlich Sammlung. - S. D. Friedr. Gii nt he r, Erhpriuz von Schwarzburg.Rudolstadi habcu cine eigene Sammlung, ebenso Dr. med. Sorg.

Dëigenwalde (Poumern). Antsraflis Goed en Samunlumg.

Raiblow (Merklenburg). Sponholz's Sammlung.

Ruscoezin (beiDanriy). v.Ticdem il

Sarmen (Cant. Beru). Roetzer's Fultersamuluug.

Salsmanca (im Königreicle Lenn). Die Sammlung der Universität soll sehr ansehulich seyn.

Salem (Massaehinsets). Die Sammlumg des Museum der East India Marine. Society.
Salzbarg. Sammlungen des Bonediefinerstiftes zu St. Peter ete, des hocliw. Abt Pater Albert (Nage. za III), welche dic Professoren und Chorherren Gebrürler Jakob und Jolinunes xusammengebracht haben. Furner laben samulungen ungelegt: Lelurer Mayburger und Dr. Spa. tacuectier.

Salop (Englanul). Sir Heinr. Barton Edward's grosse Sammlungel.

Salzwedeln (Mngleburg). D a min e il's Coleopterencollection.

smenar (Maine et Loire). Courtilli e r's Collection.

St. Albans (Giafseliaft Hertford in Englaud). Die kustbare Sammlung W. Swainson's.

St. Antouio do In Florida (bei Malrid). Almosempflegers D on Sil vador de Lopez Sammlung von Exoticis.

St. Dartuélemy (Antillen). Forstström's gesammelte Schätze.

St. Dewis (a of der maskarenl. Inarl Bourbon). Proprietair Roudie's Sanımluı!g.

St. Florian (Steyemark). Die Naturalionsammlung des Stiftes, - Chorlicrrns Joh. Schmidberor Sammlung von dem Obste schädlichen Kerfen.

st. Cuallen. Die Sammlung in der Stadtbibliothek (vereinigt mit der Schlnepfer's sel. in Trogeu). Die Simmlumgen der HH. Professoren Scicitlin und Z ollikoffer, Hartu a 11 u. M a ye $\mathrm{s}$.

fiaint Germain en Laye (bei Paris). Graf Amedee L. Mich. v. Lepelletier de Saint Fargean's nusgezeichnete Hymenopterensammlu1ın.

Saint-Savenr (Yonue) Dr. uned. J. B. Robilleau-Desvoidy's Sammlung.

Saint-Sever (Dept. des Landes): Dr. Lè u - D u four's Collcetiou.

san Jago de Compostella (iin spanische॥ Künigrejelie Gralizien). Unbedentende Naturaliensamulung der Unives silät.

St. Tueouliaral (bei Marburg in Steyermark). Colcopterensammilung voul J. N. Spitzy.

st. Metersinrg. Museum der Akaleuie. Conservator: Hr. Eduard vi Mè uétriès. (Vassili-Ostroff, 7. ligne. maison de l'Academie $\mathrm{Nr}$. 1) und $\mathrm{Hr}$. Goorg Schtader (Vassili-Ostroff, 
Hôtel de l'Aeademie, place de la Bourse, No. 2). Gross und prächtig. Mit! scheut nichts, alles zusammenzuhringen und des förderlirhen Eifers liebei ist ribmlichst z.u erwähnen. - In mineralogiseben Museum der k. k. Akademic der Wissensehaften trilf tev Zoologe eine Menge der interessantesten Petut: fakten. - Muscun Romanzow (an) engliselien Quai). - Die Samunlung des pädagogiselıen lnstituts. - Die Simmulung der freien ökonomischen Gesellsclıaft.-Dic Sammlung de 'Gräfin S trogan off. - Nach der bequem alphabet. Orduung seyen aufgefulut die Puivatsammluugen von Prufessor Di. v. Bä i, Hofrath Dr. Bartels, Dr. Blas seloke, Dr.u. Professor B ra nd I, Flottenkapitän v. Etholèn, Ohergiartuer Fr. Fald er. un un n, Leibarzt Dr. S. F iselse r (wovon Fl eseliuez. Virles kafufte), Stuatsrath v. Haine 1, Kollegienrath He nu i ug, Bened. Jä ger, v. M ar tini, Director J. Fr. v. Mínetries, v. Meyendorf, Gardeoftizier Victor von Molschulsky, Joh. Oberts, Dr. Gerard v. Pinder, Ganf Piccolonin i, Dr. med. Feruinand $S$ a l I b e $\mathrm{g}$ (Suln), Custos Schrader, Schreuk, Slovitz, Emil v. Stjernwall, Professor Stschegloff, Tscheruikh.

Gankt Polten (Stadt an dev Tratsen, in lem Viertel ob dem Wicnerwalde). Sammlung des Benediktinerstiftes daselbst, aufgestellt in der Bibliothek des Klosters.

Sankt Urban (Kiloster iu Cant. Isuzern:. Lang'sclie Sammlung von Naturgegenstäuden.

Shnta Fudi Wogota. Das Stadtnuseuin.

Earagosar (Aragonien). Eine kleine Sammlung befinlet siclı auf u'iner der üffeutliclıen Bibliotlueken, als Unberrest der eingegangenen Universität, welehe vor der Belagerung existirt hat.

Sarepta. Wilh elms' Sammlung voll Coleopteren.

Sassari (Sardinien). Oeftentlicle Samulung.

Achrofinamsen Sammlungen der Hil. Zollik ofer, Ammon, Seiler und $\mathrm{Z}$ wirk.

Schamdan (Sachsen), K, v. Tiselie r's Falteicollection.

Relnieck (in Curland). Pastors Biittner Sammlung. . Schleisshein
Samulung des $k$. Inndwirthsehaftlielıen Instituts.

Schmiedeloerg (in Schlesien). Kờ hler's Sanumlung.

Schnéprentlial.

(Herrogtluum Sachen-Gotha). Naturaliensammlung, begriindet van $S$ alzmann.

Schtipfen. Rote n b a ch's Sanm. lung.

seen (bei Winterthur). Pfurer Rudolph R w rorf's Käfersammlong. Seitenstridten rim Viertel Oberwienerwald, an der Gränze von Steyerwark). Die Samunlung des dortigen Benediktinerstiftes.

Sencegat. Commis Dumoli u's und Capitain Foucon's Gesummeltes.

Sevilla. Sammlumg der ProvinzialUniversität. Nieht sehr bedentend. Dir Colleetion des Apotheker Ca mpos. Siegen (Westphalen). Dr. जund Dirckior's Suffriun Käfersammlong. Siena. Muscum der Universität. - Professor's Giuli Cullection. Die Sammlung des Padre Ricea im Kloster der Barfïsser. - Dic Collection des Di. Maz\% i.

Sharn (bei Stocklıolm). Sammiung des Gymusiums. - Commerzien ratlis Cp. Jos. Selï̈ u li er selıätzhare Sammlung von Käfern.

Semyxur. Di. Il elfer's Sammloing.

Solnranenmor (bei Görlitz in Schlesien). Leluer Er(mann Stiller hat hier eine Sammiung.

Solothra:n. Das Museum der Stadt, gegriiudet durch den berïhunten Hogi in. Jahre 1820.

wondershansen (Fürstentlum Schwazhurgr). Naturalienkabinet in Residenzschloss (auf dem Berge).

*persuter (bei Marienstadt in Westgothland). Sammlund des hoeliberiihmten Entomologen Selı̈ulıer (vgl. Skara).

Speyer. Steuereimelumer Linz' sche Collection.

stemdal. Seliot ts ted t'sche Sammlunir.

Stettin (Pommern). Die Samnlumgr des entomolagischen Vereims. (Vgl. licrüber die entomologisehe Zeitung vou Stettiu.) - Ausserdem finden sichî noch Sammlungen von Dr. und Medirinalassessor Behm, Priasiclent W. v. Bonin, Oberforstmeister Bulow. Rieth, Stadirichter Cram er. $\Lambda$ potheker Dicklioff, Reutier Dohrn, 
Regierungsreferendarius D reger, Pro. fessur Hering, Lehrer Lincke, Regricrungsrath Selı idt vor.

Stoclsholna. Musemur. Sanunlungen der Akademie der Wissensrhinften. Mejist die fauna Enropae botealis. - Die Sammlungen der HH. Geyer (Director) und des Leibarztes $\mathrm{Haden}$ berg, damn des Prafessors v. Bezel, Cameralrath Billbere, Lientenant Bohemann, Wali berg.

Stollberg (bei Aachen). Jugendlehrer David Benrad, Dr. med. Korf um, Messiugfalurikant J y ue n. Lelsrer J. W. M e i ge u, ferner dic Tuchfabrikanten $P$. " Clur, Offermann, Messingfulurikant J. A. Pelzer, J. A. und M. L. Schle iclice, Tuelfabrikant J. W. Selımitz, Seeger, Gebrïler Stoltenh of hesitzen entomologisehe Cabinete und Sammhungen.

Stralsund. Dr. Hecht's entomologisehes Cabinet.

- Strnssburg. Universitn̈ts - nud Stadfmuseum (Conservator Dr.S r. h i mper, Aduinistrator Gust. Silbermann). Reich, selıön und instruktiv. 2000 Käfer, Falter 2600. (V vil. Isis 1844, Heft IV, p. 246.) - Die HH. Catoile de Biancourt, Barth, H. Franck, Steuerbeamter Goubert, I, erboullet, Sa Selim per und V.Zeysse loff halien ansserdeu noch Sammlungen zoologiselier Gegenstäude.

Stuttgart. Noturalienknhinet (Vorstand Obermedizinalrath . Dr. J äger, Conservator Dr. F.K rauss). Geride die Classe der Kerfe ist am schwärhsten. Die grösste Partic hildeu südafrikaniselie Küfer. - Museum des Inndwirthsehaftliehen Vereines (z.untioclist der Thieratzueiscluule). Conservotur und Sekretiir Dr, und Professor Plipninger. Sehr schiïn, vollständien und iustruktiv. Gestiftet von der grossen Naturfremdin Känigin Katharinu. - Dic Petrefaliteu-Sammlung des Major v. Zieteı. - Die Sammlung des Graten v. Seckend orf, kün. wïrtt. Regierungsraths. - Die Sammluug des $k$. Hof domainenratlos und Garten-Inteudanten v. Se yffer: - Die Sommlung des Bergrallis und Dr. med. Hehl (wïnscht sie z.ı verkaufen, da er noch eine zweite Sammlung hesitzt). Alle entlalten melir oder weniger versteinerte Kerfe, sowie Krabben. - F. Berge,
Dr. Ha u f f, Prof. J ̈̈ger, Hofökonom Koeh, Scliwertfeger Kohl, Lehrer Kol h, Dr. und Custos Ferd. K i a uss, Professor Kurr, v. Marteus, Candidat H. Nördiluger, Dr. Plie"I inger, G(d). Legationsrath C. L. F. v. Roser, Otto Seyffer I, Jowelier Josepla Trinker, S. Erl. Wilhelm Graf von Würtemberg, Präceptor Vol\% und Professor Zcuner.k haben unturhistoristlie Sammlumgru.

Stregniis (Sehwedeu). GymnasialSammlung.

Swansea (Wales, England). IV. C. Mr r ray's Entumologieal-Calinet.

Swinnem inde. Justizcommissarius' E rllard Sammlong.

Symphoropol (Taurien). Dr. med. Casim. Rostang's entomologi-. sche Srlıätze. - Clır. Ritter v. Steve u's Sammlung.

Tanzarlel (Thüringen). Pastor Kraus e's Sammlung.

Tavira (im Königreiclı Algarvien, Purfugal). Sanunlung einer niederläudischen Dane, welehe am Hafen wolunt.

Teneswar (Ungarn). Dr. Filldel's Cahinet.

Tharand (bei Dresden). Sammlung des Forstinstituts. - Dic Collection (von Petrefakten) des ( $t$ ) Hrn. Oberforstrnths v. Cotta. - Hieru komineu noch dic Collertionen des Prof. Kruzseh und Prof. C. A. Rossmässler.

hung.

Thorn. v. Nowicky's Samml-

Thmin (Schweiz). Browne's, des reishen britiselien Pliytologen entomologisme Sammlungr.

Toledo. Kleiue Sanmlung der Uuiversisät. Instructiv und eigentlich unr zum Gebrauche bei Vorlesungen.

Toul a (Russland), T e le ertk of f's Sammlung.

Tonlon. Pharmazeut's Banon Sammlung. Reich und schr schön. Flottenconmissair Gall e's, S e llier's und des Marineoffziers S a u le y (Broder des 11. S. in Ronen) Sammlungen.

Toulonse (Dept. de In liaute Garonue). Sammlung der UuiversitätsAkademie.

Tournay. Naturhistorisches Mu. secum dasclbst. 
Tremilug (bei Mühlstadt, Kiirn. then). Cooperator Gusse uhauer's (vgl. Flattach) Summlung. Vel ibi vel ubi.

Trient. Das Naturalienkabinet des Pater Taxis, Camoniens in dieselStailt.

Trier. Die Sammlung der Gesellschaft niitzliclier Uıtersuchungen.

Triest. Die Samulumgeu der uautischen Schule und der Academia reile, des Dr. und Apotheker Biasoletto, Egge uhöf fuer, Apotheker Napoli, des Fïrsten Alphons Gabr. v. Porcin und Lehrer L. Rol et t.

Troppan (öst. Selılesien). Museum der Stadt (Custos Hr. Faustinus Eus); danu die Sinnmlung des Custos und Professor Eus.

- Tray (Now.York). Das Museum des Lyceum of natural history. - Dic Sammluug ules Renselaer-lustitut.

Tilbiugon. Das Museum der Universität (bereichert dıreh v. Lıd. wig und Kiauss). - Dr. and Pri. vatdocent Hcrm. M p yer besitzt einc Collection von Naturalieu, sowie Stadlrath Nördlinger.

Turin. Sammlung der Akademie der Wissensehaften. Unbedeutend. Das Museum der Universität. - Nachstehende Naturfor'scher habeu Sammlungren: Bon afous, Prof. Steffano Disderi, Dr. Ferrero, Prof. Gè í, Giorna, Matteo Losauil, Ritter Graf A. de Marmora, Prof. Moris, Kriegscommissair G. L. Peiroleri, Carlo Perolli, Laur. Ponza.

Ufrenheiun (Rhciubnyeru). Condilor's Bullenh fimer Sammlung.

UIn. Die (zum 'Theil petrefietologische) Sammlung des Hru. Grafen von Mandelslohe, k. w. Forstraths.

Upsala. Naturalienkabinet der Stadt und der 1476 gestifteten Universität. - Museum der Akadeute del Wisscnschaften. - Des herühniten Afzelius Sammlung. - Die Sammlung des Dr. Falirne usund Nieolson, welche vermuthlich sich hier befindet. Utica (New-York). Das Museunı des Lyeeum of natural listory.

Utreelnt. Physikalischem und na. turhistorisches Muscum der Universitiit (1636 gestiftet). - Dr. und Director Numann's unil Prof. Schrö. fer vau der Kolk's Sammlung.
Vallarolid (Königreich Leon). Sehöne und interessante Sammlung der sehr busurlyten Universität.

Vandoenvre (bei Nancy). Pro.

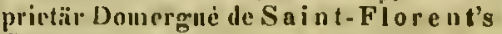
Sammlaing.

Veideslneinn (Rheinbayern). Hospitalarzi C. H. Selultz hat eine phytologistle "l. zoologische Sammlung.

Vencedig. Naturalicnknbinet in Lyceun. - Die Sammlung des Grafen Mantrini, des Dr. Andreucetti, Graf Nicolo di Contariui, Dr. med. Nurdo (anf der Insel Chiogria).

vendône. Monlet de Laroche hat eine Sumulung vou Coleoptereu.

Verous. Die Sammlung in $\mathrm{Pa}$. lazzo Rotari (Versteineruugen; nilier beknnut geworden durch Catullo's. S(chrift). - Die Sammlune vou Iguaz.. Bevilacyua La zise, - Die Sammlnng. des Grafen Orlando, des Bcru. Angelini und Benedetto da Campo.

Versgilles. Professors Baudry de Balzac und Airhitekt's Bloudel' Sammlungen; ferner dic des Cavallerie. Capituin Louis Emanuel Graf von Jousseliu, Professor St. Julien, Le roux und Akademiker Jul. César Savigny.

Vevay (Canton Wuadt, am Genfersee). Die Doctor La vater'sche Sammlung.

Vicenza. Sammlung des berühmten Forsthers Catullo.

Virginia Museum der Universität.

Voluratshofen (bei Memmingen, bayr. Schwabeu). Die Samulung des Hru. Baron v. L up in.

Wuldenberg, (Breslau). Markseheider Boeksoh's Sammlutg.

Wallenstadt (Schwciz). Pastor Eiseuring's Kerfsummlung.

VWalterslannsen (bei Giesseu). Gelieimer Sekretiil Fr. v. Selienk besitzt hier rine uette Sinmmlung.

Warasilin. Postoffiziant Natl y's Sammilung.

Wurmbrumn (in Schlesien). Antschiory Mnuger besitzt eine Käfercollection.

Warschnu (Czaarthum Poleu). Universititssammlung. - Das Museum der physikalischen Gesellychaft und der königlichen Gesellschaft der Freunde der Wissenschaften; die Sammlung des Hofrath Drzewieki und des Entonologen Waga. 


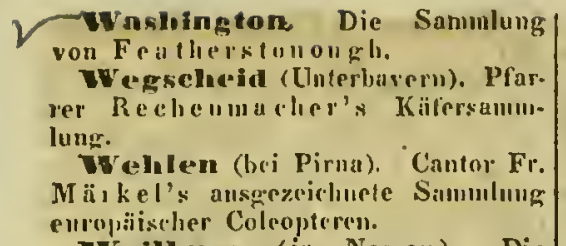

Weilburg (in Nassau). Die Sammlum der gesammten uassanischu'n Naturpiodukte des Professur Sa udberger:

Weinar. Dr. med. Froriep's (Sohn) Sammulung. - v. Göthe's Sammlıme.

tereissenfeld (a. d. S.). Sipre. intendent Heidenreicli's Sammlung und des Graveur Rudolplis Schmidt.

veentelsalorf (Cant. Solothurn). Fine Summlnug von Verstciurrungen vou Juta, Hot. IV allier gelıörig.

vEernigerode: (Prenssen). Die Sumulnuge im griflichen Residenzschlosse, gleich inber der Stadt (auf cineIII 8.2 $7^{\circ}$ loblen Berge).

Wresterdis (Silwerlen). Sammlung des Gymmasinms.

Wwest-rovint (Niw.York). Die Collectinn der Militiir-Akadenic. Dis. Sammlnng des Lycem of natural listorv.

whitelna!l (England). H. Downes' Sanminlug vou Coleupteren.

Wriazsna. (Russland). Maslenuikotr's Sunmlung.

Eviburg (Festung in Finnland). Carl Gust. Giaf von Munerlueim's Cabinet der Entomolugie.

wrien. Kaiselliches Naturalienkibinct. (Direktor: Ilr. P. v. Sclurei. bers: Cnstoden: Hr. Meperle von Mülllfeld und Hr. Kollar). Eine der grössten Simmulungen in der Welt. Die Samulungen der Universität. Besonders merkwïrdig sind die Pitrefacten des k. k. Hofmineralientiabinets. - Die Sanmlungen des k. k. polytechnisehen Instituts. - Die Sammlnig des k. brasiliselien Museums. - Dis Nafuralien- MInsenn der k. k. mediziniseh-ehirurgischen Josephs-Akademie (Josephinum). (Custos Prof. Dr. Fiselier, ein tiieltiger Gelchrter.) - Die Sammlungder k. Theresianiselien Ritterakademire (Theresiammu). - Dic Sammlung der k. k. Landwirtlıselhafts-Gesellsiliaft. - Die Sammlnng des Grafen Breuner. - Dic Sammlnug res
Fiirsten Paul von Esterliazy. - Dic Samulung des Marquis voi Malaspill und der Fıan v. Bartu\}. Dir Sammlnne des Angustiuevilosters. - Das Cabiuet res Servittenklusters. - Die Sammlung Si. k. k. Hoheit dey Evzliegzogs Rainer (iu der Burg. Custos $A$ bbe $G$ a pp von Ta an a bu rg). - Dis sanmlung der Fiirsten Alois und Johanu v. Lichtenstein (im Pilaste gol N.).-Die Samulung Sr. k.k. Hoheit deg Erzhorzogs Carl. - Die Sammlung des Di. Rolett. - Au diese reilien sicll die vielen Sammluncen der Privaten: Staulsfieldarzt v. Biscluoft, Burger, Dirsing. D): und Custus lindlielier, Graf v. Feriri. Fityinger, W. Foekr. Bauingenienr Gruber. Gruner, G iir ler, Dr. und IInfiatl $H$ a m nelselinidi, Di: med. Hin pr. Dr. wed. Jaequi (Solun). Dr. med. Fr. v. Kolen ati, Inspector V. Kollar, Dr. Krackowitzer, lir. Graf v. Knenb ura, Julins Lederer, Hofsilanespieler la zuwe, Miler Jos, Mann, Graf v. M ars s l a 11. Custos Joh. Carl Megerle v. Milhlfelde, Tecluiker Mille r, der Grafen Andeas und Grore Mniszech, Jol Natterer, Director Pat rsert, Naturalimuatudler Isudw. Palreyss (nur Vorrätlee), - Podewin, Dr. med, nnal Adjunkt Redtenbaclier. Sillott, Direct. v. Sclirej. bers, Seng, Di. Sipber, Carl Stenz, Reclimungsofficial Ulliricli, Weidnau, Friedr. We l witseli, Dr. Wu uluam, Fraur. Ziegler ( $\mathrm{Hy}$ menopteren).

Wiesbanten. Die Sammlung des Vereins fiir Noturkunde. Director liferiber ist Hr. Dr. "Tl on $\ddot{a}$, weleler cine Geschichte des Vereins fïl Natulkilnde im Ileryogthum Nassan und des naturhistoristelen Museums z.n Wieshaden (mit Vignetten. Wiesbaden und Sipren 1842. 8.) her ausgab. -1686 inlïndische Gattungen! der allgemcinen alls 3183. - Beeker, W. BInm, Kammerrevisor Groos und Dr. Ind Dircktor Thomi habeu nicht unansc-lunliche Collectionen.

Wilua (Lithamen). Das Musemn der katholischen Universitüt und des Etatsiotlss von Eicliwald.

Crindshein (Franken). Stadtpfirrer Grossm n un's Sammlung.

Winterthur. Dr. J. N. Sauter's 
Thisby (Gothland in Sehweden). Gyunasialsammlıng. Kabinet des Dr. K olw aliu.

Wiirzbura (in Franken). Die be. deutende Simmmlung der Universitait, gestiffet int Jahre 158:2 Irich an vaterlïudisrloen wie exotiseben Kerfen. Unter ihrem Couservator, Hrn. Dr. und Prof. Leiblein. wird sie zul einer der vorzägliehstenUniversitätssammlungen sirh erheben (vgl. dessen Sehrift: Grundziige einer mpthodischen Uebersiclit des Thierveiehes ete. Würzbury is39. 1. Bd. 8.). - Dr. Dallinger, Dr. mesl. und Physicus Laubreis und Dr. und Prof. C,ibleiı haben überdiess nncl, Simmulungen.

Tolfrathshausen (Oberbu. yern). Krümer K e rl loat eine Collection von bayrisehen Küferu.

Tarmoutli (Norfolkshire), Moseum tler Stadt.

York. Das Naturalienkabinet des Institutes.
Thhirovy in Bülımen). Bergmeister Prevssler's Kertiamunlung.

Ziurich. Die öfr.ntliche Sammlung Jer Stadt (in der Wasserkirche aufgestellt). Merkwï̀dirg Iurch dis Sammlung vou Courad Gessuev, des Dr. La vater etc. Entlialt etwas melir als dic von Bern; meist helvetische Kerfe. Wie man viruimmt soll Dreclsslermeisters Bremi Sammlung von Kunstwerken aus ller Insektenwelt der Sammlung cinverleibt werden. - Als cine alfentliebe Sammlnue ist zu betrachten die des Partienliar Escher-Zollikofer, mnsergeichnet reirh und nur von wenigen Musren an Vollständigkeit übertroffeu. Vou den mristen Gattungen siud ganze Suiten Doubletten vorhan. den, wis wissenschaftlich besondera wiehtig ist. - Folgende Privatsammlungen sind noch vnrlianden, als von Prnfessor Dr. He er, Dr. Alb. Köllieke i, Hofrnth wid Professor Oken (wollhe wir in rinem Sommer hindurelı geordnet und bestimmt haben), Cnspar Schulthess. Eseher. 


\section{Syllogistische Schriften, oder von der Kunst des Sammelns, Aufbewahrens etc. der Kerfthiere *.}

\begin{abstract}
Abbildumg und Beschreibung der Schmetterlinge nach ihren drei Lebensperioden; nebst Anweisung, wie sie z.l trzielien, z.u fangeu, z.u behandeln und aufaubewahren sind. 1. Hft. IMit 10 snuber illnm. Kupfert.). 8.

Anveismmg, kuze, Naturalicn zu sammeln. Aufgesezt von Joh. Jak. Bossart. Barby 1774. 24 S. 8 .
\end{abstract}

Clésins. Anleit., Insekt. z. snmmelu.

Bromn. Anleitung zum Sammeln, Znbereiten und Verpacken vou Thieren, Pflamzen und Mineralien. Heidelb. 1838.

Esiselt, J. N. (Dr. med, in Polima). Kurze Anweisung zom Aufsuchen, Faugen und Conserviren der Lepidoptera, Neuroptera etc. (In Opiz's Beitr. 7. Naturg. 11, p. 312-322.)

Erxleben. Auweisung Insekten zu sanımeln (Hamüv. Magaz. 1765. 30, 40. St. 609).

Gürtner, M. G. Gesanmelte Ge. heinmisce und Vortheile für das Iusek. ten - und Selmetterlings-Kabinet, für Schmetterlingssimmler und Liebhaber derInsektenkunde. Mit 1 Kupf. 8. Leipz.

Teier (Joh. Dall.). De veruice ad eonservanda insecta et animalia (Mise. Aead. Nat. Curios. Dec. 2. A. 8. 1689. p. 297).

IXihu (A. C.). Anwcisung Insckteu z.n summeln. Eisellach 1773. 8.

Meinelsen (J. G, F.). Aulcitung Insckteu zu sammelu (Naturf. I St. S. 229).
Fezold. Lepidopterisclie Anfangsgründe zuII Gebrauch angrohender Schmetterlingssammler. 8. Colurg 1766.

Schmetterlings-Kabinet fiir Kindel, oder kurze Besclireibung der eurupïischen Schmettrulinge; nebst Anweisung, solclie zn fangen, aufzuhewahren und ans Raupen zu zichen. Mil 91 illum. Abbild. 8. Leipz.

Schmiedlein. Tasehenbuch füj Insektenfrenude. Leipz. 1784. 8.

Stein (Kiıl Phil. Clırist.). Hand. buch des Zubereitens und Aufbewahrens der Thicre aller Classen ete. Frankf. 1802.

Snckow. Das Naturalienkabinet, oder gründliche Anweisung etc. Mit Abbild. gr. 12. Stuttg. 1835.

Thon, Dr. T., die Naturgeselichte der in - und ansländ. Schmetterlinge nit einer Allweisung zum Srlumetterliugsfang u. z.u deren Auflowalırung. Mit 66 Tafeln Abbild. Schumal gr. 4. Lvipz. 1833.

Vogel, C. F., vollstïudige Anwei. sung über das Aufsuchen und zweck. mässige Erzichen d. Raupen, das Ausgrabeu d. Puppen, Einfungen und Aufspamuell d. Schunctterlinge ete. gr. 8. Berlin 1838.

Waltl. Winke zur Aquisition sel: tener Insekten (Gistel's Fanums).

Westerhanser (Josepli). Bemerknur zom Habhaftwerden seltener Insekten (Gistel's Faunus Bd. I, Hefi 2, p. 119).

* Anleitungen zum lnsektensammeln enthalten die Sichriften von Gärtncr (vgl. Lexilkon p. 31), Ingpen. Mallunwsky (p. 50), Pechioli, Rennic. KIrby uns spence, Oken, Rockstroh. Sclimldi, F. J. Schmldt, Shick a $\mathrm{k}$. Struve, v. Tiselier,

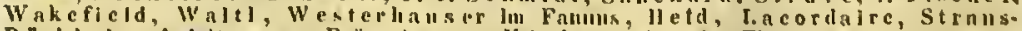
Dil rkhelm (Anieitung zum Pröpariren). - Vgl. das Lexikno der Thertologen. 


\section{Register der Wohnorte und der dahin gehörigen Personalien*.}

Aachen (Niederrhein, Preussen). Debey. Förster. Lesoine. Kaltenbach. A bo (Fiunland). Alistedt. Dammert. Saliberg. 'Tams.

Aduont (Steyermark). Angelis.

Aix (Bouches-du-Rhône). Buyer. Boyer de Fonscolombe.

Mix (Provenee). Saporta.

Alexandrien (Aegypten). Cèrisy (Lef: de). Vyl. Lefehvre.

Aigier (Afrika). Bedeau. Meiss.

Alteuburg (Saclsen-Gothi). Apetz. Waitz. Kanoldi.

Altona (Holstein-Dänemark). Sommer.

Ansterian (Nordholland).

Franke, jur.

Andover (Massadusetts. Nordamerika). Gray.

Amsbaeh (Mittelfranken. Bayern).

Kiishler.

Arusdore (bei Sclımiedeberg in Srhlesien). Teirhgriber.

A rusberge (Westphalen. Preussen).

Varendorff.

Arras (Pas de Calais). Clinu-

veant.

\section{Ascliatrentomres}

Bayeru). Dühnes: Kittel.

A aclatoncli (bei Inrgwintheim in

Frauken). Kress.

\section{Aschersleben}

Pieussen). Holmung. Liben. Nellling.

Acka (Ungaru). Pronay.

Aub (bei Würzburg in OberfrankeII). Römer.

Augs bures. Biseloff. Fagerrotl. Freyer. Petry. Stadtmiiller. Wagner. Anriol (bei Marseille). Muriog. Avignon. Requien.
Tamberg (Franken). HauptKireliner. Sponsel. Weisenfeld. efarby a. d. Suale (Magdeburg. Pleusseu). Hermes.

IEnrcellous. Campa. Grülls. Janel. Jurdano. Sans. Yauez.

Fuxluan (Eugland). Lïrby.

Earusoul (Sibirien). Gebler.

IBarthél cuy (Antillen). Foı atıöm.

Basel. Hauhart. Imhoff. Labram.

Meissner. Mieg. Mïuch. Werthenแเamก.

Entignolles (Seine), Foulques de Villaret.

Eennenire (Dep. du Gard). Baridon.

IBanme (Doube). Girod. Chantrans.

Bantzen (Sclilesien). Gerlıardt.

Lecricliem (am Odenwalde). Wider.

Meichlingen (Sehloss in Thürin. gen). Ramidolir.

Berlin (Braudenburg. Prenssen). Anderscli. Bemlated. Bonché. Dierbacl. Elurenberg. Erichson. Fintel. mann. Gabriel. Godet. Gruff. Gurlt. Hemmerich. Hertwig. Isensee. Humboldt. Klug. Körfe. Knnewku. Krassow. Kriiger. Leyde. Link. Mevers. Miilles. Olfers. Otto. Phillippi. Philippi (R. A.). Rei(l. Ruthe. Schiippel. Schultz. Sehulz. Stanuius. Steffuliny. Streubel. Troselsel. Virmand. Zimmernum॥ (?).

Berm. Benteli. S. Brunner. St. Brumer. Ougrshurger. Perty. Shuttlewortl. Stettler.

Bingen (Grossherz. Hessen.) Wogwer.

Eiruinglaam. Marshall.

- Vgl. dle Tafei der Museen. - Diese Ueberalcht wird auch den resp. HH. Buchhindo. lern bei firen Verwendungen voll Nutzen seyn. 
Ditclue (Elsass), Sehultz.

Bolderau (bei Rign). Gimmerthal. Bologua. Bertoloni. Negri.

Hown. Goldluss.

Bopprard (am Rlıcin). Bach.

Bordenux. Blanchard. Dirgelas.

Lalande. Lamberthod. Perrières. Ruger.

Borentin (bei Ratibor). Zebe.

- IBostour (Vercinte Staaten). Greene.

Harris. Peek.

Botzen (Tyrol). Büttner. Kil'clier. Knoflanch.

Arandenburg (Potsdan. Preussen). Vieweg.

Ernsilien. I dotzky.

Fraunsberg (Königsberg. Preusseil). Sagere.

Fraunscliveig. (Braunschweig, Wolfenbrittel.) Hartig. Lachmaum. Lindner.

Bremeu. Norwich. Pagenstecher. Schmidt. L, Clır. Treviranus. Wilkens.

Bresian (Sclılesien. Preussen). B arkow. Beruldingen. Döring. Dylırı. F igulus. Frank. Friedrich. Gloger Gravenhorst. Jacnscl). Klopseh. Kornatzki. Isetzner. Lübbert. Matzck. Nees von Esenbeck. Nerustadt. Otto. Reudschmidt. Richter. Rotermund. Rüdi ger. G. Seluilling. Schilling (Just.). Schmidt. Schummel, Steffens. Uechtritz. Wimmer.

Brest. Garnot.

IBrieg. Döring.

Bristol. Mrillard.

IBromley (bei Londou). Forster. Britun (Mähren). Diebl, Kupido. Müller. Rau.

Brifssel (Südbrabant). Leopoid. Bus. Drapiez. Schayer. Schuermum. Wellens. Wesmael.

Burgfield (England. Bird.

Burassiorf (Cinton Bern.) Meyer.

Buregrvintlueim (Franken. Bay. eru). Schaedel.

Caen. Blot.

Culcutta. Pearson.

Cnmbrilge. Babington.

Caphanu (Eugland). Bennetf. wigr.

Capustndt (Atrika), Krebs, Lnd-

Carlsrulıe (Baden). Aekermann. Brann. Schrickel. Waldmer.

Cussel (Clunf. Niederluessen). Glasewald. Gundelatel. Junker. Landgrebe. Möller. Niepold. Richl. Schwarzenberg.
Castelnamary (Aude). Andréossy. Encely. Gauzy. Viala.

Castelumovo (bei Cerboli). Luciami.

Catanin. Ferrara.

Cayenne (Franec èquinoxiale; isle). Jacuier.

Chnilous-sur-Mnrue (Champagne). Dagonet. Mahicu.

Clntohs-sur-Saône (Bourgogne). Arvisenet. Bitstard.

Chamereclk (bei Cham, Unterbayern). Prablberg.

Clnarlottenburgo (Brandenburg. Prenssen). Stein.

Clanctres. Guenè (vgl. Paris). Clnartres. Marehaud (sen.). Villiers.

Châteandun (Eure et Loire). Guillaumé (vgl. Paris).

Chelsen (bei London! Vigors.

Chenée (bei Lüttich in Belgien).

Robert.

Cln ristianin (Norwegen). Rasch. Sicbke.

Clnur (Schweiz). Wredow (vgl. Neapel.)

Citta-Vechin (anf der Insel Lesina in Dalmatien). Niseteo.

Clanstinal (Hannover). Saxesen.

Coln (am Rhein). Peill. Rave.

Zanders.

Colniar (Elsass), Cantemer.

Colonubier (Canton Neucnburg). Gelien.

Concise: (Schweiz), Mellet.

Constantinopel. Cachorn.

Countanz (Buden). Leiner. Macaire. Marmor. Nenning. Seyfried. Crefeld (Cleve-Berg. PreusseiI). Bruck.

Crebmenczk (Vollynieu), An. drzejowsky.

Cremoun. Bertazzi,

- Cubar (Antillen-lusel. Westindicu). Gundlach. Alex. Macleny.

Damzig (Preussen). Berendt. Bock. Schmilt.

D) nenustndt (Grossherzogthum Hessen), Caemmerer. Glaser. Höplner. Kuup. Klingelhöfer. Reissig. Sehleiermadier.

Dn rufy (Vogesen). Lepaige.

Del cen ont (Delsberg. Cant. Bern. Jura). Verdat.

Delnenlorst (Grossh, Olden(burg). Oppermann. 
Deptroral (England). Newmann. Digme (Dept. dles Basses-Alpes). Honnorat. Yvin.

Gijou. Cluristol. Lacoste. De Lamarek. Lanier. Lacordaire. Vallot. Mouno d'Bssola (Picmunt. Upber'u Sinuplon). Biedermann.

Doruburg (a. d. Saile). Fack. Dorpat (Lictland). Asinus.

Dortunund (Westplalen), Charpentier.

Dresden (Künigr. Saclisen). Corberun(?). Carus. Ficinns. Gruner. Harzer. Kaden. Reichenbarh.

Boublin (Irland). Jones.

Ditrben rin Kurland). Sehoen.

Diiren (Aachen. Preussen). Sehüll. Steffens.

Ditsseldorf (Cleve-Berg. Preussen). Hildebrandt.

Duminove (bei Kowal in Polen). Alers.

Fclirilans (Canton Wadt). Chavaunes.

Edlinburgla (Siidschottland). Duucall. Makenzie. Stark. Walker (S. P.).

Conterinoslawl (Russland).

Freirriss. Hampt.

Wibenstoek. Thierseh.

pisenberg (in Sachsen). Kyber. wiberfelid (Cleve-Berg. Preussen). Cornelius. Fulilrott.

Wldenn (bei Greifswalde). Grebe.

wistbethgrnd (Gouv. Cherson).

Borliniak. Verleiı.

Fuglaud. Rees.

Finoutekis (Lappland). Grape.

Eppiug (England). Doubleday.

Erfurt (Preuss. Sachseu). Kefer. stein. Selımidt. Strübing. Weissen. horn.

Firlangen (Franken. Bayeru). Handsiliucl. Küster. Rosenhauer. Sipbold.

Escliveller (bei Stollberg). Graeser. Schlehek.

Essex (Ungarn). Hauschka.

cupen (Aachen. Preusseu). Fassbender.

Furnsburg (bei Augsburg). Muyer.

Falnise (Dept. de Calvados). Frenaye (de la).

Finsterbergen (in Thïringerwalde. Sichsen.Gotha). Kellner.

Fiattach (in Oberkärnthen). Gusscuhaner.
Fiorenz (Toscana). Antinori. Corti. Guiciurdini. Iseopold II. Passerini. Pechiali. Spenee (W. B.). Spenee (R.).

Frnulifurt a. M. Heyden. Kietsehmar. Riese. Riippel. Srhotf. Zrller. Nrankfurt $a . d$. 0 . Metzner. Rilliter.

E'rnazensiond (Böhmen), Palliardi.

Freiburg (im Breisgau). Bougine. Menzinger. Perleb.

Nricelwald (Niederlessen). Bauer. Friscliviller (bei Strassburg).

Stransi-Duirckhrim. (Vgl. Paris.) Fihufurchen (Sindungarn). Nendtwicli.

wida (Churliessen). Sehmerfeld.

Fadnluning (England). Rusticus. Gransen (bei Brieg. Wallis). Anderegge

Frinutz (Steyermark). Erzlierzog Jolıanu, K. H.

Gর̛̃zz (Pommern). Triepke.

Fanuwitz (Schlesien), Ehren. lians.

Cemarke (Cleve-Berg. Preussen). Stein.

Fenr. Chevrier. Clooisy a. Choisyb. Huber (P.). Juriue (Fils). Iasserre. L'Huillier. Mielzinsky. Pictet. PrévostDuval. Wallner.

renara. Durazzo. Spinola.

Cenrgitefsk (Gouv. de Caucase). Grjiboflisky.

(x́ern (Fïrst. Reuss). Sehmidt.

riessen (Oberliessen). Balser.

Engelber's. Seliweigger. Wetter. Gelasegove (Selootlaud). Hooker. Cxiatz (Schlesien). Pohl.

Ğoeduitz (Kärntlıen. Gurkthal). Baclier.

Calogan (Sellesien. Preussen).

Zeller (P).

Ciirlitz (Selulesien. Preussen).

Weirel.

Cîbtiugen (Hannover). Bartling.

Berthuld. Fehler. Koller. Wagnes.

Folulis (Schlesien). Spröde.

reotlir (Sachisen-Gotha). Baumbael.

Desberger.

Cx'rea. Runiy.

Giratz (Steyermark). Grimmer.

Haltmeyer.

freifs unald (Pommern. Preussen).

Hornsehuch. Rosenthal. Selilling.

Schultze. Weigel.

Grénoble (Danphiné). Crepu. 
Grindelwald (Canton Bern). Mïller.

Crrosswardein (Ungaru). Farkas. Grnnwitz (in Schlesien). Minkwitz.

Gisteig (bei Saarnen i. d. Sclıweiz). His..

Ainmzenlnamsen (Baycru). Fehr. Welser.

Muarle:en (Nordholland). Anslyn. Hage (bei Friesack). Moritz.

Malberstadt (Magdeburg. Preusseil). Troschlie.

Henll (in Tyrol). Russegrer.

Pralle (a. S.). Bulle. Burweister. Gepnar. Kanlfuss. Münter. Nicolai. Runde. S.haum. Schlüter. Slrack. Sinckuw (G.). Zschorn.

Hamberg. Beske. Dregè. Jaegev. Lelomann. Steetz. Struve (H. v.). Thorey. Winthem (W. v.). Winthem (A. v.). Wiebel.

Hameln (Hamover. Calenberg). Pflumer.

Hithan. Leisler.

Mannover. Hausmann. Hegewitsib. Krösmann. Mühlenpfordt. Spangenberg.

Havama (auf Cuba. Antillen). Mauleay (Wilh.) (Vgl. Cuba) und Solın. Raymumt Je la Sagra.

Heidelberg (Baden). Bischoff. Brotz. Frantzius. Kilpp. Ledebour.

Mexenneker (bei Regensburg). Weidenbald.

Mildesletim (Hannover). Leunis. Santer.

Mirschiberg (Schlesien). Felırle. Flotuw. Langer. Stillfried.

IIof (säebs. Grïıze. Franken). Bassch. Sthün.

Holnenelfoe (am Fusse des Riesengelirges). Hankil.

Folnenfurt (bei Budweis in Böhmen). Nenning.

Hohenhein (bei Stuttgart).

Mögling. Silbüle.

Inmilisbnras (bei Magdeburg). Natbusius.

Ingenbroieln (bei Montjoie). Stollberg.

Ingoistadt (Bayern). Bauer.

Iu-Itell (bei Traunstein. Bayern). Mayer.

Inswich (England). Jerm.

Trknzik (Russland). Thermin.

Irsingen (bei Türkhcim). Mayr.
Jacohsl iagen. (Hinterpommern). Tielle.

Jassy. Crihak. Mayer. Sturdza. Eava. Fischer.

Dend. Huschke. Koch. Krituse. Thon. Voigt.

Jolimont (bei Bern). Schuttlewarth (vgl, Bern).

In wiserslautern (Rheinbayern). Bernhein.

Hamichlof (Gouvern. Perm). Somuff.

Ianatschatka. Kouzmichtchef. Hornstando (in Lappland). Grape (vgl. Enontekis).

Hasai. Eversmanu.

Autharinenhof (bei Flensburg in Holsteili). Jordt.

Terensk (Gouv. Penza), Beklemirlieff. Ranzoff.

Herzthely (Gran. Ungarn). Rumy. Iharliof (Russland). Krynicky. Kiel (Holstein) Boie. Drewsen. Meves. Trentepohl. Wiedemanu.

Kiew (Russland). Besser, Czekanowsky. Hochut.

Kitzingen (Franken). Seelig.

IIngenfnrt (Kïnthen). Hüber.

K Ianseninure (Ungarn). Teleki.

Mleinrotosetzt (bei Turnau in Bölmenen). Hering.

TLeinselamalkalden (Churhessen). Hölzer. Hofmeister.

Flibtz (bei Stettiin). Richter.

Iunigzsherg (Preussen). Hagen. Ledebur. Rathke. Riehter. Rockstroh. Kopenlengen. Beck. Colsmann. Diewsen. Jacubson. Lerke. Kaas. Reinhardt. Schiödte. Seliouw. Stäger. Thienemann. Westermann.

Kotolow (?) (bei Friedland in Mecklenhurg-Strelitz). Muschl.

Hrackan. Estreicher.

Mranchthal (bei Bern). Jaegri. Hrensutinster (Oberöstreich). Altwirth. Erlacher. Hartenseluneider. Schocuberger.

I rentl (im bayr. Oberlande). Krämer.

Drenaberg (Schlesien. Preussen). Mever.

Lrenzmacln (an der Nahe. Hesseni). Orlich.

Lronstralt (im finmiselien Meerbusen). Kyber.

La Tassée (bei Lille). Alavoine. Laler (Breisgau). Laugsdorf(Vater). 
Lancashire (England). Norris. (VRl. Rotrales.)

Lausanue (Scluveiz). Bugnion. Chavannes. Forel. Godet. Huber. Laybach (Krain). Coronini. Freyer. Graf. Hohenwarth. Kokei]. Koss. Sehmidt.

Leertii (bei Lüneburg). Reosmanu.

Leipzig. Bayrhoffer. Ehrlardt. Frunke (sen.). Gärtner. Kiesenwetter. Krutsch. Kunze. Langstengel. Runtzao. Sachsc. Schicuereck. Scholze. Schwaegrichen. Tilesius. Vogel. Will. Wiukler. Zeiz.

Lemberg (östı. Gallizien). Zaeher.

Leobscluitz (Schlesien. Preossen). Fiticker.

Leyden (Südholland). De Haan. Tipinwardt.

Liebenstein (Schloss bei Eger iu Böhuren). Richter.

Ginoges (Haut-Vienne). Fray.

Linz (Oberöstreich). Erlacher (vgl. Kremsm.). Knörrlein. König. Pirngruber.

Misspibon. Macedo.

Uiverpool. Melly (vgl. Manchester).

Lodenau (Schlesien). Haopt.

Coirent (Frankreich). Sganzin.

Gond on. Ainworth. Bainbridye.

Barchard, Barklcy. Beckwitl. Bell.

Benuett. Bingley. Blundell. Boud.

Bowerbank. Browne. Cantor. Chane.

Christy. Children. Clark. Curtis.

Dale. Davis. Delta. Dillwyn. Don.

Don. Espinasse. Flemming. Goodal.

Gould. Gray, Griffitlı. Haliday.

Hanslow. Hanson. Hardwicke!' Heys-

ham. Hobhouse. Holme. "Hope.

Horsfield. Howitt. Ingpetı. Jackson.

Jardine. Jenyns. Kirkop. Lees. Le-

keox. Lewin. Lewis. Loddires.

Markwik. Marsham. Neale. Nuttal.

Pickering. Petit. Read. Renuic.

Ronalds. Rodd. Riley. Roe. Rogerson. Roget. Samouelle. Saonders. Slieppard. Shockard. Sillimans. Smee. Skrimshire. Sowerby (J. ner.

E.). Sowerby (G. B.). Spence CHIidlletown (Connetifut). Jolın. (W.). Stevens. Stcpliens. Sykes. ston.

Todd. Unterwood. Wailes. Wallich. Walker (Fr.). Wakefield. Walford. Walton. Waterhouse. Westwood. White. Wilson. Wood. Yurrcl. Lucen. Alberti. Bonaparte.

Lümeburg (Hannover). Heyer.
Eilticin (Belgien), Carlier. De Konink. Morren. Selys de Lougchamps.

Ennd (Sïdgothland. Schweden).

Dalılboun. Düben. Fallén. Fries. Lagemann. Munk of Rosenschold. Petterson. Rosenschiöld (vgl. p. 54). Sundewall. Zetterstedt.

Mnzeru (Srhweiz). Baumann.

Hyon. Clardiny. Donzel. Fontenay. Foodras. Seringe.

Wratras (britisch.0stindien). Heath. Mredrid. La Gasca. Villanova.

Magdeburg (Preussen). Banse. Damm. Fest. Matz. IKrasper.

Wniland. Bassi. Betta (Fratelli). Bonola. Branibilla. Carmagnola. Co. molli. Fermario. Galeazri. Jan. Longhi. Maggi. Marietti. Medici. Nicoli. Perroud. Ponzoni. Porro. Vechi. Villa (A. et G.).

Masiuz. Schınitt.

Malxens (Grauböndten). Am Stein. Mrnchester. Mrlly.

Tranutucim (Barlen). Suckow. Van der Wyck. Vogt.

Mrerbarg (Steyermark). Holzer. Marburg (Korhessen). Gundlaci. Herold.

Mariuge (Dep. Poy-de-Dome). Beaudet-l_afarge.

Trarseilie. Barthélemy. Famin. Leantier. Roox.

Mazurgues (prìs Marseille). Solier.

Meckienbars (Strelitz). Mussehl (vgl. Kotolow).

Meiningen (Herz. Sachsen-Meiningen). Baumbach. Lomler.

Meissenleisn (am Rhein). Hoffminn.

VI êsıugeu (Churhessen). Wäckel. Hemuingen (Sclivaben. Bayern). Wachter.

Mergentheim (Württemberg). Prinz Pinol.

Messina. Arrosto. Giacinto.

Metz (Dept. Moselle). Cajré. Hollandre.

Preyringen (Cautou Bern). Brön.
Militscl. (Sehlesien). Hartlieb.

Mitau (Kurland). Fleischer. Lich. tenstein.

Madling (bci Wien). Heeger.

Montfoie (Aachen. Preussen). 
Noutmorency (sur Seine). Boll. dier.

Montpellier. Aubé. Chabrier. Gasparini. Honnaud. Salzmann. Serres (M. de). Villiers.

Moskau. Bahr. Fischer von Waldheim. Khloustine. Laveau. Lovetzky. Marin. Olnhausen. Bascaut. Zubknff.

Nihilinusey (Defore haute Rlin). Broch. Wor line. Wapler.

Trahlheiny Rhein. Cöln. Preusseni). Wenig

Iriincles (1 (Bayern). Debruyn. Drary. Erd. Fleschuez. Gemminger. Gistel. Hefner. Held sen u. jun. Helmsaner. Kriechbaumer. Kummer.Ledebour. Muess. Mayer. Mayerhofer. Merkel. Morawitzky. Oberleitner. Roth. Schenkenberg. Sehubert. Scliwab. Stark. Steinkirclmer. Stoliz. Tascher. UIlersperger. Wagner.

Nantes. Cailland. Dubuison.

Naunburg. Känufer.

Faurod (in Nassau). Ilgen.

reapel. Briganti. Chiaje (delle). Costa. Piazz. Tripaldi. Wredow (vel. Chinr).

Neden (bei Cleve). Macuss.

Nertchinsk (Gouv. Irkouzk). Vladsimilsky.

Sencn burg (Schweiz). Agassiz. Cartier. Coulon. Godet. Nicolet. Wavre.

Teulnaldensleben (bei Magdelsurg). Fickler.

Teuliansen (bei München). Garduus.

Nensiedel (am See). Stenz.

Nensolul (Ungurn). Zipser.

Nenstadt (a. d. Aisch in Frankeu). Brand.

Nenstait-Wberswalde (Potsdam. Preussen). Ratzeburg.

-Nev-Maven (Nordamerika. Conneeticut). Harris. Herrick. Hutton.

New-York. Leconte.

Nijni-Novgorod. Pelatier.

Nixuorf (in Böhmen). Fischer v.

Röslerstamm.

Nizza. Maecary.

Yufring (bei Gmünd in Oberkürnthen). Gotsch.

Norwich. Denny. Wilkin.

virnberg. Jaeckel. Lechner. Sturm.

Nutrenen (Schweiz), Felix.

Ddeubach (bei Mainz). Müller./novsky.
Ddessa. Bertholdy. Mühr. Nord. mann.

Dedenburg (Ungarn). Gallus. Ockskay d'Orksko.

Deluringen (Hohenlohe). Oesterlen.

ofen. Kindermann (sen. u. jun.). Neuscliel. Pyday.

Dlilenbure. Schloifer.

(Dravitza (Banat). Wicrzbicki.

Dremburg. Karèline.

Drleans. Boulard.

Dstende. Sehnabel.

Palermo. Grohmann.

Paris. Amyot. A. Aymard. Bar. det de Longuesse. Barker-Wepp. Berneaud (vgl. Thiebaut). Biot. Blainville. Boisduval. Boitard. Bonpland. Bory de Saint-Vineent. Botin-Desylles. Brebisson. Brulle. Buquet. Mdme. Caillard. Chevrolat. Comte. CuvierDebraut. Defiance. Dejean. Delamontagne. Delessert. Descourtilz. Desliayes. Desmarest. Desnonlins. Desvoidy. Doguero. Doumerc. Dugès. Dujardin. Dumecil. Duponcliel. Dupont. Elwards (Milne-). Duvau. Der Herzog von Esslingen (vgl. Rivoli). Eydoux. Fristlammel. Gaimar. Gaubil. Gaudichaud. (Isid.) Geoffroy Saint-Hilaire. (Etienne) Geoffroy Saint-Hilaire. Geoffroy de Villeneuve. Gervais. Gory. Goudot. Goureau. Guenèe (auch in Chartres). Guèrin-Mènèville. Guillaunè (vergl. Clıâteaıdun). Hoffmam. Hombron. Jacquinot. Javet. Julien. Lachat. Lacoste. Lamarck. ILanier. Luporte. Laueret. Ledoux. Lefebvie. Lepelletier de Saint-Fargau. Leprieur. Le(quien. Lechenaud. Lessan. Lneas, Luczot, Marquart. Malo. Martyn (pere et fils). Miger. Milbert. Moux-Deloehe. Mulsint. Nodier. D'Orbigny. Paulet. Pereheron. Pierret. Pouret. Poey. Puzos, Qnoy. Rambur. Rejele. Rivoli. Romand. Rousseau. Sainson. Saint-Hilaire (Aug.). Sallè (Augusta). Sallé (père et fils). Serville (Aud. de). Strauss-Dürkheim (vgl. Fröschwilè). Madènois. Thièbaut de Berneaud. Ars. Thiébaut de Berneaud. Truchet. Vasselet. Vaudouer. Verreanx. Villaret. Walkenaer. Parana. Bertè. Monici. Rondani. Fossan (Unterbayern). Waltl. Envia. Brugnatelli. Zendrini. pechau (bei Mlagdeburg). Mali. 
Terpignan. Compagun. Farines. Pertitsclief(in Volliynien), Chaudoir.

Pestlu. Frivaldsky. Sadler. Sattler.

Plniladelphir. Barton. Bul-

dry de Lozieres. Collins. Fnggo. Godman. Hentz. Jenison. Latrobe. Ponceau. Rensslaer (vgl. New.York). Zimmermann.

PPisa. Clicsa. Savi.

Poblet (Abtei von, in Barcellona). Jordano.

Point al Fétre (auf Guadeloupe).

L'herminier.

Policza (Böhmen). Eisclt.

Pontarlier (Doubs). Ecoffet.

Poseu (Posen. Prenssen). Löw.

Potulam (Brandenburg. Preusgen). Türk.

Prng. Ceel. Corda. Demel. Dietricl. Feldner. Fieber. Hinciss. Mikan. Nickerl, Opiz. Pressl. Riedl. Schuidt. Seidel. Swobodit. Trautmann. Wastel. Weidlenhoffer. Wimmer. Zillieh. Zippe.

Protz de Miolo (Dept. des Pyrences or.). Xatart.

Pressbura. Dorner.

Przennysi (ostr. Galizieu), Zawadzky. A.).

Pulkova (Russland). Struve (G.

Pyrmont. Brüder Speyer.

Quenstedt. Nimrod.

Raumennu (bei Dresden). Hoftmannseg.g.

IBntibor (Schlesien. Preussen). Guttmann. Kelch.

Ravenun. Tilesins.

Regenshurg. Angerer. Emesti. Fürurohr. Geyer. Herricll-Srlueffer. Hoppe. Korlı. Sehueh, Zienler. Tegaio. Brignoli.

Teluin (Mecklenburg). Daniel. Reiclıstadt (in Bölumen). Mann. Remand près Vendôme (Loire-et Clier). Dutrochet.

Menthendorf. Brehm.

Tentlingen. Keller.

Rlueine (ivestplialeu). Murdfield. 耳inzan (Russland). Vozdvichiusky. IBibenuville (Dept. de HautRliii). Sclureiner.

utiga (Liefland). Jensen. Sodofisky.

: Mifferswyl (Canton Zürich). Hegetschweiler.
Fochester. Dewey.

Ridgen (bei Montjoie). Müller.

IRour. Metuxa. Rulli.

RotenIs cls. Schiipfer.

Eotrales (bei Manchester). Norris (vgl. Lancashire).

Rotterilam. Brants. Van der Hoeven.

Tonen. Lebas. Maille. Sauley.

Itigenwalde (Pommern. Preus. sen). Güden.

Htiilow (in Merklenburg). Sponholz.

Budolstant. Friedrich Gïuther, Erbpriuz von Schuvarzburg-Rudolstadt. Sore.

Ruscoczin (bei Danzig). Tiedemallu.

Ryssel. Macquart.

Sarnen (Cant. Bern). Roetzer. Sangritz (bei Döllach in Kärnthen). Rotl.

sit. Albens (Country Hertfort). Swainson.

St. Bnrtluéleny (Antillen). Forstroem.

St. Denis (Insel Bourbon. Masearenhas), Rondic.

Srint Ferimnin en Tnye (bei Paris). Isepelletier de Saint Furgeau. Suint rericur (Chamonny). Balma. Saint-Géver (Dept. des Landes). Dufour.

Saint-Snveur (Yonne), Robineau. Drsvoidy.

salop (England). Edwards.

Salzburg. Gries (Jae. u. Joh.) Mayburger, Nageznun. Spatzenceker. Salzvedeln (Mngdeburg. Preus. sen). Dammeil.

Ganunr (Maine et Loire). Courtillier.

Sin Antonio de In Floride (bei Madrid). Loplez.

St. Florinn (Steyermark)。 Schmidberger.

st. Rallen. Hartmann. Meyer. Zolliklınffer.

St. Leonlonrilt (bei Marburg in Steyermark). Spitzy.

St. Petcersbure. Bacr. Bartels. Blasclake. Brandt. Etholén. Falderinanu. Fischer. Hamel. Henuing. Jaeger. Martini. Menitrics. Meyendorf. Motschulsky. Oberts. Punder. Piecolomini. Sahlbcrg (Sohu), Selırenk. Slovitz. Stjernvall. Stschegloff. Tschernikh. 
Sareptr. Wilhelms. Shumur (Maine-et-Loire). Courtillier.

Scha Thansem. Seiler. Zwick.

Schandau (Säclıs. Sehweiz). Tiseher.

Schleck (Curland od, Sehoouen?).

Büttuer.

Sclnmierleberg (Sehlesien). Külı. ler.t Marbary.

Sclicipren. Rothenhach.

Seen (bei Winterthur). Rordorf.

Senegal (Afrika). Dumoliu.

Folleun.

Sevilla. Campos.

Siegen (Westphalen). Suffrian.

Siena. Giuli.

Skisa (bei Stoekholnı). Sehoenherr. Sunyrna (Natolien. Asien). Helfer.

Solil (Ungaru). Radvanki.

Solnruenudorf (bei Gürlitz in

Schlesieu). Stiller.

Sparschter (bei Marienstadt in

Westgothlaud). Seliöuherr (vgl. Skara).

Apeyer (Rlseinbayern). Liuz.

Stemdal (Magdeburg. Preussen).

Seloottstedt.

Stettin(Ponımern. Preussen). Belım. Boniu. Bulow-Rieth. Cramer. Dick hoff. Dolsru. Dreger. Hering. Limcke. Selumidt.

Stochlnolin (Selweden). Bezel. Billberg. Bohemanı. Retzius. Wahlberg.

Stollinerg (bei Aachen. Preussen). Benrad. Kortum. Lynen. Meigen. Offermnnı (P. U. Chr.) Pıllzer. Selsleicher. Schmitz. Serger. Stoltenhof (Gebr.). Strnisund (Pommern. Preussen). Hecht.

Strasslumrg. Catoire. Duvernoy Franek. Goubert. Selimper. Silbcrmann. Zeysseloff.

Stutterert. Berge. Duvernoy. Hauff. Jäger. Koelı. Kohıl. Kolb. Krauss. Nördlinger. Plieninger. Roser. Sryffert. Trinker. S. Erl. Wilhelm Gr. v. IV. Voly. Zenueck.

Swransen (Wnles). Murray. Rees.

Swineminde (Pommern. Preus. seil). Ehrlardt.

Symplioropol (Tauricn. Krimm). Rostung. Stèven.

Tauzndel (in Thüringen?). Krause.

Tsclnelnbinsk (Orenburg). Wangeim.

Temeswar (Lugan). Findel.
Tharand (Sächs. Erzgebirge). Kruzsel. Rossmaessler.

Thurn (Bez. Marienwerder. West-

prenssen). Nowieky.

Than. Browne.

Touls. Teliertkoff.

Toulan. Banon. Galle. Saulcy. Sellier:

Trefrling (bei Mühlstadt in Oberkã ruthen). Gussenbauer (vgl. Flattirlı).

Triest. Biasoletto. Eggenhüffner. Napoli. Porcia. Rolett.

Troppar (Schlesien. Preussen). Ens.

Tihbingen. Meyer. Nördlinger. Turin. Bonafous, Disteri. Ferrero. Gẻne. Giorna. Losana. Marmora. Moris. Peiroleri. Perolli. Ponza.

Uffenlıein (Rheinbayern), Bullenheimer.

Upsila. Fahraeus. Nieolson.

Utrecht (Sïdliolland). Numaun. Seliroeter van der Kolk.

Vnudoeuvre (près Nancy). Domergue de Saint-Florent.

Veidesliein (Rheinbayern).

Schultz.

Vendone. Monlet de Laroehe.

Vemedig. Andrcucetti. Contarini. Nardo (Chioggia).

Veroma. Angelini. Campo.

Vershilles. Baudry de Balzac. Blondel. Jousselin, St. Julien. Leroux. Savigny.

Vivaldentomeg (Breslitu. Schlcsipil). Bocksch.

Valleastadt (Schweiz). Eisen. ring.

TValtershausen (bei Giessen). Schenk.

VTrasadin (Croatien). Natly.

Tra rsclian (Polen). Drzewicky. Waga.

Uarantorun (Schlesien). Manger. $t$.

Vegscheil (Unterbayern), Reehenmacher.

Telnlen bei Pirna (Säch. Scloveiz). Märkel.

Veimar (Sachsen-Weimar). Froriep (juu.).

veisseureis a. d. S. (Provinz Saelssen). Heidenreieh. Schmidt.

Whitelnall (England). Downes. Wirzana (Russland). Maslenuik off. Wiburg (in Finnland). Manner. heim. 
Wien. Bischoff. Burger. Die-l TWindsheim (bayer. Franken). sing. Endlieher. Ferrari. Fitzinger. Foeke. Gruber. Gruner. Gürtler. Hanmersehmidt. Hampe. Jacquin (Sohn). Kolenati, Kollar. Krackowitzer. Kuenbure. Lederer. Löwe Mann. Marschall. Megerle. v. Mühlfeldt. Millır. Mniszech (Brüder). Natterer. Parreyss. Partsch. Podewin. Redtenbaelier. Sehott. Sehreibers. Seng. Sieber. Stenz, (Vater und Sohn). Ullrich. Weidmann. Welwitseh. Wundram. Ziegler. vieshaden. Beeker. Blum. Groos. Thomä. vilua. Eichwald.

Grossmann.

Winterthur. Sauter.

Wissgoldingen (bei Selıwäbisel.gmüud in Wïrttemberg). Kunkel.

Wolfratlashausen (in Obcrba. yern). Kerl.

vill rzburas (Franken), Dallinger. Laubreis. Leiblein.

Zhbirnv (in Böhmen). Preysler. Zuticich. Bremi. Eseher-Zollikofer. Heer. Kölliker. Oken, Schult. hess-Eseher. 


\section{Aufzählung aller entomologischen Schriftsteller von Aristoteles bis auf die neueste Zeit*.}

Die vollständigen Listen dar Selıriften der grössten und mehrer nusgezeiehueteu Naturforscher slehcu hler mu lihen geeigneten Urlen und dürten ganz iusbessndere deu Lalen zu einem hiterarisehen Behelf dienen, welcher anch den Eingeweilıten der Plnge oft vergebliehen Naclischlingens und des damit veribundenen Zeilverlustes ïberhebl.

Wir hahen hies versuchsweise nur die Chefs d'ocuvre nod die in verschiedenen akndemiaelen Meunireu und nndern periodischen Sclarifien zerstrenten Abhandlungen von $A$ u d o u i n, Bounet, Clere, George Cuvler, $\mathrm{U}$ a iman, Fabriclus, Geer, Lain arek, Latrellle, Leach, Le uweuloeel. Lepelletierde St. Fargeau, Vander Ianden, T.Inné, ollvier, Panzer, Paykul, R éuunr, Redi, Rüsel, Sny, Selimldi, Sclirank,

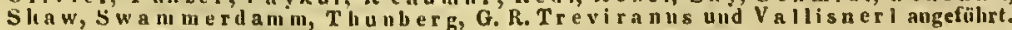

[Die Nummer $n$ a Sternleia aber vor del Nanen zeigt an, dass die Namenträger noch leben.]

Aalborg. 1. Däne.

Abluot, Johu. 2. Engl.

Fixtracts from the Mimmle Boock of the Limucan society (Linn. Trans. Vol. V, p. 276. Nov. 6. Pnpilio PanIscus).

Acerbi, Giuseppe. 1. Ital.

Acharius, Erik. 2. Schwed.

Aclirelius, Dan. 1. Scliwed.

Ainm. I. Deutsch.

Adnus, Georges. 2. Engl.

Adaus, Michel. 2. Russ.

Adrenson, Michel. 1. Franz.

Berïlımter Botaniker; geb. in Aix (Provener); grst. 1808 .

Adlerhein, Pehr. 1. Sehwed.

Adleriunack, Cay Gustav. 2. Schwed.

Adnuiral, Jacob l'. 2. Niederl.

Aclianus. 1. Gricelse. Lebte uls Arzt gegen das zweite Jahrhundert.

Afzelius, Adam. 1. Seliwed.

Observalinns on the Gemus Pausus, and Description of a new species (Linnean Transact. Iol. IV, p. 243, m. XXII Tab. 22).

1) Pausus m le rocephnlus p. 255 et p. 268, Tab. XX11, fig. $1-6$. -

2) P. sphnerocerus p. 260 et p. 270 , og. $1-7$.

3) P. ruber Thunb, p. 272 ,
4) P. Iineatus id. sp. 272.

5) Cerocoma $r$ uficullis Fabr,p. 273.

A Arassin, Louis.

Alilers, F. B. P. 1. Deutseh.

Alnews, Aug. 4. Deutsch.

Käfer auf Salzbodeu (lsis 1833, p. b42-48).

Alireins, von Augsburg. 1. Deutseh. Alurems, G. Fr. 1. Deutseh.

Aigner, Aut. 1. Deutsch. (Oestr.).

* Ainvortl, W. 1. Engl.

* Alberti. 1. Lucca.

Albin, Eleazar. 2. Engl. Maler.

lusectorum Angliac nuluralis historia: illusiratn iconibus in centum Tabulis acneis eleganier aul vivum expressis, et istis, qul id prosuti, aecurata etium coloratls, ib autoce Eieazire Albin, pictore. Hic nccedunt amnotationes fumplae, et observationes plurimas insignne, n Guil Der ha m hahitae. Londinl impensis G. Irrys. 1738. 4. 100 Paga. in 100 PIntass plet.

Albinus, Bernhardus. 1. Deutsel. Eigentlich Weiss: Medicus; geb. in Dessaul 7. Jumi 1653; gest. zu Leyden 7. Sept. 1721.

Aloreclit, J. F. E. 1. Deutseh. Zoolom. Ind physienl. Entdeekungen vnn den Biencu elc. Gntha 1775.8.

Albreclnt, Joh. Sebast. 1. Deutseh.

* Dle Zälulung der Lebeuden (und Todten) in der Vorrede zun Lexikon Ist unvollsttindlg, weil Im Laufe des Druekes Eluschalungen sowle Deleationen genachi wurden. Dieke Uehersicht nber enthinlt alles und sogar was in den Suppleunenten, beigezehen zum Lexlkou der Phytozoologen, noch nachgetragen worden Ist. - Auch ist die Berichtigung mancher Wohnorte in dle Ortotafel übergegangea. 
geh. 7.11 Coburg in Franken 4. Juni 1695 ; gest. ebend. 8. Oct. I774.

Albrecht, Joh. Peter. 1. Deutsch. Geb. in Hildeshein. Mitgl. d. Aead. Nat. Cur. I 681 .

AIdrovendi, Ulysse. 2. Ital. Geb. 11. Sept. I522 zu Bologna; gest. das. 10. März 1605.

Alexander. 1. Eugl.

Algren, M. 1. Schwede.

Aliponsonl, Giuseppe. I. Ital.

Allen, Benjamin. 1. Engl. Medieus des 18. Jahrb.

Alloni, Carlo. 1. Piemont. Prnfessor. Geb. 1725 und gest. 1804 in Turin.

Aloattl, M. I. Deutscl.

Alpinus. Prosper. 1. Niederl.

Amoreux, Pierre Jos. 1. Franz. Geb. gegen die Mitte des 18. Jahrh., gest. $18 \% 25$.

Amorenx, N. Von Montpellier, wo er Arzt war.

Notiec des Insectes de la France réputes veninteux. Par. 1786, Fol. Av. fig.

Ansteln, Joh. Georg. 2. Srhweiz. Geb. 174,1 zı Hauptwyl, gest. 18. Febr. 1794 in Pfeffers.

\section{* Anyot.}

Anderson, James. 12. Engl. Engl. Agronom; geb. 1739, gest. 1808.

Anderson, Johann. 1. Dentsel. Geb. 7. Hainburg 1674, gest. 1743.

* Audrenccettl. 1. Venet.

Andrews, James-Petites. 1. Engl.

Angelinus, Fulvius. 1. Ital. Lebte za Ravelina.

* AngelInI, Bernardino. 1. Veron. Anonynus.

Abbildung und Beachrelbung der vorzïgl. enroptisehen selimellerllıge, getreu naeh der Natır. Mlit 8 ausgentalten Kupfertaf. 8 . Frankfurt (Döring).

\section{Amonymus.}

Zerstörung der Eiehen im Walde von Vincennes durch die Larve ron Seolylus pygnания (L'Inst. 1836, Nir. I58. - Isis I8.37, 769).

\section{Anonyinis.}

Kerfbewegung (Isls 1822, p.561).

\section{Anonyunks.}

Bletien (Froriep's Notizen XXXI, 138).

Anonymas.

Coelienlle (Tillser Zeitung, Nr. I- 3 ).

\section{Anomynus.}

1) Insekteujäger (F. K.).

2) Nuturg. der Ins.

"Amslyu. 2. Niederl. Lebt zu Haarleem.

Anthoine, d'. 1. Franz.

\section{Antlgonus.}

llist, mirab. eolleet. (Cap.II. De ciendis)

* Antinori. I. Ital.

Aristoteles. 1. Grieche. Geb. z.ı Stagyra (an Thracieus Grouze) unı 384, test. in 63. Jalire. - Der Vater der Zoologie.

Artinre. 1. Framz.

Ascenius, Petrus. 1. Schwede. * Asumas, Herm. Mart. 1. Liefländ. Atze, Christ. Gottl. 1. Deutsch. * Aubé, Charles. 3. Franz.

Audowin, Jean-Victor. 38. Franz. Prof. d. Eutom. am Mus. dhist. nat. de Paris. Geb. 2. Apr. 1797 zu Paris, gest. 1842 das.

1: Auswnclis vou Stich"elner Cecidnuyla. - Kältedulden der Kerle (L'Instit. 1838, Nr. 226, A pr. p. 110 . - Isis 1830, 360 \%.

2) Kerfskelet (Isis 18??, ए. 80),

3) Veber Seolytus (Amiat. soc. ent. de Fr. 18.37. - Isis 1839,531$)$. Vgl. Feistli $\mathrm{nmel}$.

4) Ueber Blewus, der elnen Theil selnes Lebens iu Mcerwasker zubringt (L'Instit. 1833, p. 38. - Isis 1837. 310). (Dabel erklärt D॥ir neluet das Athmen der Plialaeul potamo. gata).

5) Ueher die ansteekende Krankhell dee Seidenwiiruer, Museardine (L'Iust. I836, Nr. i68. - Isis 1837, 78?). Vel. Mo I tague.

6) Veber ein Kert, las lang iu Neer. wasser lelst (Anial. d. sc. 11at. III, 1835, p. 30-40. Bleruns fulvesecus. - Vgl. $0 \mathrm{k}$ en ${ }^{\mathrm{s}}$ Bemerkıng lilezu und Isis I836, p. 521, Gött. gel. Anz. i807).

7) Einfaches Auge an der Stirne zwlselien den beilen zusaumengesetaten Augen von Anthreins, Trogodermin (Vortrag 18.35).

8. Coecus catl und dessen liortpfianzung

(LiInst 1836. Nr. 19!, 1837. - Isjк 1837, 793).

9. Kerfe in Mehl: Larven von Pilnus Fur und Pyralls larlnalis (L'Inst. 1836, Nr. 189. - IsIs I837, 793).

10) Ophion dosltheae (L'Inst. I83, 42. Isis 1837, 315).

I) Cantliariden (Isls 1834, 896).

12) Ueber das Welbelien von Cebrio glgas (L'Inst. 1833, 251. - Is is 1837, 314.

13) Ueher elnen in Meerextiefe wohnenden apteren Blenus mit zweilelluliel Respiration i Vortrag 1835). - Veber Larven von Sitavis und Melise (Vorlr. 183j. - Isis 1836, p. 763) - Nest der Formica smaragdina (ibid.).

14. WrkläruIg der Kerfe in Deser. de I'Eg ypte (partion Isis I83', 970).

i5. Ueber Gallensteine (!!), gefunden In den Galleugringen elues Weibchen des europ. Feuersehroters (Lueanus cervin, (Aunal. des se. nat. v, p. 129), - Naelitrigliche Bemer. kungen iiber die Ilarngallengfiuge (UrinoblIlalres, der Inseclen (lbid. p. 134).

16) Museardine der Seidellwiiriner (I, Inst. I837, 22 ? - Isis 1837. 858). - Ueber dle den Relien sclitidl. Kerfe (ibid. - Isis a. c. 859 ). (Tinea roserann.)

17) Benerkungen ïber die Befruclıtungen der Kerle (Is Is 1841,643 ).

15) Ueher den sehtidiclien Kerf Pyralis vltana (ibld. 673).

19) Proctolrapes-Larve, eine Selımarotzerin 
20) (avee $L$ a chat) Analomie d'une Larve apode (Conops), trollvée dans le unurdou des pierres (Mem. de la Soc. d'hist. wat. de Parln. 1, p. 1. 329. - Jomrn. de Pliys. I pl. T. 85. 1818. 228 ).

21) Letures sur la génciration des insectes,

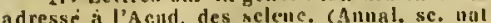
I1, p. 281). - Reclierches matomiques sur la famille diu Drilus farencens et sur le mile de celte espece (ibid. p. 443, fig.).

221 let M Edwards) Vnlersucliungen iber die wirbellasen Thicre der Clanser-luselu (Ammal. sc. mat. 1828, XV, 5. - Ixis 18.31, 10291.

23) Rechercliem anatomigues sor te thorax des animux articules et celul des insectes bexapodes en particulier (Extrait des Amul. sc. 11at. T. I, 15:8, 97).

24) Prodrome d'une listolre niturelle, chimlque, pharunceutique el inedienledes Cantharldes. Thise. Paris 18?6. 4.

25) Resume dEntomologie, ou histnire naturelle des animanx articules Paris 1829 8. 2 Vol. in 32. 'Allas de 48 pl.).

26) Explicalion sommaire des planclies d'insectes de lourrage de la commissiou d'kingte. P'aris 1825-27. liul. Ct. Nir. 14.

27) Recherches pour servir à I'listoire des Caullarides (Anu. sc. n. IX, 1826, 31).

29) Uiservalions sur m insecle qui passe ell graude partie de sa vie sous la mer (Fixtr. des nouv. Amal, du Mus. d'hist, nal. 11, 117). Vgl. Nr, 4 und 6 .

Aveliu, Gahr. Eman. 1. Schwed. * Aymaral. A.

Azzarn, Dou Felix de. 1. Spanier. Geb. 1746.

* Ereli, in Bopparl. 1. Deutseh. IBacon, Lord Verulame. Ber. engl. Philosopl. Geb. 1556, gest. 1626.

In lick, Abrahnm. 1. Sehwede.

DBichner, Mich. Andr. 1. Schwed.

* Ester, C. F, V. v. Deutsels. Zu St. Petersburur.

Inier, Joh. Jak. 1. Deutseh. Gob. zu Jenis 1677, gest. zu Altclorf 14. Juli 1735.

Batrer, Heinr. 3. Engl. Physi. kus. Geb. 7.u London geren Anfing des 17. Juhrhı, gest. 25. Nov. 1774.

Baldaute, Antonits, 1. Ital. Lehte in Bulngun.

Bancrort, Liwurd. 2. Engl.

Bruister, John. 1. Engl. Botaniker.

\section{Finntas, Sir Jos.}

Buprestls quadridentata (Llun. Trans. I, p. $255, \mathrm{~N}$. XXVIl. - Extr. from Min. Bock. Nov. (788).

* Inuse, in Magdeburg. 3 .

verlioteru. 2. Franz.

Turbut, J. 1. Engl.

* Harchard. 1. Engl.

Diarelay, John. 1. Engl.

Brarluau. 2. Engl.

Baricelle, Giuglio Caesare. Ital.
Geb. in der Diöe. Benevent; lebte z.u Aufang des 17. Jahrlı.

Merluer-WVepp, $P$.

* GarkIey.

Maron. 1. Franz.

IArrelier, Jar. 1. Fran\%. Geb. zu Puris 1606. Dominikaner. Botanikel. Gist. 1673.

* Berrow, John. 1. Engl.

" Is artinélicuey. 1. Franz.

Eartholimes. Thomas. 1. Däne: Geb. 20. Oct. $1616^{\circ} \mathrm{zu}$ Kopenhagen, gest. 4. De\%. 1680.

arertina, Joh. Math. 1.

- EBartou, Benjanin-Smitlı. 1. Engl.

Lebt in Amerika.

Bartrasu, William. 1. Philad.

Bsartran, Moses. 1. Amerik. Medieus in den vereinigt. Staaten.

Ulservations on lhe native Silks Wormx of Norih-America (Trans, of Phllud. l, p. 294 $-301)$.

Bartram: John. 5. Anerik. Geb. Penusylv. 1701.

* IBssi, Curlo. 4. Ital.

Baster, Job. 1. Niuderl. Geb. 7u Ziriksee im Zicland 1711, gest. 1775.

Enttirn. Giov. Anton. 1. Itat. Medieus zu Rinimi. Gest. das. 1739.

* Bamary le Inzicres. 1. Engl.

i. An.

Diandet de Ia Fage, MaricJean. 1. Franz. Lebte zu Clermont. Bumbia : Johnum. 2. Siluweiz. Geb. za Basel 1541, gest. zu Muntpellier 1613.

Hamenten, J. 1. Schweizer.

Danuer, Johammes 'aulus. 1. Deutsch.

DBumbiracer. 1. Deutscl.

Nonvelle classification des Mouches di denx alles. Paris 1800 . 8. (Extralt dı Dlpiéres de Mi I ge II).

Itanter, 1. Franz.

Baxer. I. Engl.

Inyle-Earelle. 1. Ital.

* Tayrleotier, Karl Theodor. Baziu, Gilles Augustin. 4. Franz. Vou siraxbusg; gest. Mïrz 1754.

Denurieu.

Abrige de l'histnlre des Invectes. Parla 1764. 2 Vol. (Brscliten ohne Namen.)

Beatlay. 1. Engl.

Feclestein, Johann Mathhäus. 2. Dritsel.

IBecle, Albr. 1. Sehwed.

Beckinanu, Johann. 3. Deutsch: Geb. 4. Juni 1739 ж. Hoya (Hannover), rest. 13. Febr. 1811.

Beckovith, Joln. 1. Engl. 
Iteirelss.

De unilitate et mecessitnte historlae aaturulls. Helmisi. 1759.

- Bell, Thomas, 2. Engl.

IBeIla rall, Lodovico, 1. Ital.

Ielon, Pierre. 2. Franz. Ber.

Medicus u. Naturforscher des 16. Jalih.

" Hernedem, $P$. J, van,

Beanett, I. A. 1. Nicderl,

IBeminett, E. 'T. 1. Engl.

Aphrophora goutdotii , Proceell. ijy Taylor.

111. Ja11. 22, p. 11. - isis 1835, 5i9. Vi.l.

Goud $0 t$ ).

Illerelins; Georgius. 1. Schwed.

Derenalt, $G$. C. 1. Deutsch.

- TBerge, F. 1. Wiirttemb.

Bergen, Carolıs Augustus n, 2.

Deutsch, Cieb. 11. Altg 1704 zu Frank-

furt a. d. 0., gest. 7. Oct. 1760.

Dergius, Peter Jonas. 1. Schwed. Medicus und Botauiker; gest. 1791.

Eergunanu. Torbeıı. 9. Sillwed, Geb. 9. März 1735 zu Katharinaberg (Westgothland), gest. 8. Juli 1784. War nur in der Jugend Entomolog.

IBersstrilsser, Heinrich Wilhelm.

1. Deulsin

Bergstrisser, Jolı, Andr. Benjgnıs. 7. Deutsch.

Berlk, van. 1. Niederl.

Derlcemliont, Johu. 2. Engl.

Medicus; rest. 1791.

Hernatovitz. 1. Genfer.

* Derminardt. W. 1. Preussc.

Dernitz, Martinus Bernardus a.

2. Deutsch. Chirurg des 17 .Jahrh.

Bermonlli, Jol., 1. Sehweizer.

Dec Archinedes seines Jalilhumelerts; geb. zu Basel 27. Juli 1667; gest. ebend. 1. Iall. 1748.

* IBerallingean, Graf. 1. Deutseh.

Merta Idi, Giov, Luigi. 1. Picmont.

Gel), in Murello.

* Bertliold. Arn. Adolph. 1. Dentselı Be rtoloni, G.

Heslex, Miclacl Rupert. 1. Deutsel.

Besse $x, W .1$. Russ.

Betti, Znecaria. 2. Italien. Poet des 18. Jalirls. Giüder der Acadeuna d'Agricoltura di Veroua.

Mevan. Edward. 1. Eingl.

Bibiana, Franeiseus. 1. Ital.

Hicluens, J. E. 1. Engl.

Bieberstein, Baron Marsclual de.

1. Rusn.

Hicerkander, Cla. 27. Scliwed,

Pastor in Westrogothien: gest. 1795.

"rilbers, Gustav Jol.. 1. Schwed

Von Stockholm.

Biberg. 2. Seluwede.
uBillardière, de la. 1. Franz,

Botaniker.

"Eingley, W. 1. Engl.

Porticula gigantea (Limmean Trans. Vol. IX, Nr. XXII, p 104. - Fixtr. fr. the mluut. Boock of the Liuncan Society).

* Biot. 1. Franz.

Kerfe im Iuftleeren Rnum (isis 1819, 276).

feircln, Thomas, 1. Engl, Geb. 17115 , gest. 1766.

BBiral, C. H. 2. Engl.

* Iiscliont, T. L. W. 3. Deutselı. Bissuti 1. Ital.

HEninille, Hency Duerotay de. 3. Frairz.

* Flanclinar, $A$.

" Bllanelinar, $\mathrm{E}$.

BI ankant, Stephan. 1. Holländ.

Geu. Blancardus; geb. zu Amsterdam. Lebte nul I688.

EJ asvidre, J. J. I. Deutsch.

Eleguy, Nicolas de. 1. Franz.

Chiruig; geb. zи Paris im 17. Jahrh., gest. zu Avignon 1722.

IEIEสgon, L. 1. Engl.

IIDell. 1. Deutscls.

FIocli, Marrus Eliezer. 1. Deutseh.

Geb. z॥ Ansluach 1723 ; yest. 1799.

BDloeme, Carol. Magnus, 2. Schwed.

Blompderth. 1. Franz.

Elomal e Will, Ferl. 1, Deutseh.

* FH DandeI, J. Hipt. 1. Finnz.

Glock, Freiheriv. 1. Deutsch.

*Elot, F. 3. Franz. Lebte ind ward veeb. zu Caen.

* Blaun. 1. Deutsch.

WIanenbaclı, Joh. Fricdr. 3.

Deutscl. Gefeierter Naturforseler. Geb.

7.1 Folla 11. MLai 1752.

Boelvex, Joh. v. 2. Russ.

rocen. 1. Franz.

Hoclinet, Sumuel. 1. Deutselı.

Eock. 1. Deutseh,

Godilacet, Picter. 3, Niederl.

Bnelinn, Moriz Jolıaun. 1. Deltselı.

Dise-K, J. A. 1. Deutsch.

Eocrinave, Hermanu, 1. Holl. Geb auf dem Landhaus von Woorhout bei I.eyden 13. Dez. 1668, rest. 2u Levden 23. Sept. 1738.

Boermer, Imanuel Carl Heiur. 8. Deutsi:h.

Brotlins, Jue, 1. Schwed.

"IBolnenanan, C. H. 4. Scliwed.

Bolna er. G, R. 1. Deutseh.

Ho ict, Fijedr. 3. Holstein.

Hoislinval, J.A. 15. Franz.

* Boitaral. 3. Franz.

Bommere, Valmont de. 1. Franz. 
Bomme, Leendert. 1. Niederl.

- Bonarous, C. 3. Piemont.?

* Bomanarte, Carlo Luciano. 2.

* Bond, William.

Bomdaroy, Anguste-Denis-Fungeroux de. 5. Franz.

* Honelli. Francesco. 3. Ital.

Bommanius oder Boununi (Philippus. 1.' Ital. Geb. zu Rom 7. Jan. 1638, gest. 30. März 1725.

Bonner, Jnutes. 1. Engl.

Bonnet, Charles. 16. Seliweiz. Naturphilosoph, Geb. zul Genève 13. März 1720, gest. 20. Mai 1793.

1) An abstract of some ncw observations upon insects ITrausactions philos, of Loud. XLII, Nr. 472, 4j8.

2) Observations sur une nouvelle partic propre à plusleurs clienilles (Aléa. de Matliem. e) Phys. des silv. cirangers í l'Acad. de Paris II, 42-52. - Oelivies de Bonict. II).

3) Sur ia urandc Clienille th quene fourchuc du Sanl (il,id. II, 276-282. - Uitgezogle Verliaadelingen. 5 Veci $226-54$ ).

4) Recicrelies sur la Respiration des Chenliles (ibid. V, 276-\$03. - Plillos. Transact. of Londour. 1748, 300).

5) Observatious sur les stignates des $\mathbf{P a}$ pillons (lbid. V, 294.

6) Sur le moyen de conserver diverses especes d'inaectes dans les cablnets d'hixtoire naturelle (Rnzler: Journal de Physique IV. 1774. 296-301. - Bonnet, Ouivres V, Part 1 , 12-23).

7) Cettre et Mimoire sur les Abeillex (imprime avec lhistoir de la lleine des Abeilles par Schir achi ilioz. Journ. V. 3:7-44 cl 418-28; VII. 23-32. - Ocuvres V).

8) Schreiben an H, R ic m, nebst des letztern Anmerkungen über die Bicnen (Berliner Samminugen Vil, 1775, :45-270-0chvr. V).

9) Aplidies yariac (Ocuvr. I, $1-\{14$ ).

10) teurres d'histoire naturelle et de Plilonophie. Nellehatel 1779-83. 19 Vol. 8. - Uebersetzmmg: Leipzig 1783. 8.

1i) La Paliuenesle philosophlque, nit Idees sur l'état passé el sur l'ctat futur dcs etres virans. Ouvrage destiué t servir dc Suppleuent aux derulers écrits de l'anteur, el qui conticnt principulement ie Précls de ses Lecherches sur le Cliristlanisme. 'I Tones. A Mnnster 1770. 8. Unsere Ansgabe, gewislmet dem Fürstbischof von kijlı, Maximilian Fried ricli, hat, laut der Dedicariou cle., Philipp lleinrich Perrenon besorgt.)

12) Contempintion de in mature. Amsterd. 1764. 8.

13) Considerations sur les corps nrganiscis. Ansterd. 1762.8 . T. II. (Uebersetzung mit Zuxitzen von J. A. k. G $6 \mathbf{z}$ e. 1, II. Lengo 1775. 8.)

14) Mimoire sir une nouvelic partie i une espere de mamciun ou de corne charme, viace sous le premler anneau, cutre in tèvre inférleure et In préuièrc pnire dex Jambesi comaune a plingleurs especes de chenliles (Mém. de Matheim. et de Plıys. II, p, 44).

Bonomo, Giov. Cosm. 2. Ital. Meliziner zu Livorno.

Bonsdorf, Gabricl. 6 . Scliwed. Booth.
Borkhausen, Moriz Balthnsar. 4. Deutsclı. Geb. z.॥ Giessen 1760, gest. z." Darnisladt 1806.

Borlace, Willian. 1. Engl.

Borowsky, Georg Heinricl. 1. Deutseli.

"Wory de St. Vhincent, J. B. C. M. 2. Franz. Gcb. zu Agen gegen 1772.

Bose d'A uti c, Lonis. 18. TFranz.

i) Descriptlon d'une wouvelle espice de puce (Pulex tasclatus) (Société phlloviat. A. 4. T. 2, p. 156 ).

2) Deseriptions of two new species of Phalacha 1Liun. Traltsict. Vol. 1, p. 196, Nr. XXIII, Tub. 17.

3) Cecylomia (1sis 1818, p. 1559).

Bose, Georg Matthius. 1. Dentsch. Geb. zu Leipzig 22. Sept. 1710, gest. Magdebuig 17. Sept. 1761.

Bottomi, Dominieo. 1. Ital. Geb. z.1 Leoulini in Sicilien 6. Oet. 164t, gest. gegen d. Jahr 170 t.

Dotiri-J Desyilés. 1. Franz.

* Bonclié, 1'. Fı. 3. Deutsel.

" Bomelne. F. C.

- Iond, Willian. 1. Engl.

Ho wdic'r, Henry-Phillip. 3. Franz. Phalmazent.

Boulewicer, Abbé. 1. Franz. Lebte zu Dijou.

Gourdelin, Louis-Claude. 1. Frnnz. Geb. zu Puris t695, gest. 1777.

Finonton, I,

* Towerbunla, James. 1. Engl. Howles, $G$. Dun. 2. Spanier.

4 Bayer, 1. Franz. Lebt in Aix.

" Doyer de Fonscolombe, $L$.

L. J. N. 5. Franz.

Boys, William. 1. Engl.

- IBrace, J.

Bröckenlamagen. 1. Deutselı.

Bratley, Richard. 1. Engl.

Mrdikus : gest. 1732.

Bralnen, Nikolaus Jos. 5. Dentsel, Bramals, Jouchim Dictrich. 1. Dentsel. Geb. zu Hildesheim 18. März 1762.

Hrandstein, P. W. 1. Skandinavifr.

HBrand, J. Fr. v. 3. Deutselı.

Brients, A. 3. Holländ.

* Eraven, Alex, 1. Deutsch.

"Irebisson. 1. Franz.

* IBrelinar. 1. Deutsel.

Bremond, François de. 1. Franz. Geb. 14. Sept, 1713 zu Paris, gest. ebend. 21. März 1742.

Ireuchel, P. J. 1. Deutsch. Ireynins, Johauncs Philippus. 3 
Deutsch." Geb. 211 Danzig 1680, gest. 1764.

Brez, Jakob. 2. Niederl. Geb. in Meddelbourg 1771, gest. 1798.

Briganti, Vincent. 1. Neapolil. Briganti, Jos. 1. Engl.

Erisson, Mathurin-Jaeques. 2. Franz, Geb, zu Fontenay - le - Comte 1723 , gest. 1806 (?).

- Broch, J. K. 1. Elsasser.

Bronleriy. 1. Engl.

Eronckes. 1. Engl.

Erongulart, Alexandre de. 3. Franz.

* Mrotz, Johannes. 1, Deutsch.

Erougton, Thomas Duer. 1. Engl. Brousse, De la. 1. Franz.

Brown, Littleton. 1. Engl.

Brown, Peler. 1. Engl.

Browne, Patriec. I. Irländ. Me-

dicus und Bolanist; geb, z.1 Crosboyne in Irland 1700, gesl. zu Rusbrook 1790.

"Browse, Thumas. Capitain.

Eruce, James. 1. Engl.

Bruckumun, Franz Ernest. 6. Geb. zu Marienlhal 27. Sepl. 1697, gesl, zu Wolfeubiillel 21 . März 1753.

* Brugnatelli, Luigi. 1. Ital.

* Bruiusuna, J. J.

* Brullé, Auguste. 11. Franz. Brumelli. Gabriel. 1. Ital.

Brumu, Friedr. Leop. 1. Deutsch.

* Mrumer, Sanuel. 1. Seliweizer. Brunmich, Martin Thomas. 4.

Dïne; vou Kopenhagen.

Entounlogla. Ilafnite 1764. 190.

Bscherer, Danicl. 1. Dentsch.

wuclnoltz, Frauz Heinr. 2. Denlsch.

buclooz, Pierre Josepl. 2. Elsass.

Einer der grössten Compilatoren des vorig. Jahrh.; geb. 27. Jan. 1731 z.u Melz: trest. zn Paris 30. Jan. 1807.

Bucluvald, Balı. Jean de. 1.

Diıe. Mediainer; geb. 1697, gest. 1763.

Bucliner, Andr. Elia. 1. Deutsch.

IBiscloing, Ant. Friedr. 1. Deutseh.

Grb. in Westphalen 1724, gest. zu

Berlin 1793.

Bittuer. 1. Deutseh.

* Enginé. 1. Deutsch.

* Buguion, C. 1. Sehweizer.

* In nilile. 3. Deutseh.

Buisson, Arnaud du. 1. Franz.

Bull emanu, Hn. Jusp. 1. Deutsch.

"Bulow-Rietli. 1. Deutsel.

* Enuuet, Lucien. 1. Frauz.

Rurcluard, Eruest Friedr. 1.

Schwed.

- Buratach, Friedr. 1. Deutsch.

Bure 1l, John. 3. Engl.

Burgloart, Godofr. Henr. 1.

Deulscli. Geb. za Reichenbach 5. Juli

1705, gest. zil Bileg gegen 1776.

Burssulorf, Friedr. Aug. Iadw'.

2. Deutsrl. Geb. z॥ Leipzig 1757,

gest. 1802.

*Bnrmeister, Herm. 1\%. Deutsel.

Buttler, Curl. Geb. zu Wicombe', 1560, gesl. 1647.

Monarchia feminina (apunı).

Cagunti, Mareel. 1. Ital. Naturalisi und Kritiker aus Verona, geb.

$15 / 3$, gest. 1612.

Caguisera. 1. Franz.

Culdani, Floriano. 1. Ital.

Canelli, Gcorg Jos. 1. Engl.

Caumerarius, Joh. Rud. 9. In

17. Jahrh.

Cauaphell, Jolın. 1. Engl.

Cannals y Marti, Don Juan-

Pabli. 1.

Canonico, Giuseppe Gaetano Ca-

ra de. 1.

"Cantener, L. P. 2. Franz.

Cantus - Pratanus, Thomas.

Ital.? 1627?

Cnpieux. 1. Deutselı.

Carlsou, Gustav von. 1. Schwed:

Carison, J. 1. Engl.

Carolus, Theodorus, 1. Deutseh.

Carpenter, Thomas. 2. Engl.

Carradori, Giovachino. 1. Ital.

Curré. 1. Nicd.

Curten, J.

Hisınr. de Cantharidum usu et operatione. Edlut. 1776. 8.

Carter, Colonel Laudon. 1. Engl: ill Amer.

Observallons concerning the Fly-Weevll, that destroys lie whent, with some useful discovertes and conclusions, coneerning the propagation and progress of thal pernlejous Insect. and the metliods to be ined to prevent the destrucllou of lie grain byil. By Colonel Laudon $C$ a $r$ ter (Trausact. of Philad. Vol. I, p. $\left.274-28^{-}\right)$- Some wubject, hy the Conmltlee of $\mathrm{H}$ u $\mathrm{sl}$ ) a $\mathrm{ndr}$ (l. c. p. 287-297).

Ceris, C. G. v. 1. Deutsch.

Cusuati, Franciseo. 1. Ital.

Casserius, Julius, 1.

Dé voels uuditusque organis. Ferrarae 1600. Fol. e. perum. tab. (Üugemeln fleissig und durchifulirend durch alle Classen des Thlerreichs. (i.)

Castaquedo, Ferdinando Lopez di. 1. Spau.

- Castollus, Petrus. 1. Ital. Medikus und Botaviker; geb. gegen das 
Eude des 16. Jahrh. zu Messina, wo er 1656 starb.

* Castelnue, Graf von (vgl, Laporte).

Castles, Jolun, 1. Fingl.

Uetier die Zul ckrrnmeise in Graulada (Pllloo. Transnet. 1790, n. 346).

Castro, Joaehim de Amorim. Spail.

Cat, Le. 1. Franz.

Catelan, L'abbe de. 1. Franz.

Chtalan, Laurent. I. Frauz. Pliarmazeut von Montpellier, wo er Jehte (um die Mitte des 17. Jahrh.).

Cateslyy, Marc. 1. Eugl. Natn. ralist; geb. 1679 orter 1680 , gest. zu London 23. Dez. 1749.

Sandilolt in dess. Cnrol. III, T. 3.

Cederhiel in, J. 1. Russ.

Cestone, D'lacinto. 2. Ital. Pharuazent; geb. zu Sunta-Maria in Giorgio in der Mark Ancona am 13. Mài 1637, gest. zu Livorno 29. Jan. 1718.

A new dlscovery of the originnl of flens (Philos. Transact. V. 1699, p. 42. - Pule x). 2) Isturia della Grann del Kermes (In Valtisneri opere. Vence. 1733, Fol. 459),

Cetti. 1. Sardinipr.

Clnabrier de Montpellier. 1.

* Cinabrier, J. 3. firanz.

Chnisnenu, Charles. 1. Frauz.

Cinambom, A. 1. Franz.

Chnut, J. I. Engl.

*Clnediny, L. C.

Charleton, Gualterno, 2. Engl.

*: Charpentier, Toussaint de. 3.

Deutscls.

* Clumuloir, Baron Max. v.

Chnmssler. 1. Fran\%. Von Dijon.

- Chevrolat, A. 24. Franz.

* Ciniaje, Antonio Delle. 1. Neup.

* Chinje, Steffano Delle.

*Clnidiren, J. G. 1. Engl.

CInrist, Jolı. Ludw. 2. Deutseh.

* Cleristy, William. 1.

Cist, J. 1. Amer.

Cyrillo, Dominir:o. 1. Neapol.

Geh. zu Grugno (Nespol.) 1734, gest. auf dem Scluaffot in Folige der politi. scluen Errignisse dieser Epuche.

Clairville. 1. Englischar Entomolog; elied. ctublirt iu der Seluweiz. "Cliris, Bracy. 2. Eungl.

CInrki:, Edward Danicl. 1. Engl. Clerk. Carl. 4. Silswed.

1) Beskrlfuing $p m$ Asp-fjärlleıl (c. tig.) (Veteusk. "cad Handl. 17-35. 278, dentsel, 283 ; lateiu. Annal, trausnlp. 11, 370-721.

2) Nägra ununarkningnr, nugiiende In scktcr.

1) Beskrifuing pa en Plialnena; 2) Beakr. $P R$

Gisıcl'i Lexlkon. en Tang, at fungn fjirlltar och Insecter; 3) Om Kark-bnttuars nytta i lusert-Cabinetter (Vetensk. Ac Hnndl. 1755, 214, dentsch 212, holländiach. Unlgerng. Verlıand, van de Soc. d. W cetenscli. Alusicrd. III, 228).

3. Icones insectnrum rarinrum, Holmbae. 1 Pirt. 1759. c. 33 tab. col. 4.2 Pnrt. 1764.

4) Tal innelıăllaude nĭgra Anmmrkningar nm Insceterne. I PI. (Vetensk. Ak. Ilandl. Srockl. 1764.)

Clevic, Le. Naturalist von Laval.

Obscrvations sur in corme du psile da Bokc (Acnt. sc. 1815) nud meliro andere selir Interessmte Anfstitze (Cuvier).

\section{Clesiug.}

Anleit. lıs. zu saumeln.

Cleyer, Andr. 1. Holl, Langr Zeit Medikus bei der holländiselıen Compaguic auf Batavia (Java).

Clnsiens, Car. 1. Nicderl.

Clutins, Theodorus. 1. Niederl.

Aueh Cluyt-Direk.

CIntins, Angerius. 1. Niederl.

De nucc med, et de Hemerobio, Insecto ephenero et majali verme opusc. 2. Amstord. 1634.

Coello de Seabra. 1. Portug. *oldstremin.

Coleris, Juhannes. 1. Deutsch. Colius, Samuel. 1. Engl.

Collimson, Peter. 6. Engl.

Colnman a , Fabius Lineoeus. 2. Römer.

Conodus, P. 1. Rouen?

* Cone olli, Antonio. 1. Ital.

Cona paretti, Andreas. 1. Ital.

* Compite:, J. Aclijle.

Consett. Mathew. 1. Engl.

* Contarini, Nicolo Conte di. I. Veuet.

Cormelocrt, Antoine-Jeau, 2. Frauz.

Illustratlo iconograplica luseetnrum, quae in Musacis parislnts obscrvavlt et lu lncem erlilit Jnh. Clırist. Fab r l cius; pracuissis ejusdeun descriptionibus; 1 ccedunt species plu. rimac vel minus alt nnndum cogultie. Auctore Antonio Jnannc C oquebert. Tabularum Decns prima. Parlsiis, typis Pelri Didot natıt majnris. All, Vil. 4t Pagg. X Tnh. Fol, mln. I. decns secunda. Paris. cort. Anno $X$ (I801) n pus. \$5-91. А Tab. XI-XX. 1. decns tertia. Pnr. A11. XI1 (1804), 2 pag. dedicall. a pag. 91 $\rightarrow$ I4? indice lucluso. A Tab. XXI-XXX. Die Dimmosen zu den Kupferu xind aus $\mathrm{F}$ aIn Irins' fintommlogin systematica. Viele Psnensarten besclareb Latreille In alesen Werke, welche Cn quebert nus alcm Bulletio snc. Pluil. excerpirle. Die Kupfer sind miltel-

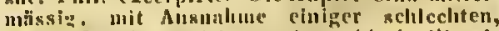
jeinch flelssig gezeichueJ. aber velilecht IIJumlnlıt. Druck murt Papier selır saclıön und da uerud.

Corlocom, Graf v.

* Cordin.

Cortingins. 1. Deutscl.

Cortl, Bonaventura. 1. 
* Costa, Gabriel da. 2. Neapol.

Cotte. 1. Franz.

Crmaner, Chr. Car. 1. Vou Kopenluagen.

Crancr, Pieter. 1. Niederl.

Kautimann zú Amsteriam.

Entomolng. ( 400 pl, III. Pap.)

Crell, Ludov. Clırist. 1. Dentsch.

" Crepina, F. E. H.

Crentzer, Christian. 3. Oestr.

Croustett, Call Jolıann, I. Schwed.

Cunrad, Jos. 1. Detutsel.

Curtis, William, 4. Engl. Planmizcut; geb. z.u London gegeu 1736, gest. zu Brompton ₹. Juli 1779.

Obscrvations nu A phides, clilefly luhensird to sliow that the principaly cause of Blights In Planis. and the ranse of ulicllonev. Dew. (Linueau Transact. Vol. VI, p. 75, Nr. II. Tab. 5, tig. 1, 11. 2. Aphis salicis.)

* Ginertis, Jolun. 5. Engl.

Cuvier, George-Léopold-CluretirnDitgobert. 11. Deutsch. Der ausgereiclmetste Nafurf. seit Limne; geb. zu Moulbelliard (Elsass) 23. Aug. 1769, gest. zu l’aris 13. Maj 1832

1) Sur la unuvelle eapece ule cartomicre (Vespa nilldulaun Falor.), av. fig. (Bullet. de la soc philomat. $1,1797,56)$.

2) Sur In manlère chon se falt la nurition dans les insectes (Bullet, Ile Ia soc, phil. I. 179x, 74. - Journ. Ile la soe. de Pharmacie l. Parls 1. 128, - Mém. de la sac. d'blst. Nit. Par. 1, 1799. 34. Av. l1

3) (lloservations sur quelgues Diptres (Journ. d likst. nat. II. an 7, 253-255).

4) Rapport sur un Mèmoire dc Mr. M. de Serren sur le tube intestinal des lasectes, impriné en tête de ce niınolre.

5) Réene anlmal, dlatribue d'après son orgauination. 1 èlt. l'aris 1817 . \&ol. 8 . 2. edit. Puria 1829. 5 Vol. 8. Entomologie par $T$, il rellle. - Uebers. mitzustitzen von 11. R. Sc IIInz, Bde. Stullg. 1821-2.5. 8 . Nacli der 2. Auflage iberseizte das Werk Volg (vgl. diesen).

6) Lettre sur I'Entomologle A M. II a rtmanI (1790). (Revnc ent. 1, 1833. 145-60, av. I pl. slesalnce parCuvier.) - Lcttic 1791, av. I pl. (1. c. 195-210, - Ces deux lettres out ćlé publicea depula la mort ule l'antenr.

7) Ubservations sur quelques Diptcres (Jourunl d'hls). I1a). 179?'

8) Mémoire sur les Cloportes (ibid.).

9) Deseripulan de deux especes Honvelles d'lusectes ( Mlagnz. encyclopéd. I, 205-7). (0) Leçous d'Anatomle comparce. $4 \mathrm{Val}$. Parin, all 8-14 (1799-1805). 8. av. fig. Ueberscizt von T.. F. Froriep und J. F. Me ekel. 1 Bde. I.elpxly $1808-10$ s.

11) Menoire sur le Pou du têtard (Journ. d'lilat. nal. Tou. II).

"Cnvicer, Frederic. 1. Franz.

* Czelfanownky, L. 1. Russ.

Czcombinalsy, D. 1. Pole.

c Cuilnalk, J. v. 1.

DuEbrok. 1. Scliwed.
Donhl, Georg. 1, Oestr.

* DaInItoona, Anelers Gustaf. 18. Sehwed. Gel. 3. März 1806.

pate, I. C. 1. Engl.

Dale, Samtel. 2. Engl.

Daluna, Joh. Wilh. I8. Scliwed. Gel. 1787 7.1 Heinseberg, gest. 11. Juli 1828 in Slorkholm.

1) Esmals d'une classificntion xystematique den Papilloux de Suede (Veteusk. Ac. Hundl.

1816). In scliwed. Sprache.

2) Chinell arancoides (Dipt.) Vetensk. Ae. 1 andl. 1816 ).

3) Notes sur le genre biopsis, nv. desc. et fig. de 3 nollv. espices ribirl. ISi7). Diopsls (I. ix 18:0, lit. Anzeig. p. 501).

4) Forsök lill Upjustälıing af lnacetPaniljen P t er o nu al 1 ni, I symuerlet med afscende pä de i sverlgue timne Arter; af J. W. I) a I m a n n (contiuuat.' (V'etensk. Ilumdl. I8:0, p. $340-326$ 1822, p. 3114-304).

5) Anmbirkuingnr on Antluribi varii lcfnadssiett oflı forvindling inuti Coccus; af L. R. N. Dalman (Vetensk. Handl. 1824, 384-39\%).

6) liaıs,k alt närmare bestäuma alïgte: Castuia Fabr. samt de iletsamma tillibrande Arter (ibitl. is24, p. 390-408, fig.).

7) Om Insekter inneslutue i Copal (c. lab. Vetcisk. Ac. Ilandl. 1825. 2. Part. p. 375. Siockh. 1826. 8I.

8) Allalecta entomologica. Holmiae typis Lindlijanis (Gryplisw.) 1S2.1. Cum tab. IV, acu. VII et 104 pp. 4liaj. (Bell. Zool. Jouru. I825. V1, 236 . - Isis 1825,87 .

9. Nya genera ocli Species af Insecter. (St. Vet. HaudI. 1821, p. 372-388, fy.) ("/ro: phoris, Plinleria (Antimac!ıs), Pिolytumus, ilymenoptera.)

10) Om nägra suenska Arler af Coccux, samı de inutl tlin fórekommande Parasltinsukter (ibid, $1855, \mathrm{p}, 330-411$, fig.). (ibid. 2 Part. 330. Stoeklolm 1826. 4.), Anliang: Nene Genern: Ilymenopt. Coleoptcra, Curci. Ilon, Articerns, Kipldins, Pausus, Ceraubyx, Tillus.

II) Insectorum nova genera. Holm. 1819. ConII I tal). 8.

I2, Ammirk. ou lchneumoniclerma et och af Pimpla atratia (Vet. llandl. 1825, p. I88198. (Gg.).

13. Kphemerides Entomologicne. I. Holmiac (l.ipsianer. 182\%. 8. 36 P'ag. (olıne Collspectins) (T'lon, cil. A reli. I, 3. p. 80).

19) Sneusk Koulngy. Herausgegeben von der k. scliwed. Akall, d. Wlssenscli. Stochh. 1826 et $\times \mathrm{eq}$. 8. IIn '2. Bd. Ileft 12 sind तle Kerfe liesclirieben und abgebildet)

(5) Prodronus Monrigrmphine Castnine, generls Lepidupterorum. Ilolniae 1828. 4. Tab. calor.

16) Méanolres sur queltynes lelınemmonides. Stockl. 1826. 8.

Dandolo, Conte.

Dancwin, Erastuns. 2. Engl.

* Darrandes.

Dauberitun (le jetuc). 1. Franz.

Daman, F. C. 1. Deutsels.

"Dnvis, A. H. 1. Engl.

Davy, Sir Humphry. 1. Engl.

Dabraw, Jolin. 1. Eitgl.

Decker, Mag. 1. Deutsch. 
DedekInd, Jo. Jut, Will. Deutscll.

* Defrance. 1. Franz.

Deegner, J. Hart. 2. Deutsch.

* He RIanz, W. 4. Niederl. Pe:lene, Juh. Christ. Courad. 1. Deutsch.

Ver-llch einer vollständigen Ablandlung vom Mawurm. Lejp. 1783. 8.

- Dejejen. 10. Franz. Delabigarre, Peter. 1. Engl.

in Anu'r.

Helantre. 1.

Delins. Henricus-Friderieus de. 3. Deutsih. Mediens: reb, in Wernigerode (Sichseu) 8. Juli 1720, gest. 22. Okfob. 1791.

${ }^{*}$ Mn:Itac. 2. Engl.

Denis. 2. Oestt.

Demming, William, 1. Engl. in Amer.

* EDenuy: H. 1. Engl.

Monthera, William, 4. Engl. Theo$\log$; Heb. 1654 zII Stawton; gest. zu Upminstre 5. April 1735.

* Desberger, A. I. A. 1. Dintsch. Desbois, Alex. de la Clienaye. 2.

Fran\%.

* Desclianins, Boinard.

* Destonritiz, 'lheodos. 2. Franz.

"Desinayes, G. P. 2. Hranz.

Desjarulins, J. Soust auf St. Maurice.

I) Alueitn xylostella deu Küchengrirten schädlich (L'tust. 1837, Nr. 218. - Isis 1837 , 845).

2) Veber Curculio (Meunelius) striga Falor. auf Isle de Funce seh: lüulig in iVstdern (Aiulil. d. se. 11. XIX, 1830, 240i.

3) Kerle vou St. Manriee etc. (Phil. Mag. IX etc.).

Deslandes. 2. Franz.

Dbesmarest, Gac̈t. 4. Frnnz.

Desnumrets, Dan. 1. Niclerl.

P Desnaricires.

"IDesmonlins, 1. Franz.

"Deworines. 1. Franz.

Hetsvoily, Rohimeall. 2. Franz. Detharding, Georn C'hristoph, 2.

Dïne. Lubte ra Kapenhagen.

Descenx. 1. Fraur.

* Dickliont. I. Deutsel.

Dicquemare. 2. Franz.

* Diebl, Friulz.

* Dierbucla, J. H. 1. Prenss.?

"Dlewing. 1. Oestr. Diggers. 1. Engl.

Dillenlns, Joh. Jak. 1. Deutseh. Botanisl; geb. zu Giessen 1627, gest.

2. Aug 1747 .

DIIIAn, Joh. Talbot. 1. Engl.
* Millwyit, L. W. 2. Engl. Lebt zu Sivanseu.

Ditlnemar, J. C. 1. Deutsch.

Dioncon rides, Pedacius. 1. Griecl.

"Divaleri, Siefluno. 4. Piemont.

Ditturarsele, W. H. E.

EDix.

Doiblos, Arthur. 1. Engl.

* Dbiner, Liduard. 2. Deutsel. D) Uriss, Reiuhold. 2. Deutsch. Dondorir; J. A. 1. Deutsch.

Donovan, Edward. 6. Engl.

1) Britislı Insect.s. I.ondon.

2) Chines. Inseet. Deuseh von Grubner. $111 \mathrm{ft}$. Leipz. Is01. 4.

* Dorner, Jos. voll. 1.

Eortlues, Jacques-Ansplue. 4. Franz. Medikus und Naturforseher. Geb. zu Nimes 19. Juli 1759; rest. 1794.

* Donbledny, Edward. 2. Engl. ponmerc, A. J. I. 2. Franz.

* Dovines, E. T.

is Dis ince. $^{2}$

Drayariand. I. Franz.

* Drapiez. 14. Franz.

Mbreivsen, Christ. 1. Holstein. Kopenhay.

Ernxy. I. Engl. Goldschmidt; grest. vor circa 16 Jahrell.

IIlusiratlons of natural histury. Loud. 1770-1782, 3 Vol. 4. (Sehr schobıe illumbirte Tafeln.)

Dincrue. 1. Franz.

Dudley, Panl. 2. Engl.

"IDiluen, Magnus v. 1. Sehwed.

* Deufane, Léon, 22. Franz.

Dinfschmid, Caspar. 1. Oestr.

Duges, Ant. 1. Franz, T.

1) Ueber dic Charahtere nnd Gattungen des Flohes (Aun, d. sc, n, XXVII. 1831. p. I4s, T. A. - Isis 1835, 62j-3\%. Taf). (Ordnum Rhimaptera)

2) Bail der zusammengesetzien Inseklenungen (Aln, d, ac. ont. XX, 341. Tuf. 12).

Dulnamel du IEomperiu, H. L. 1. Frallz.

* Dnméril, Audré.Maıie-Constant. 7. Franz. Geb. zu Amiens 1. Jan. 1774.

* Homal.

Dinnlor, W. 1. Engl.

* Duncam, James. 1. Sehottländ: Dnnker, Joh. Heinr. 1. Deutsch.

"Driponchel, A. J. 15. Franz.

Dupent. 6. Franz.

Dutficid, James, 1. Eugl.

* Dintrocluet. 3. Franz.

Denval. 1. Deutseh.

" Duvan, Anguste. 1. Fran\%. Dwlgulosky; J. 1. Role. 
Eberhard, J. Peter. 1. Deutsch. Geb. 1727, gest. 1779.

* wiverle.

Wberlinus, Georg Wolf. t. Deutsel. Eheruerus, Erasuus. 1. Deutseh. Wbert, J. J. 2. Deutscli.

Edwards, George. 3. Engl. Ber. Naturalisı; geb. 7. April 1694 zu Stratford (Grafsch. Essex); gest. zu Plajston 23. Juli 1773.

wa wards, Milne.

Egede, Hans. 1. Norw.

* Elrenberg, C. G. 2. Deutsch. Elaret, Geura Denis. 1. Deutsch. Maler und Botanist; geb. 1710 ; gest. zu Londoll $\$ 770$.

Eicluhoru, Joh. Conr. 3. Dentsrh. Eiclustuidt, Lorenz. 1. Deulseh. Mediers: geb. $1596 \mathrm{zu}$ Stettin; gest. zu Danzig \&. Juni 1660.

- Eiclewaid. 1. Dentsch.

"Eiselt, Joh. Neponuk. 3. Böhmp. Ellis, Johı. 2. Enyl. Negoeiant. Lehte un die Mitıe des 18. Jalırh., gest. 5. Okt. 1776 z.u Loution.

Ellis, Daniel. 1. Fngl.

Eneric. t. Franz.

* Engelhere, J. M. 1. Dentscl. Engramelle, Marie-Dominique Joseph. 1. Franz. Augustiuermönch: geb. zu Nedouchal (Artois) 1727; gest. zu Paris 1780.

sErll, J. 1. Bayer.

Ericlison, Wilh. Ferl, 20. Preuss. irnst. 1: Dentsch.

Erxleben, Joh. Christ. Polykarp. 4. Deutselı. Geb. zu Quedlinburg 22. Juni 1744, gest. zu Göttingèn 18. Aug: 1777.

Eschscholtz, Friedr. 7. Lief.? Gest. 19. Mai $183 \mathrm{t}$.

1) Ueber die Sippe Parnalus (aus Mèm. de Mnose. I. - Anu. d. se. no XX11, 33\%. - Lis $1835,184)$.

2) Kerfakelet (IsIs 1822, p. 52).

Espaigutet. 1. Franz.

Esper, Eugen Johann Clıristoplı.

4. Deutseh.

- Espoinasse, J. 1. Eugl.

Estiund, Olof. 1. Schwed.

- Eversanan, E. 3. Russ. (?)

Evaldt, Benjamin. 1. Dentscl.

Geb. zu Danzig 1674; gest. 1719.

* Eydoux, Forfuné. 1. Franz.

Eyrich, Joh. Leoulaatl. 2. Deutsch.

Waber, Jol. Matth. 1. Deutseh. Geb. zu Augsb. 17. Jahrl.; gest. 1702. Eaber, Jo. Casp. 1. Deutscl.
Fabriclus, Joh. Christian. 25. Schwetl. Eiller del berülınt. Entomologren, Sthuiler Linne's, geb. 7. Jan. 1748 ill Tuudern (Seliwedeu).

1) Nova insectorum gencru (Skrivter af anturhist. Sielsknbet BI I. Ilell 1. 213-2\%, dentsch 191 .

2) IIII Skrivter I Insekt (Ibid, 3. Bd. 1. HIt. $145-156)$.

3) Beskrivelse over den skïdellge Sitkker, og Bnunldz-orin i Vest-Indien, of om Pluntaena sneharnliv. Noetua Gossypli, et Zygaena Puglonis cibld. Bd. 3, If eft 2, 63).

4) ('ychrus, ell ny Insect-Slacgt (ibid. III, Heft 2, ti s).

5) Index enlomalugleus. KIliae 1802. 8.

(b) Philosoplia entounloglea sistens seielltIac lindamenta adjectis telint tlouibus, exemplis observationlbus, ndumbratlonibus. Hambural et Kilouil, impensis Curol. Eruest. Bolınil 1778 . 8 S. Vurr. 178 S. Text. 8.

7) Determinatio generis los nffinlumque (Aetes de la soe. d'hist, unt. de Puris. I, 27 $-35)$.

8) Systemin Autliatormm. Brunsvig 1805. 8. 9) Systemn entomologine. Flessh 1775. 8.

(10) Genera insectomu. Kitonii 1781. 8.

II) Fintomologla systematien. 5 Vol, ilnfu. 1792-98. 8.

12) Species Inseclormn exhibentes eorum differentins specifieas, syunyma auetorum, Loea uatalla. Metomorpliosin adjectis observationihns, descrlptionilus. Tom. 1. Hanburgi et Ki o 13 jupensis (:irol. Eruest. Bohmli 1781. 552 S. 8. - Taml. 11 ibici. 517 S. 8 .

13, Systema Riyugot orum. Bruusv. 1803.8.

(4) Systema Fleutheratorum. 2 Vol. Kilon. $1801-2$. 8 .

15) Reise nach Norwegen, mit Bemerkungen aus der Naturhistorie und Oekonoule. f̂aub. 1779. 8.

Fatoricins, Ottn. 3. Deutsch. Fabricius, Philipp Kourad. 1. Dentst:l.

Fahroni, Atamo. 2. Ital.

Facklnins, Audr. 1. Deutsch.

"Nageo, John. 1. Amer.

"Fahrïng, $0 . J$.

Diptera suce, a Fallen, Lundae, 1, 11. 1814-2) (Isis is37, 759).

Fairfax, Nathaniel. 1. Engl.

* Falderannin, Fr. 4. Deltsed.

* Halién, Carl Frietr. 12. Schwetle.

" Farinew. 4. Franz.

" Finviari.

"Felir.

Feige, Carl Theolor Lutwig. 1. Delltseli.

* Feistlanuel, Baron von. 2. Franz. Felici, Giovanui Battista. 1. Ital. welton, Samuel. 2. Engl.

Derniu, Philipp. 2. Niederl. Medicus; Leb. z.l Ausgang des 18. Jalurh.

F'érunsne, Baron d'Audebert dr. 1. Frauz. Oberoffizier, Ritter ett. in Paris. Der berillumte Nafurforscher 
und der Dírektor und Gründer des Bulletin des seiences uaturelles.

* Ficiuns, Heinr. v. 1. Drutseh.

* Ficher, Fr. X. 4. Bühme. Niehig, Joh. 1. Deutsels. to Findel.

* Fintelinaun, L. 1. Deutscl. Firums.

Heusclirecken (Is Ls 1820, 913).

Fisclier, J. B. 1. Deutsclı.

Fiscluer, J. E. Edler von Röslerstamm. t. Oestr.

Fisclier, Joh. Leonlıard. 2. Lief.

Wisclier v. Waldheim, Gotthelf. 28. Deutsch. Geb. zu Waldheim 15. Okt. 1721.

Fiselnerstrion, Jos. 1. Scliwede.

* Witzinger. 1. Oestr.

Finda, Jol. Daniel. 1. Deutsel.

- Nemuing, John. 1. Engl. Fleisclıer, Esaias. 1. Däıe.

- Flelsclier, G. Th. 1. Russ. Fodin (conf. Rosenblad). 1 .

Floyd, Edward. I. Engl.

"Focke, W. 1. Deutsih.

Warster, Arnuld. 2. Deutzcls. Noseo, John. 1. Engl.

Fontriur, Felice. 1. Tyrol. Pliysicus, Naturforscher und Anatom: geb. 15. Apr. 1730 zil Pomarolla (Tyrol); gest. zu Floreuz 9. März 1805.

Norlses, James. 1. Engl.

Forel. 1. Waadtländer.

Forellins, Lars. 1. Sehwed. Lebte in Gothenburg.

Forskaol. 2. Sehwed. Reisend. und Naturf. Geb. 1736, gest. an der Pest zu Djerim (Arabien) 11. Juli 1763.

Forssberg. 2. Srliwed.

Forsten. Rudulphus. 1. Niederl.

Diss. Cautbaridun histuriam naturnleu elienilcam et medican exhibens. Lugd. Bat. 1775.

Forster, Georg. 1. Engl.

Forster, Johanı Reiulsold. Deutsch.

* Eorster, Albert. 1. Engl.

Forsyth, William. 1. Engl.

* Foudras. 1. Finnz.

* Nonlifues de Villnret, J. H.

J. 1. Frialz.

Foureroy, Antoine François. 2.

Franz. Berilimter Chemicux. Geb. zu Paris 15. Juni 1755, gest. 16. Dez. 1809.

* Wouruel.

Frnnchevillo, de. 1. Franz. Francillon, Jolu. 1. Engl.

* Franek, U. 1. Franz.

Franklia, Williau, \&. Engl.
Franzius, Wolfgang. 1. Niederl. Fraula, v. 1. Frauz.

* Fray. 1. Franz. wrenzel. 1. Ungar.

* Freyer, C. Fr. 8. Bayer. Frezan. Alltonio. 1. Ital.

* Friedrielt. 1. Deutscl.

* Fries (Bened. Friedr. 3. Schwed.

Friscle, Juh. Leonh. 6. Bayer. Geb. 19. März 1666 zu Sulzbach (Oberpfalz): gest. $1743 \mathrm{zu}$ Berlin.

\section{Fritsclt.}

Geschichte der Hundswutl, Iammt Beolsachtungeu n̈ber die Wirkungen der Meloe. Wien 1781.8.

Fritze, Jol., Gottl. 4. Deutsely.

* Fivrldslcy von Frivald, Em. rich. 2. Ungar.

Friliel. J. Alois. 2. Dentscl.

Friblitich, Fr. 3. Wiirtt.

Enmmeratin tortricun L. Regno Wïrttemhergico indigenarum, sistens speeiernm dillerenilas, symonyma selecta, earnu domicilia et tempus cuin descriptionibus. Tubinga 1830.8.

Frounageot, de Veriax. 1. Franz.

Froenuanu, J. C. 1. Dentscli. Frorien, Vater.

Wroriep (Sohn). 1. Saclise.

* Firrerolur. 1. Deutseh.

Fisssly, Jolı. Casp. 10. Sehweiz.

"Tabrlel, C. H. 1. Drutseh.

Gadel, Pelsr Adrian. 4. Finnläud. Zu Abo gest. geren Ende des 18. Jalırhunderts.

Cüille, Heiur. Moriz. 4. Holstein. (oder Luittielı?).

1) Beiträge zur Anatomie der Inzekten. Altona 1815. 4.

2) beiträge z. Anat. U. Physiol, der Medı. sen elc. M. '2 Kupf. Berlin 1816 .

3) Gallengefäse der Kerfe (lsis 1820, it, Ai12. p. 652).

"Giirtner. 1. Deutrel.

Galurlieb van der Metilen,

5. Guslav Casimir. 4. Preuss. Greb. 24. Dez. 1630 zu Grymsholm bei Stockbolm, gest. $1717 \mathrm{zu}$ Altenlandsberg bei Berlin.

* Frimard, Joseph Paul. 1. Franz. Galentins, Dominicus Gusman. 1. Ital.

Giabli, Francesco. 1. Ital.

Trallo, lietro Paolo da San, 1. Ital.

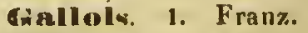

Sี่ a rulen, George. 3. Engl.

Crardes, F. 1. Srhwede.

* Finarduas. 1. Deutselh.

Treridlel, Pierre Joseph. 1. Franz. Geb. zu Manasque 1. Aug. 1659; gest. 1737. 
Hlst. de plant. (251, t. 54, tig. 9. Cynips ehernis?).

कRrenot. 1. Fr.

Cỉ্arvie, Jolu Mr. 1. Eugl.

Gineticr. 1. Deltsch.

* Gebler. 6. Jiuss.

Tieer, Carl de. 20. Schwed. Reicher Privatmann; geb. zu Finspang 1720 ; gest. zu Stockholm 8. Mäı\% 1778.

1) Ohnervatinus sur l'auatomle de la chenIlle à deux quewes du saule (Mciu. de l'Aead. des sc. de Paris 1748, 29).

2) Tipulil fusca descripta (Nova act. Ijps. I, $65-77$. - In seinen Méuolres T. Vi, $35 i$ -359 ),

3) Observations sur la propriète singuliè qu'ont les grauds clienilles à quatorye pattes et à double quene, dil sinule, de seringuer de la liqueur (Mem. de l'Acad. des sc. de Paris. Sav. èrang. I, 5:30).

4) Meunire sur un ver luisant femelle ef sur transformation (ibid. $11,261-75$ ).

5) Observations sur les Eipheneres dont l"accoupleuent n eté vII eu partie (iuld II, 461-69. - Ultgezogte Verhandel. ing. 7 Dceh $271-(84)$.

6) Obvervations sur les Pucerons du prumier, ef en particulier sur leur nucouplemeut (ibid. II, 46y-13. - Ses Menoires III, 50-53. - Uit gezogte Verliandel. 5 Deel, 255-62).

7) Rön och observation ófver suma Insecter som hulı, lıitppn i högden (Velelık. Acad. Handl. 1740, 265-281; dentsch 27!, lateinisch. Act. U Us. 1740, $49-67$. Aunlect, trans. I, 46 -56; tranzösisch: Meim. de Mith. et de Pliys. lle l'Acad. Par. Sinv. Ctrang. 1750, hist. 39. In ejusd. Men. VII. I8-3I:

8) Beskrifning pa en thiea kallnd clet. Ac. Handl. 1740, 464-69. - Lateiu.: Analect. trausalp. I, 106i-109-Ia ejusd. Meum. I, 505).

9) Beshrituing pa en lusect, soul lefver uppa maest alla orter ock trien itti et kwittskuin, och kalliıs (Vel. ne. Haudl. 1741, 22136. Deitsels 557 ; Int : Anal, trinsalp. 1, 166 -176. - lu ejusi. MIem. III, I63-I8(1).

10) Beskrifning pn en liseet kullid Porlura (I. c. 1741, 293-303. Deutscli 239; Int.: Anul. transalp. 1, 273. - II (Len Meum. VII, 35-39).

II) Beskıfinlıg pa ell Insect af ett nyit saupte kallad Pliysapus. c. lip ,Vet. nc. Handl. 1793, 1. Delitkcli 3; Iat.: Aual. Irans, 1, 277 $-81 .-M$ im. III, 4-II).

12) Tal ou uiftan vom Insccterue, ocli deras skurs kädaule tilkkynda oas (Schwed. Acad. Abliandl. 1, 325. Sioekh. 1744. 8. et Ibid. 1747. - Ueber den Nutzeu (ler Kerfe).

13) Beskrifuing of maskur, som fortïra upaunemalen I mngazluer, aumt forsök at utroti deu (Ueber Cnlnudra I. c.).

14) Lyckte - Illasken frnn China iVetensk. acad. Hnudl. 1746, 60-66. An. iransalp. I, 475-79)

15) Beskrlfning ofver en clilnensisk och en lulnendsk fjöril, jnmfe nagra aumürkuingur blver ljörillarme I genı ell (Vet. ak. Handl. I798, $208-230$ )

16) Roen om Mask-Lejonet (Vet. ak, IIandl. 1752, 180-192 et 261-265 Deutsch 187-"66. Latelu. : Amal. trausalp. II, 462-70. - Mём. $V 1,160-183$ ).

17) Tnl on Insects adstrlag (Stockholm 1754. Deutscli: Stockli. Mag. 1, 239. d. n Sauml. ver. siclir. d. fel. In 'Schwed. I. Naturf. 5 St. Nr. 13, 207-3j6. - Mem. II, $17-51)$.
18) Mémolres pnur servir ǹ l'hlstolre des Insectes. Tome I. A Stockliolui. De I'Impri. merie de L. L. Grefiug. 1732. XIV et $707 \mathrm{pp}$., 37 plinches (tig. ncueo incis., 4. - Tome ii, 1. partic (l'recciles de discours sur les Inseetes en gèneral). A Stocklı. Del'imprimerie da Pierre 11 is selberg. 1771. XII er 616, pp.. 15 pl. Tom. 11, 2. partic. Ibid. 1771. Il el 617-1175 pp. 16-42 - Tome III. Ibid. 1773. VIII t1 1.96 pp., 44 pl. - Tome IV. Ihisl. 1774. XII et 456 pp., 19 pl. - Tome V. Ilicl. 1775. VIII el 418 pp., 16 pl. - Trime VI. Ibid. 1776. VIII et $5: 22 \mathrm{pp}$., $30 \mathrm{pl}$. Creier, Joh. Daniel, 1. Dertsch.

* Gené, Giuseppe. 3. Ital.

Cincolfroy, Claude Joseph. 2. Franz. Pluiluazeut; geb. zu Patis 8. Aug. 1685 , gest. 9. Miirz 1752.

Cieofroy Saint-Brilaire, Etienuc. 4. Franz. Geb. zu Etampes 15. April 1772

* Cicofroy Saint-BHilaire, Isi. dore. 1.

"Tdeofroy Snint-Izilnire, Auouste.

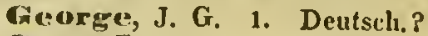

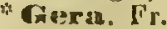

Tix (:F)i, Ranievi. 2. Ital.

Storin naturale di un nuovo iusctlo. Fl. renze 1798. 8. (Rhinocyllus antlodoninl:̈lcus.)

Crerdes, Olof. 1. Schwed.

ficrales, Fredrik. 1. Schwed.

Gieriunr, Ernst Friedrich. 50.

Deutsch.

Cycsenins, Wilhelnı. 1. Deutsch. Versuch einer lepidopterolog. Eacyclopädie. Erfurt 1786.

Aycierns, Jol. Daniel. 1. Dentsch. freyer, Carl. 1. Deutsch.

Tractatus de cautharidibıs, Francof. 1687.4.

* Cieycr (Augsburg).

cibeliu. 1. Franz.

Ciil, Jayune.

Declar. de lus provechos grindes de Ina Colmenas y Alibaucas de lus Abujas. Zuragoca. 1621. 8 .

* Giannertinal. 3. Russ.

Gímunni, C. Francesco. 1. Venez.

* Ciormas, Spirito. 2. Ital.

Account of " singulnr conformation In the wings of some specles of Mothe (Iolnnean Transact. 1, p. 135. litb. 13).

(구으르, Figlio.

Cnlendarlo entomologleo. 8. Torlan 1791.

* Histe1. Fr. X. J. 45. Bayer.

Cxingrion, Citoy.

HIstolre d'un T'ctanos, avec symptômes d'liydropliobia, produits par le Polson de Can. tharlde (Mem. de Tur. 1803, p. 15).

Cind bacle, Georg Jac. 1. Deutsch. Medicus; geb. zu Frankfurt a. M. 1736 , gest. 13. Sept. 1796.

Glasser, Johaınes Friedericus. 2. Deutsch. 
* Flaser, L. 1. Deutsch.

Sileditselı, Joham, Gottlieb, 10. Dentsrl. Geb. zu Leipzig 5. Febr. 1714, gest. 7.11 Berlin 5. Okt. 1786.

Treichen, Willelm Friedrich v., genamut Russworm. 3. Deutscl. Geb. yı Bayreuth 1717, gest. 1783.

Gricelin, S. G. I. Deutsclu.

Gineliu, Johanı Frichr. 12. Natu'forscher. Geb. yu Tiibingen 8. Aug. 1748, gest. zu Göttingèn 1. Nov. 1804.

Godnet, Jean Baptiste. 3. Franz. Proviseur unter des Kaiscrregierung an Jyerum 7.u Bonn. Gest. 1825.

llistolre naturelles sles Lepidnpieres, ou Pnpiltons de France. thurage base sur la methode de M. Lntreille. Avee tes tigures te chnque expece, desslnees et colorices diapres unture par. 1). D II men il. Paris Imprime cliez Laclievnidièc, fils. (Uugefihr I00 Lieferumen In 6-7 Böndeil in 8 . Prels der Liefer. 3 Fr.)

"Godet, Louis. 1. Franz.

Gocdirert, Juhann. 1. Molländes.

Naturforseber und Maler. Geb. 1620, grest. 1668.

Conzancr, Andreas. 1. Dänc. S ฺลก.

Foncalo, De Las Casas. 1.

Crocze, Johann August Eploraim. 30. Deutscl. Beröhmit, Natırforscluer. Geb. zu Astiersleben 28. Mai 1731, fest. 7.I Berlin 27. Juni 1793.

* Golifuss. G. A. 2. Deutscis. Croldsmitl, Olivier. 1. Engl. Coroing. C. R. 1. Engl.

* Cory, Hippolytc. 30. Franz. Cinotivald, Christoplorus.

Dentscli.

* Aoradot.

fononfler, de. 1. Frauz.

Cionald, Will. 1. Engl.

Aconnt of eluglish Ants. Lond. 1747. 8 (Ameisen).

* Tomronar. 1. Franz.

Thoz, Geortr Ficds. 1. Deutsch.

* Iraclis, Mariano.

* cyncils, Vater.

* Coraf. 1. Deutsch.

* Crositin.

* Crenvenhorst, Jol, Ludw, Carl. 14. Deutsel.

Array, Stepli. 1. Engl.

Arny, J. L. 4. Engt.

* Coñay, Gcorge Robert. 4. Eugl. Tregory, G. 1. Engl.

Greve.

Niturg. d. sehädl. Insektenarten ete, 8.

Grev, Nehenial. 1. Engl. Geb. zu Coventiy gegen 1628, gest. 1711.
Côriesmbach, Alexander William.

1. Engl.

* Giriffith, E. 1. Engl.

* Cinnuer, Carl Heinr. B. 1. Oestr. Soñorineve:Id, Jo. 1. Engl.

(x) ronan, Carl Ludw. 2. Deutscli. Côromovius, Lmurent Theodor. 3. Hollind. Gest. z.⿲ Leyden.

Frosier, L'Abbe. 1. Engl.

Estuluer, Mich. 1. Deutsth.

"Gruithuiseu, Fr. v. P. 1. Bayer. Grundigs. 1. Sachse.

fin riindler, Gotthi. Aug. 2.

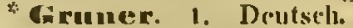

Givitzonuin, Jolı, 2.

* Tuénée, A., de Clıâteaudun. 2. Fran\%.

Guenean de Montbeillard, Plibibert. 1. Franz. Geb. zu Semux en Auxuis 1720, rgest. zu Paris 1785.

Evinutlece, Jo. 2. Deutscl.

* Gacériu, L. Fỉjix Edounrd. 46. Fıanz. Geb. zu Toulon in Novemb. 1779 .

Cucteril, Jcan Etienne. 12. Franz. Geb. zu Etampes 2\%. Sept. 1715, gest. 7.⿲ Puis 8. Jal. 1780.

Cuilding, Laudsdown. 7. Engl. Gest. 1832 auf der Insel Sinnt-Vincent.

1) The Naturol-llisiory of Lanla nmpuintor of Palurielus (Trans. of Linn. Soc. XIII P. II. p. G04, Twlo, $X X X)$.

2) Ameisen16uen (Ibid. XV1, 1. - Isis 1830, p. 897 ).

3) Crepltaculnm Locnstac ete. (1sis 1829, p. 1287)

4. Xvlocopa, Horia (1s|a 1829, p. IIII).

5) Clinidlum, Clitunidae (Isis 1831, p.716).

6) Plinima et Ascalaplins (1sis 1829,p

1212). (Alle Abh. nus Linuenn Transacts.)

* Cunillanué. 1. Franz.

Cumander, Pehr. 1. Sehwede.

* Condelach, Ferd. Will. 1. Deutselı.

Gratise, Conr. Dietr. 1. Deutsch.

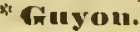

Gyldential, Lconlıard. 2. Sclived.

DInns, Joh, Adamv. 1. Deutsch.

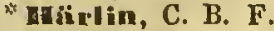

"ylagen. Herm. August. 1. Deutsch. Ingemach, Jakub Johaun. 3. Sebweiz. IVar Conservator am Leydencr--Nusenm und starb 1820.

Mormolyce (Ann. d. so, n. I1. 1825. T. 21. p. 500. Durch $S$ tor m publleirt).

Hagendorn, E. 2. Deutsel. Geb. z.l Wolau in Sehlesien 22. Iaı. 1640 ; gest. zt Gülditz, 27. Febr. 1692.

Fingendorplinus, Chr. 1. Hagenat 1526. 
Hagreted an, Joham Otto. 2 Scliwed. Inton, Carl Wilh. 3. Bayer.

Die wanzenarlgen Insekten gelreu nach der Nitur aligebilelel und besclirleben, Nïrnberg (Zelı). - Brachte das Werk bis zu Bd.3 1838. 8. Heft 2, 3; daranf siarl er llsis 1836 , 6:7).

2. lcones Cimienm. - Abbildungen der wanecunticen Innckren. I. Liefg. gr. 4. Mit 4 illum. Tat, Nïrub.

3) leones Orthopternrum. - Abilidungen der hinulligeligen lusekten. 1. Liefg. gr. 4. Mit 4 illunb Tat. Nürnb.

Halı, Pefrus. 1. Abo.

Hale, Gulichmus Puscy. 1. Holl.

* Anlialus, A. H. 4. Engl.

* Musuel, J. v. 3. Deutsch.

Mnmilton, Carl. 1. Engl.

Hasmuer, L. 2. Franz.

"Inumunersclimint, C. F. 12. Oestr.

thancock.

Hanow. 11. Dentsch.

Hansemann, J.M. A. 1. Deutsch. Mnrol wicke, Thomas. 5. Engl.

Ist todt (p. 37).

Anıclsen und Binttläuse (Zool. Journ, XIII. - 1sis 18.311, 1248).

Harrer, Gicorg Albrecht.

1.

Dentsch.

* In rris, Th. W. 1. Nordau. Engl. Marris, Moses. 4. Engl.

I The Aurelian: or natural hist ory of english luseels; namely Mollis and Buttenllies. Together will the Plunes an which they Teed ete. By M. If arris, Secrelary lo the Aurelian Siciety. London priuted thr the Aulor. 1766. I Titelkupf. I0 S. Vorr., $2 \mathrm{~S}$ RegtsI.,

80 ×. Text, 4t Kupfertaf. Follo.

2) Expnsition of british lusects.

IItarison, Jolm. 2. Engl.

* Mrtig, Th. (6. Dentsch. IFartlielos. 1. Engl.

Bartumun. 1. Deutscl.

* Mnrzer, August. 1. Dentsch.

Inssel quist, Friedr. 2. Silhwed.

Geb. z.u 'Tacruvalıa (Ostgothlaud) 14.

Jan, 1722, gest. zu Snyrna 2. Febr. 1752.

Hasselt, Wilhelm Hendrick van.

2. Nirderl.

Iintclict, J. 2. Engl.

Hattorf, Joh. 1. Deutscl.

* Muvfr. W. 1. Dentsch.

* Mumlorert. 1. Selwciz.

* Hunaran, G. H.

* Enupt. 1. Deutsch.

* Hinguannin, Joh. Firicdr. Ludw.

2. Deutsh.

Havens, Jonnthan N. 1. Lebte in New-York.

. Wavorth, Adriall Hardy. 8. Engl.

Gest. 1834.
Mnymann, Chr. Jolı, Golt. 1. Denisch.

Melorenstreit, Johann Firnst. A. Nutnrf. und Mredikus; grb. zu Neustadt an der Urla 15. Febr. 1203, gest. zu Lejprig 5 Drz. 1764.

Debenstreit. 2. Deutsch.

Mecount, Philippe. 2. Franz. Geb, zal Abbeville 11. Febr. 1661; grst. zll Paris 11. April 1757.

" Heeger, E. 1. Oestr.

* Heer, Uswald. 8. Sehweiz.

* Hegetscliveiler, Juhann Jakob.

2. Selwivizr.

IIteinglkeנ, Carl. 3. Engl.

Enlomologische Nolizen (Zonl Jnurn. V, 1830, 12. 191. - Isis 183:, 661. - Spinnen und Ceratifis cilriper(in). †े

Ireise, Joh. Gottl. 1. Dentsch.

Meister, Lanr. 1. Dentsch.

* Held. Niex. 1. Bayer.

* MeIfer, J. G. 1. Oest.

IHell. 1. Schweiz.

- Eellenins, Carl Nicolas. 2.

Schired.

Mell wig, Joh. Clirist. Judw. 3 .

Deulsth.

Heunert, C. W, 1. Dentsch.

Menuiclie, Ciaton Godofr. 1. Dentsclt.

"Hentz. N. M. 2. Amerik.

Hexhst. 1. Dentsch.

Herbst, Joh. Friedr. Wilh. 13. Beriilhut. lintom.; geb. 1. Nov. 1743 zil Petershagen, gest. 7u Berlin 1807.

Beschreibung einiger höehsl seltener Hcu. schrechen (Nene Schritt. d. Ges. Malf. Fr. IV. p. $\mid 11-21$. 'T'nl) I el II).

"Mering. 4. Deutsch. (Stettin).

Mermanu, Joh. 2. Deutsch.

Hermaun, Jean - Fridẻric. 1.

Merinlistiuit. 1. Deutsch.

Herusulez, Don FranciscoGarcia.

1. Span.

IIIst. Mex. 78 (Cochenille).

\section{IIeroil ot us.}

l.it, $V$ ete. Uebers, von $G$ oldhagen p. 383. (Bieneu.)

"Heroli, Friedr. 6. Deutscl.

Merold, Manritins, 2. Dentsch.

* Merricli-Schiirter. 6. Bayer.

* Mertivig.

Mettlinger. 1. Franz.

Meuvil, Van der. 1. Niederl.

"Meyuleu, C. H, G. v. 3. Deutsclı.

* Meyer. I. Deutsoli.

Heysliam, Jolin. 1. Engl.

Hierne. Urb. 1. Schwed.

Fildanus, Fabr. 1.

* Itild reth. 
AHIt, Thomas. 1. Enort.

1111, John. 6. Enarl. gel, gegeu das Jabr 17I6, gesl. zu Londoul gregen 1775.

Eirire. de la. 5. Franz.

rirscli, F.

Ankiindigung elnes netuen Mitlels, des Cyulps Rosarum, zur scluncllen Vertreibung der Zahns chumerzen (Hufeland's Jnuru. d. praki. Arıneikunde IX, St. 4. Jena 1800, p. 171).

* EII.s. 1. Seliweiz.

*Holuhonge, Jolu Lam 1. Engl. Dodierne, J. B. 1. Ital.

Ilosefungel, D. Jak. 2. Nirderl. IEogutrdin, Peder. 1. Dïn.

" Indpruer. Nic. 1. Dentsels. IIdiss, Prof. 1. Deutseh.

* Eloeven, J. van der. 6. Niederl.

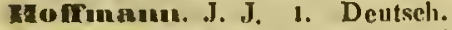

* EnTupusegg, Johann Centurius v. 4. )eutscli.

Hoditum anatex, Godofredus Daniel.

1. Deuiseh.

" Holnenwarth, Siegmund vou. 1. Oestr.

Eoister, Laurentins. 1. Deutsch. IInIlar, Weneclaus. 1. Bölıne. Geb. zu Prag 1607, gest. zu Loudou 1677.

Holnberger , Petrers. 1. Zu Norkü̈pping.

ITombers, Will, 1. Geb.zıt Batavia 8. Jal. 1652, gest. zu Palis 24. Sept. 1715.

\# Hoinbron. 1. Tranz.

Home, Liverard.

I) Dnrstellungen les Nervensystems der Ihunucl (Krltik Iliriiherin T I e d e mi a n II's umd iler Brïter Trevlra u u Zeitselor, f. Pliyslol. 11, 1. Hft.).

.2) Fusslsau der lusekten, welche kleben (Plill. Trnnsacl. 1819, p. 322).

Heneyers 1. Deutsch.

Itooke, Robert. 2. Geb. auf del Insel Wight 1635, gest. zu Londou 1703.

.Hooker. 1. Engl.

मIDIf, K.

2. Tope, F. W. 6. Euml.

Hop D, Joachim. 1. I. ntseb.

* Hoppe, Davil Heims. 2. Geb. zu Vilien in Hannover.

EOPDe, Tolijas Conrad, 5. Deulsch. Tyoreln, Friedr. Wilh, I. Diutseh

Thorubustel, H. C. (Melittophilus Theosebastus.) Der Euldecker der

Absonderumg des Wnches der Biene (Hamburg. verm. Liblioth. 11,45 ). Vgl. Il inter

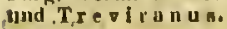

- Mornigng, E. G. 4. Deutsch.
Eorrabov, Nic. 1. Dän. Geb.

zu Kopenlangen 1712. gest. 1760.

Horncinuch, F. 1. Deulsch. Hormstedt, Cles, Friedr. 1. Dentsch.

* Torkfeld. Thomas. 3. Engl. Hotton, Peler. 1. Holl. Geb. zII Amsterdam 18. Juni 1648, fest. 10. Jan. $170 \%$.

Ellotz. Johannes. 1. Deutseh.

Hont, 2, Oestr.

Hoyer, Joh Gearg. 2. Deutsel.

Wuber, François. 1. Schweiz. Geb. z.n Genf 1780. Correspondent der Akarlemie ru Grneve. Diess ist der brribmte seluarlsehende blinde Forveluet.

Nonvelles observntions sur les Abeilles. Parls et Geneve 1814. 2 Vol. 8. Avec fig.

Inber, Pierpe. 5. Ein Soln des François Huber.

1) Memoire ponr servir a l'histoire de la s.lenulle du IIamac. TInea Hnrrisella LIn., Deco* phore de Lntr. (Mém. de Genève VII, p. 121161, 2 planch.).

2) Veber eine Wanderung vou Sclimettẹr. lingen (Min de Gelleve III, 2. 1826. p. 217).

3) Obxervations on severnl specles of the Genus ApIs, known by the Name of llumblebees, nul cnlled Bombinatrices by LAnuweus (Iinn. Trans. V'1, p. 214. Nr. 19, Tnb, 25. 26. 27. Vollatïndige Nalıreisch. d. llum. meln (Bombus, Fabr.. Bӟеuи Jur.).

a) Tome 2, de Nour. Olis. sur les A beill. de son perre. - Recherches sur les moeurs de. fourmis Pnr. 8. Av. fig.

Hibuer, Jak. 4. Bayer. Gest. 1827 .

1) Abbililungen mud Beschrelbumgen noch unbeschrieliener und noch nicht abgebildeter Sclinetterlinge, Augsturg 1785, 8.

2) Beitrige zur Gesclichte der Selımetterlinge. $1786-94.8$

3. Simmling auserlesener lägcl und Schmetterlinge. 1792. M. 100 ill. Kinpf.

4) Verzeichniss beknnter Sclimetterlinge. 1816. 8. . II. 4. $41 \mathrm{kr}$.).

5) Sammlung europäischer Sclimelterlinge, erriclitet von J. H. In A. 1805. 4. Titelbl. 27 Bog. Text. 700 Bl. Abuild. ,Zusaumen 1l. 24\%. $27 \mathrm{kr}$. rhcin.) (Wird fortges. v. C. Geyer:

(6) Samulung exotlschar Sclumetterlinge, errichtet von J. H. in A. ISti6. "\$tit 12 Text. blötterı und 339 Abbild. (Dax Gnnze fl. 198. 9 kr. rh.). Wird fortges. v. C. (i e yer.

7) (iesclichtte europäischer Selumetlerlinge, gesnmmelt von 'J. H. 1806. 4. TItelbl. 5 Bog. Text. 406 BI. ausfoun. Abbild Jedes Blail kovtet 14 kr., xusainm. A. I63 33 kr. rhein.1 (Wiral Jonth. v. C. Geyer in Regensb. - Thun a ent. Arch. I. ?. p. 30.)

8) Siystematisch - nlphabctisches Verzeich. niss zar Samml. enrop. Selmetterlinge, 182'? 8. (54.kr.)

Irinner, Johamn, Gottfried, 2. Dentseh.

"Iluell.

Ixilpseh. 1. Deutsph. 11 : 
Irufnagel. 10. Dentsel. Hughee, Griffith. 1. Engl. Huller. L'.

Abeilles imén. de l'Acad, rov. de ste et bell. Jettr. A Berl 178I. Selir interessant).

MuIsh, Robert. I. Engl.

Mopel, Aug. IVilh. 1. Russe. Illuttuy.⿲. L. Niederl.

- Minuboldt, Alex. v. I. Preuss. Neuxpanien. III.

Elummel, Arvid David. 3. Russ.

Eusals entom. Nr. 1-5. 1821-1826. 8. (1sis 183.3. 498. Enthitit daraus: Blatta lapponicll. Poisterachabe Ganze Naturgesch.)

Hitumel. Anton. 1. Deutseh. Hinter; Jolnin. 1. Enml.

I, Benbachtungen iiber dle Blenen (Philos. Trana. 1792. p. 128-194).

2) Absoud. des Wachees der Bienen (Ibid, p. 14.3) Vgl. Hornbostel, Trepirall us.

"nuwehke, E. 1. Deutsch.

nnuger.

Inle, Joh. $\mathrm{Abr}$.

Iliger, Jubaun Carl Will. 25. Dentscil.

"Iminofr. Ludw. 6. Schweiz.

Inperato, Ferrante. 1. Ital.

"Ingpen, A. 1. Eugl.

- Isemsee, Emil.

Iser, Carl. I. Scluwede.

Jeblonsky, Carl Gustav. 1. Deutsch.

- Jacksom, James Gray. 1. Engl.

- Jacobson, Jar. 1. Däne.

Jacobaens, Oligerius. 3. Jiitläıd. Geb. 6. Juli 1650 z.॥ Arhıseแ in Jütland, gest. zu Kopenlagen 18. Juni 1701.

Juequin, Nikolaus Jos. 2, Oestr.

"Jncerninot. 1. Frin\%.

Juger, Christ. Friedr. 1. Württ. Grb. z.. Stuttgart 13. Okt. 1739.

- Juger, F. W. 1. Dentscl.

Mediain. An welsung wegen der tollen llunde. *iutl. Tübing. 1782.

- Jinisel.

- Juthenstela, M. v.

Jaion, Paul. 1. Deutseh.

- Jrimeson.

- Inm, G. 1. Ital.

- Inrdine, Sir William. 1. Engl. Jarocki.

- Jensen, Henning. 1. Curländ. Detzens, Paul. 1. Deursch.

* Jerm. 1. Engl.

Joblot. 1. Franz.

Joerileng, J. H. 1. Deutsch.

Jolın. 1. Eugl.
Beschreibumg einlger ostindlmelien Insekten (N. S.lir. d. Berl. Fr. I, II 347-53, Tab. IV). Heusclirecktn. I spinne.

- Jones, William. 2. Kngl.

Jolnensten, Juhunn. 1. ['ule. Geb. zo Sambter in Polen 16u3, gest. 1675 zu Zielsendorf.

Jomsselln, Juln. I. Engl.

Doyese, J. B. X. 1. Frauz.

Jnmu, Don Gcorge. 1. Engl.

Jucln, C. W. 1. Deutscl.

* Inllen, Stanislans. 1. Franz.

JnIIn, Jolın. 1. "Engl.

Jung, Conrad Cluristoph. 1. Deutsch. Inungims, Joarhiu 1. Dentsch.

"Dnuker, F. C. 3. Deutsel.

Enrine, Louis. 3. Silnwiz. Medicus und Naturtouseluem: reh, \%u Genf 1751 ; grest. gegeu das Eude Olt. 1819.

Xenos vesparum iMen, di Tor. XXIII. In is 193\%, p. 76I, T. 131.

Justel. 1. Lngri.

Insti. 1. Deutsch.

HUnp fer, Engelliert. 2. Deutseh. Medicus und Reisender. Geb zuL Lemgro (Westplaten) 16. Sipt. 1651, gest. 2. Nov. $17 / 6$.

MnIm, Pehr 8. Seluwed. Geb. 1713 in Osterbotluien, gest. 16. Nov. 1797.

* IL atenhuch, J. H. 1. Dentsel. Inmen, Georg Iosepl. 1. Engl.

" Fumoli. 1. Deutsih.

* In nipe, Christ.

Ir jије. 1. Deutsel.

* Knriline. 2. Russ.

Inrsten, D. L. G. 1. Divtsch. "Inaje. I. Deutscli.

Wheferstein, Clur. 9. Dentsch.

" Irelch (von Ratibor). 1. Deutsel. Heiler, Joh. Christ. I. Dentsch.

"Ielliner, A. 1. Deutseh.

" Temmedy, $A$.

Ierner, J. S. 1. Deutscli.

Ierr. Rubert. 1. Sehotlländ, Chi"urg; gest. zu Edimburgh 1814.

Ierr, James. 1. Eingl.

Nat. lise. of the Ins. Wleli producen the Gum Lacea ivon Coceus Lacea) (I'JiI. Trans. Vol. 7ı, p. 374. fig.).

Keys Joln, 1. Lngl.

* Iitil, J. 1. Engl.

* Lienenwetter, II. v. 2. Dentsch.

Ylug, Edmund. 3. Engl. Medieus des 17. Jahrl.

Exirby, William. 30. Engl.

History of Tipula Triticl, nnd Iclinetmon Tipulae, with some Observations IIpon otler Insects that attend the Wheat, in a Lelter to 
Thoms Marsham, (Llnn. Trans. IV, p. 230, Nc. X:..)

Mirelsdore. Michael. 1. Deutsol. Klreher. Athaunsius. 1. Deutsich. Jesult. (jeb. 2. Mai 1602 z.ı Grysa bei Fuldu, gest, z.ı Rom 28. Nov, 1680.

MIrinaler, Tbcodor. 1. Deutsch.

H Irump. 1. Engl.

IIrsten, George. 1. Deutsch. Geh. 20. Jail. 1613 7u Stettin, gest. 4. Mii 1660.

* Elttel. 2. Dentsel.

Iltenamm, Christ. Friedr. Carl. 1. Deutsch. Miniaturualer; geb. zu Altrlorf bci Nïruberge 1789.

Mlein, Jakob T'heodor. 1. Deutselı. Beribmter Nuturfoiseluer; geb. 7.u Kämigsberg 1685, gest. 27. Febr. 1760 z.) Ditmzig.

Mletisins, Jolı. Jak. 1. Deutscl.

Lleomdns. I. Deutsch.

4 Iopsele, J. E. 1. Deutscl. Klitgel.

Betrachtungen viber den Bau ver Bienen. zellen (l1anubov. Magaz. 1772. St. 23).

Klang, Fiedr. 19. Dettsil.

W Iniglnt, Th. Ant.

Oekonomle der Bienen (Phil. Trans. 1807, p. 234).

Mniphor, Joh. Hier. 1. Deutseh.

Inoch, August Wilh. 3. Deutsclu. Beitrige zur Incektenkunde. Leipz. 17811783. 1.-3. St. 8. M. K. $\rightarrow$ Neue Belträge. Leipz. 1911, 8. 1. Thl. Mit 9 illum. Kupferi.

Xnorr, Gcorg Woltging.

Dentseb. Graveur; greb. 1705 zu Nïnbero: grest. 1761.

Hnox, Robert, t. Engl. Reisonder des 17. Jalimh.

Lob, J. A. 1. Deutseli.

"Iucli, K. I. 2. Buyer.

* Hucle, Kail. 1. Deutsel.

Mishler, Joh. Gottir. 1. Deutsu.

* Itibelnin. 1. Elsass.

क IL ilbing.

Hibliclcer, Albert. t. Scliweizer. Id I renter. Jus. Gottl. 1. Deutsch. Geb. \%u Sul\% am Nrekar 1733; gest. 11. Nov. 1806 .

Itidnig: Jul. Gerluard. t. Liefl. Geb. in licflaud 1728 , gest. un $\mathrm{Ma}$. dras 1785.

Kdinlas. 1. Deutsch.

Kding; Samuel. 1. Franz.

Itanigy, Emamuel. 1. Drutsclt.

Hibte, P. F. 1. Preusse.

Lissmes, Abraham Gutthelf. 1. Deutsclı.

Holceil. Fuivdr. 1. Oestr.

I olk, Seluröter vun der. 1: Nied.
Mollar, Vincent. 9. Oestr.

Wuller, G. Gottl. 1. Deutseh.

Wornatzki. 1. Deutsch.

Hortain, C. A. 1. Deutsch.

Ioy, Tubias. 1. Deutnch.

* Irpehowitzer. 1. Opstr.

Mrliner, M. Jos. 1. Bayer.

Erntrt, Ahs. Fricdr. 1. Deutscl.

Aramer, Wilh. Heinr. 2. Deutsch.

Iranuer. A. de.

Irantx, Davill. 2. Däne.

* Irsaper. 1. Deutsch.

" 1 rasenv, C. R. A. Frhr. v. 1.

* Erause. Jul. Wilı.

Hriechbaumer. 1. Bayer.

* Krliger. M. \$. 1. Drutseh.

Krilintz. Joh. Georg. 1. Deutsil.

Doctor und Medieus und einer tler Heissigsten Sehriftsteller; geb, z, Ber.

li) 1729 : gest. 1796.

Apis. Eneyel. W, f. 166-68 (roh.).

"Ir ruzsel.

Mrynicky, F. 2. Russ.

Killu, Aug. Christ. 3. Deutach. Geb. zu Eiscuach 1743, gest. 24. Febr. 1827.

Iuthingr, Giaf v.

Milster, J C. 1. Bayer. Kugelanm, J. G. 1. Deutşeh.

Kuluma, Joh. Ad. 2. Deutsch. Medicus und Anutom; geb. zu Breslau 18. Mär. t689. gest. 29. Mai 1745.

Menduname, Johann Clırist. 1. Deutscin. Geh. zu Breslail 1684, gest. 11. Nui 1751.

Mnnze, Gust. 2. Deutsch.

Iuntzmanu. 2. Deutsch.

"Iyber, Joh. Fricár. 3. Deutsch.

Labllinrdidre, Jcan Julicn. 1. Fran\%. Botanist vou Alençon. Mitule Reisen in Englaud, Syrien. nut den Lihanou, die Inseln der Tiirkei, in Corsika und Sardiuien und zulezt nuch Neuluallaud.

Note sur les Moenrs des Bourdons (Mem. dı Nıs. T. 1, p. 55. Boubun sylvarunı).

* Intoram, David. 2. Scliveizer. Inacedes. 1. Franz.

- Irecleat. 1. Franz.

Haclnuman, F. H. A. 1. Dcutsel.

Tanclumand, Friedr. 2. Deutscl. Mcdicus; geb. zu Hildeshein, grst. (676 im 42. Jahr').

Iuncordalre, J. Theodore. 5. Fianz.

* Tuacoste. 1. Franz.

Lacmann, Ch. Aug. 1. Deutsch. 
Waet, Joh. van. 1. Flämniscli. Genzrapli; geb. zu Antwerpen gegen das Ende des 17. Jahirh., gest. 1690.

- Ua Cíncer, M. 1. Span.

* Lagerunan, Johian. i. Schwed.

Hafelinuting: 1 . Tyrol:

* Latsunile. 1. Franz.

Calaine. 1. Franz

Cisumack', Jean Baptiste Pierre Antoinelte Monette de. 5. Franz. Geb. 1. Aug. 1744 \%) Barenlon (Dept. Somme), rest. 29. Dex. 1829 .

1) Histoire naturblle dev Animax saus vertebres, presentaut les caractères yeuernux et parlieulier's de ews animanx, leur Deslribution, Icurs classes, lemis fimilles, leurs genres, et la cltarlon des prlneipalex espeees qui s'y rapportent; pricedee diune Introdncion ete.

Tome i. Paris, Verdiere, Libraire. quai den Allaustins, Nir. 27. Mars 1815. 462 s. 8. Tome 11. Parls 1816. 568 S 8. - Tome III. Waris 1816, 569 S. 8. - Tume IV. 1817603 S. 8. - Tome V. 18is. 61:2 S. - Tome VI, 181!. $2.32 \mathrm{~S}$.

2) Philosophie zonlogique. 2 Vol. Paris 1800. \$.

3) Kerfe (1s/s 18i:i, p. 20581 i

Irimartiniere. Französisiher Natuforscher Ind einer deo muglücklichen Berteiter de la Perunse's,

A nimanx parasites (Jouru. d: Pliys. 1787).

Lnenbert, Jolu, 1. Eni.l.

Land rinni, Marsilio. 2. Ital.

Eamdus, Obertinus. 1. Bekannt unter dem Namen: Atelino Leuteoliano. Lan's, Heiur. Gottlob. 1. Bayer.

hunnge, Johann. 1. Detitsch.

Lunglians. 1. Bayer.

Cungrias. Clırist. Joh. 2. Dentsch.

Langlois. 1: Pratu.

* Istinier. 1. Franz.

Laviorte, aîné, J. L. 1. Framz. t Laporte", M. F. Id. de, Graf von Castelipan.

"Lrisurice."

Laspeyres, Jakob Heimrill.

Deutsclı. Stadtritl.

1) Tinea sociklta und enlonella t.in., dle belden Greselilecliter eiller Art (Migh. 11. Ges nistf. Fr. In Berl. I1, p. $256-61 \%$

2) Vorselılas un cinter tenen In Mr Klass der Glossuf.n émsulithrenden Gattung (Nene Sclur d. Ges, natf. Fr. lı Berl: |V, 23-5!l).

3) Sesine europaenc leonibus et deseriptionibus illustratae. Brrolini 1801. Sumptihns nuetnris itypls Weunerianiss. (6 pp. Vurrede ele 3: pp. T'ext Tlielblat gi-stuchen. I Kupfortalef geuinit in Fullis mln. (Opus pulelicirlmum, clurta iconibusque splemilils).

Chasmiaric, 1. Franz.

Iatreille, Piewr Audri.

66. Franz. Geb 1701, gest: 1833. "Entomulugorium princę̧ฯ".

1) Mutllles deenivertes en Franee (Aet. de Ia puc. d'lost, uat. de Par. I, 1792. Poirt. I. (t)-12).
21 Descrlption de devix nnuvellex espices de Mutilles (Jnurn. d'hist. uat. II, 98-10I).

3) Description d'une nonvelle espeee de Tiphiн Magis. eney clop. I. !ํ.

A. Mamuite sur le Phalente eulleiforme te l'éclaire ( $G$. Aleyroiles) (Magas. eneyel. 1795, Nr. 8. IV. 304)

5) Memrine sur le fenre Dinpkis de Llıné (Journ. de lit voe. de Medie. et u'hist. "1, de Bnrlealix 1, 77. - Misas encyel, Vi, i79\%, 433).

6) Précls des earacteres féuériqucs des insectus disposes dons mo ordre naturel. Brives 1796. 4. Extrait: lullel. de la soe. phit: 1, 1797, 118. - Mig. ene. V1. 17!)7, 5.50)

7) Descriprion dil kelmes unile de lorme (Mig. encrel 11. 1796. 1415. lieimpiniec at In snite de l'liivlnire dex fontmis. 180?,326-332).

8) Deconperte de nids de Thermes (Mag. eltcycl. 1797, V1. 531\} bullet. de la soe. phil. I, 1798. 84.

9) Letle sur le genre Rhinomícer (Jonru. de la soe. de Mivdle, et d'lils uat. de Bordenix (1, 3.31).

10) Memnire sur une nouvelle expcee de P'svlle (Livia) (Buliet. d. sne. philou, I. no 6 (1798), 113: Rcimpr. it la sulte de l'luls. des foumls. 321 )

II) Observatimu sur la Raplidla nplinpsls (Bullet. Ane, philonll, 1, 1798. 133. Itv. tig)

1.2) thoervations allr l'hivulr: maturelle de In Puce (Rupport géneial de la soe. phil. 11. I'ar. 1798)

(3) Hasuls sur l'histoire dex fournic de la Prince. Brives all $6 \cdot(17158)$. I Pasc. 1'2mn. (Fxtruit Joura de sinuté et d'hiss. II. de Bordeillx (liapelle). 111, 130).

14) Observation sur une Teigne de la cire décrite par Réumun daus aes mémi)lres (Jouru. soe. Ile nicitice el d"lisis. IIat. Burd. III, I9).

15) Duservations sur la louruul finguense de Fabriclus. Av fig. (Bullet ane plit. 11, 17 J9, 1. Exir. Mag. ene. 1. 1799. 93.

ifi, Observations sur jibcille tapissicre le Rénulutr. Av, fig. (Bull, soe, phil. II, 1799, 3.3. Rrimpr, it in suite de l'hiss. des (nourulk. 297).

17. Bémulre sur un Insecle qui noturrt ses petits de l'ubeitle Anmestique (Plillanthms apivorns). Av. fig. Bull, soc. phil, I1, |79y), 4) Réinipr. ì in sul]e de ['lllst. J. Jourmls. 307 .

is) Sur nue nonvelle espree d'lelineusion (J. Pendulatur), Av. lig. (l3nll. $\times 01 \%$, plillom. 11, 179J. 134).

19) Dexcripilon d'un wouvelle genre it'll. sectes. Peleciuns) (Bulle]. soe. philom. II, $1799.15 .5)$.

211) Observatinus sur ics mneurs el d'indusirie d'une petite Abeille (Mag. cneycl. 17199 IV, 2:3(1).

21) Dexeripllon d'un nonvenu genre dilnsectes (Gen. Filmis). Av. fig. (Bull. snc. plail. II, I840, I5.5. Reïupr. ḋ ti sulte de I'hist. d: fourutis. 3y(i).

2.2) Memoiresur In V'rilette strice (Rapport des travallx de la soe. philoul, 1791)- i800, IV).

2.3) Ilintoire naturelle lles crustnces et lusectes falivant partle du Buffon de Somulni.

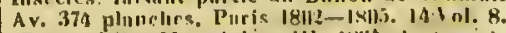
(Exir.: Illig. Mon. Il. Ins III, Isills. I el scq.).

21 Histoire inatirille des lourmls. Av. 12 pl. enl Pur. all 10 (180). 8. (Avee dlivers m(imoires).

25) Observatlous sur le geure Ihlein, et sur l'espece qui vil parasite sur le paou (Illst. de fourmis' 350 - !fi).

26) Ordie nnturel des lusecles déslgnts 
generalément wous le nom d'Abeilles (H|xl. d. fouruis, 401).

17) Description dinne nouvelle expece de fonrml, Bull. soe. philom. 111, 1802, 6.3).

28) Otsetvalinus sur quelques Guepes. Av. fiu Aun. du Mux. d'hist. mat. 1, 180?, 287. - Bull xoc. Hhil, 111, 147).

29) Deserlptiou d'une larve et d'une espee Inedite du genre des Cassidles. Av. fig. (Ann. dil Mus d'hlst. 11. 1, 1802. 295'.

30 ) Observitlon anr I'A heille pariétlue de Fabriclus. et Considérutions suy le genre all. quiel elle sc rapporte (Ann. d. Mus. d’hist. " 1804.11 . 251.1

3) Mémoire sur un gotenu de ruche d'une Abellle des (irindex-ludes, et atur les differences ules Aleilles proprement dites, viviant on eraule socintio de l'anciun contluent et du monvent. Av, fig. (ibitl. IV, 1804. 383).

32) Notice des espirees d'ibeilles vivant en grnucle soclété nu Aleilles proprement tltes et deseripllon d'uspèces nonvelles. Av. fig. (ibid. V, 1804, t61 .

33) Dlculonmalre d'histolre naturille de Délerville. I. rilit. I804 \& ,Plusienrs artieles

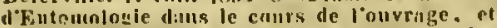
dans le 24. Volume un tableau méthodlque des Inscetes, et'aures auimaux'.

39) Generid ('rusticcortuil at Inseetnrum ceeundum ordineut malural'm in Famillas dls. posita, ivoulbus exempllsque pluriuis explienta. 4 Vol. Putrls!is el Argentornti (könig) 1806-7. 8. Av. XVI Tab. (Fol. I. XVIIt et 311? pp. II $280 \mathrm{pp}$. 111. 2.2S. 1Y. 399 pp.).

35. Notice hingraphlque sur J. C. Fabricius (Anis. du Mus. d'hist $n$. I809, 11, 393).

36) Memoire sur le genre Anthidlo de Enlivielus. Av. I pl. (ihid. 1809. XIIl. 24 et '207. Dentsch in (jermar's Mug. d. Ent. II, [81 5 ; $40-103$ )

37) Nouvellex observatlonsine In manlere dont plusicurs iusuetes de l'ordre des llymenopteres paurvoicul a la sulisiatance de lenr postritite (Anu. du Nus. 1809. XIV, 41\%. Extr. Siniry Buil, sne, pliti. :810. 11. 75.

38) Consldirutions generales sur l'ordre nuturel, des animtalix cumposiant la clnsve des Crubles, des Ariclinidcs el des liusecles. Paris 1810. \&.

39 (Ohisersatlons de \%oologle et d'Auntnmle par Mr. de llimmbolit (la Parlle dés lıx(cles). Av, pl. co!. Pat. Isil et sulv. 2 Vol. 4. (1)le enlounologisclie Partle iilierset at voi

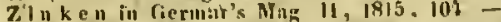
Irrén wir nichif. so fiulet sich ein Auszug davou' in Wiedemanis Atchiv ofler Magarin. das wir cluen nicht bel der lamul hnhrn.)

40) Enryelopédle nitilnodiyue Beaucoup diurlleles ñ daler de ISII, et les planiclocs' quil en depenstent

41 Menolire sur mu lusecle que les nneicns

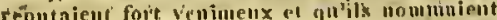

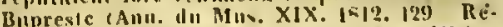
imprimé dans l'hist. nut. de Plins, editinn

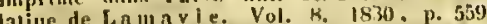
Rielupr. dans le mene onvragr, edlit. frauc.

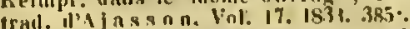

49) Nouveiu Dicllonnaire d'histoire mat"relle, 2. edition (Toul les Artieles d FutnmoIoglei. Parla 1816 et siliv, 8.

3) Rurle mulioul par (uvier. $(15 \mathrm{pl}) 1$. idulon Puris 1817. Vols $\$$. - Le 3, Vol. contennut I'Hucumologle. Av a pl Fxitr. contectit. Par. I829. 5 Val. 8! Av. 20 pl. l.ew Vol. 4 et 5. 1899 Av, 4 pl

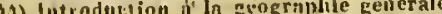
de's irnetinldes et des linscéter,' out des Climats

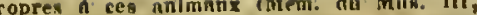
t817. 391. - Elnteltutuz'zu: elner altgtmelnet: Genuraplie der Araelı. 11. Ins, ouler Bestimmillik ler klimat. Grenzer. welche diesent Thiergatungen eigen sind. Isis 1818, p. 1015 $-104 !)$.

4.1 Considératlons nutuelles et eétlerales wur Ies inseetes vivant en socielé (Nein, du A1Is. 1II. $1817,391-410$ ).

46) Des lisectes peints onl-sculptes nur les monuments de l'Fispte. I pl. (lbld. V. 1819, 249-270. - Copris Nidax. - Unile eperoni d'(llivier. - Atenclınsacer. - Atcuelus punclicollis. - Celoine.

17) Nemoir "x sur divers sujeta de l'histnire natitrelle des Insectes et uuires. Parls 181.4. 8. (ce sont: une notice sur' les penples nncicus, nomes Seres, 113-118. - Des Insecter peiula on sinlpues s. I. Im. de l'buypte, InI roductiou i' la géographle des inscrtes etc.)

48) Pasqage dex aninhux lnvertétres' allx minanx verfébres. Parin 1820.8.

40) Des rappurfis generalix de l'organisintion extéricure lles mintactivertebres aricules, et comparaioun dos Anuelidos avec les Alyrin:podes Mrin. du Mus. VI, 18!0, 116-144).

5ii) De quelques eppenilices particuliers di) Thorax de divers Inseetco (Ihid. V11, I821. I-21. - A'un. gin. des sc. pliys. Brux: Vi; 3.32

51) De la formntlon des ailes des Insertes. Fase. 8.

5:-) Dewerlption des Insectes de Ia Nubir, reeurillis par M. ('allliand: I, P'. 4. Par. 8.

53) De lormane minsienl des Criguclx et Io Trutuales, et de sh comparaison avee Ini des monles' des' Cigales (Mlui. du Mtus, vilt, $(21-23:)$

5i) Belairecissements relatifs d l'opinion de $M$. IIuber fils, sur loorigine et" l'issne exté rieure des Aluellles (ibld. VIII. 133- 148).

5j) Oliselvation sur l'organisulion extrrlenre les aulminux artlcules ñ pirda urticulé. (ibid. 1111, 182\%, 169-202).

36) De l'origine et des progrès de l'Fnto. inolisgle (ibirl. 461-482. - Ursprung 11. Fort seliritte ler fontomolingle. IsIa IN23. p. 864):

:7) Iletioumine clissique d'histolre natu= relle. Divers artlcles generiux. P'ir, $1822 \mathrm{et}$ duiv 8 .

58) Ilisloire naturelle ef leonograilite de Coleupteres d'Europe (avec' Deje $\pi$ il).

59) Nolice sur III Insecle liyménoptere de Ia faullle des Diploleptires, connu daux yuclques parties lu Brésil. sous le unm de Guéplo

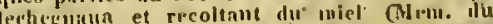
Mus. 11, 184. 313-318. Fig. sur une planclie lli T. 12. - Hetr. Aiti. d. sc nat. 11, 1825, 235).

(itl) Note sur ull Memoire de M. le Comle connce Nielzinsky, relatlve î In larve du Uri: IIIs' Ravesechs' (Ain. d. se. n. I, 1824. 78).

6) Fusuisve d'une distribution du rigñ atrinal. Fasc: 8 . 1 N24?

62) Ripport aur un ouvrige de Dalminn? inritule: Auntectn cntomolugiea (Amn. d. sc. 11. 111. $1.24,374)$

(ij) Fimillos naturelles du' regue aulual. Parls is?3. 8: - Deutsch vou therthold. Gött. 1815 .

6) l'ours d'Futomolngle.' 1. Année. Ar. 24 pl. l'aris 1831.8 ,

65) De l*ursanisation extritenre drs Thy sannures (Nouv. Allal. du Blus d'hist: nat? (, 161$)$.

(ii) Dlatrliurion inethotique dé In firitlle des verricorncs (ouvraet postlume). (A'mi'. suc. (iltoul, de Prance, 1!1!, 1834, 1.13.) 
67) Aufkläruıgen ither elulge Stelleıt der Alten über die Sieldenwürmer, oder diejenigen Kerfe, welche duselbst Bombyx oder Veimlx heisuen (Ann. d. se. nat. XXIII, 18.11. - Isis 1835. 26.3).

65) Myrmeclum rnfum (Ixin 1831, p. 7\$1).

69) Licurvect (Isis 1818, p, 1018).

"Iatrobe, Hemy. 1. Amer. Enerenzio.

De cichd, corumque ovis (Comment. lıst. boan. 1745,11 ).

- Inacret. 1. Eranz.

- Istencen, George Cumte de. 2.

Laxinaun, Eilc, 4.

Leach, William Elford, 15. Engl.

1) Eucyclopredia. Fdinb. 1810 et seq 4.

2) An arcollil of two species of Clytra (Meni. of llie ent. soc, Lond. 1812. 243).

3) An essuy on the brlitili species of the genus Meloe. with descriptlons of lliwo rxo. tlc peries. (Trulls. noc. limn. Lond. XI, P. I, 1813, 35. '2 T'alb. col.)

4) Descriptinns of some new genera and specien of auiulals. discovered in Afica. By T. C. Bodwlch. (Lin halluel Bogen in 4.)

5) On the genera and speciex of proboscldeous Inxects; and on the nrringemenl of oestrideous Insects. Elinb. 1817. 8. c. tig. (Mull. of Wernerian suciety, printed by Ncili et Comp. 3 Tab. col.)

6) On the atirpey and genern composing the famlly Paclaphidae, wlih descriptions of some new species (Zool. Joliri. II, Nr. 8. $1825-26,445)$.

7) Zonlogical Miscellany. 3 Vol. gr. 8. M. viel III. Kupf. Loudon 1817 .

8) History of Entomology. Edlnburgh 1819.

9) Malacovtraca podoplithalma Britanulae (C. pulch. tigur. ill.). 4. Loidinl. $181 j$ et 1816 .

10) A general arrangement of the claskes Cruatacen, Myriapoda aud Araclonida Llunean Transact. XI. Extr. Bullet. de Ia Soc. phll.).

ii) Appendlx 110, 10; a general notlec of the anlmals taken by M. Johin Crattch, during the expedition to explorc the nource of the river Zaire. 4.

12) Chnracters of a new genus of Coleopterous linects of the family Byrrhidae (Trins. of IIIII. Soc. XIII, P. I, p. 4I, N. V (Murmidius Icrrilglneus. Isis 1829, p. I073).

I.l) On the classificalion of the natural Trlbe of Insects Notonectidie. witl dencrlptions of the britislı specles (L/nin. Trins, XIt, p. $10-19, \mathrm{Nr}$. III. (IsIs 1829, p 787).

14) Further Obxervations on the Genus Melae, witl Veseriptions of sIx exotic ipc. cies (linneal Transact.292. XXIII. Tab. XVIII, 15 specles].

Yeblomd. 1. Franz.

Lebreix, F. I. 1. Franz.

Weche, Johann. 2. Sihwed. 1790.

1) Novae Inscetorinı species. Norloul.

2) Geschlehte des IIonIgthaucs iSchwed. Abh. 1762, p. 931 .

Heclere, 1. Franz.

- Heconte. John. 1, Amer.

Tedolius, Samuel. 1. Deutsch.
Iacteruutler, Martin Frobenits. 4. Destsch, Geb. zu Niittbery 22. Aug. 17 II: gest. das. 16. Mai 1769.

Hedonx. I. Franz.

Le*ma, Kund. 1. Norweg. Geb. in Norwegen 1647 ; gest. zit Drouthein 1774.

Lecuweminoeck, Antony van. 17. Holl, Physicus; geb, zu Delft 21. Oi. 1632, Gest. 28. Altg. 1723.

1) De ovls apum (Philos. Trans. Nr. 94. fil37. - Deutscli von t.eske I, I Thl. 98. M. Kin,f.).

2) De Pediculis (Philos. Trans. 97. 6116).

3) Af the uniunlcula in Ratin, Yca, nud Snowwater, ulso In Pepperwater Ilbid. Nr. 133,821, Badd. 11, 73. - Deutselı von I, es ke I. 2 ThI. 51).

4) The manuer of observing the antimal. cules in several soris of witer (Phil. Trims. Nr. 134. 8t4. Badd. II, 78. - Dellsch von Leske, 1. '2 This. 51).

5) Abstrncl of a Letter concerning generation by nu Insect (Ibitl. 1685. Nr. 174. 1120).

6) De Boubycum evocix, earunaue ovum (Verfolg der Brievell. Lcyden I689. 4.).

7) An cxtract of a Letter continiaing the listory of the neneration of an lnsect (Phil. Tин11. 1694. 199).

8. De ovirio et cornea ocull Llbellulae. (Vyfde Verfilg der Brieven. Delphis. 1696. 4.).

9) Circulionis generntio (Phllos. Trans. Nr. 2(3).

10) Dé oculis scarabnel Cervus volans dict! (Ibid. Nr. 280. 196).

11) Letter concerning some Insects olserved by hial on tirnil-trees (1bid, $1700, \mathrm{Nr}$. 266, 6i59).

12) Concerning green weed groulng In water, and some animalcula found about theern (tbid. Nr. 28.3. 1304. Badd. 4. 197).

I3) De Cochenllla (c. fig.) (Ibld, XIV, Nr. 292. Ifila-28. Badd. IV, 327).

14) OLservitio mleroscoplen de Proboscide Culicis (Phil. Trans Nr. 307. 230j).

Ij) Arcana Naturae detectn ope mIcros. coplorum. Delplis Butavorum 169j-96-97 et 1719, \& Vol. \$. - Deutsel. Delft 169ti. 4. - Ilolländisch: Delft, 5 Vol, Fol. 1, 1697; II. 1719; III. Amsterd. 1719, IV, 172.2; V; 1722. - Lateln.: Lilgd. Batav. 1696. 4. Finglisch: Loudan 1698 2 Vol. i. - Andere Allsgatie: Delplis 1722. 4 Vol. 4. - Andere: Leydell. Vol. 1722. 4.

16) Von den Bicuen (Arcana Naturne $c p$. 33) Uekounm. phys. Ablaudl. II. 648).

17) Vou den Sehlupfiwexpen der Blattalnse (A rean. Nit.) (Berliner Sammlungen IX, 341. M. Kp f.).

Lefelonre. 2. Franz.

"Inerabure, Alexandre. 14. Franz. Grb. 7.t Paris 1797.

Lethmann, J. G. C.

Insectorum speclea nonnullinc vel novae vel minus conultae, in agro Hamburkensl raptae, ex ordiue Dipleromu. Deser. et Iron. IIlustr. (Artu Acad. Natur. Curlos. Tom. XII P. I, B. "3s, Tab. XV. I) LeI a W int he 1 i i Lchn. p. 24I, I. I; 2) DoII c h opus n expo* II , Wiedin. p. 22k, f. 2; Dol. di s cipes, f. 3; 3) Dol. punctatus, Id.p. $24 . l, f .4 ; 4)$ bol. praceox, p $24 . J, f .5 ; 5)$ Phora pleta, Id. 
p. 244. f. 6; fi) Selara abdomlnalis: fehm. p 244, f. 7 ; 7) A edes.

Icelnanann. Mnt. Christ. Gottlub. 5. Dentsch. Mincralog; gest. \%u St. Petersburg 20. Febr. 1767.

Further Oloservations on the Curculio Trifolli, In a Lelfer to William Mark. wick (Llunean Traus. Vol, V1, p. 147, Nr. XII).

tuekenx. 1. Engl.

Heiner. 1. Badenscr.

Lenupriere, William, 1. Eugl. Lenz, Joh. Georg. I. Deutsels.

Lea, Jommes. 1. Spanier. Araliselies Geograph des 16. Jahrls., Kcmunt der Afrikaner; gcb. zu Granadi. War lange in iuneru Afika und clhiclt vou Leo X. den Namen als ev Christ geworden; ging noth Leo's Tud nich Afjiku zuriok und starb, unbekunut wo. Sein Werk: Descriptio Alricae euthïl 9 Bicher.

Lepechin, Iwau I wanowitsch. 3. Risse. Geb. 1739, gest. 1802.

tuepelletier de wint-barGean, Comte Amedée. 10. Franz.

1) Obscrvalions sur quelques espéces d'lıкеcten de la sectina des ll ymennpteres. appetes porte-tuax (Cleptes, Clirysls, Hedyclirum), av. I pl. col. (Anmal. dı Mlıs. d'hist. Ini. V(1, 1806, 115- $1 \cdot 29$ ).

2) Observations sur l'uecouplenent d'lnsectes despeces vilferentes (Analyse les travillx de iAcnd. roy. d. sc. pour 1827. Wliysique 56 ).

3) (avec Andinet-Serville) Paune tranculse. Einlomologie.

4) Monographia Tenthredinum șuonymia extricata. 1'aris 1823.8

5) Meimoire nu० le genre Gorvtes de Latrellle (Annil. snc. entom. de Fraice. I, 18.32, 52-79. 1 tabl).

6) Observations sur louvrage de M. Dahlbom sur les Bombus de la Suede, avec les caracteres lles genres Bomlus et Billyrus (AIII. sne. ellt. fr. 1, 1832, 366-38\%).

7) Description de trois nonvelles espéecs de cimbex (Aunul, soc. elt. 11, 454).

8) Remarques sur les caracleres donnes par il. Klug au genre syzigonia (Hymellop(iures) (Aul, sne, ent. Fr. 11, 1833, 436, c. fig.).

* Iuequien. 2. Frauz.

Hermine, Claude. 1. Franz.

* Iercinx. 1. Franz.

Leske, Nathaniel Gottfr.

5. Dentseh. Geb. 22. Okt. 1757 zit Muskau (Oberlausitz), gest. 25. Nuv. 1786 zu Marburg.

Lesser, Fricdr. Christ. 3. Detitsch. Grb. 29. Mai 1692 zu Nordlbausen, gest. 17. Sept. 1754.

Lessou, R. P. 2. Franz.

Tettwom, John Coakley. 1. Engl.

- Letaner. 1. Dentsch.

- Leunis, Johnmes. 2. Deutsch.
Wencknrt. F. S. 1. Dentsch.

Leviode. 1. Waudtl.

Leveille, 2. Franz.

wevett. 1. Engl.

* Leviun, Johı Willian (Cuvier schribt Lewius). 4. Engl. War auclu Ornitholug.

Natural History of Lepldopteraus Inect of New Sonth Walck. Lemd. 1805 With. Plat. col. 4. - Oder: Prodrouns Fintamology. NaInril Hixtory of Lepicinpterous lusecta oi New. South-Walex. Collucted, eligrareul und taith fully peinled ofter nature, liy J. W. Lewiu, A. L. s. of Paramalt:i, Londou, 180j, V'l und 19\%. M. $19 \mathrm{~K}$. in gr. 4. 135 Thlr.). (Thon Eitt. Arcli I, p. 5.)

wervis, Richard.H. 2. Engl.

* Lezale, L̇. I. Deutsch.

*'Dlerminier, Ferd.

"Llenilier: Simon. 1. Frunz.

Libanis, Audreus. 1. Drutsch. Geb. zu Halle (Saclss), gest. zu CobuI' 1616.

Hichtenberg. 1. Deutsch.

Hiclutemstein, Aut. Ang. Heinr. 4. Deutscl. War Professor der orieutalikcheu Sprachen zu Hamburg; feb. 1753. Sebricb iber Mantis etc.:

1) A Dissertarion on two natural genera hitherto coutounded unter the maue of Mantix. Trauslated from the Gomar by Tho. mas Young (limenu Trums. Vol. II, p. I, Nr. 1, Tab. I, If. Pliasma, 25 Speciem; Mantls, 43 Species. Neu davon slnd von Lichtenstein: 1) Phas ma hectic $\mathrm{cm}$, p. 14, Nr. 13. Tab. 1. f. 2; 2) Ph. O॥॥I แ1 и сi, p. 17, Nr. 23, Tab. 11, f. 1 ; i) al a $n t \mathrm{i}$ к $t$ itum, p. $19, \mathrm{Nr}$. 1, Tat. I1. f. 2 ).

2) Kissay on the Eyc-like spol in the Wings of the Lne $n x t$ of Falurichs. as indicaliug the Male sex (Linnean Tranmact. Vol, IV, p. 51. Nr. V, Trb. 5. Loell ta alvifolla $\delta$ et +.)

Iuex, Carl van. 1. Nicd.

huevig, Friederike, geb. v. Berg.

Hintieck. Eriv Gustav. 1. Suliwed.

Hizom, Richard. 1. Eugl.

Timok, Joh. Wilh. 1. Deutsch.

Hudem, P. L. van der. 7. Niederl.

1) Fswai sur les Insectes de Juva et den iles voisines. l'rén. Mén. Clcludel, (Mem. de Brixell. $V$. 1829.)

2) Observalions sur lex Hyménoptẻrea d'Europe ue la Famille des fonisveurx. Prem. Part. Scolietes, Siapygiles. Pompiliens et Sphogldes (Mén, de l'Äcal. de Brux, IV, 1827 , p. $27 I-368$. Denxivm. Part. V , 1829 , p. $1 \rightarrow$ 126. Beubecides, Larrates, Nyssunieax et Crabronites).

3) Nole sur denx insectex de I'ordre dex hyménopteres (Tentyres el Methoque) (Annal. d. *c. llat. XVI, 1829, 48. - Isis 1834. (041).

4) Notlce sur une coipreinte d'Inseete, renfermée danx un echantillon lle calcalre sehistellx de Sohlenlunfen, cn Baviére (Mcm. de l'Aead. de Bruxell. IV, 1827, P. 245-251, ar. fig. Libellula). 
5) Aeshnae bonouienses deacriptae. cum tabula ncuea, adjecta ejus auunfatione ad agrionew bononienses mb ipwo descriptice. Bononise $18: 0$.

6) Alonosiaphine bibcllulinirum enropaearmm specimen. Bruxell. 1828. 8 .

* Indmer, F. W. 1. Deutsih.

Hindenbera. 2. Detitsch.

Einke. 1. Dentsill.

Inumé, Cull v. 32. Siliwede.

1) Syntema Naturae. Leydae (Granoviln) 1735. Fol imper. $80 \mathrm{pp}$. In Tabelleutorm.

b) Fidit. lloliniac 17 in, $80 \mathrm{pag}$. 8. (anctum nomenclituria aulnalium, charaeterihus). c) Hulae (Langen) 1740. 4. obl. 70 pgg (lateinisch-dentsch).

d) Purisiis (per B. Jussleum) 1740. 8. 108 prg. Imit beigelugtell französisclien Nanen. Uebrigens wie Fditio b.)

e) Halic (Aguetliles) 1747. 8. 80 pgf. (Wic Filltio b. nur mit deutselıen Namen bereicliert.)

fi Ilolmine 1748. 8. 232 pgs. (von LInne selbal durchuesehen. Die essentiellen Pllanzencharactere, die Species, der Thiere sund Steine inflialtend).

g) Lipsiae 1748. 8, 232 pgg. (iibrigens wie Fiditio $\mathrm{t}$.

1) Holmia 1753, 8, $-136 \mathrm{pgg}$, (Dle Vegetabllicu durch.J. J. Hanrtmanu, das Steiureielı durelı II. Möller zı Skarı).

1) Leydae (dureh Gronotina) 1756. 8. 226 pog (wie Fitit. $)$.

k) Holmiae 1758, 8. (Vicl relcher). druck).

1) Lipsiae 1762. 8, (Betrigglicier Nach-

iu) Holulae 1766 (?) (von Llane selbut besorgte Auggalse; ganz relch gepeu die lirulieren. - Ein Nichdruck ersalien 11 Wien: 1767 -1770. - St. Miiller's Uebersetzung 177.s1776. 8).

II) Jipsine, eura Joh Fr. Gmeliu (editio decima tertín, ancta, reforminta), 1788 (Becr). 8. ill III Tomin nnd IX Pirtilus nelist Index.

2) S. M. Adolphi friderlei Reuls Museum. T. 1. Holm. 1754. Hol. Prodronums Toml 2. Ilolmlae 17bi 8.

3) ste. Lonisue Ulricue. Reginne Museum. Hola. 1764. 8. (Conchoiogikelı und entouographlarli).

a) C. G. Teasin Mnacun, 11 olm. 2i:3.3. Fol. (1'riorlitlges Werk iiber l'oncliylieu und Mineralien. War dus erkte Muster).

5) C. de Geer Mluxemu (reich an Lurchen,

Kerlen und nudern Selteulselten, wie G m e Il u slch musdriicki).

6) Upanliensis Muscum Aeademlac.

7) Stockholmiensls Musenu Academine Seienthrum. (Wegen dleseu. Mukealdencriptloneu wurdeu naelnateliende Reigen internomuen :)

8) leer lapponicum (1732) illedituun.

9) Iter Dalekarlieum (1734) inedlum.

Iiv) lier oelandlenu (1711). Ilolmine 1745. 8. - Ueläuska och Goillitiusa resa. Stock. holm 174;, 8. - Uebers. von Sclirelier. Ilalle 1763. 8.

11) leer Rothlandicun (1741). Holmine 374.8.

i2) iter westrogothlcum (1740). Holmlate 1747. 8. - Wḧstgota resa; afritisens ständers Defolalng förrittad. Stockh. 1747. 8. Schireber' Uebersetzung. Halle 1763.8.

13) Lter scanleun (1749). 'Holm, 1731, 8 Skänsha resa, Förrktted, 1749. Sloekh. 1751. ' 6 .
IF. wird nibht anparseud seyn, luler der

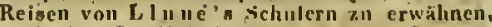
unch der Gmelin"schen Aufiollung (Systema Nafurae Tom. I, P I Praelint.):

a) (c. Terusetoonı if in Asiam (verun. glüekt in l'ouliciudor) 1745.

b) P. K a I m i in pellugyvanian et Canadam 1747. - ler in Americani septentrlo. nulem. Ilolu. 1753-17bit. 8. 3 Vol. 1749.

c) Mon1iuf ill lapponian Lulensem

d) F. Has selquIs I i In Eegyptum et Palaestinam. 1749.

- lter Palpislisun. Ilolm. 1757. 8.

e) 0. Torenil in Malabariain et Su. rate. 1750

i) P. Osberkii In Chinam et Javan. I751. - Iter Indive arimt. Holm. 1757. 8.

g1 l" Loellingi in llispanjam et Americam. 1751. - lier lispluienm. $110 \mathrm{~m}$. 175s. 8.

175:2.

I) J. Bergli in Gotlaudiam insulam.

i) M. Kaelueri in Italiam et Apu. 11am. 175.2

k) D. Sola udri in Lapponlam Pitensem et Tornenseia. 1753.

1) D Rulandri in Surinam et Eusta. tium. $1755^{\circ}$ 1758.

m) A K. Martini in Spitzbergam.

ii) C. Alstroeineri In Enropan austrnlen. 1760

o) J. Falki in Gotlatudiam. 1760 .

1) J. C. Nabrieli in Norwegiam 1779.8,

14) Fantin succira. Holmine 1746. 8. Editin 2(k, dullo alletior illa 1761. 8.

15) Amocnitute* Hendenicae. Holm 1749 -1763. 8. F Vol. (V'II, I769, Vill, IX cuir. Se.lı c ber 0. Erlangae 1785.)

16) Dissertaliu tie curionitate naturali (Amoen. acidl 1. 4?9. (Uebersetzt in L inues auserlesenen Aluandhuzen ans der Nalurgeachichte. Pliysik und Arzuelwissensehalt. l.eipzig 1776 , wo auch meinrere der folgenden stchene)

17), Diss. clli bono (Anoen. III. 231).

18) Diss. 1 sus historine naturalia (Amoen. acnd. V(I, p. 403).

19) Disa. sounus plantarum.

20) Instructio musel rerum naturnllum. (Amoen aciul. III, p. 4 36. Uebersetht In den Nr. 16 citirfen. Alshandlungen", desgleiehen anch in vou. Murr. A hiantil. vou Naturallenkablucten. Leipz. 1772 8. - Vel. aur J Sammlumen ans der Namrucuelichte, Oekonomle ete. Dreaden 1774. 8.)

21) Dlss. instructio, peregrimatoris (Amoen. nead. $v$, p. 298).

22) Diss yua peregrinationum intra pa. trlau aseritur necessitus (ibld. .11, 373).

23 Versuchr efiner Natur-, Kinst- und Oeknnomiehlsiorle von einigen scliwedischen Provluzen. leipzig 1756. 8.

24) Disñ.,Peloria (Ammen. acad. I)

25) Dlas. Generatio ambigenil (Ibid, VI, p. 1).

46) Diss, opennomia Naturae. Upsal. 1749. (lbid. 11, 1. - Select. ('x Amoru, dis. 1, 260), 27) Diss. Polilia uaturne. Upsal. 1760 (Am, acad I, i7).

28) Dias. mileratlones avlum (ii). IV, 505). 29 Diss. funclamenta ornlthologicn, Ups. 1765 (ibid. VII, 109).

480) Diks. surinnuneusia grilllann, (ibld, I, 31) Disn, .Auphibls Gylleaberglana (Jbld. 250). 
32) Fundamenta entomologlae. EUpsal 1767. 4. (ibid. VI1, 129).

Iisle, Guillaume de. 1. Franz.

Iister, Murtin. 6. Engl. Medicus; geb. zu Radeliffe gegeu 1638 , gest. zu London 2. Febr. 1711.

Tyjumglk. 3. Silluvede.

Tachuer, Michael Friedrich. 2. Deutsch. Medicus und Botaniker; geh. zu Fürtlı bei Näruberg 28. Febr. 1662 ; rest. 18. Okt. 1720.

Wudit, Ereole. 2. Ital.

Liber, Enanoel Christ. 1. Dentseh. Geh. 1696 zu Orlamande, gest. \%o Jena 1763.

Lonfling, Peter. 1. Schwede. Lieblingssehïler vou Limue; geb. 31. Ja11. 1729 zo Tollforsbruch bei Walbo, gest. 1754 in den spanischen Kolonien.

Tibv, H. 12. Deutseh.

* Libve, Christinnus Ludovicus. 1. Deutseh.

Tronitoral, C. F. 3. Franz. Geb. 1743, gest. z.u Palvis 1824.

* Tusigini. 1. Ital.

* Lomgranu, WVill.

Lopez, Don Salvador. I. Span.

* Torey, Felix.

* Iscinira. 1. Piemont.

Dasclage, Friedr. Heinrich. 3. Deutsch. Geb. za Ansbaeh 16. Febr. 1755. p. 3\%).

Cantlar. vesic. (Naturforsch. St. 23,

Iondon. 1. Engl.

Howtluep, John. 1. Engl.

Toyal, lidw. 1. Engl.

Tuncas, de Verdun. 1.

* Incas, H. 4. Franz.

Iunce. 1. Franz.

* Inciani, F. 1. Ital.

* Ianczot, F. M. Jal. 1. Frauz.

Tind o Ir, Job. 2. Deutsch. Orientalist: geb. zu Erfurt 1744, gest. zu Frankfint a. M. 1704.

Tundvis, $V$. 1. Deutsch.

Iundiving, Christ. Friedrich.

Deatselı, Geb. zu Leipzig 19. Mai 1751.

* Hilben, Reetor.

Iunual, Niels Tönder. 2. Sehwed.

Lyonnett, Pierre. 3. Franz. Advokat; grb. zu Maestrieht 21. Juli 1707 , gest. 1789.

\section{II., in Rouen.}

Betragen der Larven von Lampyris (Anu. d. sc. nat. VII, 1826. - 1sis 1834, 850).

Mac-IBrille, James. 1. Engl.

Gisfol's Lexikoll.
TInceney, Auge, 2. Franz.

Trac-Cnilocin, J. 1 .

"Irackenzie, G. S. 1. Engl.

Mac-Xinnew, Dauicl. 1. Sehottl. IInc-Ianurio, Colliu. 1. Sehottl.

Mathonations. 1698.

IIac-Leay, William Sharp. 10.

Engl.

II uc-Ieny, Alexander, Solm. 1. Engl.

Iraciuart, Jean. 6. Franz.

Nacumer, Pierre Jos. 1. Franz.

Chemicus : geh. z.u Paris 6. Okt. 1718, gest. 15 . Febr. 178\%",

Mrader, Joh. 1. Bayer.

* Nöirlkel, Friedr. 2. Deutseh.

M vari, Conte Carlo. 1. Ital.

Traile. 1. Fianz.

Mriret. Vrl. Merian (Syhille).

Minjor, Jolı. Daniel. 1. Deutseh.

Geb. zu Breslacu 16. Aug. 1634, gest. zu Stoeklıolm 3. Joli 1793.

Majoli. Entomolor (alter).

Mains, Joh. Ileim. 1. Deutseh. Gebor. zu Pforzhcim 1653, gestorb. 1719 .

Mralezicu, Nicolas de. 2. Franz. Geb. भı Paris 1650, gest. 1727.

1. Ialinowsky, de. 3. Deutseh.

* Mal co, Charles. 2. Franz.

Mnlpiglie, Maicello 2. Ital. Geb. zu Crepalenore (au den modenes. und bolognes. Grenzen) 10. Mirz 1620, gest. 29. Nov. 1694.

Inndeville, Bermard de. 1. Holl. Geb. zu Dort gegen 1670, gest. zu Loudon 1733.

* Masucerierim, C. G. de. 12.

Ma raldi, Jaeq. Philippe. 1. Franz. Astronom; geh. 1665 in ter Giafsehalt Nizza, gest. $17: 99$ 7. Paris.

Marclund, Nicolans. 1. Franz. Medicus; gewt. 1678.

Mnregral, Genry. 2. Deutseh. Geb. 20. Sipt, 1610 zu Liebstatt, gest. an der Küste vou Guinea. Seine Schriften grab La èt helaus.

* Inekvielk, Willinm. 1. Engl.

* Vramern, Cunte A. de.

Mincsclanil, A. F. Giaf voll. 1.

Oestr.

Mrarselnlius, Ulysses Salis v. 1. Seliweiz.

Nrarsha11, William. 1. Engl.

Marshner, 'Thomiss. 8. Engl.

1) Some Account of all Insect of the Geuits Buprestls, takell alive out of Wood composing a Desc. which liad been made above twenty Years. Lua letter fo Alexander Mar-Ienay, by Th. Marshain (Limean Transtet. Vol. X, Nr. 
XXI, p. 399, Tab. 32.) (B "prest I spJendenk. Paykull. B. splenditla. Wohm in Uppland.)

2) Observatinn on the cureulin Trifolii, or Clower Weevil, n small Inscet which infests the Jeats of lise cultivated Clower, and dextroys lie Seed. In a Ietter in Thon. Marshan, by Will. Marchwick, with additimal remarks by M. M a $r \times$ li $\mathrm{n}$ uा I.immean

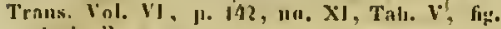
i. A. b. B. A.

3) Oh,ervitions on the Insects that infested the Curn in the Year 1795. By Thomas M ars h a ni (linn. Trans. Vol. III, p. 292, no. XXII!, Tab. '?! tig. 1-I2. Imingo samnt Metamorplone. (Tlirips.)

4) Deseription of Notoclea, a new Genus of Coleopterous Insects from New Hollaud 1Linı. Trals. IX, po: 283, no. XXI, Tnb. 24, 25. 20 Specics von dem Genus Notoclea, einer Käfergattuug nus der Fanilic der (lurysnum len).

5) Furfloce Observations on the Wheni Insect. in $n$ Lepler 10 lhe Snmucl Ginode" 0 ug l (Linn. Trans. IV, p. 224, no, XIX.

Marsigli, Luigi Fordinaudo, 2. JaJ. Geb. 10. Juli 1658 ztl Bologma, gest. 1. Nov. 1 iso.

Ouservazioni maturali intorno al Mare ed alln (iman dedle Kermes. Venez. 171 .

Martin, pire, 1. Franz.

IIntin, filk. I. Franz.

Virtinet, Jonun. Florcut. Nicilerl.

Martini, Fricdr. Heinr. Will. 1. Deutsclu. Medicus und Naturforsclocr; mob. 3 I, August । 729\% OJirdruf(Gotha), gest. \% Berlin 17. Inu 1778.

Martini, Clr. 1. Ital.

Martinifere, do la. 1. Franz. IIal.

Mnetiuo, Gio. Battista da Sau. 1.

II axtym, Thomas. 2. Engl.

Mnrtyn, Mattew. 1. Eigl.

* Mrertym. 1, Frauz.

II axtulier, Jos, 1. Destr.

Matlues, Jakob. 1. Russ.

Nattluioli, Petr. Audr. 1. Genannt Matthiole: geb. zu Roul 23. März 1501, grst. 1577.

*Intz, vou Magdeburg. 1.

* IIntzock, Dr. 2. Drutsch.

Inadult, Israel. 1. Engl. Geb. 1708 , gest. 1787.

Manduyt, P. J. E. Delavarenne.

1. Gest, zu Paris 1792.

Nhurer, Fel. 1. Deutsch.

IInyer, Jnh. 2. Bölım. Geb, zu Prag 1752. War Medicus.

IVInz, Domingo. 1. Span.

IVeckel, Joh. Friedr. 1. Deutsch. Der dritte dieses Namens aus ciner min dic Amatomic und Medizin horhverdienteu Familic: geb. 1781 ₹11 Halle.

Geschlechistleile der Käfer (dess, Beitr. aur vergl. Anat. II).
"Mediel. 1. Ital.

Micerburela, Nirlas. 1. Nicderl. "Megerle v. Millelfeld, Joh. Call. 2. Oestr.

Bemerkungen. BerJchtigungen und Zusätze zal Fabricit Systemn Fleuther. LInz I812. 8. 44 ล. I6 Gr.

Mendiuger. 1. Deutsel.

* Meiscen, Jolı. Will. 4. Drutsclı. Meincehe, Johanu Friedrich. 3. Denisch.

MeIly. 1. Engl. MeIsheinucer, $\vec{F}$. V.

I) Malachins, Junltion (Isis 1830, p. 608).

2) Calalngue of Inscrts of Penngyivania. Hannover 181\%. (Fin nuderer Autor?)

Mesunder, Carl Fricdrich. I. Schwede.

* Venetries, J. Francois. 6. Russ. Menouville, Nicolas Joscpli Thierry de. 1. Frall. Vgl. Thierry. Wentze], Jolı. Clirist. 9. Deutseh. Mrdirns: Eesl. 1718.

IVerbitins, J. Val, 1. DrutscJ.

Merian, Marin Silıylla. 4. Deutsel. Grafs Gattiu: Minialurmalerin: gelı. zu Fraukfut 1647. Zeiclute in Sulinam (10 Julie) Hud stah 1717. Hore. Toehter (Dorollic: Marie Menriette) gab ihr Opus postlumum lieraus.

Histoire des Insecls d'Enrope, trad. en france par Mniret. Ansifcrd. 1730. Fol.

Metaxn, Telemaro. 2. Ital.

"Wetzuer. 1. Dentsch.

Mey, Johann de. 1. Medicus; zel. zu Middelburg (Zèland), gest. 1678.

"Neyemilore, von. 1. Dentscl.

* IN e:ye: r, H.

M Myer, in Wien.

II eyer, Fricdr. Albr. Anton. 7. Dentsch.

Tentnmcu Ordinum Insectormu, Götting. 1792. 4.

Meyer, I, R. 1. Schwciz.

IIteyer, J. 1. Deutsch.

Mepyer. Nikolaus. 2. Deutsch.

Mies, J. J. 1. Seliweiz.

" MieIninsky, Iguace Conte te. 1. Der Geufer.

Niger, Filix. 1. Fran\%.

* Niknen, Joh. Christ. 2. Oestr.

Hillard, S. W. 1. Engl. Miller, Jolıi. 1. Engl.

Milliu, Aubiu Louis, 4. Frauz. Archïolog und Naturforsclier; geli. 1759 zu Paris; gest. das. 14. August 1818.

Mills, Jolı. 1. Engl. 
* Mil use, $\mathbf{G}$.

* Mitclioll, $S$. Inodecer, Adolph. 10. Sclivede. Geb. \%1l Stockholm 1738, gest. 1799. IIIodenen, Carlo. 1. Ital.

* Migling, 'Th.

N\&Her, Daniel Wilhelm. 1. Ungar. Geb. zu Pressbure 28. Mai 1642, gest. zu Altdorf 25. Febr. 1712.

MibIler, Georg Friedr. 1. Deutseh. Mitller, Ch. H. 1. Deutseh. II direm, Jos. Ch. 1. Deutsch. Moln $\mathbf{r}, \mathrm{D}$. M. II. 2. Dänc. Molima, J. Ignatius. 1. Eingl. Ilolimenx, Thomas. 2. Engl. Molinemx, William. Molitor, Urb 1. Deutsch. Moll, Carl Elirenbert voll. Deutscll.

Abl. über dle Schüdlichkelt der Insekten. Salzl. 1783 .

* Montregne.

Momtragin, Grorge. 1. Engl.

Celeripes Vespertilionis et Nyeter. 1 . (Linu. Trans. IX, p. $166, X \mid, I I, T .3$, f. $5 .-1 \times t$ N. biarticulntil).

Monti, Imnaz. 1. Deutsch.

VIontins, Cajetauns. 1. Ital.

Morama. Jean Francois Clement.

3. Frauz. Geb. zu Paris 28. April 1726, gest. 1784 .

Mineier, James. 1. Engl.

* Moritz, Id.

* Mormingr, Richard.

Murtimer, C. 1. Eugl.

* Misrrein. 1. Belgier.

IIosclicetti. 1. Ital.

* Notsclumizisy, Victor von.

Russ, Vel. Victor.

Monfet, Thomas. 1, Engl. Medicus des 17. Jahil.

Honx-Deloche, Fr. 1. Fran\%,

Milller, Otto Friedt. 9. Däne. Geb. «u Kopenhageu 11. Mürz 1730, gest. 26. Dez, 1784.

IViiller, Phil. Ludw. Statius. 1. Deutsch.

* Miiller, Johanues. 3. Deutsel.

"IIILer, Phil. Willbrand Jakob. 8. Deutsel. War Pfarrer in Odeubach.

Entomologische llefte, enthaltend Beiträge zur weitern Kenteniss und Aufklärumg der Inseklengeschichle. Fiuc lorniluelt zu chier künftigen Fruna des Depnrteucnts vom Donuersberge und den angreuzouden Gegenden, der Departemente von der Saar, von Rhein und Mtosel. Ansgearbeitet von ciaigen Fremnden der Naturgeschichte (J. J. lloffmanu, P. W. J. Mïller, 3. D. W. Koch, J. Nt. Lius). Erstes lleft mit I Kupfertaf. v. IIrn. Sturm. Frankf. n. M. bei Friedr. Fsslinger. 1803. 119 S. 8. Zweites Heft uit 2 Kupfert. v. Sturm. 1803. 130 S. 8. (Hister ete.)
Miller, M. Friedr. 5. Deutseh. Milller, C. L. 1. Deutsch.

* Tilller, D. E.

Milnter, Gust. W. 1. Deutscl.

*ilsant. 2. Frinz.

* Irnicik of Desenscholid, Eh. 1 .

Minralt, Joh. v. 33. Sehweiz. Medicus; geb. zu Zïrich gegeu 1645, gest - 1733 .

inurafielal. 1. Deutseh.

Mineray; Joh. Andr. 1. Deutsch. ' Miruray, W. C.

Mrascliel do Moschae, Joh. lgnatius. 1.

Mrnqueli, $S$. 1. Deutsch.

"Mirse, Jolı.

Milizcheralil, v. 1. Deutreh. IIIylius, Christlob. 2. Deutsch.

Nizen, Daniel Eric, 2. Selwed. * Narao, Giov. Dom. 2. Deutsel. * Natterer, Johann. I. Oestl.

Nierle, G. P. I. Eingl.

Necollanin, Jolı 'fobervilte. 3. Ener. Berïlunt durch seine mikroskopischen Untersuchungron als Physikus; greb. zu Loudou 1713 , gest. z.u Briissel 30. Dez. 1781.

* Negri. 1. Ital.

Neícleart, Jolı. Mich, 1. Deutseh. * Nees v. Hisenlueck, Clnist. Gottfried. 5. Deutscl,. Dr. der Heilkuude, Prof esisor der Botauik \%u Bonu; gel). 14. Fobr. 1776

Nomeniog, Kajet. 1. Badens.

NenTorie, Jos. Graf Stockar von. 1. Diviscli.

Nevilan, Bridelle de. 1. Franz. Nenfuille, de.

Une savante dissertalion filr la Coclienille. 1736 (bel Réanmur).

Neumene, Caspar. 1. Dentsch. Geb. 11. Juli 1683, grest. z\| Halle 1727.

Nenwied, ['rin\% Maxim. Alex. phil. Geb. 23. Sept. 1782, Brude:r ales jest regierenden Fiirsten Angust vou Neuvied.

* Nevrmume, Ldwald. 5. Engl.

wewport, G.

Nicolni, A. H. 1. Preuss.

Nicolni, E. A. 1. Dentsicl.

* Nickeri, Franz Anton. 2. Bölıme. Nieolas. 1. Franz.

"Nicolet, H. 1. Frauz.

Niebulir, Carsteu, 1. Dentsch.

Niesen, Christian. 1. Deutsch. 
Ninnrod. 1. Deutsch.

Miremhere, Johaun Euseb. 1. Span. Jesuit: gel). zu Nadrid 1590.

Missole, Guill. 1. Franz.

Dissert sur lorigine et la nature du Kermes (Jlist, de l'A(ind. rny. Par. 1717. 4.).

Nitzsclı, Ghrist. Ludw. 2. Deutseh.

Nodier.

Bibliogr. entom. Par. 1840.

Noess.

Nollet, Jean Alltoine. 2. Abbé, Physirus: geb. 1700 zu Pimpré (bei Noyomnais), gest. zu Paris.

* Norimanu. Alex. v 2. Deutscl. Sorfolls, In. 1. Engl.

Nozemanu, Cornelis, 1. Niederl.

* Ninsiramn. 1. Holländ.

Nysten, Pierre Ifubert. 1. Geb. 2.u Littich, gest. zu Paris 3. Mitr\% 1818.

Dehsenheiner, Ferdinand. 1. Deutscl, Gest. 2. Nov. 1822.

* Deliskay d'delisho, Franz Freiherr von. 2. Ungar.

Odhelins, Juhanı Lorenz. 1.

Schwede.

Dalier, August. 1. Franz.

Dedmanu, Samuel. 1. Scliwed.

- Okeu, Lorenz. 6. Badeuser.

DIafrean, Egrert. 1.

- DIrers, J. F. M. v.

Dlivier, Antoine Guillaume. 13. Reiscuder Naturfor'scher; geb. bei Toulon 19. Jan. 1756, gest. zu Paris 11. August 1814.

1) Folomalngie, wn lisinire uaturelle dess Insectes, avec lenrs enracteres géuériques it epceitifues, leur descripilon. Icur synonymie, el leur ligure enlumine. Coleopteres. Tome I. 1 Paris, de l'imprinerie de Bandnuin, inprimenr de l'Assemblée nutiouale rue du Foin.Snint-Jacques, Nr. 31. I7ng. XX, 191.

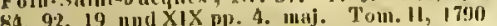
16. 8. 22. 10.10 .12 . 12. 10. 4. 8. 6. 12. 4. 4. 16. 8. 22. 10. $10.12 .4 .6 .54 .92 .3 \%$ 8. 1114 XIX pp. Planches 203. 4. - Im fimzen slnd es 5 Bde. Puris 1789-isos. In Ful. mit ill. Kupf. - Uebersetalug vonk. Illiger. Ba. 1-2. Braunscliw. 180\%. 4 III. Kupf.

2) Insertes qui inugent in farine (Jouru. ehemique de Fourerny. 1, 204-6).

3) Deseription d une nonvelle espece de Cetoine (Journ. d'hist tat. 1, 92-94).

4) Sur l'utilise de l'etude ilex lusectes relativencul a l'auriculure el anx arts (Ibid. $33-5 \mathrm{fi}$ el $241-53$ ).

5) Catalogue des lasectes envnyés de Cayentre í lat socléle d'hist. nat. de Paris par í. Leblond ret. de la snc. d'hist. nat. Paris. 1, $1,120-125)$.

6) Mémolre sur les parlies de la lmurhe des Insecles (Jnurn. de Phys. T. 32, 462474. - Opriseoli seelil 11, 422-29. - Extrnit d'nn mémolre gur lea párt. do J. b. d, las. Juin 178;3).
7) Sur quelques nourelles especes de Co. leopteres (Jnirn. d'hlst. nal. 1. 26?-268).

A) Sin mie nouvelle espece de Scarnbéo (ibid, 11, 292).

9) Ohservations genérules sur les Clienilles fileuses, et discriplion d'une nonvello espece de Bumbyr (ihid. I. 344-358).

10) Observatious sur le genre fulgore (Ib. $11,31-34)$.

II) Sirr me nouvenn genro de Diplères ctablis snus le nom de Nenestria par M. Latrcille (Nouv. Bull. des sc, pliil, n. 33. 1810.

3. nnu. T. 11,93$)$.

* Diftz, P. M. 1. Bölıme.

(D)perminani, A. F. 1. Deutsch. * Drbigny, Alcide Dessaline d'. 1. Franz.

Arreary, J. Earl of. 1. Engl.

Drtı, J. G. 1. Deutsch.

Getlob, Joaumes Christopli.

1.

Deutsith.

Dsbeck, Peter, 7. Seliwed. Reisender Naturforseher; geb. gegell 1720, gest. 1805.

\section{* Dinchakort}

Bverloeck, J. A. 2. Deutsel.

Gedanken von Acr Bugoula der Allen. (Ilaub, veru., Selurift. III, St. 3. Apis.)

bwenn, George. 1. Engl.

IPnley, William. 1. Engl, Theo. log: geb. zu Peterhorough 1743, grest. 7.u Suiderland 1805 .

Enlisot fle Esenuvais, Ambroise Maric Françuis Joseph, Baron (v. B.). 2. Botanist; geb. zu Arras 27. Juli 1755 , rest. zu Paris 21. Jar. 1820.

Insectes recucilles en Afrique et en Amérique. dans les royaumes d'Owire et de Benin, i) St. Dnminizue et dans les Etats-Unis, pendiut les aunces 1786-1797. 17 Livrnisons. Rivellyée par M. Audinet-Serville.

Fadias, Peter Simon. 7. Deutseh. Reisenuler Naturfor:sher; geb. zu Berliı 22. Sept. 1741, gest. 7. Sept. 1811.

* In Ilinadi, Anton. 1. Böhm.

Dalsucr, M. Dudley. 1. Engl.

Pafterun, Guillaume Louis Formanoir de. 1. Franz. Gob. in der Diöcese vou Sens 1712: rest. gegen Eude desselben Jabrliunderts.

"I ininar rol.

* IPuder, Gerard. 1. Deutsch. Pnizer, Georg Wolfgang Franz. 13. Drotseh. Geb. \% Etrelwang (Oberplalz) 1755, grest. geğen 1825.

1) Dentschlands Jnsektenfnnma oder culomologlsehes Taselienbuel fïr das Jahr 1795. Nïruberg in der Felsecker'sclieu Bughliandlung. lGmo. Aucl unter dens Tirel: Eutounulogia Germaulca exhibens lusecln per Germnnian ludigena secundum elasses, ordines, genera, species, adjeclis syuonymls, locis, obnervalo. nlbus. 1. Eleulbernla, cum Tabulis senels. 
Norlmbergne upud Felueckeri laercdes. 16. $370 \mathrm{pp}$. et 13 fig.

2) Beiträge zur Geschichte der insekten. Niirnb. t790. Edlt. alt. 1791. Edis. tert. Erlang. 1811. 4. Mlt 12 lllıs, Kupfert.

3) Index entomologicus, sistcus onnes Insectorum npecies. In G. W. Panze rI Fauna Inxectorum germanica descriptas atque deli. neatas; secundun methodum Fabriclanum : ndjectis encndationibus, observationibus. Pars 1. Ficutherata. Not imb. 1813. pp.216. 8. (Ger. mar's Mag. d. Ent. 1. 2, p. 179).

4) Ritische Revislon der lusektenfauma Deutkchlands nach deli Systen liearbeitet. 1-XCVI. Ileft, I. Băudchen. Niiruberg in der Frlaccker'schen Buchlaand. 1805. It4 bez. Seilcn In 8. 11. Bündcheı. 1-C ifeft, mil 2 IIIım. Kupfert. Niirnb. 1806.271 tocz. S. 8. (bieser 2. Bul luat auch deu Titel: Entomolo. plecher Versilch dic jurinischen Gattengen der Lime'schen Ilymenopleren nach dea Fabricius'schen System zu prïfen etc).

5) Abbllduing und Beschrolbung exotlscher Insektell. Nürnberg, 1791. 4. (Uebersetallng oder Allszig ans Drury.)

6) Novae inscctorna specles. Norintb. 1790. 4 .

7) Fannc insectorum Americae borenlis prodromus. Krlangac 1794. 4.

8) Symbolate entonologicae. Erlang. 1798. 12. (vide Infra).

9) Beitrüge zin ler Schrift von Kob: Vebes die Wurmutockniss. Err. 4.

iti) Denulschlauds lusekten. (Fortges, voul Dr G.A. W. ller rir.h-Se hilfer von liett ill nu ) Regensb I. -109 . Ileft kosten à I fl, $36 \mathrm{kr}$.. alas 110.3 tl. $9 \mathrm{kr}$. Sie erschiench zu Niirıberg 1793-1823. - luclus. dessen kritisclic Revision der lisektenfiuma. 2 Bde. Mil ? IIIum. Kupf. Ehend, 1805 und 6 ( $3 \mathrm{fl}, 36 \mathrm{kr}$.) und: Index eutounologicus erc. Pars I. Elelltheratia. Fisend. 1813,2 fl. $24 \mathrm{~km}$. werden alle 130 tlefic zul IG0 fl. I's lir. erlasken. Der Text dieser 110 llelte ist anch allein tiir $60 \mathrm{tl}, \mathrm{zu}$ erhaltcu. Das Duzeul chizelner Blätelicn Abbildungen tïir I f. 36 kr.

Parkinson, John. 1. Eugl. Botauist; greb. zn fondon 1567.

Parciletti. 1. Franz.

Parrenuin, Dominique. 1. Franz. Jesuit; grb. 1665, grest. ztl Pecking 1741.

Parrenuiu, Pèc. 1. Fr.

* Passerini, Carlo. 7. Ital.

* Pnul Veriluelau, Priuz in Wirttemberg. 1.

* Panlet. 1. Franz.

Parkinson, Jolin.

Description of the Pliasna dilatatum (LInn. Trans. V, p. 190, no. XV, lig. 2, 3, 4, 5).

* Iouli, J. 1. Dïne.

Paulini, Cluist. Fr. 5, Deutsch. Geb. zu Eisenach 25. Febr. 1643 ; trest. cbend. 10. Jnni 1712.

De pullcibus in ovo (Mllscell, Acad. Nat. Cnr, Dec. 3. A. 3. 1695 et 1696, p. 310$)$.

\section{Involl.}

Coclicuille (Annal, d. se. inat, Vill, 105).

Pey Itul1, Gustav v. 12. Seliwede.

1) Mlonographia llisteroidun. Upsallne 1811. 8. 13 Tab. $112 \mathrm{pp}$. (Germar Mlag. 11, p. 300$)$.
2) Beskrifning ofver et uytt suensk nattfly. Phalacna noctua tellfern (Veteisk. Ac. "1y a 11 andI. I756. 60-64. Dentsch: 1786. 58).

3) Monographia Curculionum Sueclae. Upsal. 179:?. 8 .

4) Anuârkuingar vid genus Cocclnella neh Beskrifning ofver de sueuska nrter al äro med fina här bestrodde. (Benerkungen iiber die Sippe Coccinella und Beschreibung der scliwedischen Artell. vou denen einige mlt llaten bedeckt sind.) (Vetensk. Ak. llandl. 1789. 14).

5) Fauma suecica (Coleoptera). I'psaliae, 1800. 3 Vol. 8. iDie 3 Bände, da die eraten 2 oline Datum sind, crschienen vou 17781800).

6) Beskrivelse over 5 Arter nye Nat.Sommerflıye: Bombyx et Noctua (Skrivt, af Nat. Selskabet. 11. 2. p. 92-10!1.

i) Beskrifalng ofver nya suenska lnsecter (Coleoptern) (Vetensk. Ac. nya llandI. XX. $1799,48-11 j 1$.

8) Monographla Caraborum Snecixe. Upsaline 1791. 8.

9) Monographla Stapliylinorum Sileclae. Uрка1. 179!. 8.

10 Beskrifning offver et nytt nattly: Plialacua Thea betulina (Vet. Ac. nya Hasdl. (78;), p. 57-60. - Delltsclt : 1765. 52).

i1) Beskritnisg ofver et nytl Nattfly: Phal. Tin. grandocvella (ib. 224-25. Dentsch: 178j. 219).

i2) Beskrifuing ofver forvandllngen, af Phalaena Noctua Partlicuias (ibid. 196-98. Deutsch: 178j. 193).

Payradeau. 1. Franz.

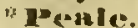

toersun, J. T. 1. Eugl.

* Peclnioli. 1. Florent.

*ecels, William Dandriderc. 3. Engl. Var l'pofessor auf der IIarvitids Uni. versitit in den Vereinigten Statent.

Ueber ciuen den Fichten schädlicheu Rũs. selk:iler (Jonru. d'agrieult. de Massaclinv. IV).

Pelletier. 1. Franz.

* Rerelieron, Achille Renuy. 7. Franz. Geb. z.t Paris 25. Jan. 1797.

I"ercival. Robert. 1. Engl.

Perlel, C.J. 1. Deutseli.

Herolli, Carlo. 1. Ital.

Ferrieres, Auguste. 1. Franz.

* enty, Maximilian. 5. Bayer.

Letanna, Inigi. 1. Ital.

Petagma, Viucent. 3. Ital.

Detazzi, Luigi. 1. Ital.

Petiver, James. 10. Engl. Gest. 20. April 1615.

Petterson, Carl Will. 1. Schwed. Pencer, Kaspitr. 1. Deutsel. Pezolat, Cl. Pli. 1. Deutscli. Pleisua. 1. Deutsel. * Plialipui, Rud. Aurand. 1. Dentsclı. E-Inipps, Contiue Jolın. 1. Eugl. Hignaner. 1. Deutseli.

* Fetet, Francois Jules. 5. Scluweiz. Pictorins, Georgius. 1. Deutsel. Geb. 1500 z Villingen im Scluvarzwald. 
Picus, Andr. 1. Deutsch.

* Ferret, A. 1. Franz. Piller. 1.

Prison, Wilh. 2. Holl. Medieus des 17. Jahrl. und Gefiihrte des Prinz Moriz vou Nassau in Brasilien.

* Pleninger. 1. Wïrtt.

Hinius, Cajus II, Major. 1. Geb. ums 23. Jahr unserer Aera, unter Kaiser Trajan, zu Verona oder Como, gest. gegen 79, als der Vesuv Hereulanum und Pompeji bcgrub.

Pluche. Noël Antoine. 1. Franz.

Geb. zu Rheims 1688, gest. zu Vareanes-

Saint-Mauren 1761.

p-lumier, Charles. 3. Franz.

Reisender Naturforscher; geb, zu Marseille 1646, gest. bei Cadix 1704. War Mönch in dem Orden Jer Minimen.

Pode, Nirolaus. 1. Steyermark.

* Poey, Ph. 3. Franz.

Poiret. 2. Franz.

Polluil, Nathaniel. 1. Engl.

Polisins, God. Lam, 1. Dentsti.

Pollicln, Joh. Ad. 2. Dentsch. Geb. «и Lautern in ler Pfalz 1. Jan. 1740, gest. 21. Fehr. 1780.

Pontedera, Giuglio. 1. Lombard. Geb. zn Vicenza 1688, gest. zu Pallua 3. Sept. 1557.

Pontoppidau, Eric. 2. Norweg. Bischof von Bergen in Norwegen; geb. 1698, gest. 1784.

Ponza, Latur. 1. Ital.

* Porro, Carlo.

Posselt, Carl Friedr. 2. Deutsch. Geb. zu Carlsrulie 1780, gest. 1804.

Poncle ct, F. A. 1. Franz.

Fonpirt, François. 4. Franz. Chirurg und Anatom; geb. zu Mans, gest. Okt. 1708.

" Ponret. 1. Franz.

Povelsens, Biarne. 1.

Pré, Joun. Friell. de. 2. Dentsel.

" Freiss, L.

"Présas, Jos. de.

* Pessi, J. S. W. 1. Bühne.

"Ereysuler, Joh. Dan. Eduard. 4. Bölın.

Pritclenrd, A. 1. Franz. Prochraslea, G.

Beobaelıtııgeı üb. Ins. iı d. Wasserblas.

Prumner. 1. Ital.

Pnget, Louis de. 2. Wranz. Physieus; geb. zu Lyon 1629, gest. 1709.

1*ullin, Sam. 1. Engl.

Furcleag, Sam. 1. Eingl. Geb. in der Grafsehaft Essex 1377, gest. 1628.

Putius, Joseph. 1.

Pntt. 2. Franz.

Puymanrin, de. 1. Franz.

Qunerin, Jos. 1. Oestr. Geb. zu Wien 19. Nov. 1733, gest. 19. März 1814.

Anensel, Conrad. 4. Schwed. Geb. zu Leyda in Skania 1768, rest. zu Stockliolm 1806.

Quinmones, Juan le. 1. Span. Geb. 1600 zu Tuldido, gext. 1650.

* Roy, Jean Rent Constant. 1. Franz.

Taben, Friedr. 1. Schwed.

Thack, Elıund. 1. Engl.

IEaf, G. Chr. 1. Deutsch.

* IEngazzuni, $R$.

* Raubur, J. P. 4. Franz.

"Tenudolar, Câl Augnst.

Dentvich.

IRanchin, 'François. 1. Frauz. Geh. zu Montpellier 1\$60, gest. 1641. 1 sango, Conradus 'Tiburtius. 1. Deutsch.

Rappoit, Carl Hejur. 1. Deutscl. Geb. z. Fisehlausen in Preussell 17. Juni 1702, gest. zu Königsberg 23. Okt. 1753.

Renpp. 1. Deutseh.

IEnthler, Ernst ludw. 1. Deutsch.

* Intinke, Heint.v. 1. Deutsch.

"IRtzelnirg, F. T. C. 12. Deutsch.

" Rave, B. 1. Dentsch.

Ray, John. 4. Engl. Auch Vray. Geb. 29. Nov. 1628; gest. 7. Jall. 1704.

Ray, Playcard Augustiu Fiudle. 2. Frauz.

Rayger, Cirolus. 3. Deutsch.

TRzonuowski, George de. 2.

Huzze. 1. Ital.

Résunnr, René Antoine Far. phinlt de. 8. Frunz. Geb. zu La Roehelle 1683, gest. zu Bremontiere (Maine) 17. Nov, 1757.

1) De la mécanique avee laquelle diversen espeecs de clenilles et d'antres insectes plicut el ronlent ales feuilles de plautes et diarbes. surtout celles du clicue (Min. de l'Ácad. des sc. de Paris 1730. Mém. 57. Ei. In 8 . A. 1730. Mèn. 79).

2) Observations rur les lnaectes quil se multiplient sans accouplencat par la neut fécondité de elangue individu (Món. de l'Ac. des sc. Paris 1741. Hist, 32. Ed, i] 8. Hist. 44).

3) Lllstolre du ver Lion (Mém. de l'Aend. 
des sc. Par. 1753. tllst. 58. Mém. 402-419. Hal. il 8. Hist. 89. Mein. 604. Deutsclı: Brem. Magaz. 1, 2 St. n. 14. Neu. eut. Mng. von Fuessly It, 1 St. p. I. - Holländlscli: Ultgezogte Verliandel. A Deel. p 177 et 226). (Aneli in seinen Memolien wiederliolı, )

4) Histritre des quepes talim. de l'Arad. des se. de Parls 1779. Hist. 13. Mén '230377. Edit. in 8. Hist. p. I6. Meul 302:

5) Memoires pour servir il l'histoire des tusectes. 6 Vol. nceompagnès d'ü grand nombre ale plauches; le 7. Vol. eat reste ell Manuscrit. Paris 1734-36-38-40. 4. Edit. contret.: Austerdin 1737. 12 Vol. grand in 8. avee beaucoup de planches).

6) Decouvertes d'une nouvelle teinfure de ponrpre ineu. de I'Acad. dess sc. de Paris. 1711, p. 169-199. - Mylius' Pliysik. Belust. 10 St. $73 i$ ).

7) Observations sur une petite expece de vers aqualıque assez singuliére (Mém. de l'Aend. Iles se. Par. A. 1714. - Atcim. p. 203. Fidit. iII 8. A. 1714; Mleit. p. 262).

8) Il istoire des Teigmess ou des lnsectes qui rongeut les laines et les pelletieres. part I-'2 (Aleti. de I'Aenul. roy. Par. 17'28, 20!439. Av. lig. (1II) 139, 158, et p. $311-337$. Deutsch: Launov. gelchrte Anzeigen 1754-58 $-59-68$ Sı̈ick. 1

Recli, Francesco. 2. Ital. Greb. zul Arexn 18. Febr. 1626, gest. 1. März 1697 .

1) Espericuze intorno alla geteruzinne degl lnsetti re. 26 tav.). Firenze 1650.4. Lateinisclı: Amsterd. 1671. 1?, (38 $10 \mathrm{~b}$.) Al1sterdu ui. 1686 12. Nipoli 1687. Fircure 1688. 4. (29 tab.) Hsirl. 168\%, 12 .

210 puseula rirenfe 1684-86-1724. 3 Vol. 4 Venez, 1712. 3 Vol. 8. Nispol. 1741. 6 Vol. 4. Austelud. 1686 . Pars i, 18. P. I1, 1b. 1687,18 .

* Redtembreluer, L. 1. Oestr.

Teew, Abraham. 1. Franz. Geb. geren 1743 im Norden des P'ay de Galles, gest. 9. Juni 1825.

Ieveve, Henry. 1. Engl.

IRertelius. 1. Seliwede.

Melu, San. Cour. 1. Deutseh.

* Reieln, Gotifr. Christ, 4. Deutseh. Reieli, Carl, 2. Devutseh.

* Ievelnembacle, Heinr. Gottlieb Lindwig. 1. Deutsch.

* IReinwardt, C. G. C. 1. Holl, Reiselius, Salomon. 1. Deutsdi. Tesumbil, .J. J. 1. Deutseh. Iteugger, .J. A. 1. Deutscli.

1) P'lysiolog. Untern. iiber A, thier. Haushalt iler Insekien (Oken dariiber: 1sls 1817. p. 1295).

2) Sandfloh (Dess. Reise 274).

"Rennie, James. 1. Engl.

* Ieenswluer, Jer. vall. 1. Niederl. in Amerika.

Etwas ïher dle Naturgeselifelite der Gegend Orange (Cienda seplendeelm. Acheta) islltion. Amer. Journ. Xill, p. 224. - Isia 1832, 1055).

Lenstribu, Carl Friedr. 1. Seliwed. Retzius, Andreas Joh. 2. Geb.
1742 zu Christiansstadt, geat. zu Lund $182 \mathrm{t}$.

Reviglins, Diego. 1.

Reynated. 1. Frauz.

Ribond. 1. Franz.

"reichardson, Willian. 1. Am. Riclue, Antoine, 1. Franz.

Eicluter, Ad. Dan. 1. Deutsch.

ricliter. 1. Deutscl.

Nicluter. 1. Deutsch. Arzmeimittellelire. $t i, 430$ (Lytta).

* Eicliter, Ludw. 2. Deutsch. Iicluter, Christopl Fricdrich. 1. Deutscl. Geb. zu Sorau (Oberlausitz) 1676, gest. 5. Oet. 1711.

Diss, de Cochlıilla. Lips. 1701. 4.

Ricord-Madiana, J. B. 1. Franz.

Eien, Joh. 3. Deutseh. Geb. 1730 zu Frankentlaal (Rheinbayeru), gest. zu Dresden 1807.

Tirand, Cyrille. 1. Franz.

Rimrod, C. G. 2. Deutscl.

Eisbers. 1. Schwed.

Teiser, Carolus. 1. Deutsch.

HEisso, A. 1.

Miville, Godeheu de. 2. Franz.

Rivinus. 1.

Robergitius, Laurentius. 2.

Scluwet.

Boberfot, Claude. 1. Geb. zil Macou 1753, crnordet aut 28. Aug. 1799 in der Nähe von Radstadt, wohin ihn der Congress 1797 rief mit dem Titel ",u des Plenipotentiaires framçais".

Robert, Nicolas. 1. Miniaturmaler: geb. zu Langres zu Ausgang des 17. Jalurh, gest. 1784.

* Ro beret, (h. 3. Von Lütich.

"Mobineru-Desvoidy, J. B. 5. Franz.

Trobimson, Tanered. 1. Engl.

Roces, Della. Wat Generalvikar vou Syra.

Traite sur les Alsellles. I, 11.

- Revelstroli, Heinr. 1. Dentsch. Rochefort, Charles de. 1.

Roener, Joh. Jak. 3. Sehweir. Botanist; geb. zu Lürich 1761, gest. 24. Jan. 1819.

Toesel von Rosenthor, August Johanu, 4. Dentsch. Geb. zu Augustenburg bei Armstadt 30. Mairz 1705; gest. z॥ Nïmberg 27. März 1759.

1) Der fliegende tlirsch oder Schröter, nebst seiuem Ursprunge aun dem prossen llolz. wilrne (Forst-Magazin III, 325).

2) Nachicht vom tholakifer (iblt. VI, 198). 
3) Vertllgung des achädllehen Speckknfers (Dermestes) (Frankische Sammlungen I, 2077).

4) Monalliche Insekten-Belustigungell. Bde. Nïrnb. 1746-6I und Ch. F. C. Kleemnnn, Bcilräge dnzu. I Bd. Nïrnb. 1761. 4. A. ill. Kupf (Es Ist noch ein 6. Bd., ller cclir selten ist, vorlianden.)

Ro Tredi, D. Mauriec. 1. Ital.

* Roger, Th. 2. Franz.

" reogernon. 1. Engl.

* Irolnands.

IRolnualer, Dan. 6. Scliwede.

* It lli. 1. Rümer.

* Romunud, de. 1. Franz.

- Momenumi.

Tomdelet, Guillaume. 1. Franz. Geb. zn Montpellier 27. Sept. 1507: gest. 30. Juli $1566 \mathrm{2u}$ Realmont hei Alby.

Rosa, Michcle. 1. Ital.

Hose. 1. Deutsch.

Roven, Nils, 1. Schwed. Geb. zu Gulhenburer 1. Febr. 1706, grest. 16. Juli 1773.

Rosemblat, Eberh. 1. Schwed. Von Luแul.

* Rnsenlinuer, WV. G. 10. Deutsıl].

* Cosentiral, Fr. Chr. 1. Deutseli.

Cieber dns Gernclisorgan der Stuben- und Schmeisflicge (Musca domesticn et camaria) (Reils und Anthenrietlss Archiv f. Pliysiol. 181 , X, S. 427. - Germar Mag. I, 1, p. 138).

* Rosensclidia. 1. Vgl, Mu nk.

IRoser, C, L. F. v. 2. Wüitl.

Roskesclunik, Joh. I. Uugar.

Rossi, Petrus. 5. Ital.

* Rossusuaissler, E. A. 1. Deutsch.

* Rotteraliucli. 1. Deutsch.

Rente-nburer, S. A. v. 1. Deutsch.

* Renullin.

* Rousseani. 1. Franz.

Ronuide, tle la. 1. Franz.

Hounx, Augustio. 1. Frauz. Geb. 7u Bordeaux im Jan. 1726, gest. 28. Juni 1776.

Rouelle. 1. Franz.

Roxhuxerl, Willian. 2. Engl. Medicus umil Naturforseler; grest. zu Edinburglı 1814.

1) Account of the Tusch nud arrindy silkworms of Bengal. (LAll. Trnus. VII, p. 33, Tnb. 2. 3 (Phalaenae pnphia et Cynthia).

2) Ueber Chermes Lacen (Phil. Triusnet. 1791, p. 229, T. 6).

- reoger.

Hozic: Jean. 3. Franz. Agronons; geb. zn Lyou 1734; stalb wälrend dem Siege von Lyou durelı einen Bombensehuss in seiucm Bette au 29. Sept. 1793.

Rucellal, Giovanni. 1. Ital. Geb. zu Florenz 1475, gest. 1528.
Burbeck, Olants, 2. Seliwed. Berilhmter Medieus: geb, zu Wesleras 1620 , gest. 1702 im Sept.

Tuni, G. T. 1. Eugl.

* Me ii puell, Eduaru. 1. Frankfurt. IRumpel, R. F. C. I. Deutsel.

Rumple, Georg Eberluad. 1. Deutsel. Botanisl : Keb. 1626 zu Solu, gr.st, auf Auboillic 1693.

IIunde, Wilh. Herm. 1. Dentsch. Ifusclueyt, Ant. I. Deutsch. Reusleu, Moses. 2. Engrl.

* Russegracer. Joseph. 1. Sillwciz. rensse: 1, Alexunder. 1. Schottl. Reisender Medicus; grest. 1770.

* Rusticus. 2. Jingl.

* Iinutice. 1. Dentsili.

IRnty, John. 1. IIİnd. Medicus; geb. 1698, gesl. 1775.

IRuasclien. MIteld, v. 1. Holl. Be. kaunt durely die Wette weren der Thierheit der Cochenille, die er gewant und 1729 die ganze Verhandlung druckeu liess.

Vgl. Réa u mur llist. d. Ins. IV, s9 und $\mathrm{Br}$ and $\mathrm{t}$ und Ratzeb. med. Zool. II, 220, not.

aussch, Heinr. 1. Holl. Gest. zu Amsleinam 1727.

IRaysclier oder Ruuseher, Micleh. (virl. obeu).

Rzackyusky, Gabr. 2. Pole.

\section{G., C. D. J.}

Inscktensamulung. Etllch und dreisslg Stïek. 1793.

* Nange (in Braunsberg). 1.

* Subiue, Edwat. 1. Engl.

Gaclis. C. W. I. Dentsch.

* Sinlderer, Carl Reginalı. 3.

Scliwid.

* Saluluerer, Fertinanu, Sohn. 1.

Mimisour. L. Paris.

Fniut-Milnire, Auguste de. 1. Fratil\%.

Sadis (verl. Marschlins).

Prngnente zur Entomalugie der Alpen (Salis Alplan 11, 58).

Galvi, Douniu. 1. Ital.

Aalzumana, lohann Rudolph. 1. Deutsch. Gest. 11. Nov. 1656 im 83. Jahr.

t Snumatie, George. 3. Engl. Saudiverá, Karl v. 1. Böhm. Ganders, Heine. 1. Deutseh. Sauders, Robert. 1. Engl. Sundwith, Thomas. 1. Engl. Sangalla, Pietro Paolo. 1. Ital. Eans, Mariano. 1. Span. 
- Saporta, de. 1. Franz.

- Sancerotte, C.

- Enumbers, W. IV. I. Engl.

sauri. 1.

t Sunter, Joh. Nep. 1. Silhwri\%.

Snuvages, Pierre Aupustin de la Croix de. 3. Geb. 1710 zu Alais, gest. 1795.

* Savi, Paolo. 1. Pisaner.

* Saviguy, Jules César. 2. Franz.

- Sracesen, W. 1. Deutsch.

Sny, Thomas. 15. Ames. Statb (in) 47. Jahr) zu New-Harmony (Indiana, Penusylvanien) 10. Okt. 1834.

1) Narrative of an expedition to the source de St. Peters-Rlver etc. Appendix. 1. Zoologia, Part. ent. p. 268-278.

2) Deseription of new species. Hister and 110 lnleptn in hitbits lle United stntes (Journ. of Acal. of Philad, 18:5. 32-47).

3) Note anr III memolre dn Capitaine Leconte, intitulee: Nollvelles expèces de Coleopteres de l'Amerique d"Nord (Contrlbutions of the Maclurian Lyceum 1, n. 2. JuI. 1827, 34).

4) Description of new spccies if llymenopteres inlabiting the United states fibid. 1, Ju1. (829, 67).

5) Descriptions of new spceies of Heteropterons llemipteres of Norih America. New Harnony lndinna. 1831 8. (Kin Fasciculum voll $39 \mathrm{Pag}$. Wiederahdruck nder Ausz118 aus seinen grössern (Verkell.)

6) Amerlen Entomology or descriplions of the lusects of Norll Auerica. Illustrated by coloured Figures from original trawings executed from nalurc. Philadelphia by Mitchell 1825. Vol. I, Vtll mul $112 \mathrm{~S}$. ar 8. Mit 18 illum. Kupfertaf. 5 Slıill. (voul $1817 \mathrm{Vol}$. I, Nr. 1?). III Vol. 1824-28. 8. (Thun. Archiv l, 2. 1. 40. - Jen. allgem Lit.-Zeit. 1826. Ang. Nr. 145, p. 195, mit Zusätzc॥ alıs Becks Repert. t827. Nr, 7.7

7) Descriptinns of new North Amerlcm Coleoplerous lnsects, and ubservations on some ulready described (Boston Journ. I, no. 11. 1834, p. $151 . X, 2041$.

81 Curculionites of $N$. Amer. with Observ. on some species alrendy known. N. Harmony 1831 .

i) Deseription of new species sulerlcan of the genera Buprestis, Tracliys et Elater (Annals of Lyeerm of nat. hist. of New-lork $1,2,249-269-1$ sis 1832 . 1077 ,

(iI) A Colossary, or Explanation of terins used in Eutomology. Philadelphla 1825. S.

II) A Monograph of Nortl American insects of the Genus Cicintela (Transact. of amer. phil. Soc. h, at Plitad, Vol. I. Ncw ner. Philad. 1818, Nr. XXXV, p. 401-426, PI. Xili).

12) Descriptlon of new Nortlı Amerlcan ln. sects, and olservations on some nlready deseribed (Trans. of Plitad. IV, 18.44, p. 409471).

13) Descrlptions of Insects of the families of Carabici and Hydrocautliarl of Tabieille, Inlabiting Nortl Anerica (Trans. of Philad. Vol. II, lew. scr. 1825, p. 1-110).

14) Descriptims d'insectes de I'Amèrlque du Nord (Journ. of Acrdeny Soc. Inat. of Plill. Vol. $1,19-23 ; 45-48 ; 63-64$. Vol. II, 1114 ; $t 02-114 ; 353-60$ ).

15) Description d'insectes de l'ordre des
Dipteres des Ftats-Unls (Journ. of Acad, Phll. Vol. III. IN23-24, 9-54; 75-101; 139-216; $238-282 ; 29 \mathrm{H}-33 \mathrm{I}$ ).

16) Describlions des inseetes Coléoptères récucilles dans la dernitre expedition anx Imonta:sues roclicuses (Journ. of Acud. Pliblacl. Vol. III, 3, 102-62; Vol. IV, I Part 83 et 1.39).

17) llemiptèes et orthopières recuelles dans la dernière expéditlon anx montignex roclicuses (iblil. IV, 2 l'ill. 3117 - 345).

15) Descriptinn of new species of Coleop. terous lnsects of Unitod stales (itid. V, $160-$ 211h; 23i-234; $29.3-304$ el VI, 149-t78; $183-$ $188 ; 225-244 ; 299-314)$

Scaliger, Julius Cïsar. 4. Itul. Geb. 1484 im Schloss Riva am Garda. see, gest. 1558.

Gcarfi, Joh. Clirys. 1. Venez.

\section{"Schinds.}

Echiiffer, Jakob Clurist. 18. Preliger; geb. zu Querfurt in Thïringen 31. Mai 1718, gest. z.n Regensbung 5. Jan. 1790. (Vigl. Fürurohr's naturh. Topogr.v. Regensb. I, 1838. 28 et seq.)

Abbildung und Beschreibung dea Malcu. wurmkiticrs. Regensb. 1778 . 4.

Sclndler, Johann Gottlicb. 3. Deutscli.

Scharfenbers, Georg Ludwig. 3. Deutsilt.

"Schaum, Herm. Rud. 2. Saelıse. schellhamuer, Günther Clurist. 1. Deutsds. Geb. zil Jena 19. Mïrz 1619, gest. zu Kiel 10. Jit1. 1710.

Schellenberg, Johann Rud. 3. Sehweiz.

Sclecenclezor, Johann Jaknb. 2. Silıwei\%. Geb. zu Zürich 4. August 1672 , gest. 25. Jan. 1733.

Scheven, Theou. Gotllieb. v. 6.

schirtermiiller. 1.

* Scliilliugr, Gustav. 4. Deutsch.

* Sclnilliugr. Sam.

"Schiotte, David. 1. Däne.

* Schibalte. J. C. 3. Däne.

Schirncli, Adam Guttlul. 12.

A pronum ; mest. 1773 \% Kl.inbutzen.

Schlacht 1. Dentsili.

Sclileclatlentuer, Math. 1.

Dintselı.

* Schlotthumher, $A$. $F$.

schliter, Fr.

Scleleger, Joh. Bapt. 1.

Schuersulit. 1. Diutscl.

Scleunicl, Carl August. 2. Von

Wernigerode.

Schunid, Joh. I. Deutsrh.

* Schunidberger, Joh. 2. Ocstr.

Chorherr zo St. Florian (Steyemark).

"Sclemide, $\Lambda$ it.

is Sclemidit, Rudulph. 
- Sclemint, H. R. 1. Deutsch.

* Schmidt, Robert. 1. Preusse. Sclnuidt, Gottfried Traugutt.

Deulsth.

"Schumidt. J. Preuss. Regierungsrath.

Schmidt. Dr. prakt. Arzt in Stellin, wriland Redacteur der entomologischen Zeitung.

Ueber die Töne, welche Paelohius Hermanni lǜren liösst (Ent. Zeit. 1, 10. (Mil A bdominalsegmenten).

2) Veber Campylus lineurls L.In., mesnnuelas Lill. Ind livens Fnbr, (Ent. Zeir. I, 35)

3) Apparal zum Fange von Waxserhälern (ilid. 63).

4) Revisiou der europäiselien Arten der Galtung Hoplia (ihill. Go et seq.).

5) Uebrr Trichiıs fascialus L., abdominaIIs Dej. und zonnlus Germ. (ihitl. Ilo).

6) Aphorismen aus den Tagebuch (ibid. 130. Kolenpterens.

7) Ueher Clythru quadripunctata L. und thre näehslen Verwundien ib II, 14(1).

81 Dir virnpinischen Arten der Gattung Aullicux rulı. iblil. III, 74 et seq.).

9) Recusinu der Fauna belvetica von 0. Heer (1lid. III. 57 ).

10) Die Verwnurllungsgeschichte des L/xus gemellalus Schiml. (ibid. 273. Jaf. Fig. 3-8,

II) Vertalirel bei der Untersuchung kleiner Kö̀pertheile der luschten (ilid. 2.37.

12) Lean Suffriani. Nene deutsche Art (ibid. 27).

i3) Brief über Gistel's Systema lusecto. rum in den A eis (Beilnge zum Faumus). Band 11 .

14) Kevisinn ler dentselıen Aphadicu-Arten. (Germars Zelisehr, f. Ent.-1i, I. 1840, p. 83176. - 76 Gattungen clarakterisirt nnd be. selirieben).

15) Verzeichulss europiinscher Krifer.

161 Reviston der deulsclicul Auisolamen (Germ. Zeltsch. f. d. Ent. III. 1.30. - 6 Sp. Leiades, 29 Anisntaua, 1 Agaricnphagus, 3 Hydnobius. I Triarthron (Märlsclii).

Gelnmidt, Joh. Andr, 1. Dentsch, * Selnuidt, IIermaun Maximiliau. 1. Böhme.

* Scluairt, Ferdiu. Jos. 4. Krainer.

Delnuidt-Gibl, H. M.

Sclnmiedlein, Gottir. Bened. 2. Deutsch.

* Gelnitt, ju Majuz.

Selneider, David Heinrich. 12.

"Sclidiulnerr, J. C. 8.

* Seliott. 1. Deutsch.

" Selionew, J. F.

Selirank. Frauz von Paula von. 20. Geb. zu Varmbach in Bayern 21. Aug. 1747.

1) Beitrhige zur Naturgeschichte. Angkb. 1776. 8

2) Ueber die Methode, die Nulurgeselichte zu studiren. Eine Vorlesnng. 1780 . Regensburg. 8.

3) Einlamologische Belträge (Sehriften der Grsellschalt naturf. Freunde zu Berlin. I. 1780. 4.)
4) Eitumerallo luseclarum Ansirlae indi. genorum. Ang. Viud. 8. \& Vol. c. lig. 1780. (Vergl. Zusilize liezu im Faluns I. Bd., neue Folge 1 1.37, llefi I). Vgl. Nr. 9).

5) Von den Minierriupchen in den Fliederhlätlerı (Neue phil. Ablandl. d. Münel. Akad. II. 1780 :

6) Nachrichl von einigen elatischen Thie. ren (ibid.)

7) Kleiuc entomalngische Anmerkungen (Sehrift. d. Ges. untf. Freunde zu Berliu. II, 1781).

8) Zoologische Bemerkungen (ibid. III. 1782).

9) Kritiselue Revision des ostreichisehen Insektellverzejchuisses (Fïsoly n. enlom, Mng. St. 2 I11 3. 178?).

10) Beltrag zur Naturgeselilehe der Motten. die sicl, lu Säcke voll Hole- oder Blätterspänen kleiden (ibid. II st. 1782 ,

II) Anleitung die Naturgesehlohte zu studiren. Mïnchen 1783. 8.

12) Entomologisehe Nachrichten, an J.C. Finssly (n. eut. Mag. II. 1784).

13) Namrhistarische Bricfe ïber Oestreich, Salaburg, Passau mul Bereliespaden cuit c. li. Fr. v. M oll). Snlzb. 2 Thle. 8.

i4) Vnn der Nulcharkeit der lissekten (Huibners Tagelluch. II lleft. 178.5).

I5) I crzelehnis, in Fïrstentlume Berehtesgacleu benbachteter lusckten (Fiissly uenes enl. Mag. 11. IBdí. lleft 4. 175ì).

16) Bnyerische Reise Minneh. 1786. 8.

17) Uviber die Küfergattung Meluloutlaa. (Borns Arbeiteu eiutrieht. I'reunde. 17\$7. 11. Bd.).

(8) Anmerknngeu zu den ersten zwanzig Stiichen des Naturlorsehers (Del Niturtarselier 23 St. 1788).

19) Euloumlogisehe Beobrelulungell (ibjd. S1. 24. 1789).

20) Abhandungen einer l'rlvalgesellselıaft von Naturforselierib und Gekononen II Oberdeutschland. I Bd. Mïnelı. 1i92. 8.

21) Akndentisclie Reise. Minneh 1793. 8.

2.) Beiratg zor Naturgeselielate der Stratiamys Chamaclean (Naturforseler 27 St. 17ग3i.

23) Mlkrosk opische Wahruehmungeu (ibld. 27 St.).

24) Naturlistorlsclıe u. ökonamisclıe Brlefe n̈ber das Damaumon. Manul,. 1795. 4.

2j) Sinnulung naturlistmrischer und yhy. sikalikclier Anfsiize. Niirnb. 1796.8.

26. Fauna baler. 1. Bu. Niiruli 1799. 8. 11 Bd. Ingalat. 180?. - III. Ingn/st 1803. (Zu. noibe liteal von des Verf. Feder eulhailt der bei Nr. 4 eltirte Jahrgang des Paunus.)

27) Grundiss der allgemeinen Naturgeselichte und Zonlogle. lírlangen 1801.8.

28. Laudshuilselie Nehellkiunden zur Farweitcrumy der Naturgesehichte angewnudt. 2 Stiieke Landsh. 1803, \&.

29) Ueber dle Raupen dar Santenle (k. bayer. Regierungublatt 18is, Desemb.).

3i) Uelier cille Sindlresseril (Malls 3. Bericht ilher dle Arheiten d, math. phys. Klasse der k. Alsad. zu Mïuehen. 1810).

31) Ueber die mit lem Erhsenkñfer behafteten Firbsen (Wochenblati den bayr. Landwhithach. Vereins II, 1811).

32) Ueber Seiclenzucht, hesonders iber de Frlaaltung der Seltemraupen Im Freíen (Ibld. II. Jahrg. 1812).

33. Uehrr deu liornwurm (ibisl. IV. I814).

34) Renatus Aulon v Reaumur (Eon, 7elf. selurlft aus Bnyern. 1818).

35) Ueber dis wattewebeurlen Elsenraupen 
izwel Abliandlungen: Denkschrlfien der k. b. Akadeule d. Wisoenselı. 1820 . Vl, warin vou des scl. Oberlieulenant v. lie heostreits (gest، 1443) Bemn̈hungen mit den Elsenraupen die merkwiirdige Rede ist).

3(i) Die Natur predigt Gott. Eine Rede. Minclien 1826. 4.

Selureber, Joh. Christ. Dan. v. 7. Geb. 17. Jan. 1739 zu Weissenseen in Thüringen.

Selireber, G, 2. Deutselı.

Selnreilier, Joh. Frichr, 2. Geb. zu Königsberg 1705 , gest. 1760 zu Petersburg.

* Sclireibers, Carl v. 1. TVien.

Selnemelnzer, Joh. Jak. 1. Sclureiz. Geb. \%u Zïrich 1672, gest. 1733.

Selnöek, Lueas. 1. Deutseli. Geb. 30. Sept. 1646 zul Augsburg, gest. das. 3. Jan. 1730.

Selordsder, Samuel. 1. Sehwed. Seliriter, Jol., Samuel, 20.

* Acloribter vain der Molk. 1. Niederl.

Selnubartles, Joh. Rud. 1. Deutsch.

- Selnildert, G. H. 1. Drutseli.

Selnaltz, Christ. Friedr. 3. Deutsel.

Geb. zu Wildenlaggen 1730, gest. zu

Dresden 1775.

Sclevitz, J. H.

Seluntze, Jobann Dominicus. 3.

Deutsib. Geb. zu Hambur 1752, gest. 22. Mai 1790. Medicus.

* Sclnultze, zı Greifivalde. 1.

Selnoltze, Joh, Heinr. 2. Geb. zu Colbitz 12. Mai 1687, frest. zu Halle 10. Oet. 1744 .

Gclennacher, Clurist. Friedr. 1. Soliwede.

* Selnumane 1, T. E. 3. Sellesier.

Gelnuster, Gotfwild. Geb. zu Jena 28. Dec. 1701, gest. zu Cliemnitz 25. Dez. 1785.

"Selewnib, C. Ludw. 1. Bayer.

* Selovil grichen, Fr. 1. Saclise, Acln ovan, Christ. Friedr. 4.

Selivinetz 3. Deutsch.

De hydroplobis ejusque specitico Meloe uajali et Proserrabneo. Halle, 1783.

* Acliverar.

" Selnivaigerer, A. F. 2. Deutselı. Selnvemelkeld, Caspar vou. 1. Geb. zu Greilenbrro in Sclulesien, gest. zu Görlitz iu Jııi 1609.

Scapoli, Jobann Auton. 5. Tyrol. Geb, z.и Cavalles in Tyıol, gest. z.ı Pavia 8. Mai 1788.

Seniba, Ludw. Gottl. 3. Deutsch.

beafomi, Francesco. 1.

Seba, Albert. 1. Pries. Plarma zeut; geb, 2. Mai 1605 zu Feltzelu (in Ostfriesland); gest, zu Amsterdau 3. Mai 1736.

Gebrstiam 1, Georg Cluistlan, 1: Drutscli.

* Aiecisig, A.

Sefstrom, Eric. 1. Scliwed.

Sezer, Georgius. 1. Geb. zu Nïrnb. 1629, gest. zu Danzig 19. Dec. 1678.

Segerins, Joh. Gottl. 1.

heguy. I. Fran\%.

seidel, Wenzel Benno, 2. Böhm.

Seligran ann. I. Mich. 1. Deutsels.

"Aelys de Lengchnans, Edur. de. 2. Beloier.

Gelle: Clirist. Grottlob. 2. Geb. zu Stettin 1748, gest. zu Berlin 9. Nov. 1800.

Serm lex, Joh. Sialumo. 3.

semalel, Nathaniel. 1. Dentsch.

Aeple, Cluristian ef Jan Cluristian. 1. Nicelcul.

* Seringat, J. C. 1 .

serres, Oliviar di॰, 2. Franz.

* Gerres, Mareol de. 6. Finuz. Sertini, A. D.

Agricoltura, pendotti ef commercio delli Sicilia. Firenz. 1777. S. 1.

* Cervigle, Audiuft. 12. Frauz.

* Aennzir. 1. Fran\%.

Slater, Thunas. I. Geb. creren 1692 iu Westumeland, gest. 15. All: 1751 .

Travels, or observatimus relating to severil parts of Barbary aud the Levant inith plat.) Oxforil 1735. Foi. Suppl. 1746. Ionill.

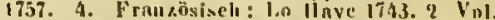
4. Av. cll. et tig. Dentselt: l,pipaig 1765. 4. Hollî̉ndisch : Utrecht 1773. "2 Vol. \&.

Alnav, (jeorge. 7. Lingl. Geb. 1751 z.t1 Bicrton (Burkinghamshire); gest. 7.11 London 1613 .

1) Vlvariun Niturae, or (lie unturalist Miseellony (lateln. Imd engl) will fig. liy P. Notter. I ande 1790-1813. 21 Vol. 8.

2) Speculua linneanum sru \%nologlae liunenua illustratio. Lonal. 1791. 4.

3) Musel Leveriaul explientio (lotine pit augllec) (e. fig. col. Iond. 179\%. 4.

4) Zoology of New-llollaul (iil $\mathrm{Sm}$ it lis Natural llistury of New- II. Linndou. 1794 el sey. 4.

* Glepjara. 1. Engl.

Silnerean. 1. Deutsely.

thlnekard, W. E. 3. Engl.

vieloea. F W. 1. Oestr.

Sienola, Robert, 1. Engl.

* Sielonal, C. 'Heodor vou. 10. Deutsel.

Siegr-1, Chuist. Friedr. I. Deutsch. Sivertorli, C. H. vou. 1. Siegwert, George Friedrielı. 3. 
Geh. zu Grossbettlingeu (Wïrttemberg) 3. Apr. 1711, gest.9. Mlïz 1795 in Tübingen.

De insectia eoleopteris nec non de plantis quibusam rarioribum. Tubingac 1755. 4.

* Gillocenamu, Gustave. 17. Els.

* Sillinum. 1. Anrevils.

Silvestre. 1. Franz.

Sianamons. 1. Engl.

Aiansou, Archibald. I. Engl.

Sitomus. 1.

Skelton, Philipp. 1. Engl.

Skene, J. 1. Engl.

* Skriuslire, Thomas. 2. Engl. Slabber, Martinus. 3. Holl.

Slomanc, Mans. 1. Ital. Geb. zu Killilenlı in Irland 16. Apr. 1660, gest, zu Cholsea gegen 1750 .

Slovitz, J. I. Piuss.

Aneatluman, Henrv, 1. Natulforsclier; lebte geogen dic Mitte des 18. Juhrh., starb 1787.

llistory of termites ,Phil. Trans. 71 Vol. - Franzbs. in Sparrinauns Reise. Die Ueliersetzung ist von Dr. Rigaud zu Montpellier).

Guellie, William 1. Starb zu Edinburgh 1725.

Sunitlo, J. Eduaid. 2. Engl.

* Aunitt.

soave, Félix. i. Ifal.

Socolori, N. 2. Russ.

* Godudisky, J. H. G. 3. Russ.

* Solier. 7. Franz.

Somacrat, Pierre. 1. Reisculer; ceb. in Lyou gegen 1745, gest. zu Paris 1814.

tioniuére. 1. Holland.

Somuind ue Manoncour (Charles Nicolas Sigisbert. 1. Naturforsclier; geb. 7u Luneville 1751, gest. 1812.

"Norg. F. L. A. W. I. Deutsch.

Gontluall. J. 1. Engl.

Goutluerue. 1. Engl.

* Gomerloy, G. B. 3. Ener.

* Nicurerloy, J. Earle. 2. Engl.

Aun rruasuu, Auders. 4. Schwed. Natnforseher nud Reisender; geb. gegen 1747 in Upland, gest. zu Stockholm 20. Juli 1820 .

Apeceloly, Willian. 1. Engl.

* Aences, William, 6. Engl.

* Auence, Rulicrt H. 1. Eugl.

* Spence, W. Blondel. 1. Engl. Sperllng;, Paul Gottfr. 1. Gest. 1709.

Aperling, 0. Fr. 1.

* Speyer, Ad. 1. Deutsch.

- Speyer, Otto. 1. Deutecl.
Qrielenberser, Dav. 2.

sipineto.

"Avimola, MIaximilian. 4.

Sprat, Thomas. 1. Engl. Gcb.

1656 zil 'Tallaton, gest. 1713.

spremgel, Curt. 1. Geb. zu Boldekow in 1'ommern 3. Ang. 1766.

Gprentrerms, Balthasar. 2. Dentscli. "Sprewitz, A. v.

Ajoribate. 1. Sehlesier. In Gohlis. Stuclkhorse, Hugh. 1. Engl.

* Striger. 4. Dän.

Sitampe. I. Däı.

Staucario, Victor François. Geb. zu Bologna 1978, gest. 1709. Ital. Zoulog, vorzugsweise Entomolog und Allatom. Selirieb iher:

Die zusanmengesezten Augen der Kerfe, ïber Cicadn (Com. Lust. Bonon. 1745, 11, c. 1748, V. 79).

stmudigel, Udalricus. 1. Dentsch. Stamluafr, Mich. 1. Dcuisch.

* Staunias, Fiedr. Hermanm. 2.

Stardigclins. 1. Deutsel.

* stario, J. 1. Engl.

Stcoluinum.

Stecels, Ioh. Heinr. 1. Deutsch.

" tevustrujp: Jap.

* Sterialnuy, Gustav. 1. Dentsch.

* stefrens, Irinr, 1. Dentseh.

"stein, Ficdr.

" Sitein. Fl., von Nicmegk. Diciur, J. F. R. 1. Deutsch. Steinlunet, J. C. 1. Dentsch. Nieinuetz, Joh. Friedr. 5. Steller. Georg Wilh. 1. Deutsels. Atentzel, Christ. Gottfried. 4. Deutsch.

De cantluridibus prosperae adversaeque nuctoribns Valetudinis. Vlleberg 1740.

* Stepluciss, Jaucs Francis. 0.

\section{Stemilderar}

Ueber den Borkenkäfer (lsis 1830, p. 313).

Stenchins, Joh. 1. Sehwed.

* Stevens, Joln C. 1.

"steveu, Christ. de. 5. Russ. stew. 1. Engl.

stieglitz. 2. Deutsch.

"sitiller, Erdmanu. 1. Deutsch, Qtilkany, William. 1. Encrl. Nito I1, Casprar. 4. Holländ.

stollers, F. C. 1. Deutscls.

"strack. 1. Deutsel.

* strums-D ifrkbeinn, Herculc. 2.

"Atreuluel, Alg. Vollr. 1. Deutsch. Sitrolvel loerger, Joh. Siephan. 1. Geb. zu Gratz in Steycrmark.

Strodaum, Joanues. 1. Franz. Stroein, Hans. 3. Däne. 
Stroem, Marten. 1. Schwede. ? alt, im dritten Jahre der 123. Olym. Astronom; geb. zu Upsala 1707; gest. piade

1770.

* Struve, H. v. 1. Deutsch.

Stunnpf, Georg. 1. Deutsch.

- Sturm, Jakob. 9. Deutsch.

Styles, Francis Eylcs. 1. Engl.

* Suckow, F. W. L. 7. Von Mannhein.

Gesehlcchistheile der lnsekten (Hensingers Zeitselir. f. Pliys. 1828, II, III).

- Suckov, G. 1. Deutsch.

Sufrolk. 1. Engl.

* Sufrrian. 8. Westphalc.

suizer, Joh. Heinr. 2. Schweiz.

* Sumdervall, J. C. 1. Schwede.

Sundicus, Petios. 1. Sebwede.

* Suraimson, Willian. 6.

Sivainmerdara, Jolı. 3. Holländ. Aluton1; geb. zu Amsterdau 1737; gest. daselbst 1630 .

Biblia Nalurae seu historln insectormm il certas classes reilacta post mortem edicllt Boerlanave; (53 Tat.). Lngul. Balav. I, 1737, 11, 1739, Fol. - Denlseh : Leipzig. 1752. Fol, 57 Taf - Englisch. mit Noten vom llell. Cond. t7j8. - Francösisch: Obscrvations de Swan. inerdam sur les iusecles Paris. 4., av. plauel. (Bildet len 5. Bd, der Mear. des sav, eitrang. (le Paris.)

Swartz, 01. 3. Seliwede.

Swayerummu, F. P. 1. Niederl. sivederas, Nils Samuel. 3. Sivintou, John. 1. Engl.

Gyliel. I. Deutscl.

- Sylres, W. H.

Targioui-Tozzett, Gioviami. 1. Ital. Geb. zu Florenz 1712, gest. 1783.

Tanseler, A. M. 4. Russ.

Ennmeratio alque descripto lnsectormm ex faullla Caullaridiarum quac in Kossia observavit, Mnsquac 1811, 4. Cum 2 tab. III.

weichumen, $F$.

Teil. 1. Franz.

* Telecki. I. Ungar.

Tellier, J. B. dc. 2. Franz.

Temipler, John, 1. Engl.

* Temuleton.

Podurina (Truisact. of tlic entoin. Soc. 1. Pars 11. t835', (Wurde im Lex, nusgelassen.)

Tenzel. 1. Deutsch.

Tertre, Jean Baptiste du. 1. Franz.

Thendosius, Jenn Baptiste. 2. Medicus; gest. im Sept. 1538.

Tluevplurastes. Griechisch. Arzt; geb. zu Eresos auf der Iuscl Lesbos, im zweiten Jahre det 16\%. Olympiadc (321 vor Christus); starb, 85 Juhre
Tliemennan, L.

Thierry de Menonville, Nicolas Joscpli. 1. Botanist, geb. 1739 zII Saint Nihicl in Lorrain, gest. 1780.

"Thierscln, Ernst.

* Thiélonut de IBernenud, Ars. 1. Franz.

Thomä, C. 1. Deutsch.

* Thomas, David.

Thomé. 2. Frauz.

* Thon, Th, 3, Deutscli. Thorley, Johm. 3. Engl.

Thosse, de. 1. Franz.

Thumberg, Carl Pet. 46. Scliwed. Geb. 11. Nov. 1743 zu Jönköping, rest. 8. Aug. 1828 zu Thumebcrg bei Upsala im 85. Jahre.

1) Novae insectorum specles descriptae (Nov. Act. Upxal. IV, 1784, 1-28. - 45 Gittiıghen aus alle॥ Urdnungea).

2) Curculin Samise, nova et singularle insecll specics e capitc bonre spel fibid. IJ, 29-3(1).

3) Generis Philanulal monographla (ibid. VII, 126$)$.

4) Allititamenta ad monographlam Philanth (llid. IX).

5) Coleoptera capensia antennls filiformubus tibicl. IX.

6) Tabaui septemdecim novae species deseriptae (ibid, IV.53!.

7) Truxalis insecli genus illustratum (ib. $1 \mathrm{x}, \mathrm{t8:27}, 76$ ).

8) Descriplion itun nnuvel lusecte. le Pantophululuus tubanicus (Nonv. Recueil de Méu. de In soc. roy. des se. et bell. lett. de Gollienbure.

y) Grylli monographia illusirata. C. fig. (Men. de l'Acad. de st. Pelersbourg. IX, 18:1. 3900 .

io. l.⿲丿丨anl Monograplia, C. tab. (Mẻm. le sne, lles Natur. de Moseon. I, $150-1531$.

ti) Dissertalio, Hovas Insectorutu species sistens. Upsaliae, 3. Pars 1. Resp. S. N. Calström. 1-28. I Tab. 1778. - Pirs 11. Resp. S. M. Eklund. 29-32. I Tab. 1783 cent latea In den Commentariis lipslensibus XXVI, 483. - Facta Atedicorum suecicorum, 1. 259). - Pars III. Resp. J. Lundali1. 53-68. 1784. Pars IV. liesp. C Engström. 69-84. 1784. Pars V. Reop. J. C. Noracus. 85-106. Tab. 1789. - Pars VI. Resp. A. J. Lesques, $107-$ 130. Tals. 1791 .

12. Perieulum entomologicum quo charaeteres gearum insectormm ete, Diss. Resp. Sam. Tümer. Upsal. t789. 2. Edit. nov. e. not. Mayer. Götting. 1791. 12. - Edit alt. sneclea all. ab A. D. Ilımmel. Upsal, 179.3. 8.) 13) Dissertutiones neademicale (Collectlo dissurtutiounu edil. Persoon. Ginting. 17991801. 3 Vol. 8.).

i1) Musenm maturaliun Academiac Upsa. liensinm, dissernationes. Upsnliae, 4. l'ars I. Resp. F. W. Radlotf, p. I-16. 1787. - Pars 11. Resp. C. M. Holmer, 17-32. 1787. - Para 111. Resp. A. G. likberg, 33-42, 1787. - Pars IV. Resp. P. A. Bjerken, 4.3-59 (1 Tab.). 1787. - Pars v. Resp. 0. Gnep, 59-59. 1787. Pars V......... - Pars VII. Resp. J Branzell, 85-J4. $1780^{\circ}$ - Pars VIII. Kesp. C. 
E. Rademine, 95-106. [Cette dissertation, colls forme de thèse, n'est, à dlre vral, qu'un catalogue par ordre de donatlons ct de matlères, mals il se trouve au bus des pages des notes deseriptives pour uI graud nombre d'especes. Je ne connais de vue que ees huit prénicires. Je trouve des notes tres-variables sur la quantité de ces thẻses, tantôt je vois une annonce de 22 , plus un suppleineut de 8 ayant paru de 1787 '̀ 1800 , tantố l'indieation est de 1? theses parues de 1787 a 1809 . Ces onvrages détaclies sont si rares dans les bibliotreques qu'il est trés-ulltielle de se livrer a allune véritication. - Je trouve encore une indieation de 9 disserintions sur differenls iuseets, de 179.1 a 1825 , et de 30 autres theses sur Ia fallue de differents pays, de 1825 a 1826 ; toltes me sont incounues. - Perclieron, Bibliograplie, 1837, 98.) (Auel wir kennen diese Raritḧten niclit dureli Antops|e.)

15) Diss entom de hemlpterls rostratls eapensitus. Upsat. 1822. 4. P. I, P. III, P. III, Resp. Jo. Eu, Rungren, I'. IV. Resp. C. U. Westerliny.

16) Pueumora ef uytt genus ibland insecterne uplackt ncl beskilprit (Vetensk. Akad. Haudl. 37. Bll. 1775, 254-260. - Deusch: 252. Ausz. Fĩssly, 11. ellt. Mag. III, 84\%

17) Beskrifinlng pa tvânne แya iusecter Paosus (let. Ak. 1уа Handl. II, 1781, 168131. Deutsclı: 1781,170 .

18) beskrifning pa en ny Silkes-nuask i fran Japan; Noetua serlel. C. fig. (ibid. 11 , d581. 241-273. Dentsels 1. e.)

19) Corilyle et sarskllcli Inseet-slïgte lse. skrifvit (lbld. 17y7, 44).

20) Natga nye Natt-Fjärilur af blalrulla re slnegtet (lbill. 1797, 165. - Naelutfilter).

21) Beskrifning of sligtet Trincus (ibid. 1804).

22) Ptyneerus et Ripidius i ibld JSO6). 1808).

3) Animerkuingar over Spliex figulus (ib.

21) Neue Arten des Gescluleclits Phenuora (ib)ld. 1810\%. Vgl. Nr. 16.

25) Neue Art"n des Gerchlechts Blatta (iv. 1510). V $\mathrm{Bl}$. Nr. 43

26) Beselireibung der zwel newenlnsekten. geschlechter Gnathoecrus und Taunacera (ib. 1814:

27) Besclurelbung vou vler neuen Arten Bruclin: (Ib)।. 1816).

28) Beselıreibung vou Midas gizantea (ib. 1816).

29) Deseriptlones lnsectorum sueclcorum (Nova Acta Upsaliensia V. 8j- 119 ).

30) De Bracliycero traciatus entomologleus (ibid. VII, 104: 150).

(1) Generls Anthren) monographia (It]d.

32 , Deseriptio Acrydii (Ibld. 157).

33 : Coleoplera eapensia antennis fusiformlbus (flid. VIII. - Alurnl species).

31) Tanyalosane 17 novac specics deserlp. tae (Ibicl. IX, 1827,63).

35. Gelis luseet] gemus doserlptun (ib]d.

199. lelunemnnes apteri).

36) Coleoptera rnstrata capensia (Mem. alc l'Aend. de Si. Peterab. (V).

37) Henipterorua maxlllosorum conera (ilild. v. - Orthoptera).

3) Coleoplera capensla antennla lamelatls sive clava tissili Instructa (ibld. Vl. Lamellicoruia'.

39. Coleoptera capensla autennarum clava nollda et perfollata (luld. VII, 1820. - Clavi. cornia).

4u) lelncumonidea, insecta hymenoplera.
Pars I (ibld. VIII), Pars II (Ib]d 1X, 1824, 285).

4], Traeliyleres, Insectl genus (ib. VIII).

42) Novne Insectorum species Rutelae ge. nus (ibid. VIll).

43. Blattarum novae species deserlptae. (Jbid. X, 1826, 275 .

44) Reisen; vgl. L i un é.

Thylesins, Ant. 2.

Thymins. 2. Doutsel.

Tliede. 1. Deutseh.

Tigny, de. 1. Frunz.

Tilesius, G. 2.

"Tilesins, IV. G. 1. Deutstlt.

Tilling, Matllias. 1. Geb. zu Jevern in Westplıalen, gest. 168.

Tillet, Mlatuhietl. 1. Apronous; geb. zu Bordeatux gegen 1720, gest. 1791.

Tinnmer, Joh. Das. 1. Deutseh.

" Titins. Joluann Damiel. 3.

Fodil, John. 1. Ëmpl.

Toda, Robert B. 1. Engl.

Torlerini, Giov. Batlista. 1. Geb. zn Veneditr 1728, gest. 1799.

Torres, Lilys NIendoz de. 1. Span. Tourette, Antoine Louis Claret le la. 1.

Tonvenel, 1. Franz.

Towuston, Robert. 1. Engl.

\section{"W'rinill.}

Trebra, Friedr. Will, Hrinr. 1. Deutsels.

Treitaclnke, Fripdi. 4.

1) Naturlistoriseher Buldersnal des Tulerreiches. Nach Williau Jardlue lierausgegeben. 24 Liefermuges. Lex 8. Pestl 1837-42.

2) Die Selumetterlinge vou Enrapa. Fort. setzung jes Delsenlieimer'selien Werks. 7. Bd. i830, gr. 8. Leipsig. F. Fleischer. I-4. Bd. Jlernasgeg. von Oclisenlieimer 807 - I6. (12 fl. ) 5. Bis, 1, -3. Alth. 82.; -27 . (10 fl. $48 \mathrm{kl}^{\circ}$ ) 6. Bds 1., 2. A bth. 827.28, c $7 \mathrm{fl}$, $12 \mathrm{kr}$ ) (Isis 18:6, p 53.) II (das. p. 1064).

Tremilley, Abralı. 1. Geb, zu Genf 1700 , gest. 1784.

* Trentepolil, Johaun Jakub. 2. Deutseli.

Tremuer, Joh. Phil. 1. Dentsclt. Trevirames, Grorg Reinluuld. 4. Geb. 7.11 Brrmen 4. Ja11. 1776.

1) Ueber dle Bereitung des Waclises durel die Blenen (F. Ticdenn viranus Zelikclir. fĩr Plıysioingie. 111, I. 1828. 62)

2) Ueber die Eutatelung der gesclilechts. losen Imilividuru bel den llymennpteren. vor. zügliclı aus den Blencugeschlechte (Ib]ı. III, $11,221)$.

3) Ueber dis llerz der lusektell. dessen Verbiudum mlt len Eirrstbcken und ilher ein Banchuetios der bepilnpteren. M. K. ciblet. IV, 1831. 11, 181-184).

4) Abiıadlungen ïlser slen funern Ban der ungeflïgelten Insekten (In seluen verniselut. Sclırllt. Bd. 1 und 11. Gätung. 1816. 4. M. Kupes). 
5) Resultate elniger Untersuelıungell über / der .Gallerla di Minerva" und In relnen Wer. den inneru Bau der Inxekien (Annalen der Wellerauer Ges. 1, 2. 1809, p. (69-78).

6) Vermischle Sehriften. Bd. I und 11. Goltingen 1816-17. 4. M. K.

7) Ueber das Satugea und das Geruchsorgan der lusekteu; ibber den Nutzen der Scluwimublase bei den Fisehen (Aan. d. Wetl. Ges. II. 1 (181'2), p. 147-16\%. 2 Tuh.).

8) Ueber ein Kerf, welelies die wilden Feigen in Oberitalien bewohn (Cynlps l'selles?). (Isls 1827, [1. 313).

Treviranus, I. Chr., Solu.

Trew, Christ. Jak. 4. Hedicus und Botaniker: geb, zu Lauf iu Frankell 1695, gest. 1769.

Trievonid, Märten. 3. Geb, zu Stockholu 1691 ; gesl. 1747.

Triepke. i. Preuss.

Tripildi, Andrca. 1. Neapol.

* Troselnel, Frriuz Hermann.

\section{Deutsch.}

Trost, Patriz. 1. Bayer.

Kleiuer Bellrag zur Entomologie In elnem Verzeiehulsse Eichstidiselier Inseklen mil Anmerkungen fiir Kenner und Liebliaber. 1 . Tauseacl. Erlangen.

'Truze-1. C. B. I. Deutsch.

prucluet, Michel. 1, Franz.

Trenuplius. 1. Deutsch.

Trelumener. 1. Schwci\%.

Titirlk, $W, v$.

Thermins, Jolı. Jak. 1. Deutseh.

Tenrier.

Relse Hach Tibet, Hamburg 1801. 8. (107; A p/s).

Turton, W. 1. Engl.

Widnmenn, Isaac. 1. Sebwede. Ultjesfort, Heinr. 1. Deutsels.

* Ullricla. Oestr.

Ungararl. C. S. 1. Deutsch.

Der Maiwnirm ein lllifsmlttel wider den tollen Hundsuiss. Ziillichaul 1783.

Unzer, Joh. Aug. 4. Geb zu Halle 29. April 1727, gest. 2. April 1799.

Upinarelk, Johannes. 1. Sehwede. Urainus, Jolı. Heiur. 1. Deutsclı.

* V., in Tübingeu.

Gemeinfassliehe Belelorumg über den Mlalkäfer ele. Tühlng. $183 j$.

Vulentin, Joh. Ernst. 1. Deutseh.

Valentiui, Mich. Beruh. 3. Geb. zu Giessen 26. Nov. 1657 , gest. das. 13. Miirz 1729.

Vallisueri, Antonio. 11. Mcdicus und Naturforseher; geb. 3. Mai 1661 zit Trasilico im Modenesischen, gest. zu Padua 28. Jau. 1730.

i) Dlaloghi fra Mlalphigi e Plinlo Intorno la curiosa orlglie de molil iasetti. Venezin 1700, 12, Patarii 1700, 8. (Eirschien auel, in

hea mit Verbesserungen und Zusătzen. 1,3 -88 ).

2) Esperleaze ed osservazlont latorno all origlne sulluppl, e cosluml di varii iasettl. Prolova 1713. 4. (Aneh In seinen Werken. I, 361 ).

3. Opere fisleo aledicle contlnente un gran numero dl traltati, osaervazlonl, raglonamentl e dissertazloni sopra la fisica, la medicina la sloria ualurale, Venezia 1733 . 3 Vol. Fol.

4) Historia nuseae roslscenc, ldea divislonis iaxeclorum in classes loco nalali de. silniplas. Padua 1725. 4. (In ejusd. oper, I, 179).

5) Osservazioni sopra la Cuntharide (In ej. oper. 1, 255).

6) Della nobilitá e utilitá dello atudio degl' insetti (ia ej. op. 1, 3117).

7) Osservazionl spetianil alla storia naturale degl' Insetli (ia ej. op. l, 361).

8) De rara quadam Loensta (Fpliem. Aead. Nal. Cur. Dec. 2. Cente. 3 et 4 . Observ. 35. $81-84$ (la e). op. $11,62-63$ ).

9) Scarafagsio nollurno marlno (in ej. op. 11, 95).

10) In meglioramenti e correzioni di aleuno esperienze de F. Redi.

II) Conclusioues physleo-medleae, abl elian de inseclorum genesi agit (In ej. op. 111, 622).

Vallot. 7. Framz.

Vondelins, Dominicus. 1. Lebte zu Padua.

Vinidre, Jacques. i. Lateinischer Dichter; geb. zu Causse bei Beziers 1664.

Vasco, Giambattista. 3.

* Vaudouer. 1. Franz.

Vemquella, Nicolas. 2. Berühm. ter Chemiker; geb. 1763 zu Saint-André d'Herbalot in der Normandie, gest. das. 14. Nov. 1830.

Velsele, G. N. 1.

Velseluins. 3 .

* Veralut, F. J.

* Verint, G. J. 1. Schweiz.

Verge, dı. 1. Franz.

* Verlinell.

Verueys. 1. Engl.

VinneIIR, Gius. I. Ital.

Vient, Philippe Rodolphe. 3. Me. diciner; geb. zu Payeru (Selwweiz) 1720, gest. zu Lausanne 1783.

Vieat, Madame.

Versuelie aus der Brnt gemelner Bieneu Küniginaen an erlabllell Mem. de In soc. ceon. de Bern. 1769. Part. 2, Nr. 2).

*ictor vou Motschulaky. Auch nur "Victor" allein.

Vina, Marius Hieronymus. 1. Lateinischer Dichter; geb. zu Cremona 1490, gest. zu Alba 1566.

Viever, Carl Friedr. 1. Deutseh.

vigors. 1. Engl.

Vills, Antonio. I. Ital.

Villa, Giovuni Battista. 1. Ital. 
- Villiret, Foulque de. 2. Franz. villermont. 1.

Villers, Charles de. 1. Franz.

- Villiers, Andrien Prudent de, 3. Villiers, de Chartres. 1. Franz. Vixey: J. J. 3. Franz.

Voet, Juh. Euseb. 1.

Vorel, Joh. Georg. 1. Deutseh.

* Vogel, Christ. Friedr. 1. Deutseh.

Vogler, Joh. Philipp. 2. Deulsch.

Medieus; geb. zu Darmstadt 1746.

Voigt, Joh. Christ. 3. Geb. zu Zoppat 22. Nov. 1725 , gest. zu Seliwarzaeh (?) 28. Juni 1810 .

Voigt, Jol. Heiur. 1.

- Voigt, F. J. 1. Dentsch. (zn Jena).

* Voiniliow, Walstlio.

Volmar, Joh. Cluristopl. 1.

Volunar. War l'rofessol zu FuIda und starb vor mehren Jaliren. Scine gediegenen Arbeiten sind in Faunus enthalten.

Volta, Gio. Serafino. 1.

Vorster, Karl Freih. v. 1.

Voye, Mignot de la. 1.

"Vagner, Moriz. 1. Deutseh. Von Augsburg.

* Vagner, Rudolph. 3. Deutsel. Uatrmer, Joh. Jak. 2.

Medieus; geb. 30. April 1641, gest. 14. Dez. 1695.

*Vagner, Andr. 1. Deutseh.

Wagner, Pet. Chr. 1. Medieiner und Naturforselier; geb. zu IIof 10. August 1703, gest. zu Baireuth 8 . Okt. 1764 .

* Wahlberg. 1. Sehwede.

Wali Iboni, Joh. Gust. 1. Schwed.

* Wniles, George. 4.

* Wnatefield. 1. Engl.

Tralelı, Joh. Ernst Emanuel.

Geb. zu Jena 1725, gest. 1778.

* Walchuer, F. H. 1. Deutsch.

* Vairisemaer, C. A. 1. Frauz.

* Ualford, Thom. 1. Engl.

* Valeler, firancis. 3. Engl. Erallenius, Johanu Friedr.

Schwed.

Waller, Richard. 1. Engl.

* Tallieli. 1. Engl.

Wallis, John. 1. Engl.

Walpurger, Jolı. Gottlieb. 1.

Deutseh.

* Enalt, Jos. 4. Bayer.

Walther. 1. Deutseh. Walther, Isaae. 1. Deutsch.

Walton, John. 2. Engl.

* Waniek.
IVaril, Samuel. 1. Engl.

Warier, Jos, 3. Engl.

vartmann, Bernhard. 1. Deutseh.

Wassali : Anton Maria. 1. Ital.

* Wastel, Gregor. 1. Böhme.

* Waterhouse, Georg Riehard. 5. Eugl.

Vatson, Will. 1. Engl. Botaniker und Playsikus; geb. 1715, gest. 1787.

velser. Fripdr. 4.

(und M. H. Molir) Arelive f. d. system. Naturgesclo. Hamb. 1804.

2) Beiträge fiir Naturkunde. Kiel. I Bd. 1805. M. $11 \mathrm{Kpf}$.

Wedelins, Georg Wolfgang. 1. Geb. zu Golsen 12. Nov. 16i5, gest. 6. Sept. 1721.

Weidlerns, Joh. Friedrieh. 1.

Deutseh.

"Veigel, Pastor. 1. Deutseh.

veiss, Emanuel. 2. Sehweiz.

Weiss, Simon. 1.

well, Jakob v. 2. Deutseh.

* Wesmnel, C. 4. Niederl.

Vesteruman, B. W. 2. Däne.

westfelu. t. Deutsel.

Westplialı, P. N. I. Dentseh.

"Westwood, J. 0. 24. Engl.

"Wetter, Joh. Bapt. 1. Deutsch. Whitalcer, Guil. 1. Holl.

White, Stepl. 1. Eingl.

* White, A. 1. Eugl.

IVIedenunn, Wilh. Rud. Christ.

t2. Geb. zu Braunschweig 1770. t.

ii Nova genern dipterorum (Isis 1820, p. 673).

2) Ansserenrapäisclie zweillïgelige Insekten. Als Forlseluung des Meigen'sclien Wer. kes. 2. Thl. mit 5 Stcintaf. 8 . Hanm. I, mit 9 Steinif.

3. Aehias dipterormm genus a Fabricio condituu; ithustratum novisque speeielus nuetum el collvenui physicorum Germanormm ob. lalum, Cum tubl. Jitl. It 8 maj. Killac.

4) Mellode Insekten seluell an tödten (Germ. Mag. 11. \$. 336).

5) Besclucibungen maihl. Dipteren in Melgeiss Werk.

6) Archiv und Megnciu.

triel, Cormelius Stalpart vau der. 1. 1. Holl. Medieus; geb. gereu 1620. vieri.

0 pp. onn. Anstetod, 1660. 4.

* Wrilbrand.

Wilcke, Sam. Gust. 1. Deutseh. vileke, Joh. Carl. 3. Sehwed. vildam, Thomas. 1. Engl.

vilde, Jerem. 1. Bayer. Aus der Pfalz.

Wildmenn, Thon. 2. Engl. Vilduann, Dau. 1. Franz.

Wilhelm. 1. Deutseh. 
whinelmi, J. G. 3. Deutsch. vilkes, Benjamin. 2. Engl.

One lumdred and twenty Copper-Platex of english Moths and Butterllies, representiug their changes into the Calerpillar, clirysalis. and Fly States, and the Plants, Flowers. and Frulis, roheron they sect. coloured with gient exactness from the Sulijects themsclyes, with a Natural History of the Moths and Butterdlex, deseribing the Method of Malnaing, Pre. serving, anul Feeding etc. Louton printed for Bew. White etc, 1773. if unbez. pp. Vorr., 10 unbuz. pp. Eilll., 63 bez. pp. Text, 4 pp index 120 kipftf. 4.

vilkin, S. 1. Engl.

vVill. 1.

WVillemet. 1. Geb. zu Norroi bei Pont à Mousson 1735, gest. zu Nancy 1807.

Williams. 1. Engl.

vallieln, Jodoe, 1. Deulsclu.

Willomelaby, Francis. 3. Geb. zu Middleton, gest. (im 37. Jahre) 3. Juli 1672.

"Wilson, J. 1. Engl.

" Vinkler, Ed.

volulfalert, Juhaun August. 1. Deutsch.

Volfr, Jakob. 1, Geb. zu Naumburg 30. Dez. 1642, gest. 4. Juli 1694. Jooln, D. H. 1. Deutsel.

voln. 1. Dentscl.

WoIf, Nathunael Matthew. 2. Engl.

Evolf, Joh. Friedr. 1. Deutsch. vollebins, Joh, Jak. 1. Schweiz. WVollenhnnlt, Georg Audreas. 1. Deutsel.

* voood, William, 2. Engl.

Worth, J. 1. Nordam.

Vottou, Eduardus. 1. Lateiniseh : Odonulus; geb. zu Oxford 1492, gest. z.u Londoul 1555.

Urey, J. 1. Engl.

UVnIfe:11, Xavierus. 1.

* Wumiran. 1. Oestu.

Wurna, Fued. van. 1. Niederl. viarzer. 1. liranz.

vytteubnch. 4. Schweiz.
Xenurchms. Bei Athenacus (über stimmlase Cicadenweiluer).

Xenoplnow.

Anabasis. Lilo, $1 \mathrm{H}^{\prime}$ (Aen).

" Tancez. 1. Span.

* Tarrell, Williain. 1. Engl.

Teates, Thomas Puttuson. 1. Eninl.

Instituions of Entommlogy. Lond. 1773.

Touge. Jan. 1. Engl.

"Yule, J.

Zanmbeccert, Gius. 1. ltal.

"Zanulers, J. W. 1. Deuts(').

"Eanetti.

Yanmoni, Antonio." 1. Agronom; trel). \%u Udiue 1696, ges!. 1770.

"Zanwadsky, Aleximdro. 2. (Mestr.

* Tebe, F. S. I. Deutsel.

Telnuprenuig, J. A. 1. Dentseb.

Zyeiss. 1. Deutseh.

veller, P. C. 6 . Diutsrl.

*aendriui. 1. Jtal.

"Zuenneck. 1.

* Zetterstedt, Jol. Willelm. 4.

Schwede. Geb. 20. Mai 1785.

zaier.

Ueber Parasiten vou Lytt.l veslentorla (Ferussac Bullet I830).

Zies. 1. Deutsch.

"Zimmermann, Cluistian. 3.

\section{Zingmerminum.}

Cochenille (dess. Taselienb. der Reisen. Leipz, 1806. 12. p. 134 (Frtrïg1.).

Zinani, Giuseppe. 2.

Tinke, Gentrottr. 1. Dentscls.

Zinken, gell. Sommer, Jul. Leop.

Theod. Hriedr. 4. Deulseh.

Beobachtungen üher die Sarktiögcr unter den Sclunetterllugen, ihre Fortptlanzang uni

bintwickelung. Mit Nachselurif vou Germar

(Mng. d, Eist. 1, 1, p. 19, 111. - Ebeud. \$. 41

-47 ).

"Zablxofr. Os. 2. Russ.

Vascinachins, J. J. 1. Deutsch. 
.

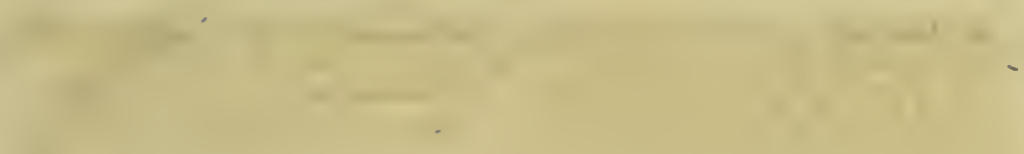

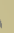

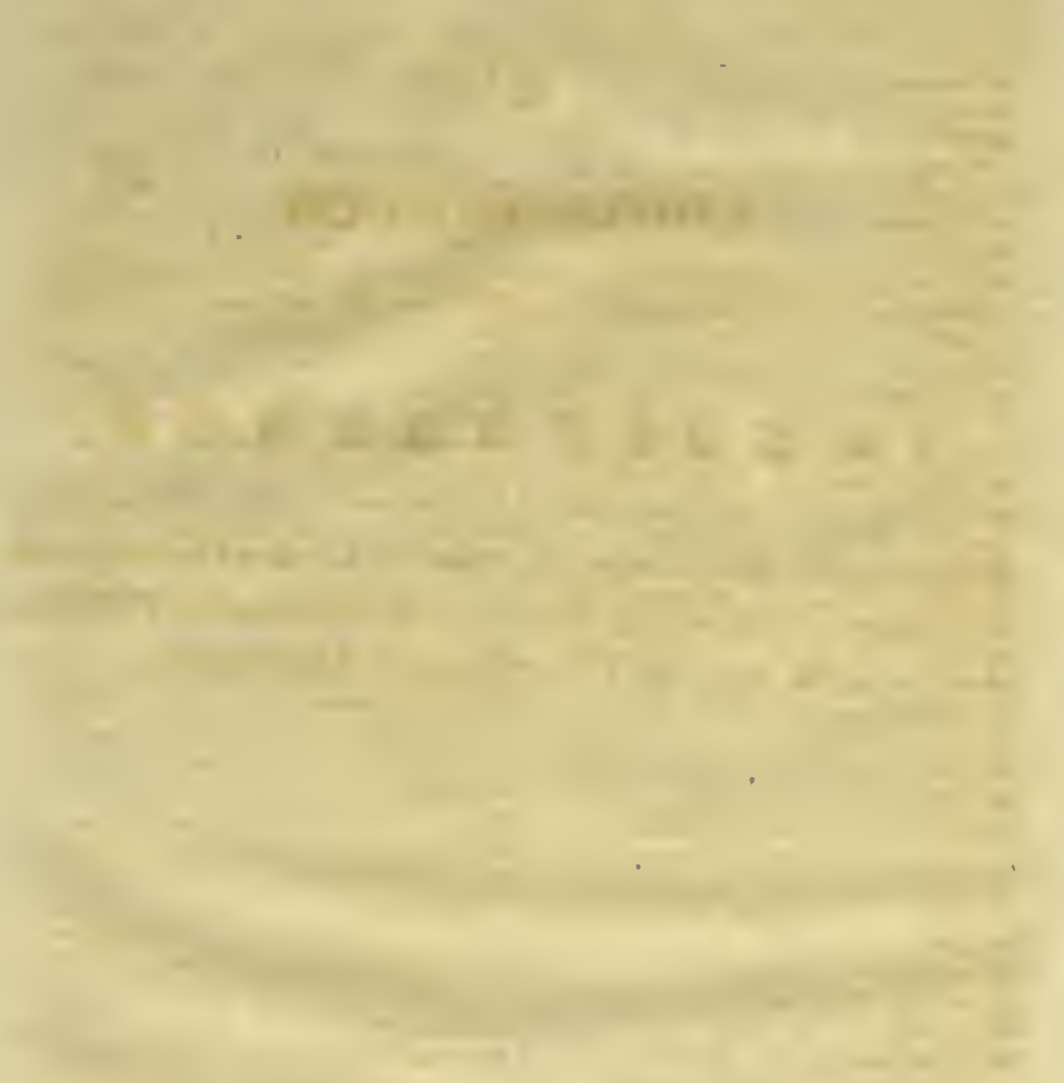




\title{
II. \\ ADRESSENBUCH
}

\author{
DER
}

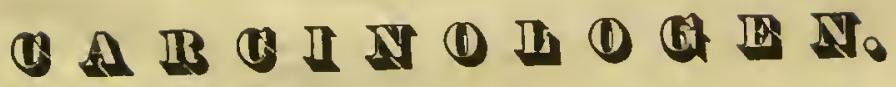

Aufzählung der Schriftsteller etc. über die Krabben (Krebse) und die Crustaceen überhaupt, dann über die Myriapoden, Paläaden, sowie über die Cirripeden oder Rankenfüsser. 
The sight of naturs in her magnificence, or in her beauty, or in her terrors, has at all times an otcerponcering interesst, which even habit cannot greatly toeaken.

Walten Scott (The Pirate). 
DEN HERREN

\section{MAXIMLLIAN PERTY}

ZU BERN

UND

\section{MILNE EDWARDS}

ZUं PARIS,

sowIr.

\section{KARL IUDWIG KOCH}

ZU REGENSBURG,

\section{K. L. KRÖYER}

ZU KOPENHAGEN,

C. W. DE HAAN

ZU LEYDEN

UND

J. FR. BIRANIDT

ZU ST, PETERSBURG. 
inarntomintrewt

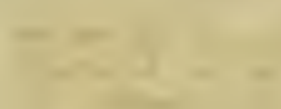

- waskno imine

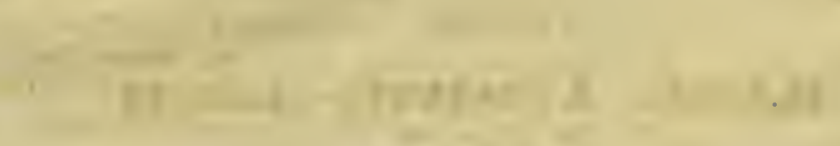

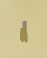




\section{Vorwort.}

Der Name Careinos (ó xapxinos, eaneer, éerevisse) ist, um die gewöhnliche Vox hybrida Crustaceologie (Leach in der Edinburgh-. Encyelopaedia hat diesen Ausdruek unter den Neuern gebraucht, wie Andere Insectologie) zu vermeiden, gewählt worden, nachdem D e sin arest ilm in der Verbindung (Careinologia) früher angewendet. Zwar legt J)esmarest (Crustac. foss. und p. 90 der Considérations) nach L e a h (Edinb. Eneyel. VII, 390 und Malacost. Britan.) nur dem Liınésehen Cancer moenas den Sippennamen Careinus bei (ebenso De II a an), und Latreille (Préeis des Caract. gén.) bezeichnete früher deı Crevette des Ruisseaux, den Flohkrebs, Gammarus Pulex des L in né, mit diesem Namen; Rafinesque endlich taufte ein Genus aus der Familie der Palaemonien also (Préeis des Déeouvertes semiolog. Palerme 1812. 18.). - Das aber hat Nichts auf sich. Carcinologia ist die Lehre von allen Krebsen.

So wie das Wort Evitomon für die Kerfe, kann Careinos für die ganze Reihe der Krebse gebraueht werden, besser, weit besser als der relative Nane $M \alpha \lambda \alpha \times 0$ saxos, molli erusta obtectus, worunter man zu den Zeiten des Aristoteles, des Athenäus, Hippocrates ete. die Krebse begriff, welche die Römer Crustata und Crustaeea nannten. Abermals wieder unbestimmte Namen!

Nach Perleb und Goldfuss heissen die Krabben Polymeria, nach Batsch Polypoda. Cuvier blieb beim Alten; aber Latreille wählte llyperhexapi, Savigny Apiropoda för dic Classe der Crustaeeen. l) uméril belegte nur die Familie der eigenlliehen Krabben mit dem Namen Carcinoiden, ganz entsprechend den Caneeriden, wie sieh ron selbst versteht. Man wird, so lange es eine Natur und also aueh eine Natureschichte gibt, sagen: Crustacen oder Krebse (Krabben). Arist.otoles (ed. trad. Gaza e) Lib. IV, eap. II. Ilist. an. sagt: Crusta intectorum ( $\tau \tilde{\omega} \nu \mu \alpha \lambda \alpha x o s \rho \alpha x \omega \nu)$, genus primum Loeusta (

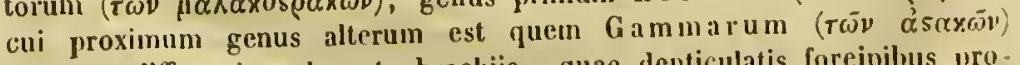
voeant: differt is a loeusta brachiis, quae denticulatis foreipibus pro.

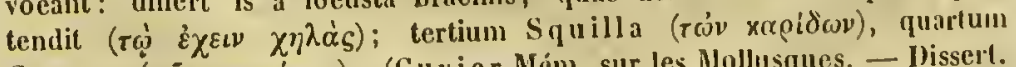

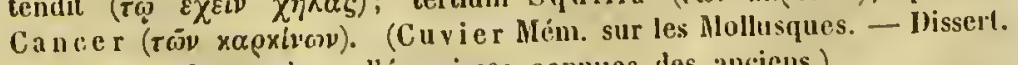
critique sur les espèces d'éerevisses connues des ancicns.)

Aspice, guam longo distendat pectore lancem, Quae fertur domino squilla.

(Juv, Sitt. V, v. so.) 
Fast sollte man ineinen, die Literatur dieser bisher fast stiefkindlich behandelten Classe sey arn; dennoch ist sic es nicht, wie Jer Anhang nachweiset. Die Arachnologie ist aus natïrlichen Grïnden un viel mehr vernachlässig̨ worden. - Ausser Jen griechischen und lateinischen Autoren, deren Anzahl etwa ein Dutzend ist, haben die systematischen Schriftsteller und die neueren Nomenclatoren, worunter Linné, KI e in, Fabricius, G. Cuvier, Bo sc, Lamarck, La treille und RafinesqueSchmaltz, so wie Leach, Haworth und Blainville, erst die Bahn zur spezielleren Kenntniss gebrochen, und etwa zwanzig andere Forscher (Otto Fabricins, I, ichtenstein, Gronovius, J. R. Forster, Oedmann, Viriani, Tristan, Adams, Denso, Spengler, Lepechin, Lesucur und Fougeroux de Bondaroy etc.) durch schätzbare Monographien dieselbe erweitert. Wahrend indessen die Anatomisten und Physiologen Collinson, Minasi, Parson, G. Cuvier, Savigny, Etienne Ge offr o y $\mathrm{S}$ t. - Hilaire, Willis etc. den inneru Bau untersucht und eine Menge Aerzte sich, medizinischer Zwecke willen, mit den Krebsen befasst hatten, wie Roberg, Libavius, Gastaldy, Ilen unger, Cartheuser, Cloquet, de Pré, Nebel, II o in berg, Schulze, Gruner, Sommer, Frank etc., lipferten uns die topographischen und reisenden Fo'scher einen Sehatz von Details über das Leben, Weben und Vorkommen, so wie die geographische Verbreitung der lirebse. - Daher netumen sie mit Verdienst und Gunst die ansehnlichsten Stellen in den Reihen der earcinologischen Literatoren wie in Jer zoologischen überhaupt ein. - Zu den Topographen gchören Olto Fabricius und Carl v. Linné, Ilans Ström, Otto Miller, Thomas Pennant und William Elford-Leach, Gicorge Nontagu, Geoffroy, Fourcroy, Domenico Risso, Scopoli, Sclırank, Gius. Olivi, Panzer, Koch, Krauss, Frisch und vorzüglich noch Thomas $S$ ay, der nicht zu vergessen ist. - Ueber Sitten und Lebensart, Fang cte. haben wir zum Theil den Scliülern des niesterlienden Nordsterntügers, theils auch andern Männer", welche unter den "Reisenden" in den Literatur glänzen, noch Wesentliches zu verdanken, und wir nemen uur die Namen Rüppel, Ehrenberg, Phipps, Eigede, Martens, Savigny, IIasselquist, Catesby, Forskaol, I, inne sellsst (Westgothländisehe Reise), Pet. Kalm, Rumph, PIanehus, Patrick Browne, Ilans Sloane, A. Parra, Rochefort, Piso, Marcgrave de Liebstadt, Krauss, Feuillée, Molina, Osheck, Schrank, Bontius, Quoy, Gaimard, Ross, Mario de Proce, den Be.lciter La Peyrousés, la Martinière etc.

Allgemeine Ahbildungen, ansser einigen in den gewohnten Museographan Kuorr, Peliver, Seba, Gronovius, Linnaeus, Ol. Worinius, finden wil wenige, ausser was Desinarest, Latreille, Il erbst, R ü p pe I, Ren a d d, Le a ch (Zoological Miscellany), K ra uss und S owerlyy bekannt gegeben; sehr ansehnlich ist dagegen die Literatur der mikioshopischen Monographien und Beohachtungen (namentlich fruchtlbar über dic Lintomostraccen), noch beträclitlicher an Schriften, welche über Trilobiten und fossile Krebse überhaupt tractiren. - Deı Namen 
Swa in merdam, Ledermüller, Joblot, Baker, O. Muller, L. Jurinc, Wormius, I, a t, Kä n p fer, André, Be ekmann, Spengler, Herbst, Du hamel du Moneeau, Rathke, Klein, Brown, $\mathrm{S}$ ch äffer,. Brand $t$, S e hulze, Cuvier, Hermann (von Strashurg), Losehge, Cavolini, Strauss, Gruithuisen, Férussae, Brongniart, Gray, King, Shaw, Sehlosser, Prévost, Ra ekett und noeh einer Menge anderer, zumeist längst sehon gesehiedener, zolleı wir noeh heut z.u Tage unsern wärmsten Dank. - Was die folgenden geleistet, geht in die Vorwelt zurück und berïhrt die Versteinerungskunde.

Es ist gewiss merkwiirdig, dass vorzüglich Adelige von alter $\mathbf{A b -}$ stammung (C. Sternberg, G. Graf Münster, Baron Sehlotheim, Graf von Ml a udels lohe, Fraih. L. v. Bueh, Baron Hübsell, S a ehs v. Lewenheim ete.) sich mit den alten Thieren und Pflanzen vorzugsweise besehäftigen und abgegeben haben. - Ein harmoniseher Zusammenlang mit der Erziehung, mit den Erinnerungen an eine alte Abstammung ist, wic unser Meister 0 ken sagt (Isis 1840, 281), offenbar nieht zu verkennen und das ist ein neuer Beleg von dem Parallelismus'der Geschichte und der Natur.

Waleh und K n orr, Parkins on (Organies Remains), Ca lecolar, Catullo, Seheuchzer, Waguer, Lesser, Mylius, D'Anone, unser Freund Frhr. v. Zi eten, $\Lambda$ gassiz, Ka u p, Spada, Ranzani, Desmarest, Torrubio, Bronn, Wahleuberg, Jul. de Tristan, Brunnieh, Guettard, Brongniart, Latreille, A udouin, Dekay, J. v. Rensslaer, Stockes, De Han ete., sie behandeln die Ueberreste einer vergangenen grossartigen Zeit.

Die Nitur hat in der Classe der Krahben krieghaft phantasirt. Von dem Wenigen, was wir aus derselben kennen gelernt, ist Alles wunderbar hizarr. Dic Crustaecen, Myriapoden und Araclıniden sind fist lauter Beine. Acht oder zehn und noch inehr Füsse sind in der Norn; sehr selten sind zwei oder drei Pare vorhanden; der Flügel fehlt, auf welchen die Gottheit die Farben der Blumen der ganzen Erde und das Licht und den Schmelz des llimmels und der Gestirne bei den Kerfen geheftet hat; das Auge erscheint zum öftern gestielt und die Fühler übersteigen die gewöhnliehen Längenverhältnisse und verıreten deutlieh die Stellen der Ohren; die Vorderfüsse der Deeapoden laufen in ries"nhaften Seheeren aus und deren Leib umsehliesst ein schicnigel Panzer, alljährlieh sieh wundersam erueuend, wie die verlor'll gegangene Glierlmasse. - Und unser Loos ist das dieser Krabbenwelt: Nasei. Pati. Mori!!!

Die Eintheilnng der Crustaecen unter den livertehraten, deren Nervensystem nicht vou linoehen oder Knorpel umsehlossen ist, sonderu von einem External-Skelet oder verknöeherter, kolılensaurer, kalksubstanziellen Haut, ist sehr einfaeh und von der äussern Bedechung hergenommen. I, atreille bildete zwei Abtheilungen: Halaeostrica und Entomostraea; jene zerfallen nach den Füssen in Decapoda (Zehnfiissler), Stomapoda (Mundfïssler), Laemodipoda (Kehlfüssler), Amphipoda (Kreisfïssler) und Isopoda (Gleichfüssler). Die Decapoden umfassen zwei Fanilien: 
kurzschwänzige (Brachyura) und langschwänzige Krustenthiere (Macrura, z. B. Astacus des Gronovius, wohin unser Bachkrebs, A. fluviatilis, gehört, the Crawfish der Britten). - Duméril theilt die Crustaceen (rückgrathlose Thiere, mit Blutgefïssen und blätterförmigen RespirationsOrganen, $=$ Kiemen, versehen ; meist mit zchn Füssen, in sieben Familien: 1) Aspidioten oder Schildträger (mit den Sippen; Limulus, Apus, Ozolus, Binoculus und Calygus); 2) Ostrakinen oder Zweischaalen (mil den Sippen: Daphnia, Cypris, Cytherea, Lyneacus); 3) G y mn on e e te u oder Nacktsehwinmer (mit den Sippen: Polyphemus, Zoea, Branehiopus, Cyelops. Argulus); 4) Asta coiden oder Spitzschnauzen (wohin die Sippen: Ranina, Orithyia, Maja, Dorippe und Leueosia); 5) Carcinoiden (Astacoiden; ganz entspreehend den Canceriden von Latreille) oder Krabbenförmige (besser wïre der Ausdruck: Oxyrhynchen) (mil den Sippen: Calappa, Dromia, Hepatus, Cancer, Matuta, Podophthalmus, Portunus, Ocypoda, Grapsus, Poreellana und Pinnotheres); 6) Macruren (Astacoiden, die mit Mühe das Land besteigen) oder Langselıwänzige (mit den Sippen: Crangon, Palaemon, Penaeus, Astacus, Scyllarus, Palinurus, Galatea, Pagurus, Hippa, Albunea); 7) Arthroeephalen (Astacoiden; völlig entsprechend den Branchiogastra des Latreille) oder gliederköpfige Krabben (nit den Sippen: Mysis, Squilla, Phronima, Gammarus, Thalitrus). - Das diehotomisehe System Haworth's * ist nur ein modifizirtes Latreille'sches. Er bringt die Crustaceen in zwei Gruppen: Brachyura und Macrura, und sondert die Brachyuren in Notatoria und Cursoria, welche naclı Gestalt und Arehitektonik des Körpers, nacl: der Fusslage und andern Kennzeichen wieder in eine Menge Abtheilungen aufgelöst sind. Das klarste, einfachste und beste System der Krabben wäre das voul D uméril; sofern es nicht einen wesentlichen Fehler an sich trüge. Dumeéril trennt nänlich dic Myriapoden (Julus, Polyxenus, Scolopendra ete.) und Polygnathen (Physodes, Oniseus, Armadillo) von den Crustaceen, indem er sie zu den Insekten in die näehste Nähe der Spinnen (Akeraten) stellt, was durehaus nicht gebilligt werden kann. Unmittelbar darauf lässt er die Würmer folgen. - Weleher Naturforscher möchte die ausserordentliche, innige Verwandtschaft derjenigen Geschöpfe mit den Krelssen verkennen, deren Gattinnen ihre Eier als ein Paqquet unter dem Sehwanze oder in der Nachbarschaft tragen, wic dic ächten Krcbse, welche sie auf das bestimmteste vorstellen. - Nach unseru Beobachtungen an mehren Oniseiden treten die Eier unmittelbar zwischen die vordern Abdominalplatten von hinten nach vorn aus deu. Eierleitern. Der Gatte treibt seinen hornigen Penis samenergiessend in die Abdominalsegmente (etwa in das fünfte oder sechste), die Eierleitern befördern diesen aufwärts und die Foceundation ist vorüber. Latreille (Ilistoire naturelle des Crustacés et d. Ins. III, 1802) sonderts gleichfalls die Asseln (Oniscideen Brandt) ab, sie zu den Inselten stellend, nahm aber diesen Irrthuin zurück in dem Buelıe: Familles naturelles du règne animal. Paris 1825, was er in seinem Meisterwerk

- Anuals of plilosopliy, Vol. LXV (1825), P. 105. 
(Genera Crustaeeorum ete. Paris 1806) zu thun noch unterlassen hatte, wo er sie jedoeh sogleieh auf die Gamariden folgen lässt unter $z$ wei Legionen: Tetracera und Myriapoda. - Indessen sagt ein gefeierter Naturforseher (Perty) von den Arachniden: Dio Verwandtschaft mit manehen Crustaceen ist sehr gross, so dass eine Vereinigung der Crustaeeen und Arachniden in eine Klasse in maneher Rüeksieht zu reehtfertigen wăre *.

Uebergehend die frühern Eintheilungsmethoden von Latreille, Fabrieius, Lam arek, Ris so, Bose, Blainville, Desmarest ete. führen wir zum Nutzen der angehenden Zoologen noeh das System des seharfsinnigen Perty ** auf, und geben einige allgemeine Bemerkungen und Fingerzeige zum Fang und Aufbewahren der Krebse an.

- Schrank, unser seliger niesterbender Lehrer, hat die Crustaceen unter die flü yellosen Insekten, zu den Spiunen, gesellt; gleich mach Chelifer lässt er die in Bayern lebenden Sippen folgen, als da sind: A stacus, Squilla (Gammarus Recentior), Ino (I. piscina. - Branehipus stagnalis Recent.), Oniseus, Triops (Tr. palustris. - Apus eancriformis und produetus Reeent.), Cyclops (C. rubens, C. minuticornis, C. minutus, C. quadricornis, C. planieoruis), A m y mone (A. Satyra, A. coeca, A. silena, A. Bacha, A. Thyone), $\mathrm{N}$ a u plius (N. salutatorius), C y p ris (C. pubera, C. deteeta, C. laevis, C. fasciata. C. vidua, pilosa), Lyceus (I.. Sphaericus, C. macrouras, L. trigonellus, L. lamellatus, l.. truncatus, L. crepidus, L. striatus), D a plu uia (D. penuata, D. longispina, D. quadrangula, D. retienlata, D. sima, D. rectirostris, D. eurvirostra, D. mueronata, D. crystallina, D. truneata). Die Myriapoden lässt er, abgerissen, wie sie überhaupt dastehen, nachfolgen。 Wir stellen dic Myriapoden zu den Crustaeren, was sic mit allem Recht sind. Sie genau zu beobhehten wird uns cine dringende Aufgabe seyn. - Lamarck hat geglaubt, die Bala»en zu den Crustaceen rechneu zu unssen, worin viel Wahrselueiulichkeit liegt, Wrelche' durch Perty's Systeal (Naturg. III, 857) zur begreifliehsten Wahrheit geworden. Perty stellt nämlieh, als Uebergangsformen von de॥ Würmern zu deu Krabben (in aufsteigender Ordnung), die Chaetopolen (Wümer mit Fussborsten) und Cirrhipedien (Rankenfüsser e. G. Balanida und Lepadicea) in dic Mitte. - Aueh den Lemaeen ist eine entsprechende Stellung in Systeme angewiesen, - und Schrauk, weleher

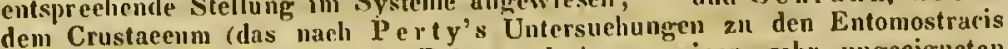
gchört) Arctiscon zwisehen Pulex und Acarus einen sehr ungeeigieten Platz anweist, äussert sich bei In o piscin a (Fauma hoie. III, 1, p. 250) oder dem Branehipos stagnalis folgendermassen: "Unmöglieh kann er unter die Krebse gesezt werdell: er hat weder den Körperhau noch die Eigeuschaften davon; die langen geweiheförmigen Fäuger des Mämmchens sind keine Scheeren, die kiemenförmigen Platten keiuc Füsse, sonderu Flossen und vielleicht Kicmen zngleieh, und das Thier ist nieht, wic der Krebs, gleieh wie es aus dem Eie kömmt, seiner Mutter ähnlieh. Aber er gehört chen so wenig in eine derjenigen Gattungen, welehe Müll er aus dem Limne'schen M n noculus gemaeht hat. - Er selnwimmt anf dem Rück"ll am liebsten, und gut, selten auf der Seite, fast nie goht or auf dem Bauche und allemal nur langsam. Reines Wasser liebt el nieht. In Sammlungen lässt er sich nieht auder's als im Weingeist aufberahren. - Eiuige Tage im Wasser, wenn es auch crneuert wird, aufbehalten, versehwindet er, nachden er immer unscheinharer gcworden. - Die Eier trägt das Weibchen in einem Sacke am Grunde des Schwanzes mit sich herum. In Wassergräben bei Burghausen und Ingrolstadt gefunden." Der fisehförmige Kiefenfuss. Schäf. Ablandl. II, 43. Mit Abbild. Allcemeine Nuturgesehiehte, als philosophische und Humanititswissensclıaft für Naturforsher, Philosophen und das höher gebildete l'ublikum bearbeitet. III Bde. Bern. 1837-41. 8. 


\section{Classis: Crustacea. Krebse.}

Ordo 1. Entomost raca.

Familia 1. Xenomorphida. Genus 1. Aretiscon (Macrobiotus). Spee. 1. A. tardigradum.

Familia 2. Lernaeina (Fischläuse). Genus 1. Lernaea. Spcc. 1. L. branchialis.

Genus 2. Lernaeocera.

Genus 3. Penella.

Genus 4. Achtheres.

Genus 5. Anchorella.

Genus 6. Tracheliastes.

Genus 7. Chondracanthus.

Familia 3. Dichelestiua.

Genus 1. Dichelestium.

Spee. 1. D. Sturionis.

Gimus 2. Nicothoe.

Genus 3. Ergasilus.

Genus 4. Carcinium.

Spec. 1. C. opalinum.

Familia 4. Caligina.

Genus 1. Caligus.

Genus 2. Cecrops.

Genus 3. Pandarus.

Genus 4. Argulus. Spec, 1. A. foliaecus.

Familia 5. I, ophyropoda.

Genus 1. Cyclops.

Genus 2. Cetochilus.

Spre. 1. C. australis.

Genus 3. Disphia. Spec. 1. D. pulex.

Genus 4. Lymeeus.

Genus 5. Polyphemus. Spec. 1. P. oculus.

Genus 6. Cypris.

Familia 6. Pli yllopoda.

Geuus 1. Branchipus. Spec. 1. Br. stagnalis.

Genus 2. Artemia. Spee. 1. A. salina.

Genus 3. Apus.

Spec. 1. A. cancriformis.

Spee. 2. A. productus.

Genus 4. Limnadia.

Spec. 1. I. Hermanni.

Familis 7. $\mathrm{X}$ ip hos ura.

Genus 1. Limulus:

Spec. 1. L. inoluecanus.

Familia 8. Trilobites (Paläaden).

Genus 1. Paradoxides.

Genus 2. Agnostes.

Genus 3. Callymenc.

Genus 4. Asaphus.

Genus 5. Ogygia.

Genus 6. Serola (alle fossil).
Ordo II. M y r a poda (Tuusendfüssler).

Familia 9. Chilopoda.

Genus 1. Seutigera. Spec. 1. S. araneoides.

Genus 2. Geophilus.

Genus 3. Scolopendra. Spec. 1. Sc. morsitans.

Genus 4. Lithobius. Spec. 1. L. forficatus.

Familia 10. Chilognatla.

Genus 1. Polyxenus. Spec. 1. P. I,agurus.

Genus 2. Polydesmus. Spec. 1. P. complanatus.

Genus 3. Craspedosonia.

Genus 4. Julus. Spec. 1. J. terrestris.

Genus 5. Spirotreptus.

Genus 6. Spiropocus.

Genus 7. Glomeris.

Genus 8. Sphaeratheriun.

Genus 9. Spacropoeus.

Genus 10. Polyzonium. Spec. 1. P. germanicum.

Ordo IIL. I s o p o d a (Asselartige).

Familia 11. Oniscina.

Genus 1. Asellus.

Spec. 1. A. aquaticus.

Genus 2. Porcellio. Spee. 1. P. scaber.

Genus 3. Oniscus. Spee. 1. 0. murarius.

Genus 4. Philoscia. Spec. 1. P. muscorum.

Genus 5. Ligia.

Spee. 1. L. hypnorum.

Spee. 2. L. occuniea.

Genus 6. Jaera.

Genus 7. Oniscoda.

Genus 8. Arcturus.

Genus 9. Idotea.

Genus 10. Anthüra.

Genus 11. Sphaerouna.

Genus 12. Cymodoca.

Genus 13. Limnoria. Spee. 1. L. terebralls.

Genus 14. Bopyius. Spec. 1. B. squillarum.

Genus 15. Cymothoa.

Genus 16. Anilocra.

Genus 17. Cirolana.

Genus 18. Anceus.

Geuus 19. Pranizza. 
Ordo IV. Microcephala.

Familia 12. Laemodipoda.

Geuns 1. Cyamus. Spec. 1. C. Ceti.

Genus 2. Caprella. Spec. 1. C. linearis.

Familia 13. Pyenngonida.

Geus 1. Pyenogonum.

Genus 2. Plıoxiclıilns.

Geuus 3. Nymphon.

Ordo V. A m plı ipoda (Flohkrebse).

Familia 14. Gammarina.

Genus 1. Typlis.

Genus 2. Plıronima.

Spec. 1. Pl. sedentaria.

Genus 3. Themisto.

Genus 4. Hyperia.

Genus 5. Corophium.

Genus 6. Atylus.

Genus 7. Gammarus.

Spec. 1. G. pulex.

Ordo VI. Stomatopoda.

Fumilia 15. Bipeltata.

Genus 1. Pliyllosoma.

Familia 16. Unipeltata.

Genus 1. Squilla.

Spee. 1. S. mantis.

Genus 2. Alima.

Genus 3. Erichthus.

Genus 4. Gonodactylus.

Genus 5. Zoëa.

Genus 6. Mysis.

Gemns 7. Cynthia.

Genus 8. Lucifer.

Genus 9. Thysamopoda.

Geuus 10. Nebalia (noch wegen der Stellung nieht klar).

Ordo VIl. De e a poda.

Familia 17. Macroura schwänzige Krebse).

Tribus 1. Carida isalikoken).

Genus 1. Pasiphaë.

Genus 2. Sergestcs.

Genus 3 Acetes.

Genus 4. Palaemon.

Genus 5. Crangon. Spec, 1. C. vulgaris.

Genus 6. Paudahıs.

Genıs 7. Nika.

Genus 8. Alpheus.

Genus 9. Peneus.

Genus 10. Stenopus.

Genus 11. Atya.

'Tribus 2. Ast ncina (Hummern).

Genus 1. Astacus.
Spec. 1. A, fluviatilis.

Spec. 2. A. marinus (Homarus, der Hummer).

Geuus 2. Neplirops.

Spec. 1. N. norvegicus.

Genus 3. Callianassa.

Genus 4. Gebia.

Genus 5. Megalops.

Genas 6. Poreellana.

Genus 7. Galathea.

Genus 8. Eryon (fossil).

Tribus 3. Loe ustae (Langousten).

Genus 1. Scyllarus.

Spec. 1. S. arctus.

Genus 2. Palinurus.

Spee. 1. P. vulgaris.

Tribus 4. Anomala.

Genus 1. Pagurns. Spee. 1. P. Bermhardus. Spee. 2. P. elypeatus.

Genus 2. Birgus. Spec. 1. B. Latro.

Genus 3. Hippa.

Genus 4. Remipes.

Genus 5. Albunea.

Genus 6. Glancotloe (nocli zweifelhaft hinsichtl. d. Stellung).

Familia 18. Brachy ura (kurzschwänzige Krebse, Krah. ben).

Tribus 1. Notop oda.

Genus 1. Ranima. Spec, 1. R. elypeata.

Genus 2. Dromia. Spec. 1. D. Rumphii.

Genus 3. Dorippe.

Genus 4. Homola.

Tribus" 2. Trigona.

Genns 5. Lithodes.

Spee. 1. L. arcticus.

Genus 6. Paetolus.

Genus 7. Inachus.

Genus 8. Leptopodia.

Genus 9. Mitlurax.

Geuus 10. Micippa.

Genus 11. Maja.

Spec. 1. M. squinado.

Genus 12. Parthenope.

Spee. 1. P. uspera.

Tribus 3. Cryptopoda.

Genus 13. Calappa.

Spec. 1. C. granulata.

Genns 14. Aetlira.

Tribus 4. Orbiculata.

Genus 15. Leueosia.

Spec. 1. L. nuclens.

Genus 16: Corystes.

Spec. 1. C. personatus. 
Tribus 5. P in u ipedia(Schwinumkribben).

Genus 17. Matuta.

Genus 18. Orithyia.

Genus 19. Podophthalmus.

Gcnus 20. Portunus.

Spec. 1. P. Dufourii.

Spec. 2. P. maenas (gemeine Krabbe).

Tribus 6. Arcuata (Bogenkrabbeil).

Genus 21. Cancer. Spec. 1. C. Pagurus (Tasehenkrebs).

Genus 22. Atelecyclus.

Genus 23. Thia.
Tribus 7. Quadrilatera.

Genus 24. Eriphia. Spee. 1. E. spinifrous.

Gcnus 25. Tclphusa. Spec. 1. T. fluviatilis.

Genus 26. Gonoplax.

Genu's 27. Gelusimus. Spce. 1. G. voeans.

Genus 28. Oeypoda.

Genus 29. Pinnotheres. Spee. 1. P. Vetcrum (Pinnophilax Antiç.).

Genus 30. Geeareinus. Spee. 1. G. ruricola.

Genus 31. Grapsus. Spec. 1. G. varius.

Vom wunderlichen Aretiscon und dem winzigen Cypris und Cyclops an bis zu dem Palinurus Lalandii, der Scylla serrata (Deh.) und dem Hummer, welch eine seltsame Plastik der Formen I llier lange Scheeren, ein Körper wie ein Churhut geformt, mit Perlen und Stacheln besezt (Lambre spinimane) und dann wieder nur rauh, wie Chagrin (Coriste denté); ein Leib, dem einer Butte ähnlich, schwanzlos, kurze haarige Fühler, zehn Füsse, die Scheeren kurz, zusammengedrïckt, reihenweisc mit Körnern besetzt (Attelé cycle à sept dents); wie yon Laich bedeckt der runde Rückenpanzer, die Kneipfüsse wie von dem Käfer Chalcimon Humboldti entlehnt (Portunne varie). Lange hat dort die Natur verweilt, dem Portune Etrille ( $\delta$ ) seine Füsse auszuarbciten, so zierlich, wie wenn es gälte, cin Gesellenstück aufzulegen und wāhrend sie dem Portune marbré borstige Fühler schenkte, pflanzte sie bei der vorigen zwei Halbmonde zwischen hinein. Kurz gestielt vergab die Mutter dort die kostbarsten Geschenke unseres Erdenlebens, die Augen; am stacheligen Podophthalmus verlängerte sie die Stiele und machte sic wie deichselförmig, mittlcrweile sie ihm die Vorderscheeren wach dem Muster des Chiasognathus ausschnitt; drohende Gebärde, wio gefrässige Rachen von Esociden machen die Scheeren des Lupée pelagique. Schön symmetrisch bildete sie den Polybie de Henslow und die Matute vainqueurir aus der Familie der kurzschwänzigen Decapoden; Kraft und Trotz offenbaren sich bei der Crabe Tourteau und Xanthe floride, in den rundlichen Scheeren und glänzenden, lanzenspitzigen Seitenfüssen. Zierliche Bänder und artiges Schnitzwerk vergab sie an Piriméle denticule und yorzüglich an Hénate fascié; machte fast dienstuntauglich die Zangen der tuberkulösen Calappa, und bildete auf dem Rücken der Oethre déprimé ein wahres Gebirgsrelief. An der Scite des schlanken, nierenförmigen Leibes des staclieligen Pilumnus dräuen, wic Sensen, die scharfen, krummen und einzahnigen Scheeren; den famosen Tourlourou färbte sie mit zierlichen Flecken und begahte ihn mit ziemlich starken Waffen, reich besezt mit Dorneı, wälırend der quadratleibige, kleine Ocyporle über den Augen Hörner trägt (Ceratoplithalme), welche sich bei der Gélasime de Marion und dem Gonoplace rhomboìdale wie Trommelschlägel ausnehmen, auf denen 
erst die Augen des Tambours sitzen. Keulenförmig ist die Hand, die rechte und die linke, bei einer Plagusia und die Stirne einer Eriphia (Front-épineux) strotzt von Stacheln wie ein Igel. Sie muss besondere Feinde haben, weil sie dic groben Hïnde mit den zahnreichen Scheeren nicht $z$ u schützen vermögen. Bei der erstern hat Natur Zeichnungen angebracht, wie man sie auf den Denksteinen der wilden Völker Indiens findet und die Schenkel längs gestreift; aber die geschwollenen Hände mit Bürsten versehen bei Grapse Porte-pinceau, dort dieselben verkümmert (Gr. peint), und bei Leptopodie sagittaire den Leib wie nichts geachtet, ihn winzig zum Ëbarmen gemacht mit einem Stirnstachel versehen, dagegen die Füsse so lang gestreckt, dass sie einem Weberknecht ähnelt; bei einigen andern hat sie die Wolle nicht gespart (Dorippe laineuse) und die Füsse pfriemförmig gebildet und das letzte Paar hakenartig eingekrümmt, eine Dromia ganz in Haaren eingewickelt. Wenn die Ranina dorsipes, die, wie der Name andeutet, ihre Beine auf dem Rücken trägt, und ganz einer Mecrassel halb, halb einem Glomeris ähnelt, so überraschen die zizenförmigen Erhabenheiten einer Orithyia auf der Oberflïche ihrer Kalkschale, von denen sich besonders 2 wei grosse auszeichnen. - Schreckhaft hat mit der Parthenope horribilis die Natur verfaliren; sie wollte ein Schreckbild vorstellen und es ist ihr gelungen. Das Herz des Krabben hat sie nicht verändert, wohl aber mag manchem Thier der Muth sinken bei ihrem Anblick, denn sie ist über und über mit Stacheln, Dornen, Höckern und Warzen bedeckt, wie cin Ast von einer wilden Rose, und ihre Hände endigen in wunderlichen Zangen oder Seheeren, welche an éiner Eurynome so zart auslaufen, wie eine ChirurgenPincette, vorn aber tüchtig gezähnelt sind. Die der Jagdgöttin, Diana von Ephesus, heilige Maja squinado (Seespinne) von fleischrother Farbe zeigt hübsche Verhältnisse und ihr ovaler Rückenschild ist warzig: wunderlich dagegen hat der Schöpfer mil einer Pisa (tétraodon) verfahren, deren Scheeren wie gewisse Zangen eingekerbt sind; eine Micippa hat er mit lauter Erbsen besäet. Die Säge des Hayfisches repräsentirt Pactolus Boscii im Kleincn und gleichsam als sässe das vegetative Leben in den Extremitäten, ist der Kürper bei Macropodie faucheur an Umfang bis zum kleinsten reduzirt. Die Scheeren des Scorpion weisen die Hünde eines Inachus (Scorpion $\delta$ ), frappant denen des seltenen Opilo Hellwigii ähnlich. Mehre dieser Sippe führen diese Waffe. Durch Farbenpracht ausgezeichnet sind hesonders die Thalamiten, die Gattungen der Sippe Lupa und viele Grapsiden. Die Beine der Lupa pelagica sind blau und roth, und der Schild von Goniopsis picta spielt roth, grün und gelb. Am häufigsten und schönsten gefirbt sind diese Thiere in den heissen Ländern.

Telphusa und die Myriaden von Gelasimus hausen im weichen Sumpfboden an den vielen Flüssen der Bucht von Rio-de-Janeiro. Letztere, beunruhigt, richten sich auf ihren Füssen empor und drohen mit ihrer dickeren in die Höhe gerichteten Scheere; sie fliehen erst, wenn sie daran sind, gefangen zu werden, während die Tourlourous sich am Eingang ihrer Wohnung halten und bei der geringsten Gefahr sich zurück- 
ziehen. Beim Graben ihrer tiefen und schmutzigen Wohnung sieht man sie ganz beladen unit Koth, den sie mit Hilfe ihrer Scheeren in einiger Entfernung aufhäufen.

An der Meeresküste dieses Landes wohnen beständig unter dem nassen Sand die lichtscheuen Hippae mil ovalem, fast walzigem Kopf; sie werden gefangen indem man blos mit der Hand den Sand aufwühlt, und gern gegessen. - Portunus und Maja halten stets in Grund der Gewässer ; von lezteren fand sich, als wir bei unserer Abreise von Brasilien die Anker lichteten, eine grosse Menge an den Tauen nebst Millionen von Nymphen, deren lange schmale Füsse an einem sehr winzigen Leibe hïngen.

Ueberall, wo die Küsten buchtig und das Wasser etwas seicht, sind die Crustaceen häufig, wie an Isle de France, den Mariannen, den Papous-Inseln, der Sechundsbay etc.; wo aber steile Klippen und kein Strand, da finden sich bloss die grossen Gattungen in geringer Anzahl, wie an Bourbon, Port-Jackson, den Sandwichs-Inseln. An Owyhyhi im Haven Toyai fängt inan mit der Angel aus einer Tiefe von 6 Faden eine grosse, rothe Ranina, deren platte und mit Ausnahme der Seheeren zum Schwimmen geeigneten Füsse den gewöhnlichen Aufenthalt im Wasser bezeichnen, so dass sie also schwerlich, wie einige Reisende angaben, das Meer verlassen, um sich auf die Gipfel der höchsten Bäume z.u machen.

Die Einsiedlerkrebse (Pagurus), übcrall häufig, am meisten an den Mariannen, Papous-Inseln, Timor, Coupangbucht bleiben bei grosser Hitze unter Gesträuch; an Abend kommen sie zu Tausenden hervor und kündigen sich durch das Geräusch ihrer Sehalen an. Bekanntlich sind ihnen alle einklappigen Schaalen recht; hier aber sahen wir die meisten in Neriten, weil diese hier sehr gemein sind. Bei Gefahr schlüpfen sie in das nächste beste Loch oder lieber unter die Wurzeln oder faulen Stämme alter Bäume, selten ins Meer, wenn es auch ganz nahe sey. Nach dieser Beobachtung lassen sie sich vielleicht in zwci Familien trennen, in solche, welche gewölnnlich im Wasser und in solche, die gewöhnlich auf dem Lande wohnen; erstere unterscheiden sich durch runde Augen auf langen walzigen Stielen.

Auf Guam und Vaigiou finden sich über 1000 Schritte von der Küste in den Wäldern schr grosse Paguren mit violetten Scheeren, in Buccinis, mit einer Frdkruste bedeckt; einige geben, wenn man sie quält, einen Schaum von sich. Sie gehen dem Lichte nach; einer kam von Weitem auf das Nachtfeuer der Matrosen zu, wurde gefangen und gekocht.

Phyllosoma trafell wir zuerst in November 1817 unter $5^{\circ} \mathrm{B}$. und $56^{\circ}$ W. L. v. Paris auf dem Wege von den Canarien nach Brasilien, seitdem bei Neu-Guinea unter $2^{0} \mathrm{~N}$. B. im Januar; in grossen australischen Ozean unter $18^{\circ}$ S. B. und bei den Freundschafts-Inseln im Oktober. Lebend sind sie ganz durchsichtig wie Crystall, mit Ausnahıne der himmelblanen Augen; die gelbe Farbe der Exemplare in den Sammlungen kounint vom Alkohol oder von der Vertrocknung. Wegen ihrer Durehsichtigkeit hemerkt man die Fussmuskeln und einige in 
einen Lăngskinal mündende Seitenkanäle, die im todten Zustande unsiehtbar sind. - Diese Thiere leben wegen iher Gebrechlichkeit ferne von den Küsten; ilıre langsame Bewcgung stach sehr ab gegen die Lebhaftigkeit der mit ilnen gefangenen Alimen, welche gleichfalls durchscheinend sind. ( $Q$ u oy und G a imard, über das Betragen und die geographische Vertheilung einiger Crustaceen: Amnales du Mus. d'hist. nat. XIV. 1828, p. 259. - Isis 1834, 1026.)

Wie schon erwähnt worden, ist die Ausbeute von Crustaceen innerhalb den Küsten sehr gering; wenigstens seheint es noch so.

An jene horrible Partenope erinnert uns der Anblick des grossund langgefussten Lithodes aus der aretischen Hemisphäre; auch er ist so stachelig wie jene und seine rechte Scheere ist grösser (q), was bei den Krabben öfter vorzukommen pflegt. - Hymenosoma ist wie cine Testudo gebildet und ihr Sehild ist getäfelt und rund; eine Egeria aus Indien hat das 2 weite Fusspaar ausserordentlich verlängert, wie ein Phalangium. Ilia nojau führt Scheeren, gleieh einer feimen Pinzette; cbenso die stachelige Areania und mehre Gattungen Ixa. Bei den geschwänzten Krabben Hippa und Albunea vertreten die Scheeren die Stelle von Rudern und sind verkümmert; es treten Fühler, lang und zierlich, auf und die Leibesform geht in Sehlankheit über; während bei Pagurus Bernhardus vorn alles streitbar ausgerüstet ist, befindet sich der Schwanz in cinem Zustand von armseliger Nacktheit. Dio Natur legte den Trieb des Einquartierens in leere Gehäuse in ihr Wesen. Läiglich und breit mit fast vie reckigem Rückenschild und platt ist Scyllarus Arctos, auf dem Schwanze voll dentritischer Zeiehnungen. Die ungeheure laangouste (Palinurus vulgaris) zeigt schönen, fast cylindrisehen Bau, sehr lange, starke, stattliche Fühlhörner und einen Sehwanz wie Astacus.

Squilla Mantis wiederholt unter den Krebsen die ganze Struktur der Mantis oratoria unter den Insekten, den gefrüssigen Urthopteren. Die vielen abenteuerlichen Bedeckungen der Crustaceen sind entweder hornig oder kalkig. Manche werfen dieselben öfters ab, namentlich die mit Kalksehalen, ersetzen verlorene Glieder. Fleisehfressend sind sie, räuberisch und oft sehr kühn; in ihren Verrichtungen äussern sie Beweise von vielem Instinkt.

Unter den kleinen Entomostraceen sind viele Sehmarotzer, vorzüglieh die Lernaeen, welehe sich an die Kiemen versehiedener Fische anhängen; so aucl die Dichelestinen und die beweglielıen Caliginen, z. B. Argulus foliaeeus, der auf Kaulquappen ete. parasitiseh lebt. Der molukkisehe Krebs so!l mit dem Stachel, in welehen sein Hornsehild ausläuft, tödtlich verwunden, weil or giftig sey. Die meisten Myriapoden gehen nur des Nachts ihrer Atzung nach; sie rauben nach unsern Beobaehtungen Alles, was sie von Insekten erhasehen, nur muss es leben. Die grosse Scolopender (Sc. morsitans) ist giftig und ihre Zangen seheinen hohl und die Giftzähne zu wiederholen. Eine Assel (Limnoria terebrans) zerstört, wie Thoms on sagt, an den Küsten die Sehlcusen, was wir nicht glauben, sondern dem Teredo zusehreiben. Monoculus Apus soll 
durch Wühlen den Reissfeldern schädlich perden (?) (rgl. Bronn's Reise I, 142). - Auf dem Wall lebt in grosser Anzahl Cyamus Ceti. - Der possirliehc Gammarus pulcx scheint von Cyelops und Cypris ctc. zu leben. - Manche Stomatopoden (Mysis, Cynthia etc.) leuchten. Die Eigensehaften, phosphorisch zu leuelıten, besitzen noch Cancer fulgens, Cyclops quadricornis, Scolopendra electrica etc. - Die meisten Decapoden leben im Meere, weniger in süsscn Gewässern; aber cinige in Morästen und Wäldern und gehen, wemn sie liehtscheu (?) sind, wie Hippa, nur zur Nachtzcit in Begleitung der andern Gesehlechtsverwandten aus. Der Schaden auf Reisleldern scheint nicht von Krebsen herzurühren. Die Gangarten sind vor- und rückwärts, nach der Seite. - Pagurus clypcatus, so sagt Milne-Edwards, lebt in den Gehäusen von Landschnecken am Meeresstrand und weiss sich geschickt ins Meer zu rollen, weun er Gefahr befürelıtet, wie unter den Insekten die Curculioniden thun (ins Gras). Die Gattungen der Sippe Grapsus schlagen, verfolgt, die Scheeren geräuschvoll aneinander, wälırend sie iın seitlichen Laufen ins Meer füchten und Gelasimus voeans soll den Eingang seiner llöhle mit der einen grössern Scheere schliessen. Manche verscharren sich schleunigst im fcuchteı Sande.

L'instinet des Crustaeés est en géneral assez médioerement développé. Les crabes et ceux qui appartiennent aux genres voisins, sont ceux chez lesquels il semble avoir le plus de finesse. Ces animaux en effet paroissent très-rusés, surtout lorsqu'il s'agit d'échapper à leurs ennemis; alors on les voit parcourir le terrain ave beaucoup d'avantage, en choisissant pour retraite les licux du plus difficile aceès. Plusieurs d'entre eux dont la carapace est très-tendre, comme lcs pinnothères, font leur résidence habituelle dans les valves de certains mollusques, tels que les moules et les pinnes inarines, ct d'autres qui ont un abdomen mou et vulnérable (les pagures et les birgus) le placent soit dans des eavités de eoquilles univalves abandonnées, soit dans des ereux de rochers, afin de le préserver; et eeux-ei ehangent de demeure à certaincs époques lorsque leur corps a grossi, afin d'en choisir une nouvelle plus commode. Quelques crustacés maeroures (les thalassines) s'cnfoneent dans le sable ou la vase pour se dérober à la poursuite de leurs ennemis. Les dromics dont les quatre pattes postérieures sont relevées sur le dos, les enploient à retenir sur cctte partie un aleyon de la nature des éponges qui, en s'aceroissant, finit par envelopper tont le dessus de leur carapace, comme une sorte de manteau etc.

Les eymothoés et les isopodes voisins; les caliges, les bopyres, qui vivent eomme parasites sur le corps des cétacés, des poissons, ou mẻme sous la tête d'autres crustacés, possèdent une qualité instinctive qui leur fait distinguer les êtres sur Icsqquels ils peuvent se fixer, et les parties de ees êtres où ils doivent se placer préférablement pour trouver la nourriture qui leur eonvient.

Les crabes de terre, appelés Tourlouroux dans les iles, ont I'habitude constante de se réunir à une certaine époque de l'année en 
troupes innombrables, et de mareher par le plus eourt ehemin, vers la mer, sans s'inquiéter des obstaeles qui se trouvent sur leur passage. Après la ponte, ils se rassemblent de nouveau, pour retourner à leur uncien domicile.

Quelques especees de différens ordres vivent toujours en soeiétés nombreuses et nous eiterons particulièrement les erangons, les talitres, et la plupart des petits entomostracés, surtout les daphnies, dont la couleur donne quelquefois à l'eau une teinte rouge assez foneée.

Les erabes sont eourageux, et lorsqu'il ne leur reste plus de retraite, ils avaneent fièrement leurs serres, et cherehent à peineer avec leurs doigts, ee qu'ils font trés-fortement en raison de leur taille. Quelques uns, en serrant ees doigts avee foree et rapidité, produisent un bruit ou un elaquement remarquable, et, eomme ils tiennent trèsélevée la serre avee laquelle ils produisent le bruit, on leur a donné le nom de erabes appelans.

Quant aux autres erustaces, ils n'ofrent rien de remarquable dans leur instinet, si se n'est dans le soin qu'ils ont d'éviter leurs ennemis. - Desimarest, Considerat. génér. 1825, p. $37 *$.

* Chapitre VIII zu Desmarest's Considerations genérales sur la elasse des Crustaris. Paris 1825 , p. 396-420, ist selır mangelhaft. Natiirlieh köınen die seit dem Erscheinen dieses kostbalen Werks (das dureh Mi In e-E d wards nenem bis auf die sebönen Tafeln so zienlieh entbelırlieh geworden ist) gedruekten Quellen nieht dariı aufgefüht seyn; aber vou den ältern, vor 18.2 und viel frïler erschienenen Werken und Abhandlungen fehlen die von Selirank (bei den Topographen), A bildgaard, Baker (n letter concern. ete), Berniz. (Chela, Gummarus leprosus), Buble (de fontibus), Brïnnieh (Del barbugede etc.), Bullivant (Letter eoneern. etc.), B ii chner (de rurioribus ete.), B г с y nius (de inseetis), B a icr (Sciagraphia), Boyle (General heads), Burgsdorf (Naturh. Beschr. ete.), Br unelli (de flumine Amazouuu etc.), B a r rere (Essai sur l'hist. unt. ete.), Cun ing ham (a eatalogue ete.). Carus (voul den Auford. ete.), Cat (An account. ete.), C a mper (rool. Bem.), Chiaje (Mémorie etc.), Claytou (Letter-gwing ete. et areonnt. etc.), Chemnitz (Beschr.), Condamine (Extrait ete. et Relution ete.), Cam a ra (Ensaio cte.), Caille (Observations etc.), Duméril (Elements et Traité etc.), Dutroehe t, D al d o r f (Uddrag etc), D e f a y (Zool. Bem.), Dul man (Nagra Petrificater), Fraucendorfer (De partu millep. ete.), Flinders (a voyage), Forster (Sperim. hist. nat. volg. ete.), Fahlberg (Utdrag af Sumlingar), Garmann (Seolopendrae), Geer (Observation sur une espeee ete, et Obs, snr me Jule), Gasea (Amenidudes), Godeheu (Olsservat.), Gunner (Nogle smaa ete.), Guéttard (Mém. sur plus. m.), Giildenstedt (Zusammengetr. Beitr. etc.), Gerlan (Essai), Grimm (Gammari in lap. collv.), Geier (de veruiee), Guyot (Buffet), Gray (Sever. micr.), Goekel (Epistola de mirab. Diese und einge andere Dissertationen konnten wohl in Desmarest's Werk wegbleiben), Grouvius (Animal. in Belg. et Observ. de aniuale.), Herbst (Besclor. e. s. sond. Seclaus), Hire (Olserv.), H a in (Experinentu), Hespel (Verliandel.), He a r ne (Memoriabil.), Helb ig (Gammari terrestr.), II a bliz.I (Observ.), Ha cquet (Ueber Verseh.), Hard wie ke (Nirrat.), Hill (Inquir.), Jones (Letter concern.), H. Jo nes (on usint. Hist.), King (a letser eoneern. crabslyes), K amel (de piseibus ete.), König (Nat. Naelır. a. Tranquebar), Kulm (Rïnoch ammirk. etc.), Limnaeus (Animal. p. suer. ob.), Löffling (Monoenlus ete.), Landes (Observ. sur la génér. des sol.), Link (Versuch), Lentilius (Addenda), Lewen heimb (Messis observat.), Lhw yd (Letter contain.), Lehmann (Onderzoek etc.), Lorimer (Letter), Lee uwenhoek 
Ueber den Fang ist nicht viel zu sagen; indessen wollen wir hier angeben, was wir aus lirfahrung wissen. - Wie man sich unseres Bachkrebses bemäehtigt, ist. bekannt und mehres mag man in lirandt und Ratzeburg's mediziniseher Zoologie naehlesen. Bei uns, in der Pfalz und im Salzburgischen, so wie um Laibach und Klagenfurt, lebt der Astacus Mlolitor (Nobis) und bei Dachan Astacus Torrentinm (Schrank). In allen Quiellenbächen Deutsehlands bemerkt man kleine Punkte mit freiem Auge, die sieh um ihre Achse langsam drehen: das sind theils Cypris -, theils Cyclops-Gattungen. In ihrer Gesellsehaft kommen die, Daphnien, Flohkrebse ete. vor, die man wegen ihrer Menge leicht bemerken kann. - In den Fahrgleisen suehe man nach Sommerregen den Branchipus, der trübes Sehlamınwasser liebt, und den Polyphemus oculus. Vom Lenz bis zum Ausgang Juni finden sich fast alle Cyclopsgattungen 0 . Müller's zwisrhen Wasserlinsen; oft sehon im März, so auch die Amymonen, die mit den Blumenpolypen und der Hydatina senta gesellig beisammen wohnen; immer im reinen Wasser und wohl öfter in den Bläschen der Utricularia und zwisehen der Wasserscheide. (Lyneeus). Die Daphnien ziehen als Aufenthalt die Pfützenwasser vor, überhaupt die stagnirenden, die nicht gar zu trüb sind; selten erseheinen

(a specimen ; Epistol. posth.), Lie (Efterretning), Latreille (Les Langoustes), Lochead (Obscrv.), Loewe (k. Naturg.), I,ichtenatein (Dic Werke von Marcorave etc.', Leconte, Lutgeus (Phys. Beschr.), Macrter (Reise und natur. Nachr.). Müller" (Anmirk., u. Ontvende smia ete.), Mayer (Collect. lap.), Miller (Account of the Isl.), Marsde n (Arcount of a plorn.), B1 a loet (Observat. sur un verete.), Miutzashefahl iNachricht ete.), Maun (Mém. sur l'hist. wit.), Mor t imer (vgl. Brown), Maecouloeli (Caucer Phalangium), Nicharlis (Oratio), Marteus (Rerise H. V.), Montagu (Remarks on Sculopendra: Deser. of several etc.), Muralto (Destr. insectorum), Malezieu (Observ. sur jes Anim.), Nicola (Au casy method. ete.), Pie ardet (Observo), Petiver iRemarks ete. Maryland; Rem. ete.), Pezolilt (De Palingenes.), Plantade (Memoire), Pallas (Tableau), Portius (De cancri fluv.), Pa r ry (Journ. of a voyatre), Pirkering (mit D a u a: ('aligns americauss), Perk (Metlod ete.), Reaumur (Moyen etc.), Reuss (Repert.), Ricluardson (Part of a lett., sev. obs.), Ramdolır (Abbi.). Ruslu (An arcount etc.), Robineau-Desvoidy (Recherches), Ratlike (Anatomie der Idotea), Roffredi (Lettres etc.), Ritter ('Tentamen; Meletemata), Razonmovsky (Essai), Reineggs (Schreib.), Sehäif er (Der Fisehf., Kiefenf.), Stewart (An account efe.), Setzen (Beitro), Sibbald (Part of a lett.), Spengle r (Beskrivelse of en nya art Kraebs), Seh lothe im (Ueber Verstein.), Strachan (Observ.), Sparmann (An account), Seilla (de corpe. Mar.), Spix (Ges. und Beur.), Stark (Elements), Stobacus (Observ. de lapill.), Sha ow (Remaiks on Scolopendla), Ström (om silde rte.), Stafford (Extruit of a letter), Scoresby (An account; - Jourmal), Stubls (An clargemeni), Tilcsius (de eancris rantscl.s, Thunberg (Nov. ins. sp. - Oniscus corntlinus: - Ett kort uddrag cle.), Thoresly (Letter eoncern), Taveruicr (Observations concerning etc.), Tow ns (Extrat of a lett.), Vandelli (Flor. pt Faun.), Vale ntini (Chela astac.), Wulfen (Descript. zool.: Winterbclust.), Weigel (Suppellex etc.), Whintrop (An extract etc.), Wris s (Olsserv. div.), IVurfbain (de oculis cancror.), Wodel (de cuncro in cunrru), Walter (necte Trilab.), Warder (newe Trilob.), Wilcke» (Naclır.), IV a rgenti (Om Zoologien etc.), Wileke. (Rön i nitural historien ete.), Zouyef (Reflexions). - Anonymus (de cancro publico). 
sie elier als Mitto Mai's. Leicht zu bemerken ist ein kleiner rother Punkt, kaum 1"'t betragend. Das ist Daphnia pulex. Sie ist so durehsichtig, dass man das Herz pulsiren sicht und an 200 Zusammenzielıungen in der Minute fühlt; sie vermehrt sich ungeheuer und fürbt Strecken im Wasser roth. - Ganz recht hat Thic neman n, der sagt, dass im Sommer nur Weibehen zur Welt kommen, im Herbste Männehen. Ob sie sich ohne Begattung fortpflanzen, muss erst untersueht werden. - Ueber Winter bleiben Eier am Leben, wenn Kälte dic Alten tödtet. - Selten, wie Branchipus, findet siel Apus eaneriformis in Fahrgleisen und in der Nähe ausgetretener Gewässer. Er sieht grün aus und macht sich durelı : sein sehnelles Sehwimmen, rücklings und gewölınlich, bemerkbar.

Die deutsehen Crustaceen lassen siclı an folgenden Orten auffinden :

a) In Teichen: Argulus Delphinus (selten);

b) in schlammigen Gräben: Apus eaneriformis (z. 8.), Lynceus sphaericus, trigonellus und alle Verwandten, etwa 6 noch; ferner sämmtliehe Cypris (s. g. conehacea und lutaria). Hr. Forstrath Koeh hat um Regensburg 18 Species entdeckt. - Branehiopoda stagnalis;

c) in Quellenbächen: alle Daphnien. Die exspinosa, pennata und ramosa sind die gemeinsten. - Cyelops in 15-18 Speeies; davon sind pietus, agilis, vulgaris und quadricornis die häufigsten. - Gamma* rus Pulex und fossarum, Onisens aquaticus;

d) in Brunnen: Gammarus pu:aneus (Koch);

e) in Altwassern, Berg und Steiubåehen; Astacus fluviatilis, tristis, Torrentium, und saxatilis (alle in Bayern); dann Molitor, Aretiscon, die Parasiten ;

f) unter Moos und Laub der Wälder: Ligia melanocephala, Glomerir pustulata, alle Juliden, Pollyxenus lagurus;

g) unter Steinen: Jtea losea, riparia, Oniseus fossor, Poreellio laevis, nodulosus, Armadillo vulgaris und variegalus und viele Julus;

h) in Keller'n etc.: Oniseus asellus, Porecllio seaber;

i) Unter Baumrinden und Ilolzmulm in Wälderu: Poreellio sylvestris ;

k) unter Erdschollen: Mancho Juliden und Polydesmus complanatus, Lithobien und Geophilen.

Zwischen den Secgewächsen der Meere marlit man gewisse Ausbeute von Krebsen (z. B. Nymplion, Caprella, Crangon. etc.). Palaemon Squilla (Garneele) lebt in grösster Menge unter Büsehen der Ruppia und Zostera an Mceresküsten, so wie auf Fueus und wird am leichtesten init dem Stossnetze gefangen. Des Ifummers bemiehtigt man sielı nit Reussen und Köder. - Eine Manier, sehnell Krabbeu zu fangen, haben wir in Dalnatien von Knaben erlernt, welche männliche Krabben mit Bindfaden am Ufer himabliessen, wo sie glaubten, dass sich welche befinden; sie warteten gar nicht lange und zogen meist zwei und mehre Weibehen zu 'Tag auf. Fs ist immer dasselbe, ob man Weibchen oder Mănnehen hinabscnkı; zu den Weibehen kommen natürlich die Männehen. - Aueh den Eidechsenfang haben wir in 
Süden qualifizirt. Wir gebrauchten gewöhnliche Fischangeln mit Insektenköder (aber lebendigem), und die kehsen liefen hungrig, was sie konnten, und fingen sich. - Zwisehen Felsen, zur Zeit der Bbbe, fingen wir die Kralbben am besten init der Hand und $z$ war so, dass wir sie mit den Fingern seitlich fest paekten und den Cephalothorax niederdrückten, zu verhindern, dass sie nieht kneipen konnten. - Als Hauptregel beim Fang merke man sich Nachstehendes. - Unsern eigenen Erfahrungen zufolge, welche wir an den Küsten der Mcere gemacht haben, sind alle Wasserkrabben lichtliebend. Dem Lichte eilen sie zu wie eine Menge Gesehöpfe aus dem Reiche der Thiere. - Das ist schon bei unserm Flusskrebs der Fall, der unter Liehtschein von den Landleuten am häufigsten gelangen wird. - Die mit Mladreporiden, verwitterten Sehalen und Gehäusen, ausgehöhlten Stämmen bedeckten Sandhänke und Terrassen beherbergen eine Menge grösserer und kleinerer Crustaceen, und man kann sich davon erst Vorstellung Inachen, wenn man bei Nacht sie init Licht freiwillig aus dem Labyrinthe von Scetang, Corallinen, Zoophyten aller Art und Grösse, den Algen ete. lockt, zwischen welchen sie so gerne leben und wo die farbgeschmückten Medusen, die zellentragenden Anthozoen, die spritzenden Anemonenpolypen (Aetiniac), Celleporen, die proteutisehen Gestalten der Acalephen, eleganten Holothurien und eine Menge der versehicdenartigsten Würmer und Mollusken ihr Wesen treiben oder ausruhen. - Es ist ein Hochgenuss für dlie habsüehtigen Forseher, die sie alle sind. Die scllauen Krabben wählen meist solehe Plätze, welehe von der Brandung nicht erreicht werden. Viele graben sich selnell in den Sand ein, hat man sie aus ihren Verstecken getrieben, wic bereits oben bemerkt wurde. - Unter Steinen und Holz sitzen die sehlechten Sehwinmer unter den Krabben, träge und still, oder in tiefen Uferlöchern, wo sie auswärts schauen, die gestielten Augen nach allen Seiten wenden und Frass erwarten. Nahet eine und mehre Balanen, so kommt es ihnen, besonders den loealen, sesslaften nieht darauf an, diese auf Lebenszeit auf ihrem Rüeken sitzen und sehınarotzen zu lassen. Auch mit deın Aasköder haben wir manehen Krebs gefangen. Sie sehlagen vergebens die mächtigen Seheeren zusammen und sehleudern, wie inanche Spinnen thun, ihre Beine weg oder stellen sieh wohl gar auf dic Ilinterfüsse zur Wehre, hat inan sie ans Land gebracht. - In ruhigen Zustand sind die Krabben oft unkenntlich, weil entweder Moos sic über und über bedeekt oder Jepaden und andere Cirripeden oder Sehlamm und Eirde ihrer Sehlupfwinkel. Kin sehr feines Gehör ist ihnen eigen und wenn sie das geringste Geräuseh vernehmen, so ziehen sie sich oft auf die lustigste und pfiffigste Weise und mil einem drüuend aufgehobenen Arme zurüek. Fin Leiehtes haben die Sehnelllïufer, die Renner (Oeydromien oder Oeypoden) unter den Krabben, wohin Gelasimus nainentlieh nebst andern gehōrt. - Einige halten sich an Gegenständen so lest, dass sie liebcr die Beine lassen als nachzugeben. - So hatten wir in der Bucht einer jonischen Inscl einmal Beweis von solcher Pertinaeitit. Die Ligien findel man fast 
úberall unter Steinen; beim Fang der Julideu, Oniscoiden habe mau Acht; dic rrstern geben einen eaustischen Saft von sich, dass die Hand davon aufschwillt, besonders die grossen in dem Süden Europa's (iı Dalmatien, Italien).

Längere Zeit der freien Luft ausgesetzt, sterben die Krabben bald; läıger leben sie, was natürlich ist, in feuchter Luft, bei Regenwetter. Wir thun sie bis auf die grössten, dic in Werg gewickelt werden, nachdem sic ausgetrocknet sind, in Weingeist, umbinden sie, wie dic Fische, fest mit Werg und packen sie dicht in Fässchen oder Blechbüchsen.

In Museen heftet man die grössern Krabben aul Pappc und schneidet den Theil unter dem Thier aus, damit der Bauch sichtbar werde. Dic kleinen Sippen und Gattungen setze man in schwachen Weingeist.

Reicher als das adriatische ist das Mittelmer an Crustaceen au und auf der Oberfläche, was wir aus Autopsic behaupten können. Die Delphine scheinen die Adria noch am meisten zu licben.

Es gibt in allen Museen einige Crustaceen, aber schr wenig Sammler und Privatsammlungen von Krebsen, wic man auch blutwenige Sippen und Gattungen in unserm deutschen Vaterlande, auf der Erde und im Wasser zïhlen kann. Das Meer ist die Heimath der stattlichsten; dieses beherbergt den essbaren, wohlschmeckenden und schönen, so wic ergiebigen IIumner, den ungeheuren, der Speise wegen hochgeschätzten Palinurus (Langouste), den breiten mittelländischen Scyllarus, die Squilla und die Maja (der Diana von Ephesus sonst heilig), den röthlichen Tasehenkrelss. den uustäten Tourlourou und den kleinen Garnat; unsere Flüsse und Altbäche fïhren den suhmackhaften Krehs. - Sind wir damit znfrieden! Was zur Nahrung dient wird nicht lang aufbewahrt und an den Fischläusen, Lernacen, Kiefenfüssen, Japhnien, Cypris- und CyclopsGattungen, ten nässelnden, langweiligen Asselı, Tansendrüssen und reisfertigen, krümmigen Scolopendern und den winzigen possirlichen Wasserföhen (Gammnarus pulex) unserer Quellenbäche findet selten ein Mensch auch nur ciniges Behagen. Sie glïnzen nicht, darum bemerkt man sie nicht. - Das Gesammelte auflowahren, was der Matrose über Bord wirft, kano auch nichts Erspriessliches bringen. Dio höchste Zeit wäre es, dass sich die sogenannten $\mathrm{Nat}$ urforscher mehr um die Natur als um das Bü cherschrciben bekümmerten. Fangen wir an zu reisen, an den Küsten der Meere und der Ströme zu forschen, wenn wir mit dem Einheimischen glauben fertig geworden zu seyn. - Coquettiren wir nicht mit dieser erhabenen Natur, von der wir so bald wieder seheiden müssen, und verhüten wir, dass die höchste, die herrlichste aller meuschlichen Wissenschaiten von Menschen, denen, wie den Krebsen, der Magen im Kopfe liegt, geschändet werde.

Ungeachtet ich wohl Werth in diese Art von Eifahrmngen zu setzen weiss, welche man in grossen Naturalien-Samonlungen erlangt durch die so wichtige Vergleichung der vielen Gegenstände, durch die genane Anatomie der einzelnen Organe, so wird man doch nicht lïugnen können, dass denjenigen, welche sich dieser Art zu studiren allein bedienen, 
im Grunde die Natur, welehe sie 7.11 erforsehen suchen, fremd bleibt. Das Wichtigste, nämlich das L,cben, fehlt hier; die Naturforscher wandern nur zwischen und arbeiten nur an todien Leibern; des Lebens manchfaltige lirscheinungen, der Organe wunderbares Wechselspiel, des Individuums Verhalien zu der Natur und dessen Einwirkung darauf, all dieses bleibt ihm unbekannt, nicht zu reden von der Ilenge Irrthümer, welche dergleichen Untersuelungen von mehr oder weniger sorgfïltig aufbewahrten Thieren nicht selten herbeiführen, so wie den Schwierigkeiten, Organe zu deuten, deren Verbindung mit andern aufgehört hat. Der Naturforscher muss daher, wenn er seinem Namen entsprechen will, nicht allein in seinem Laboratorio arbeiten, sondern häufig und vornehmlich in der lebenden Natur*.

Die Krebse sind crst ungefälir in den Zeiten des K. Carl IX. nach Sehweden und zwar in die zu dem k. Tafelgute Gripsholm gehörende See gekommen. Wie rar sie noeh 1649 daselbst gehalten sind, erhellet aus dem Briefe der Königin Christina an den Landeshauptmann zu Upsala, worin aller Krcbsfang um Upsala herum verboten wird, damit man keinen Mangel bei der bevorstehenden Krönung haben möchte. (Stockholm Magaz. III, 187. - Beckmann de Histor. n. Veter. 1766 , p. 28. Nota 10.)

Die Rankenfüsser, Kopf-, Augen- und Fühler-lose Thiere, die eine vollkommenc Metamorphose durchlaufen, stecken in Kalkschalen oder Ilauthüllen verkehrt, den mit drei Kieferpaaren bewaffneten Mlund nach unten geriehtet. Die sechs Leibesringe, welche auf den ersten, mundtragenden folgen, haben 6 Paile gespaltener vielgliedriger Füsse, Ranken odler Cirren. Sie halten sich an oder sitzen an Krebsen, Conehylien, Walen, Holz und Gestein, auch aufeinander im Meere; auf den Kiemen von Palinurus vulgaris (Gymnolepas) und der Maja squinado (Pentalepas Schlegelii nach Cantraine). Da die hichergehörigen Bernaeles oder Entenmuscheln (Lepas) zu den Mollusken gerechnet wurden, so verweisen wir hier auf das Lexikon der Conchologen.

Cirripedes kommt von den lateinischen Wörtern cirrus und pes her; daher muss man Cirripeden und nieht Cirrhipoden schreiben. Das priechische Wort xıழ́’os he"eichnet eine fahle Farbe und hat keine Verwandtschaft mit dem Wort cirrus der Lateiner; also ist die Benennung Cirrhipoden nicht synonym mit Cirripeden **.

Die Zahl der Kerbthiere ist 80,000 , die der Crustaceen 1,600, die der Araehniden 5,100, nach unserer Zilhlung. - In Bayern haben wir 70 Crustaceen, 36 Myriapoden und 1000 Arachniden entdeclit; der geniale $\mathrm{Koch}$ zu Regenslurg noeh viel mehr.

Iin leichtes Verfahren, Entomostraceen aus der Priestley'schen Materie zu erzeugen, ist folgendes: Man übergiesse ein halbes Loth

* Sars, Nyt Magazine of Naturvitenskab. III, 2. 1833, 139.

$*$ Lut eille, natirl. Famil. d. Thierr, ïbers. v. Berthold. p. 220, Not. (Diesen Fehler beging Cuvier, Regne au. 1830!! anch RymeI Jones.) 
prïparirter weisser oder rother Corallen (Mladrepora oeulata oder Isis nobilis) mit 6 Unzen destillirten Wassers, stelle diese Mischung in einem etwas grösseren Glas in die Sonne, rühre sie melhre Male des Tags um, giesse nach 14 Tagen die Flüssigkeit vom Bodensatze ab und stelle sie den Wirkungen des Sonmenliehtes aus. - Binnen 14 Tagen wird Priestley'sehe Materie und aus derselben nachher Conferven entstehen, aus denen sich, besonders im Sommer, binnen 3-4 Monaten Cyprides deteetae entwiekeln werden. Wird die Flïssigkeit in einem engen und hohen Cylinder einer starken Sonnenwirme ausgesetzt, so entstehen Ulven-artige Gebilde, aus denen sich in längerer Zeit Daphniae longispinnae entwiekeln.

Die Mleerkrabben sterben im süssen Wasser und der Sammler muss sie also dahin bringen; denn im Weingeist werfen sie die Füsse weg. - Um die Farben zu erhalten, nehme man Kulkwasser (vgl. Bronn's Ergebnisse).

Denjenigen, weleher hin und wieder ein Werk oder eine Abhandlung in diesem Buche vermisst, und es darum tadelt, entgegnen wir, dass das Lexikon kein Handbueh der Literatur, sondern nur ein Lexikon von Personen sey, zu denen die Schriften beigegeben wurden, um den Tyronen der Wiss'nsehaft etwas mehr als diese in die Hïnde zu geben.

Im Vorwort (pag. 4) ist Helas zu streiehen und Alás zu setzen.Milike berichtige man mit: mé like. - Pag. 6 ist L. van der Linden (nebst mehren später zu nennenden Verstorbenen) anzuführen; - auf pag. 9 der Vorrede möchte die Zahl der Lebenden zu erhöhen seyn, da spätere Einschaltungen hinzugekommen sind. Libenda Zeile 20 von oben setze nach Spalten "Namen"; - p. 9 ist statt Seolegiologen zu Insen Skolekologen. - Im Vorwort (gleieh die 5. Zeile v. o. p. 3) muss "ausgesprochen" gelöseht und „erklärt" dafür gesetzt wreden.

Der gelehrte bayrisehe Naturforscher, Joseph Westerh a user, starb zu Münehen am 16. Juni 1840. Er sammelte 30 Jahre und war ein tïehtiger Careinolog und Arachnolog.

Zusätze und Berielıtigungen, so wie Supplemente überhaupt zum Lexikon der Eittomologen folgen im Lexikon der Echinodermatologen als Anhang nach. - Ueber die Sammlungen der Carcinologen vergleiche man den Nachtrag.

Im Alphabetikon ist auch die Nachlese aufgefülırt. - Ein Alphabetikon der Materien einmal spïter.

Wie im vorangehenden entomologisehen Theil waren wir gleiehfalls wieder streng bemüht, Alles aufzuführen, was unserem sterblichen Auge nieht entgangen war. Jlier wie dort können wir versichern, haben wir den grōssten Theil der angeführten Literatur selbst durchgesehen und verglieben. 


\section{Adressenbuch der Carcinologen etc.}

Agrsaiz, Louis, Professor zu Neueuburg. - Nomenelator zoologieus, contineus nomina systematien generum animalium tam viventium quam fossilinm anetore L. Agnssiz. Solodıri (Jent et Gassmann) 1843. Fasi. III, IV. Crustacea, Vermes, Hemiptera et Infusoria (Isis 1843, p 622. Die Crustacen von Burmeister, die Wiirmer von Mieseler, die Hemipteren vou Ericlison und Germar, dic Infusnrien von Elirenberg revidirt.)

Alberti, in Wilhelmsholl. Unt(e)stützte durch Beiträge H. v. Meyer's Werk ïber die fossilen Krebse.

Alessandriul, Dr. med., in Bologna. Schrieb iiber Seolopendra norsitans.

Alexauler, John E., zu Edin. burgh. - Ueber Krabben - und Echiniteneoralleray z.n Sudbonrue (Proceed. of genl. soe. 1838).

Althans, in Dürrhein. Mitarbeiter al Mayer's Werk jiber die fossilen Crustacen.

Ause, Martiu Saint-, in Paris. Mémoire sur l'organisation de Cirri. pedes. Paris 1835. 4. Av. Planch. Ueber dell Ban der Cirripeden (Guerin Migas. V. 1825. 1-28, T. 2, aus Recueil des Savants étrangers. - Isis 1842, 209-211. Mit Oken's Zusätzen). - Ablandlung über die Cirripeden (Annil. d. se. nat. III, 1835, p. 316, 2 PI. - Isis 1836, p. 532\% - Uebes die Cirripeden (L'Institnt 1833, p. 226. - Isis 1837, 314). (Stellt sie zu den Gliedertheren, naeh Kiefer'n und Füsseil.)

Apetz, Gymnasiallehrer in Alten. burer.

Arcet, Félix d', in Paris. Entdeckte 1830 cime neue Artemia (Crust.) mebst Besclureibung dreier neuen Gatin den Salzseen von Goumplidich, tungen (T'ransact. of zool. soe. 1, P.
Almmaralı und Bedach (vgl. L'lnstit. 1836, Nr. 183. - Isis 1837, 790).

Bubingtou, Caıl Cardale, Esq. Professor am Johus-Collegium in Cambridge.

Iner, K. Fr. voli; Rifter, Profesnor ete. zil St. Petersburg. - Ueher die sogenamute Emeuerung des Magens der Kroluse nnd Bedcutung der Kredosstcine (Mïller's A rehiv 1834). Ueber Entwickelungsareschirhte der Thiere. Beobaclitune und Reflexion. 1. Thl. Mit 3 eolor. Knpfert. gr. 4. Köuigsherg, Boruträger.

Malleustedt, G. J.. Prediger in Papstdorf bei Halberstadt. - Die Urwelt. Quedlinb. 1818. 2 Bde. 8.

In relny, A., in Jamaica. - Ueber die Landkrabben anf Jamniea (in dessen View of Slavery und Ediub. new phil. Journ. VII, 280. - Isis 1832, 817).

Barker-Wepp. P., in Paris. (et S. Bertluclot) Histoire naturelle des îles Canuries. Paris et Frihoure ehez Herder. 1835-41. 4. Atlas in Fol. (Isis 1837, 246. 1839, 700. 1\$42, 698-7 t6). (Lnrehe, Fisehe, Seluneek n, Käfer, Müeken, Spimnen, Krobhen, Echinodermin. Die Entomologie von Brulle. Selu viele Genera und Species. Die Dipteren lat Mitequart bearbritet. p. 97-121.)

Eurthélamy, Conservator dex Musfums in Marseille. - Neur Kıabbensippe (Ranilefta Edwardsi) (Atti dollu terza Riunione. 1841. p. 313. - Isis 1843,403 ).

Be-11, 'Tlıomas, Esq., Prisident ete. in Lombon. - A liistory of britisls Crustaera. Lond. 1842-43. - Beobaelatungen ïber die Sippe Caneer (Leach) 
4, Nr. 33, p. 335, Taf. 43-47. Ixis 1837, 265). - Ueber Cancer Leael (Platycarcinos Latr.) (Proceed. III, t835, Juni. - Isis 1837, 135). Crustaceeı von den Küsten Südamerika's, welehe Cuming und Miller mitgebracht haben (Trausict. of zool. soce. 11,1 , IV, p. 39 , T. 8-12. Isis $1838,33-47$. - 150 Gattungen. - Pruecedings III, Nov. 1835, - Isis $1837,196)$.

Beneden, P. J. van, Professor 21 Löwen. - Exereiees zootomiques. Bruxelles, Fase. I. 1839. 4. 53 tab. 4. II. 1839. 55 , tal 10 et seq. (Mém. Acad. Bruxelles. XI et seq.).

Perthelot, Sabine in Paris. Histoipe naturelle des iles Canaries (avee Barker-Wepp). Paris et Fribourg 1835-39 (Isis 1837, p. 246; 1839$, p. $700-7+3)$.

ibertholi, Arnold Adolph, Dr. Professor z.ll Göttiugen, - Apus Cancrifurnís (Isis 1830, p. 685, Taf. VII),

IBeske, C. H., berïhnter Naturalientuändler in Hamburg.

Isigot de Morugues, in Poitou (vil. Tristan).

IBIgsloy, in New-York. - Versteinerungen in Canada (Trilobiten und Corliylien) (Sillim. Amer. Journ. VIII, 1821, 11. 60 . - Isis $t 332,1045$ ).

Mlainvile, Henry Duerotay de, Professur der Naturgeschichte zol Paris. - Principes d'Anatomic comparé. Paris 1822. 8, - Ueber cinige T'hierchen, welche die dureh Vertroekunng verlorne Beweyng in Wasser wieder erlualten (Ann. d. א., nat. IX, p. 104. - Isis 1834, 897. - Rotifer, Tardigrada). - Essai sur' une monvelle classifieation des animax (Bullet. des zc. par la soc. philom. t8t6, p. 123, 4.). - Le même essai inséré avce quelques modifications, dans les principes d'Auatuuip romparée. Tom. 1, Tab. VII. Paris 1823. 8. - Sur la roucordanee des anmeaux de Entomozonires liexipodes adultes (ibid. 1820. Mais 33 ),

Blanchard, A., in Bordeaux. Bearbeiter ales entomologisehen Theils (mit A udouiu) zu der neuen Ausgabe von George Cuvi er's Werk: Le Rêgne animal distribue d'après son organisttion. pour servir de bases a l'histnire uaturelle des animaux et d'introduction a l'anatomic compare. Edition aecompagnée de planches gravèes, ruprésen- tant les types de tous les genres, les enracteres distinetifs des divers gronpes et les modifications de struetnre sur lesquelles repose eette elassificution; par une Réunion de Disciples de Cuvier, MNI. Audouin, Deshayes, d'Orbigny, Dugess, Duvernoy . Lan rillard, Nilue Edwards, Roulin et Valenciennes. Paris (Fortin, Musson et Cie).

IBanding, Dr. W., in Camden.

BIrslus, J. H., Professor in Halle. - Ueber cinen meuen Sehaalenkrebs, fimnetis Wiegmanni, von Braunscliweig (IX. Versamml. des Harzvereins. Isis 1839,667$)$.

Balek, Christian, Leetor z.ı Christiania. Nahm an der französisclsen Expedition nach Spitzbergen Theil. Uebersicht der bisher in Norwegen gefunderien Formen der Trilobiten. familic (IKeilhau: Gaea norvegica. I, t838, p. 138). - Ueber die Trilubiten (Füıhaudl, Kjöbenh. t840, p. 289. Isis 1843, 295). (Stellt sie z.n Limulus.)

Hory de Gaint-Vincent, J. D. M., Hajor ete. zn Paris. - Voyage aux isles Cillariens (dentseh in Auszuge voll Bergk).

irranalt, J. Fr., Professor, Ritter, Dirertor elc. in St. Petersburg. Ueber die Gattungen von Seulopendra (Bulletín scientifique public par l'Aeadomie imperiale des sciences de St. Petersbourg. VII, 1840 , p. 147, Nr. 1t). - Tentaminum quorundam monograplicornm insceta Myriapoda ehilogunatha Lat'cillii speetantium prodronus (Bullet. mose. ed. leq. V, t833, p. 288-97. Tab, - Anual. d. se. nat. 2. ser. VilI). - Bericht ïber die Gesehichte, Anatomie und Physiologie des Glomeriden (Bull. se. de l'Aead. de St. Petersl. VI, 1839, Nr. 19. - Isis 1841, 70). - Ueber die Gattungen von Glomeris (ibid. VII, $1840, \mathrm{Nr} .4,5$. A pril. - 8 newe Gattmugen). - Innerey Ban von Glomeris (ibjol. IX. 1842, 1. - Miiller's Arehiv 1841, p. LXXXVIII und $1843,(X)$. - Allgemeine Bemerkungen iber die Ordnung der Myriapoden (französ.: Bull, se. de l'Ae. imp. de St. Petersb. VII, 1840 , P. 293. lsis 1844, 121) - Generis Juli specierum Enumeratio (ihid, VIll, p. 97, Nr. 175. - Isis 1844, p. 124). - Beobarlitungen iber das Jeben und Weben der Gattungen von Glomeris (lbid. p. 343. - Isis 1. e. $t 26$ ). - Kritische Bemerkurgen iber. die Sippen Sphate- 
rotherium et Sphaeropaeus (ibid. p. 357. - Isis 1. c. 129). - Nachtrag (ibid. 665). - Bemerkungen uiber dic Mund-, Magen - oder Eingeweidenerven (Nervus sympathicus siu Nervus reprodurforins) der Evertebraten (Miem. de l'Acarl. de St. Pétersb. 1835, 561 -611. Tub. $1-3$ ). - (und $R$ a i zeb u гg) Astacus fluv. (Med. zool. 1I, 28, Tab.). - Gehörsäekehen bej Astacus fluv. (Meckel, A rchiv). - Ueber eine neue Ordnung der Myriapoden (Vortrag z.u Jena. - Isis 1837, 501). - Conspectus monographiae Crustaceorum Oniseodorum etr. Mosquae 1833. (Mémoir. de l'Acad. Il. Isis 1834, p. 317). - Ueber Myriapoden (Amllicher Bericht über die Versamml. deutscl. Naturforscl, in Jena. $1836,135)$. - Reeveil de mémoires relatifs à l'ordre des insectes Myriapodes, el lus a l'Acad. imp. des se. de St. Petersb. T. V-IX. gr. 8. 1841. - Urber die Classification der Sippe Polydesmus (Bullet. sicient. V, p. 307. - Jsis 1839. 659). - Naclitrag über die Gattungen vori Polydesmus (Bullelin scientifiqu. IX, 1841, Nr. 193, p. 9.

Eravais, in Algier. Crustaceolog. Sammrlte in Nordafriki Ciustacren.

IBriguoli, Giovanni di, Professor der Butanik anf der Universitijl zu Reggio. Schriftsteller. - Ueber fos sile Crustacea und Animalia radiaria. Rrgegio 1836.

IBroulerip, William John, Esq. in London. Zoolog von Ruf. - Deseription of fossil Cinstacea and Padiarin found to Lime Regis, Dorsetslive (Transact. of geol. sur. 1835). - Pagurus, Bullinus (Zool. Journ. XILI. Isis $1830, p .1256$ ).

Bronu, Heinr. G., Professor der Naturwissmschaften ete. zu Heidelberg: - Lethaea geognostiea odes Abbildung und Beschreibung der für die Gebirgsformationen bezciehnendsten Vrrsteinerumgen. 2 Bde. in 8. mit 47 Taf. in 4. Stuttg., Schweizerbart. - Gesch. d. Natur. gr. 8. 3 Bde. - Ergebnissc meiner naturlistorischen Reisen. 2 Bde. lleidelberg. 8.

Brulle, Auguste, in Paris. Bearbeitung der Crustaceen und Kerfe in P. Barker-IVeppet S. BertheI ot's Histoire naturelle des iles Canaries. Paris 1835-41. 4. Atlas in Fol. (I.sis 1842, p. 6.98).
Brumner, Sigmund, Dr. med. und Arzt zu Bern. - Der bekannte Schuellreisende. - Streifaur durelı das östliche Ligurien. Elba, die Ostküste Sjciliens und Malta, zunächsl in Bezug auf Pflanenkunde, im Sommer 1826. gr. 8. Winterthur (Steiner).

Buch, Lropold Freiherr von, in Berlin. - Reise durch Norwegen und Lappland.

Bucking hnu, John S., in London. Seine in engliseher Sprache erschienenen Reisen stehen im Magazin von merkwürdigen neucu Reisebeschreibungen, ans fremden Sprachen übersezt und mit erliinteruden Ammerkungen begleitet. Mit Kupf. u. Kart. 37. Bd. A. u. d. 'T.: Reisen in Mrsopotamieu von Aleppo über deu Euphral nach Orfah, dem U1 in Clıaldian, durch die Ebenen der Turkomanjen, nuch Diarbeke in Klein-Asien. A. d. Engl. iibrrs. gr. 8. Berlin, Voss. (1.-36. Bd. 1790 e1 seq. - Kosten complet 128 fl. $6 \mathrm{kr}$.).

Buckinud, $W$., Professor z.ll $0 x$ ford (vgl. Lex. d. Theriol.).

IBurineister, Herm., Med. et I'h. Dr., Prof. der Naturgeschielitc zu Hallc a.d.S. - Artikel: Cinstacen et sperialia ad $\mathrm{Cr}$. spert. (Ersols and Gruber's Encyclopedie). - Britrige zur Naturgeschiclitr der Rankenfüsser (Cirripedia). Brplin (Reimer) 1834. 4. 49, 2 Tab. (1sis 1836, p. 554). - Die Respirntionsorgane von Jolus und liepisma (Isis 1831. 134, Taf. I. - Vgl. Agassiz). - Besclircibung riniger neuen oder weniger bekannten Sclumarotzerkrebse. M. 4 Kpf. $1833,46 .(2.4 \mathrm{kr}$.) (Nov. Aet. Aearl. Leop. Car. XVII, 1, und Isis 1835, 930). - Zuologisclier Haud-Atlas. 6 Liefge. (Surhe, Lurche, Fische, Kerfe). Imp.-4. Berlin 1839.

Canus, Carl Gustav v., Medizinalrath, Lribarzt etc. in Dresden. Anatomic. - Von den Anforderung(') an eine küuftige Bearbritung der Naturwissenselaften. Leipl. 1822. - Leheushedingungen weiss- und kaltblitiger Tluiere. Leipz. 1824 4. (80 Astar. fluv.). - Versucle mit Arctiscon (Muillers Arehiv 1834, p. 551). - Erlïute. rungstafilu \%ur vergleichenden Anatomie. Fol.

Caresna, Hiacintho, Professor in Turin.

Cammont, de, in Caen. Der 
Stifter der Ver'sammlungen der Naturforsclier in Caen.

Cerr, Lr, Professor zu Caen.

Charpentier, Toussaint de, Oberbergrath zu Dortmund in Westphalen. - Bemerkungen ïber einige Crusta. enen des unittelländischen Meeres (Verh. der Berl. Ges. I, p. 152-173).

Clarannues, Jak. August, Direktor de's Naturalienkabinets in Lausamne.

Clainje, Steffano Delle, Professor in Neapel. - Mímoires sur les animaux saus vertébres du royaume de Naples. Naples 1823-25. 2 Vol.

Cinristol, Jules de, Professor zu Dijon (verl. Jex. d. Theriologen).

Ciapperton, Esc. in London. Berülumter Reisender. - Travels. Drutselı: Clapperton's zweite Reise in das Innere vou Afrika; nebst dem Tagrbuch seines Bedienten, Richard Linders. A. d. Engl. gr. 8. Jena, Bran. 1830 .

Colsmunu, Professor in Kopeluhแgerl.

Collstrenm, John, zu Leith. Ueber den Bau und die Jebensart von Limnoria tecebrans, eine kleine Krabbe (Edinb. 1. phit. Journ. XVI, 316, Taf. 6 und Vol. XVIl, 310. - Isis 1838, $40-45$, '(af. 1).

Canate, Achille, Professor der Naturgesehichte an der Akademie zu Paris.

Contarini, Nicolo Graf von, in Venedig.

Corrin, A. J., Dr. med. und Custos des Nitionalmuseums in Prag.

Costa, 0. G., Dr. med. und Prof. in Neapel. - Fauua del Regno di Napoli. Fuse. J, XXX. - Lrzioni di Zonlogia. Napoli 1838. - Ueber den Zustand der Naturwissenschaften im Königreich Neapel am Ende des Jahres 1840 (1sis 1843, p. 651). - Fauna sicilian 1839, 1 .

Con:lom, Louis de, Direktor des Museums der Naturgeschielite iu Neuen. burg (au Faubourg près Hospital).

Creduer, in Giessen. Careinolog.

Crepu, Conservator des Muscums der Naturgeselichte zu Grenoble.

Crosse, A., in Counecticut. Hervorbringung von Krystallen und Insekteu durch die galvanische Säule. (Sillim. Americ. Jouru. XXXV, Nr. 71,125 , fig. - Die hier entstandenen Insekten siud meines Eraehteus niehts anders als Eichhorn's Wasserbir, Sehrauk's Arcticcon, Sehultze's Maerobiotus, wrichen man bis jezt zu den Infusorien gestellt hat. Die Sache giug also olne Wunder zu. Oken.)

Cunuing, in London. Grosse Crustaceensanumlung aus dem Tropenland.

Dana, John, in Philadelphia (vgl. Pigkering).

De Tran, W., Dr. med. ete. in Leyden. Gefeiert als Carcinolog und Entomolor. - Fauna japonica, auctore Plı. Fr. de Siebold. Cristacea elabo. rante IV, de Hann. Lugd. Batav, apud auctorem et Amestelodami. Decas IIV, 1833-39. Fol. Tab. 32, 2 et 8. (1sis 1842, p. $386-391$ ).

Delony, J. E., Dr. med. in NewYork. - Ueber Eurypterus, cinte neue Versteinerung ans den Branchiopoden (A unals of nat. hist. of N.Y. I, 'T. II, 1825 , p. 375 , t. 29 . - Jsis 1832, 564 , Taf. IX. - Vgl. Amer. Monthly Mag. Ill, p. 291). - Zweitos Exempl, von Eurypterus (Annals of Lye. II, 273, T. 5). (Mit Ammoniteu.) - Observations on the structure of Trilubites, and deseription of an apparently new genus. - Bau der Trilobiten und neue Sippe (Isotelus) (Ann, of Lyr. NewYork. I, p. 174. 1824. - Isis 1832, 1072).

Hengel, K., Dr: und Lelurer zit Königsberg. - Naturzeschichte des 'Thierreiehs, für höhere Bürgerschulen bearbeitet. 8. Köuigsberg.

Deslnayes, G. P., Dr. med. in Paris (vgl. Lex. der Entomologen).

Desunrest, Anselme Gaètan, père, Professor zu Alfort. - Article: Crustacis fossiles du Dict. d'hist. nat. Secoude edition. Tome vill. 8. 1817. - Note sur une espèce de Cypris fossile (Nouv. Bullet. des sc. par la soc. plilom. Annee 1813 , p. 259. 4. fig. Entomostraeum). - Histoire uaturelle des crustacis fossiles sous Irs rapports zoologioues; les Trilobites par M. A. B ro»guiart. Paris. 4. 7 Planch. 1820. - Considerations générales sur la classe des Crustanes. Paris 1825. gr. 8.

Desmarest, Sohn, in Paris (Rue Saint-Jacques, Nir. 161).

Desmonlins, in Paris. Berühnter Anatom und Scliriftsteller.

Deworn, V. J., in Cobleuz. Naturbeschreibung der Gelenkthiere. 
8 Hefte. 12. Coblenz (Grelehr. Buchhandi.). 1. Heft 1822.

Dierbach, J. Heinr., Dr. med, in Heidclberg. - Uebersicht der febräuchliclisten Arzneimittel des Alterthums, mit hesonderer Rücksicht auf die Werke des Dioseorides und Plinius. Ein pharmakologischer Versuch. 7. Kapitcl. Medikamente und Nalurungsmittel aus der Gruppe der Krebse oder Zehnfüsser, Decapoda (Isis 1841, 728).

Diesius, M., in Wien. Bekannt als Scliriftsteller über die wirbellosen Thiere (Annalen des Wiener Mu. seums. II).

Doubledny, Edward, zu Epping in England.

Doyére, Professor der Naturgeseliehte am College royale de Henri IV in Paris. Mitarbeiter an dem neuc. sten Dictionnaire universel d'histoije naturelle. - Ueber Macrobiotus (L'Inst. $1839, \mathrm{Nr}, 267$, p. 45 . - Isis 1839, 538). - Versuclie ijber das Wieder. aufleben der Tardigraden Rotif. (Infus.) (Aunal, d. sc. nat. T. 18, p. 5, L'Inst. 1842, p. 289. - Froriep's N. Notiz. Bd. 24, p. 232).

Drapiez, Professor der Chemie und Naturgesehichte und Direktor des Muscums zu Brüsscl. - (avec C川vier, G. A. Richard etc.) Cours complet d'histoire waturelle, medicale et pharmaceutique ou ressuné des divers ouvrages efc. Tom. I, 1. Livr. gr. 8. Aixla-Chapellc. (Melır kam uns nicht zu.)

Drzewicki, Dr., k. russ. Hofrath in Warschan.

Dubuison, Conservator des Naturalienkubinets in Nantes.

Dufour, Léon, Medicus zu SaintSéver (Dept. des Landes), Hochberïhmter Naturforscher. - Ueber Scu. tigera araneoidcs (Ann. des sc. nat. II).

Dulk. - Ucher chemisehe Bcschaf. feuheit der Krebssteine (Mijiller's Arch. 1835).

Muméril, André Maric Coustant, Professur der Pliysiologie zu Paris. Cuvier: Leçons d'Allatomie comparće. V Vol. 8. Les deux premiers publies par M. Const. Dumè ril, en l'aul VIII, et les trois derniers par M. G. L. Duvernoy, cu l'an XIV ou 1805. Cunsiderations génerales sur la classe des insectes. Paris 1823. 8. 60 tub. aen. - Oniscus (Dictionnaire des sc. nat. III, p. 117). - Seiu und Cuv.'s Berieht iiber Audouin's und M. Ed. wards' Abhandlung: ron der Luft. respiration der Crustaceen und den Modificationen des Kiemenapparats bei den Landkrabben (Ann. d. sc. n. XV, 85. - Isis 1834, 1032\% - (mit Cuvier) Bericht iiber Andouin's "Kreislauf in den Crustaceen" (ibid. $\mathbf{X}$, p. 394. - Isis 1834, 925-26).

Dumal, in Bordeaux (?). - Ueber die Algen der Salzteiche (wegen Artemia snlina) (L'lnst. $1837, \mathrm{~N}_{\mathrm{r}} .221$. - Inis 1837, 854).

Duncrue, Capitän, in London. Entwickelu»g der Crustaceen (L'Inst. 1838, Sept. Nir. 245. - Isis 1839, 377). - (Die Behauptung Thompson's mnd Duncane's, dass die $\mathrm{Cr}$. sich pleicl andern Kerfen verwandelten, läuguet Ratlike.)

Dupont, Andre Pierre, Naturalist in Paris. Von der $k$, Gescllsrli, zu London. - Mémoire sur le Glaucus (Phil. Trans. LIII).

Durazzo, Don Carlos, in Genua. Entomolog.

Dutrochet, H., Mitglied des Instituts von Frankreich, in Rcnaud bei Vendòme (Loire et Cher). - Ueber dic Respiration der Kerfe (Annal. de la soc. ent. Franc. II, 183.3. Bull. 10). - (Arctiscon) Sur les Rotifères (Mèm. du Mus. d'hist. nat. XIX, p. 331, t. $18, f .17$ ).

Divernoy, Dr. med., Professor etc. zu Strassburg. - Bau vou Squilla mantis (L'Inst. 1837, Nr. 212. - Isis 1837, 838). - Bau des Limulus Gigas und Classification der Crustaceen (L'Inst. 1838, p. 305. Nr. 247. - Isis 1839, 379. - Revue zool. Sept. 1838).

Edwrerels, Milne, Professor in Parik. - Caliers d'histuire maturelle (avec Ach. Com te). 6 Cahiers. - (et Audonin) Untersuchungen über die wirbellosen Thiere der Chausey-Inscln (Ann. d. sc. nat. 1828, XX, 5. - Isis 1834, 1029). - Ankrüdigung und Einleitung zur Histoire naturelle des Crustacés (ibid. 1, 1834, p. 26n-312). Histoire naturelle des Crustacés. Paris (Roret). I, 1834. 8. 468. II, 1837, 331. III, 1840, 605, 19 'Taf. (Isis 1842, 139-144. Ucbersiclit des Systems). Suites à Buffon, formant avec les oeuvres de cet auteur mu cours complet d'histoirc naturelle. Collection, accounpagne de piauches. Paris (Roret). 8. 
Auf as Bde. berechnet. Anfang 1834. - Verbreitung der Crustaceen (L'Inst. - Introduetion à la Botanique par 1838, Sept. Nr. 245, p. 290. - Isls Alph. De Candolle. Il Vol. - Hi- 1839,375$)$. - Ueber dell Bau und stoire naturelle des Végétaux phanèro- dis Classifieation der zehufïssigen gumes par L. Spaeh. Vol. 1-V. - Krebse (Aun. d. se. n. XXV, 298. Introduction a l'Entomologie par Th. Isis $1835,470-71)$. - Ueber die La eorduire. Vol. 1, II. - Histoire Eutwickelung des Limulus (L'lnstit. Haturelle des Insectes Diptères par t838, Nr. 258, 397. - Isis 1839, 392). Ma equart. Il Vol. - Histoire natu- - Kiemenapparat bei Crustaceen: Thyrelle des lnsectes Hyménuptères par sanopoda (Aun. d. se. 11. XIX, 451, A. Lepelletier de St. - Fargeau. Taf. - Isis 1833, 615, T. 191. - Ueber I Vol. - Histoire naturelle des lu- den Mechanismus des Athmens der seetes Lepirlopteres par Bo isduval. 1 Vol. - Histoir Maturelle des Insectes Apteres par Wolkenaer. I Vol. - Histuice naturelle des Crustaeis par Milue Edwards. 1 Vol. - Hist. uat. des Reptiles par $D$ umeril et Bibrou.111. - Citilespr. Cuvier.) (mil Victor Aud ouin) Anatomie und Physiologie der Crustaeeen. 3. Abth. t. 2-6 (Aunal. des se. nat. XIV, 1828, 77. - lsis 1834, 1023 oder Mèmoires pour servir a l'histuire naturclle des Crustarés. Paris 1829). Memoire sur le Nirothoe, animal singulier, qui snee le sany du Homard (Ann. d. sr. llat. Tour. IX). - Ueber den Bau des Mundes der saugenden Crustacren (Amn. d. sc. I1. XXVIII, 1833 , p. 78, Taf. - Isis 1835,983 ). - Untersuchungen über die Natur. gesslichte der Crustaeea amphipuda (ibid. XX, 353. - Isis 1834, 1127$1145, T_{\text {af. }} \mathrm{XV}$ ). - Ueber den saugenden Krobs Pandarus (L,ench) (ibid. XXIV. - Isis 1835, 466). - Ueber Paguriden (ibid. 2. sér. V1). - Observations sur le genre Mithrax. Paris 1835. 4. - Beschroibung der Sippen Glaneothoe, Siryonia, Sergestes und Aretes (ibid. XiX, 333, Tf. - Isis 1833, 608, Tf. 16-17. - Gnérin Mag. II, 1832, lig.). - Nene Crustneecn: Rhoea, Cuma, Pontia, Nebalia (Loplsyropuda) (Annal. sr. nat. XIII, p. 287, T. $13,14,15 .-1$ sis 1832, p. 756 ,'T'.13). - Beobachtungen über die Verände. rungen verschiedener Crustaeeen (ibid. 111,1835, p. $32 t$. - Isis 1836 , p. 534). - Verwaudlung der Crustacecu il'Inst. 1833, 243). - Ueber Crustacea unipeltata (Anunl. d. se. nat. XIII, XIX). - (mit Audouil) llipponoe (ibid XX. 156, Taf. - Isis 1837, 100, Taf. 1). - Ucber Nehalia (Crustaeen) (ibid. 111, 1s35, p. 309. - liss 1836, p. 530). Neuer Stomapod (Amphion Reymaudii) (Ann. soc. cut. fr. I. 1832, 336, fig.). Crustaeeen (L'Inst. 1838, Nr. 250, p. 329. - 1sis 1839, 384). - Ueber den Bau der Athemorgane von Oniseus und Tylos (ibid. 1839, Nr. 280. - Isis 1839, 592). - Neue Clustaceen (ibid.). - Uiher die Charaktere der Anemuren (K1 abbeli) (Aun. d. se. n. XXVI, 255. - Isis 1835,510$)$. - (mit A 11 douin) Ueber die Cireulation in den Crustaceen. Physiologie (ibid. XI, 1. 283). Anatomie (ibicl. 352 , Tf. $24-32$. Isis $1834,936-944) . \quad$ - (nit dems.) Ueber das Nervensystem der Crustaceell (ibicl. XX, 181. - Isis 1834, 1111).

Ehrenberg, C. G., Hofrath ete. in Berlin. - Ueber Macrobiotus und Fureularin: Wiedcrbelebuny etc. (1 sis $1834,710)$. - (mit W. F. Hemprieh) Naturhistuisclee Reiseu durel NordAfrika und West.Asien in den Jahren 1820-25. Herausgeg. vou Elirenberg. Mit Kartel und Ansicliten. A. u. d. T.: Reisen in Egyptell, Libyen, Nubien und Dongala. Mit Landcharten und Ausichten, gr. 4. Berlin 1829 et seq.

Whrhardt, Justizeonmissarius in Swinemünde. Sammlung.

Eichwaid, Ed, Dr. und Prof. zu Wilna. - Die Thier - und Pflanzenreste des alten Ruggensaudsteins und Bergkalks um Nowogorod (Bullet. se. de l'Acad. de St. Petersb. VII, 1840, Nr. 6, 7. - Aufzälılung der Fisehe, Krebse, Kopffiissler, Sehulthicre, Ringwürmer, Strahlthiere). - Ideen zu ciner systematisehes Oryktozoologie (aus den .lahresverhandlungeu der kurländl. Ges. f. Lit. und Kunst abgedr. gr. 4. Mitan). - Zoologia specialis quam expositis animalibus tum vivis, tum fossilibus potiss. Rossiae in universum, et Polonine in specie in usum Icetionum publ. in universitate Vilnensi habendarum edidit. Pars I et II. Pro- 
paedeutieam zoologiae atque specialem Heterozoormm et Podozoorum expositionem eant. Cum IX icon. Pars III, Lex.-8. Vilnae. (Lipsiac, Voss.) 1829 -31. - De Trilubitis. 1825.

Wigluts, in Albany. - Bronguiartia trilobituides (L'Inst. III, 1835, $\mathbf{N r}$. 105. - Isis 1837, 718. - Transactions of the Albany Institution 11, 1833, p. 53).

Wklon, Dr. med, ill Hamburg. Reise nach dem Kap.

Frall, Jos., Dr. med. und Professor zu Müncheı. - Èntwickelıñg des Hunmereies von den ersten Veränderungen in Dotter an bis zur Reife des Embryo. gr. 8.4 col. Taf. Müneh. 1843.

Erichson, Wilh. Ferd., Prof. etc. in Berlin. - Ueber zoologisehe Charaktere der Iusekten, Arachuiden und Crustaceen (dess. Entomographien. I, 1840,1 ).

Escluvege, L. W. v. - Brasilien, die nene Welt in topograph., grognost., bergmämmiseher, natuhistor., polit. und statistischer Hinsicht, während cines 11 jährigen Aufenthaltes von $1810-21$, mit Hinweisung anf die neuern Begebenlıeiten. In 2 Thlen. mit Kpfn. gr. 8.! Braunsehweig (Vieweg).

Aschér-Zuminofer, Particulier in Zürich.

Dydonx, Fortune, Marineehirurg in Pasis. - (mit Soule y ct) die Bearbeitung der Crustace en in Capts. Vail. lant Voyage autour du monde 1836 et 1837 sur Ta Corvette la Bonite. Paris (a Bertrand) 1840. 41. 8. Atlas in Fol. ill. (Isis 1872, 623).

Ferrario, G., in Mailand. SammIung.

Ficinus, Ilofrath etc. zu Dresden. - Mit Hofiath v. Carns, Uebersicht des gesammten Thierreichs. - Voll. kommenste Darstellumg der Idee der Animalitit durch Entwiekelume hüherer iuncrer Ejnheit (Selbstanschauung, Vernunft, Freiheit) in rriusten Gegensatze zur ldee dos Vegetabils. 2 Tab. in gr. Roy-Fol. Dresden und Leipzig. 1828.

Fischer v. Waldheim, Gottlıelf, Viecpräsident der Akademie, Direktor, Professor ete. in Moskau. Zoognosia.

Hinders, in London. - A voyage to terra australes. London 1824. 4. Tom. I, 11, 111.

Fleming, J., zu Edinburgh. Aehrenlese in der Naturgeschichte an der seholtischen Küste (Brew. Edinb. ph. Journ. VIII, p. 294, IX, 248. Isis $1832,599,600$. - Crustaceen.)

Focke, W., in Wien. Schriftstellel. (Amalen des Wiener Museums d. Naturgesich. Wirluellose Thiere. Abthl. II des Werkes. 1841.)

Norster, in Aachen.

Franke, sen. Naturalienhändler in Leipzig.

Franke, jun. Naturalienhäudler in Amsterdam. Besizt vorzüglich ostindische Naturalien.

Freminuille, in Paris. - Ueber einen neuen Krehs, Eryon caribensis (Ann. d. se. n. XXV, 273. T. 8 (Isis 1835,467 ). - Ueber die Landkrabben Tourlouroux auf den Antillen (ibid. III, 1835, p. 213 . - Isis 1836 , p. 526-529).

Freyer, Heinrich, Magister Pl. und Custos zu Laibacli in Krain.

Primard, J. P., in Paris. - (et $Q$ u oy) Deseription des animaux reeucillis dans l'experdition autour du monde, commandie par leCapituine Fr y y in e t. Paris 1825, Fol. Av. Planehes.

Golle, Flottencommissair bei der Marine in Toulon.

Crarduas, in Neuhausen bei Müıcher. - Die wissensehaftl. Sanmlungen Münchens (Isis 1844, Heft V und VI, Unsehlag-).

Garmot, in Brest. Bekannt als Reisender (um dic Welt mit der Corvette Coquille).

Craubil, Offizier in Paris.

Teinitr, H. G., in Dresden. Charakteristik der Sehielten und Petrefakten des silehsisehen Kreidegebirges. Dresden (Arnold). 11, 1840, 4. (Isis 1840， 799). (Euthäl Fische, Krehse, Conchylien.)

Geomroy-St.-milnire, Isidor, Prof. der Naturgesehichte in Paris. Bericht über V. Alldouin und M. E d wards anntomische Untersuchun. gen über das Nervensystem der Cru. stueeen (Anı. d. sc. nat. XIII, 1828, 218).

Geofroy-St.-Hildire, Etienne, Professor in Paris. - Mémoires aur 
l'organisatiou des inseetes; le troisième surtout ayant pour litre: sur me colonne vertcibrale et ses eôtes dans les insectes apiropodes. Janvier et Fivrier 1820 (Isis 1820, lit: Anz. pr. 527). - Considirations pliilosophiques sur la determination du systeme solide et ilı système nerveux des animaux artinules (Ann. I. se. 11. II, 395). Bericht über Milne. Ell wards Veränderungen, wolcle die Crustacen in ihrer ersten Lebeuszeit erieiden (ibit. $\mathrm{XXX}, 1833$, 1. 360-373. - Isis 1836, p. 476 ).

Trervais, in Paris. - Polydesmus etc. (Anu, de la suc. entomol. de $\mathrm{Er}$. V, 1836). - Bemerkungeu ïber zwei Flohkrebse um l'aris (Gammarus P'ulex et Roesclii) (Ann. I. se. nat. IV, 1835,127 . - Isis 1837,148 ). - Ueber scolopentra (Lilnst. 1837, Nr. 202. Isis 1837,797$)$. - Uebel die Myria. potlen (Gıèr. Magas, de Zool. V, 1835. - Zufille, wem Grophilus eleetricus in die stimhöhle kriceht - Besthreibung von vielen Geophilen und von Polydesultis. Fip. Galeotles C'ubae). (AnI. d. se. 2. ser. VII - - Ueber die Metumorphosen Uer Myriaporlen (Ann. sor. eut. Fr. 1837. - Isis 1839, 532). - Serliszelun Myriapoden, wormuter 5 uene, um Paris (L'Inst. 1836, Nr. 190. - Isis 1837, 793). (Spriehı auch ven! halber Metamorphose derselben.)

Gircinto, Padre Carlo, di G. M. Carmelitano Scalzo, Professor der Botanik zı Messinu. Selıriftsteller.

Gistel, Dr. pluil. in München. Rivise tes Grufen R. v. Je u i sou - IV a Iwo ort li unil Dr. Gistel tlurelı Sïrlde utschiansl und Italieu. Bd. 11, 1835. Mit Kpfi. (Astaeus Molitor etr.) - Beobarlitungen und Bemerkongen (Fanus 1], 1835: Fossile Hummern (87:; riesiger Hammer (97). - Artikel zum Tusehenconversations-Lexikon, heransgeg. von ciner Gesellschift Gelehten XII., XIII., XIV. Bd. A॥gsburg (Schlosser) 1832. 16. - Sehellilarr. Das Lleilbat und die Ummebunn. Gedrukemein für Kurgriste. Miñchen 18.37. 8. (Geognosie, Flura untl Fauma).

Fiodet, Louis, in Berlin.

(x) ldiruss, Geurgh Angust, Profess. cte. 2u Boml. - Grimulriss der Zoologic. 2. Auft. Nämberg 1834 ('räcision unt Klarheit walten in diesem gediegenen Werke). - Britrïge zur Familie der fussilen Custacen (deta

Gislel's Lexikon.
Acad. Leop. XIX, 1, p. 35:, fig.). Petrefactu Germaniae tam ea, quae ill Museo Universitat. Reg. Boruss. Eridericiae Willolmiae Rlienanae sorvantur quan alia quaecunque in Muscis Hoeninglusiano Muensteriano aliisejue extant etc. Abbilunngen und Beschreibungen der Petrefiliten Dentschlauds and der angrinzenten Lisinder, inter Mitwirkung les Hin. Graten Georg zo Münster herausger. Roy.-Fol. Dïsseldurf, Aruz. 1827-36. - Uebel die Eimreilung der Trilubitrn (Ann. d. se. n. XV, 83). (Gehören, "rach G., zwischen die Branehiopoden und Isopoden.) - Naturhistorisclee Allas. 15. Lief. Mit 20 litl. Taf. in Roy.Fol. und ausfiihıl. Erläıt. iı gr. 4. Disseldurf, Aruz u. C. 1.-14. Lief. $1824-29$.

Govilsir, in Edinburgh. - Meta+ morphosil: Iler Decicputlen (Edisub. new phil. Journ. XXXI11, 1842, 181). Caligus (ibił. 178. - Miill. Archiv 1843. - All1. d. se. nat. 18, p. 181). - (uit Ms. Bell) The Crustucea in Zoology of the voyage of Erebus and Temor ( $\operatorname{Ros} s$ ).

Gondot, Kerfhäudler in Paris.

Gräre, Heimich, Dr. phit, Prof. der Philosuplie und Direktor der Realund Birgersehule in Jena. - Nalurgeschichte der Ulei Reiche. 2 Bïnte. Berlin 1841. 8. (Kostbar). - Naturgeschichte nách nlleu drui Rrichen für Schule mut Haus. In Verbindang mit J. F. Na um in $n$ bearb. g\%. 8. Eisleben.

Grant, Professor der Zuotomio und Physiologie an ter London. Universitut. - Oatlines of rompacrative anitumy etc. (vpl. Lex. d. 'l'heisol.).

Gravenliorst, J. L. Clir, Gelieimerrith, Prof. und Direktor yu Breslan. - Vergleichenule Uebersicht de's Linne'schen und einigir nrueren 7.00logischen systene, nebst dem eingeschalteten Verzeichuisse der zoologiseheu Sammlung des Veifassiprs unt ten Beschreibungen nener 'Thierarten, die in tletselben vorhanten sind. Güttimen 1S07, bri Heiur. Dietrich. 8. 476 S. - Vergleithende Zoologie. Breslan 1843. 8. 687 (Isis 1843, 627).

CAray, George Robert, Esty, Custos an b ritischen Muse on \%u London. - Synopsis dl. Cirvipenlen (Amu. of Pliil.XXVI, 1825.97. - Lsis 1834, 4s8). - Ueber die Reproduction der Cirriperlen (Tay. 
lor Proceed. III, Oet. - Isis 1835, of a new speeies of the Genus Asta548). - Cirripedes (iu K in g's Survey to Australes. 11. Append. 469).

Crustaceen in Griffith's Anim. King. dom.

Green, Jakob, M. Dr. und Prof. in New.Haven. - Monugraplyy of the Trilobites of North-An. Philad. 1831 (mit gemalten Modellen) (Sillim. Am. Journ. XX111, 395. - Isis 1835, 95). - Ueber cinen neuen Trilobiten (ibicl. $\mathrm{XXXI}, \mathrm{Nr} .63$ und Nr. 66, 343).

Gù riffith, Edward, Esq. in Lnudon. - (Cnvier) The Animul Kingdom.

Goxinithaisen, Franz v. Paula, Dr. und. Arzt, Prof., Ritter etc. z.u München. Genialer Forseher von exorbitantem Ruf. - Ueber Daphnia Pulex (Nov. Aet. Aead. Leop. XIV, 1). Zu Wiegmann's Ablıandl. über Entstehung von Entomostraceen ete. (ibid. XI, II, 723). - Infusarien, Würmer, Entomostraea (Isis 1820, lit. Aureig. p. 247).

Gǘrin, François Etienne, Prof. in Paris. - Mèmoire sur l'Eurypodr, nollveau genre de Crustacés (Mém. du Mus. d'hist. nat. T. 16. - Ann. d. sc. XVII, 319. - Isis 1834, 1085). Ueber Latreille's Notopoden (Aun. d. ค.. 11. XXV, 1832, 288. - Isis 1835 , 468). (Dronia: Dymomene, Cymopuliı (Roux), Caphyra, Dorippa, Ethusa (Roux); leztire nit neuer Species.) Crustaceen aus der Familie der Hyperinen (Guèr. Mug. VI, 1836, T. 1720). - Ein neuer lírebs zwiscluen Paguriden und Thalassiniden : Isea elongata von deı Mariannen (Annal. soe. ent. Frunes: I, 1832, 295, Tf. 10). - Bemcrkungen über Limnudia (Daplınia gigas Herus. pte.) (Guer. Magas. VII, 1837. - Isis 1842, 223). - Teunographic du Regue animal (Crustareil). - Abhandlung über den äusseren Bau der Phyllosomen, welehe Lesson und Reguud mitgebracht haben (MIag. I11, 1833, IIft. 5. - Isis 1834, 273). - Temisto, neue Sippe der Crustaceen (Mèm. de la soe. d'hist. nat. Paris IV, 1828, p. 379, T. 13. - Isis 1832, 486, Taf. IX).

IfaItmeyex, Georg, Dr., Prof. und Custos um Joanneum z" Gratz.

Hamel, J., k. k. Staatsratl etc. in St. Petersburg.

Hnxlan, Richard, Dr. med. und Prof, in Philadelphia. - Description cus (Trans. of Philad. III, 1830 , p. 464-(i6).

velmholtz, A., Dr. med. in Berlin. - De labuicui systemat. nerv. evertebrat. Diss. inang. Berol. 1842. (T'üeltige Arbeit. Vgl. Müll. Areh. 1\$43, CXCVII). (Bestatigt, dass dic Elemente des Nervensystems hei den Crustaeeen, Arachniden, Kerfen, Wii rmein und Mollusken diesclben sind, wie bei den Wirbelthiereu.) (vgl. Siebold in Mullers Arrl. 1843. 11). Nervensystem de's Flasshiebses (nente Sclurift. von Danzig 111, 4. 1842, 120).

Merold, Mauritius, Prof. zu Marbug. - Disquisitiones de mimalium vertehris carentium in ovo formatione. Franc of. Il, 1838, Fol. 'T. 6 (Isis 1839, 1. $231-240$. Ripension).

Derrich-Schiofter, G. A. W., Dr. med. und Stadtgerichtsurat zul Riegensburg.

Hildebrnual, Prof. in Diisseldorf:

MofTannum, Naturalist aus Hanburg in Paris (Quai des grauds Augustins Nr. 19!. Verkauft hillig.

Toeninglamas, Friedr. W., Priisident in Crefeld. - Ueber Calymene mac roplithalma (Isis $1 \times 24$, p. 464, 986). - Unber Trilobiten uns dem Ueher. grangskalk (Vurtrae 1834. - Isis 18.36, 1. 224). - Beitrau zur Monographie der Guttung Crania. 4.

Doevea, J. van der, Professor in Rotterdam. - Recherclies sur l'histoire mafurelle et l'Auatomie des Linules. Leyde (Luchtmans) 1836, Fol. 48, 7 ì. (1sis 1839, 397) (Prachtwerk in juder Hinsieht). - Uebor die Zahl der Luftlïclier bei Scolopendra Jeach ('T'vilsehr. f. nat. Gesclied, IV, 1839, Taf. - Isis 1840, 395). - Tahula reguis animalis, udditis classium ordinumque elaracterıbus, quam edil. in usum auditorum. Imp. - Ful. (Lugd. Bat. Cyfverr) Lips. Weigel.

Hombres, D', Baron, Firmus von Alais.

Engi, F. J., Prof, zu Solothurn. Schöne Crustaceensammlung.

Jnequinot, Marine-Chiıtrg zu Puris.

Jïger, F. W., Fr. phil. und Lehrer zu Hamburg. - Grundriss der fesammtell Naturgeseliehte. Hainburg 1840. 8. 
Jasche, in Werningerode. Carcinolog.

Jones, Rymer, Professor zu Loudon. - Myriapedat (Cyclopaclia of anatumy and pliysiology by Todd. Pait 24. 1842, p. 544).

Dolau, Prof. zu Cork. - Ueber die Cirripedien (Tlompson Zoological Resenrehes and Hlustrations etc. Cork. - Dann in den Philosophical Transaetions. 1835 , II).

Johnntou, G., in Edinburgh. Beitrige yur brittischeı Fauna (Leacia lacertosa. Crust.) (Janı. Ellinb. philos. Jnurn. XIII, 1825, p. 218. - Isis 1832. 611). - Ueber Caligus (Eliuh. pls. Jnnru, by Brew. X, p. 292. Isis 183:- 603). - Orythii rocrinei (Zoological Journ. 1838).

Doly, in Paris. - Nenes Entomostracon, Isaura cycladoides (Aumal. d. sc. nat. XXII, 293 und 349. - Müll. Areh. 1842, CXLV und 1843. V).

Exnup, J., Dr., Prof. und Cinstos zu Darmsiadt. - Allyemeine Zoologie in ilıren Gattumgrseprisentanten naelı den nenesten Untersuebungen. Darmstadt, Leske. 1827 et seq.

Killaw, Prof. in Mannheim. Sehr verdient um das dortigr? hiibsche Mu. seum.

MAxby, Willian, Esq. Rev., Rector von Barliam (Englinul). - (mit William $S_{p e n e}$ (e) An Introduction to Entomology : or elements of the natural history of insects. With Plates. Third edition. Vol. I. Lonlon; printed for Lougman. Hurst, Rees, Orme and Brown, Paternosterrow 1818. 8. (Second edit. 1816, I, XXIV $॥ 11519 \mathrm{pp}$. I1, 1817, 1. 329. 8.) Dentsilh: Einleitung in die Éntnmologic, oder Elemente der Naturgeschichte der Inserten. Mit Abbild. Bd. I. Stuttgart, in der Cotti'sehen Buchhandiung 1823. (Die Uebersetzung ist unter Oken's Leitung veranstaltet. $22 \mathrm{~S}$. Vorrede der Verfass., $4 \mathrm{~S}$, von Otell, $2 \mathrm{~S}$. Inhalt, $563 \mathrm{~S}$. Text, $17 \mathrm{~S}$. Register von Oken. Bd. II, 1824, \& S. luhalt, 392 S. Text, 14 S. Register v. 0. 8, a Binde, mit Kupfern, im Ganzen.)

Iixchuer, T. W., in Sorau. Die Versteinerungen und Fossilien, welche bei Surau und der Umgegend gefinden werden. Eine deutsch geschricbeue Abhandlung, gr. 4. Sorau, Julieı. - De Petrefactis et Fossilibus, quae Soraviac et in vieinis agris reperiuntur commentatio. Cum immagin. lith. 4 mij. lbid.

Inlidicn. F.. Direetor (?) 7.n Berlin. - Die Versteinerungen der Mark Brandenburg, insonderlicit diejenigell, welche sich in dell Rollsteinen und Blöeken der südbaldischen Ebene findeu. Mit 10 Steindrucktaf. gr. 8. Berlin (Lüderiz).

面I las, Friedr., Dr. Ined., Medizimalrath, Dirertor des k. Museums der Natu!geschichte eft. in Berlitu. - La. treille's genera Crusticemrum et Inseetorum: angezeigt von Dr. Fr. K I u (Web. und Mohr's Beitr. z. Naturg. If, p. 2(7).

Foch, K. L., pens. Forstiath in Regensburg. Tüchtiger Zoolog und Schifisteller. - Deutsehiants Crustacern, Mriapoden und Arachniden. Regensb. IX. 16. (a Heft 24 litl. Kpf., 3 H. o kr.). (VI, 1836. Isis 1837, 68).

Woch, F. C. L, in Rrna. - (mit W. D u $u$ ker) Beitr. z. Kenutu. des nordileutsch. Oolithengebildes und dessen Versteinerungen. Mit 7 Taf. gr. 4. Braunschw. Oehme. 1838 (4 fl. $30 \mathrm{kr}$.).

Filollar, Viucent, Dr. ph. und Custos an k. k. Museum in Wien. Beiträge zur Kenntuiss der lernacenartigen Crustaceen (Anual. d. Wien. Mus. I, 1, 18.35, p. 79). (Von Oken zu den Sehmarotzerläusen gestellt )

Lomiuck, de, Professior zu Luittich. - Hemoirs sur les Crustacées fossiles de Belgique (Extr. des Mim. de l'Acall. de Brux. XIV).

Lote, B., Islorer zu Mardeburg. - Das Thierreich. Nach A. F. Schweigger's Systeme. Als Leitfaden beim Unterriclite in der Naturbeselureibung der Thiere. 8. Magdeburg.

In rassow, C. R. A. Freil, von, in Berlin. - (mit Di. Ed. L e y de) Lehrbuel der Naturgesclichte fiir Gymmasien. I. (Zoologie.) Beilin 1842. 8.

Hexness, Ferdinand, Prof. Dr. phil, Custos au k. zonlogiselup Museum iu Stultgart. Sammelte willuend seiues Aufentlualtes im südlichen Afrika in den Jahreu 1838, 1839 und 1840 die Crustentluire mit besonderer Anfwerksiankrit. Seine Eifalırungen sind niedergelegt in der empfohlungswiirdigen Schrift: Die sijdafrikanist'l. Crustaceen. Eine Zusammenstellung aller bekannten Malacostraca, Bewerkungen liber 
deren Lebensweise und geographische - Des Limnadies (Bullet. de Moseon Verbreitung, nebst Beselirribung. Ind ed. Leq. (V) 1833, 1. 357-364, Tab. Abbildung mehrer neuen Arteu. Mit (Crusturea, Daphuia rte.)

4 lithogr. Taf. Stuttgart (Scliweizerbart) 1843 . gr. 4.

Iñ⿻i口yer, Henriek, Prof. in Kopenhagen. - Geryon tridens, fine neue Krabbe (Naturhistorisk Tidskrift. I, 1, 1836. - Isis 1840, 947). - Monogratiok fiemstilling of Släıten IIippolyte's nordiske Arter ete. Mled Bidrugtil Dekapodernes udviklings historie. Kjöbeulavu, 1812. 4. 152, 1.6 (Recer1. sirt in Oken's Isis 1844, p. 157). Grönlauds Amfipoder beskrevie af $\mathrm{H}$. K. Kjöbenhavn 1838, 4. 94, T. 4 (Isis $1842,475-78)$. (Zur Crusticeenlelure selu' wichtig.) - Conspectus crustaccorom (iroenfandiae (Naturlı, Tïlskr. V, 1841, 249-261. - Isis 1841, 408). Vier urue Arten der Crustaceengaltung Cunta Edw. (T'idsk. III, Hft. 6, 18.11, p. 503-534. - Isis 1842, p. 915938). - Monografi af Ilippolyte. Kjübenl. 18.12, 4. 152, T. 6 (Oersteris Oversigt 1841). - Uebersieht der nordischen Arten der Galtung llippolyle (Tidsk. III, p. 570-79. - Isis I. c. p. 938). - Munua, ciue nece Krebsernttung: besclur. und abgeb. (ibid. II, Hft. 6, T. 7. - Isis $1811,427-29)$. - 130pyrus abdominalis (ibid. III, 1, 1840, p. 11:-112, Taf. - tsis $1841,1.693$ -699 , Taf. II, I!I, 2, 3, 1. 289-99. 1siv 1. e. $707-713$ ). - Anzeiclunnngen und Bemerkungen zur diiı. Fauna ib. I, K. 4,1837, 1. 40.1-15. Mysis). Ueber die Verwandlungen der Pyewogoniden (ihid. III, 1840, p. 299-306. - Isis 1841, p. 713). - Uebri dic Selumarntzerkrebse, mit hesonderer Rürcksclut unf die dänische Fanna (ib. I, H. 2, 1836, p. 172-208, Tf. II. Isis $1840,702-724$, 'Tf. und 738). Uiber die Selimarotzerkiebse feruer (ih. I, II. $5,1837,476-509,605-78$, Tf. - Isis 1841, 89-115, 187-200). Schmarotzerkrehse (Forts. ibid. II, II. 1, 1837 , 'Tf., p. $8-52$. - Isis 1841, 281. - Schluss. ibid. 11, 1838, 2, 1). J31-157, - Isis 1841, 333-347).

Krohn, August D., Dr. med. ete. zu St. Petersburg. - Gefïsse und Nerven des Krebses (Isis 1834, p. 518 -33 , Tf. XII).

Irriger, Fr, Dr. med. und Medi zinalrath iı Pyrmout. - Selö̈uc $z 00$ logisclie Sammlıng.

Inister, Pof. an der Gewerbsscluale zu Erlaugen (Nr. 284) - Reiseberichte aus Dalmatien ind Montenegro (Isis 1842, p. 283-301). - Naluilist. Reiseber. II (ibid.609-616). - Reiseb. III cibid. 743-52). - IV (ibid. 847 $-50)$.

Lachunanu, F. IH. A., Dr. med. in Brantuseltweig. Seluriftsteller.

Cummarck, Mademoiselle (fille ainée du Clovv. J. B. Lamarek) z.11 Paris. Mitnubeiterin an Cuvi e r's Règne animal. P. 1830. Classe der wirbellosen Thinre.

Eeconte, Joln, Ingenicur-Capitain in den vereinigten Siaten \%u New. York.

Lebas, Paul, zu Ronen. Entomolog und Reisender in Columbien, Carthagrua u. s. w. Gronse Sammlumg.

Eesonold I.. Se. Majestit, ICünig der Belgier, in Brinssel.

Deesson, Mariur-Pharmnzeut etr. in Piris. - Crustacen in Duperuey's (Coguille) Vovagr (Isis 1833:57). Illustrations de Zoologice - Centurie de Zoologie. Paris 1830. 8. Av. planel.

Lesmenr, Clarles Alexandic, Prof. in New. York. - Sur une nouvelle esplece d'insectes du grure Cymothoa dé Fabricius (Cymothoa bopyroides) (Nouv. Bullet. des seiences pur la soc. philomat. 181., p. 45).

Edenis, Joln., Prof. in Hildesheim.

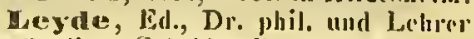
zu Berlin. Schiftsteller (vgl. Krassow'.

Hherminier, Ferd., Dr. med. zu Point-ì-Peitre aluf Guadeloups.

Eiclitenstein, Hofrath und Prof. in Berlin. Gab die Gattung Leueosia (im Nagraz. der natur. Frenude VII, p. 135 - 145) als Probe einer teuen Beatrlicitung der Krabben und Krobse liernas. - Die Werke vou Maregrave und Piso vitier die Naturgesclichto Brasiliens rrläutert (Abhandl. d. Beyl. Ak. J. $1814-15$ und $1816-17$ und 1820 -21 ).

Diemned, d J., auf St. Moriz. Bischreibung von meuen Crustaceen auf' Maurire (Phil. Mag. IX).

Link, H. F., Hofrath ete. in BerIrynicki, Joh., Prof. jn Kharkoff.|lin. - Die Urwelt und das Alterthum, 
erlïutert dursh die Naturkunde. Berl.|Bonn. - Analekten für vergleichende 1834. 8. (2. A॥f.)

Anatomie. - Dichelesthion sturionis

Loven, S. E., in Storkholm. Evadue Nordmanui, ein bisluer unbrkanntes Entomostracon (K. Voi ieuskaps Handl. 1835, 1-29, T. 1, II. - Isis $1838,4-5$. - Wieumanu's A rehiv $I V$. Bd.. 1. 1. 143. - L'lustit. 18:5, 262).

Funul, Peter Willelm, Dr: med. und Prof. in Kopenhitgen. - Ueber die Cireulation der Crustaceen (pekrünte Preisschrift). Kopenh. 1824. 8. - Zweifel an dem Daseyn eines Circulationssystems bei den Cinstacen (Isis 1825, 593). - Blutlanf der Crustilceen (ibid. 1829, p. 1299). - Kreislanf der Crustaceen, Forts. (ib. 1830, 1222-1228).

Nacleay, William Shap, Fisq. zu Hobartstown in Vou Diemensland. Besilurieb die Crustaecen in Smitl's Illustritions of the Zoology of SonthAfrica (Amulosa). - Ueber die kurzseluwï̈niyn"u Decapoden (Amnulosa III, 1838. - Isis 1843,932-35). - Lebensart vou Gripssus ruricola Deg. (Zool. Trans. I). - Ueber Urania (mit Nebenhemerkungen üher Krabben anf Cuba) (Trians. of Zool, soc. I, p. 197. - Isis 1836, p. 410).

Misuien, E., Arzt und Couservator des Cabinets zu Chalons sur Marue.

Maruura, le Cnlonel Albert do Ia, iı Genua. - Voyage en Sardaigue on Description statisticue, plysique et politique de eette île, avec des recherclies sur ses prodnctions mafurelles et ses antiquitès. Seconde édition. 'Turin (Bocea), Paris (Bertrand). I, 1839. 8. 528. Atlas. Tab. 10, Fol. II, 1840, p. 695, Atlas, T. 41, Fol. (Recells. in Oken's lsis 1842, p. 626).

Marshall, Thomas, Esq. in Birmingliam.

Min rtens, vou, Kanzleirath in Stutt. gart. - Reise nach Venedig. UIm 1824. 2 Bde. 8.

Martlus, W. C., Dr. med. und Professor zu Erlangen. - Lelirbuch der pharmaceutischen Zoologie fiir Apotheker etc. M. 3 Taf. Abbild. Stuttg. (Balz) 1838.

Mayer, Franz von, Ritter des französisehen Verdienstordens derTreue in Miinclien. Vorstand des zoologisehen Tauschvereins (Adalbertstrasse $\mathrm{Nr}, 9$, parterre). Vyl. Lex. d. Entom.

Mayer, F. J. C., Professor zu

Fenke, Hofrath in Pyrmunt. Prachtvolle Sammlung; ditvon vieles nen.

Meyer, Hermann v., in Frankfurt a. M. - Nenc Gattungen fossiler Krebse aus Gebilden voun bunten Sandstein bis in die Kreide. Gezeiclnet null beschrieben. Stuttg. 1840. 8. 28 pp. 4 Taf. Fol. - Palinurus Sucrii (Acta Leopold. XVI, P. 2, p. 517, Tf. 38). (Versteinert.) - Beitrïge zu Eiyuu, einem Geschlechte fossiler langschwänz.iger Krebse (ibid. XVIII, 1, p. 261, Tuf. 1-2).

Pichelotti, in Turin. Fand zwei Trilobiten in oberu tertiären Boden bei Turin (L'Inst. 1838, Mai, p. 177). (Isopodel1?)

MEikan, Johann Christian, Dr. med. nnd Professor in Prag. Berülmmter Foisender. - Entomolngische Beobachtungen, Berichtigungen und Entdeckungen. Coleoptera. Oniseus pustulatus (p. 128), Pedienlus Andrenae (136) (N. Abhandl. d. bölın. Ges. Bd. III, Physik, p. 108-136). - Brasilische Julus - Arten (13) (Isis 1834, 741 ).

Mullet, in Paris. - Hippolytus desmarestii an Wasserpfanzen (Aunal. d. se. nat. $\mathrm{XXV}, 460$. - Isis 1835 , 472).

Moler, M. H., Professor zn Göttingen. - (mit F. W eber) Naturhistorisclie Reise durch eimen Theil Schwedens. Göttingen 1804. 8.

II tiller, Johannes, Dr. med., Director etc. 2u Berlin. - Arehiv fïr Anatomie, Plyssinlogie und wissenschaftliehe Nedizin, in Verbindung nit mehren Gelehrten. Jeder Jahrgang 6 Hefte, mit Kupfert. gr. 8. Derliu. - Unber die Angen und das Selien der Insekten, Arachniden und Crustaceen. Forts. (Anu. d. sc. n. XVII, XVIII, 1S29, 73, t. 19. - Meckel's Archiv, 1829). - Ana. tomic vou Scolopeudra morsitans (Isis 1829, 549, I, II). - Ueber den Bau der Augen bei Argulus foliaceus (Tiedem. Zeitschr. IV, 1, p. 97. - Isis 1832,1024 ).

Milller, Dr. med, in London. Bedeutrule Crustaceensammlung. Schrieb iiber sïdamerikanische Crustaceen.

Munter, Gust. Wilh., Dr. philos. und Aufseher des k. anatom. Muscuus 
zu Halle. - Allgemeine Zoologie. Halle 18.10. 8.

Nolder, S., Dr. phil. und Maler in Leyden. Bekanut durch dic vortrefflielien Darstellungen derjapanischen Crustaren in Siebold's Fauna Japonica. Dreas I-IV, 1833-39.

Murcluisou, in Ediuburgh. Ueber die tertiären Siisswasserformationen in der Provenes (Ediub. new phil. Journ. Vll, 287, T. s. - Isis 1832, 819). (Vicle fossile Kerfe, bestimnit voul J. Curtis.)

II use, J., zu Canbridge (Maryland). - Thiercheu im Schuee (Garncelen?) (Sillim. Amer. Journ. XVIII, 1830, p. 56. - Isis 1832, 1060).

Napoli, L., Apotheker in Triest.

Nomuann, C. F., Dr. ete. zu Leipzig (?). - Beiträze zur Kenntniss Norwegeus, gesammelt auf Wanderuilgen wälırend der Sommermonate der Jalıre 1821 mol 22. 2 Thle. Mit Profilen und Charten. 8. Leipz. (Wienbraek) 1823. - (mit Dr. H. Grä fe) Naturgesehiehte nach allen dree Reichen fïr Srhule und Haus, gr. 8. Eisleben (Reichardt).

Nenming, Professor und Dr. phil. zu Coustan\% - Ueber die Naturgeschichte der Ungegend von Constanz. gr. 8. Const.

Newport, in London. - On the organs of reproduction and the development of Myriapoda i Pliilos. Trans. 18+2, Part. 11, 99. - L'Inst. 1842, 98. - Froriep's nene Notiz. Bd. 21, p. 161. - Müller's Arelı. 1843, VII. - Lond. a. Ediul, phil. Mag. n. 127, Suppl. 1842, Januar.

vietselı, C. F., Lehrep in Hanau. - Lestfaden zun Unterricht in der Naturheschreibung und Naturgeselicilite, durch Tabellen und deren Erliuterung. gr. 8. Hunau 1835.

Nordunaus, Alexandor vou, Profrssor zu Odessa. - Mieroscopische Beiträge zur Naturgeschichte der wilbellosen Thiere. Berl. 1832. Heft 2. M. K. (Crustareen.)

Noyes, Professor in New-York. Carcinolug.

Desterlen, Friedricl, Dr. mel. in Murllardt (Wiirttemlierg). - Ucber den Magen des Flusskrebses (Miller's Arch. 1840, p. 387, Taf.).

Dleire, H. d', Med. Dr., geh. Hof- rath ete. in Bremen. Sammlung von Sephicren rte.

GIfers, lgn. Fr. von, Grsandter in Berlin. - Ueber die Liune'srlien Gattungen Cluiton und Lepas (Mag. der naturf. Fremule in Berl. VIll, p. 163 $-178)$.

Bken, Lorenz, särhsisch. Hofratl, Dr. med. ef I'lil., Professor der Natur. grescliclife ete. in Zuiricl. - Naturerselticlute föir alle Stionde. 13 Bde. in 8. M. Atlas. Stuttg. (Hoffmaun) und eine Menge anderer gediegener Schriften.

Ardoigny, Aleide Dessalines d', in Paris. - Curophimm (Cancer grossipes) in Cuvier Rı.gur animal. IV, 121.

Deto, Aslolph Willolm, Ohermedizinalrath ete, zu Breslan. - Nene Crustacen uns dem Mittelmere (Nov. Act. Acad. Leop. Carol. XIV, 1). Ueber melire Petrefakten iKrebse. Museloeln, Anmoniten, Palaronisens, Schle. sipns (Voutrag 1836. - Isis 1837, 433).

Oudart, in Mïllhansen. - Vgl. Lex. d. Theriol.

(D)en, Richard, Professol zu London. - Crustaeern von Clili (Taylor Propeed. P. Il, 1832, Jan. - Isis 1835, 367).

Perry, Willian Edward, Capitain in Loudon. - Jomnal of a voyage for the discovery of a North.Wrot.Passage from the Atlantic to the Pacific; reformed in the years 1819-20, in lis Majesty's slips Hrela and Gripper, under the orders of W. E. Pary, R. N.. F. R. S. rte. Lond. John Murray, Pnblisher to the admiralty, and board of Longitude 1821, 179 bez. S. 4. 20 Kupfert. - A Supplenent to the appendix of papitain Parry's voyage for the discovery of a North-West Passagre, in the years 1819-20, contuining the Zoologiral and Botanipal Notires. Londou, John Murrily. Albemale-Street. 1824. 310 S. (mit dem Werke selbst, $6 \mathrm{Kupfert.4.)}$.

Pral Alexander Leopold, Fïrst von Lippe-Sehaumburg, Durchl. zu Lippe. Zoolog.

1*anu, in Marignane. - Ueber die rothe Färbung der Salzsïmpfe im suidl. Frankrcich (L'Iust. 1836, 182. Isis 1837, p. 789). (Brauchipus salinus?) Peneada, in Agnano. - Monocolo agnellino. Mem. fis. anat. (Atti dell' Accad ital. Liv. 1810, I, 127, fig.).

Perlek, Carl Julius, Dr: phil. und 
med., Professor der Naturgesclichte und Botunik zu Freiburg in Baden.

Porrières, Auguste, Particulicr in Bordeaux. Schöne und instructive Sammlung.

Perty, Maximilian, Dr. med. et phil., I'rufessor del Natureseschichte ete an der Hoclisclumle \%u Bern. Bevibuter Zoolog und Schriftsteller. Allurueine Nalugeselhichte als philosopliseche und Hunanitatswissenschaft. 3 Bitille. Bern 183i-41. 8 . (Isis 1841, 369-379). - Delretus Animaliun articulatorum, quac in itinere per Brasilian antus 1817-20 jussu of anspiciis Maximiliani Josephi I eti. peracto collegerunt Spix et Martius. Digessit, descu ipsit ef pingenda (uravit Dr. Max. Perty. Monachii imp). Edituis 1830-34. 4. - Zur Fanna monacensis (Crustaceriu) (lsis 1832, ร23-728). - Einige Brmcıkungrn uber dic Familie Xenomorphidae oder die Sippe Arctiscon Sclirank (Macrobintus Schultze, Trionyclium Elnemb.) (Isis 1831. 1241-1246).

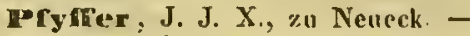
Skizzin vou der Insel Java und doreselber verschied. Bewohmern. 3. Hft. mit gemalten Bildern. Fol, schati:lausen (Hurter) 1830.

Hoielnering, Ch.. in Philadelphia. - (uit J. Dan a) Culigus americamms (Fisclulaus) (Silliu. An. Jouru. XXXIV, IV, 70. - Isis 1840, 201).

Presl, Carl Borziwng, Dr, med. und Professor \%u Prak. - Di. F. X. R am is ch's Naturgescollichte lles Thierreichs. Fortsetzung. M. Holzsehnitten. gr. 8. Prag 1835.

Prectumllea. N., in Neapel (?). - Brsthrihung eines neurn kicbses im MIere hei Messina (Cryptophthalums Costa) (II Progresso XVIII, 1837, 141. - INis 1843,53 ).

Purluinfe, Dir med. und Professor zu Breslau. - Eutstchuner des Macro. biotus uus Saud, den man mit Wasser beucr.t und stehen lisss, wo danu warll einizen 'Tayen mohre davon siclı ze.igell (Vortıag zu Prug. - I sis 1838, 594).

enix, Chr., Professor in Aachen. - Natubesclureibung der Thiere, oder Zoologie nuch deu Haudbuche der ZooInvie von Di. G. A. Goldfuss. 1. Bd.lı. 2. Abthl. Naturbeschur, der nilgeflugelten Kerfe, Halbdeskflïgler, Kä. fer, Zweifligler, Hant-, Grad- und
Netzflügler. 8. Aachen-Cöln (Dumont). 1830. Die 1. Abthl. Ur-und Pflanzenthiere. 1824.

Quoy, Jean Renè Contant, in Paris. - (mit Gaimard) Ueber Sittell der Crustacea Decaporla (vgl, deren Reisebesclireibung). - Ueber das Betragen und dic geographische Vertlıeilung cininer Crustaceel (AnI. d. sc, 11. XIV, 153. - Isis 1834, 1026).

Fufrelsberger, F., in Wien. Gemälde aus dem Naturreiche beider Sicilion. Mit 8 Kupf. und 1 Vignette. gr. 8. Wien 1830.

IRafinesque - SmaItz, C. S., Professor der Butanik und Naturgeschicbte au der Universität zu Lexington (Nurdamerika). - Précis de Déconvertes semiologinnes. Palerme. 18. 1812. - Anilals of Nature. New.York. 8. Nir. I.

Ramdohr, K. A., Dr. med. zu Schloss Beichlingen in Thüriuren. Beiträge zur Naturgeschichte einiger deutsclien Monoculusarten. Halle 1805. 4. c. tab. - Ueber das Erscheinen der Monoculusarten an manchen Orten Germ. Magaz. 1, p. 189). - Ueber die Gattung Cypris Müll, und drei zu derselbell gehörige ncue Arten (Mag. d. Ges. naturf. Fr. थu Berl. II, p. 83 -93 , Tab. III). - Micrographisclie Beitriige. 1805. 4.

IRsselt, Heintieh, Dr. med. und Conservator an k. Naturalienkabinet zu Cliristiania.

Thathlce, Heinrich, Professor etc. zu Königsberg. - Beiträge zur verplcichenden Anatomie und Physiologie: Reiscbemerkungen aus Skaudinavien (neueste Schr. d. natf. Ges. zu Danzig III. 4. 1842. - Decapoden und deren Lintwickelung, Mollusken, Spinnen, Crustaceen. Vgl. Mhïl. Archiv 1843, CLXIX). - Bemerkungen über den Bau des Dirhelesthium Sturionis und der Lernaeguoda stellata (Acta Leop. XIX, 1, P. 125, T. 17). - Anatonie der Ilotea Entomon, oder des Scluachtwu'ns. Danz. 18:0. 4. - Entwickelung des Flusskrebses (Isis 1825, p. 1098). - Untersuchungen über die Bildung und Entwickelung des Flusskrobses. Mit 5 feil illum. und sclowarzenl Kujpt. gr, Fol. Lejiz. (Voss) 1829. (Isis1829, p. 429). - Krebs(Auszug : Annl. sc. 11. XX, 442, T. $5-8$ ). $-E_{1}$ 。 
bryon von Astneus marinus (neue Selir.jstaees des environs de Nice. Paris 1. matf. Ges. in Danz. III, Htt. 4, 23). 1816. 8. Av, fig. (impriméc en 1813). - Entwickelung der Crustareen (nene - Obscrvations sur quelques nonvelles Not. v. Froriep. XXIV, 181). - Nymplion femoratum (Naturlistorie Selskabets Skrilter, V, 1, p. 201. Talo.). Entomologiske Jagitingclser : Nymphon, Pyenngronum ete. (Skrivter af naturhist. Selskibet. Bind 5, Hefte 1, p. 191).

Tatzebure, J. T. C., Professor zu Neustadt-Eberswalde. - (mit B ra $\mathrm{ndt}$ ) Medizinisehe Zoologie. Berlin 1834. 4.

IR ave, B., Dr. med. in Köln.

Redtentuacloer, L., Dr. med. und Adjunki am Museum zı Wien.

Helumauu, iu Donanesclingen. Bearheitet ivissensehaftlieh die fossileu Crustaceeu.

Meichenbnelk, H. G. Ludw., Dr. med., Hofrath, Professor ete. iil Dresden. Weitberiuhmter Botaniker, Zoolog und Schriftsteller. - Der Na. turfreund,oder: praktisch-gemeiunitzige Naturgesehiclute des In- und Auslandes durch eine mögliehst vollständige Sammlung unit grösster Solgfalt ausiefülırte:, von vorzuiglichen Kinstlern gezeichmeter und in Kupfer und Stalul gestocliener treurr Naturgemälde erläuteıt, bearb. und herausgeg. Lex.8. Lripz. - (W. Hemprieb) Grundriss ler Naturgesehichte für löhere Lebranstali'n, 2. Aufl. Nach dem Tode des Verf. umgearbeitet vou R. 8. Berlin (Riieker).

Meichenbrach, M. A. B., Lehrer in Leipzig. - Bildergallerie der Thierwelt oder Abbildungén des Interessantesten ans dem Thierreiche, mit ausfïhrl. Besehreib. 14 Hefte. gr. 8. I,eipzig. - Nenester Orbis pietus oder die sielitbare Welt in Bildern, rin Universalbilderbueh mit vielen Stahlstichen. 4. - Naturgeschiehte für Volksschulen uml die unteren Classen höherer Bürgerschulen, mit 150 Abbild. aus dem Thierrejele. gr. 8. Leipzig (Hermauı und Langbein).

IReinluarit, H., Professor der Zoologie in Kopenhagen (nieht Stockholin).

Iticmamn, R., Lelorer in Magde. hurs. - Naturhistorisclu-tec:mologiseher Isciffaden fïr Büiger - nud Vorberejtungsseliulen. Magdeburg. I. Tlicekunde. 1838 . 8.91.

IRisso, Domeuico, Apotheker in Nizza. - Histoire naturelle des Cru-

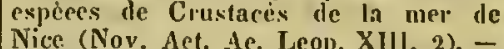
Productions V, p. .22, 'T. 3, f. 10 (Plorosyne?.

IBtgen, F. Aug., Professor in Marlungr. - Ueber dic Aufrinanderfolge des ersten Anftreteus der verschiedenen organischen Gestalten. Marburg 1828. 8. (Auch in deu Seluifteu der Marhurg. Gesellscloaft ete. 2 Bile.)

Ixivoli, $S_{i}$. D. der Herzog voll, und Esslingeu, Solu vou Massena in Paris. Kostbare Sammlungeu.

Robincau-gesvoidy, J. B., Dr. merl. in Saint-Saveur (Yomne). Recherehes sur l'organisation vertebrale des Crustaces, des Arachnides et des insectes. Paris 1828. \&. Av. fig. (6 fr. 50 c.)

Roctuel, Ant., in Pestl, - Naturbistorische Miscellen iiber die norrwestlichen Karpathen. Pesth 1821. 8.

Roenner, Fr. Ad., in Hannover. Die Verstrinerungen des norddeutschen Kreidegebirges. Hannover, 1, 1840, II, 1841, 4. 145, T. 16 (Isis ! K42, 240). (Conchylieu, Ceplualoporlen, Rluizopoden, Anneliden, Constaecen, Fiselie, Mosasaurus.) - Die Versteinerungen des Oolithgebirges.

Toss, Capt., in London (vgl. Le a e lu hinten). - Relation of the voyage of H. M. S. S. Erebus and Terror nuder the command of Captain Sir James Clark Ross ete. during the Yenrs 1839 , $40,41,42$ et 43 . Edited by John Richardso n and John Elward Gray. (Ersterer bearbeitete die Fische, Lezterer die Sucke, Reptilien, Conchylien und Coralleı, G. R. Gray die Vönel und Bell und Goodsir die Crmstaceen.) Deutsch: Zweite Eutdeckungsreise narh den Gegenden des Nordpols 1829-33. Aus dem Engliscluen von Julius vou der Gröb cu. 2 Theile. Mit 17 Stahlstielsen und 5 Karten. gr. 8. Berlin (Reimer).

Tossmissler, E. A., Professor iu Tharand. - Beitrige zur Versteinerungskunde, mit litl. Abb. 1. Hft. - A. I. d. T.: Die Versteinerungen des Bramukohleusandsteins nus der Gegend vou Altsittel in Bïlumen, litl. und besclur. m. 12 litls. Taf. gr. Lex.-8, Dresden und Leipzig 1840.

Ronssel de Vauzème, in Paris. 
- Cetoehylıs australis, cin noues Crustacenum branchiopolum (Ann. d. sc. n. 1, 1. 333, T. 9. - Isis 1836, P. 514-16). - Uellי" Cyams Ceti. Anatomic (ilsid. I, 1834, p. 239, T. 8, 9. Isis $1830,11,505-5(0)$.

Eivpluel, Eduard, 1)r, etc, in Frankfurt i. M. - Reisen in Nubien, Kurdofan und dem petriiiselten Arabien, vorr.igglich in geocriaphisch-statistischer Hinsiclut. Nit 8 thupt: und 4 Karten. gr. 8. Frankfurt a. M. - Desclireibung und Abbildumg voll 24 Aiten kurzst-bwünziger Krabben, als Beitrag zor Naturgeseluichte des iollien Mepres. Fiankf. a. M. 4. M. 6 Steintaf. (I Rtlulr. 12 gr... - Abbildung und Besclureibung einiger nenen oder wenim bokanntru Verstcinerumgen ans der Kalk (dhieferformation vou Soluleuhofous. Finufurt. 4 Iaf. in Eol. (qr. 20).

Eumpr, in Würzlurg. Bearheitet die Cisulinecen and lieferte Mehreres zil v. Ne y er's Werk.

Itunde, Wibloln Hernann, Dr. med. in Halle.

Thutles, Oberlehrer in Berliu (sonst in Frankfurt a. d. Oder). (Vgl. Trosclicl.)

Snclk, Dr. in Halle. - Einige Versteinerungen aus der Grauwacke von Dallu in der Eifel (neue Trilohiten) (Vortrag 1836 z.l1 Jena. - Isis 1837, 476).

Saint-Rilnire, Auguste St, in Paris. - Upher die rothe Farbe der Sálzsïmpfe bei Montpellier (L'Instit. 1838, 251).

visus, Mariano, Proprictajr in Barcellona. Scluriftsteller.

ciars, M., Pfarrer zu Kiud bei Bergen in Norwegen, - Ueber cinige newe Trilubiten (Isis 1835, p. 333-43, Taf. 8,9).

Snvi, Paolo, Professor zu Pisa. De Julu ('ommunissino et fortidissimo (Memorie seient. Dee. I, Pisa 1820. 8. fig. - Isis 1823, p. 224).

Selnilling, Sam., Lelorer in BresJau. - Austülırliche Naturgesehiehte des Thier-, Pflauzen- und Mineralreichs. 4 Bde. Mit 276 Abbild. 2. Ausg. Breslau 1843. gr. 8.

Schinz, Heinr. R., Dr. und Professor ill Züricls. - (Cuvier) Das Thierreich, cingetheilt nach dem Bau der Thiere, als Grundlage ihrer Natur- geschiphte und ler vergleichenden Annfunic. A. d. Franz. 3. Bd. Krebse, Spime1, Inscktel1. err. N. Stuttgart (Cutta). 1. Bd. 1821, 2. Bd. 1823.

Schlottlesuber, A. Fr., iu Göttinger. - Naturforsclier.

Schmidt, Jos. Herix, Dr, med. in Birliu. - De corporum locterogenicorun in plantis animalibusque genesi. Berul. 1825. 4.

Sclnuidt, A., Privatlelırer z.u Erfurt. - Haudbuch der Naturgeschiclite. Mit gegen 400 erläut. but tredruckten Abbilal. Erfmit. gr. 8. 1843 und fruber.

sclnuidt, H. R., Dr. phil. in Danyier. - Cinundriss der Nafurgeschirlate fiir die oberen Classen hölierer Bingerscluuleu. \#. Abtlul. Danzig 1843.

sclumidt, Dr, med. in Mohrungen bei Uracls in Württemberg. Petretaktensammlung.

Sclnuide, F. C. 'Th, Oberlchrer an Gymnasium zu Halberstadt. - C. A. Schmidt's Blicke in den Haushalt der Natur uder Darstellung aus der Tlierwelt. Herausgregeb. \&. Halberstalt.

Scliolz, Dr. P, Professor zu Breslau. - Werke der Allınacht oder Wunder der Natur. VIII. Bd. M. Steint. Jahrg. 1832. 4. Breslau (Grass).

schubert, Fr: W., Professor zu Potsdam (?). - Reise durch Srliweden, Norwegen, Lappland, Fimnland und lugermanuland, in den Jaluren 1817, 18 ond 20. 3 Bole. (I. Bd. Reise durel das westliche Scliveden, Norvvegen und Finnland. Mit $1 \mathrm{Kpf}$.) go: 8. Leipzig (Hinrichs). Der 1. Bd. 1823.

Schuch, Dr. med, in Regensburg. Sammelte iu Gricchenland.

Schultze, Hofrath, zu Greifswald, - Bekannt dureh die interessante Wiederentdeckung cines in der Daelrimnenerde lebenden Thiercluens (Macrohiutus Hufelaudii). - Macrobiotus Intelandii, animale Crustaecornm clisse novum, reviviseendi post diuturnam aspliyxiam et ariditaten potens. Berol. 1834, c. tab. 4. - Macrobiotus hufelandii (Vortrag. - Isis 1834, 708, Taf. Ist Trionyelium ursinum Otto). Ueber Wiederbelebungsfähigkeit und Maerubiotus (Vortrng 1835. - Isis 1836, 765). - Ueber Maerubiotus Ilofelundii und noel 4 andere Species (Vortrag. - Isis 1838, 593). Vgl. Purkinje, Perty. 
Sehultz, Carl Heinrich, Dr, med. und Professor in Berliu.

Schmlz, J. H., Oberlehrer in Berlin. - Grundriss der Zoologie und Butanik. Zum Gebr. in Gymnasien ete. 2. Aufl. Berlin 1843. 8.

Scores by, Will., jun., Schiffeapitiin in Edinhurgh. - Ueber die microscopiselıen Thiere un Gröıland (Brew.ediub. pl. Iourn. IV, P. 111. - Ixis 183:, 591). - An Accomnt of the Arrtic Regions, with a listory and description of the Northern While - Fisliery. In two Volumes. Ediuburgli 1820. 8. (Enthilt: Ganmarus arctieus, Cancer' Boreas, Cancer Ampulla, Oniscus Ceti Liun.) - Journal of a voyage to the Northern Whale-Fisherv; inc.Inding Resparehes and Discoveries on the eastern coast of West-Greenland, made in the Summer of 18:2, in the ship Baffin of Liverpool. Edinliurgh 1823. 8. - Deutsche Uebers, : Tagbuclı einer Reisc auf den Walfiselufang, verbunden mit Untersuchungen und Entdeckungen an der Ostküste von Grönlund, im Sommer 1822. Aus dem Englischen iibersezt mnd unit Zusiitzen und Anmerkungen verschen von Friedrich Kries. Mit 9 Taf. Abbild. mnd einer Landkurte. Hamburg, bei Friedrich Perthes. 1825, XVIII und 414 Sciten in $\mathrm{gr}, 8$.

siconler, J., Dr. med. und Professor der Grologie in Dıblin. - Eudotea, foss. Cinstacengeschlecht.

Seirriea, von, Gelicimer Hofrath, auf der lnsel Reichenau (Rhrin. Baden). Sammlung von seltenen und wiclotigen Versteinerungel der Krridcformation. in der Trias. und Liasformation und in) Jurakalkschiefer.

serres, Marcel de, in Vontpellier. - (mit Duméril) Ueber M. St. Ange's Bau der Cirripedeu (L']ıstit. 1834, 236. - Isis 18:37, 710) (Ok c n Anmcrk. hiezu1\% - Rï̈rkengefüss der Kerfe (lsis 1819, p. 593).

Smith, Andrew, jn der Capstadt. - Illustrations of Zoology of SouthAfrika etc.

siebola, Ph. Fr. v., Dr. med. Ritter ete. in Lcydeu. - Fauma japonira, sive descriptio animalium, quae in itinere per Japroniam : jussu ct auspieiis superiorme, qui summum in India Batava imperinm tenent, suscepto, annis 1823-1830 collegit, notis, observationibus et adumbrationibus illu. stravit. Conjunctis studiis C. J. Temminck et H. Schlegel pro vertebratis atque $W$. de $H$ a $a$ ul pro invertebratis cliaborata. Reg. ausp. ed. Leydell. Fol. - Fauna Japonica. Ć́rustaera

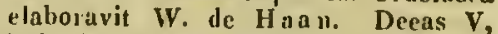
Fol. M. 7 Taf. Leydell. 1842.

Sictoold, C T'ı., Dr. med. nud Prof. in Freihurg, sonst zu Erlangen. - Seine Britriggr zur Naturgescliclite der wirbellosen Thiere. Danzig 1839. 4. 94, T. 3 enthatten 4 selır merkwürdige Abhandlnngen: a) über die Olırenqualle (Mednsi aurita); b) über Copulation des Cyclops Castor; c) Samenschiäurche des Loligo vulgaris; d) äber Helminthen zum Genus Gregarina gehörig, und e) iber Xenos splecridarum und dessen Schmarotzer (vol. Oken's Recens. Isis 1840,310$)$. - Ueber dic rothen Beutel des Apus eancriformis Latr. (Isis 1831, p. 429-34).

Sillimans, in London. Redacteur des Anpriean Journal of sciences.

Sowerby, James, in London, British Miscellany. Lond. 8. W. Plat. - Malacostraca Podoplithalmata Britanniac or descriptions of the British Crals, Lobstens, Slurinjus and other Crusticea with purdumenlated Eyes, deserihed by WV. E. Leeach M1. D. M. Cl.. illustrintedby highly-fineshed Figures. by S. will be publislied on the lst of December 1817 (Price 75, 6 d. eueh colonred 65 plain.) - Cirripeden (Zool. Journ. VIl, p. 1029).

Spence, William, Esq. in London. Aperece, William Blondel, Esq., auswärtiger Sceretär der entomologischen Gesellscluaft in Loudon, Künstler etc. in Florenz.

Starlk, J., in Edinburgh. - Ele. ments of natural history, adopted to the pressent state of the seience. Ellinburg. 182s. 2 Vol. 8

Stremstrup, Jap., Professor in Kopenlıagen. - Beitragr zur Geschichte der Cirripeden der Vor - mud Jeztwelt (Kröycr's nat. 'Tijlsskr. II, Hft. 4, p. $396-415 .-$ Isis $1841,413-416)$.

Stein, C. G., Dr. plit. und Professor au pranen Gymusium zu Berlin. - Naturgeschichte fiir Reill- und Bürgurschulen unit besonderer Hinsicht illf Geographic ausgcarb. 3. verm. und verb. Aufl. Mit 21 color. Abbild. gr. 8. Leipz. Stein, Friedrich, Dr. med. in Ber-
lin, - De myriapodum partibus geni- 
talibus, nova generationis theoria atque intruductione systematica adjertis. Ber. 1841. 4. Acced, tab. 111, in aes incis. (Mill. Arch. 1842, 261).

stokes, C., in Boston. - Asaphus platycephalus fin J. J. Binshy's Note ou the geograpliy and geology of lake Huron. 'I'rans. Soc. geol Lond. VI, 208. 1824).

Stranss-Wiirehleim, Hereule Engene, in Pais. - Momoire sur le genre Cypris, de la elasse des Crusticés (Mem. du Mus. dlicst. nat. VII, p. 100, av. planch.). - Mémoire sul Ies Daphuia, de la classe des Crustacés (Mein. du Mus. V, p. 38, PI. 29) Anatomie du Dapluia).

Strnve, H. v.: Kais. w. Staatsrall und Ministerresident in Hamburg.

Gykes. Will. Henr., Oberstlicutemant ete. in London. - (and Westwood) Some Aceounts ol the Yanderabes of the Dukhan (Trans. of the Entomologieal Society of London. Vol. I, part. 3).

Tetzmer, W., Dr., Lehrel zu IIannuver. - Handbuch der Naturbeschreibung zum Sclut. und Privalunterrirlite praktisel bearbeitet. gr. 8. Hannuver.

Tillesius: W. G., Holiath, Dr. med. etc. zu Leipzien. - Ueber das Geschlecht der Mrereicheln, der sogenamuten Entenmuscheln, Sectulperu und Lantrhälse (Lipas L.) (dessen Jnhibuch der Naturgesch. 1, 1802, 22:-420). De cantris cantschaticis, oniscis, entomostracis ef eancellis marinis microscopicis noctiluscentibus, cum appendice de acaris et ririnis eamtsehaticis (Mém. de l'Acal. de St, Petersb. V: p. 331 ).

Thienemann, Friedr. Aug. Ludw. Dr. med. und Inspector am k. särchs. Naturalicumuseum in Diesden. - Lehrbuch der Zoologie. Berlin 1828. 8. (Vortuefliticli.)

Thompson, W. Vaughan, Generalinspector der Spitiler zul Cork. Ueber die doppolte Metamorphose der zclunfüssigen Ćrustaceen in cinem Beispiel von Caneer Mochas (Phil. Trens. 1835 , p. 359 . - Isis 1836 , ․ 62224. - Virl. anch Thoupson's Zonl. Journ. p. 1 und 63 , wo dieses zu erst mezeigt wurde). - Mrtamorphose der Crustaceen (Zool. Journ. Nr. XllI. - Isis 1830 , p. 1261, - Pliil. Mag. IX, 1831, 140, - Isis 1834, 816), - Entdeckung der Verwandlung der Lepaden. (Phil. Trans. 1835, 1. 355-359. - Isis 1836, 1). $62 \mathrm{i}-22$ ). - Metamorphose der langschwänigen Kr(b)se (Jameson Edinb. Joutn. XXI. p. 221. - - Isis 1838, 126 -127). - Zoologi(al Rescitrches and Illustralions. Cork (Cirriped.).

Todld. Robert B., Di. med. in Loncion. - The Cyclopaedia of Anatomy ete. (vgl. Lexik. der Entumologen).

Tristan. Jules de, in Poitou. (et Bigot de Morogues) Mémoire sur in Crustace renferme dans les schistes de Nintes ef d'Angers (Journ. des Mines. XXII1. Tom., Nr. 133, p. 21. - Orygirs, Trilobites). - Mémoire sur quelques insectes trouves sur les côtes du Poitou (Aum, dı Mus. d'hist. nat. XII!, p. 371. Av, pl. - Sphaeroma. Inotea).

Troschel, Franz II erm., Dr, med. in Berlin. - (init J. Fr. Ruthe, Wiegmann's und Ruthe's Handburh der Zoologie. Nach den Fortschritten der Wissensthaft ïberarbeitet und vermehrt. Berlin 1843. 8.

שulressier, in Besançon. Careinolom.

Valentin, Professor zu Beru. Versuche mit Áretiseun (Isis 1834, 713). Voigt, F. S., Hufrath ete. z.u Jena. Vgl Lixik. Il. Ent.

Voitl., J. v., Oberstbergrath in Regensburg. - Geognostischer Theil (unit den Petrefakten) der Fümolu'schen Topographic von Regensburg. Bd. I. 1838, p. 177.

Wagner, Rudolph, Professor zu Götlingen. - Ueber die Rankenfüsser (Mull. Alch. f. Anatomie, Plyssiologie ctr. 1834).

Dagner, Moritz, Dr. med. zu Augshurg. - Rrisen in der Regentselatt Algier i. d. J. 1836-38. Leipz. 18:1. 8. 3 Bde. M. Kupferatlas.

Wallt, Josepl, in Passan. - Krnbbell etr. in seiner Reise durch Spanien.

White, Adam, in London. - Beschrieb die vou Capitain Grey im König-Gcorgs-Sund (Australien) gesammelten Insekteu in dessen Journals of two Expeditions, dann auch (mit Edward Doubleday) die des Werkes: T'lor Zoology ete.

Wedall, John, in London. Travels. Deutsch : Reise nuch dem Südpol, in dell Jahreu 1822-24. (Aus 
d. ethogr. Arehiv abgedr.) gr. 8. Wilckens, H., Dr,med. in Bremen. Jena (Brau).

Worbe, D. M., in Loudon. -

Westplonl, Joh. Heinr., in Dan- Note sur unc Scolopeudre d'Afrique zig. - Nafurwisseuschaftliche Abland- (13ull, de la soc. phil. 1824).

lungton, I. Heft. Dinzig 1820 (Nettest. Sehritt. d. Danz. natf. Ges. 1).

vestwood. J. O., in London. Ueber Unipeltata Crustacea (Phil. Trams. 1835, I1). - Ueber dic Sippe Praniza Leach (Ann. d. se. nat. XXV1I, p. 316, Taf. 6. - Isis 1835,633 , Tf. XII). On the supposed existencc of metamorphoses in the Crustacea. Ueber die vermuthete Metamorphose der Crustaceen (Phil. T'rans. 1835, 11, p. 311 , Taf. 4. - Isis 1836, p. 617-620. - Vol. Thompsou).

Whibrand, J. B., Dr. med, Hofrath und Protessor zu Gicsseu. Haudbuch der Naturgesehichte des Thierreiehs; mach der verb. Limnè'sehen Methode. Mit 1 T'ub. Gicssen 1829. 8. (Sehr gut.)

Fadilacle, E. G., Dr. phil. in Bonn. - De Apodis caneriformis Anatome ef Historia evolutionis. Bonuae $1841,4.72$, t. 4 (Isis 1842, p. 869).

Eanders, J. W., in Köln. - Bci. trïgre zu einer Geschiehte der Thiermetamorphose. Köln 1807.

Tiegler, im Steinbere zu Winterthur. - Saminlung vou Versteinerungen (Sucke, Vügel, Kerfe, Fische, Lit'che' des Jurakulkschiefers.

Timatermann, Christ., in Berlin. Sammelte, cin grindlielıer wissensehaftlicher Eutomolog, in Auerika und wird Grosses leisten fiir die Folge.

kiviels, von Glarus, in Scliaffhausen. 


\section{Ortstafel der Carcinologen.}

Anchen. Förster. Quix. A guano. Penada.

Alais. Hombres.

Albany. Eights.

Alfort. Desniarest (Vater).

Algier. Bravis.

Aitenbirg. Apety.

Anstercinu. Franke.

Angsbinrg. Wagner.

Frarcelloun. Saus.

Denrtinan. Kirby.

Heichlingen. Ramdohr.

Berlin. Buel. Elireuberg. Erichsot. Zimmermann. Godet. Troschel. Helmholty. Klöden. Klır. Krassow. Leyde. Liehtenstein. Link. Müller. Olfers. Ruthe. Sack. Schmidt. Scholz. Sehultz. Schulz. Stein (2).

Eern. Brunner. Perty. Valentin. Birninghnn. Marshall.

Besn nçon. Udressier.

Holograna. Alessandrinci.

Bann. Goldfuss. Mayer. Zaddach.

Bordeaux. Blanchard. Dumal. Perrieres.

Boston. Stoekes.

Arnnmscliweig. Lachmann.

Bremen. Oleire. Wilkens.

Dreslan. Gravenhorst. Otto. Purkinje. Schilling.

IBrest. Gamot.

IB riissel. Drapiez. Leopold I.

Caen. Caumont. Cerf.

Cambridgre. Babington.

Canulen. Blanding.

Copstadt. Smith.

Clnalons sur Marne. Mahicu. ster. Münter. Runde.
Cluristianin. Böek. Raseh.

Coblenz. Dewora.

Connecticnt. Crosse.

Constanz. Nemuing.

Cork. John. Thompson.

Crefeld. Hoeningliaus.

Danzig. Solımidt. Westphal.

Garmstadt. Kaup.

Dijon. Christol.

Dortunud. Charpentier.

Drevelen. Carus. Ficinus. Gei-

nit\%. Reichenbach. Thienemann.

Duhliu. Scouler.

TDifrrlneian. Althaus.

IDisselororf. Hildebrandt.

Nanburarlt. Alexauder. Flemming. Goodsir. Sowerloy. Julunston. Murchi sull. Scorrsby. Stark.

Epping (England). Doubleday.

Erlinet. Sclumidt.

Erlangen. Kiister. Martius.

Fiorenz. Spenee.

Fronlfurt a. M. Meyes. Riippel.

Nreiburg. Perleb. Sicbold.

Gemua. Durazzo. Marmora.

Ciessen. Credner. Wilbrand.

Aittingen. Berthold. Molir.

Schlothauher. Wagner.

Aratz. Haltueyer.

Crevifswalde. Schultze.

Grénoble. Crepu.

Gundelonpe. Lherminier.

Malle a. d. S. Blasius. Buruci- 
InIherstadt. Schmidt.

Hamburg. Beske. Ecklou. Jäger. Struve.

Hinau. Nietsch.

Hannover. Roemer. Tetzuer. burg.

Meideberg. Bromu. Dierbach.

Mild eshoin. Leunis.

Jamnice. Barclay.

đena. Gräfe. Voigt.

Kharkof. Krynicki.

Miud (Norwegin). Surs.

Misin. Rave. Zanders.

Kunigrberg. Dengel. Ratlike.

Kopenlongea. Colsmann. Kröyer. Lund. Reinhardt. Stcenstrup.

Laibacl. Freyer.

Throusaume. Chavaunes.

Leipaig. Franke. Reichenbach. Tilesius.

Gritl. Coldstreau.

Lexington (Nord-Amerika). Rafinesque.

Leyden. De-Haan. Mulder. Sicbold.

Lippe. Paul Alexander.

Ediven. Van Beneden.

Lokdon. Bell. Broderip. Buckinghan. Clappertun. Cuming. Dunciuse. Flinders. Grant. Gray, Griffils. Jones. Miller. Newport. Owell. Pary. Ross. Sillimans. Sowerby. Spence. Sykes. Tudd. Weddell. IVestwood. White. Worbe.

Itittich. Koninck.

Magdeburg. Riemann. Kote. malliugd. Ferrario.

Manulein. Kilian.

Marburas. Herold. Ritgen.

Marigunne. Payan.

Marseille. Bathélemy.

Mnryland (und zwar zu Cam-

bridge). Mıse.

IIewsius. Giacinto.

Mohrungen. Sehmidt.

Montpellier. Serres.

Moskau. Fiseher v. Waldheim. Mihhllausen. Oudart.

Munchen. Erdl. Gistel. Gruit. huisen. Mayer.

Murrieardt

Oesterlen.

(Württemberg ).

Nantes. Dubuison.

Tiempel. Chinja Costa. PrestAndrea
Neneck. Pfyffer.

Neucentburar. Ag:issiz. Coulon.

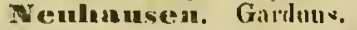

Nerstadt-Hoernovade. Ratze.

Niev-guren. Green.

New-Iurk. Digsby. Dekay.

Lesurnr. Noves. Leconte.

Nizza. Risso.

Ddessa. Nordmann.

Bxforal. Burkland.

Papstarf (bei Ilalberstadi).

Ballensiedt.

Dris. Ange (M. Saint-). Areet. Barker-Wepp. Berthelot. Blainville. Binlle. Comte. Deslayes. Desma. rest (Solı1). Desmoalins. Duyère. Dumiril. Dupont. Edwards. Eyduux. Freminvill., Gaimard. Gaubil. Geoffroy-St.-Hilaire (Isidor und Etienme). Gerviris. Goadot. (juerin. Hofmamn. Jaegninot. Joly. Lalmalek. Lesson. Millet. Orbigny. Qıoy. Rivoli. Roussel d. V. Sint-Hilaire. StrunssDorklieim.

ก"assan. Waltl.

Fentl. Rochel.

Mlidadelplise. Dana. Harlan.

Pickpring.

EPis It. Savi.

Puitor. Bigot de Morogues.

Tristals.

Potsdam. Sehubert.

Preng. Corda. Mlikan. Presl.

Pyrunont. Kriigre. Menke.

贾egensiburg. Herrich. Koch.

Siluth. Voitl.

Itergero. Brignoli.

Teichenan. Scyfried.

Eena. Kocl.

IRenand. Dutrochet.

Rotterdam. (Van der) Hoeven.

roven. Lebas.

Saint-Maurice. Lienard.

Srint-suveur. Robineal.

Suint-Séver (Landes). Dufour.

sanlat Petersburg. Baer.

Brandt. Hamel. Krohn.

Scleaflinauseu. Zwick.

Solotiurn. Hugi.

Aoran. Kireliner.

Stockluolu. Lovén.

Strasberg. Duvernoy.

Stuttyert. Krnuss. Martens.

Swluenthnde. Ehrhardt. 
Tharand. Rossmissler. Toulon. Gulle.

Turin. Carena. Michelotti. TrIest. Napoli.

Van DlemensIand (zu Hobartstown). IV. S. Macleay.

Venedig. Contarini.

Warscliax. Drzewicki.

Werulngerode. Jasche. wrlen. Diesing. Focke. Kollar. Raffelsberger. Rrdtenbacher. -VIllefimeliali. Albcrti. TVilna. Eichwuld. vinterthur. Ziegler. Viirzburg. Rumpf.

ZiebIgk (im Herzogthum AnhaltKüthen, 1 Mrile davon und $2 \frac{1}{2}$ Meilen von Dessiu). Naumaun (Sohu).

Zilirlch. Escher-Zollikofer. Oken. Schinz.

Unbekanut sind die Wohnorte der Herren:

Dulk. Esclnwege. 


\section{Die carcinologischen Schriftsteller des Alter- thums, des Mittelalters und der neuesten Zeit. Nebst der vollständigen Angabe ihrer Werke *.}

\begin{abstract}
Abillgari, Peter Christinn. Beskrivelse af tvende nye insekter lienröreude under den linueiske slacul Monoenlus, on den Müllerske slaegr Caligus: 1) Caligus prassus: 2) Culigos oblongus (Skrivier af Naturlist. Selskaleet, Bind 3. Ilefte 2. p. 46).

Artans, Jolur. Deseriptions of some marine unimals on the const of Willes. Onisens bidentalus (T'vansact. of the Linnean soeicty. Vol. V, p. 7, 8, T. 2, tig. 3).
\end{abstract}

Acliunus, Clandins. De notura animilinm Lib. XVIl, gracee el latine, Petro Gullio et Courado Gesuero interpretibus. Tiguri. 1556 , Ful. Genovae 1611. 16. (Lib. 12, Cap. 26; lil. 14, Cap. 15 de Constaceis). - Ex Aeliani Llistoria por Petrum Gyllimm, latini facti. ifemque ex Porphyrio, Heliorloro, Oppiano tum eodem Gyllio aneti. Lih. XVI. Lugdoni 1533, 4.

Aprssiz, L.

Ahrens, Aog. Fauna insectormm Europis. Nr. 25, enm descript. sistens. Halae apud Kinmel 1824.

Alberti, Miel. Dissert. de Squilla. * Aluerti.

AIdrovandus, Ulysses. De animalibas exsanguibus. Bononiae 1606. Cum fig. Fol. (De erustatis. Squilla).

* Alessandrini.

* Alexnuiler, John E.

"Althuns.

André, Vater. Briefe über die Seliweiz.
Androb, William. Description des yrox du bimule grant (Transaet. sor. Pbilos. 1, p. 239. - Xiphosurit). Bemerkte zuerst die kleinen Angen an Limulus (Philos. T'rans. 1782, II, T. 161.

* Ange. Martin Saint.

Annone, D'. Cillsticea pétrificata (Ariti helvetiea. 111, fier.).

Amonyanc. Leoplitende Crustacen (L'lust. 1838, Mai, 11, 228. - Isis $1839,366)$.

Anouymus. De rancro pulice, Limnaei; Gammaro, Fabricii (Suec. Grundmirgla) et noxa, quam retibus piscator'nu infert (Nova Aeta societ. Upsal Vol. VI, p. 89).

Amonymus ( $S(h \| l z e)$. C'harakterisiruug piner kleinen Art von Tasehenkrebsen, deren Rürkenschild ein Menschengesicht vorstellt etc. Hamburg. 4. Mit i Kupf. (Dorippa).

Arcet, Felix d'.

Argenville. Conehyliologie. $\mathbf{T}$. XXX, F. H. (Lepas).

Aristoteles, De naturnanimalium: libri novem. De pirtibus animalium: libri gnatıor. De generatione animalium: libri quinque. Interprete Throdoro $G$ aza. Diese Ansqabe triggt widerDruckort nut Jahrzahl (Venetiis; entweder 1497 oder 1476?). Fol. Tradoction français (distingne) de $\mathrm{C}$ am is. 2 Vol. Paris. 4. - Lib. V hist. anim. e. 16, IV, e. 4.

Aurlomin, Vietor. Recherehes sur

* Da dic meisten älteren Schriftsteller die Crustaceen zll den Kerfen rechneten, so er. gänze uan nie Lüicken dieser Tafei durch dic Tafel der entomologischen Autoren, wle itureh das Lexilon der Eitomologen den Text voul deul der Lebellell. 
Ics rapports nalurcls qul cxistent cntre les 'Trilobites et les animaux articules (Ann. d. sc. pliys. et na. de Bruxelles. VIII, p. 253), -- Mémoire sur les ripports des Trilobites avec les animaux articulés (Ann. gènér. d. se. pluys. VIII, p. 233, av. fir. - Trilobites: Isis 1822, p. 87). - Ueber Trilobiten (Vortrag 1835). - Ueber einigc Ciustaceen aus Nord-Afrika (Ammal. soc. cnt. Fr. 1837. - Isis 1839,532 ). - Text zu Crustacea in Destr. de l'Egyple XXII, p. 249 (Isis 1832, 968). - Neues Crustaceum (L'Instit. 1837, Nr. 192). (In Nälic von Lymuadia). - Ncu cuidecktes Crustaceum von Chili (Vortran 1835. - Isis 18:6, 763). - Ueber Dichele. stina (Amn. d. se. "1at. IX). - (nit M. Edwa rds) Anatom. Innd Pliysiol. der Crustac. III (ibid. XIV, 1828, p. i7. - Isis 1834, 1023). - Ueber die Circulation in den Crustaceen (Ann. d. se. nat. $X I, 283-352$. - Isis $X, 394$. 1834, 936). - Nicothoë, Oniscus thoracicus (Isis 1831, p. 1228, Taf. 8.) - Hipponoë et Euphrosyne (Isis 1831 , p. 100 , T. 1. - Zool. Journ. XIII). - Aclilysia (Isis 1827, J). 751 , T. IX),

Athenaens. Deipnosoplistarum lilı. XV; cura et studio Isaaci Cas anboui auctiores cmendatiorcsque editi; cum iuterpictatione laliua Jacobi DeIc champi cadomensis. Ajecti sunt indices rerun, seriptorum, proverbiorum, voeum accuratissimi (libli 3 ). Heidelbergac 1597. Fol.

Wadier, De. Observations sur la reproduction des pattes de crabes (Journ. de Pliys. II, p. 33-34).

muer, K. Fr. v.

Buier, Johanu Jakob. Oryctographia norica. 1730. Fol. mit. c. fig. (Crustacea). - Sciammphin musei sui et supplementa Oryetographiac Noricae (Acta Acad. Nitt. Curios. II, Append. p. 65 ).

Baker, Henry. A letter concerning an extrnordinary fisl, ralled iu Russia (ual, and the stones callod crabs-cyes (Pljil. Trans. 1748, p. 174). - Of microscopes and the discoveries made therc. 2 Vol. 4. fig. 17 (Stïswasser-Lutomostraca).

* Bnillenstedt. G. J.

Bankroft. Guiana.

"Iarclay, A. Vicw of Sclatuery (Gecarcinus ruricola).
Warker-Wepp, P.

Barrelier, Jacubus. Irones aeri clegant(1) incisice etc. (PIerumgue fide. les ligurac, ralae descriptiones.) Paris. $1714, \mathrm{fol}$.

IBarrere, Pierre. Lssai sur l'histoirc naturelle de la France cquinoxiale (Mèm. de l'Acad. des sc. de Puris. A. 1741, Hist. p. 23, Ed. oct. A. 17\$1, Hist. p. 32),

\section{" Harthelemy.}

Taster, Jab. Opuscula subeesiva, observationes miscellancas de animalculis et plantis quibusdan marinis corumque uvariis et seminibus continentia. Harlcmi 1702-65. 2 Vol. 4. C. fig. (111, 144, T. 12. Pycnogontum).

Manhinus, Caspar. Pinax (p. 513. Lepus an.).

v IB aม usanu, Jakob.

Eechanmu, Joscph. Bcitrag zur Naturgesclichle des Kiefenfusses. ( $\mathrm{Na}$ turforsclier VI. St. p. 35-40).

" BeII, Thomas.

Eelon, Picre. La nature ct diversite des poissons. Paris 1555 . 8. obloug. - L'histoire naturelle des estranges poissons marius. Paris 1551. 4. - De aquatilibus libri II. Paris 1553. 8. obl.

* Bemeden, P. J. van.

Denmett, Edward Turner.

* IBerenit.

* Meruliard, $W$.

Berninarali, M.

Berniz, Mart. Beruhard a. Chicla astaci marini monstrosa (Miscell. Acad. Nat. Curios. Dce. 1. A. 2, 1671, \%. 174, 175). - Gammarus leprosus (ibid.). - Cancer Moluccanus (ibid. p. 176). (Monoculus polyplieuss.) - Ol. servation sur une trés grande avilité de manger des écrevisses, accompannè de sympiomes surprenants (Collect. aead. III, p. 163).

"BertheIot, Sabin.

wertiold, A. Ad.

\section{Bickmanu.}

migot de Morogues, P. M. S. (vgl. 'l' ist an).

* Bisistoy.

* II Ininville, Henry Ducrotay de.

* Ianchari.

DIasius.

BIasins. Anatomc aniun. p. 269, Tab. 18 (Astucus fluv.). (V $\mathrm{V}_{\mathrm{L}}$. W illis).

IInmenbach. Iohaun Friedricll. Abbildungen naturhisloriveloer Girgell. stäucle. V. T'hl., T'ub. 50 (Cillyumenos, 
Trilobites). - Vergleichende Anatomie. - Handbueh der Naturgesehiehte (Lepas etr.).

\section{* Buek, Christian.}

Eonani.

Bontius, Jacohus, Historiac naturalis et mediene Indiae. 1658. Fol. (Entlualten in den Werken l'iso's.)

* Bory de St. Vincent, J. B. M. TBose, Lonis Augustin Guillaume. Histuire naturelle des Crustaces, contenant leur description ef leurs moeurs. 2 Vol. in 18.; faisant partie de l'èdition de Buffon, dite de Castel. publiec par Deterville, An $X$.

* Boverbank, James.

Toyle, Robert. General heads for the natural history of a rountry, great or sinall (Phil. Trans. Y. 1666, p. 186). "Trandt, v.

Brumue, Fr. A. v. Salzburg und Berehtesgadeu! cin Taschrubuch für Reisende und Naturfeunde. Neue Ausg. mit 1 Ansidlt von Salzburg mul 2 Taf. gr. 12. Wien (Beck). (Bot.)

irébisson, Mitglied der Linnèsehen Socirtät vou Calvalos. Catalogue methodique des Crustarés terrestres, fluviatiles et marins, recucillis dans le dẻpartement do Calvados. 8.

Breynins, Joh. Phil. De. Insectis (et vermibus) quilusdam rarioribus in Hispania observatis (Plil. Trans. 1705, p. 2050. Ephem. Acad, Nat. Curios. Cent. 5 et 6 . Append. p. 101).

Brignoli, Giov.

IBromguiart, Alexandre. Histoire naturclle des T'rilobites. Paris 1820. 4. Av. planclı. (Kam gemeinsehafilich mit dem Werk von Desmarest über die Naturgesehichte der tossilen Crustaceen heraus). - Mémoire sur un nonveat genre de Crustacis (Limnadia) (Mém. du Mus. d'hist. nat. VI, av. pl. - Isis 1822, 212).

* Iromu, H. G.

"Erotz, Joh.

Brovva, Littleton. A letter conrerning the same sort of inseets found ill Krnt; - with an addition liy Cromwell Mortincl (Plil. Trans. 1738, p. 15.3) (Monoeulus Apus).

Browne, Patrik. 'The eivil and nalural History of Jamaica. London 1756. Fol. With Plites.

Trimnich, Morten Thrane. Den barbugede Pampelfisk (Coryphaena apns) en nye art, of deus giarst. Skrutike. trolden (Oniseus Eremita (Skrivter det)
Kjöbenl. Sclskab. Nye Saml. Deel 2, p. 319. Deel 4, p. 406). - Trilubns canlatus (ibicl. I, 1781, p. 392, Nr. 3 (Irilobites, Assuplies).

* IBrulle, Augnste.

Trumeili, Joaunes Angelus. De flumine Amazonum (Commentarii ho. monienses. VIl, C. p. 23, 0. p. 39).

* Brumuer, Sani.

* Inech, Leop. v.

* Inckingliam, J. S.

* IRncliland, $W$.

Biichner, Johann Gottfried. De rarioribus quibusdam unimalibus in Voigtlandia quondim natis ae degentibus (Arta Acarl. Nat. Curios. Vol. IV, p. 261).

Bihttuer, J.G. War Pastor zon Schlech in Curland. Zoologisrlue Bemerkungen (»u Ok e n's Naturgesclichtite) (Isis 1838, 360-71). - L'ebre Krebse; Hippobosca, Mrioken, Falter, Käfer bes. schädliche, Lu rehe, Vügel, Fisclıc, Sucke. Lcbensart von mehroren Käfe'n. (Interessiant.)

wnule, Joh. Gottl. De funtibus unde Albertus Magnus Libris suis XXV. de animalium materiam hauserit (Commeut. soe. Goetting. Vol. XII, P. 3, p. 94).

Bullivant, Benjamin. Letter concerning some natural observations, made in New-England (Phil. Trans. 1698, p. 167).

Burgsalorf, Friedr. August Iudw. v. Naturhistor iselie Beschrcibung NellOstpreussens iN. Schr. d. Ges. naturf. Fr. zu Berl. Bd. 3, p. 56i).

* Burmeister, Hermann.

Cnille, Abbé de la. Observations faites a l'lsle de. l'Asernsion. sur le loeal, sur les oiscaux, In pếelır, la longitude et latitude (Mím. de l'Acad. des sc. de Paris. A. 1754, Hist. p. 110. Mém. 1) 126, Ed. Ort. A. 1754, Hist. p. 163, Mem. p. 195).

Celceolnrins. Musarum veroneuse: Sect. 11I, p. 429, 1625. Fol. (Crustincen).

Canuara, Manoel Furreira da. Ensaio de descripcaso fisira e reommiea dit comerca dos illieos na Ameriea (Vemorias cronominas da Arad. de Lisboa. I, p. 304).

Canuper, Peter. Zubulogische BBemerkiugen (Sclur. d. Berl. Gres, Matf. Fr: Bd. VIt (Beoharlit. Bd. 1), p. 479).

\section{Cardanus.}


Cartheuser, Johau Friedrirh.|unter die Seltenheiten. Gistel.) Prueside. De Millepedis. Resp. J. (Squilla p. 91 unserer Ausgabe.)

Dan. Begero. (lı Dissertationibus ejus physiro-chemiro-medieis.) Franeof. ad Viadi. (Erfurti) 1774. 8.

*arus, C. G.

Cutullo, Tomm. Ant. Sargio di Zoulogia fossile (delle Provineic AustroVenete). Inp. 4. (43! Bg., mit $8 \mathrm{Tf}$.) Pidoval 1827.

Cat, Claude Nicholas Le. An acromut of glasse's of a new contrivance for preserving pieces of natural history in spirituos liquars. Translated from the fremeh by T. S. (Phil. Trans. 1749, p. 6. 88).

Cutesby, Marr. The natural histor'y of Carolina, Florida and the Bahama Islimds. London 1731-43. 2 Vol. Fol. Witl plit. (Gelisimus vorans).

Cavollni, Filipp." Riflessioni sulla memuria del signor de Termeyer sopra il Puler acquajolo (Opureuli Seelti I, p. 178-190. - Dathnia pulex). Sulla Generazione dei Pesci e dei Grauclii. Napol, 1787. 4. (Auch deutsi:h.)

* Chnrpentier, Toussaint de.

Chesunitz, Joham Hicronvmus, Besetoreibung einer Reise naeh Faxoe und Stevens Klint (Beschiift. d. Berl. Gis. natf. Fi. Il, p. 197). (V V. O. F. Miille r.)

* Clinje:, Steffano Delle.

* Claristol?

* Clapperton.

Claytou, Jolu. Letter-miving III arraunt of several alservables in Virginia - of the beasts of Virginia (Pliil. Trans. 1694, p. 121).

CIayton, William. Aecount of Falkland Islauds (Plil. Transact. 1776, p. 99).

Cloruet, Hippolyte. Faune des mélecius, ou histoire uaturelle des animaux et de lrurs produits, consideress sous li rapport de la bromatologie et de l'hygienc en geincial de la thronpeutique, de la phurmacologie et de la toxicologic. Paris 1822-24. 8.

CIusius, Carolus. Exaticarum libri decem. Autverpiac 1605. Fol. (Limulus). - Curar pasteriores. Raphelengii (Plant.) 1611. 4. (Mit diesen werden erst die Werke des Clusius romplet und de 13 ure hat Unreelit, wem er in sein. Bibl. Inst. 2 Bd. S. 326 sagt, dnss die Werke dieses Autors mit deı 2 Folianteı complet seyen, Es gehört

"Coldivtrean, Johil.

Collinson, Peter. Anmerkungen von dem Seekrebs (Hamburg. Magaz. 11, 476-82). - Some farther observations on the Caneer major (Philos. Transiet. Vol. XLVII, p. 40-42. Y. 1746, p. 20. 1751, 1. 40).

Culumna, Fabius. Aquatilium e't terrestrium aliguot animaliun, aliarumque naturalium rerum observationes (In Ecphrasis lib.) 1616, 4. - Phytoh. 40.

* Conte, Achille.

Coudauine, Charles Mirie de Ja. Relation abregre d'un voyage fait dans l'intirienr de l'Amcirique meridimale, depuis la cote de la mer du Sud, jusques aux cotes du Brisil et de la Guiane en descendaut la tiviere des Amayours (Meill. de l'Acad. des se. de Par. A. 1745, Hist. p. 63, Mièn. p. 391. Ed. Ort. A. 1745, Hist. P. 90, Mem. p. 556). - Extrait d'un Journal de Voyuge 'ou Italie (ihid. A. 1757, Hist. p. 6, NIèm. 1. 336. Ed. Oet. A. 1757, Hist. 11. 9, Mím. p. 536).

Coek, James. Cancer vocans bei Rio (dess. erste Reise, 17 ).

Coquelbert, Anloine-Jean. Nuturalist zu Rlueims.

"Costa, O. G.

* Crosse, A.

Crusger, Daniel. De oenlis Caneri factitiis corumque notis (Eplı. Acad. Nat. Cur. Der. III, Anu. 3, 11. 262 -264).

Cuniuglean, James. A catulogue of Shells etc. (t)lants, carths) gathered at the Island of Ascension (Phil. T'runs. 1699, 11. 295).

burtis, Jolu.

Cuvier, George Liopold Chritien Fredérie Dagubert. Dissertation critique sur les espeees d'Ecrevisses coulnues des auricus, et sur les nous, qu'ils leul ant donnes (1803) (AnII, du Mlus. d'hist. nat. 11, p. $36 s$ ). - Ohservatious sur quelques Diptires (Journ. d'hist. nat. 1792). - Mémoire sur les Cloportes terrestres (ibid. II. 8.). Tablean elementaire de l'histoire naturelle lles unimaus. Paris 1798. 8. (et D u meril) Tahleau des Crustares, annexe an Traité d'Anatomie romparé. Par. 1799. 2 Tom. 8. - Ueber Darm! und Herz vou Limulus. - Ueher die Rankenfüssler (Cirripedia Latı.) (Ann. 
du Mus, d'hist, nat, Vol. 1). - Mémoire sur Ia maniè dont se fait la notrition dans les Insectes (et Crusticres) (Wëm. de la soc. d'list, mat. de Par, an VII, p. 47). - Leguss d'Anatom, comparè IV, lce. 27, seet. t, art. 2.

DaIdorf. Uddrag af lans daghom paa "'l reise fra Kiöbenbivn til 'Tranquebar, sidst i Aaret 179009 färsı i Aaret 1791 (Skrivter uf Naturl. Selskabet, Bind 2, Heft 2, p. 147).

Dealuan, Johaun Wilhelu. War Director des Museums z,l Storklıolı!. Nagra Petrifieater, fundue i Orster. götlinds övergiingskalk, aftecknade och beskrifue at J. W. Dalmiı (Sitock. Vatens. Handl. 1824, 11. 368-78). (Maselieliu.) - On Palieaderna, eller de sa kallade Trilobiterna (Kougi. Vetensk. Acad. Handl. 18:26, 1. 1t3-153. Cont. 1. 226-295). - Uelier die Palacaden oder die sogen. 'Prilobiten. A. d. Schwed. übers. v. F. Eugollhart. M. $6 \mathrm{Kpf}$. gr. 8. Nüruberg (Sclımer) 1828.

Hinna, Jolın.

Defuy. Loologisclıc Bemerkungen (Sehrift. d. Berl. Ges. natf. Fr. VI, 1. 427).

Degreer, Charles. Mèm. VII (vgl. G cer).

"He Wnan, W.

* Delsay, J. L.

Delabigarre, Peter. Exeursions on oor blue mountains (Transact, of the soe, of New-York. Purt. 2, p. 128).

* Delle Clniaje (vgl. Clitaje).

"Dengel, K.

Desiso, Johanu Daniel. Von der Walfisclilaus (Cymothoa) (in dessen Beitr. y.ır Naturkunde. 12 Stärk, p. 10.14-1060).

"Deshayes, G. P.

"Desmurest, Anselme Gaëtau.

"Desumoulins.

* Devwora, V. J.

"Desvoilly (vgl. Robineau!

Dicapermare. Description de l'aetif (Oniscus) (Jouru. de l'hys. Tour. XXII, S(r.. Part. p. 29-3.t).

" Dicerbeh, J. Hoinr.

Dondorft. Uebersetz. Senebiers p. 41 (Arctiscon).

Domovan. Insect sof India (Cıst.).

" noosbleday, Edward.

"Doyere.

"Draplez.

* Dnfour, Léoll.
Doulhninel da Noncenu. Ob. servations sur les insurctis qui sattaelient aux poissons, ef description d'uu insecte qui sattache aux saumous (Arts rt Meticrs, pêches. 2. Partie, 2. édit. p. 293, 1769. - Caligus).

TDuIr.

" Dunuéril, And. Mar. Const.

* Dirmal.

"DBum cam, James.

* Dnesane.

thenelser, $W$.

mupent. Audre Pierre.

"Duveraoy.

* Ditrochet, $\mathbf{H}$.

"Ed varals, Milne.

Filwerals, J. Natural history of birds. London 174i, Vol. II, T. 286, f. A. (Lepas anita!!)

Fgede, Hans. Det gramle Grön. lands nye perlustrution aller naturel historice. Kjöbeuls. 1741, - Traduction français (Description du Groenland). Gencve 1763 (Cymothoa).

"Wlu renlugers, C. G. v.

cheriert, Balthasar. Physicalische Nachricht. Unbers. von Moro's „De Crustaceis etc." Bucl. Memuiugen 1745. 4.

wichloorn. Beitrige zur Naturgrescliclute der kleinsten Wasserthicre. (j. 74, t. 7 , f. E. Der Wasserbär, Macrobiotus). - Microseop (Cyclops et Daphnia .

"Eiclnwald, Ed.

\section{wiglets.}

Whis. John. An necount of several rare spercies of Barnaeles. Lond. Dee. 1758 ('luil. T'rans. Vol. L, 2, 'J. 34, p. 8.5 ).

Wivert. Dissert. sistens Millepe. des. Argent. 1711.

Encyclopénlie methodique. Crustaces.

" Erril, Mi. $\mathrm{F}$.

" wirichson.

Hiscliselooltr. Crustaceen in Mèm. de Moscon, VI. 1812.

* Eversiuman. Reise nach Bu. charu. Berlin 1:23. 85. 4. (Insecten, Mammalien und Vörel ete.)

"Eyilonx.

Nabricins, Johaun Christ. Systeин entomologiae. Kil. 1775. 8. Speries insectorum. 1781, 8. - Mantissa juscetorum. 1787, 8, - Entoınologria systematica. Il Tom. 1793.8. 
Ton. V, Suppl. Daldorff 1798. (Tom. II Crostine.)

Fabricius, Otto. Fana Groen. landica, systematiee sistens animalia grocnlandiae occidentalis luacturus indagita, quoad nomen specificuu, triviale vernacolumque, symonymin uuctormu etc. $e$. fig. IIafuine et lips. 1790. 8. - Brskrivelse over den store rıönlandske Krabbe (Sktivter det Kjöbenl. Selskab. Nye Sanl. Deel 3, p. 181) (Cancer IIaja).

Fnhlbers, Simuel. Utdrag af samlingar til natural-historien öfwerön St. Barthelemi i Vest-Iudien (Vetensk. Ac. Nya Handl. A. 1786, p. 215, 248).

Frujas-Saint-Fond. Gummarus? (Ann. du Mus. XII),

Nérussac, D'Audebard de F., fils. Mémoire sur deox nouvelles esperes d'entomostracis et d'hydiaenes (Anu. do Mus. VII, p. 212. - Cyclops Molleri; Cypris renifornis).

Fenillée, Louis. Joumal des observatious pliysiques, mathématiques lt botaniques, faites par l'ordic du Roi sur les eôtes orientales de l'Amérique meridiunale ete. Paris 1714, 3 Vol. 4.

\section{* Nicinus.}

* Nischer von WValdhein, Gott. helf.

vinemuing, $J$.

Forstarl, Peter. Deseriptiones auimalium, avium, amplibiorum, piscium, insectorum, vermium, quae in itinere orientali observavit. Post mortenI auetoris edidit Carsten Ni c buhr. Hatinia 1775 . - lcoues verum naturalium quas iu itiuere otientali depingi euravit. Hafuiae 1776.

Norster, Johinn Reinhold. Specimen historiae naturalis volgensis (Plic. Trans. 1767, p. 312). - Nachrieht von einem ntuen Insekte (Naturforselier, XVII. St., p. 206-213).

Fongeroux de Bondaroy, Augoste Denis. Sur un insecte qui s'attnehe a la chevrette (Mém. de l'Aead. des se. de Pal. A. 1772, P. 2, Ilist. p. 1. Mlénı. p. 29. - Oniseus; Bopyros).

Fonrceny, Ant. François. Entomologia parisiensis sive Catalogus iusertorum quae in agro parisiensi repe. riuntur. Paris, 2 Vol. in 18. 1785.

Frank, Georg. l'raeside. Ovır. roypacıa, Dissertatio de Asellis seu Millepedis. Resp. Dan. Birr. Heidelbergac. 1679 . 4 .

Erankemat, Georg Fricdr. Fran. cus ule. De canero marino rotundo majori variegato (Acta Aead. Nat. Cur. Vol. I, p. 315).

Fraumdorffer, Philipp. Oniseographia eoriosi. Brunae 1700. 12. (132 pp.). - De jartu millepedaron (Misc. Acad. Nat. Cur. Dee. 3, 1695 et 1696, 1). 3).

\section{"Fréminville.}

Freycinet. Stomatopoden (Dess. Reise werk).

Friscli, Joh. Leonhard. Bexchreibung von allerhand Insekten in Deutschland, Berlin 1720-33. 13 Hefte in 4. M. v. Kpf.

\section{* Trabriel, C. H.}

Fiide. Ueber Apus eancrifurmis (Wiedeluanu's Magazio 1817, 87).

"Triumad, J. P.

* Ararderies.

Cx̀̆aruann, Clırist. Friedr. Seolopendrae lux inuata (Mise. Ae. Nat. Cur. Dee. 1, A. 1, 1670, p. 307).

Gaser, Marian La. Amenidades naturales de las Espaunas. En Orel. nela. 1811. 4.

Cinstalily, Johann Baptist. An Salinate sanguis constitutioni Cancri fluviatilis. Diss. Avenione. 1713. 4.

Geer, Charles, Baron de. Mimoire pour servir a l'histoire des insectes. VII Vol. 4. av. fig. Stoekliolm 1778. (Vol. VII cont. cutom. et èrev.) Observation sur un Jule ou millepied cylindrigue, brumoitre, a deux raies feuille-morte tout le long do dos, et yoi est pourvu de deux eeus jumbes. II vit ordinairement daus la Terre. C'est relui qoe Limacus appelle Scolopendra teres, pedihus utringue ventum. I'auna suce. 11. 1260 (Mim. de Mathèm. et de Phys. 111, j. 61) (Julus terrestris). - Ohservation sur nue espece singulière de millepied ou de seolo. pendre, qu'on trouve sous l'irource des vieux urbres et dans la mousse (ibid. 1, p. 532) (Pollyxenus lagurus). Memoir. T. VII , p. $365, \mathrm{pl} .20$, f. 1. Eiue gote Natorgesclichte des Flusskrebses.

* Feinitz, II. G.

Teofroy, Claude Josepl. Observations sur les éerevisses des rivieres et sur quelques particolarités des icrevisses (Mëm. de l'Ae. d. sc. de Puris, A. 1709, Hist. p. 15, Méı. p. 309. Ed. oct. A. 1709, Hist. p. 19, Mèm. p. 404). 
laquelle ces animaux sont rangés suivant un ordre miéthodique. Paris 1762. 2 Vol. 8. Av. planch. - Sceonde Edit. an $\mathrm{X}$.

"Geofroy-Saint-Hilnire, 1sidor.

" Feofroy-Snint-Rilaire, Eti. enne.

Georgi. Reise.

Terlan, Legoux Je. Essai sur I'listoire naturelle (Memoir. de Dijout. T. 2, Mèm. p. 41).

* Fervais, Paul. (Anual. de la soc. ent. V. 1836.)

S'eseke. Dissertat. de Cancri astuci quibusdam partibus. Götting. 1817. 4.

Aesmer, Conradus. De piseinun et aquatilium animantiun Natura. Tiguri 1568. - Nomenclator aquatilium animantiun. Ieones animaliums aquatilium in mari, et dulibus ayuis degentium, plusquam 700, eun none:nclaturis singulormm, latinis, graceis, italicis, hispinieis, germaniris, anglicis, aliisque etc. Tiguri 1560. Fol. (p. 10. Aslac. fluv. m. gut. Holzs.; p. 121 Balanus.)

Axisler. Lernaea salmonea (Act. surc. 1751 et Ëncyel, meth, vers. pl. LXXVIII, f. 13-18).

" Gistel, Johannes.

Siockel, Joh. Georg. Epistola de mirabilibus quorundam animalium (Mise. Aeat. Nat. Cur. Dec. 2, A. 5, 1686. Append. p. 101).

Codzze, Joham August Ephraim. Reproduktionskruft bri den Insekten (Naturforscher, XII, St., p. 221-24). - Von dell Fischlermaen (Leipz. Mag. f. Natur 1784. 1. St.). - In Bonmet's lnsect. Abls. 1773, p. $367-375$, t. 4 , f. 7. Das Bärthierelieu (Maerobiotns). - Idem in 20. St. des Naturforscluers j. 114.

Gollehen, de Riville. Observation sur un animal conricux tronve dans les mers de Ceylan et noumi paou de mel (MIem. de Math. et de Plıys. T. 3, j. 275. - Monoeulus Pavo marinus).

"Gínoldfuss, $A$.

* Goorlsir.

(T)rif. De lapidibus eancrorum citratis. Altorfii 1735. 4.

* Früfe, $H$.

* Grant.

* Cüravenhorat, J. L. C. v.

* Tirнy, Georg Robert.

Gray, Stephen. Several microsco. pical observations and experiments (Phil. Trans. 1696, p. 280).

* Fín reen, Jakob.

* Giffith, Edward.

Grimu, Hermaun Nieolaus. Gammari in lapides couversi (Mise. Acad.

Nit. Cur. Dec. 2, A. 1, 1682, 1. 367). Friselini, Fr. Observitions sur la Seolopendre mariue ete. Venet. 1750. 8. Groef, Johann Erhard Anton. De lapidibus Caneroruu citratis. Diss. Altorffi 1735. 4.

Grironovius, Laturentins Tleodorus. Deseriptio Astaci norvegici enriosi (Acta helvetiea IV, J. 23-26). - Aujmalium in Belenio labituntiun Centtria 1 et 2 (ibid. Vol. IV. p. 24.3): Cent. 3 et 4 (ibid. V, 120); Cent. 5 (ibid. V, 353\% - Observationes de animalculis aliquot marinae aquae inuantibus atque in littoribus belgiris obviis (Beroe, Medusa, Squilla, Hydra) (ibill. IV, p. 35). Xiphcosura. - Znophylacium Gronovianum. Fase. 11. Lugdun. Bat. 176!. Fol.

5) Tritlenisen, Fr. v. Paula.

Arrmer, (Jurist. Gottfi. De febre urticuta, a Canris fluviatililus et fragariae veseac fructu. Progr.

Fualticri. Testarum conrlyylioruin index. Florentine 1742. Ful, T. 106 , f. e. (Lepis Scalpellum Liun. Wir mïssen hier lounerken, dass das Meiste der alten Literatur der Cirripeden hei den Conchologen an suchen sey. Gistel.)

Criillenstedt, Johann Anton. Zusammengetrageuer Beitrag rou Reisegeschichte nacl den caucasischen Gebirgen und Georgien (Sclur. d. berlin. Gesellsceh. naturf: Fr. 111, p. 460).

" Añérin, François Etienue.

Cuettaral, Jean Etipume. Némoire sur les arduisicres d'Angers (Mem. de l'Aead. royale des sc. de Paris 1757, p. 59, fig. (Trilobites otc.), - Minoire sur plusienrs morecallx d'llistoire naturelle tires du rabinet du Duc d'Or. leans (Mém. de l'Acad. des sc. de Par. A. 1753, p. 369).

Guilding, Lansdown. Besclurieb den Seyllarus carinatus, Jeptopodia rufeseens und Seyllarns aequinoetialis (Linu. Tians. XIV, P. If). - Macro. podia oceidentalis (ibid. XIV, I. II, P. 335, Ni. 1). - Westindische Crustaceen (Isis 1829, p. 1112).

- Gunner, Johann Erust. Nogle snia rare og mestendeclen nyc norske 
aöedyr beakreven. Monoculus finmarchicus; annulis postice quinque; capite obtusu: pedum anteriorum quatuor; posteriorum vero quinque paribus: cauda recta, bifida (Skrivter det Kjöbenliavuske Sclskiab. Deel 10, S. 175) (Monoculus). - (Ferner noch 2 Ascidiac, 1 Nereis, 1 Doris, 2 Asearides, ausserdem Monoculus finmarchieus enthaltend.)

- Waman (vgl. Dela ano'.

MalolizI, Carl. Observations d'hi. stoire naturelle foites sur les rotes de la mer rispienne (Aetin Acarl. Petrop.).

Hancquet, Balthasal. Ueber verschiedcne auf eincr Reise nach Semlin gesimmelte Beobachtungen (Abhandl. ciner Privat-Gesellscl. in Böhmen. II, p. 230). - Blicke über dis menschliche Wissen in der Naturkunde (ist unter keineu Namen ersclienen). 1813. 8.

Hagonincin. Symbola Faunae insecturum Helvetiac. Basileae 1822. 8. min. c. tab. color. 15.

Haisu, C. W. Grïndliche Anweisung, Krustenthiere, Vielfuisse und Asseln, Arachniden und Insektell aller Classen zu sammeln, 7.1 prïpariren, autzubewahreu und zu versenden. Nach mchr als zwanzigjülıriger Erfahrung und eigener Ausuibung für Simmuler und Licbliaber bearbeitet. Nürnber.r (Zeh) 1834 (12 gr: od. $54 \mathrm{kr}$.).

Haiu, Jobaun Paterson. Experimenta mieroscopica (Miscell. Ac. Niat. Cur. Dec. 1, A. 2, 1671, p. 53).

\section{"Irarlan. Richa'd.}

Hardwicke, Thomas. Narrative of a journey to Surinagur (Asiatic Researches VI, in Oct. p. 309). - Cermatia lougicornis (Scutigera Lam.) (Transactions of the Limean Socicty XIV, 1. 131).

IRasselouist, Fredrik. Rcise nach Palästiua, Aus d. Sehwed, übersetzt. Rostock 1762. 8. - Voyage en Palestine. Traduction franç. 2 Vol. 12.

Menrne, Urban. Memorabilia nonnulla lacus Vetteri (Phil. Trans. 1705, p. 1938).

\section{Hebenstreit.}

Teinecken. Cerascopus, Hegeter, Cermatia (Zool. Journ. Nr. XIII. Isis 1831, p. 101, T. 1). - Nachwachsen der Crustaceen (ibid. XIII. - Isis 1830, p. 1264).

- Helbergins, Otto. Observations aur les cuabes de terre, traduit de l'al- lemand en français (Collect. Academ. III. p. 446).

Telbig, Johann Otto. Gammari teriestres (Misc. Ac. Nat. Cur. Dec. i, A. 9 et 10,1678 et 1679, p. 453).

Helmioitz, $A$.

Helmontins. De lith. c. $7, \$$. 31 (Krebsuugen).

2x Teuminger, Johann Sigmund. Praesicle. Disputatio sisteus Millepedas. Resp. Jos. Phil. Elvert. Argentorati 1711. 4. (30 pp.)

Trentscli, Gust. Friedr. Epitoue Entomologiae systematicae secundum Fabriciun continens genera et species insectorum Europaeorum. Lipsiae sumtibus officinae publicae inservientis lit. teraturap. 1804.4.

Herbst, Joh. Friedr. Will. Versuch ciner Naturgesehichte der Krabbeu nnd Krebse, nebst einer systematisclien Beschreibung ihrer verseliedenen Arten. Bd. I. Zürich 1782. Bd. II und III. Berlin nnd Stralsund 1796. 4. M. viel. Kpf. (Niitzliche Compilation, untermischt mit mclureren neuen Figuren.) - Bcschrcibung der Flinderoder Hellebuttenlaus (Selur. der BerI. Ges. natf. Fr. Bd. III, p. 94) (Monoculus, Caligus piscinus). - Natursystem aller bekanuten in- md ausländischen Insektcu, als eine Fortsetzung der von Buffou'schen Naturgeschichte wach dem System des Ritters Carl v. ILinni bearbeitet von Carl Gustav J a b lonsky. Der Käfer 1. Theil, mit 6 illum. Kupfertaf Berlin (Joach. Pauli) 1785. 1 Titelkpf., $24 \mathrm{~S}$. Vorrede und $310 \mathrm{~S}$. 'Text, 8. 2. Thl. waeh dem System des Ritters v. Linne und Fabricius, z.川 arbeiten angefangen vou C. G. Jablonsky und fortgesezt von J. F. W. IIcrbst. nut 17 jllum. Kupf. Berlin (Pauli) 17א9. 64 S. Vorr. und Einl., 330 S. Text, 6 S. Rer.; 3. Thl. mit 16 ill. Kpft., Berl. 1790, 14 S. Vorr., 325 S. Text; 4. Th. nit 12 ill. Kpft. 1792, 8 S. V., 197 S. Text ; 5. Th. m. 16 Kpft. 1793,16 S. V., 392 S. T.; 6. Tl, m. $38 \mathrm{Kpft} .1795,24$ S. V., 520 S. T. 8. - Beschreibung eimer sehr sonderbaren Seclaus vom Hemorfiseh (Sclır. d. Berl. Ges. natf. Fr. Bd. 1, p. 56. Monoculus productus).

Termann, Jean Frèdèric. Mèmoire aptérologique. Strasb. 1804, Fol. Pl. col. (Argulus, Limnadia, Di(helesthiun.)

"Heruld, Manr. 
- Merrich-Schürer.

Hexpel, Hendrik van den. Verhandeling over Gods goedheid in de bepaaling omtrent den dood der meeste Dieren (Verhandel. A. Grenootselı. te Vlissingell, Deel 12, St. 1, Bl. 313).

mhi, Abralam. Inqoiries for Quiny (Pliil. Trans. Y. 1667, p. 472).

Hire, Philipp de la. Observation sur le ramalissement des eerevisses de mer et des crabes, mis, pendant 15 jours, dans l'eau dooce (Mém, de l'Ae. des sc, de Paris, T. 2, p. 62).

Moefingel, Jacobus F. Arche. typa studiaque Patris Georgii Hoefnagelii Jacobus F. grenio duee ab ipso scculpta. Fraucof. 179)2. 1 Part. is 12 Taf. 4. (Geistreiche Zusammenstellung vieler" Thiere aus allen Classen mit ainnigen Motto's. Gistel.)

"Hocainghans, Friedr. W.

* Hoeven, J. van der.

HofTannu, $\mathrm{D}_{1}$ : Einige Bemerkungen iber die Vegetation und die Fauna von Helgoland (Verh. d. Berl. Ges. I, p. 228-261, Tif. X).

Molt. (Nat. Selskal. Skrivt. V, p. 135).

Homberg. Olsservations sur les yeox d'Eerevisses, (ni); pris en poudle: guérissent la maladie appelèe le fe $\mathbf{r}$ chaud (Mém. de l'Acad, des se. de Paris 1708. Hist. p. 66).

\section{Impernti.}

- Jüger, J. W.

Joblot, L. Deseriptions et usages de plusienrs mieroseopes, avee de nouvelles observations sur une multitude d'insectes. Paris 1754. 4. Accompagne de beaocoup de planehes. (Einige Sï̈sswasser.Eutomostraceen.)

* Joha.

John (Missionär). Nuturhistorisehe Fragmente von John zu Trancuebar an Herbst (Mag. der Ges. natorf. Fr. xu Berlin, II, J. 63-74. 1) Caneer lanatus; 2) Cancer Diogenes; 3) Plellronectes candida; 4) Lenur tardigradus; 5) Lacerta puleherrima; 6) Spinneu; 7) Raja Rlinobatus; 8) Negerflagge (Conchyl.); 9) Astaeus homarus.)

Doly.

- Jones, Rymer.

Jones, Hogh. Letter concerning several observables in Maryland (Phil. Transact. Y. 1699, p. 436) (vgl. Petiver).
Jones, William. On asiatic history, eivil and natural (Asiatic Researches Vol. IV, p. 1).

Jonston, John. Historiae nutura. lis de exsunguibus aquaticis libri IV. Amstelodani 1665, e. tig. Fol.

Dolnuston, G.

Inrine, Lonis. Histuire des Monocles qoi se trouvent aux environs de Grenève. Grandve 1820. 4. Av. pl. col. (Oeuv. posthume) (vicle Entomóstraceen). (Isis 1825 , P. 86). - Sur le Monocle Pues iBullet. des scienc. par la soc. philomat. de Paris, II, Nr. 53. - Daphuia Pulex). - Sur le Monoculos quadrieoruis (ibid. I, p. 116. - Cyclopss. - Mimoire sor le monncle rastor (ibid. A. 3, T. 2, p. 73. - Monoculus Castor). - Mémoire sur l'Argul. foliare (Anu, du Mus. d'hist. nat. VII, p. 431. Av. planeh.).

Thäuprer, Engelbert. Beselıreibonz von Jajan. Französ. Uebertra. gung : Histoile naturelle, eivile ef eecléniastique de l'empire du Japon. La Haye 1729. 2 Vol. Fol. (Xipheosura).

LaIno, Pehr. Rön och anmärknin. gar oti natoral historien och oceonomien giorde orh siincle $j$ frï॥ Norige (Vetensk. Arod. Handl. A. 1748, p. 185). - Besclireibong der Reise uach dem nördlichen Amerika. Göttingen 1759. 8. 3 Bde.

Ifianuel, Georg Josepl., De piscibus, molluscis ef crustaeeis philippensibos (['hil. I'rans. 1705, p. 2043 bis).

\section{Anand, J.}

King : Edward. A deseription of a very remarkable aquatick insect, found in a ditcli of standing water near Norwich in the Spring of Year 1762 (Phil. Trans. 1767, p. 72. - Nenes lan̄ol. Mag. 41) (Caneer (Branchipus) stagrualis Lin.).

KIng, Cliarles. A letter concerning erabseyes (Pl,il. T'rans. 1700, p. 672).

Kirhy, William.

Dirchner, T. W.

Kirsten, Joann. Jakob. De lapidibus Canerorum. Diss. inaug. Alt. dorfii 1735. 4.

KIeln, Jakoh Theodor. C'ancer quasimodogenitus oder naekter Taseluenkrebs aus der Insel Wight (Naturforsel. Ges. in Danzig. Sclor. Il, p. 187-208) (Reproduktion). - Inseetum aquaticun antea non dexeriptom (Phil. T'rans. 1738, p. 150. - Monoculus apus). - Prae- 
ludium de erustaceis (in seinem Werk: Summa dubiorum circa Linnapi elasses quadrupedum et amphibiorum). - Ostrac. T. 12, f. 100 (Lepas).

*Idulen, F.

- InIng, Fr. v.

Knorr, Gcorg Wolfgang. Deliciac Naturae selectae, oder auserlescnes $\mathrm{Na}$ turaliencabinet ehcmals herans regeben. Nürnb. 1766. 2 Bde. Fol. M. col. Kpftaf. - (et Waleh) Recueil des monuments des eatastrophes que le globe a essayecs. IV Vol. Fol. fig. color. (Crustacea et Trilobites).

$\because$ Kocln, F. E. L.

* Koela, K. L.

Irahier. Ueber Cyclops (Naturforscher X, p. 104, Tab. 2, fig. 10 -11).

- Hiblicher, Albert.

Kduig, Johann Gcrlard. Naturhistorisclee Nachricliten aus Trancuebar (Beschäft. d. Berl. Ges. natf. Fr. III, p. 427). - Reise fia Traukebar til (de Palliacattiske bierge) Zeylon (Skrifter det Kjöbenl. Selskab. Deel 12, p. 383).

"Iollar, Vine.

"Ixominh, de.

- Kote, B.

* Irassov, C. R. A. Frhr. V.

* Krauss, Ferdinand.

"Irdyer, Henrik.

* Krolun, Aug. D.

- Irynicki, Johaun.

- Irister.

Laet, Jcan do, von Antwerpen. Novus orbis seu deseriptionis Indiae oceill cutalis. Lugd. Bat. 1633. Fol. tLimulus).

Lamarck, Jean Baptiste Pierre Antoinc de Monnet, chevalici de, Professor am Muscum der Naturgeschielite, Akademiker etc.; geb. 2.u Basentin in der Picardie 1743, gest. (blind gevorden) zu Paris im December 1829. Zahlreiehe Werke: Systeme des Animaux sans vertebres. Puris 1801. 8. - Extrait du Cours de Zoologie sur les animaux suns vertèbres. Paris 1812. 8. - Histoire naturelle des animaux sans vertẻbres. (1. Anfl. Paris 1801.) paris 1805-1822. 7 Vol. 8. - Histoire naturelle des animaux sans vertêlıres, prèsentant lcs earactères genèraux et particuliers de ces animaux, leur distribution, leurs elasscs, leurs familles, leurs gemres, ct la citation dcs principales espèces qui s'y rap- portent; précédéo d'une Introduction offrant la determination des caractèrcs essentiels de l'Animal, la distinction du végétal et des autier corps naturels, enfiu, l'exposition des principes fondamentaux de la Zoologie. Tome I. Paris, Verlicre, Libraire, Quai des Autustins Nr. 27. Mars, 1815. XVI et 462 pp. 8. - Tom. Il. Mars, 1816. $568 \mathrm{pp}$. - T'. III. Août, 1816. $568 \mathrm{pp}$. - T. IV. Paris, chez Deterville, libraile, rue Haute feuille Nr. 8 et Verdièce ette. Mars, 1817. 602 pp. - T. V. Juillet, 1818. 612 pp. - T. VI. 1. Part. Paris, chez lauteur, au Jurdin du Roi. Fevricr - Juin, 1S19. VI ct $343 \mathrm{pp}$. - 2. Part. Avril, 182\%. $232 \mathrm{pp}$. T. VII. Août, 1822. $711 \mathrm{pp}$. - Histoire naturelle Ilcs animaux sans vertebres (Dcux. édit. T. 3, par Des hayes etc. Radiaires, vers, insectes. Paris 1840.8 ). Vgl. Deshayes. - Philosophie zoologique. Vol. I, II. Brux. 1837.

tumarcl, Mademoisclle de.

Landes, Des. Observation sur la géueratiou des soles et des chevrettes (Mém. de l'Acad. des sc. de Paris. A. 1722, Hisf. p. 19. Ed. oct. A. 1722, Hist. p. 26).

Lungins, Carolus Nicolaus. Me. thodus testacea marina distribuendi. Lucernae 1722. 4. (Balanus). - Historia Lapidum figuratorum Helvetiae. Venetiis 1708. 4. fig. (Crustat:.).

Lantilius, Rusinus. Addenda ad P. I. Saclis a Lewenlicimb nessem observat. mierosc. (Misc. Aead. Nat. Curios. Dee. 2. A. 10.1691. Append. p. 46).

Intreille, Pierre Andrí. Histoire naturelle générale et particulièrc des Crustaces et Insertes (zu Soun in i'k Buffon, Bd. III-VI). - Ueber Prosopistoma (Lophyropoda?) (Nouv. Ann. do Mus. II). - Encyclopédie méthodique: planches de Crustaces ct iliusectes avec leur explication. - Table encycloped dique et uncthodique des trois règnes de la nature. 24. Partie. Paris 1818. - Précis des caractèrcs gćnériques des inseetcs. Brives 1796. 8. - Regue animal, distribué sclon son organisation le M. Covicr. Ill. Vol. rédigé par M. Litreillc, et comprenant les Crustaces, les Araclunides ot les Iuseetes. Paris 1817. 8. - Genera crustaceorum et insectorum. IV Vol. 8. $A v$. fig. Par. 1807. - Mlènoirc sur les Trilobites (Mèm. du MIus. d'luist. nat. T. 
VII). - Mémoire sur le passage des animanx invertibres aux vertêbres. Jmprime avec un nutue memoire sur la furmation des ailes dans Ies insectes J'aris (Diterville)! 8. (44 p. .). - L'éerevisse conmune in Cuv. regul, all. ed. 2, T. IV , p. 90), - Des Langonstes dil Nuscum mational d'hist. nat. (Aun. d Mns. d'hist. nat. T. III, 1. 389. 1) Palinurus vuleraris, 391 ; 2) P. guttatIIs, 392: 3) P. argus, 393: 4) P. polyphagus, 393; 5) P'. versicolar, 394.)

Heitrobe. Onisens pliysudes (Pliysodes Latrobei Gistel) (T'rans. of Philad. V, p. 79. fix. - Faums III).

We-acln, William Elforl, Zoologiral Misecllany. I.ondun. III Val. 8. W. col. Plates, - Myriapoda (Zoolog. Miscollany. III, p. $32-49$, Taf. Jinn. Trans. XI. - Eucyclopacedia Irritanica I. - Edinhurghlı Eine.) Cliaracters of Micippa a new genus of Bracliyous Malarostraea, witl descriptions of two species (Ej. Zoolog. Misecll. III, p. 15, 16, T'. 128 (*tc). Doclea Rissonii (ibjd. II, p. 42, T. I.XXIV). - Atylus cariuatus (ibid II, F. 22, T. I.XIX, f. 11. - Atya scabra (ihill. III, p. 29, Ni. XI, T. 131). 'Ilıalassina scorpioides (ilijd. III, 1?.28, T. 130). - Mictis rmcifera (ibid. I, p. 92, T. XL). - Monugraph on the Genern and species of the Mialacostracous family Icurosidea (ibjl. III, p. 17-26). - I.иpa forceps (ibid. I, p. 123, T. IJIV, f. 1). - Ieptopodia sagittaria (ibicl. II, p. 16, T. IXXIII, f. 1) - Ibaens (ibicl, at in Trans. Isinn. II : p. 345). - Egeria indiea (ibid. II, p. 40, T. ILXXIII). - Dexamine spinosa (ihid. Il, p. 24 et Edinb. Enc.). - Pactolus Boscii (ibid. III, p. 19, Tah. I,XVIII, f. 1) - Nebalia Herhstii (ibid. I. p. 100, T. XIIV, f 11. - Macropodia Plalangium (ibid. 11, p. 17. Ist Macropus longirostris Jatr. gen.). - Matuta, IV kp. deser. (ilicl III, p. 13, T. 127 (1 seq.). A tabular view of the external Chavacters of four classes of animals, which I inné arranged under Insecte: with the Distribution of the genera composing tluree of these rlasses inta orders etc. and descriptions of several New genera and species (Linnean Trinsact. XI, p. 206. - Bullet. sor. Philom. 1816). - Artiele: "Crnstareology" in Edinburgh Eneyclopaedit vou Brewster. Tom. VIl, 1813
-14. 4. - Malacostraca Podophthalma Britumiae. Nos. 1-17.culı. Lond. 1815 -20. 4. lig. color. - Descriptions of new Species of Aumals diswovered in the Voyage of His Minjestys Ship lisabella to the Arrtic Reginns. I,and. 1819. 8. - Notice sur les animaıx recueillis par Ie papitaine lioss dins soll voyage à la baie de Bassin, par M. Lesclu. (Englisch.) - Onisei (Brewst. Encyel. VII. P. II; 406 partim.). A general arrangement of the classes Crustaeca, Myıiapnda and Aracluoida, with elescriptious etc. ('Trans. of the Linuean Soeicty. XI). (Vicle nene Species.) - Essay on Cirripedes. Edinburg 1812. 4. (Zool. Jonrn. VI. - Isis 1019.)

Geers, Arn. Catalogue svstemstique don Cabinet de Coquillages et Crinstaces. Ansterd. 1767 (holliand. und fru(uz.).

Hecuwenlobelk, Antony van. A specimen of some observations made by a mieroseope contrived (Phil. 'Traus. 1673 , p. 6037, 6116). - Eepistula (postlumala) de generatione animalium of de palpitatione diapluragmatis (ibid. 1723, p. 138). (Cyclopis.) - Epist. p. 102, fig. (Scolopendra). - Areana. 464, fig. 7 .

Hederminler. Mikroskopisehe Augeu- und Gimiiths-Ergötzung. Auch französ. Nürulerg 1764. 4. M. v. Fig. (Einige Entomostraca).

Celunane, Johann Gottloh. Onderzoek van een Zeker vnor bederflewarend poeder in Vrankryk witge. vonden (Vorluandel. der Mautsclo. te Haarlem. Deel 11, St. 1, B1. 324).

Mémery. Analyse des Cloportes Mim. de l'Acad. des scieuc. 1770, p. 240).

Lepeclnin, Iwan. Tres oniscorum specius descriptare: Oniseus aculealus, Oniseus seorpioides, Oniscus cnspidntıs (Arta Acad. Petrop. A. 1778, P. 1. IINm. p. 247).

Heske. Anfangsgr. d. Naturgeseli. 602.

Lesser. Testaceotheologie.

Hesson.

* Lesuciur, Cliarles Alexandre.

Idenclust, F. S. Ueber die Verlireitung der ibrig zeluliebenen Reste einer vorweltlielien organiselien Schöpfung. Freiburg. 4.

" Tiemnis, Joliannes.

Hewenluciub, Philipp Jakob Saclos a. De Gammaris amaris silesincis at aliis miris (enucrorum (Mise. 
Aead. Nat. Curios. Dee. 1, A. 1. 1670, p. 176), - Messis observationum mi. crosepucarum c variis authoribus collectirnm (ibid. Dec, 1, $A, 1,1670, p$. 40. Dec. 1, A. 2, 1671. Append. p. 3) (Conf. Lentilius).

Lieyile, Ed.

HInvyd, Edward. Letter containing several observations in naturil listory, made in lois travels trough Wales and Scotland (Philos. 'Trans. 1712, 1. 4C2, $466,467,500.1713$, p. $93,275,276)$.

Iabavius, Andreas. De Gamnaris, quos vulgo perperan Cancros appellant. Diss. Coburgi 1610. 4.

\section{* Lichtenstein.}

Lic, Ole. Efterretning om nogle naturalier i en skrivelse til Biskop Gunnerus (Trondhiemske Selsk. Skr. Deel 3, 1. 571).

* Lienard, d. j.

Cimmens, Carolas. Animalia per sueciam observata (Actu Litt. et reient. Sueciae. A. 1736, p. 97). - Fauna Suecica. Edit. 1. Lugd. Batav. 1746. 8. Edit. 2. Stocklı. 1761. 8. - Amoenitates acadrmicae. Nr. 57, Tom. 8. p. 75. Sodrrherg, Propos. Pandura et Flora rybiensis (drei Crustaceen citirt). - Johansson (Boas). Propos. Centuria Insectorm, $\mathbf{N r}$. 121, T. VI, p. 384 (sieben Crustaceen). - Tonning (Henric), Prop. Nr. 149, Tum. VIl, p. 466 (25 Aptera marina). - Sparrmanu (Andr.), Propos. Iter in Chinam (Onis. cus anulus, Cancer Neptuni). - De Entomolitho paradoxo (Acta Hoim. 1759 , t. 1 (Paradoxides, Trilubites). Cancer Phalaneiuu, from D. Maceulocl (limnean Traus. Vol. VI, p. 389. Nr. XXV, T. XXI). - Reisen durd। Westgothland. Ans dem Schwedisch. Halle 1765. 8. - Fundamenta cutomologiae, quae venia Nubilisseti Pracsule ete. Iro Doct. C. v. I i un é puhlice defend, evecunabitur Andreas Joham Bladh rte. in andit. Carol. Muj. die Jınii 1767. Upsaliac apud Jolı. Edman». 34 pir. 4.

Jink, Joh. Will. Versuch riner Geschichte und Physiologie der Thicre. Clımuit? 1805. 2 Bde. 8.

\section{\# Jink, H. F.}

\section{Hister.}

Littleton. Sinr le fossile de Dudley (Philos, 'Prans. 1750, Tab, 46-48 (Culymenc, Triluhites).

Hochend, William. Observutions on the natural history of Gniana (T'rans. of the Suc, of Elimburgh. Vol. IV. Pliys. p. 41).

Lochuer, Johann Heinriclı. Mnsacoul besleriaumm. 1716. Fol. p. 95, pl. 83 (Petrefuctu).

Limmimg, Peter. Monoculas cruda foliacea plana, descriptıs (Aeta Upisiliellsiu. A. 174t-1750, R. 42) (Mv)11culus piscinus).

Lisselke. Mater. med. (p. 25y. Millep.).

Hodve, Jall. Carl Christ. Kurze Naturgesthichte vou IVrigelsdorf in Schlesion (Halliscl., naturf. Ges. 1, 69).

Lorincer, J. Letter from WestFlorida containing some remartis on the climate, verituble productions ets. (Trans. of the American Suciety. Vol. 1, p. 250 ).

Hosclige, Friedr. Heinrich. Le. obichtungren in dem Monoculus njus, Linn. (Naturforscher; 19. Stuck, S. 60 $-69)$.

*overit, S. L.

Jumd, Niel Tonder. Jagrtagelser' (il insecternes historia. Slaegtun Seyllarus elc. (Skrivter af Natuil. Selsk. Bd. 2, Hifte 2, p. 17).

Cun und, Peter Willelm.

Lutatens, G. N. Pliysische Besclureiburir P'ennsylvaniens (Neue Sehr. d. Ges. natf. Fi: H, p. 362).

Fuyal, Edwards. Memoire sur les Trilobites (Philos. Trans. anı. 1698).

\section{Nre-Cullocls.}

Minrklim. Mcdizin. 'Thierbuelı (p. 3551 .

Ir dirter, Franz loseph. Reise vou Europa bis nach Plhiladelphia in NurdAmerika (Physikal. Arbeit. der eilltrïcht. Freunde in Wipn. Jahrer. 1. Quart. 3, 1. 53, 66). - Naturhisturischo Nachrichten aus den bahamischen In. selu (ibicl. II. Jahrer., Quint. I. 1). 58). - Niturhist. Nachr. aus Virginieu und aus Ost-['lorida (ibidl, Quart. 4, 1. 93 -101). - Nischrichten iiber die natiirliche Geschichte Penusylvanicus (ibid. I, Qı. 4, 1. 20).

Mnjor, Joh. Dal. Dissertatio epistolical de Caneris et serpentibus petrefuctis; et epistolia respunsoria Philippi J. Sachs a Lewenleeimb de mir. laj. Natura. Ienas: 160.1.8.

Malezien, ie. Observations sur des animax vus an microscope (Mim. de l'Acad. des se. de: Parris. A. 1718. Hist. p. 9. Ed. uet. A. 1718, Hist. 1. 11. 
Maloet, Observation sur un ver de l'espece des scolopendies terresties, reudu par le nez (Scolopendra) (Mèm.| le l'Acad. des se. de l'aris. A. 1733. Hist. p. 34, Eu. oct. A. 1733 , Hist. j. 46 ).

N⿴囗十u, Theodore Angustin. Mèmoire sur l'histoire naturclle de la mer du nord et sur la pêche qui s'y fait (Memoires de Bruxelles. II, p. 157).

Marcerave de Liebstad (Georgins. Historia rerum naturalium 13 rasiliae. Jibri VIII. Lugdumi Batavorum et Austelodami. 1648. Fol.

Mariou De Proce. Sur plusienrs especes nouvelles de poissons et des erustaces observecs dans un voyage a Manille pu 1819 ct 1820 (Bullet. 80c. philom. Septembre. 1822).

Irarsden, William. Account of a phenourenon obscrved upon the island of Sumatra (vast quantities of fislies thrown on shore during a very dry scason. - Phil. Trans. Y. 1781, p. 383 ).

Martens, Friedrich. Spitzbergisde oler Grönlaindische Reisebeschreibung. Hamburg 1675.

Mn rimidre, La. Mćuoire sur quelques animaux parasites reneillis dans le voyage autour du mumle, sous le commendement de MI. du la Peyjouse (Jouru. de Phys. Novenube 1787. Onistus Physodes).

"Martius, IV. C.

Mary. Krebsaugen (Jouru, britaunique, April 1750.

Trattluiolus, Petrus Andruas. Commentaria ill sex libros Dioseoridis, Veuretiis 1565. fig.

Mrtty. Sur le Caneer salinus (Diar. britann, 1756).

Mayer, Gothofredus David, Collectionis lapiulum (oculorum) cnnerornuth listorica cxegesis, veraque methodus (Ephemer. Acud. Nat. Curios. Cent. 7 et 8, p. 417).

ingyer (Boin).

Tryerue, Theodore de. Vgl. Mufietus.

Meckel. Trophi Astaci (dessen vergl. Anat. IV, 149).

Merret. Pin. (Daplinia).

Mertens. Uebcr den inncrn Bau von Lepas (Müller's Arrhiv. 1835).

Mezen. Careiniumopalinum. Asselartiges Thier (In sein. zool. Beobacht. Acta Leop. XVI. 2. Suppl, 125. Taf. 27).
Ieyer, If. v. ( $V_{\mathrm{gl}} \mathrm{l}$. M ̈̈ n s te r.)

Irclunelis, Joluanu David. Oratio de defectibus listoriac naturalis ac plilologine itinere in Dalaestiuam Ara. biamme suscepto farciendis (Commentarii Göttiugens. III, ]. V-XXX).

\section{Michelotti.}

Miller, Charles. Account of the Island of Simatra (Phil. Transact. $\mathbf{Y}$. 1778, p. 160 ).

\section{"Inillet.}

Mikan, Johann Clristinn.

Dingsi, Antonio. Dissertatione seconda su de limpanteti dell udito scoverti nel Granchio l'aruro, e sulla bizarra di lui vita. Napoli 1775.

* In olir, M. H.

MUlina, Giovanni Ignaxio. Essai sur l'histoire naturelle du Chili, publice eu Italic, traduit eu français par Gruvel. Pitris 1789. 8.

Nontagu, Gcorge. Cancer gammarus (T'ins. of linu. Socicty II, p. 3. - Ist Dexanine spinosa Leach). Monoculus rostrutus IJiun. Trans. II, j. 14, T. 2 , f. 5. - Ist Caneer bipes Otto Fubricius und Nebalia Herbstii Lpaill). - Descriptions of several new or vare Animals principal marine, discovered ou the South Coast of Devorshire. (Linn. Traus. Vol. X1, Tab. I1, P. I, p. 7, f. 4. 1) Cincer Hippo 7.dentatus, 1, Tab. I, f. 1 ; 2) C. aculcatus, 2, f. 2: 3) C. Gammarus spinosus, 3, T. II, f. 1; 4) C. Gamm. Galla, 4, f. 2 ; 5) C. G. nonoeuloides, 5, f. 3 ; 6) C. G. obtusatus, 5, f. 7 ; 7) C. G. pedatus, 5, f. 6; 8) Monoculus rostratus, 4, f. 5. - Vol. VII, p. 61, Nr. VIl. 1) Cancer rhomboidatlis, 13. 65, Tub. VI, f. 1; 2) C. muxilluris, 65 , f. 2 ; 3) C. Pliasma, 66, f. 3 ; 4) C. palmatus, 69, f. 4 ; 5) C. seorpioides, 70, f. 5 ; 6) C. articulosus, 70 , f. 6 ; 7) Ouiseus hirsutus, 71, f. 7; 8) 0. cylindraceus, 71, f. 8. Ferner verseliculene Thiere aus undern Classen. Vol.I X, p. 81, N1. II, T. II - V. 1) Cancer floridus, Tab. 2, f. 1 ; 2) C. lummefactus, f. 2 ; 3) C. denticulatus, f. 3 ; 1) C. Astacus subterraneus, T. 3, f. 1, 2 ; 5) C. A. atcllatur, f. $5 ; 0$ ) C. A. inultipes, T. 5, f. 3: 7) C. A. gibbosus, f. 91; 8) C. Gaumarus Locusta, 92, T. IV, f. I; 9) C. G. Pulex, 93, f. 2 ; 10) C. G. Saltatur, 94 , f. 3 ; 11) C. G. littoreus, 96, f. 4 ; 12) C. G. prossimanus, 97, f. 5; 13) C. G. Talpa, ง8, f. 6 ; 14) C. G. rubricatus, 99, T. 
V, f. 1; 15) C. G. faleatus, 100, f. 2 ; 16) Plıalangium spinosum, 100 , f. 7 ; 17) P. aculeatum, 101, f. 8 ; 18) P. Testudo, 102, f. 5; 19) Oniscus gracilis, 103. f. $6 ; 20$ ) 0 . thoracicus, 103 , T. 3 , t. 3,$4 ; 21$ ) 0 . squillarum, 104.

Mnrgensteru, Friderieus Simon. Descriptio Caneri marini vulgo Eremitue (Nova Acta Acad. Nat. Curios. I, p. $375-379)$.

Mi oro, Alltonio Lazzaro. De' Crostacei e degli altri Marini Corpi che si truovano su' monti libri due. Venezia (Monti) 1740. 4. Con 8 tavol. (Conf. Gronov. Bibl. p. 191. - Wir haben dieses seltene ins Deutsche übersezte Buch nie geseheı. G.)

Mortiner, Cromwell. Vgl.B row u. Mosen ril. Musacum. 1635, Fol.

Irinler, Otto Fred. Om tvende smaa Eell-oier (Skrivter det Kiöbeulı. Selskab. Nye Saml. Deel 1, p. 406. Monoculus pilosus). Von dem mopsuasigten Zackenfloh (Schrift. der Berl. Ges. nlatf, Fr. Bd. 6, S. 185, - Naturf. VI, 299-307. - Monoculus simus, Daphnia sima). - Observatious ou sone bivalve insects, found in common Water (Plıil. 'Trams. 1771, p. 230. Monoculus). - Aumerkung zu J. H. Chemnit\% Beschueibung piner Reise uach Faxoe (Schrift. d. Berl. Ges. natf. Fr. 11, p. 126). - Zoologiae danicae prodromus. Hafuise 1776 . Fol. c. fieg. coloratis. - Zoologia Danica (Aufzilli. d. dïu. Krabben. In Texte ciuc Notiz über einen Trilobiten, des auch abuebildet ist.) - Vou dem Birthierclien, Acarus ursellus (iu Füssley's Iusectenges. 6. Heft, p. 25, Tab. 36. - Matcrobiotus). - Entomostraea seu Insceta testacea quae in aquis Daniae et Norwegiac reperit. Lips. et Hafn. 1785. 4. (Grosse Am\%ahl von Entomostracinen des siissen und salzigen Wassers.)

"Miller, Johanues.

Mihuster, Geory. Graf v., in Bayreutl. $\uparrow$. Ueber cinige neue Versteinerungen in den lithographischen Schicfern von Bayern (Vorwetragen iu der Sitzung der Vers, d. deutseh. Naturf. und Acrzte zil Pyrmont 1839. - Vgl. Isis 1840 , p. 900. - Neuc Reptilien, Pterodactylus, Sepien, Krebse, Fische, Kerfe, Lurche, Pflanzeu). - Beitrïge zur Petrefaktenkunde von Herm. v. Meyer und G. Graf z.11 Mr. Mit XX nach der Natur gezeiclıneten Tafelu.
III Hefte. gr. 4. Bayreuth 1840. Decapodis macroura. 4. 88 T. 29. Bayreutlı (Buehmer) 1839, II (1sis 1840, 281).

* Munter, Gustav Wilh.

Mituscluetalu, von. Nachricht vou einigen Wasseriusekten an der Bartsch, nacls weleluen sich dic Fischer bei iliren Winterfischereien richten (Notonerta glauca, Onisens aquaticus et Nepa linearis, Dytiseus marginalis et semistriatus) (Oekonom. Nacliriclst. d. Gesellscluaft iu Schles. Bd. V1, p. 39s, Bd. VII, p. 2).

Vufretus, Tliomas. Englischer Niturforscher und Arzt des Hauses Penbrock. Gest. gegen 1600. Insectorum sive minimorum animalium theatrum. Londini 1634. Fol. Cun 500 fig. ligu. in. (Dev Herausgeber war Thieod. de MInyerne, cin Franzose und Leibarzt Jikoby I.)

Munsey. (Bei MI r y.)

Muralto, Johanues dc. Descriptiones insectorum variorum. Squilla mollis lacustris (Miscell. Acad. Nat. Cur. Dec. 2. A. 2, 1683, p. 189207).

\section{* Murehison.}

(IInxr, C. v.) Ablaandlung von Naturalien-Cabineten. Leipz. 1771, 8.

* Iuse, J.

Satterer, Johann.

"Nanturin, C. F.

Vebel, Daniel. De Mrillepedis. Diss. Heilelbergae. 1716. 4.

* Nenuing.

Newport.

Nicola, Lewis. An easy method of preservinur subjects in spirits (Trans. of the american Society. Vol. I, p. 244).

Nietsch, C. F.

Nietzscla. Ueber Macrobiotus Hufelondii (Wiegnann's Arelniv 1835).

"Nordunam, Alex. v.

Dedunau, Samuel. Grundmärglan, Cancer Pulex, beskrifven (Vetensk. Aknd. Nya Ilandl. A. 1781, P. 163. - Der Flohkrebs, heschriehen. Neue Scliwed. Ak. Abhindl. 1781, p. 165-71).

" Desterlen, Friedr.

* Dken, Lorenz.

* Difers, Ign. Fr. v.

Olivi, Giuseppe. Zoologia adriatica. Bassano 1792. 4. Con tav. 
DIfrier, Guill. Antoiue. Entomolog, Mitglied des franz. lustituts; $\mu \mathrm{eb}$. 7.u Frejus 1756. Wurde in 17. Jahl, Doctor der Medizin. Voyage. T. 30, f. 2 (Thelphusa fluviatilis).

Oppisans. Halieuticon Biblia etc. Latine J. C. S : la neidero edirl. Alquentorati. 1776. 8. - Edit. Edf. Rittershusii. Leyd. 1597. 4.

" I) roigny, Alcide Dessalines d".

Dsbeck, Peter. Voyages aux Indes orientales et à la Chine en Suedduis. Stoekholm 1757. 8. Deutsche Uebersetzung von Georgi. Rostoek 1665. 8. Auch franziisisch uibers. (82, 103 Lepis an.)

\section{(1)to.}

Dvidins Naso. Halienticon poematicum jer Gryphium. Lugduni 1535. "Diven.

Palisot de Benuvois. Insectes d'Afrique.

Pallas, Petrus Simon. Miseellanea zoologiea quibus novae imprimis atque obscurac animalium species describuntur, et observationibus iconibusque illustrantur. Hagae comitum. 1766. 3. - Spieilegia zoologicu quibus uovae imprimis et obscurae auimalium speeies, iconibus descriptionibus atrue commentariis illustrantur. Berolini 1767-80. 4. (Fase. IX). - Tableau physique et topographique de la Tauride (Nova Acta Ac. Petrop. T. 10, Hist. p. 235, Mem. p. 257). Eine deutsche Úcbersetzung besitzen wir in unserer Bibliothek (Petersburg 1798. 8.). Reise.

I'anzex, Georg Wolfrang. Fuunn insectorum Germaniae initia. Fortgesezt vou Herrich-Selläfer und dic Crustaces benrbeitet durch Koeh.

\section{Paracelsus.}

Prominson. Organic rmmains. Vol. 1IL. London, 181t. 4. With Plat.

Harra, Don Antonio. Amerikanischer Zoolog. Deseription de diffèrents moresax d'histoire natorelle, principalement de produetions marius. La Ifa. vane 1787. 4. (In span. Sprache: Descripcion de différentes piezas de Historia vatural las mas del ramo muritimo representalus en setentu y cinco laminas. Havana 1787. Darin sind abgebildet und beschrieben viele Fische und Crustaceen).
Perry, Will. Edw.

Parsons, James. A letter cull. rerning the shells of crabs (Plitos. Transact. 1752, p. 438).

\section{* ayen.}

Lecelk, William. Method of preserving animals ind their skins collected from various autloors (Collections of the Massaclousetts Histor. soe. Vol, 4, l. $(0)$.

"penda.

Pemuant, 'Thomas. British Zoulogy. IV (Crustacea). - Zoologia britaunica. 4. - Edit. alt. in 8. 4 Vol.

" Ierclieron, Achille Reny.

* perleh, Curl Julius.

Pernetty. Voyage rux lles Malouines. (Iernaea.)

"Derrin. Schöne Meertulpe (Dessen Reise durch llindostan, p. 19).

"N"erty, Maximilian.

Petiver, James. Piemarks ou some animals, plants seut to him from Ma. rvland. Sect. 1. Crustaceous animals. i) T'estudo terr. an.; 2) The Molucea crab. (Pluit. 'l'rans. 1698, p. 393, 398). - De aninalibus crustaceis caudatis etc. (Memoir. for the curious. 1708).

Tetiver, Jacob. A(juatilium nnimalium Amboinue icones et nomind. London 1713. Pol. C. fig. - Edit. secundu. Lond. 1764. - Gazophylacium naturae et artis. Lond. 1702 . Fol. C. fig. (T. VI, f. 10. Balausus.)

Pezoldt, Adamns Priedericus. De Palingrnesin (Ephemer. Ac. Nat. Cur. Cent. 7 et 8, 1. 31 ).

* Iy fer zu Neucek, J. J. X.

Plipps, Constantin Jean, au Lord Mulgrave. Voyage an Pole boréal, fait rn 1773. - Trarluetion française. Paris 1775. 4. (Cimpedia. - Cuncer Boreas, A mpulla, mugax.)

Picaralet. Observntions zoologiques, metéorologigurs ete pour le 1. et 2. semestre de l'année 1785 (Nouv. Ménoir. de Dijon A. 1785. Sumestr. 1, p. 232, Sem. 2. p. 467).

t" ickering, Ch.

Pinkerton. Petrology; a Treatise on Roks, 2 Val. 8.

I’ison, Guilejun..s. Delndiae utriusque re naturali et medien libri XIV. Amstelodani 1658. Fol. C. fig..

PMlaclnus, Janus, oder Giovanni Blanchi. Des conchis minus notis. Edit. 1. Venetiis 1739. 4. C. tab. - 
Edit. 2. Liher duplici appendice auetus. Romac 1760.

PInutade, de. Mèmoire sur I'listoire naturelle de la province de Languedoe (Memoir. de Montpellies T. 1, Mem. p. 266 , T. 2 ; Hist. p. 35).

Plinius Secundus (Cajus).

Poda, Nicolaus. Inseeta musci graccensis, 1761. 8. (Cyclops).

Poutoppidaw. Norwegen.

Portins, lucas Antonius (Porzio). De camcri fluviaibilis partibus genitalibus. (Misc, Acad. Nat. Cur. Dec. 5. A. 6, 1687 , p. 48 . - Ban Ier Gesclileehtstheile des Flusskrebses. - Ephem. Acad. D. II, A. 6 , p. 49 ; daraus in Valentioi Amphitheat. Zoot. P. II, p. 138, T. 77).

Fré, Jean Frederie de. De Millepedis, formicis. - Resp. el Lumbrieis terrestribus qualem usum hace inseeta Labsant in mediena. Disis. Erfordiaf 1722. 4.

" Presi, Carl Borziwog.

" prestundrea, $\mathrm{N}$.

Prévost, Benedict. Mémoire sur le Clirociphale (Journ. de Pliys. Tom. LIV. - Branchipus. Auch in Jurine's Werk über die Monoculiden von Gent).

\section{"Purkinje.}

Quéronic, de. Deseription d'un Inserte singulier trouve dans la cade de Lomariaker (Cancer linearis) (Mèm. de Mathem. et de Phys. IX, p. 329. Sav. Etr. IX, 329-30).

" Quix, Clir.

Q Emoy.

Teneluett, Thomas. Observations on Cancer Salinus (Trans. Linn. Sne. XI, 2. Part. p. 205. 181\%. - Artemia).

\section{* It affelsberacer. \\ 4enfuesque-Gunaltz, C. S. IRajus. \\ Mnumolir, K. A.}

ITnzani, Camillo Abbé. Memoric di storia naturale. Deca mima. Bologua 18:20. 4. (p. 73. Rani fossile). System der Cirripeden (Isis).

* Intulie, II. v.

"Inatzebing.

Razounowsky, George de. Essai sur l'histoire nafurelle du cercle de Bavière (Mèn. de Lausanne, T. JII. Hist. p. 34, Mém. p. 237). - Ueber die Trilobiten und ihre Lagerst. (Neue) (Annal. d. sc. nat. VIII, 186).

Theaunur, Rènè Antoine Ferehault de. Girb 1683, gest. 1757. Sur les: diverses reproductiuns qui se funt dans les éerevisses, les omars, les crabes efc. et entre autres sur celle de leurs jambes et de leurs ceailles (Mèm. de I'Acad. des se. de Paris. A. 1712, Mist. p. 35, Mim. p. 226. Ed, ort. A. 1712 , p. 45, Mim. p. 295). - Additions anx observations sur la mue des érevisses (ibid. A. 1718, Hist. p. 22 , Mem. p. 263. Ed. oet. A. 1718, Hist. p. 27, Mein. p. 333).

Recli. Animali negli Animali vivente $\left(O_{\text {puse. }} 111\right.$, Tab. 16, f. 5 et T. 13, f. 5. - Daphnia).

" Ie eichenbreln, A. B.

* IReicluenbrelr, G. H. L.

Reiuras, Samuel. Ueber die Triebe dev Thiere. I Bd. 3. Ausg. Hamburg 1773. 8 .

Reinceggs, Jakob. Schreiben aus Teflis vom 21. Jan. 1780 über naturlaistorische Gegenstände auf seiner Reise (Schrift. der Berl. Gesellseh, naturf. Fr. III, p. 398).

Itenard, Lonis. Poissons, écrivisses et prabes de diverses couleurs et figures extraordinaires, que l'on trouve autour des iles Moluques et sur les ciotes des teries australes. Amsterd. 1754. Fol.

Reuss I mer, Jevenial, Etienne van, Dr. med. und Professior der Geologic am Allenäum zu New-York und Priasident des Albany-Institurs. † in NewYork. - Notice of fossil Cruxtacea from New-Jersey (Anual. of the Lye. nat. hist. of New-York I, Nr. 7, p. 195. 1825!.

Retzius, Anders. Beskrifning üfver en ny Skandiunvisk Lermaca fran Nordsjön, kallad Lecmaea Dalmanni (Kongl. Vatensk. Acad. Handling. 1829, p. 109-120, Tab. VI), - (Cirr. Lib. Bar. de Geer) Geucra et species inseptor. extr. ab. Andr. Retzio. Lips. 1783.

Reuss, J. D. Repertorium conmentationum a sucictafibus litterariis editarum. Sceundum disciplinarum ordinem digessit. Seientia naturalis. Tom. 1. Göttiogae (Dicterich) 1801. 4 .

Tieharason, Riclard. Several Observations in natural history, made at North-Bierley in Yorkshire (Philns. 
Truns. 1713, p. 167). - Part of a letter, concerning the squilla aquae dulcis (Plil. Trans. 1734, p. 331).

Richter, Nusacum richterianum. 1743. Fol. fig.

"Inemann, R.

- Misso, Domeniro.

s Titgen, F. Aug.

Iitter, Jolınm Jakob. Meletemata ad historiam uaturalem svidniccusem (Nov. Act. Ac. Nat. Cur. Vol. VII. Append. p. 103). - Tentamen historiac naturalis ditionis Ricdeselio-Avimontanae, in quatuor partes, nempe floram, milueraloriam, faunan et commentitionem de acre, aquis et locis etc. divisum (ibid. Vol. 10. Append. p. 21).

Robers, Laurentius. Pracside: Diss de fluviatili Astaco, cjusque $ו s u$ medico. Resp. M. Osander. Upsal. 1715. 4. (32 pp.)

* Roblneav-Desvoidy; J. B.

Rocherort. Histoire naturclle et morale des ilcs Antilles de l'Amérique. Rotterdan 1665. 4.

* Tochei, Ant.

* Rocumer, Fr. Ad.

Roemuer, Jol,. Jak. Genera Inkectorum Limnaei et Fabricii, iconibus illustrata n Jolıamı Jacobo Rocmer. Vitoduri Helvetiorum prostat apod $\mathbf{H}$. Steiner et socio. 1798. 4. - 86 bez. S. und 37 illum. (treffliche) Kupfertafeln.

Ribel vou Rosenhor, August Johann. Maler ru Nürnberg; geb. 1705, gest. 1759. Gcuialer Beobacliter und Darstcller der Natur. - Der Flusskrebs (in dessen Insektenb. III, p. 305, T. 54 ff.) (Classisch noch jezt.)

IRofredi, D. M., Abbé de Casanova. Lettres sur les nouvelles ob. scrvations microscopic|ues de Needlam et ses notes sur les recherelies de SpalIanzani (Misc, soc. Tumbinens. IV, p. 109).

Rondelet, Wilhcln. Libri de piseibus marinis. Lugduni 1554. Fol. C. fig. - Universae aquatiliun historiac pars altera. 1555. - L'histoirc entiere des poissons. Lyon 1558. Fol. C. fig. (p. 210 Astacus fluv. Mit Holzsclınitt.).

Iosenthal, Fr. Chr.

- Ieoss, Capt. Sir Janies Clark.

Rossi, Pietro. Fauna Etrusea sistens Insecti quae in Provinciis Florentina et Pisana pracsertin collegit Petrus Rossius ete. T. I. Cun XI
Tab. pict. - Mantissas priore Parte adjecta, interdum edita et annotatis perpetius aucta a D. Jolı. Christ. Lud. Hellwig. Helmstadii litteris C. G. Fleckeisen. 1795. 8. 457 pp.

" Lossmäissler, E. A.

* Tousael de Vauzéme.

IRoux, Polydore. War Conservator zu Marscille. Crustacès de la Méditerrance et de son littoral. Av. pl. Mar. seille 1827-28. III Fase. - Nener fossilcr Xantho (Amn. d. sc. nat. XVII, $1829,84)$.

Rud olphi, K. Asm.

" Mihppel, Eduard.

Rumpluius, Gcorg Evcrard. D'Amboinische Rarität(ukanmercte. Amsterdar: 1705. Fol. M. Kpf. - Thesaurus imaginum ete Hagae 1739. Fol.

\section{"IEImur.}

" Rumde, Will.

IRush, Benjamin. An account of Dr. Hugl Martins Cancer pownder with brief observations on Cancers (Transact. nf the Americall Socjety. Vnl. 2, p. 212).

"Inthe.

Tuysein, Heinrirh. Theatrum universale omnium animaljum etc. Amstelodami 1718. C. fig. 2 Vol. Fol.

"Gabiue, Joln. Crustacea in Parry's Voynge.

Snchs a Jewentheiub, Philippus Jacobus. Gammarologia, seu Gimmarorum vulgo Calicrorim consideratio pliysico-philologico-historicomedico-chymica. Francof. et Lipsiae 1651. 8. (Tab. VIII etc.) („Enthält alle's Frïlicre und manches Gute." Med. Zoolog. II, 58.)

* Snck.

"Saint-Ange (vgl. Ange).

* Gint-Hilaire (vgl. Ge offroy).

"Gnint-Milnire, Auguste.

* Sars, M.

* Savi, Paolo.

Saviguy, Julcs César. Mèmoires sur les Animaux sans vertibres. Av. pl. Pajis 1816, T. II. - Fresswerkzellge der Crustaceen (Isis 1818, p. 2075). - Fresswerkzcuge Iler Hexapoden (Isis 1818, p. 1405). - Description de I'Egypte, publiee par ordre du gouvernement. Planches des Crustacés.

Say, Thomas. On it new genus of the Crustucea, and the species on which it is etablished (Jouru. of Acad. Philad. 
I, p. 49. 1817). - Ohservations on some of the Animals deseriber in the account of the Crustacea of the united States (ibicl. 1. 1818, p. 493). - Appendix to the Cirustacea of the united States $(1818$, I, p. 4.15). - An acconnt of the Crustacea, of the united States (Ueber die Crustacen der vereinigten Staten) (Jomrn. of the Academy of nut. scienc. of Philad. $1817-18, \mathrm{P}, \mathrm{P}$. $57,65,97,155,235,313,374,423)$. - Polydesmi (ibid. II).

schselit, Mattl. Henr. De tribus Caneri specicbus e mari balthico (Nov. litter. mar. Balth. 1699).

Schnifrer, Jakob Christian. - Apus pisciformis insecti aquatici species noviter detecta. Norimbergae 175\%. 4. (Branchipus). - Der krebsartige Kiefenfuss mit der kurzen und langen Schwanzklappe. Rerensburg 1756. 4. (Limulıs, Apus). - Der fischförmige Kiefenfuss in stehenden Wassern um Regeusburg. Regensb. 1762. 4. Elementa entomologiea. Ratisbonae. C. fig. color. 1766. 4. - Die geschwïinzten und ungeschwänzten zackigen Wasserflälıe. Regrensb. 1755. 4. MI. Abbild. (In seinen Abluandl. vou d. Ins. I, p. 251-298. - Daphuia Pulex et Sima).

Schenchuer, Jak. Joh. Piseium querelac ete. 1708. 4. C. fig.

* Schilling, Samuel.

* Sclinz, H. Rod.

Schlosser, Joseph Albin. Auszug aus einem Briefe wegren ciner neucn Art von Insekten (Hamb. Min. XVII, p. 108-112. - Artemia. - Französ. im Journ. britumique).

Sichlothein, vou. War Prïsident ete. Petrefaktenkunde. Gotha $1820-$ 23. 2 Bde. 8. M. ill. Kpf. - Upber Versteinerungen im Höhlenkalkstein von Glüeksbrum (Mag. Il. Ges. natf. Fr. zu Burlin. VII, 1. $156-59)$. Eehinosphaeriten und Trilobiten (Isis 1826, p. 30?). - Trilobites cornigerus (Leouhardt's Tasehenbuch IV, tab. 1).

* Sclinidt, Jos. Herm.

* Sclmialt, $A$.

Schmidt, H. R.

"Schmidt, C. A.

Schneider. Ueber dic von Aristoteles beschriebenen Gattungen und Arten von Krebsen (Mag. d. natf. Fr. zu Berl. 1, p. 164-185).

"Scholz, P.

Sclirank, Franz vou Paula von. terranca (Trans, of the Linucan society.

Fauna boira. III Vol. Landslut 1803. - Buyriselse Rieise. 1. (Lepraea).

sclirbter. Abliandlung. 1, 352 (Scolopendi:i).

"Schubert, Fr. W.

schitibler, G., Professor in Tiibiagen. - Beobaelıtıngen vilıer jährliclie periodisels wiederkeluende Erstheinumen in Thicr- und Pflanzenreicls. Tibing. 1831. 8. - Beitrüge zur Naturgeschiclite Oberseliwabens. 'Tïbing. 1832. 8.

Sclnulze, Christ. Friedr. Der krebsartige Kicfenfuss in deu Dresdner Gegenden (Neu. Hamb. Magaz. $68 \mathrm{St}$. S. 99-132. - Ap(is).

Schulze, Joanu. IIenr. Pracside: Diss. de Cincrorum fluviatiliun usu medico. Resp. Sam. Deublinger. Llalae 1735 .

"schalze (..u Greifswalde).

"Schulz, J. H.

Schweigarer, A. F. Ermordet in Sieilien (vol. Lex. Il. Ent. 65). Beobachtungen auf naturlistorischen Feisen. Berlin 1819. 8. - Hundbuelı der Naturresehichte der skeletlosen ungegliederten Thiere. Leipzing. 8. (llerabgesezt z.u 1 'Thl.)

Scilla, Aug. De corporibus marinis lapidescentibus. Ronate 1752. 4.

Scopoli, Jounnes Autonius. Carcinolor.

"Scoresloy, W.

* Scouler, J.

Seba, Albert. Locupletissimi Rerum uaturalium Thesiuri aecurata deseriptio ete. Amstelodami 1734-65. Fol. 4 Vol. (Die Crustacecu ia III. Band.)

Sencbier. Mikrostiop. Entdeck. (Tardigrade).

Serres, Marcel de. Mèmoire sur les yeux lisses et les yeux composès des lusectes cte. Moutpellier 1813. 8. (Ueber die Angen der lusekten. A. d. Franz. von Dicffenbach. Berlin 1826). - Observations sur les usiges du vaisseau dorsal daus les mimiux articulés (Mèm. dı MIıs. IV, 149-192 et 313-380. 2 pl. V, 59-147. 2 l.).

setzen, Ulrieh Jasper. Beitrïge zur Naturgescluchte der Herrschaft Jever in Westphalen (Nene Scluiften der Ges. natf. Fr, zu Berlin. Bd, I, 140).

Shaw, George. Remarks on Scolopendra electrica and Scolopendra subGistel's Lexilkoa. 
Vol. 11, 1. 7). - Elements of NaturalHistory. II. - Description of the Callrer stagualis of Jinmurus (Trons. of the Linnenn Society. I, p. 103-110. Flat. (Bramehipus).

sibbald, Robert. Part of a letterentuerning a second volume of his prodromus histonine naturalis Scotine (Pliil. 'Trans. 1706, p. 2314).

Gicher, F. W.

"siebold, Fli. Fr. v.

* siebolu, C. Th. v.

*silliunans.

SIabluer. Pliysikal. Belust. (Taf. IX, f. 1, 2. Seit. 37, 1775, ist Praniza morina). - Mikroskopische BeobachtıI)gell.

Slonue, Hans. A voyage to the Islauds Madera, Batbados, Nieves, St. Christophers and Jamaica. Loudon. 2 Vol. Pol. 170\%.

* Amitla, Andrew.

Somner, Carl Gottlieb. Medieamentum quoddan Swietenianum, lapides nempe Camrorum vino intritos ete. Diss. inaug. Pracs. P. J. II a r t m a u 'I 'rajecti ad Viall'um. 1787. 4.

Sammer, Joluann Georg. De latpidibus Cancrorum veris of factitiis (Eplien. Ac. Nat. Cur. Dee. III, p. 268 -270 ).

Sorg. F. L. A. W.

- Sowverloy, Jumes.

Spada, Giov. Giac. Corporam lapidefarturnm ugri veronensis etc. Veronae 1744. 4. (Besilhrieb die Ranina des Aldrovandus, vou welelier $R$ a \% in $\mathrm{i}$ wieder später eine Beschreibung. kab.)

Spadanzani. Opuse. de Pliys. nnimal. et vegete trad. de Senebier, p. 252 sef. T. 4, f. 7,8 , T. 5 , f. 9 (Lee Tardigrade, Macrobiotus).

Sparrubau, Audicas. An acconnt of a journey into Afica from the Cape of Good-11ope and a deseription of a new species of Curkow (Honey-Guide or Cuculus indieator) (Phil. Trans. 1777, p. 38).

* Speuce, Will.

sperlius. Zool. pliys. posth. I. V. C. XVIII, $\$$, 3, bei Saclis Gaumarol.

Spengler, Loren\%. Besclureibung des besondern Meer-lnsekts, welches bei den Islïndern Oskabiöm oder anelı Denskebiörn, Wunsebbiir, Wunscluk aifer lurisset (Oniscus Psora Limnaci) (Besclıäft. d. Berl. Ges, nalf. Fr. J, 292).
- Einige neue Bemerkungen tiber die nolukkisclie Krabbe tibid. II, 4.16). Beskrivelse af en nya art Kraelss, Seyllindus guincensis (Nye sianl. af det dauske Yetensk. Selsk. Skr. Doel 5, p. 33.3 ).

Spix, Johannes. Gesehichte und Beurtheilung aller Systeme in der Zoologic nich ilurer Entwicklungsfolge von Aristoteles bis anf die gegenwärtige Zeit. Nïruberg 1811. 8.

sprengel, Kurt (vgl. Läwe). Commentarius de partibus quilsus insecta spirifus ducunt. Accedunt tabulae III aeri incisae el piefae. Lipsiae in libraria Weidmanniana. 1815. 4. шнј. p. 38 (Germar Mig. II, 301).

Stackloonse, J. Nereis britannica, Fusciculus III.

Stafford, Richard. Extract of a Ietter, writter from the Bermudas, concerning the tydes there, as also whilles, sperma ceti, strange spiclerswebbs, some rare vegetables and the longevity of the inlabitints (Plilos. Tirans. 1668, p. 792).

"steenstrup, Jap.

"stein, Friedr.

* Stein, C. G. D.

Sternbere, Caspar Graf v. Berichtigung der Doppehnamen in Böeks Notizen z.u deu Trilobiten (Seluwedisch nacl, $Z$ e u k e r's Abl. darüber) (Verlandl. d. vat. Nus. in Böhm. f. 1832-35). Uebersicht der in Bölsmen dermalen bekannten 'Trilobitem. 1825. 8. 20. 2 Stciutaf. (Aus d. bölını. Velhandl. Isis 1827 , p. 623 . - Isis 1830 , p. 516 , Tat. V).

Steward. Elen. Nat. Hist. 2 (Glomeris marg.).

sitewart, Joln. An nccount of the Kinglom of Thibet (Pliil. Trans. 1777, 1). 465).

Stolonens, Kilian. Observatio de lapillis astuei marini vulgaris (Aeta litfraria sneciae. A. 1733, p. 79).

"Stolises, C.

* Struk, Jolın.

"Stralc. Naturgeschiclite in Bildern, nit crläuterndem Text. Düsseldorf. giv. 4.

stvacban. Observations mode in the Islatud of Ceilan; on the ways of enteling-fowl and deer, of serpents, of the inthene aud of cinsmon (Phii. Trans. 1702, p. 1094). - Ohservations made in the lstand of Ceilan: on $\mathrm{Co}$ ral, large oysters, rubies, the growing 
of a sort of fieus indica, the gods of the Ceylanese etc. (ibid. Y. 1703, p. 1248).

* Strauss-Didrkhein, Hereule. Eugène.

Stribur, Hans. Insecta norwegiea (Acta nidrosiens. III). - Drscriptio prefecturae Sondmörie in dinecösi bergensi. - Physik oo oeconomisk lieskrivelse over fogderied Söndmor, beliggede $i$ Bergens stift ndi norge. Seroë 1762. '2 Vol. 4. C. fig. - Om silde-eller Räd-Aat (Nye Saml, af det Norske Selskabs-Skrifter. Bd. I, p. 185). - On silde-eller Rod-Aat (ibid. Nye Saml. Bd. 1, p. 185-192).

Gtubl, Henry. An enlargement of the observations imported by a enrious traveller to Jamaica: wherc do occur diseourse, about the steans of the sea, the colour uf the sea; various plants and animals in Jamoica und other Islaurls of Caribus; the qualities of Tobaeco growing in nitrous ground: hurricans: a way to make a boad ride at anchor in the main sea; the effects of the change of climate in liuman bodies; a probable way of preventing and curing Fickness in travellers tu the West-Indies (Phil. Trans. Y. 1668, p. 699, 717).

Ancliow, F. W. L. Das Naturalienkabinet, oder gruindl. Anweis. wie d. Naturfreund bei naturhistor. Excursionen und bci dem Sammeln, Ausstopfen, Skeletiren ete, verfülnt, wie cr sie versenden und in Sammlungen dauernd sclı̈n eonserviren kanu. Nebst lith. Abbild. cler erforderl. Werkzenge. gr. 12. Stuttgart. - Anatom.-physik. Unters. d. Krustenthieı e. Heidelberg 1818. 4. I Bd. Hft. 1.

"Surriray. Medieiner in Havre de Grace. Observations sur le Foetus d'une espuce de Caligus (Ann. gen. d. se. pliys. III).

* Svainson, Willian.

Swanumerdam, Jolıan. Biblia Naturae. Lngdumi Batar. 1737 und 38. 2 Vol. Fol. (Lntein. und liolländ.) Histoire naturelle du Cancellus ou Bernard l'hermite (Recueil des Voyages de Thevenot. Par. 1681).

sylies.

Tavernicr, Jean Baptiste. Observations concerning some of the most considerable parts of Asia (Phil. Trans. 1676, p. 711, 751).
Termeyer, Raimond Marie de. Memoria per servire alla compiutu storia di Pulce aequajuolo arboreseente (Seelti di Opnse. interess. Vol. XXVIII, P. 79-10\% - Daphnia Pulex).

* Tetzner, W.

* Thienchumn, Friedr. August Ludw.

Thon, Theodor, Dr. Phil. und Professor zu Jena. Bekannter Eutomolog und Selo riftsteller, so wie Kertzeichner.

* Thompson. W. Vaugdthan.

Thoreshy, Ralph, Letter eoncerning several observables in his mustenu near Leeds in Yorkskire (Pliil. Trans. 1702, p. 1070).

Thunbers, Carl Peter. Ett kort ntdloag af ('n juurual, hällen päi en resa til och uti keisa redomet Japan (Phil. Trans. Y. 1780, p. 143). - Translation of a slort extract from a juurual during his voyage to and residence in the empire of Japan (ibid. 1780. Append. p. 1). - Novae insectorum speeies deseriptae (Ouiscus eoralliuus (Nova Aeta Upsal. IV, p. 1).

* Tilesius, IV. G.

* Todld, Robert 13.

Torrubia, Josef. Apparato para la Historin natural espagnola. Maclrid 1754. I, Pl. 13, f. 1 et 2. - Beschreibung zweier Crustacecn (Decapoda brauchyura vou Indien).

'Towns, Thomas. Extract of a letter, containing some observations made at Barbarlos (Pliil. Trans. 1675, 1. 399).

Trembley. Polyp. p. 248, Tab. 6, f. 11. - Daphnia).

Treviraums, G. L. Ueber den inucru Bau der ungeflïgelteu Insekteu (s. s. verm. Schr. I, II. Myriapoden). - Kreislanf der Crustaceen (Tiedem. Zcitselrr. f. Phys. III). - Vermischto Schriften (A'sseln).

Treyling, Joh. Jak. De eancris hormmque partium natura et usu. Diss. inang. Resp. P. H. A. Sehrader. Ingolstadtii. 1721. 4.

tristan, Jules de.

trasclicel. Frauz Hejm.

valentin.

Valentini, Joh. Ernst. Clicla astaci fluviatilis tribus apicihus pracdita (Aeta Acad. Nat. Cur. Vol. II, p. 285). - Auphitheatr. Zoot. (vgl. l' 0 r. (i i s).

Vundelli, Dominicus. Florae et Faunae Lusitauicat speoimen (Mem. 
da Acad. real les sc, de Lisbon. T. I, p. 64). - Diss. de nonnullis insectis. Palavii 1758, p. 77 (Balanus). - Apologia. Patav. 1760 (p. 25).

- Van der Hoeven (vgl. Hocve 11 ).

*anzime (verl. Rousscl de V.).

Vilters, de. Eutomol. linn. IV.Les Insectes de Fruncc, dirrites et classis splou la mithode de Mr. Lin. ue us, en quatre Volumes in 4. 1783. 232 Planch. Livi: 12-15, plancls. av. descr. (120 Livi.) (2500 Ins. a. Fikl.).

Vivihni. Plosplorescentia maris quatuordeciu luresecutium animalculorum novis speciebus illustrati. Genuae 1805. C. fig. 4.

Voigt, F. S. Syst. d. Natur. (537. Astacus).

* Voltz. in Paris. Carcinolog.

Vormins, Olaus. Museum Vormia. num. Lugd. 13at. 1656. Fol. (Xiphcosura.)

Vosmater, Amoul. Memoire sur un nouveau genre de Crabes de mer (Notogastropus) qui a des pattes sur le ilos et sous le veutıe (Mèm, de Mathen. et de Phys. T. 4, p. 635) (Uit. Rezogtc Verlauidel, 10 Dicl. 119-35).

Wngner, Petrus Christianus, Historia naturalis Helvetiac. 1715 (p. 331 und p. 222 vou rothen Krebsen um Olten" (Solothurı). (Haben wir nicht lort gefisuden, (i.)

* Irasuer, Moriz.

* Waginer. Rudolph.

Evilumber.tr. Petrificata tellu$\checkmark$ is suecanac eximinata (Nova Acta socirt. Icg. se. Upsal. Vlll Tom. Trilobitcn. - Ucbers, vgl. Jourmal de Plivsique).

ivalch (vel. Kn or r).

Walter, J. Neucr 'Trilobit (in Warder's Aufsaty).

- Waitl, Joseph.

Varder, J. A. Nene Tribubiten (einer mit Antennen) (Silliman Amer. Jouru. XXX|V, Nr. 70, 377).

Wargentin, Pehr. Onı Zoologien i gemen, och our fyrfntade diuren i syunerkel (Vetenst. Ac. Handl. A. 1751, S. 81. - Von der Zoologie iberhanpt und vou vierfissigen Thieren insbesondere. (Sclowed. Abl. 1751, p. 85).

weber, Fr.

vedel, Georg Wolfoang. Di' cancro in cancro masculo (Misc. Acad. Nat. Cuy. Dec. 1. A. 6 ct 7, 1675 et 1676, p. 160).
Weigel, Ehrhard. Supellex arte fuctorun quotidianus usibus adaptatorum (Misc. Ac. Nat. Cur. Dec, 1, A. 3, 1672. Append.).

Weiss, Emanuel. Observations di. verses de l'histoire naturelle (Acta Hclvirita Vol. 5, p. 340).

Welutey.

"Westwood, J. 0 .

vlnintrop, Joln. An extract of " Ietter concciling sone nafural curiosities (of Counecticut in New-Lugland) nf those parts especially a very slrange and very curiously contrived fish (Phil. Trans. 1670, p. 1151).

White, Allaw.

Wievlemanu, C. R. W.

Wiegmann, A. F. Fortsetz. d. Deobic(l)tungen viber die Entsteluung vou Eintomostracinen aus der Priestleysuluen grimen slaterie (Nov. Act. Al. Nat. Cur. XI, 11, 541). - Entomostituen zu cr\%eugen (Isis 1822, 1. 432). * Wilbraud, J. B.

Dilcke, Jol. Carl. Tiön i naturallisturien: 1) Beskituing pii et litet valtularik, fumuet pii unga grodor (p. 285) : 2) Silt nt finga livarjehamdia smii Valten-Inselter (1.289); 3) Annuiilkning om Snäctors-parnings sütt (290) (Vetensk. Ac. Handl. A. 1761, 1. 285-290. - Untersulhungen aus cler Naturgesclichlite: 1) Besclircibung rincs hlvineu Wasserinsekts, das auf jungen lö̈s ben gefunden worden (p. 287); 2) Wie man allerhand hleiuc

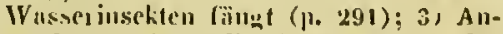
merlung uber die Parmessitt der Sclunekeu (p. 291). - Sehwed. Aliad. Abliandl. 176 I, p. 2S7-291). - Naclıvich vou seltenes Versteinerungen. Dinlin 1769. 8 .

Willis, Thomas. Deseriplion de l'irerevisse (Collect. Acad. IV. p. 593). - Anim. brut. (Cip. 3. Gefïsssystem les Plusskrebses) (daraus in Bl as ius Anat.).

" Worbe.

Wormins, Olaus. (VgI. Vor. mius.)

Vvright, Edward. Microscopieal obscivations (Phil. Trans. 1756, P. 653 ).

Enuren, Fr. Xavier de, Descriptiones \%oologicae ad adriatici littora malis eoneimuatac (16 Spec. Nantili, 1 Argonauta, 1 Hirudo, 3 Ascidiae, 4 Actiniae, I Lnplysia, 1 Holothuria, 3 Nercides, 1 Aphrodita, 1 Melusa, 4 Sepiac, 5 Asteriates, 5 Echini, 1 Julus, 
3 Onisei, 1 Monoculus, 21 Caneres, 1 Seorpio). - Winterbelustigungen (Klagenfurt) (Selir. d. Berl. Gesellsehaft waturf. Fr. Bd. 8, p. 83).

Turficein, Joh. Paul. De oenlis ancrorum monstrosis (Mise. Ac. Nat. Cur. Nee. 2, A. 1, 1682, p. 379).

* Zailiacli, E. G.

Handers, J. W.

Venker, J. C. De Gammari pulieis historia naturali atque sunguinis cireuitu commentutio auetore Zenker. Jena (Mauke). C. 'Tab. 1832, 4. VIII und $28 \mathrm{pp}$. (10 ger.) (Recensirt in der Leipzig. Liter.-Zeit. 1833, Nr. 81. - p. 76).
Isis $1832, p .1114$ ). - Das thieriselse Leben und seine Formen; ein zoolog. Handbueh zum Gebrauehe akadem. Vorträge und $7.1 \mathrm{~m}$ Selbststudiuu. „rr. 8. Jeua. - Beiträge эur Naturgeschichte der Urwelt. Organische Reste (Pctrefakten) aus der Alteuburger Braunkolilen.Formation, dem Blan. kenburger Quadersandstein, jenaischen bunten Sandstein und bölımisehen Uebergangsgrebirge. Mit 6 illum. Kupfertat: Roy.-4. Ebend.

Zonyer, Basilius. Refluxions sur Ic territoire tanuique et ses envirous (Nov. Aeta Acad. Petrop. III. Ilist. 


\title{
Oeffentliche entomologische und carcinologische Sammlungen.
}

\author{
Zugleich Supplement zu der Uebersicht im Lexikon der Entomologen *
}

Wasel bewahrt die Käfersamınlung des berilimten Clairville.

Berliu. Dic eutomologiseluen Jahrbïelıer Klugis werden allein im Stande seyn, dif Sehitzo des Miseams zu skizziren, rvovon der fleissige and gelelute Hr. Prof. Eriehsou schouso Vicles mitgetheilt lıat.

Werm. Stadtmuseun. Kerfe wenig; einige Kristen voll (die ehemalige Studer'srlue Collection).

EBomn. Dic entomologische Sammlum des Universititsmascams ro Poppelsclopf besteht ans dreien Collectionen: a) welelie nur 1 oder 2 Gattungen der vorzïgliehsten Gencía sutlült, eingeschlossen in Glaskïstchen zum Collegialgebrauch; b) eime mit einlseimischen uud exoten, (irca 8500 Individuen, in rrossen Sehüinken unter Glas: c) ciuc Siammlang von enropitischen, 25,1100 Individucu, gesammelt clurelı Nees v. F. in 20 Jahren. Alle lehmeumoniden des Nees'schen Werks.

Borderan (Giroude). Das uaturhistoriselıe Cabinet ist arm au KeIfen (iin Ganzen rtwa nur 200 inlaindische). Die Lepidopteren des Departements sind dagegen fast alle vorlanden und das grouint.

Bribsel. Ungeheure. Sammlungen.

Crerlsralue. Das Naturalienkahinet enthält d.Gesichenkesom mersela ue h's zo Oachiru in Mrxiko. Eine selyr interessilute Kerfsammlaug. Damu pine Summlong Küfer ind Falter, welche Arker. un a $n$ in Brasilieu gesammelt, cndlich eime Sammlung vou fossilen Kerfen a os der Molasse von Oeningen, eimzig in ilirej Art,

Cligloma-smr-Virne. Die entomologische Section des Nut.-Nhuseums verdankt die Stadt dem Eifer Hrn. Dr. Malica's. Diese enthält gegen 2000 Species Käfer, Falter, Hymenopteren, alle bestimut und meist im westlicluen Frankreiel, bei Bordeanx gesamamelt.

Darumstadt. Stadtmusenm. Mit Exolen bereichert durch die grosse Dotation des Hrn. v. Höpfner.

Drestena. Direktor Kaden's Sammlone von Selmuetterlingeu. Die Aufstellung derselben ist herrlich. Alle seine Sehmetterlinge befinden sich iu Glaskissten, welele unten und oben Glastufen laben, und sind auf dömen, a of der untern Seite angeleimten Korkstöpseln aufresteekt. Man braucht desswegen nur deuKasten mmandrelıen, เm dam die. Selmmetterlinge vollstindig vou minten za seben, worauf, wie jeder Kenner weiss, of selur viel ankommt. Dif obere Glastafel ist in einen gut cingefalzten Ralumen grespannt und bildet mit diesem einen leiclıt z.॥ öfinenden, aber demorli selor gat sehliessenden Deekel, dass kein sehïdliches Insekt hinein kriechen kann. Alle diese Kiisten steluen wie Schichkidsten in ciner Kommode in cincm Siluranke und werden dam dorle dic Thüren dessolhen verselulossen und vollkommen geschïzt (B ro h II). Auch cine reiche Käfersanmlung befindet siel in scinem Besitze:

- Ueber Sammier und Sammlunge॥ vgl. M a cquar t Dlptires cxotiques, 
Drelssigheluer.

Forstiısektensamulung.

Griamgen. Ia ukadeuiselien MTısenu sind die Originile zu Esper's Sclumetterlingen das Bedeotendste.

Giclestudt. Grosse brasilianisehe Insektousammlung Sr. k. k. Huheit dem Grossfirsten Maximiliun von Lenchtenberg geliörend.

Florenz. Die entomolngisehe Sammlung des Grossherzogs ist unter Passerini's Leitung im Entstelıen. Bis dato meist Exoten, durel Farbenselimuck ausgezeichnet.

Fronkfurt s. M. Die Sammlung der v. Senkenbergisclien Stiftung. Eine dicr griössteu und wisscuschaftlich ceorduetsten in Deatseliland. Von Rïpell dureli ungeheure Scliätze bercichert. - Vorzïglieh sehenswerth ist dic lusekteusammlumg des Hrn. Senator vou Heydeu, welcher durch spine zoolugischen Arbeiten den Freondea der Naturgesulichte schon hinläıglich belianut ist. $\mathrm{Er}$ hat nicht mur die ganze weite, an Insekten so reiche Ungegend vau Frankfurt, soudern auch den Rlein auf dics Surgfiltigste ontersoeht und eine grosse Mengr: Seltumeiten, unter diesen viele neve Arten aufrefunden. Er bat cine zweckmåssige llud wollfeile Art, spime Korhthiere anfzubewnluen. Diese siud uïulich in Selıu(-hteln aufgesteckt, deren Deckel so gut schliesseu, diss kein zerstötendrs lnsekt hinzukommen kann. In jedro. Sohnchtel ist pine ginuze Sijple orler cin "Theil dersclicn enthalten und auf den Rande der Selanelitel befindot sicl, eine iber deu luhalt Anskunft gehumble Aufschrift. Diese Schachtelu sind wie die Bürher in einem Bücloryhrette so aufgestellt, dass man die Mufsoluilten alle lesen nud sich also in ihmen selu' leicht zurecht finden kamo (wie soldhes auch leei den Silumlungen vou Hervicla, der dureli uns geordneten wud bestimmten elonumligen Jenison'schen, mu Hericliselien; der Clicvi i e r'scheu und Sordet'schen zu Genf, der nusvigen u. s. w. der Fall ist. Gistel.) Alles diess ist sehl schön und zweekmaissig; alleiu v. Ileydeu's Hanptstiirke (!) bestolot in Brlanullung der Kleinflïgler (Mieroplteren). Diese labe ich nirgends in dieser Vollständigkeit und I'raclit aut- gestellt gesehen. Man begreift kaum, wie es nöglich ist, die kleinsten Schmetterlinge, 2. B. Motten, welche nur $2^{\prime \prime \prime}$ bis $3^{\prime \prime \prime}$ hreit sind, - deren Flïgelstaob niclit uur bei der leisesten Berĭhrung aboewiseht wird, soudern deren Flïgel sogar bei nielit ganz zarter Belıandlumg Löcher und Risse bekommen, so z॥ spammeu mud so sehön zu erhalten, dass sie wie im Leben anssehen. Er hat zu diesem Behote cinen hesonderen Triehter vou Blecli erfunden, dumb welchen er die gefangenen Kleinfliggler so durchfilleu lässt, dass sie unten in eine Schachtel mit Kork zu licgen kommen und oben unt eimeu zarten Netze von Filet oder Spitzengrund ïberzogen werden, damit man sie in dieser, olne sie mit den Fingern z.ı berïhren, aufstecken könue. Allein mit gewöhnlielıen Insektennadeln wire: diess doel uicht zu crroichen. É nimmt desswroren zu diesen Kleiuflinglern die zartesten Nadeln vou Silherdialıt, und steckt sie in Schaehteln ein; deren Boden unten mit Hollundermark, deu Cylindern vou dem Marke der cinjibrigen Triebe vam Flicder (Sambut:ls) belegt ist. Diese ganze Behandhumsart der kleinen lusekten ist so vortrelflieh. dass sie nicht unr meine, sondern aller in Mainz Ind Strasburn anwexenden Zoologen Bewunderung in höclistem Grude anf sich mog. (Brelı.) Afrikil breitet ilıce entonologischen Selpitze ans und alle sind vou Ruppell resammelt; Brasilicn (durrl Freyreiss selig), Europu (Schmetterlinge von Rath Cordie ${ }^{\circ}$ ). Reich an Crustiecen ans dem rotl. Meer. Freilumror (Baden). Sammlung der Universitit. Nielıt sehr rejchhaltig an Kerfen.

Finenf. Stadtmuseum. Selu reiche und brillaute Sammlnng unter Pi ete t's Cunservaturims. tsas vermelut dureli Ankanf vou Prol:. Luis S a ledet's Samulumy. - Alles dorch forie Beitrigge seinv Bïrger 7.ısmumen getragen!! Horliclies Genf.

Giessen. Uuiversititssammlung.

Knisersinuterin. Snumlung des rhoinisilien $A$ pothekervereins.

Ixie: Die Sammlung des beríhmten Fulrieius in stiukeuden liapkästelu (vgl. Sillormanu Eumure. si2).

Kopenlnagen. Die lusehten- 
אammlung des Stadtmusemins bestelit aus deu Sammlungen vou $L_{4}$ " n $d$, Selostedt, Seltousloce. Hesse, Krieger und Mayer. Man kamb sich daher tenken, ditss sie zahlierich an Gattungen seyn muss. Faliririus hat Alles bestimmt. - Die Summlung der Linn's'shen Sncietät ist kleiner um ein Bedentendes und uach $F$ abricius arıangirt.

Edille. Das Stadtmuseum ist reieh au javuniscluen Kerfen.

Eidven. Universitätssammlung. Nicht seltr wesentlich; min alurel B a um ha ue r's Simmtung reiels.

Mondon. Callegium of Cliturgs. Praelitvolle Sammlune. - Das k. Mluscum lat sible wenig Kerfe. - Missum der Limnean Society. Dic Sammlung von Limne und B a uks. Die Simmlnng der indischen Gesellswhaft. Weniger Kafer (von Java), desto xalt reielser an sehönen Faltern atus Java.

Irittich. Universifitssammlung: Vicles: doch schr einsertig.

Lund. Universititsmusemus. Mreisteus Exotica, wovon Fabricius hesehricben.

Lyon. Stadtmuseum. Vieles rülutt vou deul sel. de Villers her. Aus Frunkreich (Provenee) nturl ltalien, so wije der Schwri\%.

Madria. Stadtmusenn. Ziemlich viel aus allen Ordumugen, doch alles fust mubestimunt. - Das k. Muscum enthält iuläudische und exotiscle Kerfe, rehtit schöı. welche es dem Fleisse Hri. Prof: Villanova verdankt.

Masilawal. Mnseum der Niturgeschichte. lusekten der Lomlarelei (nerist von Silla, Marietti, Porvo, Jan, Cristofori).

Thurseille. Naturlistorisclies Mnsemim. Alles atrs der Danthine. Der hiesige Conservateur du Cabinet dhistuire naturelle, Hr. P. Roux, lont 1831 sclual riur; in 230 vergoldeten, 18 Zoll hreiten und 13 Zoll holen Glassidieiunken, aufizestellt, seler reicls ausgestalfete Simumluug zum Verkatif, die alle Inscken im Limméschen Simn (d. I. Crostarem, Aracluniden und eigentlielse Insekten) in sich hegreift, und ans etwa 15,000 Arten und 32.000 Exemplaren, und zwar zum dritten Thejl exotisilien bestelut.
Moslageu. Das liais. Museum der Universitiit euthält dic Sammlung Steven's, vou illm geselueulat. Sie rillult groen 5000 Gattungen, aber zirnoliclı schler lit crlalten. Einverleibt ist Demi il of t's Sunumluug.

Minclnen. Sammlung drs Stantes. Unbedentend: Käfer meist brasilisel und die iblurgen Ordunngen veruachliissigt; indessen ist eine praehtvolle Faltersammlung (Geschenk des sel. v. MnIzer) vorloanden und einiges Hiibsche ans den Tropen. Sammlung der polyteclunischen Schule. Kleiu.

Nomtes (Loire-Infeiricure). Reich au mineralogiselsen Objecten ist, blutarm an Kerfen, das Starltmiscum.

Nenentouras. Unbedentend; jedoeh manches Schöne darunter (Onyptorigia ete.).

Dew-Iorlk. Columbian-College. Grosse Sammlung fast aller amerika. nisehen Kerfe.

Earis. Ieh glaube keinen bessern Begriff voll denl gegenwïrtigen $\mathrm{Zu}$ stamde dieses Museums mehen \%u köıueu als dureh die Witthrilung des vollstimdigen Inhailtes fles Briefes, den Hr. A ud nuiu, Professor der Entomologie an lotanischen Garten, den jeh "mi unstiondlielıeren Aufselılıss n̈ber diese Anstalt ersucht hotte, an miels geschrieben latte $*$ Der Brief ist folgender :

\section{Ijeber Antsbruder!}

Wie ich schon die lihre gehabt Ilınen 2.u seluriben, branchte ieh wohl einige Wochen, un groürig anf die Fratge, die Sic an uns stellew, zu antworten; denu um cinen geüigeuden Aufschlıss zu gelsen, mösste irh notluwendier Zeit dazn Io:ben, die Verhalprocesse der wöclentlichen Sitzungen, wrlehe die Verwaltung des Museums ltailt, cinzuscheu, un cinen Ausang der verserliedenen Geschinke, dis man ethalten, und der g.slitreichen Erwerhungen, die sich dout aufire\%eichuet finden, herzustellen; da Sie aber vou meiner Freundschaft fordern, diss ich somlejrh \%ur Feder gueife, so verziclite ich darauf, nur bedauernd. dass Sie mir niclit die Frist zugestauden luabew, un die ich

- Hr. G. Siluerman. 
Sic ersuclite. Unsre Sammlungen sind, wie Sie wissell, zahlreich; sie enthalten vicle Anucliclen, Crustaceen, Araehnideu und lusekten aller Ordumgen.

Leider sind alle diese Geyenstïnde noch bei weitem nicht klassibcirt, mel wilurevd einem laıgen Zeitraume haben sich diese Seltät\%e bei uns aufgehaïuft, ohve dass mall siclı mit deren furtgesezter Anordnung hätte beschäftigen könneu. Verschiedene Unstiinde tragen die Sehuld dieses Zustandes; der hauptsichlichste ist aher gewiss der geringe Platz, deo ban der Eutomologie beim Unterricht in den hölemen Wissenschaftel anweisen zu müssen geglaubt hat. In der That wude erst im Jihr 1830, kurz bach dem Tode des Hiv. La a a r e k, cin Lehrstuhl der Eutomolngie errichtet und Hr. Latreille diraut berufen.

Erst III) diese Zeit auch komnte ich mich mit der bis dalıin gunz veruach. lässigten Classifieirnug der Saınm]nngen besehtifligen. Einen Rückstand von melir als 30 Jahren vor mir, musste irh vor Allem anf Ausscheidung und Trenuurg der Classen, Ordnungen und vorzingliehsten Familien denken. Die Crusticeen, Arachuiden und Insekten sind vertheilt, und die grussen Genera gruppirt worden.

Allein erst im Jabre 1833, wo ich auf den durch den Tod unseres grassen Lelirers erledigten Lehistuhl der Ëntnmolugie berulen wusde, hahe ich dem von wir eulworfenen Plan, wit dessen Ausfülırumer ich indessen bereits begonnen hatte, einen freien Aufschwung zu geben vermocht.

Dieser Plan hestelat in der Vertbeilung der genau beycicboeten (abimaux articules) Thiere in drei Sammlungev. 1) Eine generiselse Sammlung, die un die Serie der vorzinglichsten Genera und der vormiingliehsten Sprecies mit pünktlicber Benewnumg entlailt: diese Sammlung ist in Glas und Filhwen gefasst tïmlich den Aunen der Studierenden anseresezt. Dem Genus voran wird eine kleine Tufel vorangesezt, die Alıbildungen der entomologischen Hauptcha ralitere darstellemd, dimit sich auf diese $\mathrm{V}$ (rise der Schüler bei Untersuehuug des Insekts Fecbenschaft gieben kimne über die Form nud die bemerkenswerthesten Eigenbeiten ler Organe, worauf sich der Bau des Genus gruindet, Beinahe die Gesammtlıcit der Crustaceeu und Arachniden ist nach diesem System klassificirt; die Insckten bilden gecignet das Gefolge. 2) Eine sperifische Sammlung in Commolen untergebracht, unfasst beziimlich der Insekten alle Genera, alle Species, alle Varietiiten nuch Bildung, Farbe und bekannten Oentlichkeiten. Dieses ist die grosse Sammlung, welche sowohl die exotischen als cinbeimischen Species entlält, methodisch nach dem Werke georluet, das Hr. Brullé und ich lierausmeben. Diese Sitmmlung besteht bereits aus 66 Scbriolien, wo. von jeder 8 Selubbladen enthailt. Lis werden jot 24 neue Sehriilke verfertigt, und jedes Jahr wird mau, hofle ich, auls weoigste eben so vicl dazu bringen. Dies wird gewiss bielut 7.1 viel seyn; dean wir laben berechnet, dlass alle die loseliten, die cinstucilen in Cartonbielisen versorght sind, Inod die wir in jeuc Scluränkc zu vertiecilen haben, aufs mindeste ihrer fïufrigg erfurdern wiirden. Irh bringe hier keiurswegs die neuen Sehïlze in berechnung: welche uns boch rukommen werden, sey es dureh Zusendunmen inserer Riejsendeu, scy es durch Cipsreheuke vou Seite edclmühiger liveunde der Wissenschalt, sey es nueb darcht liaufliche Erwerisung:- -3 ) Endlich cine Sammlumg der Insekten aus Frankreich, deren Auorribun bezïglieh des coleopteren begonmen ist und die, in Cartonbiehsen mit Bücherribrlica versurgt, in unserm Laboratorium zanr Verfï:-"ung der Personen blciben wird, welehe etwa die in der Ungenend ron. Patis oder in irnend einem anderu Orte des Künigreiclıs gesammelten Speries bestimmen wollen.

Sritdem nun dic Entomolonisten Zengen der in die Sammlnngen cingefihhten Ordnung ich unter moviler Leitung habe, scitdem sie zur Gewissbeit gelang sind, dass alle Mitiel z." deren Liha!tum annewendet seyen und besouders dass mau sich thitie mit dee Classificinum beschaftine, haben dieselisen sich auch becifert, dem Rufe zu enteprechen, den ich an sie habe ergeben lassen, in die Nationalsammlung dic Speries viederzulegen, ïber welche sic verfïgen küuntell. Mehrere, ich muss es singen, waten so grossmiithig, eiuzige und nete Insekten abzutreten, damit der Typus der Genera und Speeies, dic sic ge. 
bildet hatten, nach Bedürfniss von allen siel darum interessirenden Personen zu Rathe gezogeu werden könnte. Ielı habe melur als einen Beweis dieser edeImüthigen Bestrebung auf der Reise erlualten, die ielı im Jahr 1833 im Siiden Frankreichs gemacht, und mit Vergniigen bezeichne jeh Ilunen als des Dankes der Verwalter des naturhistorischeu Museums zu Paris wüıdir: die HH. Laporte, Roger, Perrond, d'A rgelas zu Bordeaux; Leo Dufour $\%$ St. Sever; Lubat zu Mont-Marsan; Dalracq in Bayomne; Baisgiraud in Toulouse; Farines, Alleron, Compagnio, Levesque in Perpignan; D'aube, Chabricr, Salzmann in Montpellier: Solier, Barthileny in Marseille; Boyer, Apotheker, und B oyer de Fonscolombe ग.1 Aix; B anon, Ey uiond'Ese levin, Varvas, Ml ittre in Toulou; Foudras, Fontenay, Perroud in Lyon; Baridon in Beaueaire. Alle diese Herren, von denen mehreve lhnen bekanut sind, haben nir freigebig ihre Sammlungen geöffuet, was mir dic Verwirklichung meines Wunseles grstntete, im Museum zu Paris pine Simmlung französischer Inselten zusammenzubringen. Olune Zweilel int sie noch weit davon, vollstindig zn seyn; aber ieh zweifle keinesweg's an buldiger Erleichung dirses Ziels, weun die Naturforsclier des Nordens ihue Elire darein setzen, mit deuen des Sijdeus zu wettrifern, und wemu sie dem guten Beispiele folgen, das Sie ihnen so eben gereben, indem Sic nus cine schöne Reihe Carahiques zuschiekten, wovon eine grosse Anzahl in jener walurluaft nationalen Simmlung iluen Platz finden wird. Ihre letate Seudung, so wic alle selhst mriner Person ge. maliten Geschenke sind gehörig ins Vermeichuniss eingetrareu; deun ich muss Ilmen sagen, dass weder iel noel Hr. Brullè. mein Naturforschergehiilfe noch irgend eine der dem Lelirstulile der Entomologie beigegebenen l'er'souru cine Sammlung anlegen ; icls habe auch nieht nötlig gehabt von denselben diese $O$ pfer zu forderı; dieselben waren vielmehr die ersten, welche fühlten, dass der Besitz cines Eigeuthums der Art auch bei bekunnter und gewiss unantastbarer Redliehkeit, nicht olme bedenkliche Missstäude bestelıen köune. Auclu bereiehert sich die öffentliehe Sammlung mit den Gegenstinden, die deren Berichte ihr versclaffen mol gewiss, Sie werden es mir zolgestelien, dies ist schou rine selor befriedigende Folge. Fügen Sie noch linzu, dass ill deren Zeit der Anoudnung der öffentlichen Snmmlung rewidnet ist, olme dass sie jemals durch irgend cine andere dringendere Surge divon ubgezogren würdeo. - Ich tomme num auf die Cataloge zu spreclien. Es ist gut, wenn Sie wissen, nach welchem System sic angelegt sind: Bliatte mit grdruck(en Titclu sind zur Aufualume der möglichst vollstündigen Bereichumng der III) zngescudefenGeg(onstînde bestimut. Am Ende cines jeden Jalres werden dieselben gebunden und so gesammelt bilden sie in der 'Thut rin ganz authentisches Inventar. Diese Cataloge sind in 5 Spalten getlıcilt zu rben so viel verschiedenen Gegenstünden bestimut, deren Wirlutigkeit cinzuschen Sie nol einen Blick anf die Abschrift zu welfen brauchen, die ich thnen lies liefese (siehe unten die Tabelle).

Sie selien es, mein lieber Amtsbunder, ielı labe die Sachen mof eineu guten Fuss gestellt, der aber eine unablissige Arbeit urfordert, wenu der Wayen iu seinem Gelcise gehörigr fortlaufen soll. Ich wiirde es daluin nicht tebrnelst laben olıne die Thutitigkeit der Prisouen, die irh un miclı lahe, und dic micls mit ciocer selleuen Ergebeulseit unterstïtzen. Dieser l'erson(') siud äbrigeus mur drei. Deu ersten ['Int\% nimmt mutirlicl Hr. Brullé, mein Naturforschergehïlle, cill.

'Tïglieh wiinssolse ich mir Glinck fiïr die getroftene Wahl, wic sic auch die allgrmeine Meinume un diese Zeit forderte; denn bei meines Limennung stand ich mit diesem jungen Gelehrten nocll in keiner Verbindung. Ileute und seit ciuem Julure dass wir boisinmen sind, labe ielı nicht nur seince treltilicluen persönlichen Eigenselsaften hemuen ge. lernt, sonder'n jech labe auch Gelegenheit gelabt, sein Verdienst mul scine

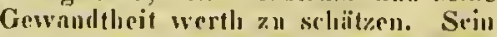
fortgeseztes Sturlinm der Speries, sein bercits gewonnener Takt, sic zu er-

- Bel uus laben dle Administratoren in der Reget mehr als über wus sie ndministrtren. Gistet. 
kemen und zu sondern sind ein slcherer Bürge für die Sicherheit der Bestimmungen, die wir nach und naeh nuter unsere Individuen bringen. Bald hoffe iel,, wird die Snmmlnug des Museums ihrer wahreu Bestimmung entsprerhen, den gegenwärtigen Stand der Wissenschaft vertreten und darum mit grossem Vortheil von den Personen studiert werden können, welehe sich mit deseriptiven Arbeiten besehäftigen. Bereits, und zwar seit vielen Jahren, ist sie von Entomologisten, die versehiedene Sehriften veröffentliehten, z.n Rathe gezogen worden; wiewolıl man dem Einen und Andern von denselben den Vorwurf maehen kann, alssiehtlich keiue Erwähnung der erhalteneu Hülfe gethan zu haben; aber freilich wohl gibt es Lente, denen die Erkenatliclakeit eine Last ist, selhst weun es sich um pine nationale Eiurichtung handelt, deren Nutzin darzuthun, dieselben doch eifersüehtign bemültt seyu sollten.

Die zweite Person, deren Zeit ausschliesslich der entomologischen Sammlung geweiht ist, ist M. Lueas, Solnn cines achtungswerthen, in unseres Anstalt ergrauten Beamten, der nun aber seit 8 Jahren von ihr durch den 'lod gesehieden ist. M. I u cas besehïftint sieh mehr im Einzelnen mit dem Mitcrial der Sammlını, was ilıı jeducls nicht hindert, in seiner Mussezeit an der Classification za arbeiten. Jeh labe seinen Hinden die Anordnung unserer Lepidopteren ïbergeben und here grosses Vertratuen zu seinem Eifer und guten Willen, wie er ihn allezit bewiesen. Ieh muss hinzufïgen, dass, da ich denselben sehı jung unter meiner Leitung hatt", \%ul' Zeit als ich in der Eigensehaft cines Naturforschergehiilfen in Museum debutiote, el darum cine wahl'hafte Anbiinglichkeit z.. mir besizt und siel es persönlieh von Herzen angelegen seyn liisst, mir bei Ausfiilurung des von mir verfolgten Planes behuilflich z.ur Seite z.n stehen. Endliels habe ich voriges Jahe vou der Verwaltung des Muscums cinen jungen Angestellten, Namens $\mathrm{B} I$ a nelinrd, erhalten, welcher golïckliche Anlagen fïr die Entonologie zeigt mod der in cinigen Juluren uns für dic wissensehaftliche Anordnung selar noitzlich werden kann. Es fehlt der Sammlung noeh sehr an Gehülfun, denn, wic ieh Ihnen gesart, ich fand hier einen Rückstand von 30 Jahren, der zı Tage gefördert werden sollte und der in einer grossen Anzahl melı oder weniger nlter Sendungen bestand, dic man vorerst auseinander legen musste und fïr die noeh kein Catnlog sich vorfand. Die vorzinglielısten sind folgende:

1) Die von Delalande, bestehend aus Gegenständen aus 13rasilien und vom Cap der guten Hoffnumg;

2) von Hrn. Angus te S i in t-HiI a ile Insekten aus den Provinzen der Minen, von Campos-Geraes und MonteVideo;

3) vou Hrn. Péron und Andern herkommend von einer Reise um dic Erde und vorziiglieh ans Neuholland;

1) die Ernten der HH. M ilb e rt und Lesucur im uördlichen Amerika;

5) einige aus St. Domingo von $\mathbf{B}$ I agird mitgebrachte Gegenstände;

6) cine grössere Anzahl nus den Antilleu mol Teneriffa von Ma ug $\dot{e}$;

7) Mehreres von Riclye anf Mindagascar gesimmelt;

8) die sehönen in Indien von den HH. Diard und Duvaueel veranstalteten Sammlungen;

9) Viele in Bengalen von MI a ssé zusanmengebraclite Insckten;

10) die Einten von Leselenault und Doumere in Guyana;

11) das uns vou Hru. B a nou nack seiner Rückkehr vou Cayenue Ueberhraphte:

12) die hedentenden in Persien, Egypten und in gauzen Orient von Olivier gesammelten Insckten:

13) die interessinten Gepenstïnde, die der verstorbene Hr. Des font aimes von seimer Reise in der Berberei mitgebracht:

14) Plidlich dic Sammlung van Bose, die das Minseum nach dem Tode dieses Gelehrten an sich gebraeht, und die darum kosthar ist, weil sie melurere von F a h ricius bestimmte Insekten und cinige selteue entlojit, die sich sonst in keiner andern Snmmlung befinden, wie das Genus Agias unter den Dipteren.

Erst im Jahre 1826, wo ielı znm Naturforseher-Gehülfeu ernmot wurde, sezte ich es dureh, dnss man naeh den lhmen oben entwickelten Systeme Cataloge mulegte nach dem Maase als Sendungen kommen und Sammlungen angekauft werden wïrden. Seither 
äussert sich die grösste Ordnung in der tägliclien Bewegung der an uns gelnngenden Geschenke und der mit Liebliabern stipulisten Tausche. Seit. loer auch fliessen uns die Gegenstiinde bei weitem reichlicher und viel häufiger z.t.

Unter den bemerkenswerthesten Geschenken nenue ich llunen:

1) Im Jihre 1826 cine xahlreiche Sammlung von $\mathrm{Cr}$ istacen vollkom. men erhalten zugeschiclit aus den Antillen und den nördlichen Amerika, von dem verstmbenen Hrı. Plè c, Reisenden des Muscums; melirere lnsektensammlungen.

2) Im J. 1827, verseliedene Inselteu mad Araclinide n ans dem süllichen Fiussland vou Hın. Bertal dy:

3) Ju J. 1828, Sendungen der HII. Lesueur aus Philadelpliai; des Hru. Bellanger aus Pondicluery; des Hrn. Go ud otans Tanger, der JH. A u d o u i u und Milne - Ed wards (auf den Küste u De la Manele zusammengesuclite Gecenslïude).

4) Im J. 1829, die bedentenden vou den JHH. Quo y und G a i mard auf ihrer Expedition mit Hru. DUrville angelegten Sammlungen. Welclie mun jene vervollstänligteu, dic vou diesen Gelehrten grossmüthig dem Museuu waren zun Gesehenk gemacht worden nach deren Reise mit dem Capitiin Freycinet. Im nämlicloen Jahre erhielt man die des Hrn. Reynaud, Chirurg an Borl des Staatsschiffes "La Chevrette", welche in der cinzigen Classe der $\mathrm{C}$ rustace e $n$ nicht weniger als 176 Species zülult.

5) $\mathrm{Im}$ J. 1830, Sendunmen von Crustacecu all.s Egyjten von Hru. R ï ppel, und von einer grossen Mruge Species aus Bengalen und dem indisehen Oecan vou H'ı. Dussumier: die schöne Reilie von in Morea gesammelten Inscktel von Hru. B ruille.

6) Im A. 1831, die Sammlum von Insekteu (Colcopteren und anderu) in Bengalen durcli die Sorge des Hru. Bellanger zusammengebraclit; dann die des Hrn. Ey d o u x, der auf seiner interessanten Ricise Giclegenheit hatte, den Senegal, die Jnsel Gniea, die Kin̈ste von Coromandel, Pondiclıery, die Inseln la France und Bourbon zu besuclien.

i) Jm J. 1832, Zeit meiner Ernennung 7.um Professor-Verwalter, verscliedene Geschenke : die vorzüglichsten davon sind: Crustaceen und Insthten aus Chili von H]ru. Ga y; Insekten aus: Philadelphia von dem dortigen Musenı; Jnsekten aus Morea vou Hin. Marlog, Chirurg der küniøl. Mariıte; Insehten aus Indien von J n c que m nut; Inseliten vou der afrikaniscluen Kúste von Ilrn.Ge rar d, Militïrchiru'g; Schwejzerinsekten ven IIru. Pict et; lusekten nus der Insel Bourhon von Hru. B réon; Iusekten aus Brasilien vnu Hro. Sylveira; aus der lusel St. Manrice von Hin. Desjardius. Fermer cine zahl. reiche Meuge Insektengeschenke aus der Ungegend vou Paris, danu ans dem siidlichleu Frankirich und dem nö̀rdliclien Italien, unter velchem sich das des IIru. Lucas, Geliilfen am Lelırstull der Entomolonic auszeiebuet, dessen Eifer übunlich zu gedenken irh selson in Falle gewesen bir. Irh muas noelı bemerken, dass aucl die VerwalIıng auf meine Verweudung hiu mehrere wichtige Erwerbungen gemachit hat. Unter diesen zeichnet sich dic Samılung vnu Crustaceen des verstorbenen Hin. Latreille aus; danu pine sehüne brasiliselıe Sanmlung, die dem Hon. Vauthier, cine andere aus nfrikanisclien lusekten, welehe dem Hru. B a v è gehört hatte: eine vierte encllicla aus mexicauischen Species.

9) Im J. 1834 war die Zahıl der Er. werlungen noch beträchtlicher; tho wiclutigste ist die vou gauz cinzigen und seltemen Speries von firn. Goudot auf Malagasear cingesammelt. I $\mathrm{el}$ ibereche die selir zahlreichen Tnuschcrwerbungen und von den vielen ulus zu. geliommenen Seuduugen menue ighl lhneu die des Hrn. d'Orbigny, die sich nur an Iusckten auf 3021 Species und 5118 Jndividueu belïuft. Die Ordunung der Coleopteren übertlïgelt ibrigens alle andern; denn wir haben uuter denselben 2006 Species und 3480 Individuen neriilltt. HI: Joanis, ein atsgezcichneter $k$. NIarineoffizier hat mia mit meluren in Egypteu eingesamuselten Insekten besehenkt. Hr. J ope, ein gelehrter engliseher Eotomologist hat uns freigubig mit cinigen Species aus ludien, Neubolland und England berciehert. M. Beruier, Chirurg der k. Marine luat uns ans Madagissear ciue selur selı̈ne Seudung zukommen lassen, die besonders aus Culeopteren bestelit; es sind an 1276 Individuen. M. Botta hat bei seiner Rïckkehr aus Nubien 
eine schöme Insektensammlung zu un- Ins serer vollstïndigen Verfuigang gestellt; sie ist zwar nieht zahlreieh aber kostlucr wegen der Selenhlicit der Species, von denen eiuc ansolunliche Menpe ganz nea sind. Die 11H. Marlog, Prevost, Dirclitur des zoologischen Laboratoriums im Muscum; Nivois, Rieord, Jourdan, Professne an der Fraceltait der Wissenselafien za Lyun: Jiavergu, Nittu, Alleron, Leo Dufour. Leelere, Canl Verroud, Solicr. Loyer de Fonsenlombe, Darthelemy. Direhtor des Musrums za Marseille; Brulle, mein Naturforsther-Grhailfe; Compaguio, Emil $B$ la ne li a r cl, inser janger Angestelltcr, laben die Sammleng mit riner bedere tenden Menge Crustacren, Arachnideu, einlıriminclien oder exotiselıen und bis dahin grossentheils uns abgehenden
Insekten bereichert. Endlich sehloss sielı das Jalur mit 2 herrlichen Sendungen, eine aus Chili und von der Küste von Pera, von Hru. Fontaines, gegenwäıtig Oberchirurg im algierisclien Hafen und die andere aus Manilla und Clina vim dem Chirurgen Ilru. Godefroy. Das Jahr 1835 beginn unter den guinstigsien Anzeichen. IIr. Dr. Cus anova, cin Spanier, der in China molicre Insckten crlanet hatte, inberliess dieselben grossmüthig dem Museam von l'aris. Wir erwarten 7.ahlreiche Sendamuen von unsern besoldeten Rrisenden, welche versehieclene ponkte der Erde hesuehen und von druen molurere im J. 1835 oder 1836 zurüekliommen sollen.

Paris, 8. Janaar 1835.

v. Audouin.

\begin{tabular}{|c|c|c|c|c|}
\hline $\begin{array}{c}\text { Numero } \\
\text { muter jedes } \\
\text { lndividuum. }\end{array}$ & $\begin{array}{c}\text { Werkunfo } \\
\text { Schenker, Na- } \\
\text { mun, Nummern } \\
\text { merlereichendes des } \\
\text { sendungs-Cata- } \\
\text { loges. }\end{array}$ & $\begin{array}{l}\text { Bestimimung (BBe- } \\
\text { sclireibuns) der } \\
\text { Species. }\end{array}$ & $\begin{array}{c}\text { Anzeigcn. } \\
\text { Geographisisthe } \\
\text { odler geognosti- } \\
\text { sche Ainschlisse } \\
\text { über Lebensart } \\
\text { etc. }\end{array}$ & $\begin{array}{l}\text { r Individuen, } \\
\text { g, Bewegung. }\end{array}$ \\
\hline 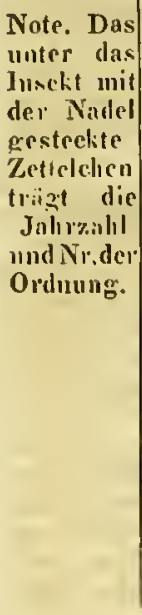 & 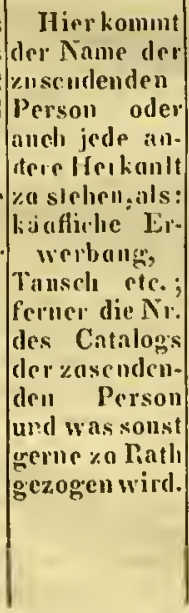 & 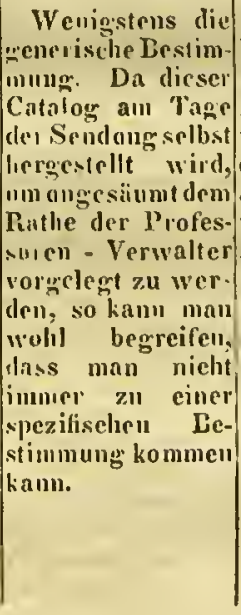 & 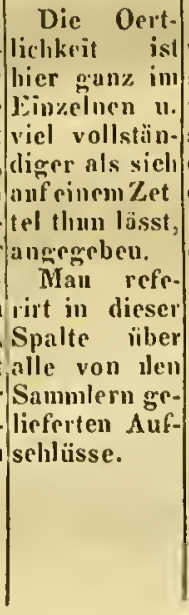 & 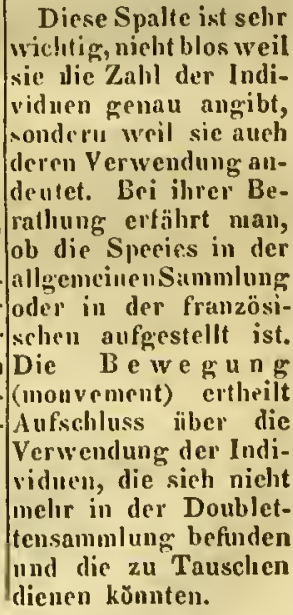 \\
\hline
\end{tabular}

Pestla. Das ungarisele Nationul-| nulosen in Ungarn vorkommt und die musc(un, eingetheilt in dic mineralo- Inscktensammlang, ctwa 4500 Species giselie (schr reiche), bocaniselıe (mit Käfer, hat grossen Werth darch Anviclen Waelsspriïaraten) und zoologi- kauf der Ochsenheimer'schen Sammlung sche Section. Die letzteré hat fast erhalten. Die Uugarn bewaluren sogar alles, was von Wirbelthieren und An- lierin Nationalität!! 
Prag. Böhmisclieg Nationalajuseum. Ausgezeiehnet.

\section{Itudolstadt. Etwas.}

Sulzburas. Benediktinerstift und Kloster St. Peter. Die Fanna Coleop. terorum Tyralcnsis bis auf das, was noels felilt.

Sanct Petersburg. Hu mme l's Cabinet kanfte die Akademic zu Petersburg (Recneil des Actes de l'Acad. de St. Petersb. 1843, p. 196).

Strnsburg. Stadtmuseum (Conservatur: Dr. Schimper). Reieh, selıön und instruetiv. - In sieben grossen Siilen sind dessen Objekte aufgestellt. Die geologischen SammIungen verdienen alle AcImerksumkeit. Le reb oullet, Di: med., ist Conservator davon, - Die zoulogiselse Section ist vorziiglicls reich durch die Falter des sel. Frank, über 6000 Gattungen, sowohl in- als anslïntisclie. Die enropitiselien Falter bilden rirca 2 Drittleil der Collection. Kiifur ete. sind in einer Menge Genera vorhanden als Basis zu einer weitern Ausdehnung. 2000 Käfer, 2600 Falter (vgl. ] sis 18.14, Heft IV, p. 246).

Stockholm. Akadeinisehes MIIseum. Meist dic Fanna Europae borenlis.

Stuttgart. Nutaralienkabinet (Conservator Dr. u. Prof. Krauss). Gerade die Classe der Kerfe ist am schwäehsten. Die griosste Partie bilden sädafrikanische Käfer. - Musenm des Iandwirthschaftlichen Vereins (Conservator mol Sekretär Dr. und Prof. Plieninger).

Thnraud, Sammlung der Forstselunle.

Turiu. Sammlung der Akademie, Unbedeutend.

Wien. Kaiserliches Naturalienkabiuet (Direktor Hr. v. Selireibers, Custoden Hr. Megerle von Mï ulf eld und IIr. Dr. K o I I a r). Eine der grössten Sammlungen der Welt. Die SammIung voll Crustacecn begreift bei 500 Gattungen in vielfiehen Exemplaren, die theils getrocknet, theils in Weingeist aufbewalırt, mit den Conelsylien gemeinschaftlich in einem Zimmer i॥ vier Glasschräıke» zuın
Tlzeil als Sehaustīcke hinter GInsthüren aufgestellt, gröosstentheils uber als sy. stematische Sammlang in den Scluub. laden, gleich den Conchylien, aufbewahrt sind. Bedentende Vermehrungen verdankte es dem gefeierten Direktor Ritter C. v. Schreihers, k. k. Ratl etc. und seinen rïlmolieh bekannten Custoden. - Die Pet refaktensammlang in der k. k. Hofburg (Mineralienkabinet) zeigt im 4. Kasten des 2. Saales versteinerte Crustaceen.

Die Simmlung von Insekten wird wobl kanm von irgend einer Privatsammlnng in einzelnen Partien, Ordnangen oder Classen und schwerlieh von einer öffentlichen im Ganzen übertroffen werden und zwar nicht blos wrgen ibrer Rriehhaltigkcit, indem sie bei 25.000 und mit Einschluss des brasilianisehen Antheils wohl äber 30.000 Gattmugen 火illılt, sondern vorziiglich weil alle Partien stets mit gleiclter Anfmerksamkeit und Thiitiokeit auf die möglichste Vervollstiondigrung, berïrksiclutigt und betrieben wurden, und fïr die vollkommenste Erbaltung und Sieherung der so selur dem Verderben muterliegenden zuhllosen línzelnlieiten mit aller Umsicht gesorgt worden ist. So zählt die Ordnung der käferartigen lnsekten odel. Coleopteren übej 10,000 , dic der Sclimetterlinge oder Ispidopteren über 5000 , die der Hemipteren, Nenropteren, Hymenopteren und Dipteren zusnmmen äber 6000 und die der ungeflägelten (Apterii) mit den Spinnen über 1000 Gattungen (Species), welehe dieHauptsammlang bilden. Daza koumen noch drei merkwürdige und desshalb fär sich abgeschlossene und besonders aufbewabrte Nebensammlungen vou Sclmetterlingen, uämlich:

a) die des verst. Abts Schifferm iiller (mit Abe Denis Verfasser desklassisehen Werkes: Schmetterlinge der Wienergegend, wozu die vorhandenen Exemplare die authentischen Belege sind, da sie mit des Autors lıandschiriftlielien Etiketten versehen sind); dann:

b) die berähmte Sammlung des in Wien verstorbenen Abbate Mazzola, die sich dureh ihren Schatz an Varietäten aus allen Ländern Luropa's, und dic uicht minder bekannte des vor wenigen Jahren in Wien verstorbenen cifrigen Sanmlers. 
c) Podevin, dio sich vorzüglich durch ihreu Reiehthum an kleineu und unscheinbsren Gattungen aus den Familien der Spanıer, Wickler, Zünsler und Motten (Geometra, I'ortix, Pyralis of Tinea) auszeichuct, und welr:he Sammlung bei einer Auzahl von melır als 16,000 Exemplaren weit über 1000 Speries entlualt, die in der Hauptsammlung selbst noch felıleu.

Die Hauptsammlung ist naelı dem neuesten Systeme geordnet und bestimmt und in genau schliessenden, mit Kork ausgetifielten und mit Glastafelu gedeckten Sehubladen in eigenen Küsten anfbewahrt. - Da die Eiurichtung dieser Sammlung schon im Jalıre 1823 geselılossen wurde, so sind alle neuen Aequisitioneu seit jener Zeit aus jeder Partie, so viel als thumlich, in systematiscleer Ordunng, als Supple. mente, auf grleiche Art aufbewahet, um cinst, wenn füglich cinc nene Eimrehtuige und Umst'elsung der ganzen Sannlung vorgrnommen werden kam, was bei dar zahllosen Menge und der Gebrechlichkeit der Objekte eine cben so muilssane und zcitraubende als gefalırvolle Arbeit ist, grehörigen Orts eingeschaltet zu werden.

Ein Theil der ungeflugelleu lnsekten, Parasilen, Epizoen etc. und diespimuenartigen, so wie die Spinuen selbst, die allein ïbet 1000 Füschchen füllen, sind ilırer Beseltaffenlseit nach in Weiugeist autbewahrt. Die ausgcacichnetsteu, hekanntesten und merkwiirdigsten Gattungen aus jeder Classe sind in grosseı Rahmen unter Glas als Schaustïcke aufgr.stellt, da man die ganze Sanımlung nicht wohl zur allgemeineu Ansicht eines grossen Publikums bringen kanu.

Diese eben so kostbare als ansgedelnte Sammlung laat iibrigens, unsser einsu Vorremach und dem Mittrlraume des anstossentleu Amphibienzimmers, nur cin kleines Zimmer zu ilırer Aufstellung, das auch noel dem Verweser als Arbeitslocal diencu muss, wie dies leider, wegen Mangel au Raum, auch bei alleu ïbrigen Sammluugen und Abtheilungeu der Fall und mit mancherlei Naehtheilen vurbunden ist. Custos Di: Vinz. Kollar.

Dis brasilianische Museum (Johaunesgasse Nr. 972) (vgl. Lexikon der 'Theriologen). Die Ordnung der
Crustaceen dieserSammlung zăhlt gegen 60 Species.

Die Sammlung von Insekten, die nach demselben Systeme und auf gleiche Art wie jene an zoologisch-botanischen Conbinet aurbewahrt ist, zühlt iiber 8000 Gattungen in mehr als 20,000, aus einem Vorratlee von melsr als 60,000 ausge wällten Individuen, die siclı durch die vortrefflielıste Erhaltung auszeichmen, so diss die zartesten Schmetterlinge das Ansehen habers, als wären sie vor Kurzen erst uud in der näclisteu Umgebung von Wien gefangen. Ein vorzügliches Interesse gewinnt diese Sammlun noeh insbesonlere dadurch, dass die Sammler niclat, wie gewöhnlich von reisenden Naturforscheru, zmulal in fremdcu Welttheilen zu geschehen pflegt, an der Menge der z.u sammelnden Objekte, der Kiirze der Zeit und der mangelnden Mittel wegeu, wohl meisteus geschehen muss, sieh auf die grösserm, sehönsten und auffallendstcil Gaffurgen, und niclit blos auf die an meisti'u beliebten und cultivirteu Partien der Eutomologie, auf die Käfer und Schmetterlinge besehränkten, sondern sich aluf alle Ordnungen uud Familien vou Insekten ansdehnten, und aus jeder auch die kleinsten und unanselunlichsten Objekte sammelten. Dies macht die grosse Anzahl der vorhandenen Gattungen sowolıl als die grosse Menge der neuen und zur Zeit noeh uubeschrichenen, die sicl wenigstens auf 4000 belänft, reklätliel.

Die Ordnumg der Coleopteren zählte schon (wie die folgrenden) 1833 bei 1000 Gattumen. Arm all Gattungel, verhältnissmässig gegen Europa und die gemissigten Zonen ist die Familie der Calabicinen (uur bei 140), wo.. runter die Gattungen der Sippen Cicindcla und Agra am zahlreichsten sind. - Reichhaltiger ist die Familie der Malacodermeu, und besouders zahl'eieh die Gattuneren der dahin gehörigen Gattungeu Lycus und Lannyyis (130). Ziemlicla zalalreieh die der Serrie orue 11 , Elater, Buprestis (220) und der Lamellicoruen: Searabaeus, Copris, Atenchus (120), MIelolontha und Cetonia (200) und verhailtnissmässig mehr noch dic der Helopiden und Tenebrionen (150). Schr zalılreich ist die Fa. milie dep Cureuliouiden (800) und der Cerambyeinen $(500)$, am reielssten aber die der Chrysomelinen 
(iiber 1000), worunter die diesen IVelttheile vorzugsweise eigenthïnliehen Gattungre Chlanys mit 50, Colaspis mit 70 und Erotylus mit 60 Gattugen, so wie die anderwärts spärlieh erscheinende Gattung Hispa mit 30, Cassida nit 140 und Galleruca und Haltica mit 200 Gattungen auffallen.

Die Ordnung der Gradflïgler, $O \mathrm{r}$ thoptera, zililt über 200 Gattungen und ist wohl am mangellaftesten, da dic Individuen am schwierigsten sirh zul verschaffen sind. Verbältuissmäissiy sehr zahlreirh sind die Gattungrn Phasma und Mantis, jede mit 26 Gattiungell.

Die Ovolnung der Ifalbfliigler, H emipteri, zählt liber 800 Gattungen, wovon die Cimiciden 450 und die Cicadarien, worunter sich die der Gattung Membracis dureh die sunderbarsten Formen auszeichnen, bei 3 ro Gattungen ausmachen.

Die Ordnung der Netzfligler, Neuroptera, ziililt bei 100 Gattumgen, worunter die Libellulinen am zahlreiclisten.

Die Ordnung der Hautflïgler, Hymeuopteru, zählt über 500 Gattungen, wovou die Familic der Ichneumonideı 170 , die der Bienes 70 , die ler Amcisen 60 Gattungen enthailt.

Die Ordnung der Selumetterlinge. Schuppenfliggler, Le pidoptera, yihlt bei 1800 Gattungen, worunter bei 700 'lagfalter und darunter die Equites (64), die IIelieonii (62) und besonders die Hesperien (212) vırlıältnissmäissig am zaliliciclisten sind; so Abendfalter, worunter 20 Gattungen der pigenthiinlichen Sippe Castuia und bei 1000 Naelitfalter, worunter dic Gattungen des Genus Lithosia verhältnissmässig ain zahlreichsten sind.

Die Orduung der Zweiflügler, D i pteril, die wohl auch, wie die vorhergehende, der Fangschwierigkeiten wegen, verhältuissmässig mangelhafter seyn dïrfte als die übrigen Ordnungen, zatilt iber 300 Gattungen, worunter die der Sippe Tabanus an zalılreichsten sint $(50)$.

Die Ordnung der ungeflügelten Insckten, Aptera, nit Aussehluss der Arachniden oder Spinuen, wovon über 60 Galtungeu vorhanden sind, und der Crustaecen zälilt ïber 40 Gattungen.

Fitter Carl v. Schreibers, k. k. Ruth.

viestomicn. Dic Gesellsehaft der Naturwisisensclualten besizt die Geruing'sche Sammlung. 1686 inläudische Gattungeu, der allgem cineu ans 3183.

vïrzbura. Sammlung der Universitit. Reiclı an vatertändiselıen wie exotisrlien Kerfen (Couservator Hr. Dr. und l'rof. Leibleiu).

Zihricl. Die Sammlung der Stadt enthält etwis melur als die vou Bern; meist helvetiselıe Kerfe. Wie man vernimmt soll B ie mi's Sammlung vou Kunstwerken aus der Insiktenwelt der Sammlnng pinverleibt werden. - Als eine offentliche Sammlung ist zu betrachter dievon Ese her-Zollik ofer, ausgereichnet reieh und nur von wenigen Muscen an Vollständigkeit übertroffen. Von den meisten Gattungen siud ganze Suiten Dubletten vorhauden, was wissensthaftlich besonders wiehtig ist. 


\title{
Akademien und gelehrte Gesellschaften und deren Schriftenangaben.
}

\author{
(Ergänzungstafel zu der Aufzählung im Lexikon der Entomologen.)
}

Amsterinm. I, a $\mathrm{nd}$ w i $\mathrm{r} \mathrm{th}$ sehafts-Gesells chaft.

Amiover (Massachusetis). Teacher-Seminarium.

Anlinit. Anbaltisebe landwirthseluaftliche Gesellschaft. - Seluriften der auhalt. laudwirthscls. Ges. zu Miihlstädt. 1 II. Leipz. 1824.

Anmaberg (Sacliseu). Oek o u o$\mathrm{mische}$ Gesellschaft.

Ascoli. Acadenia truentina.

InItimore (Maryland). Med iciniselre Gesellschaft. - A cademy.

Inrcelona. Akademie der Wissenseluafteu.

Basel. Bericht iber die Verhandlungeu der waturforschenden Gesellscliaft zu Basel voni Angust 1834 bis Juli 1835. Basel 1835. 8. 89.

Hatavia (Juva). Genotschap der Konsten en Wetenscliapen. Ward 1778 gestiftet. - Verlıandelingen. Batavia $1779-86$. 8. 4 Bde. (Deutselı: Leiprig 1782. 8.) - Der Stitter wnr Reinier de Clerk, damaliger Generalgouverneur der holländisclien Besitzungen in Asien (vgl. Allg. Lit.Zeit. 1801, Nr. 301, p. 164).

miella. Ernu uterugsgesellseliaft.

Binis. Société des sciences et lettres.

Terrin. Gesellse haft u a turforsclicuder Fueunde. - Verbandlungen (seit $18 t 9$ neu). 4. M. Kpf. Die Scliriften des Berl. Vereins: 1) Beschïftigungen. 4 Vol. 1775-79. 8. - 2) Selnrifteu. $11 \mathrm{VDl}$, 8.1780-94 (die 5 lezten fülven den Titel: Beobaclitun. gen und Entdeckungen). - 3) Nente Gistet's Lextkon.
Schriften. 1795-1806 (?). 4. -4) Migaz. Seit 1807. - 5) Verlandlungend. Ges. naturf. Fr. zu Berl. 1. Bd. Mit XVI Kpf. Berl. 1829 (G. Reiuer). XXVI et 418 pp. 4 . - Verein $\%$ ur B cförderung des Garteubaues in den prensis. Staten. - Verhaudlungen. M. Kpf. 4. Berlin (Nicolai). (In Lieferungeu. Von 1824-1830 sind 14 Lieferumgen oder 6 Bände ersclicnen).

mremcin. Museum der ploysi$\mathrm{k}$ a lischen Gesell schaft.

Brescia. Atheneum. - Commentari del Ateneo di Bresciu. Perl' ammo 1835,1836 pte. 8. - A c e a demia. - Commentari delia Aecademia di scienze. lettere, agricultura, ed arti del dipartimento del Mella per l'auno 1808. Bresc. 1808.

Bresinn. K. Gesellseluat der Wisseuseliafteu und Künste.

Brihne. Miihr.-schles. Gescllsehaft der Natur- und Landeskunde. - Literar. Gesellschaft: Concordia.

Caen. Societéd'Agriculture. Cucrmurtlien (Wales). Cambrian-Socicty.

Calcutta. Jonrnal of the asiatic Sociefy of Bengal by Princep. Calcutta. Cambrilge. Jolin's Collegi 1113.

Chy. Die (zoologische) Gesellscliaft der Naturforscher zur Erforscling von Central-Afri$\mathrm{k}$ a. Eine Frucht ilurer Expeditionen ist A. Smitl's lerrliches Werk: "Illustrations of the Loology of SouthAfrica". - Ferner: Reporter of SouthAfrican Expedition (nit Appendix). 
1836. (Monatlich.) - Smith, Proccedings of the South-Afriea Institution. 1828. - Smith, Proceedings of the xoological Society. Part J. 1830. South-Afican Quarterly Journal. iser. 1833. 2. Ser. 183.4. - Magazin of uatural History. New series. Vol, 1.

Cassel (Itessen). Gesells chaft des Ackerba nes.

Catamen. Akad emie; gegriudet 1824. Hat bis 18378 Bïnde heransgegeben. - Atti dell Aerndemia Gioenia delle Seienze naturali 1825. 27,30 . 4. fig.

Chanbery (Savoyen). A ckerb a I gescllsehaft.

Celle (Hannover). Landwirthschaftiche Gesellsehaft.

Dïnemarli. Förhandlingar vid det af Skandinaviska naturforskare och' Isäkaı hallua inöte i Göthchorg Ar. 1839. Götheburg (Norberg) 1840 . 8. 188. (Erste Versammlung der nordischul Naturforseher Anuo 1837. Vol. Isis 18.13. 207). - Förhandliugar ved de skandinaviske Naturforskeres audet Mlödc, der holdtes $i$ Kjöbenhavn fra den 3 die til den 9de .Inli 1840 (Verhandlungen bei der zweitenZusammenkunft der skandinavischen Naturforscher, welehe in Kopenhagen vum 3.-9. Juli 1 \$40 gehulten ward). Kjöb. 1841. 8. 424 S. (Isis 1843, 265). - Versammlung der dänischen Natu'forscher (Bericht in Fries' Arsberïttelser, p. 18). - Physikalisehe, chemisehe, natur. historisehe und mathematische Abhandlungen aus der neuen Sammlune der k. dünisehen Gesellschaft der Wissenschaften, übersezt von $\mathbf{P}$. S $\mathrm{chccl}$ und C. F. Degen. Kopenhagen 1800 . 1 Bd., 2 Abtheil. II. Bd. 1. I. 2. Abth.

Donzig. Écke rmann (Nic. Gottfr. Chr.) Commentationum Gedanensium. Fase. I. Berol. 1813. 4.

Designe(New-York). Nation a lAcade iny.

Deutschlend. Versa m $\mathrm{m} / \mathrm{ung}$ derdeutschen Landwirthe. (Hielt 1844 ilıre Sitzungen zu Müneheu.) Vorträge gehalten in der Versammlung der deutschen Naturforseher und Aerzte 2u Frankfurt a. M. vom 18. bis 23. Sept. 1826 (Isis 1826, p. 263-293 und Heft XI, 1825). - Al. v. I u m bold t und H. Liehtenstein: Amtlicher Bericht über die Versamml. deutsch. Nuturforscher und Aerzte zu Berlin 1828 .
M. Faesimil. 4. Berlin 1829. - H u mboldt's Rede 1828. 4. - Tiedemann (F.) Redc bei Eröffuung der Versammlung deutscher Naturforscher und Aerzte in Heidelberg an 18. Sept. 1829 gehalter. gr. 4. Heidelberg. Johuston (J. F. W., zu Ediuburgh) Bericht über die Versammlung der Natuforscher z.u IIauburg (Brewster Ediob. Jonru. IV, 189. V, 271). (Intcressant.) Anul. v. Munke darijber (ib. p. 271). - Kraus (L. A.) Ueber die Versammlung der deutschen Naturfol sclicr und Aerzte. Aus d. Freiheften f. W. Natur- u. Heilkunde abgedr. gr. 8. Göttingeu. - Bericht über dic neunte Versammlung der dentseheu Naturforsclicr und Aerate in Hamburg. 12. Hamburg. - Versammlung zu Wien (Isis 1833, Heft IV, V, VI. "Die Weisheit sass beim kaiserlichen Mahle und hat des Kaiserschutzes sich erfreut", sang Castelli, und wahrlich, die Oestreicher haben wie Briider geluandelt vom Kuiser herab bis zimm Postillon und wie die grössten Gelelurten sich offen gegeben. Es lebe Orstreieb!) - Mittheilungen über Wien in naturwissensehaltlicher und ärztlicher Beziehung und die sämmtlichen allgemeinen und speeiellen Verhandlungen der daselbst stattgehahten 10. Versamml. deutsch. Naturforseher und Aerzte. gr. 8. Berlin. - Bericht üher d. 11. Vers. d. d. Naturf. in Breslau 1833 (Isis 1834, Heft VI ete.). Ve u d t (Jol., Dr. med., Medizinalıath und Professor zu Buslan, Geschaiftsfülırer bei ter Bresl. Versammlung) Autlieher Bericht über die Versummlung d. d. Natf. 1833 (mit A. W. Otto sclig). Bjesl. 1834. 4.68. 19. - Vcrsammlung des Naturforscher zu Stuttgait, am 18. September 1834 (Isis 1836, Heit 111). - Versammlung der Naturforscher mod Arizte zu Bonn in September 1835 (Isis 1836, H fl. IX, X). - Versammlung der Naturlorscher und Aerztc zu Jena in Sept. 1836 (Isis 1837, Heft $v$, VI und VII). - Versamul. d. Naturf. ete. zu Prag im Sept. 1837 (Isis 1838, Hft. VII und VIlI). Versamml. d. Naturf. ete. zu Freihurk im Breisgau im Sept, 1838 (Isis 1839, Ileft XI und XII), - Grätz. Tagblati der Virsammlungen der Naturforscher und Aerzte z.11 Grätz 1843, Nr. 1-7. 4. S. 91. - F. K. v. Stroubeck und Dr. med. M a in seld: Amtlieher Be- 
richt über die neunzehnte Versamml. demie nützlicher Wissenschaften. 1804 deutsch. Natf. etı. zu I3raunsehweig -1809.4.

im Sept. 1841. Braunschweig $184 \%$. 4. 259, T. 2.

Deventer (Hollanu). A thenaeum.

Dietendorf. Thüringiseher Gartenbauverein.

Doncaster. Aqricultural Association. - Report of the Comittee of the Doncaster Agricult. Assoeiation. 1834. (Entlıält über selıädliche Larven manehes Nïtzlielıe.)

Dresden. Auszüge aus den Protokollen der Gesellsehaft für Naturund Heilkunde in Drisden im Jahr 1832. $1 \mathrm{Hft}$. 8. von 1833. Dresd. 1834. 8. 205, 2 Taf. (Die Miseellen ausge"Iommen sind auch siezu uusern Zueck benizt.) - K. ökonomische Gesellsehaft fïr das K. Sachsen. - Srlıriften und Verhandlumgen. Seit 1818 in Lieferungen in 8. erscheinend (a $54 \mathrm{kr}$.).

Drontlueim. Scluriften der Dront. beimer Grescllschaft. 3 'Theile. 8.

Disseldorr. Akademic der Wissenschaften.

Edinburgle. Caledouian Soe iety. - Memoires of the Caledonian Soeiety. Edinb. - Memoires of the Wernerian natural History Suciety. VII $(1831-1837)$. 8. 1839, 550, 36 Taf. (Abbild, von Fisehen).

wisleben (Merseburg. Preussen). Mansfeld. - Liter. Ges elIsehaft. Didena. A eademi e.

Eugland. Versammlung der englisehen Naturforseher. (In Hoeven's Tydsehrift for naturl. Gresehied. III, 1836, 107 fintlet sich ein Berieht über die sechste Versanmlung derselben.) - Amtlicher Bericht über lie Versammlung der enir. lisehen Naturforscher zu York (Brew. ster Elinb. Journ. VI. 1832, 360). J. Johuston (in Edinburgh). Erste Versunmlung der brittisehen Naturforseher zu New-York am 26. Sept. 1831 (Brewster Edinb. Jourı, V1, 1831, 1 -77). - Bericht über die erste Versammlung 1. engl. Nuturf. zu York 1831 und die zweite zu Oxford 1832 (Lond. a. Elinb. philos. Magnz. II, 455). - Bericht über die dritte Versammlung der engl. Naturf, zu Canbridge (L'Inst. 1833, 131).

Erfurt. Abhandlungen der Aka-
Erlangen, Abhandlungen der physikalisch-medizinisehen Soeietät. 1, 2. Frankf. a. M. 1810 und Niirnb. 1812. 4. - Neue Denksehr. d. phys.mediz. Soe. 1, 2. Nürnb. 1812. 4.

Fsslingen. Wü rttembergiselier Reiseverein. - Selriften desselben, 1834. (Macht meist in der "Flora" Ausschreibungeu.)

Florenz. Aceademia della valle tiberina toseana.

Forli. Aecademia lle' Filergiti.

Frankreich. Congres seien: tifique des rance. (Weelisclt jährlich seine Versammlungsorte.) - GeologiseheGesellsehaft vonfrank: reich. - Ani Bou é: Bulletin de li soeiété greologique de France. Paris (Soeicté). Cirea 15-16 Vol. (Ungeheurer Literaturschatz.)

Fraulkrurt a. II. Jalırbueh zur Verbreitung naturwissenschaftliaher Kenntnisse, veranstaltet vom pluysikaliselıen Vereine in Frankfurt a. M., für das Jahr 1831 ete. gr. 8. Frankf.

Franendorf (bei Pussau). Die praktisehe Gartenbaugesellse haft.

Crenf. Mémoire de la Socièté de Physique et d'Histoire naturelle de Geneve. Tom. I. 1821. - Tom. II, prem. Part. Genive (Pasclioud). Paris (Mém. mais.) 1823. IV et $203 \mathrm{pp}$. II Pl. sec. pait 1824, 255 p p. 6 Planch. 4. Tom. III. 1825, prem, part. IV et 235 pl. 6 Pl. Seeond. part. 260 pp. 1826, $8 \mathrm{PI}$ - T. IV $, 1828,520 \mathrm{pp} .18 \mathrm{PI}$. - T. V. 1832 , XIX et 360 pp. 15 Pl. - T. VI. 1833 , VII et $608 \mathrm{pp} .36 \mathrm{PI}$. - T. VII, 1. part. Genève (A. L. Vignier, maisou de la Puste) 1835, IV et 264 pp. 16 Pl. 4. - Compte rendu do la socicté de pliysique et d'histoire naturelle de Geneve lus les 1. Juillet 1833 au 30 Juin 1834 ete. - Société des arts ete. - Bulletin de la rlasse d'agrieulture de la Socitite des arts à Genève.

Gent. A then öum. - Aunales Aeademiae gaudaviensis. Ann. 182027. Gandavi, 4.

Chasgow. Mechanical. Institut.

Adrlitx. Abhandlungen der naturforschenden Gesellsehaft z.l1 Gürlitz. I. Bd. Görlitz 1827. 1, 2. II, Heft 1. 
1836. 8. S. 131, T. 2 . Hifte 2. 1838. 1.13, T. 1. Bd. III, 1, 106 (lsis 1842, p. 606).

Greirswalde. BaltischerVerein fïr Förderung der Land. wirthscliaft. - Bericht über die Vurliandlungeu (1842) von Pabst und Crebe. yr. 8. Greifswalde 1843.

Gröningem. A eademia Scientiarum (Athenaeum frauquequeranum).

Hanrlem. Tayler's Institut. - Die äleste batavische gelelurte Gesellschaft ist die Gesellsehaft der Wisse uschaften, gestiftet 1762 . Scluritien derselben in 30 Bäuden in 8. unter den Titel: Verliandelingen van de hollandsche Maatschappy (lev Wertenscliappen te Haarlem. M. K pf. Huarl. Int Amsterd. 1 554-98 (36 fl.).

Hallocestarlt (Magdeburg). Li. terarisebe Gesellsehaft.

Hambires. Der naturissenschaftliche Verein (vgl. lsis 1839, 66!. - Gestiftet 1837. Erste Versammlung 18. November dess. Jalors. - Prüsident: S. Exr. der k. russ, w. Stantsiath Hr. Minister v. Struve; Vicepräsident und Secretär: Hr. Pastor Miiller: Dr. Zimmermann Gesrlıäts- und Cassenfülırer.

Inimu (Arusberg). Gesells c haftzurBeförderungder Ockononic.

Hannover. Naturwissensohaftlicher Verein des Haraes. (Unstäte Versammlungsarte. Versammelt sich alljährlich). - Bericht iber die vierte Vers, des Harzvereius zu Claustha) 1834 (Isis $1835,56-60$ ). - Bericht ïh. d. 5. Vers. d. Havzv. zu Stolberg 22. Juli 1835 (jbid. 846). - Bericht ïb. d. 6. Vers. d. Harzv. zu Nordbausen 27. Juli 1836 (Isis 1837, 18-11). - 8. Vers. d. Harzv, zu Goslay (lsis $1838,802-806$ ). - 9. Vers. d. Harzv. zu Blankenburg (Isis 1839, 666).

Idstein (Nassau). Landwirth. seliaftliche Gesellschaft.

Ttulicin. Reunione de $\mathrm{Natu-}$ ralisti e Medici italiaui. - Atti della prima Reunioue degli Scienziati italiani tenuta in Pisa 1839. Pisa (Nistri) 1840. 4. - Versa m lung der italie iil Florenz, 1842 in Padua: 1843 in Lnera. - Atti della terza Rinnione degli Seienziati italiani, tenuta in $\mathrm{Fi}$ renze nel Settembre del 1841. Firenze
1841. 4. 791, t. 3 (Auszug in der Isis 1843, Heft VI, ]. $401-425)$.

Inesbruck. Ferd iu ande un. Mersi, v. Pf a umller und Rögrel: Beitrigc \#ü Geschichte, Statistit, Naturkunde und Kunst von Tyıol und Vorarlberg. 1.-18. Bd. 8. lunsbr. $1826-44$.

Irdigsberg. K. deutsche Ge$\mathrm{s} e \mathrm{II} \mathrm{sel}$ a $\mathrm{ft}$.

Kopenlnagen. Det kongelige danske Vilenshaberni's Selskabs unturvidenskabelige $0 \mathrm{~g}$ mathematiske Afhandelingar. VIIl. Til. Kopeulı, 1841. 4. M. 33 Taf. (Auszug in der Isis 1843 , p. $732-89$.

Enmsamme. Soeictivauloise d'utilite publique. - Journal de la Socicté vaul. ete. Laus, 1834. (1833 par D. A. C la avanues.)

Leipzig. Jahlonovsky'sche Gesclls ehaft. - Acta Societalis Jablonovianae. 4. Lips. Neu seit 1802 (bei Aue in Altona). I, 11 und 111 (1810 und 12) zu Leiprig. - Dic ältern Acta datiren sich vou 1771 und 72 . Occonomisclie Societät. - Naturforsclunde Gescllschaft. Acta societatis Naturac Scrutatorum Lipusiensis, Jom. 1. Lips. 1823. 4.

Lobru. Derlandwirthscliaftliche Verein.

Homane. Royal Philosophical Soriety. - Abstracts of the Papers printed in the Pluilosoph. Tran. sactions of the royal Society of London. Vul. I, 1800-1814.516. Vol. II, 1815-1830. 448. London by Taylor 1832. (Selir bramehbaler Auszng aus den Transactions.) - Geological Society. - Raleigh-Society (zur Unterstuituug gelelirter Reisen). Royal Institutiou. - The Jornal of Science and the arts, edited at the Royal Institution in London, by Will. Thomas Brande, Esq. - Society for the Encourageme ut of Arts ctc. - Transactions of the Society for the Eneonragement of Arts, Nanufacfures, and Commerce. Vol. 22-23. Loudon. 8. - Il ortieural-Society. - Transactious of the Hortieultural Socicty of London. Vol. 1, Purt. 1. London 1807. 4.

Imgaro (Tessin. Scliveiz). Soeietáticiuesc d'utilitánublica. - Atti della Societa ticinese d'ut. pub. dell 22 Genajo 1829 al 13 Agrosto 1834. 
Nincliester.

Literarischphilosopliselıe Gesellschaft. Mrmoires of the Literary aud Philosophical Society of Manchester, 2. Se. ries, Vol. 3. Mautliester 1819.8. Vol. 2 erselien 1813. - Geologieal Socicty.

Mranilla (Insel). $O \mathrm{ck} 0 \mathrm{nom}$ ise he Guselisehaft.

Manmheim. Zweiter Jahresberieht des Manmlıeimer Vereins für Naturkunde, 1835. 8. 30 (Lsis 1836, 333).

Irantua. Akauemie der Wissensehaften. - Memorie dell' Aecadeuia di Mantova.

Metz. Memoires de l'Aeademie ro. yalr. Tome XIV, XV, XVI. 8. (Bis 1836).

Mitau. Jahresverliaudlungen der kurliudischen Gesellschaft für Literatur und Kunst. Bd. I, 1819. II, 1822. Mictau. 4.

Montevmelai A ceademia Vald' aruese.

Moriz (Insel; auch IsI e de France). - J. Desjurdins: Vierter Bericht ïber die Arhoiten dier Gesellsehaft der Naturgeschiclite auf ler Insel Mauritius (Proceed. III, Der, 204. - Isis 1837, 206. - Ueber Sucke, neue Vögcl, Fische). - Fünfter Bricht (I. c.) (Zugrögel). - Arbeiten der Gesellsibaft auf der Insel Moriz (Tisylor Procerd. 11, Mar. 22, 111. - Isis 1835, 429). (Vögel und Fische.)

Nustan. Societé impèriale des Naturalistes de Moseou. Bulletin. I'ublie par Gotth. Fise her de Waldheiu. Tom. 1-VI et sey. Aceomp. de Panehes in 8. Masc. 18:9 ct scq. Iscipz. (Voss). (Eine Fortsetzung davon sind die "Mèmoires".)

MIinlstidt (Mnbalt). Vgl, Aulalt.

New-Grleans (Louisiana). Gesellschaft zurVerbreitung ü $\mathrm{t} z$ lielor Wisseuscluaften.

Nizza. Cliambre royaled'arrieulture et le eommerce de Niec.

- Bulletin de la Clambre ete.

Derebro (Sehweden). baugesellsehaft.

Padua. K. Ackerbaugesell. sch aft.

Ponris. Mímoires de l'Aead. des se, de Paris. Vou 1700-1790. - Mèm. des Savauts ettr, 11 Vol. rou $1750-$
1786. - Histoire et Mémoires de l'Aca. demie royale des seieners depuis son Etablissement en 1666 jusqu'a 1786, 103 Tom. Palis 1708-1786. 4. - Table alphabetique des Maticres contenues dans I'llistoire et les Mremoires de l'A eadenie royale des sriences. Anués 1666-1780. 9 '1'om. Paris 1734 -86. 4. - $\mathrm{C}$ uvier: Beriehl über die Arbeiten der Akadmic 1829 (Am). d. se. 11. XXI, 209-282). - Memoires lu Museum d'hist. natur., par les Professeurs de cet Etahlissement. Ouvrage orné de Gravures. Dédic au Roi. A Paris, ehez, A. Belin, imprimeur libr. ete. 1822. 4. - Anuales du Museum national d'histoire naturelle pas. les Professeurs de cet Etablisse. ment. Paris 1802-3 et seq A. Guevin-Mènéville : Revue zoolugique par la soeirté cuverienue. Paris Rue de Saint-Germain, Nr. 13). 1838. 8. Nr. 1-3. 48 (Isis 1838, 229). Mo. natlich 1 Hefl. - Soeiéled d'histoile naturelle. - Actes de Ia Soeiéte d'listoire uaturelle de Paris. Tume 1, l part. Paris 1792. Fol, - Memoiles de la soeièté d'histoire maturelle de Paris. Nur t Vol. 4, 1799 (Baulonin). Unter diesem Titel erselicn aber ein audres Werk in VII Bäuden in 4 . 1823 u. flo. - Societé an a tomi i we. Pecking (China). Akademio der Wissenseluafter. - Mediziwische Gesellscluaft.

Pesaro. Aceademia agraria. Seit 1830. - Esirritazioni.

Flniladelphia. Geological Soeiety.

Prag. K.k. Patriotiseh-ökonomisehe Gesellsehaft f. B. - Franz Fnss: Gesehichte der k. k. äkonom.putriotisehen Gesellach. in Königreich Böhmen, von ihrer Entstchung bis nuf das Jahr 1795. Prag 1797. 8. - Jahr. bücher des bölumisclieu Muspums für Niatur- und Liinderkunde, Kunst und Literatur. Scit 1830 iı 8 . Prag. Frülser unter dem 'litel: Monatselurift der Ges. l. vaterl. MIus. 1827-29 (a 12 Hefte). 8.

I'otciam. K. preuss, - mäikisehe okonomisehe Gescllichaft. - Monatsblatt. Jalurg. 1843-44. S. Potslau,

Totterdam. Gesellsehaft der Experimeutalphilosophie (gestiftet 1769). - Verhandelingen. Seit 1774-99. Nienwe Verlondelingen. Seit 1800.

gronen. Suciétélinéenne. - 
Vémoires de la Socièté linéenne de Normandie. Par. 1842. VIII Vol.

Snchsen. Wandergesellsehaft. - Verhandlungen der Wandergesellschaft sächsischer Landwirthe und Naturforscher in den Jahren 1834 nnd 1835, vou Dr. C. F. Groh. Dresden 1837. 8. 68 (Isis 1837,662 ).

San Cruce (Teneriffa). OckoHomische Gesellschaft.

Sambt Petersburs. Memoires de l'Academie. Chronologisch: Commentarii 1726-1746. 14 Vol. 4. Novi Commentarii 1749-1775. 20 Vol. Acta 1777-1782. 7 Vol. Nova Acta 17831802. 15 Vol. Mèmoircs, bis 1809. Fuss (P. H.): Coup d'oeil historique sur le dernicr quart-de-siecle de l'existence de lacadćmie impériale des Scienees de Saint-Petersbourg. St. Petersb. 1843.

Schuelzerische (Allgeme inc) Gesellschaft für die gexammte Naturwisseuschaft. (Gegrindet durcli den verstorbenen Dr. Gosse zu Genf.) - Protokolle der Sitzungen der schwrizerischen maturforsch. Gesellsch. voll 1815-45. Fol. Mspt. (m Bern). Auszung Jer Verhandlungen vou 1822 -45. 4. Mspt. (eo, 1.) - Neue Denkschrift. d. altg. Srbweiz. Ges. Neuchat 1837, I, 4. 10 T'al. (Isis 1837, 83335). - Neue Denksrhr. d. Sthw, Ges. Ncuch. Il, 1838, 4. 9 'Tab. (Isis 1839, 86). - Neuc Denkschr. d. Schw. Ges. Ncuel. III. 1839. 4. 62 Bogen, 27 Taf. (Isis 1839, 726). - Verhandlungen $d$. nllg. Schw. Ges. f. d. g. N. (Versaumml. zu Luzeru 1835). Luz. 1835. 8. 114 Seiten. - Verh. d. Srluw. Ges. in ihrer 20. Vers. zu Aarau (1835). Aar. 1836. 8. 177 (Isis 1836, 565). - Verh. d. Scliw. Ges. Soloth. 1836. 8. 134. T'selu ud i: die Versammı. d. Schweiz. maturforsch. Gescllsch. in Neuenbur, $1 \$ 37$ (1sis 1837, 762). - Verl. d. Schw. uatf. Ges, bei ilur. Vers, zu Basel 1838. s. 242 (Isis 1839, 675). - Merian (Prafessor z.ı Batscl) : Erölfunugsiede iiber die Leistmugen der S(bweizer in Gebiete der Naturwissenschaft (Verb. d. Schweiz. natf. Gesellsclı, zu Basel 1838).

Sevilla (Estremadura). Gesellsehaft der Vaterlandsfreunde.
Suntrua. Grichisclic Gesellschaft zur Aufmunterung der Wissenschaften und Künste.

Spainto (Dalmatien). Gesell. schati des A ckerboues.

Stockholm. Uebersetzung der K. V.Handlingar: Abhandlungend. Sehwedischen Akademie 1739-79. 41 Bde. Vom 3. Bande un übers. v. Käst ue r. Mit 2 Hauptuegistern, gr. 8. Leipzig 1742-79 (Brocklıaıs). 31 Rthlr. Zweif. Regist. über 1 und 25 Bd. ibid. 2641 Bd. - Neue Abhandl. 1-12. Bd. von Kästuer, Brandis und Liuk. gr. 8. Leipz. 1784-94. 12 Thlr. 16 gr. - Jigerverein in Stockholm. Tidsskrift för Jägare, Naturforskare, utgifveu af Jägare-Förbundet i Stockloolm. I., II., III. (und lezter) Jalirgang. 1832-34. - Jahresbericht der Schwedischen Akademic der Wissen. schaften über die Fortselıritte in der Naturgeschichte, Anatomie und Physiologic der Thicre und Pflanzen. Aus dem Schwed. mit Zusätzen von Dr. J. Müller. 1824, der Uebersetz. erster Theil. gr. 8. Bonn, Marcus.

strassburs. Sociéted des sciences, Agriculture et Arts du Departement du Bas-Rhin. - Mémoires. - Nouveaux Mémoires. 8.

Utrecht. Gesellsehaft der Kiinste und Wissenschafteu. Verhandelingen. 1781-95. 6 Bde. in 8. M. Kpf. Band 9 erschien 1801. (Melur salien wir davon nicht.)

Vlissingen. Seelïndiselı e Gesellschaft der Wisseuschaften (gestiftet 1766). - Verhaudelingen, vitgegeven door het zceuwsth genotsehap der Wetenschappen te Vlissingen. 17ti9-93. 8. 15 Bde. (Deutsch vou And. Böhme. Giessen 1775. 8.)

wien. K, k. Gartenbaugesell. sehaft. - Verhandl. d. k. k. Gartenbaugesellsclı. in Wien. Scit 1842. Wien (Beck). gr. 8. - K. k. Landwirthsebaf's-Gesellschaft. - Verhandl. Scit 1820. Jeder Quart-Band besteht uus 2 Hefter.

wilrzlourg. K. philosophisehmedizinische Gcsellschaft. Jahrbiiehel. - Nene Jalırbücher. Abtheil. für Natur- u. Heilkundc. Seit 1830. 8. 


\title{
III.
}

\section{A DIRESSENBUCH}

\author{
DER
}

\section{$\triangle \mathbb{B}$ A I I I I D D ID}

oder der Schriftsteller über dic spinnenartigen Geschöpfe. 
"In der Thierwelt ist daher die ganze moralische Welt in dor Form der. Naturindifferens schon viel deutlicher dargestellt, als in der P/ansenicell. Wir sehen in jener ein tausmudarmiges, durcheinander vimmelndes IIeer vou willkürlich sich bewegenden und handelnden Wcsen von den verschiedensten Formen, Kräften, Eigenschaften und Bestimmungen. Alle sind in ihrer Art collkommen: aber einige schtach, sahm, Milleid erregend, andere schrechlich durch Kraft und Hlutdurst, Waflen und Schnelligleit; diesn sierlich von Gestall, Farbe und Zeichnung, undere lächcrliclı oder schcusstich, wie namentlich viele von vielgliedrigen Typen, ₹. B. Cephalopoden, Npinnen - -."

Pinтy (Naturgeschichte). 


\section{SEINER DURCILAUCHT}

DEM

\section{FÜRSTEN UND NATURFORSCHER}

\section{IF U кTC. ктс.}

SEINEM

WOHIGENEIGTEN FREUND UND SCHÜLER,

$$
\text { DEัa }
$$

UNERMÜDETEN ARACHNOLOGEN,

AURICHTIGST IND ERGLBENST

DER 



\section{Prolegomenon.}

\section{"Dnnli mihi artem, duln gloriam."}

Wenn noch einige Hunderte von Dilettanten sich Sammlungen von Kerfen aller Art anlegen, so sind es allezeit die Spinnen, welehe iu diesen vermisst werden. Die Zoologie verliert hierdureh nichts. Das Vorurtlıeil der Mcnsehen spielt, zum Glück für diese Thiere, eine wohthätigc Rolle. Die Spinnen gehören zu denjenigen Creaturen, deren Körperbau für die Menge ekelerregend und absehreekend, deren Lebensweise einsam und unheimliel, näehtlich, deren Charakter grausam und räuberiseh ist. Hiezu kommt noch als Hauptursache mensehlieher Antipatlie gegen sie der Geruch des Giftigseyns, in dem sie alle stehen. In dieser Antipathie lag von je der Grund," dass selbst Naturforseher sieh nicht herbei liessen, die wunderbare Welt dieser Geschöpfe näher in das Bereich ihrer Beschäftigungen $z u$ ziehen, was schon der gemüthliehe Pastor Göze gesteht. indem er, was von den meisten gilt, sagt:

Vor ein Paar Jahren' war der Ekel bei mir noch so gross, als er irgend bei einem Menschen seyn konnte. Sobald ich aber anfing, diese Insekten absichtlieh zu untersuehen, je mehr ich ihren künstliehen Bau und andere Unstande ihrer Oekonomie studirte, je mehr ich immer. in Absieht des ersteren, bei cinzelnen Gliedern anfing und zum Ganzen fortsehritt, desto mehr verlor sielı der Ekel, bis ich ihn endlieh ganz überwunden habe, und im Stande bin, eine Kreuzspinne ohne Schauder in der Hand $z u$ behandeln und zwisehen den Fingern zu zergliedern. Eine Erfahrung, die mich gelehrt hat, dass dergleichen Ekel und Abseheu vor gewissen Insckten und andern an sich unsehädlichen Gesehöpfeu ein blosser Erzichungse hler sey, und auf nichts, als sinnlich dunkeln unangenehmen Vorstellungen beruhe. Man muss also keinem Kinde einen Abselıcu vor einem Insckt oder Gesehöpf beibringen oder denselben wurzeln lassen, sondern bei Zciten dureh vernünftige Erklärungen seines göttlich-weisen Baues aus der Secle wegzuschaffen suchen. Vernuthlich entsteht bei den meisten auch die Furcht ror den Spinnen aus dem Vorurtheil: dass sic giftig oder schädlich wäreil. Ihr Saft ist ctwas eorrosivisch, aber lange nicht so beissend, als der Saft einiger Raupen und dic Ausdünstungen eines Hecres der Processionsraupen. Aus einer bloss sinnlichen Fureht und aus Vorurtheilen entsteht der Aberglaube auch bei den Spinnen, der dic Werke Gottes so entsetzlieh misshandelt und keinen Geg̣enstand in der Natur übrig lïsst, ohne ron 
demselben gewissc unnatürliche und ungereinte Wirkungeu zu erwarten. Wenn im Herbst aus der Oeffnung eines Gallapfels eine Spinne auskriechet, so soll es Pest und Seuchen bedeuten. Mir ist ein lixempel bekannt, dass eine alte Matrone nach dem Verhalten einer Hausspinne über ihrem Armstuhl in der Stube ihre ganze Oekonomic einrichtete. Diese Spinne war allezeit erst das Orakel, welches befragt wurde, ehe man im Hause etwas vornahın. War die Spinne lustig, lief sic in ihrem Netze munter unher (gewiss aus Hunger), so war es ein gutes Zeichen, so wurden Brautage, gckauft, Malz bereitet, Gesellschaften angenommen, Besuche gegchen u. s. w. War dic Spinne aber still und schicı sie in ihrer Höhle traurig zu sitzen (vermuthlich wenn sie satt, oder das Wetter trüb war), so war es ein böses Zeichen. Dann wurde kein Handel geschlossen, kein Gesinde gemiethet, keine Stube ausgèfegt, kein Besuch abgestattet, sondern ein Busslied gesungen und immer nach der Spinne gesehen. Kam etwa die Sonne hervor und die Spinne regte sich, so wurde das Gesangbuch bei Scite gelegt und die ganze Oekonomic empfing neues. Leben. Ein kleiner Beitrag zum Grabe des Aberglaubens.

Dass aber auch eine Spinne einem Gefangenen in seinem öden Ker ker Gesellschaft, Wohthat und Freude seyn kann, beweiset das rührcnde Beispiel im 7. Bande des deutschen Mcrkur, p. 154 (G oeze in der Vorredc zur deutschen Ausgahe von Dr. Martin Li ster's Naturgeschichte der Spinnen überhaupt und der engelländischen Spinnen insonderheit aus dem Lateinischen übersezt und mit Anmerkungen vermehrt von Dr. Friedrich Heinr. Wilh. Martini; nach dessen Handschrift aber zum Druck befördert und mit neuen Zusïtzen versehen. Mit $5 \mathrm{Kpft}$. Quedlinburg und Blankenburg 1778. XXVIII und 302 und 18 pp. mit V Kpft. 8.).

Die Spinncnthiere oder Arachniden sind Drossel- und Brustthicre, und stehen in so grosser Verwandtschaft mit den Crustaceen, namentlich mit den Myriapoden (besonders auffallend wegen der Kiefer! Man betrachte die Sippe Acrosoma!), dass eine Vereinigung beider Classen woh! zu billigen wäre. - Perty trennte sie jedoch und bildete nachstehende Ordnungen :

I. Holetra.

1. Familic. Phthiracarea (Pty).

2. Familic. Acarina (Milben).

3. Familic. Phalangida.

I1. Pedipaipia.

4. Familie. Chelifera.

5. Fanilic. Solpugina.

6. Fanilie. Scorpionida.

7. Familie. Theliphonida.

III. Arancida.

8. Familie. Mygalida.

9. Familie. Tubitcla (Röbrenspinnen).

10. Familie. Inaequitela (Weberspinueu).

11. Familie. Orbitela (Radspinuen). 
12. Familie. Laterigrada (Krabbenspinnen).

13. Familie. Citigrada (Wollsspinnen).

14. Fanilie. Saltigrada (Katzenspinnen).

Gräfe rechnet sie zu den Kerfen und stellte sie in die zwölfte und dreizehnte Ordnung nach den Myriapoden:

L ungenspinnen (Pulmonariae).

1. Familie. Spinnen.

2. Familie. Scheerenfüsse.

Traeheenspinnen (Tracheariae)

und lässt die zehnfüssigen Krabben darauf folgen. - Carus stellt sie nach den Krabben als eigene Ordnung (IV): Achtfüsser, Octopoda, zwischen jene und die Kerbthiere. Und so die Meisten.

Der Spinnen Leben ist ein beständiger (Guerillas-) Krieg und ein hinterlistiges Wegelagern. Wilder Raub, Umgarnen, Umzingeln und Tödten - ist ihr Geschäft; ihre Waffen sind zwei giftige Dolche resp. Gifthaken (Mandibulae) oder ein Stachel, der wie der Biss der Malimagnathe (Theridion 13-guttatum) und der Schwanzstachelstich der Scorpionen (besonders der afrikanischen) oft bei Mensehen grosse Besehwerden nach sieh ziehen kann, beim Thier den Tod.

Die Spinne vermag dureh ihren Biss nur Thiere zu tödten, so unter ihrer Organisation oder auf gleicher Entwiekelungsstufe nit ihr stehen, und es scheint gewiss, dass diese Behauptung, in Europa wenigsteus, statthaft sey. Die Stiehe der Scorpionen in Afrika entzünden und vergiften das Blut mit llife der erhöhten Temperatur desselben und der Atmosphäre. Fs findet hier dieselbe Vergiftung statt, wie bein Biss von einer Giftschlange, eine organische direkte Vergiftung (Zersetzung) durch aus den an der Spitze durchbohrten Gifthaken (sc. die lezten abwärts einschlagbaren Glieder del zwei Oberkiefer des Maules) hervorsickerndes Gift.

Wir sind von Dysdera erythrina, gemein im Pogebiete unter Steinen, oft gebissen worden. Wie Wespenstich, jedoeh heftiger, vergeht; aber nicht wie bei der Tarantel. Die Angst macht viel Umstände hicbei.

Insectis pascuntur, rapinaque vivunt; propriam in specien etiam saeviunt; a Sphegibus et Ichneumonibus plcctuntur; senectam exuunt; aetherea seandunt; Penes in palpis, vulvae ad axillas; ancipitia conjugia, ubi repulsa et peraeta eum periculo vitae fiant. Palpis clavatis mares sunt, adeoque sexus, nec speeiei differentiae -; schreibt der grosse Lin a e us.

Sehion von Jugend dem Gewöhnlichen entwöhnt, haben wir auch dell Spinnen unsere Aufmerksamkeit zugewendet gleiclı den übrigen Geschöpfen und sie gesammelt, gemalt und beschrieben, endlielı aufbewahrt, und wir können uns rühmen, die grösste Sannlung von Arachniden zu besitzen, die in der Welt vielleicht anzutreffen ist. - Weun die Verfolgung diese Gesehöpfe nirgends duldet, bei uns finden sie Sehutz in Freiheit und leiehter Gefangensehaft; bei uns spazieren sie zahm im Scriptorium hin und wicder und viele haben da ihren Webstuhl erriehtet und geben uns manehe Entdeekung ab, während 
sie anderwärts die Bibliotheken berölkern und den Hausrath und die Geldtruhen der Reichen in wohlverriegelter Einsamkeit unit ihrem Gewcbe überzichen. - Wir beobachten die Aracluniden in ihrem Leben und bewahren sie nach ihrem Tode in Barometergläsern voll verdünnten Alkohols auf; das beste Verfahren. - Tödten kann man sie auf diese Art schnell und auch sonst noch, wenn man sie unter ein Glas mit etwas Schwefeläther bringt.

Es ist gewiss, dass die zunehmende Landkultur die ZahI der Kerbthiere vermindere und ein und dasselbe Thier da nicht melar gefunden werde, wo es sonst sogar gemein war. So ist es auch mit den Spinnen und Kerfen in unserer Gegend. Vor 10 Jahren sammelten wir da noch den Atypus Sulzeri und Opilo Hellwigii, die wir nun nimmermehr finden können.

Die Spinnen bilden unter den lebenden Wesen eine mächtige Dynastie, und deren Anzahl ist so gross, wie ihre Mannigfaltigkeit; ihre Zahl ist in Verhältniss zu den Kerfen dennoch gering, aber gegen die Mollusken etc. sehr hoch und gegen die Myriapoden und Crustaceen so ansehnlich, dass es wirklich scheint, dic Myriapoden müssten den Arachniden als entfremdete entführte Körper diescr Classe noclı beigesellt werden. Ucbrigens möchten wir die Myriapoden ganz von den Crustaceen abtrennen. - Um München gibt es cintausend Gattungen Spinnen, fast cben so viel als phanerogamische Pllanzen (1100).

Die Zahl der Spinnen der Welt zu ermitteln ist wegen der mangelhaften Pflege der Arachnologie noch zu früh. - In vielen ältern Werken sind die Spinnen ob der allzu lakonischen Diagnosen uubestimmbar. - Fabricius hat 5250 Spec. Coleopt. (1801). - Dejean 22,399. L a p o rte schäzt die in Frankreich aufbewahrten auf 30,000 und glaubt, es gebe 150,000. - In ganz liuropa sind 6194 Spec. Käfer nach D eje a n. In der ganzen Welt befinden sich (nach $\mathrm{SundevalI)} \mathrm{100,000}$ Arten.

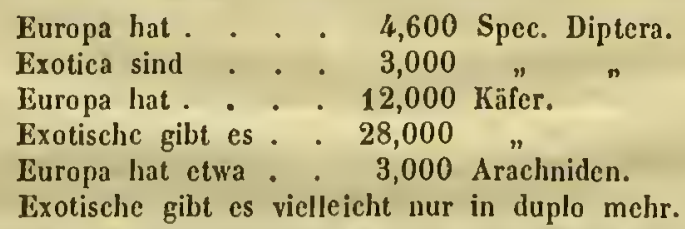

Es ist eine natürliche Eigenschaft des menschlichen Geistes, seine Aufmerksamkeit nur allmählich von dem Grossen und Ausgezeichneten auf das Kleinere und Unbedeutende, wenn es überhaupt in der Natur etwas Unbedeutendes gibt, zu lenken. (Vinc. Kollar.) - - So ist es denı geschehen, dass man aın spätesten wohl die Aufmerksankeit auf jene Wesen lenkte, welche uns so vielfach zur Bewunderung leiten durch ihre Kunstfertigkeit sowohl als durch ilhr grausames gefrässiges und banditenhaftes Wesen. "Le cerveau pense, le cerveau médite". Melıre tüchtige Männer bearbeiteten diese Classe mit Glück und Nutzen.

In der Arachnidenwelt treten mit einem Male 8 Füsse auf, eine bei allen normal ausgebildeten Kerfen unerhörte, nicgezällte Zahl. 
Den Auslüufern jener Familien, welche man unter Araelıniden, Scorpionen und Phalangiden etc. begreift, nähern sich die Gruppen, welche ganz different dem Auge erscheinen; sogar die Körperbedeekung hilft mit, die Uebergänge herzustellen.

Junge Leute mit den Namen, Sitten und der Beschäftigung der Kerfe vertraut zu machen und in ihre Classification einweihen, ist gewiss eine ebenso vortrefliche Methode, das Geschick im Beobachten, die Aufmerksamkeit und das Gedäehtniss zu verstärken, als irgend eine andere Geistesübung; und dann würden sie in ihrem hohen Alter nicht Hos einen Gegenstand haben, der im Stande wäre, sie vor dem Lebensüberdruss zu bewahren, welcher so oft mit dem Zurüekziehen vom thätigen Leben unzertrennlich ist; sondern auch eine nie versiegende Fundgrube von unschuldiger Unterlaltung, einen Anreiz zur körperlichen Bcwegung und mithin zur Erhaltung der Gesundheit und Fröhlichkeit*.

Der Kriegsdirektor Re ichard sagt, indem er die Spinne als den sichersten Wetterpropheten erklärt: Am feinsten fühlen die Spinnen die Verānderungen der Atmosphäre, und an ilınen kann man das Wetter am längsten vorhersehen. Diese ihre Ligenschaft, deren Kenntniss man den Beobachtungen des IIrn. Quatremere d'Isjonval verdankt, habe ich selbst mehrmals erprobt und mich auf ilse Richtigkeit vcrlassen können. Hier folgt cine Uebersicht der Kennzeichen, wodureh die Spinnen die bevorstehende Witterung mehre Tage voraus verkünden. (Sie mögen hier als Barometer für Excursionstage dienen!)

Hängespinnen. Lis deutet auf schönes Wetter, wemn es a) viele Hängespinnen gibt, oder b) wenu dieselben ins Grosse arbeiten; c) wenn sie in der Nacht ein neues Netz verfertigen; d) wenn sie die alte Hlaut abstreifen. - Bestïndig schönes Wetter, wenn die Hängespinnen die Hängefäden neuer lewebe lang und weit spinnen. - Veränderliches Wetter, wenn es nur wenige Hängespinnen gibt, oder wenn sie nur schwach und im Kleinen arbeiten. - Regen, wenn man gar keine Häıgespinnen sieht oder wenn sie gar nicht spinnen; wenn sie die Hauptfäden nur sehr kurz anknūpfen. - Wind. Wenn sie gar nicht spinnen, deuten sie auf Regen und Wind. Spamen sic nur die Speiehen des Rades, ohne die zirkelförnigen Fäden un den Mittelpunkt desselben herum anzulegen, so ist der Wind vorübergehend und wird sich in 10 bis 12 , Stunden wieder legen. Zerreissen sie plötzlich ein Viertel oder ein Drittel ihres Netzes und kriechen dann in ihren Sehlupfwinkel, so wittern sie einen Sturmwind, der aber bald nachlassen und das schöne Wetter wieder herbeiführen wird.

Winkelspinnen. Es deutct auf schönes Wetter, wenn die Winkelspinne aus ihrem Gewebe uns den Kopf zeigt, und die Füsse weit hervorstreckt; wenn dieselbe ihre Eicr legt, welches sie in heissen Jahren sieben Mal thut. Beständig schönes Wetter. Je weiter sie die Füsse

* Einleitung in die Entomologie von Kirby und Spence, deutsclı vor 0 ken. 1. Vorrede $\mathrm{XV}$. 
hervorstreckt, desto schōner und anhaltender wird das Wetter, wenn sie in der Nacht ihr Gewebe vergrōssert. Regen. Wenn sich die Winkelspinne in ihrem Gewebe ganz umkehrt und uns ihren Hintern zeigt, folgt anhaltender Regen.

Winterspinnen. Kälte. Wenn die in den Wohnungen über winternden Winkelspinuen hervorkommen, stark hin und her rennen und um die bestgelegenen, schon fertigen Gewebe kämpfen und davon Besitz nehmen; wenn dieselben ganz neue Gewebe spinnen, stark arbeiten oder wenn sie in der Nacht ein oder mehre Gewebe übereinander verfertigen, deuten sic auf heltige anhaltende Kälte mit Eis, die gewöhnlich nach 9 bis 12 Tagen eintreten wird. - Bekanntlich gelang Pichegrü's Einrücken in Holland auf cine solche Eis - und FrostProphezeihung einer Spinne.

Schon lange hatten wir vor, unsere Spinnenthier-Fauna von Bayern (wahrlich keine lcichte Arbeit, wenn man bedenkt, wie schwer es ist, die Jungen, Mann und Weil etc. zu unterscheiden; desshalb allein man sie lebend halten muss) herauszugeben, wenn uns die Nittel nicht gefehlt hätten; denn sol che Specialia Naturac finden bei uns keine Unterkunft. Haben wir doch ein Werk nicht an den Mann bringen können in Joco, das Sr. K. Hoheit dem Kronprinzen Maximilian y o $\mathrm{n}$ Bayer $\mathrm{n}$ seine anfängliche $\Lambda$ usrüstung verdankt, und welches unter dem Titel:

„Systema Insectorum auctore Dr. J. Gistel. Monachii apud

Fleischmann. 1837. I. Coleoptera. 8. 64, 1 Taf.“

erschienen ist, wovon $\mathrm{O} k \mathrm{k}$ n sagt:

Wer wird an dem grossen, ja unerlässlichen Bedürfniss eines neuen Systems der Insekten zweifeln, da wir seit mehr als 30 Jahren nichts der $\Lambda$ rt erhalten haben? Dass sich dalıer einmal Jemand daran machte, war höchst wünschenswerth, und man muss dem sonst schon als fleissigen Naturforscher bekannten Verfasser die Gerechtigkeit wiederfahren lassen, dass er hier mit ungewölnnlichem Fleiss sich an dic Arbeit gemacht hat. Es sind eine Menge Schriften verglichen, deren Daseyn, wir müssen es gestehen, wir nicht einmal kannten; bei jeder Gattung sind alle Schriftsteller citirt und alle Abbildungen angegeben, welche vorhanden sind; ausserdem hat der Verfasser die zahlreichen Sammlungen in Münclien und Regensburg selbst durchstudirt und zum Theil bestimmt, so dass man viel Neues in dem Werke findet.

Die Charaktere der Ordnungen, Abtheilungen, Zünfte, Familien, Sippen und Gattungen sind vollständig mitgetheilt und durch guten, abgesezten Druck mit verschiedenen Schriften herausgehoben; auch die sonst mit Unrecht so vernachliissigte Grösse des Leibes ist angegeben. Es ist daher in dem Werk alles beachtet, was man davon verlangt.

Fine Kritik über das Einzelne anzustellen ist bei einer so neuerlichen Erscheinung nicht möglich und kann überhaupt erst nach einer Reihe von Jahren stattfinden. Es sollten sich aber mehrere mit dem Verfasser vereinigen und sich in die Ordnungen theilen, wodurch das Werk gewiss schneller befördert werden könnte. Indessen kann man erwarten, dass die Käfer hei dem bekannten Fleisse des Verfassers nicht lang auf die Vollendung warten lassen werden. 
Diese Lieferung enthïl die Sippen Mantichora, Amblyeheila, Omus, Platyeheila, Megacephala, Oxycheila, Kiresia, liuprosopus, Cieindela mit einer Menge Gattungen. Man kann auf jede Seite 5 reelnen, mithin bis jezt ungefähr 300 . Da die Ileftc so sehmell folgen sollen, so wird man in kurzer Zeit im Besitze der Küfer scyn, und das ist vor der Iland eine schr grosse Erleiehterung für die Entomologic.

Die Tafel enthillt in guten Umrissen Muster von 7 Sippen mit den einzclnen Theilen, liresswerkzeuge, Fühlhörner und Füsse, in Kupfer gestochen von A. Schleieh. Min darf dem Werk glüeklichen Fortgang wünschen. (Isis 1837, p. 25S-59).

Wir inüssen gestchen, dass wir keine Hand mehr angesezt laben an dem System seit dem Erseheinen der zwei Hefte, wovon das erste auf líosten S1. K. H. des Kronprinzen von Bayern, das zweite 1838 zu Bern gedruekt wurde. Beide enthalten die Familic der Cieindeliden vollstindig mit vielen neuen Gattungen. - Neid und Schmïhsucht luaben auch dieses mit ihrem Geifer übergossen, wie Alles, was wir, seit wir sehreiben, ans Lieht gebracht. - Darüber wollten wir immer noeh nicht klagen, denn das geht in der "spinnenfeindlichen“" Welt nieht anders. - Wir kennen aber anderes Gelichter, dis so gefïhrlieh Ist, wie jener Wicht, von dem Westenrie der silgt: Absentem qui rodit amieum, qui non denfendit, quodam eulpante, malignos qui eaptat risus hominum, famamque dieacis, fingcre qui non visa potest, eommissa treri qui nequit - - Darum baben wir nirgends Rulse noeh finder liönnen, stets im offenen Kampf mit den Feinden begriffen, wenn wir heimgekeht waren vou unsern Fihrton dureh das Leben, dureh die Welt. Wie fern liegt uns (viell richt kommt sie aueh nie) die Gclegenhcil, unscre Sammlungen gehörig aufstellen zu können, damit die ganze Naturgesehiehte uns ungebe, wie "Bacon's grosses Salomonisehes Haus". - Alles im Kopfe, aus dem Kopfe! - Doch hoflen wir aueh auf diese Zeit! Wärnend durehziehen Cieero's Wort? unsern Geist, unsere Seele: Ihaec studia adoleścentian alunt, senectutem obleetant, sccundas res ornant, adversis perfugium ac solatium praebent, delectant domi, non impediunt foris, pernoetant nobiseum, percgrinantur, rusticantur * * "

Lady GIanville wurde nach ihrem Tode noeh für himverrüekt gehalten, und man wollte auf den Grund ihrer Verrüektheit, welche dureh niehts anders bewiesen war, als dureh ihren Eifer im Kerfsammeln, sogar das Testament dieser Dame umstossen **.

* Das Elend wird mir nach und nach so prosaiseh, wie ein Kaminfeuer; aber ich lasse doelı nicht ab vou meiuen Gedanken und ringe nit dem unerkaunten Enerel, und sollt' ich mir die Hiifte ausicuken. Es we is kein Menselu, was ich the und mit wie viel Feindeu ieli kinpfe, um das IV enige hervorzubringer. Bei meinem Streben und Streiten und Bentilien bitt' ieh cuch, nicht zu lachen, zuschanende Gütter. Allenfalls lieheln mügt ihr und mi॰ beisteheu. Göthe an B ätty.

$\Rightarrow$ So Harris in der Anrelian: The Aurelian: or natural history of english Inseets; namly Moths aud Butterflies. Together with the Plates on whieh they Feld ete. By Moses Harris. London, printed for the autor 1766. $1 \mathrm{Pl}$. 
Solehes gesehah in dem glüeklichen (metamorphutisehen) 7eitalter Swammerda m's, Willughby's und $\mathbf{R}$ a y's, welches melire grosse und eigenthümliche Geister, Le euw en hoek, Hooke und Martin Lister in Jingland, Redi und Malpighi in Italien, in Holland dic Frau Sibylla Merian; Hans SIoane, Petiver, Dalé, Bopart, Bradley und D andridge, hervorbrachte, die dem gegenwärtigen verbesserten Zustande der Wissensehaft das Thor öfneten, ehe der grosse L in geboren ward (1707) und dis Dietum: „Omnia ex ovo" dureh den unsterblichen Harvey die Lehre von der Generatio aequivoea zuerst stürzte.

Auch jezt wäre solches noch möglich in dem Zeitalter halbwissentlieher Oberfliehlichkeit, des Herzensbankerott, der Sellstsucht, religiösen Intoleranz und Irrthums, voll alter tiefgewurzelter Vorurtheile und Verachtung alles dessen, was die Wenigsten verstehen und unter diesen ein Hïuflein nur!

Die Spinnen zu sammeln, gilt man noch für hirnverrückt, als mit den Parzen selbst umzugehen, denen diese Creaturen heilig. - Wir aber, lireunde der Natur und Du liebenswürdiger Prinz, wir wollen uns nicht irre machen lassen von dem gemeinen 'Trosse und nachfolgen dem Beispicle Clerek's, Martyn's (Swedish Spiders), Albin, Cuvier (im Magas. eneyclop.), Pall a s(Spieileg.), Villers, R oesel, H erbst, Rossi, liriseh, Degeer, Sehrank, Lister, Seopoli, Schrank ete. und allen neuern, die diese verschmähte Thierclasse zur einzeln belielsten Wissensehaft hereits erhoben.

In Betreff des Lexikons selbst haben wir nichts mehr zu erinnern, als was $\mathrm{Oken}$ sagt: „Es wäre gewiss ein sehr verdienstliches Werk, wenn cinmal Jemand ein systematisches Verzeichniss aller derjenigen Kerfe verfertigen wollte, die in nicht systematisehen Werken vorkommen" (Isis 1834, 278). Und den Wunseh auszudrüeken sey uns erlaubt, dass ein jedes Land ein eigenes ausführliehes Adressenluch hahen mōehte. Nie wird ein allgem eines frei von grossen Irrthümern scyn.

Die Herren Naturforseher, Sammler und Händler von Naturalien werden wiederholt aufgefordert, ihre Adressen vollstïndig an den ReJakteur dieser Sehrift einzusenden, die Irrthümer darin anzugeben und das Fehlende $2 u$ ergänzen. Es darf wohl nicht erst bewiesen werdenl, dass solch ein gemeinschaftliches Verfalıren Nutzen für das Gesammte und das Individuum bezweeke. Man ladet daher zu thätiger Mitwirkung freundlichst ein.

$\mathbf{X}$ and $108 \mathrm{pp}$. 40 Plat. Fol., unter Papilio Cinxia. Der berihmte Ray († 1705) musste darüber zu Exeter vor Gericht als Zeuge wegen ihrer Gesundheit erscheinen. 


\section{Adressenbuch der Arachnologen.}

Alt, Dr, med. in Bonn. De Phthiriasi. Bonnac 1824. Fig.

Be-11, Thomas, Esy. in London. A ranea domestica (Zool. Journ. 1. lsis $1830, \mathrm{p} .418$ ).

MIack wall, J., zn Edinburgl. Beschreibungen von Spinnen (Brewst. Lond. ant Ed. plit. Magaz. V, 1s34, p. 50). - Charactere never Spimuen (ihill. VIII, p. 481 et $X$, p. 100. 1833 , III, 104. - Isis 1835, 574). - FlieKrnder Sommer (Isis 1829, p. 1277). - Ueber die Zahl und den Bau der Spinnwaryen (Linnean 'Trunsact. Vol. XV.III, P. II, 1839， p. 219-225. Isis 1843, f1. 437-39). Vgl. feruer uber die Untersuchungen dieses unermiidlichen Forsehers: Thirl meeting of the british Assoeiution at Cambridge 1833, p. 445. - Researehes in Zoology, 1834, p. 298 und lie Transaetions of the Liunean Soeiety XVI, p. 473, T. 31, F. 2, 3. - Ueber die Huusspinnen. Bemerkungen (Brewster Lonı. n. Ed. phil. Man: Tayl. ete. I, 1832, 95. Isis 1835, 559). - Beschreibung einer neuel Spiune (ibid 190, - Isis a. e. p. 568). - Vertheiligung gegen Virey (Bullet. d. se. 1. 1829, p. 131) wegen der luftsegelnden Spinnen (Phil. Mag. X. - Isis 1834, 827). - Ueber die Art, wie die geometriselıen Spimmen ihre Netze bauen (Loolog. Journ. V, $1830, p .181 .-$ Isis 1832,659$)$.

Bory de Sriat-Vincent, J. B. M., in Paris. - Note sul une espéce d'Aearide qui vit sur le corps lumain. 1823. - Neue Hautmilbe ( $A$ un. Il. sc. $\mathrm{XV}, 1828,125$, T. 1. - Isis 1832, p. $904-5$, T. 18).

Braudt, J. Fr., Akademiker etc. z.u St. Petersburg. - (mit Ratzeburg) Arachuiden (deren Med. Zool. II, 1833, 84).

Irauts, A., in Rotterdau. - Beitrag zur Kenntıiss der einfachen Augen ller grgliederten Thiere ('Tydseluift for natuurlyke Geschiedenis, uitgegeven door J. van der Hoeven en W. H. de Vriese. Amsterd. 1836, IV. Bd., p. 135. - Isis 1840 , p. 379. Mygale ete.). Irelbissou, in Paris. - Catulogue des Aranrides (in Mèm. Soe. Lim, de Normintie. 1826).

BBurmeister, Herm., Professor 7u Halle. - Ueber die Gattung Aclysin (Aud.) (Sehmarotzemilbe, Hydraraclme, an Schwimmkïfern) (Isis 1834, 138, Taf. 1). - Grmmiriss der Naturgesehichte. Für Gymmasien und höhere Bürgersehulen. 2. verb. Aufl. gr. 8. Berlin.

Cancellieri, in Neapel. - Tarantelstich (1sis 1833).

Corolan, iи Ediuburgh (?). Die Kunst der Spinme (Tlompson's Annals of philos. Apr. 1817, - Uebersctzung im Morgenblatt 1817, 13. Aug.)

Cnras, C. Gust. v., Medizinalrath etr. in Dresden. - Lebensbedingungen kalt- und weissblutiger Thicre. Leipzig 1824. - Vgl. alle Lexika.

Corila, $\Lambda$. J., Dr. und Custos zu Prag. - Eine fossile Gattung der Afterseorpionen, welchesteruber g in einem Steiubruthe anf seiner Herrsehaft Ralnitzontderkt lat: Mirrolabis Sterubergii nebst Obisium ranroides (Verhandl. d. Ges. d. vaterl. Mus. in Bölımen. 1839, p. 14, T. 1. lig. 1-6). Cotta, B., Forstratl in Tharand. †. 
- Ueber Julus terrestris als jugemiliche Versteinerung (v. Lemohard und bromis Neurs Jalubuch 1833, Nr. 4. 1. 392-394, Tal. V. f. 1, 2. - Fionue Cephalopodimm ant tritarum concome. rafum Genus? Gottl. Fiseher von Wallheim: Bibl. Pillaeontol, p. 372 ubi etian de Gistelii Insectis in Copalo inclusis ment. fact. p. 372 et iu v. Leonh. mul Bromn's N. Jahrb. 1833, Nr. 6, p. 712).

Cross, in Ellinhurgh. - Dor Er. zenger "mikroskopisther Insekten" aus morganischen Suhstanzen, \%. B. Alsenihanfliismugen, Kiesslsimue. unter Mitwirkung starker elektriselorr Ströme, etwa 1836 (Morgenhl. 18.37, p. 269)! (Sarcoptes ouler neur; maeh Perty)."Iurpiol hat lit: van Cross hervorgebrueliten Millen Aeari horridi benannt (Compte rendu. Froriep's N. Noti\%. Ni. 89).

Dama, in New-Haven(Nordamerika). - (Bit Welploy) Ueher zwei amerikinisclie llydiarhisae, gofunden in eines Siismassormuschel (Sill, Amer. Jonrn. $\mathrm{XXX}, \mathrm{Nr} .62$, p. 275 , fig.). - (mit Herriek) Besthrcihumg les Argulus Catastumi (ibill. XXXI: Nr. 63, 1837, fig. - Isis 1840, 173).

HDenuy, in Londou. Monographia Anoplurorum Brittaniate. Lond. 18.42 (Apteren).

Deshayes, G. P., Dr. med. in Paris.

Desmngest, Gaëtan A., der Vater, Professor an lur Veterinäirschule zu Alfort ete. zu Paris (Ruc Saint-Jaeques Nr. 161).

Dierbnch, J. Heinrich, Dr. med. ete. z.l Heilelberg. - Uebersicht der gebräuehlichsten Árzneinittel des Alterthums ete. Neuntes Kapitel. Mediramente aus der Classe der Seorpionen, Spiunen und Asseln (Isis 1842, 103105).

Dix. Frl. in Philadelphia. - Ueber Aranea aculeata ete. (Sill. Am. Journ. $\mathrm{XIX}, 1830 ，$ p. 61. - Isis 1832, 1061).

Doubledny, Edwards, in London. - Bearbeitete mit Adam White die Insekten zu lem Werke: The Zoology of the voyage of II. M. S. S. Erebus and Terror.

Donue, Dr. med. in Paris. Reeherches mieroseopiujues sur le nature des mueus et la matiere des di. vers croulements des urganes gemito. uinuires clic\% l'homme et ilie\% lat femme. Paris 1837 (Acarilla).

Doveses, 11., am sirhiff. Ind Vi. litilimusenm zu Whitehall (England).

MuTour, Lébu, Arat zal Saint-Siver im Dept. Iles fandes. - Ueber Lyocosa tarantula (Anu. d. sc. nitt. III, 1835, p. $95-108$. T. $5 .-1$ sis 1836 , p. 521). - Nine Spinue (Epeira spinivulva) (ibitl. 110 , Tf. 5 , f. 5 . - lsis 523 ). Naturgeschichte der 'larantel (L'lustit. 183.3, 14). - Beschreibung mind Ab. bilubug des Ptcroptus vespertibionis (Ain. d. se. nat. XXVI, 1832, p. is. Tith, - Isis $1835,500-511$. 257). A ranca (Aun. d. se. nat. 1830 . - Isis 1830, p. 776). - Neur Milbe, Tetranycluns lintearius (ibill. $\mathrm{XXY}, 1832, \mathrm{p}$. 756 , T. 9. Isis 1835, 467), - Carrillus erhinipes (Zwischending von Platlanginm und Milbe) (ibid. p. 289. 'T'. 9, โ, 1-3. - Isis 1835, 169). - Nirue Aralleiden (ibid. XXII, 355, T.,10, 11). - Beselurcilumg der Nycteribia Vespertilionis (ih. 372 , 'Taf. 13. - lsis $1835,185)$. - Einige Phalangia ans Spanien (ih. 385, T. 10). - Finlen van 'l'lomisus (L'lnst. 1834, 206.' Isis 1837, 708. - Mit Walekeuaer und A ulouin angestellt.) - Sirlumarotzer-Instekten. Athmung deroshen (L']nstit. $1836, \mathrm{Nr} \cdot 165$. Isis 183\%. 777).

Donja rollien, Dr. med. unul Mitglied der Suciete philomatiyue in I'aris.

Eillwarals, Präsident in Plıiladelphit, - Spinnentiilen an Bäumen (in dessen "Wurks" 1829).

Elerenhorers, Hofrath etr. in Berlin. Der Rerisemule in Afrika, Sihirim etc. - Symbolac pliysicae (mit 11 em prich). - Vorläufige Ueber'sicht der iu Nordafrika unt Westasien eiulneimiselien Srompione unt deren geo(rophliselse Verbreitung (Verlandl, d. Ges. Iatf. I'r. Berlin. I, Heft VI, 1829, 348).

Eichurald, l'rofessor in Wilua. Ueber Strabo's okroliykes (Sehneewiirmer) (Isis 1844,686 . Teleph, fusc. larv.). - Veber ulie caucas. Seoppione IIId PhaIangien (Isis 1834, 686). Zoologia cameas.-l'aspira.

Figluts, James, M. D. in Boston. - Deseriptions of a new mimal helonging to the Alaclonitles of Latir: discoreren in the sca along the Shores 
of the new South Sluetland Islands (Bestou. Jonro. I, Nr. 2, 1835; l. 203 .

E'ischer von Wraldheiu, Gottbelf. Staltsath, Ritter, Professor in Muskinu - Notice sur l'Argas de Perse (Rlyyncloprion persicum) (Isis 1830, 1. 397). - Bihliographia Palacontliologica. Nosquac 1834. 8. (p. 305 unl зi2, wo der Vf. Julus zu den Arachniden zählt.) - Zoognosia. Mosquac.

Fitsingrer, Dl. med. und Custos an k. k. Mluse(un) in Wien. Ausgereicluneter Arachuolog.

MIourens, in Paris. Mémoires d'anatomic ot ploysiologie comparee. Par. 1844. - Anntomic rinirale de la pean et des membrane; muqueuses. Paris 184.3.

Forströgr, schurdischer Entomolog anf St. Barthèlemy (Antillische Inseln).

Grachet, zu Bordenux. Beschuítigt sich mit den Acariden.

Créne, Josephli, Professor derNaturg(sidhichte in 'Turiu. Sichriftsteller.

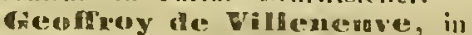
Paris, Solun des Verlassers der "Histoire abroge des lasectes, qui se trouvent atux envirous de Paris". Hiclt sich lange Zcit ant Sinnegal anf und brac!le vour dalher atuselunliche Sinmmlungen, unter deuselluren viele Sipinnen.

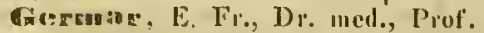
dor Mincralogie etr. zu Halle. - Fortsetzumg vout A. Alsensii Jaunal luseetorum lutapac (etum F, Kaulfuss).

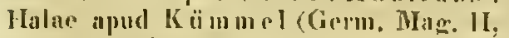
1. 331). Virle Ilefto ill 16. - Reise in Dilmation und it das Grbiet vou Rnguser. M1. () ill. Kpf. und 2 Chart.

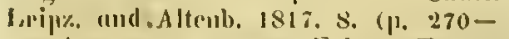
72. Arauca 13 - guttatia Fabr., Turan. tula. Ar. 4-guttata Rossi. Scorpio europicus und (arpathicus Limu.)

Gervanis ill Palris. Hist. Mat. des liseetes Apteres. Par. 1844 (3. Bd. zu Walckenacr).

Gistel, Dr: phil. ete. zu Müuchen. - Beobachtengen und limmerkungen ete. (Faums Bel. 1), 1835: TJvegale avienlaria (85). - Krenzspiunconindastrie (sib) - Tod an Senpionssticl (97). - Tarantel zälmubar (158. Silleditaru, das lleilbad und Enge- bumg. Gedenkemeiu für Kurgüstc. Mincli, 183\%, 8. eft.

Trirife, lleiur., Di: und Direhtor etc. zu Jona. - Natnrgesehichte nach allon drei Reichen fiir Srluule und Haus. In Verbiudung mit J. F. Nanmann bearbeitet. gr. 8. Eislebel. 1834 -18.88. - Naturgeschichte der drei Reiehe. I. Bd. Berl. 1841 (Hermes). 8. (Ausgrezeichnet).

Frebills, in Barecllona. - Biss und Sitten der Malmignatte (Theridion 13 guttatım) (L'Inst. 1834, 243. - Isis $1837,712)$.

Curess, Albin, Dr. phil. und Spitalassistent in Paris. - Rrecherches sur l'Acarus de lit Gale. Paris (Déehet) 1834. 8. 35 (1sis 1837, 225. Surcoptes Scalbiei). - Ueber die Jrätmmilbe (Sarcaptes bominis, Cirou) (L'lustit. 1836, Ni. 169. - Isis 1837, 784).

Gìraventuorst, J. L.. Geheincl Rath rte. zu Birslan. - Mädelıersummer (Isis 1823, p. 378). - Abstossung ciner luaus (Isis 1824, p. 799).

Firny, Georg Robert, Esy., Custos am britischeu Museum ete. zu London. - Arachniden in King's Survey to Australasiı.

Grolanann, Eranz, Naturalist in Pallerimo.

Trasue, D., Professor zu Dorpat. - Palpen der Spinuen etc. (Müll. Arels. 1842, 300. - Preuss. Provinzialblïu. 1842, 1. 3.13. - Froricpis 11. Nutiz. Bd. 24. p. 343).

Guérin-réneville, L. E., Protessor zar Paris. - Besrlarcibulingen und Abbildungrn vou Spinn(') und Kerfen (Guer. Hiar. 11, 1832).

Gine.lt, Professor zn Berlin (vgl. Lexik. Ier Theriologem). Vergleirlsende: Untersurlungen riber dic Hant des Menschron mol der Itanssïugrothiere und äbro die Kräizmilben ofe. Berlin 184. - Ueber die anf den Haussãugethieren uul Jinusvögelu leboudenī S.lumarotzorinseliten und $A$ rachniden (in dessen und Inctwig's Magraz. f. d. ges. Thic plukde. VIlI. Jahrgeg. Ileft 4 und IX. Jahrg. H. 1).

Tran m, W. de, Custos ete. in Leyden. Heraukgebor van J younet's Opris posthumum. (Vgl. L, yan et.)

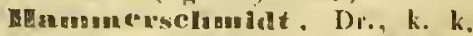
Ilofliammerprecuratur-Beamter in Wirn. - Nene und beliannte Spimuen vou Bia. den leci Wirn (Jsis 1834, 746). 
Treuson, Sanuel, Esq. in Lnudon. Hartwig, Prof.iu Berlin. - Uebrer die Schafrandenmilben (Mag. f. d. ges. Thierlieilkunde. 1835).

Heeger, Magistratsrath zu Wien (vgl. Kollar).

ixenle, Dr. med. in Zürich. Acarus folicularum (Oeffentl. Beob. Ziirich 1841. - Mïller Arch. 1843, XV). Vergl. S im n n.

Hentz, N. M., Professor in Philadelphia. - Note concernant l'Araignec dont la toile est employe en médieine. (Journal von Pliiladelphia. 1821.) - Ueber nordamerikanische Spinnen (Silliman's Amer. Journ. XXI, 99-109. - Isis 1835, 89).

Hering, E., Obermedizinalrath, Professor etc. in Stuttgart. - Dic Krä̈zmilben der Thiere und einige verwandte Arten, nacl eigenen Untersuchumgen besehrieben (Verhandl. der kais. leopold. earol. Akademie der Naturforscher. Boun, XVIII, p. 573, Taf. $33-45$, ill. - Isis 1839,441 ).

Merold, Martius, Dr. und Professor der Medizin in Marburg. Exercitationes de animalium vertebris carentium in ovo formatione. P. 1. De generatione arauearum iu ovn. 1824. - Untersuchnngen viber die Bildungsgesehichte der wirbellosen Thiere im Eie. 1. 'Thl. von Erzeug. d. Spime in Eic. M. $4 \mathrm{Kpft}$. (deutsch. Marhurg 1824, Fol.: latein. Franeof. 1835. Fol. - Physioloyische Untersuchungen iiber das Riiekengefïss der Insekten (Schrift. der Ges. жII Marburg 1823, I (Bd. 41).

ILerick, iu New-Haven (N.-Am.). Vgl. D a

Heyden. C, v., Senator in. Framkfurt. - Acuriden (1́sis 1826, j. 608).

Hoceven, J. van der, Professor in Rotterlam. - Phrymus (Tydsclur. vonr nat. Gesehicd. 1842, p. 68. - Müller's Areh, 1843, XIV).

Hope, $\mathrm{F} . \mathrm{W}$., Rrv. in Londnn. Nenes Kerf misehen Gonyleptes mod Phalanginm (Trans, of linn. Soc. XVlI, 111,1836, p. 397 , Tab. 16. - Isis $1838,413)$.

IIorm, in Heidelberg. (Archiv f. med. Erf. I, 1812, 1. 544)

Hussey; Th. J., in Edinburgh. Ueber die Acrutewnne (Acarus autumnalis) (Brewst. Juond. a. Ediub. phil. Mag. IX, 15. - Isis 1838, 90).

Jückel, J., Stud. Theol., in Nürn- berg. Küferkenner. Sanmelt feissig und zeichuet und malt wunderschöı.

Jofre, Henri, Dr. med. "n Villpnenve-de-Berr (Artèche). - Reelierehes sur la propriéte qu'à la toile d'araignèe de conper les fievres intermittentes.

Jordano, Don Iguazio, in der Abtej von Poblet in Barcelona. Sammelt fleissig auch die Spinnen.

King, Phil. P., Capitain in London. Sammelte auf der Expcrition nach den Küsteu des Polarmeeres (Capt. G. Back). Vol. Reise durch Nordanerika etc. Ueber's. v. André. Leipz. (Weber) 1836. - (Auszing: Faumus 11 ll (1), 1. 28-82. Parasita).

Iirby, William, Rector von Barham, in England. Beruhmter Entomolog und Scliriftsteller. - (mit S pe ure) Elements of Entomology. - Sitten der 'There (Bridgrewater Stiftung).

Iittel, B. M., Prof. in Asehaffellhurg. - Mèmoires d'histoire maturelle. l'aris 1826. 8.

IĹpsch., J. E., Gymnasial-Lehrer in Breslitu.

Kucln, C. L., Forstrath in Regensburp. Gefeierter A rachuolog; der Co. ryphäel einer der gesammten \%oologischen Forsehungen. (Vgl. Fïrnrohr: natırh. Topogr. v. Regensb. I, p. 57-60.) - Deutschlands Crustareell, Myriapoden und Arachuiden. Regensh. 1835, 16. (eirca 40 Hefte; a 20 col. Abh. - Auch ill HI errie li's Fauma, 120-134). - Dis Arachuiden. Getreu naeh der Natur abgebildet und beschriebell. Nïrnber (Teh), (Fortsetzung des hek. Weriss von Ha hu). Bd. 1II. Heft 1, 2, 1836. 8. (lsis t836, 628). Bis jezt geyen 20 Helte (a 6 Taf, col.) - Uebersiclit des Arachnidensystems. Mit vielen Kpf. Nürub. 1837. - Crustacea, Myriápoda et Arachinides (in Fürnrolir's, des gediegenen Botanikers, naturh. Toponr. Regensburgs. Bdl. 11, 1840 , j. 387459).

I ols Irancelı, Dr. med. in Rinteh. - Weise, zn beohachten, wie Spinuen Fitien aus ihren Warzen treiben (Pyr. mouter Versammluner 1839. - Isis $1840,923)$.

Ifollar, Vine., Inspektor zu Wien.Ueber Monographic der Phalangien (mit He eger herautsgegeben) und Vorzeig. von 50 Taf. (Wien. Vortrag. Isis 1833, 394). - Brasilicns volzüy- 
lich lasstige Insekten von Dr. J. E. Pohl und $Y$. K. Wien 1832, 4. 20, T'. 1, Fol. ill. (Isis 184\%, 238). Aufgefülurt sind: Mymale Blondii, Thelyphonus proseorpio, Scorpio, Scolopendra, Pulex, Ixoles, Thermes ete. (vgl. Faunus vou Gistel).

K Fdom nun, D. W., Lelırer aı der Garnisousschule zu Hannover.

Krymicky, Jeal, Professor ro Kliarkoff. - Arachuographiae rossicae Deens prima (Bullet. de Moseou 1837 , V, j. 73, T. 5,6 , ill.).

Comloatte, H., zu Brüssel. Amatomie der Aranca guttata (L'Instit. 1838, Nr. 229, p. 163. - Isis 1839, 368).

Hangsulore, G. H. v., k. k. russ. Consul zu Freibury (13.). - Sehrieb über die Vogelspinne (Mygale), wenn wir nicht irren, in seiner Reise.

Lefedove, Alexander, zu Paris. Ueber 'Theridion und andere Spinnen (L'Instit. 1834, Nr. 243, - Isis 1837, 712).

Mapricent, Plıamazent in Paris. - Reisender und Sammler in Afrika (Senegal).

Mewis, R. II., in London. - Explanition of the sodden appearance of the Webspinning Blight of the Apple, Hawthori ete. (Transs of ent. Soe. Loud. I. p. 7 ).

Lincke, Lehrer an der höhern Biirgesschule \%.u Stettin.

Hopez, Don Silvador, Almosen. ptlegec in St. Antonio de la Florida bei Madrid.

Muvetzlyy, A., in Moskau.

Howe, R. T., in Jundlon.

Lucas, H., Maler und Assisteut an Mustum zu Paris. - Essai d'uno Monographie dı 'Tlhclyphone (Guér. Magas V, 1835. - Isis 1842, 2111. Beselurviluung und Abbildung von llersilia caudata, indica, Saviguyi, Pacliyloscelis fulvipes, rufipes et nigripes, Audoninii, tarsalis, Attus venator (ib. 1836, V, Taf. 12-14).

Mncenry; Ange, Dr. nued. in Nizza. Entomolog und Schriftsteller. - Mi. moire sur le Seorpion de Cette. 8.

Mac-Heny, Alex., in Havanual. - Ueber Urania und Mygalf (Thericphosa Roclsefort) ('Trans. of Zool. Soe. 1, 1833, p. 197. - Isis 1836, p. 412). - Unher die Naturesehichte der My. gnle anf Cuba (Lond. «. Lidinb. phil.
Magaz, IV, ¡. 460. - Isis 1835, 604).

Vraile, Arsenue, zu Rouel. Sehöue Sammlung vou Kerteı und Spinnen.

Marclictti, Salvagnoli, Dr. med. in Florenz. - Ueber eine giftige Spinne (Dysdera crythrina) (Isis 1842, 254). - Ueber Alanea Savi (Aranea guttala Rossi; Theridion) (Isis 1842, p. 262. Vorgetragen iı der Turiner Versamm(1115.)

Mnerlog, C. P. J. B., Medicus il Auriol bei Marseille.

Metaxa, Telemaco, il Rom. Milben der Schlangen (Isis 1827, 491).

Micucher, Professor in Basel. - Acarus tolliculormu (Bericht vou Basel, 1843, p. 191). Vtrl. Heule, Si 110 il.

IIIII er, in Londou.

Milinleupfored, Dr. philos. und Lehrer der Zoologie mol Botanik an der hölern Gewerbschule zn Han. nover.

Minler, Johannes, Dr. med., Professor und Direktor za Berliu. Ueber dit Augen and das Sehen der Insekteu, Aracluniden und Crustacesu (Ann. d. sc. llat. XVII ef XVIII, 73, T. 19). - Allomoruane derSpinue (Isis 1828, ¡. 707 , T. X). - Netzbau dev Spinnen (Isis 18:28, 711 ).

Vordimfun, Alexander v., Professor der Naturgesehichte am Lyeeun Fichelien in Odessa. - Mikrogrnplsi. sche Beitrige \%ur Naturgeschiehte der wirbellosen Thiere. 2 Hefte. Mit 20 Kpftaf. gr. 4, Berlin.

Dken, Laurentius, Hofratl, Akn. demiker und Professor in Zürijl. Preisinforabe (Bestimmung des Kerfe iu Réa um a r's Mèmoires, ,ler Bibel niber die Insekten" (Isis 1835, 479) and die bekannten horlucesehitzten Verke. (Lis ist löchst autfallend, dass bisher Rean. mu's elassisches Werk wieht sowohl völlig h r a ac lo fïr die IVissenschaft als auch (bis in die neneste Zeit durch Oken's Anregung die Lepielopteren durch Hrı. Z elle r's vortuefflicie Bestimuungen) ganz ungedeutet geblieben. Man sicht hieraus deutlicl, dass es wolıl eine Menge Sammler, aber wenige Beobneliter grebe, Unserur Wenigkeit scluwebt es glrich eiuem Zau. bertraune vor, eimmal su glieklich 
acyn zu künnen, "u der Beohaeltüg zu leluen. Dii fiuvent! G.)

(D) foiz, Plilipp Maximilian, vieler gelchrten Gespllsedaften Mitglied cte. zu Prag. - Jiner der häitigsten Minner Bälomena für Verbreituror roologiveher Sammlunges: nod pliytolog-isclier zumal, dessen "Beitrige" zur Niturgeschichte (aud Nituralientausch) die מoliegrenen Arbeiten des genialen Co r d a selımücken.

Perleb, Call Julius, Professor zu Freibure im Brcisgan. - IIaudbuclı der Zoologie.

perty, Maximilian, Professor ete. zu Bern. Grosse Arachnidensammlıng. - Zır Fanma monacensis (Araclunideu) Isis 1832, 728-733). (Die Fortsctzumg ist $111 \mathrm{~s}$ der geistige Verf. seluuldig ge. blieben, da sie versprocile war. Wir haben in frühern Zeiten auch Manelies versprachen, was wir bis jezt nicht haben halten kömen. Die Jeutchen hinterm Ofen schmähen dariber! Man grehe iluen rii) su stiir. misches vielbervegtes Lebrn und sehi danu, oh sie je noelı das leisten, was wir Unbedeutendes in die Welt gesehickt habru scoit muserm 15. Jahre his jezt. wo wir 35 zählen. (Gistel.) - Delcetus amimal. artic. Brasil. Allememue Naturesenichte als philosoplische und Humanitätswissenscluaft. 3 Bile. u. Suppl. urr. 8. Bern, Fiseher. - Die Welt daif nur Grosses vou diesem Forscher erwaten.

Henumblex, F. A., Dr. med. \% Selıloss Beirlaling(n) (Thiiringen). Vel. l.ex. dor Eutomologen.

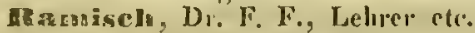
in Prag. - Naturepeschichte des Thier-

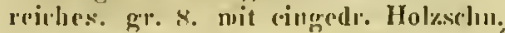
Pritg. Hiaks. 18.30.

Framzorf, in Kelensk (Gouvernement von Ponza). Eintomolog.

Easpris, F. V., Dr. med. in Paris. - Memaire cumparatit sur l'histoire nafurelle de l'insecte de la gale. Paris 1834 (deutselı, mit Zusiitren und a॥gefiulten benucrumgen vou, "G. K." (Isic 1837, 224 Sarcoples Sc.)

JR athlice. Heinrich, Professor ete. zII Königsberg. - Lycosa saceala (Froriep': neue Notizen. Bd, 24, 1, 165). - (et Berendt) Inscetir in Suceino inclusa diligontissime collegerunt, ita ut Colenjtera 700, Hemiptera 57, Pa-

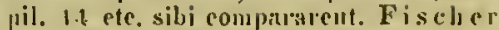
Palacont. P. 305 (Isis 1829, 41.i). Zur Morploologie. Reiscbemerk. ans Tauricu. Riga etc. 1837 (Entwickelung (les Seorpions). Froriep's Not. p. 517 $-18$.

lentzelonrg, Professou zu NeustadtEbcrswalde.

Reuss, Adolph, Dr. med. in Frankfunt. - Ucher Arachuiden (wenn wir niclit irren iu Musemm Senkenbergian. enthaltin. M. Kjuf.).

Inichter, Limsi Heiur., Lebuer \%u Fraukfurt n. d. 0. - Leitfaden der Zoolorie für höhere Büigerschuleı. . Friıkf. í. d, O. 1843. 8.

DEobinean - Pesvoidy, J. B., Arzt zu Saint-Saveru. - Ixodes melliuns und auricularis auf Dachspelz (L'Instit. 1836, Nı: 187).

IRosenheya. Uebej den flicgenden Sommer (Froriep's N. Notizen. p. 184).

HRossmüsster, E. A., Professor an der Forsischurle zu Tharand. Gallerio der Thierwelt, ein Atlas zur Uebersicht dis Thierreielss. 12 lith. Tafeln. Nacli der Natur eoloriot.

Gavi, Panlo. Professor in Pisa.

Sclamide, Joseph, Lelirer der Naturgeschichte in Ninelsen. - Die Spinuen als die besten Wetterjroplartimen. Minclt. 1801. 8.

Gelnaeibers, Carl v., Ritter, k. k. Renicrumosiath und Dircktor der velciujerten Hof-Naturalienkabinete, Akiademiker ete. 7. Wien. Eiu Jieblingssturlium dieses lunchgefeierten Forschers sind dic Spimnen und sciue Sammlung (und Abbildungen davou) ist die grösste in der V Velt.

Selnultz, Carl Heinr., Dr. med., Arzl und Profossor in Berlin.

Serdillot, Dr. med. in Paris. Bestandebeile des Spimgewrbes (linc. period. de la soc. de sante de laris. Tome XXI, p. 111).

Sicres, Marel de, in Montpellier. - Ueber die fossilen Ararluniden und lusekten der Süsswasserschichten von Aix in der proverre (Anu, d. st: nat. 1828, XV, 98. - I sis 1834, 1032). Tableau des instetes fossiles (ibid. p. $104)$.

Giehold, K. 'Tl. Is. vou, Professor in Freiburg (Breisgan). - Parasiteu, iu deu Handwörterbuch der Pliysio- 
logie, mit Rïcksielit auf pliys. Patho-|des societes et travaux scientificues de logie vou R. Wamner. Hief. XI. la France et de l'Etranger 1838, Nr. Branuscliw. 1645 (p. 659, Arachnida: 245. Sept. 1. 296. - Isis 18:9, 377). Acarina und p. 657 , Aptera).

Ginon, Dr. mrd., in Basel (?). Acarus folliculorum (Miill. Arch. 1842, 218. 18.13, XV. - Froricps 11. Notiz.. Bd. 21, \%. 2 (8). Vgl. Henle und Mifsellivil.

Stranss-bilrelkleciu, Hercule, Professor eti. zil l'aris. - Mémoire sur le Systeme timmmentaile ot musculaire de l'A raignce nvieulaire (MIygale de: Le Bloud, Lattr.) (Acarlém. des seient.).

Sinterdewall, Carl J., Dr. und Professor der Oekonomic, Intendant in Lund. - Conspectus arachnidum. Loud. Goth. 1833. 8. 39 (Isis 1834, 139. Seliwid. Arad. Sclirift. 1829 und 1832). - Specimen academicum general araneidum succiae exhibens. Lundic 1823. - Supnska Spindlarues beskrifuing (Kongl. Acarl. Haurll. f. 1829, f. 188220. 1831 . p. $108-149.1832$, p. 172 -273. - Isis 1831, Heft 12,6, 1358). - Ueber die Krätzuilbr (dessen Plıysiografiska Sällskapets Tidskrift 1,1837 , p. $28-36$, Taf. I, fig. 1-13. - Isis (84:, p. 440, p. 5).

Fineis, Charles de, Attache im Mimisferimu dos Answätigen \%u Paris. Arachologe Soust in Iaran. - Ueber ciuire Hydiachuac und Clocliferi (Anu. d. sc. unt. XXVII, 1\$31, 1. 57.)-1 sis 1835, 614-61\%. (Unbigens selucibt man Hydraratma, Rlịidicera eto.)

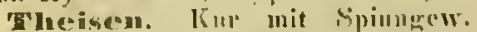
(Russ. Maga7. Xil. Sit. 2. \$. 351 ).

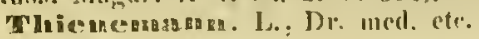
in Kopruluagen. - De Araro quodau iu Alęan nivali (Chiousplue deusa 'T'h.) vivente (Fö̀namallinual ved de skumdiuavislic Naturforsliere's andet Möde, der holdtes i Kjöhoubavn fra den 3 dic til deu 9re Iuli 18t0. Kjübent. 1841

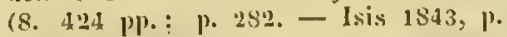
291 ).

Thicencunanu, Fr. Aug. Indw. Custos ete. zu Diesilen. - Vgil. Lex. d. Theriol.

Tilesines, W. G., Iofrath, Dr. med. und Arzt in Leipaig. Beriilumter Reiscrúler, Naturforsclier und Sehriftstoller?.

Traill, in Ediuburgh. - Ueher Argas persitus (1gl. Isis 1819, p. 1567, Tal. 19. - L'lustitut. Journal peneral| Dan a.)

trosclet, Fran\% Heruaul, Dr. med, in Berliu.

wifrcla, von, Scluulrath iı Potsdiul.

Null. Anatomie von Plalangium Opilo (Foriep's N. Notiz. 645).

remelin, Dr. med. in Paris. Ueber die lumfürmigen Auswülsce der Lindenblätter (Tilia platy plıyllos) (L'Inst. 1533, 187. - Isis 183\%, 31. (Sareoptes Gallurum 'Tiliae).

Einderwood, Mediens in London.' - Sammelte Arachuiden. - Soll todt seyu. Ob aber auch derselbe?

Vassetet, M., in Paris. War in Mexilio.

Vanthier. C., Maler in Paris. Description d'une nouvelle espdec d'Araduides ete. 1814. - Epeira eurvicauda (Isis 1830 , p. 385 ).

Deragner, Morix, Dr. med. in Augsbutr. Der. Reisende in Afrilia, Russland und Asien.

Evallzemaer, Baron Carl Athau. vou, Prösident des Imstituts re. \%u Paris (Rue de Paubourar-Poisonnidre Nr: 87). Der horibute Araclunolog. - Faume parisienur, inseetes, on histoire abrégece des insertes des cuviuous de paris, classces diupres le systeme de Fabrieins, precedie d'an disenus sur les insectes pru gencial. pour servif d'iutroduction

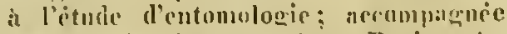
di scef plabelies gravies: Paris. Au XI ( 1502 ). 2 Vol. Cl.X of $301-438$. \$. 8. - Histoire maturelle des Alanciles. Paris 1806. 4. Livr. - 'Jableau dis Alancides. Paris 1805. 8. - Mimoire sur une nouvelle elassilication des Araneides in den Anualen der cutomol. Socrictä. - Classilication der Aruncidou (filust. 1833. p. 158. - lsis 1837, 311). - Araucides de France. (Ouvrage faisaut patie de celui intitule la Fiume liançaise public par MIM. de I3lainville. Desmarest, Vieillot ete. G. Cuvier. - IVit habeu ('s noch nielit geselieu.)

Desegles, Var de, in Paris. - Seorpio curopaeus (L'lnst. 1834, 199. lsis 1837, 708).

velpley, in New-Haveu. (Ygl. 
Westring, Zollverwalter in Gro- A praetical and theoretieal treatise on thenburg. - Ueber tönende Organe bei ciner Spinne.

the diagnosis, pathology and treatment Westwood, in London. - Be- (p. 368, Acarida).

fruchtung der A rachniden (Zool. Journ. WVinthem, W. v., Kaufuanu in XIII. - Isis 1830, p. 1257).

\section{Hamburg.}

Wlnite, Adam, in London. (Vgl. Doubleday.)

Wider, F., Pustor in Beerfelden am Odenwalde.

Wood, William, lisq. in London.

Zalpser, Professor zu Neusohl in

Wilson, Dr. med. in London. Ungaru. 


\section{Wohnorte der jezt lebenden Arachmologen.}

Ascinf Tremburg. Kittel.

Angsbures: Wagner.

Aurial (bei Marseille), Marlog.

Giarcellona. Grölls. Jordano. Bnalnave. Kirby.

Basel. Miescher. Simon.

IBecrfelden (Odeuwald). Wider. dolir.

Beiclningen (Thuingen), Ram-

Berlin. Ehrenberg. Gurlt. Hart wir. Müller. Seholtz. Troschel.

Izern. Perty.

Imonat. Alt.

Bordeanx. Gachet.

Baston. Eights.

Bresin u. Gravenhorst. Klopsel.

Irisser. Lambatte.

Doruat. Givbe.

Dresden. Thienemann.

Edinlourglı. Blackwall. Caroluu. Cross. Hussey. Traill.

Floreaz. Marehetti.

Froulfurt a. MI. Heyden. Reuss. Frankfurt a. $d .0$. Richter.

Freiburg (Breisgau). Langsdorf. Perleb. Siebold.

cyothenburs. Westring.

Iralles (a. d. S.). Burmeister.

Germar.

IEamburer. Wintliem.

Manuover. Krosmann. Mühlenpfordt.

Havanualı. Mac-Iseay.

Feidedberg. Dierbacl. Horn.

Jenan. Grïle.

Ierens 1k. Ranzoft.

HIoneketr: Krynicky.

IXionissinerer. Rallike.

Xopenduaten. 'Thiencmanu.

Tcinain. Tilesios.

Leydem. Hunn.

Houdou. (Th.) Bell. Denny. Double. day. Gray. Hausou. Hope. King. Lewis. Lowe. Miller. Underwood. Westwood. White. Wisson. Wood.

Gund. Sumdewall.

Nin dirid. Lopez.

inarlonith. Herold.

Matseille. (Vgl. Auriol.)
Mintpellier. Scrres.

Moskan. Fiseher von Waldheim. Lovetriky.

Munchem. Gistel. Sclumidt.

Neapel. Cancellieri.

Nensoll (Ungari). Zipser.

Venstadt-Wbersiralde. Ratzeburg:

New-Daven. Dana. Herrick.

Welpley.

Nixza. Miceary.

Niluraberg. Jückel.

Qdessa. Nordmann.

Palerumo. Giolımann.

Paris. Bory de St. Vincent. Brébissou. Deslayes. Desmarest (Vater). Doume. Dujardin. Flourens. Geoffroy de Villeneuve. Gras. Guérin.Mineiville. Lefebvre. Lepricur. Lueas. Raspail. Struss. Theis. Turpin. Vasselet. Vauthicr. Walekenatr. Weghe.

plilnelel fuia. Dix. Edwards.

Hentz.

Hisn. Savi.

IFotsda un. Türck.

Praz. Corda. Opitz. Ramisel.

Hegenshoreg. Koch. Seluuch. Rinteln. Kohlrausel.

ERon. Metaxa.

Eotterdan. Brants. Hoeven.

Ix onem. Maille.

Gaint-martluéleny. Forström. Naint-savenr. Robineau - Deyvoidy.

Sint-Séver. Dufour.

Sant Antonio lla Florida (vgl. Madrid).

sandi peterkineg. Brandt.

stettin. Lincke.

Stuttgart. Ileriug.

Tivaraud. Colta. Rossmässler. Thurin. Gèné.

Villencenve de Berge. Joftic. Virteliall. Downes.

WVien. Fitzinger. Hammerschnidt Heeger. Kollar. Silireibers.

vilnn. Eichwald.

Eiirich. Heule. Okeu.

Uubelannt. Roseuleyu. Theisen. Tulk. 


\title{
Tafel der arachnologischen Schriftsteller.
}

\author{
(Die mit * bezeichueten leben.)
}

Adaus, John. Descriptions of nome marine animals on the eoast of Wales: Phalaugium grossipes, Phalangriun hirsutum (T'rans. of the Linnean Suciety. Vol. V: p. 7).

Adamson. Reise nacli Senrogal; übers. von MI artini. Brandenb. 1773 . 8. (p. 246: Spinuen als Feinde der afrikanisehen Kakerlaken).

Adolplí, Clurist. Michael. De flis sic dietis metcoricis seu flamentis Nariae (Eplıcm. Aead. Nat. Cnrios. Cent. 7 ef 8, 1. 83).

Aln rens, Aug. Fauma Inscclorum Enopae Fisc. un., icon. 25 cum descript. sistens. Halae apud Kümmel. 1814 (il 1 Thlr.).

Albers, J. A. Bemerkungan ïber den Зsи der Augen verschirener Tlziere (Denksehr. d. bayı". Akad, di. Wissensch. Jahı.g. 1808, p. 81).

Albin, Eleazar (uder Eleiser). IVar Maler. A Natural history of Spiders and other curious Insects, illustrated with fiftythe copper plates. Lomkl. 4. 1736. (Rolı's phys. Bibl. 474). (Tab. 39 Arancac; t. 40 Seorp. et Pediculus Gallinae, $4 t, 42,43$ ad 53 Pediculi.)

Alhines, Bernl., Diss de Tarantismo. Franeof. ad Od. 1691.

Adrovandi, Ulysuc. Insceti Lil. V, c. 13, p. m. ed. Francof. Fol. 1626, 23; Tab. XJ. - De auimalihus inscetis. Libri septem. Bononia 1638. Fol. - Auch ïber Krätzmillıe. - De reliquis animalibus exsanguibus, utpot: de Mollibus, Crustaceis, I'estaecis et Zooplytis libri IV. Bononiac 1605. liol. e. figr. ligru.

Alpinus, Prosperus. Rorum aegyptiacurum libri IV. Lugd. Bat. 1735.4. c. tub. 25 acu. (lib. IV).
Amonecenx, $N$. Description methodicfue d'une espece d" Srorpion, commune a Sauviguargues, "u Lung'nedoe (Journal de Plıysique XXXY). Inscetes de la France venineux. 1789. 8.

Anowyone. Cours d'histoire naturelle ou tablcau de la nature. Par. 1170. (Toun. VI, p. 110-147.)

Anomyme. Abrege de l'Histoire des Inseetes, dedic au jeunes personnes. Par. 1764. (Tom. I. 8. p. 133 - 173.)

Anomyene. Arigne (Pliysikal. Abl. d. Paris. Akad. III, p. 706).

Anomyenes. Vou den Feld- und Grasweben dieses Nonats, und was selbe vol eine Gemeinsıluaft rehabt mit deu Filamentis Mariac oder Sommerfaden (Bressl. Samml. 1, 1717, Oct. Art. 2, Class. IV, p. 210). - Vou den vieleu Erd- und Grasspinusu in $\theta_{(-1)}$ ber libid. [. 2 t4. Art. III. - Curictse Observationes von rince Art Spinnen in Preussen und deren mannigfaltigen Verimalermureu (ibikl. 1 is, Ort. Class. IV, Art. VIII, p. 1745). - Spinnenei (Eie rsitckchen, Jullieuhs) jungér Sipinnen (ibid. 1720, Jumi, Cluss. IV, Art. 13, Ni. 2). - Vou der Begittmor der Spinnen (ibid, 1722, Oct. Art. \$). Von Spinnenfiessern (ibid. 1725, Art. 10, p. 416 ).

Anonymus. Der Aryt, was dep Stich der Spinuen wirke (Plıys. B̈kan. Auszïge, II, 436. III, 533). - Spinnenfresser (ih. II, 196. III, 135).Ob ilır Sticlı Gellssuclıt maclıc (ibid. VI, 306). - Vom Tamantismus (ib. II, $473,64 c .111,406,526-5.33$ ॥ud 36 ).

Anomynes. Araignce: Minicre de les mouril: preparation de leur soie; 
leur veuin (Encyclopédie ceonom. II; p. $140-149)$.

Anoby isus. Aranearm lombyce antipathia (Misc. Acad. Nat. Cur. Der. 1, A. 1, 1670, p. 278).

Anouymus. 'Tarnnteltanz (Mèn. Aead. de P'ar. 1718).

Anouyuns. Nene Wundtafeln der Naturgesehichte, oder Abbildungen aus der Naturgeschiclute, zum Gohauch beim Unterrieht in Schullehrerseminarien. gr. Fol. Breslau.

Anonyums. Von unterschiedenen Spinnen (Lryähl.Gesellsrhaftl. 1I, 1.203). - Der Tarantelbiss cine Betrügerei (Nrue ges. Er\%. III, 307).

Anonymus. Spinnen in Guinea ete. (Ally. Reisen. IV, p. 275. V, 200. VIII, 515. XII, 625. XV, 13. XVI, 301 ).

Anouymus. Die grosse fïircliterliche Spinne (Reisen eines französiseh in Oftiziers naeh den Inselu Frankreiclı und Bourbou, dem Vorgeb. d. g. II. Altenb, 1774. S, (p. 100).

Anonyumas. Vom Gewebe des Spinnen (Stuttgarter Realzeitung 1766, p. $730-731$ ).

Anonyums. Brasilisehe Spimen, Biss der eeyloniselien, toscanischen, bras. Winkelspinue, faustgrosse Spinnell, selfenes Gewebe, russiselar $A$ rachniden, zwcihöruige: Frass der Spinneneier in Siam (Berlin. Samml. 4. Bd., p. 283 . 5. Br. 58,66 . 6. Bd. 426. 7. 13d. 408. 8. Bd. $510,512,626$ ).

Anonymus. Ob eine an einem Farleu hangendespiune nicht zerquetseht werde, wenn man mit Gewali an sic selılïgt? (Frïnkische Samml. I, p. 228).

Anonyuns. Nachrichten von Spinnen (uns Leenwenhöeks Arcan. Nat, Epist. 138. - Steht in den Leipzig. phys. ölon. Abhandl. I1I, p. 871-902).

Anonymans. Wic man die Spinnen zur Betrachtung fangen und ernähren sull (Abl. d. selwwed. Akad. Ueber. XXIII, p. 241-244).

Anonymus. Von den spinnen (Neue Anmerk. ans allen Theilen der Naturlehre, I, p. 443). - Von den Spinmengeweben (ibid. 444). - Von der Art und Weise, wie dic Spinnen Fliegen fangen (ibid. 447). - Von der Erzengung der Spimen (ib. 451). - Von den Augen der Spinnen (ib. 453). - Von der Tarantel (ib. 457). Anonymis. Is Liqueur d'une $\mathrm{N}$. 33. p. 526).
Araignce fuit perdre la vûe (Biblioth. univers. Il, P. 266). - Araignee monstrueuse (ib. VI, p. 248).

Anouymns. Von den kleinen Spinner, welehe auf der Erde das Gewebe machen, das bei uus den Namen Metgen- oder Mät pensonmers fülıret (Hamnövr. Anzeig. 1754, Nr. 16).

Anouymus. Aranein ingens venenata testacea (Comment. lips. de rebus in seientia uat. et mediear grestis. IV, p. 229). - Aranci sub aqua vitae (ib. 237). - Eorum histor. 11at. (Vol. IX, p. 278, 535. III, 237. Suppl. 279. Vol. XII, p. 580). - Araneate eubieule s. parand. (ib. XV, p. 505 de usu serici araneorum).

Aronyemus. Aran. Usus medin!s (Comnereium litter. Norimb. 1734, p. 318.1735, p. $14,23,248.1737$, p. $183,341) .^{\prime}-$ Araneas inter Bufones Anlipallia (ibid. 1738, p. 373). - De excretione arancarnm cum urina (ib. 1743, p. 391).

A ucuryurs, Spinne (Drontheim. Gesellseh. Sehr. III, p. 388, Nr, 60).

Anonizuns. Gewebe nordaner. Spinnen; Spinnen in Minorca, in Peru (Samml. neu. u. merliwüıd. Reisen zu Wassel und zu Land. Gütting. 8. 10 Th. p. 63, VIII, p. 164. III, 134).

Anonymus. Vom Gewebe der Spinnen (Rejelı der Natur und Sitten. Nr. 219, p. 20, 28, Nr. 292, p. 45).

Amonyums. Spinnen (Reisen der Chineser II, 689. III, 4(6).

Anouymus. Mlikroskopische Versuche mit allen merkwürdigeu Theilen der Spinuen (Vollst. prakt. Werk von der ganzen Optik. Lübeck und Altona. 1758. 4. (1. 483).

Anonymus. Curiose Nachrieht von einer nenen Art Seide, welehe von den Spinneweben zubereitet wird und wovon der König L udwig XIV. eine Weste trïgt. Leipz. 1711. 8.

Anonymes. Von deu Fühthörnern der Spinnen (der Naturforscher III, p, 202), - Ilure Begattung (ibicl. V, 223). - Eier in Sticken (ibid, $V$, 242).

Anonymus. Aranea ete. (Mimoires de Toulouse. Tom. I. Ilist, p. 145).

Anonyuns. Von Spinnen (IIausvater, II. Bd., p. 742).

Anonymus: Wiefern die Spinnen giftig mol schidllieh sind? $\mathrm{Ob}$ sie den Tobili lieben? (Hannover. Magaz. 1770, 
Anonynus. Merkwürdigkeiten der Spiun rummen (Berl. Manuig-faltigk. III, 494). - Feines Gefühl der Spinnen (ib. IIl, 607). - Nhamdu, der Kolibrifresser (ib. IV, 315).

Anomynus. Uuständliclse Besclıreibung der Iausspinne (Matière medicale i, p. 230 Suite.

Anomymus. Von Spinmen (Neue Berl. MIaumigfalt. II, 166, 330, 72. III, $236,265,700,742,747.1 \mathrm{~V}, 89,99$, $138,233,258,281,297$ ).

Anonymas. Springspinue (Dresdener Maguz. II, p. 499).

Amonymus. Anmerkungen ïber die lieben Frauenfäden und fliegenden Spinuen (Bremisches Magaziu. 1V, p. 634).

Anomymus. Tarantel (System. Isehrbuch jiber die drei Rejcle der. Natır. Nïrub. 1777 . 8. (1 Thl. 1. 387.)

Amonymus. Diss. sur l'utilite de soye des Araigueses (Journ. des Scavans. Toin. XI,VIII, p. 407. T. CXLVIII, p. 305$)$.

Anomym ı.s. Spinnen (Kern küriöser Wisseuschaften $1, p .284$ ).

Anonymas. Vou der Tarantul (Allr. Geseh der neuest. Entdeck. von verschied. Gelelurten im russ. Reich und Persien. Bern 1777. 8. I. Thl. p. 368).

Anomymns. Uebersetyung im Hamb. Magriz. I, 63 aus Mèm. de l'Aend. de Paris 1707 , p. 438. - Tagenaria domestica).

An ony mmns. Merkwïrdigkeit der Kreuzspiune (Berl. Samml. Bd. 7, 417). Anonyanis. Aran. domestica (Acta Ups. 1736, p. 38, n. 7).

Anomymus. Von Spinnrn (Staatsund Reisegeogr. XIII, p. 766).

Anomynum. Vom Raube der Spinnell (der Freimilures, Wochenschr. p. 270$)$.

Amonymuns. Aranea aquatica (L'lust. 1884, Nr, 240. - Isis 1837, p. 711).

Aruleron, William. A supposition how the white matter is produced, which floats about in the air in autumn. (Plilos. Trans, Y. 1747, p. 428).

Aristoteles. Ilistor. animal. Lib. I, c. 27.

Armololus. Ameriea (p. 995 et 1294).

Asse ler, Thomns. Viridariam Adriaticum. Aurspurgi 1686. 8. (deutsch). Andonin, Victor. Ueberdas küust- liche Nest einer Spinne (Ann. d. sc. nat. XXX, 1833, p. 330-35). - Ueber cin Nest ciner Manrerspinne aus Neugranada (L'Inst. 1837, $\mathrm{Nr}_{1}, 212$. - Isis 1837, 838). - Bricfe zur Geschichte der insekten. Ueber schmarotzende Milben (Ann. d. se. nat. XXV, 401, T. 9, 14. - $1 \operatorname{sis} 1835,471$ ). - Textzu denAraclniden in Description de l'Egypte XXII, p. 191 (Jsis 1832, 969). Nene Sippe der. Aracliniden von Frankreich (Vortrag 1835. - Isis 1836 , 763). - Mémoire sur l'Achlysie, nouveau genre d'Arachnide (Mim. soe. d'list. nat. I, p. 98. Av. fig.). - Note sur une espece nouvelle d'Achlysie (ibid. II, 497).

\section{* Hacis.}

Inarlivi, Georg. De Tarantula : annex. lib. Il de praxi medica. Diss. I, de Anatome, morsu et effectibus lurantulae, c. fig. Romae 1696.8.

Bnker, Henry. The Microsrope made Easy. - (p. 196, claap. XXV of Spiders.) Lond. 1769. 8. - Uebersetzung: Das \%um Gebrauch leicht remachte Microseopium. Zürich 1753. 8. (p. 208 von dell Spinien; p. 531 über eine grosse Spinne.)

abanderoft. Niturgeschichte von Guiuna. Aus d. Eugl. Frankf. mnd Leifz. 1769. 8. (p. 148. - Mymale).

Barbint, J. Les genres des insectes de Linni. London 1781. 4. Av. pl. enc. beauc. (Tab. 19 Araclin. pl. 20 Crustata, Die Kupfer z. Th. lerzlich schleclit. Das Werk ist selten.)

Baricellns. De araneorum tela ejusque usu.

Baster, Joh. Opuscula subecsiva. Harlemi 1762. 4. (Tom. I. Lib. 2, p. 86, 87. Vou wandklopfenden Spinnen IIJ, 144).

Tennvois (Siehe Palisot).

Beclustein. Voigts Magaz. 1789. (Beck.) Beiträge zur bairischen Insektenfauma oder Beselueibuner und Abbildung neuentdeckter Köfer, mit angeluängtem Namensverzeiclmiss der Eleuteraten des Landgerichts Zusmars. hausen. Augsburg bei J. Wolff. 8. pp. 45. 1817. M. ill. Kj)f. (2 fl. $12 \mathrm{kr}$ ).

Beckandnu, Joh. Anfaugsgriüle der Naturhistoric. Gött. und Bırm. 1767. 8. (p. 118, Ni. 8.) - Caroli a Linné Syst. Nat. in epitumen redactum. (j. 182, yен. 268.)

IBeI1, Thomas? 
Eerner, Gottl. Ephr. Exereitat. de applicatione mechanismi etc. cum obs. de punctura araneac et ejus medela. Amstel. 1720.8.

\section{* II lekwn II, Jolin.}

Blanls aret, Steph. (auch B I a n ckard). Seliauplatz der Raupen, IVtirmer und Maden, übers, vou Rodochs. Leipr. 1690. 8. (p. 97, 33. Hptst.) Theatr. lus. T. 17, B. (Pliryous).

Bloclı. Verzeichniss merkwïrdiger in ' Kopal cingeschlossencr Insekten (Besclüft. d. Berl. Ges. watf. Fr. II, p. 164, f. 2, Nr. 2. Aranea pilosa; f. 5. Nr. 5. Aran. abd. globoso). Vgl. Scindelius (list. sucein.).

IBInemionch. Abbildungen naturluist. Gegenst ïude.

Boccone, D. Sylv. (sonst Paulus). Museo di fisica c di Esperienze ete. Venez. 1677. 4. (p. 92 de Tarantula). Deutsih: Frankf. und Leipz. 1697. 12. C. fig.

Ioclumetus, Samuel. Hierozoicou seu de Auimalibus S. Seripturae opus bipartitum ctc. Lond. 1638. Fol. II Vol.

Bodal aert, $P$. Ucber die Spinnen (bei Disjouval).

IB Umer, M. Imman. Karl Heinr. Samml. aus der Naturgeschichte ete. 1. Diesd. 1774. 8. ( 5.536 Phalangium, Aramen).

Tomare, Vallmont de. Dictionnaire universellc d'list. nat. ( 1, p. 317 -354$.

Hou. Diss, sur l'Araignee, avee une Icttre sil le même sujet par $M r$. Poutret. Paris 1710.8. |tal.: Siena 1710. 12. - Diss. sur l'utilite de la soye des Araignes avec l'analyse chimique de la même soye. Montpellier 1710. 4. et 8. Englisch: A discourse upon usefulness of the silk of spiders (Plìl. Trans. XXVII, Nr. 325, p. 2, c. fir.)

Bounce, Charles. Betrachtungen jiber die Natur. 2. Aufl. Leipz. 1772. 8. p. 377 (vou dec Sorgfult der Sackspinne für ihre Brut). - Abhandlügen aus der Insectologic. Ilalle 1773. 8 . (p. 385 voun Spinuenfaden und Spinnwarzen. - Betrachlungen ïber die organisirten Körper. 1I, 91 . Von ihren Genitalieu).

Bomono. Schricb auch tiber die Krätzmilbc. 1687.

Eouthus, Joh. Bei Piso.

Borclli, Petr. Obs. microscop.
Centuria. Hag. Comit. 1656. 4. (p. 10. Obs. 9, Oculi 8 Alanearum).

" Bory do Snint-Vincent, J. B. M.

\section{HBritel.}

* Irreuts, A.

Herélvision.

Irex. Spinnen (in dessen Flore des lusectophiles p. 15, Praef.).

Brochinums, Dominicus. De ve. neno Animantium naturali et aequisito tractitus, Florentiae 1752. 4.

Browne, Patrik. A civil and natural History of Jamaica, in three Parts. Lond. 1756. Fol. c. 50 tab. (p. 409, TR. 41, Phryus).

IBrowin, Thom. Essai sur les erreurs populaires. Amsterd. 8. 1733 (I, p. 219. De quelques Insectes et des propriétés de quelques Plants).

Brtickunne, Fr. Ern. De fabulosissimae origrinis lapide, Arachneolitlo dicto. Wolfterbittelac A. C. 1722. 4. - Epist. itinerar. Centur. 11. (Epist. 7: de araneis eorumque oculis; in Martiui's Uebersetzung Lister's p. 9-16 cuthalten. G.).

Britumicli, Th, Entomologia (p. 84, 85. Arunca).

Bittmer. Colleg. curios. (p. 449, vou ceylomischen Spimen).

Bifsclings, Anton Frieds. Eirene Gedanken uıd gesammclte Nachrichten von der Tarantel. Berlin 1772. 8. Ueber dic Tarantel.

Bufron. Allgem. Naturgesehiehte. Uebers. vou Martini. (VI, p. 314, von Spinnenfressern. V1l, p. 171.)

Bullnumu. Spimen (N. Schrift. d. halliselı. Gesellsch. 1810).

* Burmeister, $\mathrm{H}$.

Calceolarius. Muscum Veronae 1622. Fol. (p. 666 de Tarantula s. Plialangio).

Cumelif, Geory Joseph. De araneis et scarabacis philippensibus (Plıil. Trans. Y. 1711, p. 310).

27 Cuncellieri.

Chputo. Schrieb: De tarantulae anutome et morsu. Lycii 1741. 8. fig. * Carolain.

* Cariss.

Casal. Schricb 1762 von der Krütz milbe.

Catesby, Marc. Caroliua (II, T. 36 ).

Catholicon, $\Lambda$. Arnignèe, p. 432. Cestoni. 
Chrareton, Gualtierus. Onomusticon zoiron Aninalium rontinens plerorumque differentias ef nomina propria pluribus linguis exponens etc. Londini 1668. 4. C. 1. aen.

Cirillo, Duninico. Some account of the manna tree and of lise taralltula ('hil. Transs. 1:70, p. 233, 236).

Clerclc, Carl. Om spindlars fäingande och tödande (Vetenskaps Acud. Handl. A. 1761, p. 24.3. - Vom Fang(' แnd Emähıen der Spinmen. Selıwed. Ahl. deutsch, 1761 : 241). - Suensk Spindlars. Mranei sueciei figuris et descript. illustrati. Holmiae 1754. 4. Tal. ill. tig.

Clusius, Carolus. Exotica, p. 76 (Aranea monstrosa; p. 353 Aranei ad moll. virulenti). - Curae posteriores. Antwerp. 1611. Fol. p. 46 (de ploalangio Americano).

Collini, Cosmann. Sur le tarantisme (Comment. Aseild. Theodoro-palatinne. Vol. V. Physs. p. 361).

\section{cordas.}

Colmarnu, G. D. Ob die Spinnewrbe eine anlifebrilische Kraft habe? (Brannsshw. Anz. 1715, p. 1611, 1993).

Colucasent. Phytob. 110.

Coupcebert. Iilustratio iconographicil Inscetorum ed. Fabricius et C. l'aris.

Cormeliux. Thomas Népholiturus. Lattre to Dr. Thomas Dadington con. eerning some observalions made of Persons pretending to be stang by Tarantulas (Phil. Trans. VII, Nr. 83, ionos.

Conus, Rudolpli Wilhelm, De fildmentis sericis singularibus in agris Hitumhurgensibus repertis (Mise. Acad. Na1. Cur. Dec. 3, A. 5 et 6.1697 et 1698, 1. 2611 .

Cricgerus, Dan. De veneno Aranei (Ephem. Nat. Cur. Dec. II, An. 4. 1685. p. 144. Obs. 66).

Cuvier, George. Mímoire sur l'organisation de quelques meduses (Acal'us Limacum) (Societé philomat. A. 3, T. 2, p. 69).

Egale, Sam. Pharmacologia, mineralia, vegetuntia, animalia in medieina officinis usitata. Bremae. 1713. 8. (p. 384).

Dana, Jolu.

Degeer, Cluarles. Geb. 1720, gest. 1778. Vol. Gecr.

Donso. Mlonall. Beitr. "st. p.
805 (von seidenen Gewauden aus Spinnemgewebe).

Derlumer. Physicotheologie. (Von den Angen p. 153. - Gewebe p. 543, 627. Gifte der Spinnen 399).

* Deslunyes, G. P.

* Dessuarest, Anselune Gaëtan.

Pictionsure raisonne el universel des animaux. Paris 1759. 4. (1, p. 149. Araignie, et 150, 151, 153, $154,155,157,161,164,165$ (Literatur).

" TDicerbacli, J. Heinr.

Dillenius, Justus, Frid, De lapide araneorum (Ephem. Aear. Nat. Cur. Cen1. III et IV, p. 97).

Disjouval (vgl. Quatremere). * Dix (Friulein).

Dortlues, Jaecjues Antoine. Medicus y Mrontpellier; grb. 1759, gest. 1794. - Mèmoire sur les Araignes maçonnes (Trans. of limn. soe. X). Obscrvations on the structure and oceouomy of some curious species of aranea ('Trans. of the Linn. Suciety, 11, p. 86).

* Dondofediay, Edivard.

"Dufoure, Leun.

Dugres. Antoine. Ueber die Ordnung der Mliben (Aeiriens) und die Sippsehaft der Trumbidien insbesondere (AmI. d. sr. n. I, 183\%, Tit. 1, p. 5-46, et 1.11-174, 'Tilh. 111, 11. Isis 1836, p. 490. - Unters. ibrr. die Ordnumg dor Milben, II $(A$ แ1, d. sr. II, 1334, p. 18-63, Taf. 7, 8. Isis 1836, p. 516-518. - Neue Bemerkungen (il). 104). - L'Inst. 1833, 206). - A themb̈̈eher der Spinnen (L'lust. III, 1835, Nr. 92. - Isis 1837, 717). - Ueber Sareoptes Scabici (Am. d. se. 11. 1II, 1835, p. 145 , T. 11. - Isis 1836, P. $529-530$ ).

Wherharal, Joh. Peter. Versuch cines nenen Entworfs der Thiergeschichte. Halle 1768. 8. (p. 225).

"Edlvarals, Milue.

* Ellerenlyerga, C. G. v.

" Eiclewalla, Ed.

*iglets.

wncyclopédie méthodique. A rachnides.

Ettuniller, Mich. Beschrieb inden Aetis lips. 1682 den Sareoptes Scabiei. - Observationes binac de Crinonibus et sironibus (Act. crudit. 1682, p. 316 et 17 , Tah. 17 et ejusd. Oper. Med. Vol. IV, p. 816, ed. Genev.) 
Fabriclus, J. Christ. Geb. 1743, gest. 1807. - Eutomol. Syst. I. II. Supplement. ent. syst. - Systema kiltomologia ‘p. 431, 4.10).

Foirfox. Tuads sud spiders in. noxious ind the latter tiuge water of a Sky colour (Pliil. Trans. Nr. 2t, p. 391).

Fermin, D. Plit. Beschrcibung der Kolonic Surinam. (II, ). 272, vun den Spinnen).

" wisclnervon Woldhein, Gott. helf.

FIüนge. Hanuüv. Mag. 78.

Forstenl, Peter. Deseriptiones Animalium ete, in itin. orient. obs. Havi. 1775. 4. (p. 855 ueue Species).

rortis, Abbate Alberto. Reise in Dalmatien. Ueber's. Bern 1776. 8. (Il TH. p. 40 vou einer sehiidlichen Spinue: Pank).

Fonreroy, Ant. F. de. Entomologia Palisicusis; sive Catalugus In. sectorum quae in agro parisiensi repcriuntur; secundum methodum Geoffracanam in sectiones, gencra et species distributus ete. Pars prima. Parisiis, Serpentini. 1785, 231 pp. 12. Pars sceunda $544 \mathrm{pp}$.

Franzins. Ilistor, Animal, p. $35 \times 5$

Frisch, Joh. Leonhard. Beschreibung von allerlei Insekten in Deutscltland. Berl. 1720. 4. (VII, p. 7, 10. vilI, p. 3, 5. X, 6, 16, 21, XI, 25. XII, 23).

Fnessly, Jolı. Kasp). Verzeicl. niss Sehweiz. Insekten. Zürich 1765. 4. (p. 60 Phalangium, Aranea).

Fahrltep, Gustar Casinir. De minutiis aninalibus euriosis seu insecto minimo novo et antehae vix, a me saltim, unquam viso, genere (Nise. Acad. Nat, Cur. Dee. 3. A. 7 it 8. 1699 et 1700, p. 256. - Pecliculus).

Grarmanu, Clirist. Filedr. Araneac aёrs: mutrimtur (Mise. Ac. Nat. Cur. Dee. 3. A. 1, 1670, p. 278). De nutritione Aranearum ex aere (ib. I, An. I. 1670, p. 214. Obs. 120). De Antipathiu Arancarum cum Bombyce (ibid. P. 244. Obs. 121).

Finser, Marian la. Aus Madrid. Amenidades unturales de las Españas. Madrid 1821. 8.

Freer, Charles Baron de. Duir som med en strache i ïudau ïro fïste vid andra lefvande diur (Acarus vegetans)

(iistel's bexiknn.
(Vetenskaps Aead. Haudl. A. 1768. j. 176. - Thieıe, die mit pinem Strange am Eude an andere lebende Thicre liefestigt sind. Schwed. Akad. Abl. J. 1768, P’. 191):- Beskrifning pä et särdeles slags flutt; Acatus avium, pedibus tertii paris mole monstrosis (ib. 1740, p. 351. - Deutsch p. 109). Mennire pour servir it l'histoire des lins. 'T. Vil. Stocklt. 1752-78. (Tom. II, Part. I, Disc. II, p. 27. - Uebers. 1 Quart. 3. 26 vou den doppelten Gieburtsgliedern der Spinnen.) Uebers. von G izzc. Nüirnb. 1783. 4. Bd.

Gemé, Giuseppe.

Geofruy, Steph. Franz. Materia medica. Leipz. 1763. 8. (Vol. VI, 1. $227-257$ vun den Spinuen und iluen Geweben).

Fentroy. Histoire alırege des Insectes. II Tomes. Parisis 1800. 4. C. tab. aeu. (Editio cum textu prioris, anni 1764, non mutato. Clıarpentie r.)

* Germar, E. Fr.

Gesmer, Canradus. De seorpione. Zirrich 1589. Ful.

Glnedinus, Fernandus Antonius. De scorpiunum aculeis (Commentar. bononienses. 1, p. 107).

"Cistel, Johannes.

Focdart, J. Metamorphosis natIIralis ed, Belg. et Gall. (Vol. V, p. 185). - Edit. eun notis Isisteri. Loul. 1685. 8. (1. 348 de Aran.).

Gibze, Johaun August Erlırain. Der Herausgeber von Li st e r's Naturg. der Spinnen. Vgl. Listur und Ger.

* Frifife, $H$.

* Frills.

* Cùras, Albin.

* Gravenliorst, J. L. C.

* Gray, Georr Robert.

Gronovins, Isaurentins Theodorus. Zoopliyt. (II, H. 217).

Ariule, Herm. De ictu Tarantulac et vi nusices in ejus Curatione conjecturae physico-medicac. Franeof. 1679.8.

* Ermbe.

- Guérin, François Etienne.

Illagendornins, Elırenfr. De Arancis (Ephem. Nat. Cur. Dee. 11. A.l. 3. 1684, p. 88. 0 bs. 30).

Malun, C. W. War Dr. pluilos. und Naturlistoriker vou grossem Tulente, begabt mit Küustlerhand. Aufbewalırung der Spinuen (Germar's 
Mag. III). - Manographie der Spinnen. 1. Heft. Niirnberer, in Verlage der J. L. S. Isechner'schen Buchlianll. 1820 \$. 4 ill. Kpft. in jedem Hefte (a 2 fl.). I. Id Seit. Einl., 2 S. unhe\%. Text. 11. Hft. 1821,2 unbrz. S., 4 Kpft. III. Hfft. 1822, 4 S., $4 \mathrm{kpft}$ IV. tift. 1826,2 S., 4 K. V. Hft. 1827 , itlem. (Dic Fortsetzung besorgt bekauntlich Hr. Forstratl v. Kocls, einer der gecnialsten Arachnologen.)

\section{" Ha anuacersclemint.}

Honneannu. Aramearmu innoxius esus et vencuum (Eph. Nat. Cur. Dec. If, an. 5. Obs. 116 , p. 231 . Memoir. de Toulouse. T. I, Hist. $\mathrm{v}$. 115 ).

Hanovs, Mich. Clirist. Seltonlicitell der Natur. Leip\%. 1753. 8. (I. Thl. ก. 411 vou Spimnen und vou dem iu der Luft ziehumlen Herbstgarne).

rovenrefter. Ueber Sarcoptes Sinbiei. 1660 .

Ifreaptanamu. Ueber dic Kriitz. millse. 1657

atemprich. Vgl. Éheuberg.

*anle.

Mentis:

QDEucher, J. Heur. Diss, araneus homini perniciosus et salutaris, Witteb. 1701.4.

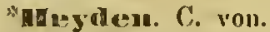

Merbst, Joh. Friedr. Will. Pastor za Berlin; geb. 1713, starb \%u Anfaug diesis Jahrhumderts. Natursystem dev ungeflïgclten Insekteu (Solpuera, Tarautula und Plalangiun). M. ill. Taf. Berlin 1797. 4.

"Hering, E.

Mermaun, Jenn Frélèic, Sohn des beriilunten Jean H.; geb. 1768, gest. vor spinem Vater 1793. - Memoire aptérologiugue. Strasb. 1804. Fol.

* Merbill, Manr. Unters. vilb. d. Bildungsgescli. der Thien: in Ei. I. Spinnel. Marburg 1824. Fol.

\section{Herrick.}

Miller. (Hufeland's Journ. Bd. 35 , St. 5, p. 115).

Hoernager. Icon. Insect. volat. l'raneot". 1692. 'Tab. 4. Eul. altera. 4.

"ITneven, J. van der.

טanuberar, Phil. Observations sur les Araiguees (Mém. de l'Acud. des se, de Paris. 170\%, p. 438. Av, fig.) - Anmerkungen uber die Spinnen ete. ((Alles) liamburg. Mag. I, 51, 173. IV, 224. XIX, 81. XXV, 27, 289. - I, 68, 69. XIII, 4. XIV, 433-436).
Hoocke, Robert. Mierographia, or some pluysiologital deseriplious of minute budies made hy mugnifying glasses, with observations and inquiries there upon. Lont. 1667, Fol. min. e. 38 Plat. (nitidis). (Examen Araucaluu p. 203).

\section{"Hope.}

Horch, Friedr. Wilh. De puliee enulriae, cum nonnullis circa runas fuctis observationibus (Miscellanen berolineusia, T. VI, p. 111) (Pediculıs).

Huihner. Von ten Spinnen als U'uplücksboten (Ejusd. Natur-Kunst ete. Lexikon).

*Inssey, Tlı. J.

Inperati, Ferrante. Histor. natur. Colon. 1695. 4. p. 901, 920 (Plıalangium, genus araneae venenatue). Das Original: Ilistoria naturale mell yuale ordinamente si tratti di diversi eondi. tion di Minere etc. Nenpol. 1599. Fol. (Auch zu Venedio 1672. Ful.)

Jablonsky. Spinmen (Allg. Lex. dev Wissensel. Känigsb. und Leipz. 1767. 4. II (1. 1437).

Jacobacus, Oligerus. De Seorpione (Alt. Ilatin. Vol. V, p. 265).

Jaksom. Spinuweb geg. Wecliselfieber (Merl. und phys, Jourm. XXI und $\mathrm{XXI1)}$.

"Jofire:, llenry.

Jalan. Besclureibung cinirer ostindischen Insekten. Orthoptera. Spinuc; der bandirte silberne Kegel (Nene Schrift. d. Ges. natf. Fr. zu Berlin. I, 3.77.

Jouston, Jolın. Historia Insect. (p. m. 131. Art. II; de araneo in genere efc. p. 133 et 137).

Inulı Mer, Märten. Anmärkuingar vill Dans-sjukan, eller den sä kallaule Tarantismus (Vetensk. Acad. Hamtl. A. 1758 , p. 29. - Ammerkungen über die Tanzkrankleit, dic man Tarantisinus nemut. Srhwel. Ak. Abl. 1838, p. 30).

Jainufer, Eugelbert. The Hiat. of Japan. Loud. 1727. Fol. II Vol. W. PI.

Iramel, Georg Joseph. De araneis ef scarubacis philippınsibus (Plil, Trans. Y. 1711, 1. 310).

ILeysler, Joh. G. Corsicanisclıc giftige Spiumen (Dessen neueste Reisen. Hannovil 1751, 4. 11, 1, 762), 一 I'arantelı (ibicl. p. 760). 
* Iing.

* Iirloy, Willian.

Irelnuaier. Grorg Caspar. Disputationes zoologieite de Basiliseo ete. nc iranea. Lat, unul Deutsch. Jenae 1736. 4. - Diss. de Arauca, imprin is vero Tarautulis. Witteb. 1660.

* Eittel, B. M.

Mleemana. Besclureibung einer sehr grossen schällicheı briuncu westindiauisehen Winkelsjunne (llessen Beiträge zu Röscl's Insclitenbel. I, p. 85).

"IIOpsch, J. E.

Hnorr. Delicinc Naturae (II. Tab. F. V, p. 19-21). Monum. Diluv.

"ucle, C. I.

" Molhiraisch.

Iolb, Pet. Beschreibung des Vorgebirgs der guten Hoffnung. Auszug. Frankf, und Leipz. 1745. 4. (p. 357 von den Spinnen).

* Mollar, V.

Neynicky.

Kundnumin. Rariora Nat. et Artis. (p. 1064).

Laet. Amerieae utriusque deseriptio. Lngd. Batav. 1633. Fol. (1. 56. Araneus marinus. - Phalangium brasiliense monstrosum p. 569, 579).

Eamarcle, Jean Baptiste Pierre Antoine Demonet. Histuire naturelle des animaux sans vertèbres, 1. Edit. Paris 1801. 8.

Lambatte, $\mathrm{H}$.

* Langsalorf, G. H.

Iantreille, Pierre Audré. Sur la famille des araignies mineuses. 1) Aranea enementeria; 2) Aranea Sauvagesii; 3) Aranea nidulans (Socicte philomat. A. 7, p. 169). - Deseription d'une nouvelle espece d'araignee (Aranea perita) (ib. p. 170). - Memoire pour servir de suite h l'histoire des insectes counus sous ic nom de Faucheres; Plalangium Linn. (ib. A. 1798, p. 113). - Des laabitudes de l'Araignee avieulaire de Linnaeus (Mim. du Mus. d'hist. nat. T. VIII, p. 456). - Deseription d'un nouveau geure d'Arancides ete. (Ann. d, sc. nat.). - Ucber dess. Caris vespertilionis (Argas. pipistrellac Aut.) (ib. XXVI, p. 260. - lsis 1835,511 ). - Histaire niturolle des Cirnstaces et des inscetes, dipendinte de l'edition des Oeuvres de Buflou, publice par Sonnini de Manomtourt. III-VI. 8. Av. fig. 180\%. - Geriera Crustaceorum fet Inseetorun. Strasburg (König), \&r. 8. T. $I-I V, 806-9$.

Tanbender. Wirkungdes Spinne. gewebes grgen Werliselfieher (Allgem. medi\%. Anual. 1801. - Korrespondenzbl. S. 74).

Haxmanu, Erie. Novac insectorum species. Coleoptern, Orthoptera, Neuroptera, Hymenoptera, Diptera. Aranea singoriensis (Novi Comment. Acad. Petrop. XIV, P. 1. Hist. p. 49. Nlèm. p. 593).

Hescle, W. E. Deseriptions of new Speeies of animals diseovered in the voyage of the Majesty's Ship Isitbella to the Aretie Resrions. Lomlon 1819. 8. - Ammothea carolinensis (Zool. Miscell. T. I, p. 34, T. XIII). - Monograph of family Anophurn (Pilrasita Latr.) (Ejusd. Zool. Miscell. III, p. 65-67). - Tliysauura (ibil. p. 62, T. 145 ete.). - Classifientio insectornm apterorum (Isis 1824, P. 169 des lit. Anzcig.).

Dellermiller. Mieroseopische Gemiiths- und Augenergötzing (p. 102, T. 54. Etwas von ciner feilnen brilbanter Spitze und ciuer halben kieinen Spinnenwebe).

Lecuwenludelk, Ant. van. Opcru. Lugd. Batav. 1722. 4. (Tom. II. Continuatio Aromor'um Nat. detect. Index Aranea. p. 311-378). - A letter conecruiug spinder's, their way of killing their prey, spiming their webls, Generation ete. (Phil. Trans. XXII, Nr. 272, p. 867).

\section{* Lefebve.}

Eelnuann, Martin Christian Gottlich. Beobachtungen über das Gewebe ler Kreuzspinne (Neue Stlur. d. Ges. natf. Fr. zu Berl. Ill, p. 147).

Lemery. Spinue (Materialien. Lexikon. Leip7. 1721. Fol. 80̈-92).

Lepechin, Iwau. Tagebull der Reise dureh versehiedoue Provinzel des russ. Reiclis. Altenb. 1771. 4. (I, p. 245: von zwei hörnigen und, Baumrimlenspinnen; p. 316: zwci giftige Spinnen, vor welehen sieh die IKalmücken selır fürehten.)

* Lepelletior le St.-Fargenu, Amélée. Naturforsclier zu Paris. Mímoire sur les Arnignces (13ullet. de lit Soe, philom. Avr. 1813, Nr. 67).

Lesser. Insectotheologie (an viclen Stellen:

"Lewis, R. H.

"Hichtenstein, von. Naturge- 
schichte der Insektengattungen Solpuga und P'halangium vou $\mathrm{Herb}$ st und $\mathbf{L}$. Berlin 1797. Bei Gottl. Aug. Lange. Ancli nuter dem Titel: Natursystem der ungeflügelten Insekten von J. F. W. Herbst. 1. Heft, ebend. VIll und 85 ip. 4. 2. Ilft. $1798,26 \mathrm{pl}$. 3. Hft. $1799,30 \mathrm{pp}$. Beide Hefte zusammen 16 illum. Kpft. 4. Hit. $1800,86 \mathrm{pp}$. $7 \mathrm{Kpft}$.

Hiuné, Carolus a. Surinamensia grilliuna, von der grossen surinamisclıen Spinue Nhanduguacu (Amoen. Aead. Vol. I, p. 505). - Systema Nat. ed. 12 (p. 1027 Plialangium, p. 1030 Araira). - Iter oeland. p. $233,239,269$, 40, 138). - Mus. Ludov. Ulr.-Reg. Suce. Holm. 1764, gr. 8. (p. 4:6 Phalangium, p. 428 Alanea, p. 429 Storpio). - Fina suecica ed. 1, ell. 2 (p. 485). - Amoenitat. Acallemiac.

Dister, Martin. Letter, confirming Jolın Ray's ohservation about musk sentel insects; adding some not's upon Dr. Swammerdam's book of insects and on that of Mr. Stens conecruing petrifyd shells (Pliilos. Trans. Y. 167t, p. 2281, 3002). - A letter, containing the projection of the threds of Spirlers and bees breeding in rases naitle of leaves, as allso, a viviparous fly and of great numbers of magrots observed at the time of the plinge A. D. 1666 (ibid. Y. 1684, 592). - Ohservations conrerning the dartig of Spiders (mit Johu W a y) (ibid. 1669, p. $1014, Y .1670$, ค. 2103). - Histor. Anim. Angliae tres Iractatus. Isond. 1678. 4. - Deutsch von Martini und Göze. M. 5 Kpf. Quedlinb. und BInnkenb. 1778. 8. A ppenl. Hist. Anim. Angrliae. Ed. 2. Loud. 1685. 8. (p. $1-3$ de Arancis). - Inquiry concerning Tarantulas etc. (Pliil. 'Trans. Vol. VI, Ni. 77, p. 3002). -. Letter containing the projection of the threacts of Spinders (ibid. XIV, Nr. 160, p. 592).

wjungli, Sven Ingemar. Nya Insceter, utur egen Samling: Scarabaeus etc.; Aranca bicornuta (Kongl. Vetensk. Acad. Nya Handl. A. 1799, 'T. 20, l. 145).

Gochner. Aranei aphrodisiacae 'ruidam deliciac (Ephen. Nit. Cur. Dee. II, an. 6. Ob. 216, p. 441).

"Enens, 1 .

Cund, Niels Tonder. Jagttagelser til insecterues historia. Slapgten Scyllarus, Aranen arcuala (Skrivter af
Niattulist. Selskab. Bd. 2, Irft 2, 1. 17).

Lyonet. Remarques sur Lesser Insectoth. 1, p. 184. 11, 1. 48. (Anclı in den neuen Berliner Mamnigfaltigkeiten.)

* Macenry, Auge.

* Mreleny (in Cuba).

Madeire, Eduard. De nova philosopia e qualitatibus occultis, accedit inandita philosophia de Tarantula. Ulissipnni. 1650. 4.

Mrefrei. Brief an H. v. Reaumur von fliegenden Spinuen und deren Gespinuste (Allgem. Magaz. der Natur, Kunst und Wissensch. VIII, p. 41, J, p. 146).

Malinowsisy. Acarus Coleopte. rorum (Illig. Mag. d. Insektenk. 4. Bd. p. $249-250)$.

Maregrave de Liebstndt, Georg. Ilistor. nat. Brasil. Ialugd. Batav. et Anst. 1648 (p. 248).

"Marchetti, Salvagnoli.

Illarmocehi. Bj-selurieb $1786 \mathrm{zu}$ erst den Theridion Malmignatte.

Martini, Friedr. Heinr. Wilhelm. Der Uebersetzer von Martin Lister's Naturareschiehte der Spimen. Vgl. Li ister.

MIn rtyn. Aranei. London 1743. 4.

Manpertins, Pierre Louis Moreau de, Prësident der berlin. Akademie ete. Geb. 1678, gest. 1759 . Berülunter Astronom und Geometer, democh auch Zoolog. - Experiens sur les Scorpions (Mèm. de l'Acad, des sciences de Paris. 1731 (p. 317. Cum tab.).

II ead, Richiyrd. Traetatus de venenis. Lungl. Bat. 1750.8. (Tarantelstich).

Meckel. Vergleichende Anatomie.

Merian. Diss. sur la generation et les transformations des Insectes de Surinam. Araignées. A la Haye. 1726. Fol. (Iranz. und latein.).

Merklein. Thierreich (p. 707).

\section{- Metraxa.}

Meyer, F. Die Spinmen um Göttingeu 1790. 8. - Naturgesehichte der giftigen Insekten. - Ueber eine Abart der Kreuspinne (Dess. Zool. Areh. II, p. $3-28$ ).

* Miescher.

Missou. Voyage d'Italic. Utrecht 1722. 8. (Tom. III, N. 58, c. fig. Tarantula 1. 368).

Todeer, Adolpli. Ammärkningur om mal, som plïgur finnas uti miöl 
(Vetensk. Arad. Ilaudl. A. 1774, S. 68. l licuticon seu de Piseatu libr. V. Argent. - Annerkungen van Milben, die im Mehle finden und wie verhütet werdin kanu, dass diss Mehl nicht mufticht wird. Schwed. Abls. 1774, 71). (Aciurus Fariuac Deg.).

Dhorgagui. Sclirieb auclı uiber dic Krït\%milhe.

7loufret, Thomas (vgl. Muffet).

Minler. Ueber die Krützmilbe. 168:.

"Miller, Johannes.

Miller, Otto Fiedrich. Fauna Fridriclssdalian (p. 92 Aranea). - Zoo. logiae Danicae Prodrom (p. 192). Hydlacluae, quac in aquis Daniac palustribus detexit. deseripsit, pingi et tabulis XI aencis incidi curavit. Lips. 1781. 4. - Mèmoire sur un nouveau genre des Inseetes aquatiques (Mèm. de Mathem. et de Ploys. 'l'. ViII, $p$. 615) (IIyclrarachua). - Von der rothen Wassermilbe, Acarus aynaticus (Schr. der berlin. Ges. naturf. Frounde III, 11. 84).

Mikller, Statius. Das Lime'sche Natursyst. V, p. 1086 (Spinnen). Verl. Slablici.

* Niniler, P. W. J. in Odenbael an Riloin. Ueber die Begrattung ete. der Ixoden (Germ). Mas. IÎ).

Ir iimster. Cosmographia (p. 1099).

Mufretus. Insectorum sive minimorum innimalium Theatrum. C. fig. Londini 1634. Fol.

Minralto, Jobannes de. Seorpionis anatome, (Misc. Acisd. Nat. Cur. Der. 2, A. 1, 1682, P. 166). - Zirolonit ste. Tiguri 1709. $\forall$. - De aranea vulgari (Mise. Nat, Cur. Dee. II, An. 2, 1683 , p. $40-60)$.

Nieremberg, Joh. Enseb. Histor. sat. mixx. peregr. exot. Lib. 13, Сap. 27 (Ostind. Spimuen). Antv.

vizwelı, Clur. Ludw. Darstell. der Familien und Gattung. d. Thierinsekten (Germ. Mag. III. Halle 1818. - Isis 1818, p. 1563).

"No rolmunin, Alex. v.

"DIGe:L, L.

DIafren. Reise durch Island. I. (p. 323 Arancae et Phalangia).

(D)enrins. Gottorfisele Kunstkammer. Langl. 4. 1666 (p. 29, T. XVII, $f$, 2, vou der $\Lambda_{\text {ran. avicularia). }}$ (1)piunus Anarabacus Cilex. Ila1534. 4. (l, v. 283).

Arping, W. Chr. Die Wetterpropheten im Thiervejel. Lacipz. 180 \%.

Dsluecle, Pelrr. Reise nach Ostind . 82.

Doven, Charles. An essay towards a natural history of Serpents: in two parts. Lond. 1742. 4. W. 7 plat. (Tarantula).

Pallas. Spicilemiazoologien. Fasc. IX (p. 41). - Ririsen durch Russlandl. Ausz. Frankf. und Lip\% 1776. 8. I. (Anls. 1r. 24, Nr. 79 (Aranca Tarantula), Nr. 8 (Phalangrum Calmueo. Bychor1.ho). II: 1) 36, Nr. 97. Ar. sprecios In Oripinaltext, l, 12. 382,413 . III. $p$. 550 (Plaalang. arinenides, dessen Bis. giflim). - Spiciler. Zoolog. Fasc. IX, p. $28-50)$.

Panzer, Georer Wolfgang. Fauna lus. Germanias.

10 andini, Clurist. Frane. De aranea rara, rubieunda, super dorsum alba lineola (Misc. Ae. Nat. Curios. Dec. 3. A. 3. 1695 et 1695, p. 316).

Dennant. Britisl, Zoology. IV, Tab. Ferleb, C. Jul.

7perty, Max.

Detiver, James. Gazophylac. Nat. (passim de Arineis). - Remarks ou some animal, plants sent to ltim from Minlyland hy Hugh Jones (Phil. Trins. Y. 1698, P. 393, 398).

Piso. Will. Arancac magnae et parvae Nlanmdu dietae (Ejusd. Histor. 11it. et medic. Ind. oec. Amst. 1658. 1. 284. (ap. X).

Panter, Franz. Diss, de Tarantismo. Basil. 1669. 4.

Plinius, Cajus, Secundus. Historia mundi libris XXXVII, opus posthunum. Parmis 1181. Fol. (Cf. de edit. Seguir ii bibl. bot. p. 141).

Pluche, Natalis. Spretacle de la Nature. Utrecht 1735. 8. (I, 1).96. Uebers. I, p. 107-139).

Poden, Nicolans. Inseeti musei graecensis. Gratecii 1761. 8.

Pontoppidnu, Erik. Naturhistoric vou Dänemark. Kopeuh. und Ilamb. 1765. 4. (p. 231. Phalang., Aranea).

Douget (vol. Bou).

Prévost, Benedict. Extrait des observations sur les toiles de lidraignce des jardins (Aranea Diadema) (Sociite philomat. A. 7, p. 170). - Memoire 
sur les alajgnées mineuses (Mim. de la soc. d'hist. nat. de Paris. Calı. 1, p. 113).

Hiteysaler, Joliann Danirl. Beschreibung und Abbildung derjenigen lusckten, welche in Sammlungen nicht anf phys. Aufsäize der Gesellsch. böhm. Naturf. 1, p. 55, Bd. II. 1. - Aranca cretata).

Quntremere-Disjonval. Araneologie oder Naturgeselichte der Spinneu nach den neuesten bis jegt unbekannten Entdeckungen, vorzïglich in Rücksicht auf dic daraus hergelcitete Angabe atmosphïrischer Verïnderungen. Aus dem Französischen der zwciten Ausgabe iibersezt. Frinkf. a. M. 1798 (Varrentr.). 8. (Das Original kenuen wir nicht.)

IRaj, J. Methorlus Insectorum etc. Londini 1705. 8. - Historia Insectorum. Opus posthnmum. Lond. 1710. 4.

"Tandohr, F. A.

* Tamiscle, F. F.

CLnnzhni, Canillo. Memorie di storia nuturale. Decad. I. Bologna 1820. 4.

IRnspail, F. V.

Henthlke, J. Entomologiske Jagtfagelser: Acarus, Scorpio, Nymphou, Trumbidium, Pyenogonum, Phalangium (Skrivter af Naturhist. Selskabet, Bind 5, Heft 1, p. 191).

"ERuthke, IK. II. v.

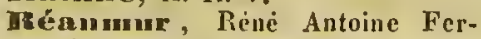
chault de. Akademiker ete.; geb. 1683, gest. 1757. - Mimoires pour servir it l'histoire des Insectes. Paris 1734. T. I-VI. 4. - Observation sur uII inseete deg limaçons (MLim, de l'Acad. des sc. de Paris. A. 1710 . Nèm. J. 305. lid. oct. A. 1710 . Mém. p. 410. A(rarus Limacum). - Examen de la Soye des araiguies (Mém. de I'Acad. loy. de Par. 1710, p. 504).

Medi, Francesco; von Arezzo, Medicus und beriilımter Literator; geb. 1626, rest. 1698. - Experimenta circa generationem Inscctorum. 3 Vol. e. fing. Anstel. 1671, 1686, 1712. 12.

MEcinarans. Vou den Tricben der Thiere. 2. Ausg. Hamb. 1762 (p. 54, 55, 98 : Netzweben der Spinnc). - 4. Ausgr. Haunl). 1798. 8. (238).

IRemueel.

* aremss, Adolph.
Michter, Eiust Heinr.

Aichter. Arzueimittellehre. (Bd. I, p. 190.)

Rivinus. De exanthematibus (figura Sarcoptis Scabici).

Teobie. De arancae ictu eum magnis inde natis symptomatibus (Philos. Trans. Nr. 382).

"Robineru-Tesvoidy.

Renchefort, Cluarles de. Histoire naturelle et morale des isles Autilles ete. Rotterdam 1658. 4. c. t. Spúter 1662. 4., dam zu Frankf. 1668 dcutsch in 12 .

Torlocles (vgl. Blanka art).

Tocmer, J. J. Genera Inscetor.um.

Fisel von Rosenhor, Geb. 1705 , gest. 1759 . Insektenbelustipungen (1 (1), P. 37 (4), p. 6. Il, 45, III, IV, 241, T. $35-40$ ).

Rolnuder, Danicl. Buschspinne (Isis 1837, p. 726 . Aus den schwed. Acten.)

Rossi, Pietro. Fanna Etrusca cum IX Tabulis edita a C. Illiger. Tom. I.I Helmstadii 1807. 8.

"MRossmiissler, E. A.

Rnmplu, G. Éver. Amboin.

Snust. Wirk. d. Spinugew, geg. Wechselficber (Allg. Anz. d. Deutsch. 1808, Nr. 176).

Snuvgges, Augustin Boissicr Abbú de. Observation sur une araignee qui sc reuse un terrier et qui le ferme exaetement avec une porte mobile (Mém. de l'Aead. des sc. de Paris, A. 1758. Hist. p. 26).

"Savi, Paolo.

Savigary. Otviage de Ia coinmission d'Egypte, partie des insectes (planclı. env. 50). 8. (Extr. Bull. soc. philon. 1814. Dec. 168? - Mènoir. II. - Fresswerkzeuge der Aptera (Isis 1818, p. 1200).

sehuiber, J. C. Icones Insectorum circa Ratisbonam indigenorum coloribus naturam referentibus expressac. - Natürlich ausgemalte Abbildıngen regensburgischer Insckten. Ratisbonae 1779. 3 Vol. (mit 280 illum. Kpft.). Element. eutom. Tab.

Schenclizer, Joh. Pliysica sacra. Aug. Vind. 1735. Fol.

"Schunid, Joseph.

Schmied er, Sigismund. De filamentis metcoricis, tempore vernali et autumnali volitantibus, vulgo divac 
virginis, grermanico Jungfer-Gurn, Sommer (Eplsem. Aead. Nat. Curios. Cent. 3 et 4, 1. 357 ).

Seloisugrast, Christ. Andr. Diss. de Enkurek Persarum et ieto Tarantulue. Respp. Petermanno. Lipsiae 1668. 4.

Sclurank, Frauz, von Paola von. Funna boica. - Beitrige (Acarus Srabici). - Eummeratio Insectorum Austriac indigenoroun. Cum figoris. Angustae Vindelicorım, apud Vidaam Eberhardi Klett et Fraok. 1781. 548 S. IV IKpft. 8.

Sclureber. Sammlnnecn. I. Thl. p. 216 (Spiunenseide). X11, 244, 276, 314 (Gewebe den Schafeı selaallieh). XV, 87 (Paarung), 98 (Surgfalt fiïr ilure Juuge).

* Gelureibers, Carl v.

Schroder. Alzueischatz. (V. Buch, p. 25,107$)$.

Sclualty, Gottfried. De ietu Araseac (Ephem. Nat. Cur. Dec. III, An. 2, 1694, p. 89. Obs. 73).

Seluniz. Sinon. De uranear: et folis antipathia et sympathia (Ephem. Nat. Cur. Dee. I, An. 3, 1672, p. 229. Ob\%. 146).

Gchweinger.

Sclawenlifeld. Theriotr. silesine. Lignie. 1603,4 . (p. 504, 505, 506-10). (Melıre Species nnd daun Lapis stellatus Vieforialis: Spimuenst(iu).

Genliger, Jui. Caesur. De subtilitite. Francof. 1582. 8. 1 Exeer. 187, 1 87). - (Ueber Krät\%milbe.)

Seopoli. Eutomol. ealmiol. (p. 392, Nr. $1077-1120$, p. 404). - A 111. 5 hist. nat. (p. 125, Ni. 158, 159). - Introduct. ad hist. natur. regn. anin. ( $p$. 405, Nr. 11(1-117).

Selon, Alh. 'Thesaurus remm natur. Amstelod. 1765. Fol. I. (an vielen Stel. len; danu 11 mind IV).

Sendel, Nuthun. Histarin suerinorum ete. Lips. 1742. in Fol. C. fig. (Besehïft. d. berl. Ges. matf. Pro.)

Seugucerdins, Wolferdıs. Rationis at(une Experientiae Commubinm ete. Acredit rjusd. Disquisitio de 'Tarantula. 3. edit. Roterodami 1715. 8. (p. 277 : Beginu der Disquis.) - Tractat. pliys. do Tarantuli. Isugd. 1668, 1678, 12. - Diss. de 'Tar. ib. 1767. C. fig. werres, Marcel de.

* simon.

Slabber, Martin. Pliysikal. Belustig. oder mikrosk. Wahrrichmungen in - und anslindischer Wasser - ond Landthicrelien. A. d. IJolländ. von P. J. St. Müller. 1775. 4. p. 1 (Schalenspiutie).

Sloane, Hans. Voyage. Lund. 1707. Fol.

* Spence, William.

spengler, Lorenz. (Vgl. Carcinol.) Sperlingius, J. Zoologia physira, et Kirchuajerus de Basilisco, Unicorun, Phoenice, Behemoth, Leviathan, Dracone et Aranea. Lips. 1661. 8. Statrord, Richard. De miris Aerneis: in Bermudis (Acta Oldenborgensia p. 664).

Stalpartus van ler gviel, Comelins. Observationum turiormm cte. Centuriae, poster. Pars I. Lecidac 1727. 8. c. lig. (Obs. 22). - De araneis comesis.

Steller, G. Will. Beschreihung von dem Lionde Kantschatka. Frankfort ond Lripz. 1774. 8. (p. 198: Spillnen valı Weibern aufgesuelst, die gerı foecumdirt seyn mörlaten).

* Stragnss-gbiirhlheim. Hercule.

Suckow, F. W. L. Anatomischphysiologiselie Untersuchungen dey lusekten und Krostenthiere. 1 Band, 1 Heft: oit $10 \mathrm{Kpf}$. Heidelb. 1818. 4. $70 \mathrm{pp}$.

Sulzer, J. II. Kenuzeiclıen der Insckten. Ziïrich 1761. 4. (1. 186, 187). - Aligekiirzte Gesehirlite iler Inseliten Winterth. 1776.4. (p. 245, 218). somilewn II, C. I.

sivaranerulau, Ioh. Bibel der Natul. l_ciply. 1752. Fol. Regist. (Art. Spinne). Originaliuusg. Leyden. 1737. Fol. 2 Vol.

\section{wharsander.}

stheris, Clatiles de.

* Thiene maanau, Fr. Allg. Ludw.

"Thicuenennu, L.

Thitunnais. Merkwïrdigliciten der Natar. Marburg 1738. 8. (p. 79. 81: Simnenseide).

Tilesins, W. G.

Tilling, Mattl. De verunim ae aranearum in fungis generatione (Mise. Ac:ad. Nat. Cur. Dee. 2, A. 2, 1683, p. 164).

Titines, Joh, Dan. Lehrbegriff der Naturgesclı, Leipz. 1777. 8. (p.357).

T'ournou. Sor la Taruntule (Villers et Capelle Journal de la Soc. de sante et d'hist. nat. de Boldeaux. I, 1. 197). 
* Truill,

Trevirnnuk, R. Nirvensystem des Sirorpinms und der Spinne (dessen Zeits(lur. IV, 1, 1S31, 89, T. 6). -Arachuiden (1sis 1818 , p. 485). Vermiselite Scluiften (il. 1818, p. 489). - Ueber den innern Bau der Arachniden. Nürub. 1812. 4. - Urber den Ban der Nigua (Acarina) (lied, und Trev. Zeitschr, f. Pliys. IV, p. 185, Tuf. 15).

* Troschel, Fr. $\mathrm{H}$.

Trost. Beiträge z. Ent.

* Turvia.

* Cndervoond.

Uuzer, D. Kranklteit der Spinnen (Dessen kl.pltysikal. Scluriften. Rintelı und Leipz. 1766. 8. p. 263).

Vnlentimi, Michnel Beruhard. Curiosa in arancis observata (Mise. Acar. Nat. Cur. Dec. 2, A. 7, 1688, p. 112). - Mira Aruncarum generatio (ibid. A. 8, 1689, p. 190). - MI1scum Museornm. I (p. 514: Tarantel). - Amplitheatr. Zonton. ete. Giessac 1720. Fol. Franewf. eod. et 1742 ; erl. ilt. v. Pars II (p. 190 : Araneate anatome).

Valetta, Ludoviei. Sclırieh: De phalangii aculeo. Neapol. 1706. 8. (Tarentola).

Tallisueri, Anlonio. De foramine aculei in seorpione Africano (Ephem. Arad. Nat. Cur. Cent. 3 et 4, p. 48).

"Vanthier, C.

Velschius, Gcorg Hieron. De punctura Vespae et $A$ ranco presentanco remedio extemp. enrata (Mise. Cur. I, amı. 8. 1677, p. 67. Ohs. 35).

villers.

Volckamuer, Jul. Genre. Obscrvv, de duello scorpii cum aranco (Epls. Nat. Cur. Dee. II, An. 6, 1. 466, Obs. 224).

\section{* Waguer, Moritz.}

Wahleuberir.

* Walchenaer, Baron Carl. Atl. v. Tableau des Arancoides. Par. 1805. 8. - Faune parisienne, insectes, ou hist. abrégec des insectes des environs de Paris, elassces d'aprés le système de Fabricins, précedée d'un discours sur les insectes en géneral, pour servir d'introduction il l'itucle d'entomolo. rgie, accompaguie de sept planches gravees. Paris. Dentu imprim. Libr. Palais du tribunal Galeries de bois 240 ; deutscla 228).
Nr. 240, an XI, 1802. 2 Vol. CLX et $301-438.5 .8$.

vallerins. De Tarautula.

Tralz. Ueber dic Kritze (nnd Nilbe) der Schuafe.

Watlius, Frane. L'exercise du Mieroscope. Londr. 1754. 8. (p. 34).

Way, John. (Conf. J,ister.)

Wedel, Georg Wolfgang. Ex ocymo non nasci scorpiones (Misc. Ac. Nat. Curios. Dec. 1, A. 3, 1672, p. 134).

Fegle, van de.

Eegrer, G. Wilh. Schauplatz vieler ungereimien Meinungen und Er. zählungen. Berl. und Leipz. 1735. 8. I (p. 436: Spinnen - und Kröteufeindschaft: III, 258: Spinnensteine).

* Tellpley.

"Westwodi.

Wrichnenam. Schricb (1786) über den Surcoptes Scabici.

* Virler, F.

Wolplnins, Casparus. Seorpionis Insecti Historia (in Libro V Gesneri Hist. in. (pui est de Serpent. nat. Francof. 1621. Fol.

Wornins. Mns. Wormian.

Wotton, Eluard. De Differentiis Animalium. Intet. Paris 1552. Fol. (p. 186-188, Cap. 211).

Woyts, Jol. Jae. Gazopliylac. medico-plsysicum. Lips, 1761. 4. (p. 213: Arau.).

Wray, Joan. Observationes de insolito limarnm gyro, et araucarum jauclatione (Acta Oldenburgensiu p. 845).

Wrede. Ueber den fliegenden Sommer (Nene Srhrift, d. berl. Ges. intf. Fl. IIl, p. 537-555).

Wnifen, Fr. Xavier de. Desriptiones zoologicae ad adriatici littora maris concinnatar. (Viele wirbellose Thiere und unter diesen auch ein Scorpion.)

Wurub, Freder.van. Goudacltig gevlakte teck, op de groote adder van t Eiland Java gevonden (Verlandel. van het bataavisel Genootsch. Deel 3 . BI. 401. - Pedieulus). - Beschryving van de groote tuin-spin van 't Eiland Java (Verlı, van liet batav. Genootsel. Deel 3, BI. 403).

Thetzel, Pelir. Anmärkning om Acari reduvii dödande med bränvin (Vetenskajs Acad. Handl. A. 1780, 


\title{
SUPPLEIENTE
}

\author{
ZU DEN
}

\section{LEXICIS DER EVTONOLOGES IVD CARCINOLOGEN.}

\section{Zum Adressenbuch der lebenden Entomologen.}

Anonyums, in Stuttgart. Biblische Naturgcschichte ete. Mit Abbild. Herausucgeben von dem Calwer Verlags-Veruil. Calw, Stuttg. 1839.

Bnianue, Ingcnieur zu Paris. Aichitccture lics ibeilles (Ann. des sc. nat. 2. sir. XIII).

isallenstedt. - Entdcckung von Inscktemnestem der Urwclt in Bern. stein (in dessen Arehiv V. Bll., [1. 28 $-40)$.

Datennn, Dr. med, nu Edinburgh. An account of the larvae of two species of Insects discharged from the human body (Edinb. med. and surgical Journ. Vol. VII. 1811, p. 41, f. 3, 4). (Pseudoparasiten.)

Bamun, Grosslı̈̈ndler in Wien. Schöne Sammlıng.

Tobe-Morean, in Paris. Mèmoires sur les tcrmites observ, ì Rochefort et dans diverses autres lienx de Ia Clarente infiricurc. Par. 1843.

Dlou, Provinzialmedicus zu Hcumora (Schweden). Insektensammlung.

Bogemaun, zu Botharp bci Grcmma.

Bonsdorfe, Professor in Lund.

Boragtribu, zu Borgo in Finnland. Kerfsammlung.

Herndis, Probst zu Skalscbo bei Westerwick (Schweden). Ostindische Vïgel und Kcrfe.

Bucic, H. G., Dr. med. ete. iu Llamburg.

Contarimi. Macrouyehus quadri. tuberculatus. C. tav. Bassuuo 1822.
Crug, Magistratsrath zu Arboga in Sehwedell. Besizt dic Sammlung scines sel. Bruders (Capitaiı) von schwed. Insekten; gross und schün.

Cnrtis. Brit. Entom. Lond. 1824 - 1839. 16 Vol. 8. 791 Plat. col. (400fl.)

Dandolo, Graf, in Venedig.

Deny. Monographia Anoplurorum Britaniac. Lond. 1812 .

Desmonlins, A., in Paris. Dècnuverte d'élytrcs fossiles de coléoptéres, dans la roche ealcaire de la montagne de Sainte Catherine prés de Roucn (Ferussae Bullet. Oet. 1826, Nr. $220,1 \cdot 253$ ).

Doyére. Darmkanal der Cicaden (Ammal. d. sc. nat. II, Sér. Xl).

Dnbois, Dr. mel. in Brüssel. Untcrsuclungen über die Zahl der Pulssehlïgc der Thiere (Encyclouriaph. des se. médic. Brux. 1840, Sept.).

Funam, in Stockholm. Dio reichstc Combyliensanmulung in Schweden ist in dessen Besitze.

Fricke, J. C. G., Dr. med. ete. zil Hamburg.

Fribliberger, Professor zu Stockholm. Kerfsammlung.

Ferson, G. II., Dr. med. ete. zu Hamburg.

Fory. Oenvies entomologicucs te Mr. Say. Reeueill. et trad. par Gory. Livr, 1 et 2. Par. 1837 (III Vol, in futurum). 
Taxrill, Madamo, zu Söderfors in|den). Sehïler Linnés. InsckteuSehweden. Mammaliensanmulung, Fi- sanmulung.

selıc, Vögel ete. (Gymnctrus Grillii Lindroth).

Fromanhl, Lcibmedicus in Stockholm. Anschuliche Sammlung von Pflauzen, Krrfen, Conchylien und Vögeln. War in Clina und an Cap, wo er sammeltc. Vicle Krebse (vgl. Webcr Naturh. Reise. p. 159).

Gibntluer, G. B., Dr. med. in Hamburg.

Hnefrner, Capellmeister in Stockholm. Grosse Sammlung lapp- uud fimländischer Kerfe.

Irast, Assessor ete. zu Wasa (Finnland). Kerfsammluug.

Hope. On insects oerasioualy foumd in the liuman hody (Trans. of ent. soc. II, p. 266. Pseudoparasiten). - Coleopterists Manual. Edinb. 1838.

Mornstedt, Arzt zu Sveaborg (Fimland). Grosse Sammlung. War in Ostindien.

Hihnendaler, Pastor in Lund. Kerbthicrsammlungund cine vouVögeln.

Jenyns. Noticc of a case in which the Larvac of a Dipterous Iuscets supp. to be the Anthomyia canicularis Meig. ptc. (Trans. of cnt. Sic. II, p. 152 , P'. XV, f. 1-9. Pseudopar.).

Killberg, Kämmercr zu Wisby (Schwedeu). Kerfsammlung.

Kolnstribn, Kapellan in Muonioniska in Lappland. Kerfsammlung.

Kolmodin, Propst in Eiksta auf der Inscl Gothland. - Vicle Vögcl, Kerfe und Conchylien.

Kotzelune, Otto v., k. k. Admiral zu Kronstadt. Zool. Reisc um d. Welt.

Lanrent. Prodrome d'anat. et pliys. compar. Paris 1837.

Lelumanu, J. G. C., ist Dr. med. und l'rofessor der Botanik und allg. Nuturgeseh, am akadem. Gymnasium zu Hamburg.

Hisse, Dr. med. in Mailand. Storia di grave affezione intestinale suseguita dall' evueuazione di una partieolare specic d'insetto nello stato di larva (Gazctta medica di Milano. 1843, 305. Pseudoparasiten).

Jittenzwey , Kerfhäudler zu Wien. Naezén, Dr. med, zu Unea (Scliwe- Marrokanergasso Nr. 516). thum Anlaalt-Cöthen (1 Ml ailc davon

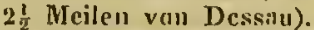

Naumamu, Julius, zu Eisleben (vgl. Gr äfe).

Dppenheim, Fr. G., Dr. med. in Hamburg.

Preifr, Capitain zu Gamla-Cárlcby (Fimulaul). Grosse Kerfsammlung.

Printz, Rathsher zu Gefle (Scluweden). Kcrfsammlung.

Rlıen, zu Carlscrona (Sehweden). Kerfsammlung.

Robert. Sitten der Ameisen (Aum. des sc. I ser. XVIII).

Enthstroim, Secretïr der ökonom. Gesellschaft zu Stockholm. Kcrfsamm)lung.

Scliaum. Amalecta cutomologica (Scydmacu. et Cetonidae). Hal. Sux. 1841.

Tengmalu, Dortor z.1 Westerïs in Schwcden. - Berühmte Vogelsammlung.

Vollenhoven, S. C. Snellen van, in Middelintrg. De sihadclijke Insecten in Tuinen, met. de mildelen tot derzelver verdelging, bencvens cene opgave der voor Tuinen nuttige lusecten. Middelburog (?).

Vrindistedt, zuStockholm. Reiscn in Griechemland. 1826. I.

Wnlulbon, \%u Calmar.

Vestrin, Secretir zu' IInllowy. stagan bei Boet (bej Grenna in Schwe(leu). Kerfsummlung.

Vestuvoad. Aremnn Entoinologica: or illustr. of new, rarc and intercst cxot. Ins. Loud. 1843. W. 4 Plat. Douovaus natural history of the insects of China. New colit ete. With 50 Pl. Lond. 1842 utd dessen Nntural history of the insects of India. W. 58 Pl. Lond. 1842 (ebenfalls neue Elition).

Will, Dr. med. und Professor der Zoologic etc. zu Würzbulø. Beitrïgc zur Anatomie der zusalumengisezten Angen mit fucettirter IIotuhant. Erlang. 1840.

Tahblorukner, Seeretair $\mathrm{Sr}, k, k$. Hoh. des II rn. Erzlicrzogs Joh a u u, zII Wieı (Renmweg, erste Gasse links, 


\section{Akademien und gelehrte Societäten.}

Berlin. Societüt für wissenschaftliche Critjk. - Berliner Jahrbiicher für wissenschaftl. Critik. Stuttg. 1827. 4.

Rologna. Accadem in Clementina. - Storja dell' Acead. Clem. di G. P. Za notti. Bolog. 1739, 11, 4.

Fombny. Medical and phyfical Socicty. - Transactions. Vol. I. Bombay 1838.

Bomm. Naturhistorisches Seminarium. - Andr. El. B üchnerius, Acad. S. F. J. Ltopold. Nat. Cur, historia, Halae 1755. 4.

Erfurt. Erfurter Nachrichten von gclehrten Sachen. Van d. Akad. nützl. Wissenselı. 1797-1801. 4 .

Wrinngen. Ablandlungen der phys. medicin. Societät. Frankf. 1810 -12. 4. und frïher Erlangen 1746. Neue Denkschr, Nürnb. 1812. 4.

Trattingen. Als Continuation der Comment. Jec.: Abhandlungen der $k$. Gesellschaft der Wistenschaften zu Güttingen. Bd. I. Gött. 1843. 4. Fr. Eekard hat ein allgemeines Saelsregister über den Inhalt der Gütting. gelelirt. Anzeigen für 1753-82 herausgegebell.

Irang. Amuales acalemici. Hagae $1830-40,4$.

Deidelberg. Gesell sch a ft fiir wissense haftliche Kritik. - Jahrbincher 1-24. t808-32 ete.

Helsingfors. Aeta socictatis scientiarum fennicae. Helsingf. 1840.

Imigsberg. Naturwissenschaftliches Seminarium. - Jerster Bericht über d. natw. Sem. Kün. 1836. 4. - Zugang: der Bibliothek. 1837. 4.

Kopenhagen. E. G. Viudinfius: Regia accademia havuiensis. Havn. 1665. 4.

Ir rolian. Anualen der gelehrten Gesellschift der Univ. Krakau. 10 Vol. Krakau 1823-25.

ceeds. Philosophical and litterary Society. - Transactions of the phit. a. litt. Soe. of Leeds. Ib. seit 1833 .
Heipzig. Acta eruditorum publicata 1682-1773. Nova. 1732-1776. Suppl. 1692-1734. Nova. Suppl. 1735 -1757. Indiees ab initio 1745 (118 Tomi). 4. - Deutsch: Leipzig 171239 (240 Theile). - Acta lipsiens. Ac. Lips. 1723-24. 16 Vol. - Comment. lips. litterarii. 3 sect. (ed. Frid. PlatI c r). Ib. 1753-54. - Miscell. lips. 1-12. 1716-23. Nova $X, 1742-52$.

Een warden. A the nacum. E. L. Vriemont: Athenaeum frisiacorum lib. 2 (Franequerae). Leovardine 1758.

Liven. Nic. Vernulacus: Academia lovaniensis. Lovanii 1627. 4.

Loudon. Report of Mecting of british Association 1832. Lond. 1833 -39 . VIII.

Littich. Akalemic der Wissenschaften. - Annales Academiae Leodiensis. 1824-27. IV. 4.

Hondi. Lind fors: Analecta hist. Academiac lundens. 1819. 4.

Miinchen. Miunch. Akad. Jahresberiehte. Min̈ch. 1808-33. 4. - GeIchrte Zeitung.

Nerucl. F. M. Avellino: Notizia di lavori dell' Aecademia pontoniana megli amni 1826-29. 4.

Prois. Archives du Museum d'histoire niturelle. Paris. Seit 1839.

Philatelphia. Notice of the Academy of nitt. sc. of Ph. Third edit. Phil. 1836. 8.

Hestock. Annales literariac Mecklenburg 1741-42. Rostockii.

Strasburg. Siance publicuc. t837. 4.

Venedig. Gelehrte Gesellsrhaft. Sonst war da cine Akademie. (Summa librorum yuos emittit Acnd. Venet. 1559. 4.)

Wilirzburg. Jahrbücher der phil.med. Gesellschaft zu Wülzburg von Friedreich. Wiirzb. 1828. - Nene Jalırb. 1830 , 


\section{Naturhistorische Ephemeriden.}

Anunli (Nuovi) delle scienze naturali del Sign. Alessandrini, Bertolonj, Gluerardi, Ranzani.' Tom. 1. Bologna 1838.

Annali letterari d'Italia. Modena 1762. III.

Arelsives littéraires de l'Europe, oil Melanges. Par. 1804-8. XVII Tomes. Contin. 1808-28. Paris 1809 -28 .

Arnoult, E. L'Institut. Zeitsclurift.

mibliothedque universelle de Genève. Nouv. série. Prem. Annèe 1836. Genève (Glaser) 1836. 8. Cah. $1-51$.

Corbyn. The India Journal of medical and physical sciences etc. Calcutta 1836 (I). April.

Eco (I') Mneeratese, di Fr. Nobilj. Giornale medico-chirurgico. Macerata.
Indientore (L') Lombardo. Nilano Seit 1830.

Maund. The Naturalist. London 1837. I.

Minervn (La) Tieinese, Giornale di scienze, letterc, arti, varièta. Pavia 1830. 8 .

Poligra ro (II) di Veroma. Seit 1830.

Review, The eclertic. New ser. Lond. 1820-35. Vol. 14-30 and third ser. V Vol. 1829-35. (Mels salsen wir nicht.)

Tromusdorf, J. B. Annalen der Fortschritte ete. III. Erfurt 1809. (Vgl. Busels und Bellermann.)

Tithingergelehrte Anzcigen. Tüh. $1783-99$. 1800. 4 .

Urban, Sylvanus. Gentleman Magazin and historical Chrunicle. Lond. grr. 8. 1757-60. 27 Vol.

\section{Bibliographien und Literaturgeschichten.}

Almanak literario ete. o catalogro general de todas las obras de ciencias etc. Madrid 1804-6. II Tom.

Antonius, Nic. Bibliotlieca hispana. Romae 1672. II 'T'. Fol.

IIalbi, Ad. Essai statistique sur les bibliothèques de Vieme. Vienne 1835.

Baldinger, Er. God, Catalogus Bibl. medieu-physicae. 1. 2. Marburg. 1805.

Barbler. Examen eritique et complement des dietionaires hist. Par is 1820.

Bnuer, 1k. Vollstïndiges Verzcicl,niss rarer Bücher. Nürnb. 1771. 6 Bde. 8.

Beckmanu, J. Jae. Pliysikalischakonomiselse Bibliothek. 1770-1780. XXII VuI.

Beilermanu, J. J. Almanach der neuesten Fortschritte etc. Erfurt 1807. VI.

ITenglenen, Corn. a. Bibliographia medira et physiea. Amstel. 1681.

Bibliographie der Bicneuzucht. Nürıb. 1800.
IBibliographie (ef.ConversationsLexikoul).

IBbliotlueen physico - medies. Writisl. 1776-77. 11.

moehmerus, Joh. Benig. Bibliotheca medieo-philosophiea. Lips. 1755.

Briquet, Fort. B. Dictionnaire list., lit. et bibliogr. des Français et des Etrang. natul, ell Franec. Paris 1804.

Brucksuamu, Fr. Ern. Bibliotheca Animalis. Wolffenb. 1743. 8. und Forts. ib. 1747. 8.

Brumet, J. Ch. Nouvelles recherches bibliographiques. Paris 1834. III. - Manuel du libraire. Paris 1810. III. Edit. 2. 1814, IV. - Ed. 3. 1820. IV. - Ed. 4. 1838-39, IV. - El. 5. 1842-44. V.

Mrnnuer, L. P. De statu rei literar. in Suecia seculo 16. Lond. Gotlt. 1831.

Bnchinullerzeitumg (j. 101). Eigrnthum des Vercints. Ej'scheint noch.

Budik. Bibliothekarstudien. Wien 1834.

Burdach, K. Fr. Handbuch der 
neuesten in - und ausländischen Lite-|lexikon. Leipz. 1793-98. V. Neue ratur der ges. Naturwissenselaft (den Aufl. 1812. 11.

3. Bd. der Liter. der Heilwissenseh. bildend?

Bure, Guill. Fr. de. Bihliographie instructive. $1-\mathrm{X}$. Paris $1768-82$. Catalogue des livies. Par. 1757.

Catalogue de la Bibliothèque d'un Amateut. Paris 1809. IV.

Clere, Jean de. Bibliothèque aneienne et moderne. Amsterd. 171127. XXVIII Vol.

Croix du MI aine ete, De la. Les Bibliutheytees françaises; nouv.ed. Par. 1774. VI Vol. 4.

Dentis, M. Einleitung in die Bücluerkunde. Wien 1777-78. II, 2. Aufl. 1795, II. 4. - Merkwürdigkeiten der garellischen Bibliothek. Wien 1780. 4.

Deuso, J. D. Physikalisehe Bibliothek. Post. und Wism. 1754-57. 1760-61. 11 .

Desessarts, N. L. N. Les sières litt. de la France. Par. 1800-3. VI Tomes et Suppl. - Nouv. clietionnaire bibliographique. Par. 1804.

Dibulin, 'Th. T'. Bibliotheea spenceriana. Loud. 1814-15. IV. 4. Deseription Catal. Lond. 1823.

Dictionunire bibliographique, historigue et critique des livres races. Paris 1791. 3 Vol. 8.

Whle, Burkl. Vers. einer pr. Gesehichte d. Anat. und Physiol. vou $1800-25$. Wien 1836.

Erxleben. Physikalisehe Bibliothek. Gött. 1775.

Ferussac. Bulletin universelle. Par. 1823-24.

Formey. La France literaire. Berl. 1757.

Gresnerus, Couradus. Bibliotheea universalis. 'liguri 1545. Fol.

cesnerus, J. M. Biographia aendemia. Gütting. edlil. J. N. Eyring: Halae 1768-69. $11 \mathrm{I}$.

Feorgins, Thcod. Allgemeines curopäisches Bücher-Lexikon. V Tilıle. M. III Suppl. Leipz. 174\%-58. Fol.

Txingrueve, P. L. Ilistoire litteraire d'Italie ('Tom. 10. cont. par $S$ alfi). Paris 1811-23. X.

Tistoire litéraire de la France. Paris 1733-1824. I-XVI Vol. 4.

Miclutentlial, Pietr. Manuale bibliografico del viaggiatore in Italia. Milano 1830.

Mongitorl, Ant. Bibliotheca sicula. Panorni 1708-14. II. Fol.

Moscluini, G. Ant. Della Letteratura Veneziana. Venez. 1806. III. 4.

Nülder, Jub. Traug. Einleitung in die ökonom. und phys. Büeherkunde. III Bde. Leipz. 1780.

Pongens, Chr. Bibliothèque fran: caise. Paris $1800-1$, à $12 \mathrm{Vol}$. (24 Bäinde).

Puy de Montbruu, E. H. J. du. Recherches bibliugraphiques. Leide 1836.

Teiu, Gabr. De rerum fenniearum seriptoribus ete. Aboae 1826. Part. I-IV. 4.

Ricle, O. Bibliotheea americana nova. Lond. 1835.

Rolır, Jul. B. v. Physiealisehe Bibliothck. Leipz. 1774. - Verbess. vou G. Küstuer. Leipz. 1784.

Salamon, A. G. II Verzeichn. iilt. und neuer Büeher $(22,000$ Vol.). Kopenh. 1845.

Sclnem clizerus, Johannes Jacobus. Bibliotheca seriptorum historiac naturalis omnium terrae regionum inserviention; Hlistoriae naturalis omnium Helvetiae prodromus; aceedit Jacob lc Long. de scriptoribus histor. nat. Galliae. Tiguri 1716. 8.

silberu, N. P. Bibliofheen historica dano-norwegica. Hamb. 1716.

Sprengel, C. Literaturac medieac ext. ricentior Lips. 1829.

Stueliu, Chr. Fr. Zur Gesehiehte und Beschreibung alter und neuer Büchersammlungen im $\mathrm{K}$. Württemberg. Stuftg. 1838.

Talblettes universelles ou resume de tous les Journaux et bibliogr. gener. P. J. B. Gouriet. 1' 1-36. Par. 1820 $-1822$.

Tartarotti, Giacop. Saggiu della Meinsins, W. Allgen. Büehcr-| Bibliotheea tirolese. Roveredo 1733. 
Und später (mit Todesehini) Venez. 1777.

Theile, J. C. Bibliothek. Leipz. 1837. 8.

Thum, Grif v. Leo. Ueber den gegenwiilt. Stand der bölm. Literatur und ihre Bedeutung. Prag 1812.

Tirnboselni, Girolamo. Bibliotheea modenese. Mod. 1781-86. VI. 4. Storia della letteratura italiana. XII Vol. Mod. 1787-93. 4. (Auch Venez. 1795-96. XVI Vol.)

Tolerini, Giamb. Letteratura turchesea. Venez. 1787. III (deutsel von Hausleutuer, Königsb. 1290. II).

Wpsa In. Catalogus libr. Bib. Aead. Upsal. 1814. 4.

Valentincllas, Jos. Sperimen bibliographieum de Dalmatin et agro Labentium. Venet. 1842.

Villemsin. Cours de Litterature française. I, II. Par. 1838.

vogel, Ern. Gust. Literatur frü- herer und noch besteliender europ. und Corporations-Bibliotheken. Leipz. 1840.

Wnchler, Iuulw. Vers. einer allgem. Geschichte der Literatur. Lemgo 1793-1801. IV. - Lehrbuch d. Literaturgesch. Leipz. 1827.

Werlnuff, Er. Chr. Arius mul. tiscius primus Islandorum historicus. Haf tr. 1908.

Wrede, E. F. Eneyelop.-plıysik. Literatur. Leipz. 1806.

Wyttenbncln, Jak. Sam. Gelehrte Nachrichten aus IVelselıland, vorz. d. Pliys. und Naturk. betr. Bas. 1783.

Znchariae, Frane. Ant. Storia Ictteraria d'Italia. Venez. 1753. Modeva 1754-55. Contio. il). $17506-59$. Suppl. Lueca. 1753-55. XIV Vol.

Zuodineus medieo-gallicus. Opera Nic. de Blegny et de la Roque. Paris. 4.

\section{Biographien.}

Adrovandi. Memorie della vita di Ulisse Aldrovandi medico e filosofo Bolognese ete. Bologna 1774.

Athenacum berïlumter velehrter Wiirttemberger. Stuttg. 1829.

Bnnder, Kl. Al. Das gelehrte Bayem. 1. und cinziger Band. $\mathbf{A}-\mathbf{K}$. Nürnb. 1804. 4.

EReutlner, Christ. Hamburgiselıes Stats- und Gelchrten-Lexikon. Hamb. 1739. 8.

Biographien. Verweisen auf das "Sachregister zum Kayser'schen Bïcher" - Lexikon. Leipz. 1838. 4. p. 220. VIIl (bis 225)" und l. e. p. 436. Ebenso Literaturgrsehiehte.

Calnet. Bibliographic lorrain ou histoire des honmes illustres ete. Naney 1751. Fol.

Cavier. Mémoires dn Baron G. Cuvier, publices en anglais par Mistresse Le e, et en français par M. Theod. Lalcordaire. Par. 1833.

Czilsann, J. J. H. Dic Iebenden Silıriftsteller Mălırens. Brünn 1812.

(Debrny.) 'Tablettes des Eerivains français. 2 Vol. Paris 1810.
Dictionnaire bistorique ou listoire abrégée de tous les honmes, gui se sont fait un nom etc. Paris 1772. $\mathrm{V}$ Tomes in 6 Vol. 8.

Ebeling, D. Memoria J. A. H. Reimari. Hamb. 1615.4.

Elovert, Ph. Nachrichten von dem Leben etc, deutseh. Aerzte und Naturforscher. I. Hildeslı. 1799.

Felder, Fr. K. Gelehrten-Lexikon der kathol. Geistlichkeit. Jandslıut 1817. Fortges, von H. J. Waitzenegfer. Landsl. 1820. II. (Darin ist Sehrank's cte. Biographic enthalten.)

Finauer, Pet, Paul. Versull einer bay'r. Gelelirtengesch. Münel. 1767. - Magazin f. d. Gesel., und Kenntu. bayı. Selıriftst. Münclı. 1775. 1-6. 4. Ind 1782.

Finsler, J. Bemerkungen aus dem Leben des Jol. v. Muralt. Zürich. 4.

Crmohumann, J. G. Neues histor.biogr. Handwörterbueh, Leipz. 1796 -1808 . X.

Fros, C. H. Memoria J. Clı. Dall. de Schreber. Erl. 1811. 4. 
Fronov's Leben. Hamb. $1723 \mid$ verst. deutseh. Sehriftst. Leipz. 1802 (von H. Wilckeus).

criildewstielt's Leben (Sehrift. der berl. naturf. Freunde. II).

namberger, G. Chr. Das gelehrte Dentsehland. Lemgo 1767-70. M. 2 Nachti, - 2. Aufl, von Mensel. 1772 - 74. Forts, vou Meusel. 3. Ausg. 1776-78. - 5. Naclitr. 1783-84. 5. Ausn. 1796-1812. I-XVI.

Kans, B. Das gelehrte Württemberg. Stuttg. 1790.

Hesse, Ludw. Fr. Verzeichniss seliwarzburo. Gelehren ete. $6 \mathrm{St}$. Rudolst. 1831-36.

Itirsching, F. K. G. Histor. literar. Ilandbuch berülumter und denkwiirdiger l'ersonen des 18. Jahthund. Leip\%. 1794-1815. XVII Vol.

Joecher, Chr. Gottl. Allgem. Gelehrten-Lexikon, nebst Forts. von Adelung. und Rotermund ete. Leipz. 1750. VII. 4.

Iñliun, M. v. Kätenstein. Nach. riehten über böhmisehe Sehriftsteller. Prng 1818.

Klecmann, C. F. C. Nachricht von den Leben und Schriften Aug. Juh. Röscl's von Rosenhof (im IV. Theil scin. Ins.-Bel. Nr. 76).

Hobalt, Ant. Mar. Bayrisebes Gelehrten-Lexikun (bis 1724). Landsh. 1795. - Ergïizungen hiezı gab G a ud e r.slof er heraus, ib. 18:4,.

Indhter, J. Fr. Lebensbeschreib. merkw. dentscl. Gelehrten elc. Leipz. 1794. II.

Roppe, J. Chr. Mecklenbure's Schriftsteller. Rost. 1818 (und überdies: Krey's And, an Rost. Gel. ib. 181-16).

Leske's Lebeusgeschiehte. Leipz. 1787.

Mrahul. A. Anumaire necrologique. 1820-27. Paris 1821-30. X Vol.

Mrarchand, Pr. Dietiunnaire historique (biogr.). Haye 1758-1759. Fol.

MireIners, Chr. Ippensbesehreib. ber. Mämmer. Zürich 1795-96. 111.

IIensel, J. G. Das grelehrte Deutsclulaurl. 5. Ausir. Lemgo 1796-1811-12. $\mathrm{XV}$. - Lexikon der voll 1750-1800 $-1816 . X V$.

Necrologe (Nouveau) français. 1800 (par A. J. Q. B.) Paris 1812.

Nitzschins, Greg. Guill. Memoria Christ. Rudolphi Guill. Wiedemanni Kiliae 1841. 4.

Perrault. Les hommes illustres. Paris 1696-1700. Il. Fol. (Editio non east ruta.)

Rnimann, Joh. Nep. Rede zur Gedïehtuissfeier des Nieol. Jos. Frlurn. v. Jaeruin. Wien 1818. 4.

Recke, J. Fr. und Napiersky, K. E. Allgem, Schriftsteller- und Gelehrten-Lexikon von Livland, Esthland und Kurland. 4 Bde. Mitau 1827-32.

Reuss, Jer. D. Das gelelurte England. Berl. 1791. II. 2. Ausg. 1804.

Saint-Benve, C. A. Critiques et portrnites litéraires. V. Par. 1841. Selnlichtegroll, Fr. Necrolog auf 1790-93. Nebst Suppl. Gotha 1791-1806. XXIII. - Necrolog der Deutsch. Gotha 1802-1806.

Sclnmidt, And. Gottfr. Anbaltsehes Schriftsteller-Lexikon. Bernb. 1830. Gallerie deutselı, pseudou. Schriftst. Grimma 1840.

Selnuidt, Fr. A. Neuer Nekrolog der Deutsehen. Ilm, 1824-37. Mit Register; Veimar 1836-48.

Sclivenckfeld, C. v. Historisehe Nachricluten über denselben. Prentz. lail 1744.

Scriloa, Hr. Ed, Biograph.-literar. Lexikon der Schriftsteller des Grossherz. IIessell. Darmstadt 1831.

Spangenbere; J. Clır. J. Handbueli dihingeschiedener Gelehrten. Jena 1819.

strnln, Phil. Das gelelirte Russ. land. Leipz. 1828.

Strieder, Fr. W. Grundlage zu ciner hessischen Gelehrten- und Sehriftsteller-Geschielıte, 18 Bde. Götting. 1781-1819. (Fortges, vou WVachler. und $\mathrm{J}$ แ $s \mathrm{ti}$.)

T'afuri, G'. Bern. Serie cronolog. degli serittori mati nel regno di Napoli dal seculo V fino al. see. XVI (in Calogiera recolta d'opuscoli T. XXIII, XXIV).

Thiess, J. 0. Gelehrten-Gesehiehte 
der Universität zu Kiel. $1800-1803$. II Vol.

Tieden, E. J. H. Das gelehrte Ostfiriesland. Aurich, 1785-90. III.

Trembley, J. Vie privece et litteraire de Charles Bonnet. Berne s. a.

Vindicianus. Elogium viri G. Franci de Frankenau. s. I. c. 3. 4.

Walehs, J. E. Innn. Leben und Charakter von Hering. Weim. 1779.
Wilckens, H. Hamburgischer Ehrentempel. Hamb. 1770. 4.

Will, G. A. Nïrnbergisehes Gelehrten-Lexikon. Nürnb. 1755-58. IV. 4. und V. (Forts. von Nopitseh. 1802-8.)

winkleru, J. B. Biograph. und literar. Nachricht. von den Schriftst. ete. Steyermarks. Grätz 1810.

\section{Realwôrterbücher.}

Cyclopaedia: or, a new Uni-|fesseurs du Jardin du Roi etc. Supplèversal Dictionary of Arts and Sciences. 4. - (Eneyclopacdia britanica.)

Bayer, Mark. Forst- und JagdWörterbuclı. Leip\%. 8.

Eochartus, Samuel. Hierozoicoll. Londini 1633. Fol. II Vol. (Auch später ibid. 1663 und Franequerae 1619. 4.)

Dictionnaire des seiences uaturelles, suivi d'une biographic des plus celèbres uaturalistes. Par plus. Pro-

Zziegre. Standrede am Sarge H. Melch. Güze's. Hamb. 1786.

\section{ment. Par. (Vol. T.) 1840.}

Dieterich, M. Naturhistorisches, ökonom.-lechnolog. Handwörterbuch. Ulin 1817. 2 Bde. 8.

Klihgel, Geo. Sim. Eneyclopädie. 6 Bde. Berlin. 1782 und 1806-10.

Schan platz der Natur. Leipzig 1775. 11 Bde. gr. 8.

Schmiedlein, B. Handwörterbuch der Naturgeschichte. Leipzig 1801. 3 Bde. 8.

\section{Oeffentliclıe und Privat-Museen.}

Brckmann, Sueno Jols. et Reinholm. Primordia musei Alexandrini. Helsiugfors. 1840. 4.

Cnrevell, Robert. A visit to the Mantellian Museum at Lewes (London Magaz. 111, p. 9-16).

Cubinet zu Heidelberg. C. Gatterer Verz.

Fischer, Gottl. Das Nationalmuseum der Naturgeselichte zu Paris. Vou seinem ersten Ursprunge bis zu seinem jetzigen Glanze geschildert. 2 Bde. M. Kpf. und P'l. Frankf, a. M. (Esslinger) 1802-3, 8.

Mirschings, F. C. C. Nachriehten von sehenswiïrdigen Gemälde- und Kupfersticlı. Sammlungen, Münz - und Naturalien-Kabineten. 6 Bde. gr. 8. Erl. 1786-1792. (6 fl.)
Comdon. Synopsis of the contents of british museum. Lond. 1823.

Molinet, Pierre. Le cabinet de la Bibliotheque de Sainte-Geneviève, contenimt les Antiqu., des Animaux les plus rares. Par, 1692. Fol. c. tab. aeneis.

Moutfancon, Bern. de. Diarium italicum. Par. 1702. 4. (von Bibliotheken und Museeu).

Praris. La Menagerie du Muséum national d'histoire nuturelle ou description et histoire des animanx qui y vivent ou qui y ont vécu; par les citoyeus Lacipede et Cuvier, avec des figures peintes d'après nature, par le citoyen Marechal peintre du Museum, graves, avec l'agrement de l'administration, par le citoyen Miger ete. Paris, ehez Miger, Patris, Grundcher, Dentu. an. $\mathrm{X}-1801.41 \mathrm{~K}$ ptaf. 
Pesth. Das ungarisclie Nationaluncum zil Pestl. Wien 1814. 4.

Stocklootm. Ueber die Sammlungen der Akademie der Wissensel. vel.: Naturlistorisclie Reise durch eiuen Theil Schwedeus von Dr. Fr. W e ber (zu Kiel) und D. M. H. Mohr (sonst in Güttingen). M. 3 K. Gölt.
1804. 8. (1) 133-142). - Degeer's Kerfiaumulumg.

Cipsala. Museum dev Gesellschaft der Wissensclaften. (Das älteste fnstitut in Schweden dieser Ait.) - Naturh. Sammlungen. Vgl. Weber's und Mloh,'s Reisen, p. 162-165. - Bibliotluck der Universität Upsala. (lb. 1. 146.)

\section{Syllogistische etc. Schriften.}

Anonymus. Memvire instructif Minerals for a plysical use. London sur la manièe de rassembler, de pré- 1672. 12. parer, de conserver et d'envoyer les diverses curiosites d'histuire naturelle etc Lyon 1758. 8. (Mit $25 \mathrm{Kpftf}$ )

Boluest, Edwurd. A rutional way of prepiting Auimuls, vegetables, and

Trenge, Fr. Farben-Lexikon. M. 48 enl. Kif. Halle 1782. 4. (Die Natuv: gesthichte lat cirea 36 Farben und deren Abstufungen.)

\section{Ortsregister.}

Arboga (Schwed.). Crug.

Forgan (Finulaud), Borgeström.

Gotharp (Schwed.). Bogemann. IBrissel. Dubois.

Cnlmar. Walilbom.

Coriscroua (Schwed.). Rhen.

Edinburgh. Bateman.

Cilosta (lusel Gottland). Kolmodin. wislefoce. Jul. Nanmann.

CAnula-Carleloy (Finnl.). Pfeiff. Fefle: (Schwed.). Priutz. riil.

MaIlongstugnan (Schwed.). West-

Lanburar, 13uck. Frirke. Gerson. Güntlier. Oppenheim.

Bhedunarn (Scliwed.). Blom.

Kronstadt. v. Kotzebue.

Gund. Bonsdorf. Hünemüder.
MIImiland. Jisse.

viddeilon rer. Volleuliuven.

Mnonioniska (Lippland). Kolıströn.

Pnris. Bulanne. Bohe. Moreau.

Stralsebo (Schwed.). Brandis.

siiderfors (Schwed.). Grill.

Stockluclin. Ekinanu. Fröhliergrı. Gröndall. Irílfıer. Ruthstrüm. Vröustedt.

Situttarart. Anonymus.

svenbory (Fiunland). Hornstedt.

Unen (Schwed.). Naezèn. .

Venedig. Dandolo.

Evasa. Hast.

Westerais (Scliwed.). Tengmaim.

IVien. Baum. Mittenzwey. Zahl. brurkiner.

WVisloy (Schwed.). Killberg.

vritrzburg. Will.

Zhebigk (bei Dessau). Nuumann,

\section{Entomologische Schriftsteller.}

Anbrosins, In Iexacmeron lib. Tesilias. Hexaemeron congressio. 5, Cap. 51 ( $\Lambda_{\text {pisis). }}$. 8. (A pis). 
IBres. Observations Entomologinges (Larve de Stapliyliı) (Mlènt. de Lau. simne. III. ts. Av. Vign.).

Caelfus minodiginus. Apis (I_mo) Lih. 22, Cap. 3, j. 848.

Crentzer. Stymbolit.

Hazzi, von, Staatsrath. Das Ganze des Seidenbaues. 4.

Mernclides Ponticns. Cap. 9 (Apis).

Holneuwarth, Frhr. v., in Jos. Reiner's hut. Reisen I. Auhang 258270. Tab. (Scarubaeus armiger. Papilio eassioides, Sphinx exulans, Phalaena chaerophyllata).

Dlorus Apollo. Hieroglyplo. ( 1 , 62. Apis).

Jamblichus. Apur Phatrum cap. 94 (Apis).

Kocl. Insekten-Iarven im menschl. Darmkanile (Ammon's Monatsber. f. Med. I. HIt. 6. 1838. 642).

Eevale. Sur l'histoíre naturelle des Guêpes (Mém. de Lausamıe III, p. 23).
4) ungh. I,ebte zи Skjïrsjo (einige Meilen von Jünkïpinğ).

Mnreinums. De Etruria metropoli (p. 182 und bei Creutzer Symbolik. IV, 1. 416. Apis).

Dlivier, M. Entomologie ou hi stoire naturelle des Inseetes. Coleoptères. Paris. I-VI. Vol. 1789-1S08. 4. (avee 360 planel. col.).

I"orpliyrius. De antro nymph. Cap. 16 (Apis).

Rossi, Petrus. Fauma etrusea sistens Insceta quae in provinciis florentina et pisana praesertion colleyit. I, 11. Cun IX et XI Tab. Mantissao priore parte adjeeta, iterum edita et annotatis perpetuis aucta a D. Joh. Christ. Ludw. Hellwig. Helmstadii 1795. 8. Tom. secmdus iterum edita et annot. perpet. aneta a D. Carolo Jlliger. Helmst. 1807. 8. (p. 196-201 Crustacer et p. 201-222 Arachnid.).

Serents Somonicus. (Cap. 13, ping. 43 seq. ed. Ackermann. A pis).

\section{Zum Adressenbuch der lebenden Carcinologen.}

Mnclumer, Andreas. Dr. med., ord. offentl. Prulessor der Chumic etr. an der Universitit zu Niinclielı. Huch. resehäzt als Lehrer, Schriftsteller und Mensel.

Burueister. Organisation der Trilobiten. Berl. 1844. - Ueber den Bau der Angeu von Branchipus palıdosus (Müll. Archiv 1835).

Crerpenter, in London. Mikroskopische Untersucluungen niber dic innere Struktur der Skelete der MolIusken, Crustaceen und Eelinodermen (Aunals of nat. list. XII).

Dnveruoy, Professor zil Strasbury. - Ucber eine newe Kienientorm, entdeckt aı dent zelınfüssigen Krebs Aristeus (AnII. d. sc. nat. II, sír. XV).
(Mit Lereboullet) Ueher Atlımungsorgane der Isopodin (ibill.).

Doyere. Tardigrades (Crust.) (AIII, I. sc. n. nouv. sér. XIV, 269361. - Valentin Repert. 1841. 2(i1).

medi, M., in München. Ueber Haarbildomg (Abl. d. k. bayr. Akad. 1841).

Fnare, in Loudon. Uchey las Gehürorgan bei den Krebsen (Pliilos. T'rans, 1843. IX).

Forbes (und Oersted). De regionibus marin. Elem. topogriphias hist. mat. freti Oeresund. Diss. imang. Copenhag. 18.14.

Germar, Professor in Halle. Reise nael Dalmatien (Crustaceen und Araclmiden). - Entdeckung cines Trach- 
neglases mit Idotea antiquissima (Ballenstedt's Archiv 1824. Vol. 6. Cah. 1, p. 79. - Férussac Bullet. Oct. 1826, Nr. 138, p. 153).

Joly, Carcinolog und Scliriftsteller in Toulouse. - Verwnndluug der Decapoden (Aun. d. sc. 2 sèr. XIX).

Koch. Crustacecn etc. Bis 184440 Hfte. - Arachn. Bis 184411 I3de.

Mnndl, in Paris. - Mèmoire sur Ia disposition en spirale des appendices tigumentrires des animaux et ile quelques autres parties de l'economie (Auı. d. sc. nat. 2. sèr. IX).

Martin St. Ange. Mèu. sur l'organisation des Cirripèles. Par. 1835.

Newport. Entwickelung der Myriapoden (Pliil. Traus, 1841.99).
Flilippl, ill Cassel. Eutomostr. d. Mittelmeers (Wiegm. Arcl. 1843, I).

Ratlake. Beiträgc zur vergleichenden Anatomie und Pliysiologie. Rrisehemerkungeu über Sknudinavien etc. Danziy 18.12. (Zur Eutwickelungsgeschichte der Decapouleu.)

Schultz. Echiniscus Bellermani. Berol. 1840.

Tulesius. Ueber das wächtliche Leuchten des Mcerwassers (Ann. der Wetterau. III, 1814, p. 360-373 und IV (oder neue Aun. 1). 1819, p. 1-11, Taf. XX und XXI, a, b (Crustaceen).

Vaientiu, Professor zu Bern. Ueber den Ban des Hautskelets der Crustaceeu (in desseu Repert. 1836, p. 122).

\section{Wolnorte der Carcinologen.}

Iern. Valentin.

Cassel. Philippi.

Mopeniagen. Forbcs.

Meipzig. Tilesins.

Yomilon. Carpenter. Farre. Newport.
Mihmelıen. Büchner.

Paris. Mandl.

Strasburg. Duvernoy. Isercboullet.

Toulouse. Joly.

\section{Carcinologische Schriftsteller (todte).}

Acostn, Josef de. Historia uatu-[tabilibus, Animalibus et quolibet ente ral y moral de las Indias, cn que sc in subsolari globo. Lips. 1488. 4. tratiu las cosas notables del Ciclo: y Elementos, Metales, Plantas, y Animales del las. en Sevilla 1590. 4. Barcellona 1591. 12. (daun in versehiedenen Ausgaber. Cf. Gronov. Biblioth. p. 2).

Adanson, Mr. Histoire naturclle du Senegel. Tome I. Paris 1757. 4. Cum tal. XIX aen.

Adlerus, Carolis Frilericus, Diss. de Noctilucn Mariua, sub praes. Car. Linnaei. Upsal. 1752. 4. C. fig. (Amoell. Aend. 1II, p. 202 . C. fig. Urban Gentl. Magaz. Vol. XXVII, p. 208.) Vgl. Griselini's Scolop.

Albano, Magister Petrus de. Libellus de Venenis, Mincralibus, Vege-
Aliertus cognonicutus Magnus. De unimalium proprictatibus libri viginti sex. Mantuae 1479. Fol. (Edit. antiquiss. et rariss.). - Liber secretorun de virtutibus herbarum, lapidum et Animal. quorund. etc. Antverpiae 1555.8 .

Albinus, Bernh. Siegfr. Academicarum annotationum liber. Lcidae 1754. 4. C. tab. VII aell. II. Ibid. 1754. C. t. VII, III. 1756. C. t. VII.

Albin, Eleäser. A natural history of Euglish Insects, illustrated with hundred copper plates etc. London 1720. 4. W. Not. a. c. obs. by W. Der h a m.

Anderson, Johann. Bescliryving 
van Ysland, Groenland èll de Straat Davis. Amsterd. 1750. 4. C t. aen. (Ais d. Deutsrh. v. J. D. J.)

Anouyuans. Vocabula rei nummariace cte. Collecta ex Budaci an. etc. IVitteberg 1558. 12.

Amouymus. Arlescription of three. lunderl Animils, viz. Beasts, Birds, Fislues, Scrpents and Inserts ele. Luond. 1753. 8. C. tab. aen. With suppl.: A Descr. of atgreat variety of Animals cte. Lounl. 1753. 8.

Anouymus. Systime naturel du Règne Animal. Paris 1754. S. 11 Tom. Av. pl. (lom. II lnsecta elc.)

Aqnacens, Steplamus. Biturieensis vili equestris. Lı ommes e. Plinii see. Naturalis Ilistorine Algutissimi seriptoris libros Commeatarii. Paris is 30 . Ful.

Armstrong. Julur. History of the Islind of Minureril. At Lundon i752. 8.

Avicenen, Abeusiuil. De animalibus A ristatelis libri novemdeciu, ex arabiea in latinum translati per Mnnistrum Michaelem Scotum. s. I. et a. Fol. (55-101 Seilen).

IEnrtholiums, Casparus (Solundes Tlumas B.). Diss, de Resjuratione animalium. Hnfuiae 1700. 4. - De maturae Mirabililus. Hati, 1674.

asidloo, Godofi: Vin den Grumaet, lirable en Kreeft. Dolft 1704. 4. C. fig.

Bigut de Morognes. Mèmoire sur un Animal aquatique d'une forme singuliere (Mim. de Matl. et plyys. Vol. XI, p. 145).

Bоunnni, Philipp. Musenm Kirherianum sive Museum a $\mathbf{P}$. Alhanasio Kirehero in Coll. S. J. jum pr. incoepfum (unuseum Kircherianum modierat jau pridem Amstelaedauni 1678 iu Fol. eom figuris. L. Th. Gronovius), muper restitutum, auctum cte. Romae 1749. Fol. - Ricreazione dell Ochio - della mente nell' Osservazione delle Chiocuole. Roma 1681. 4. Cum fig. 540 aen. (Später latein. Rom 1684. 4.

Bontius, Johannes mieht Jacob. wie p. 242). Historiae naturalis et medicae Indiae oricntalis libri VI, in Guil. Pisonis: De Indiae utr. re - niturali et medica libr. XIV. Amstel. 1658. Fol. c. fig. lip. ine.

Eorluce, William. The riatural history of Cornwall. Oxford 1738. Fol. With 28 plat.
Trossclee, Giuilielın van den. Historia medica, in qua libris IV Animalium uatura, et eorum medica utilitas exaete el luculenter tractantur. Bruxellis 1636. 4. C. ieon.

(B) rekumun, Fr. Ern. Verzeichniss der vornelenisten Stioke, welehe: in dem Curiosit. - und Naturalin-Cabinet vin J. Chr. Olearii befindlicls gewesell siud. Jena 1750. 4.

IRerandius, Vineentius. Spreulum qoindinplex, uaturale, doetriuale, ancale, histuriale, in quo totius naturae historia: annium seientiarum lincyclopaedia; mortalis philosophiae thesaurus; temporum el Actionum linmanarum Theatrum amplissimum exhilietur ete. Duaci 1624. Fol. Vol. IV.

Ceralmus, Hieronymus. De rerum varietale libri XV11. Avignoni 1558 . 8. et Lugd. 1580. 8. et Busil. 1664. 4. (Lib. VIl de Animalibus tractans).

Clegleoru,' Genrge. Observations on the Epidemiral Diseases in Minoren. Lond. 1751. 8.

Colueman, Falius. Lymenens Phytobasanus sen plant. histor. ele. Nealp. 1592. 4. c. tab. et Florent. 1744. 4. C. tal. XXXVIII aen.

Costa, Emanuel Mendes da. A Description of at curious fossile Animal. (Geutlen. Magaz. Vol. XXV, 1. 21, f. 3. - Trilobites?).

Cuba, Joh. Ilutus sanitatis, seu libri IV de Auimalibus at Replatilibus, de Avilus et Volatalibus. de: Piscibus et Natutililus itc. Argentor. 1536. Fol. tab. limn.

Cysut, Jolıann Lcopold. Beschuejhung des herulunten Lueerner odpr Vierwaldstitter See's und dessin fiirureflichen Qualiteiten und sumderbaaren Eygenscliaficu etr. heselir. in 1645. Jahr. Luecrune 166t, 4.

Dampicer, William. An account of a new Voyage round the World. Lond. 1697 . 8. IW. 5 plat. und auel 7.11 Hang 1698. 4. (belgisclı. p. 245. Milleped.).

Derleam, William. Plysico-Theology, or a Demonstrntion of the Being atid Atributes of God from his Works of Creation. Lond. 1714. 8. C. t.

Detherding, Georg. Disquisitin physien Verminu iu Norvegil atc. Hafnine 1742. 4.

Denblinfer, Sannelis. Disser- 
tatio de Cancrorum fluviatiliun usu medico, sub praesidio Joh. 11. Schulze. Hilae, Magdeh. 1735. 4.

Dioncorides, Pedacius. De Materia medici. Paris 1549. 8. Joanne Ruellio interpr. libr. VIII. Gr. et latine.

Welwards, Georg (nicht J. wie p. 214).

Francese, Gio Germano. Trattato nlle figure anatnmiehe della piu primeipali animali terrestri. Neapol. 1625. Fol. C. fig.

Trevelre. Ueber Astacus.

Ginefeld. Chemiselıe Analyse der Decktheile der Entomostraciten oder Trilubiten ISeluwrigg. Jahrb. - Isis 1831 , p. 276-278. - Leonlı. und Bromn's neues Jahrb. 1833 , Nr. 6, p. 709).

Fuariuonins, Christoph., vnn Verona. Commintaria in primm lib. rum Arestotelis de Historia Animalium, in quibus unimantium differentiae cons. spieuar sunt, et sedula nd certam divisiun is norman ordinantur. Francof. 1601. I.

Gunttinus, Michaelis Angelıs de. (Et Diunysios de Placenza). Observations of some Animals, and of a strange Plant, made in a Vnyage into the Kingdom of Congro (Plit. Trans. XII, Nr. 139, p. 97\%).

Inrris, John. Some mieroscopical observatiuns of vast numbers of $\Lambda \mathrm{ni}$ malcula seen in water (Plil. Trans. Vol. XIX, Nr. 220, p. 255).

Anrveius, Guilelmus. Exercita. tinnis de generatione animalium: quibus accednut quaedam de Partu, de membranis, et Humoribus, et de Coneeptione. Austelaedami 1651. 12. (versebicdene Ausraben).

Masscelduist, Fred. Iter Palac. stinum : eller Resa til Heliga Landet fürn̈ttad ifriin är 1749 til 1752 , med Beskrifningar, Röı etc. utyifven af Carl Linnacus. Stockl. 1757. 8.

Mebenstreitius. Joh, Eru. Commentaria in Musenm Riehterianum etc. Lips. 1743. Fol. (Text latein.-deutsclı).

Hildegardis, Abbatissa. Hortus sanitatis, IV libris qune subsequuntur compleetens; de Animalibus et Reptilibus, de Avibus et Volantilibus, de
Piscibus et Natatilibus, do Gemmis elc. Argent. 1436. Fol.

Jacobaeus, Oligerus. Musaeum regium, seu Catalog. rer. nat. Hafnia 1696. Fol. C. tab. aen.

Klein, Jac. Theod. Von Schaalthieren, Conclue allatiferac, EntenMuselieln und beiläufig van Pholaden orler Stein-MLuscheln (Act. Gednn. II, p. 349).

Leers, Arn. Catalogue systématique d"un Cabinet de Coquillages et Crustacès (holländ. und frainz.). Amst. 1767. 3.

Leenwenhoeck. Ondervindingen en beschouwingen der onsigtbare geschapene waalheden. Leidae 1684. 4. C. ien. - Vervoler der Brieven. lb. 1688. 4. C. fig. - Vierte Vervolg. Delphis 169.. 4. C. fig. - Vyfile Vervoler. Ib. 1696. 4. C. f. - Areana naturae. Delphis Batav. 1695 et 1697. 4. C. tab. aen, (3. Ausgabe. Leyden 1708. 1.)

Lesser, Fr. Clir. Tesiaceo-Theologia. Leipz. 1744. 8. M. Kpf.

Lonicerus, Adanus. Vollständiges Kräuterhuch, nebst Beschreibang der furmelunsten Thicren, Erden, Vögel etc. Francf. a. M. 1582. Fol. M. Kpf.

Majerus, Michaelis. Traetatus de Volucri alborea absque patre et matre in insulis Oreatum forma Ansereulo. run prnveniente etc. Francof. 1619. 8. (Bernueles.)

Matthiolus, Petrus Andreas. Commentarii in libros VI Dioscoridis de materia medica. Venetiis 1569. Fol. C. fig. (Die gewünliche ist die Ausg.: Basileaı: 1674. Fol. Lib. II.)

Michnelis, Joh. Theoph. De Crustaceo ill 'l'estaceo, seu Cancello intra substantian Conchae margaritif. delitex. (Act. pliys. med. Vol. V, p. 85. Obs. 19).

Moscendi, Ludovicus. Note overo memorie del Musco di Ludovico Moseardo dal Medesimo descritte et in tre libre distinte ete. Padova 1656. Fol. C. tab. (Später Verona 1672. Fol.)

Needhamus, Theodorus. Nouvelles dèconvertes faites par le mieroscope, traduit de l'Anglois etc. Leide 
1747. 8. C. 8 tab. (p. 110 de Conchis anatileris).

Nozemann, Cornelius. Besehryving van de Ganzen-mossel (Concha) anatifera) (Uitgez.om. Verhand. Vol. II, p. 576, Tab. 18).

Robinson, Tancred. Observations on the fiench macreuse, and the seotch Bernacle (Plilos. Truns. Vol. XV, Ni: 172, p. 1036).

Sesins, Petrus Paulus. Catalogus omnium animalium testaecor. ete. Genevae 1046. 4.
Valeuty'n, François. Beschryvink van Oud en Nicuw Oost-Indien. Amst. 1714-26. Fol, V Tom. C. tab. aen. (Crustacea).

Wagner, Joh. Jac. (nicht Petr. Christ. wie p. 260 des I.ex.).

Thanniclnelli, Johannes .Jacobus. Euumeratio rer. uatur., quae in museo Zannich. asserv. Venet.1736. 4. (Crust., pisc(es etc.)

Zorgdrager, C. G. Bloeyende opkumst der aloude en liedendaingsclie groenlandselse Visscherye ete. Amsteld. 1720. 4. C. tab, aen. 


\section{Inhaltsverzeichniss.}

Vorwort . . . . .

1. Adressenbuch der Entomologen Nachiträge dazu

Akademien und gelelurte Gesellschuften und deren Schriften

Ephemeriden . . . . .

Bibliographien und Literuturgeschichten . . . . . . 100

Biographien etc. . . . . . 108

Realwörterbücher . . . . . 113

Uebersicht der öffentlichen $\mathrm{Mu}$ spen und Privatsamulungen

Syllogistische Srhritiell etc. .

Ortstafel der Entomologen der Gegenwart (Register der Wohnorte

Anlang. Anfziblung aller entomologischen Schriftsteller.

11. Adressenbuch derCarcinologen Dedication und Vorrort ? Lexikon der Carcinologen. Ortstafel der Carcinologen .

Seite
5
11
77
82
93
100
108
113
116
145

146
155
195
197
218
237

Seite

Die carcinologiselien Schriftsteller des Alterthums, des Mittelalters und der nevesten Zeit ; nebst der vollständigen Angabe ilırer Werke. . Carcinologische und entomolngischeSammlungen. (Zugleidı Supplement zu der Uebersicht im Lex. d. Ent.) . . . .

Akudemien und gelehrte Gesell. schaften (Eigänzungstafel z.u der Uebers. in L.ex. d. Ent.

11). Adressenluch der A rachnolo. gen; Dedication und Prolegomenon . . . . . . 279

Jexiknn der Arachnologen . . 291

Wohnorte der Arachuolugen • 299

'Tafel der arachnolog. Schriftsteller und d, arachu. I,iteratu $\mathbf{r}$ Supplemente zu den Lexicis der Entomologen und Carcinologen . . . . . . 


\section{Verbesserungen.}

(Sonaltgo Belgaben, Irrthifmer, Sterbefälle, Wegfallendes n. .. W. Ieso uran gefilligat im Anhang $z$ un Lexikon der Phy $60 z 00$ ogen nach, worin lïr Berlchigung all mögliclier Fleiss ungewendes worden lst.)

Deluxtur p. 14, lin. 6 et 3. - Del. \%. 1.5, lin. 29. - Ad p. 16. lin. 19 setse: lkls 18.35, 412. - Zu Clievrolat selze: Zollbeanteler. - Del p. 21, IIn. 40 von obeu. p. 23, lin. ? setze Heidelberg at. B. - p. 25. lia. 5 v. n. setze: T. XII, PI. I, 2, I56, PI. 7. - I1. 29 zu Fischer P. R. getze Wlen (Vorsladt Hundsthurm, Johainamasse Nr. 154), - H, zul Färster: 4. H., IS., 47 Taf. (Isis 1841, 314). Bei forel setze: Vgl. Bugnioll. - p. 30. lin. 9. 1. - p. 34, setsc b. Gotdf.: Handbuch d. Zool. - p. 39, del. liti. 10 und 28. - p. 40. III. heiss. claleri. dous. - ib. HI. 12 v u, del. - Fr. Jacquin t. - p. Al (Kнip) setze Errallgen. - p. 49. Macy. III Lille. - p. 55, Nodier t. - p. 58 (Perty) setze s. 3941 (Isis t841, 369-379). p. 60 Rensl, del. - p. 61 sonens. vgl, p. 54. - Roux del. - p. 62 Salılb. zu Helsingtors. p. 6.3 Savlginy t. - p.65 Scliweigger del. - p. 68 Stannills, mull in Rostork. - p 70 Suckow zu M. ist t. - p. 71 Thon t. - p. 102, Jin. 17 v. 11. setze: 1826-17, 111. - p. I09, lin, Iti v. n. Percunes, - p. 217 , lin. 20 v. o. st. diene jene. - ib. del. lin. $21-29$ Incl. - p. 227 fehlt: King, Plitipp. Cpl, in London. - Narralive of a survey of the elo. Coaston Australmin. I. Lond. 1827, p. 69 (Cirripeden).

\section{Zur Naclnricint.}

Die privilegirte Naturalieulandlang zu Münclıen ladet sämutliche Naturfurscher ciu, mit ihr in Verbiudung z.u treten. Der Zweek iluer Bestrehungen ist in sofern ein vorthuilhringender, als dic Handlum (zoufolge äfentlicler Ausschreibang) sowohl Naturalieut aller Reiche als cinsiblägige Werke in Cotinmission, Kanf und Tauscli alluimmt und prompt hulb- and ganzJälorige Abrechumug dieröber slellt. Sic giht selbst anf Credit vou $\frac{1}{2}-1$ Jalıt Böcher und Faturialien. Das mit der Handlung in Verbindome steluvide

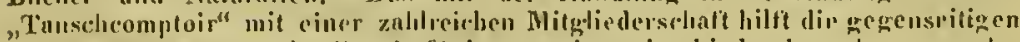
Iuleressel un so schueller hefürlern und es ist hieduch und wegen der grosisen Vorräthe Handlung und 'Tansehinslitul in vollkummenem Stande, allen Wünsthen entgegen ou kummen. Sie besorgt Speditionen und madit in Anf-

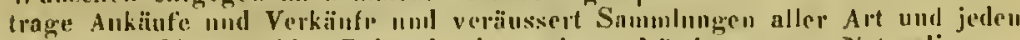
Unfangs. Sie besoldet Reisende in meluren Liinderu, III Naturalien жu sammeln. Man wenlet sir.l in portofreien Briefen nath Mïnchete, an besten direct an den Redactenl dieses Lexicons (Theresimustrasse $\mathrm{Nr}$. 20, l, im Hause. des Grafen Kuhn oder (in Sommer) Latudgut Geisenbrunt ant Parsberc, wohin auch Beiträge z.ım Lexikon zı senden sind), welehet mit Vergü̈ren Auftrïge ïbroninmt. Derselbe crucht bei dieser Gelegenheit alle Herren Antoren, ilue Schriften au ilın za sendın, wenn ihneut daran liegt, andere daför cinzutauschen, oder dieselben in hayrischen Blätert bekunt gemacht und beurtheilt \%u selien. 



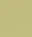


ser.tis

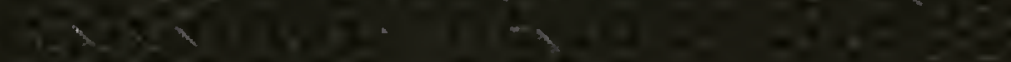

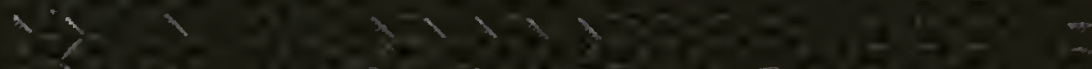

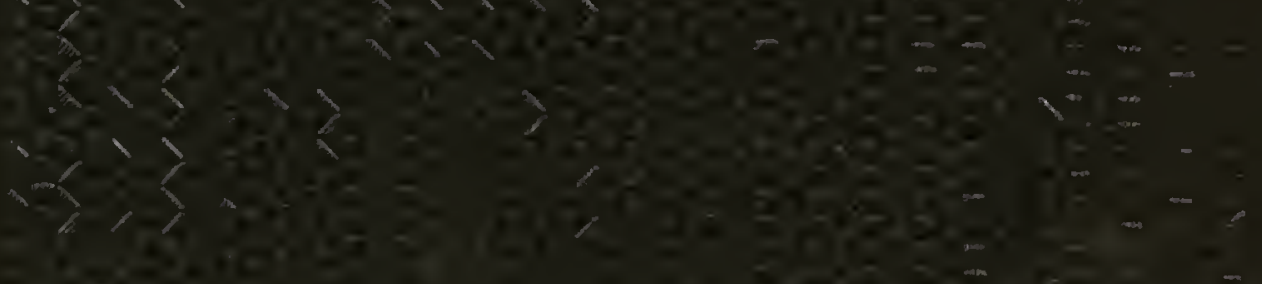
$\rightarrow>>>>$

$x+\frac{1}{2}$

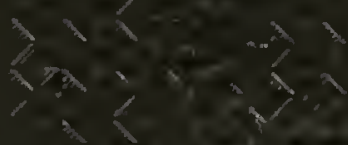

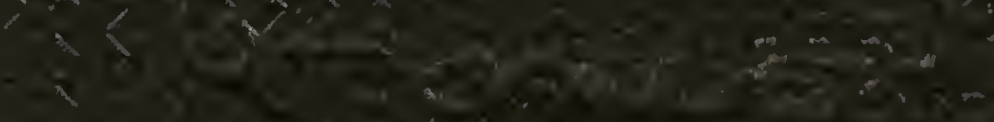

$v^{2}>0+2>3$

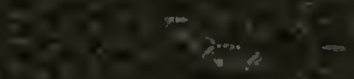

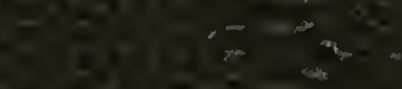

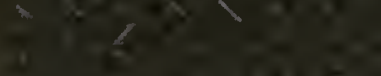

